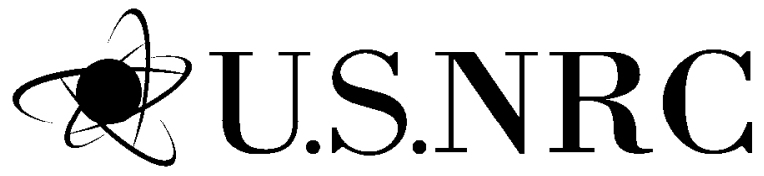

United States Nuclear Regulatory Commission

Protecting People and the Environment

\title{
Evaluations of Structural Failure Probabilities and Candidate Inservice Inspection Programs
}



United States Nuclear Regulatory Commission

$\overline{\text { Protecting People and the Environment }}$

\section{Evaluations of Structural \\ Failure Probabilities and \\ Candidate Inservice Inspection Programs}

Manuscript Completed: November 2008

Date Published: May 2009

Prepared by

M.A. Khaleel and F.A. Simonen

Pacific Northwest National Laboratory

P.O. Box 999

Richland, WA 99352

D.A. Jackson and W.E. Norris, NRC Project Managers

NRC Job Code N6398 


\begin{abstract}
The work described in this report applies probabilistic structural mechanics models to predict the reliability of nuclear pressure boundary components. These same models are then applied to evaluate the effectiveness of alternative programs for inservice inspection to reduce these failure probabilities. Results of the calculations would support the development and implementation of risk-informed inservice inspection of piping and vessels. Studies have specifically addressed the potential benefits of ultrasonic inspections to reduce failure probabilities associated with fatigue crack growth and stress-corrosion cracking. Parametric calculations were performed with the computer code pc-PRAISE to generate an extensive set of plots to cover a wide range of pipe-wall thicknesses, cyclic operating stresses, and inspection strategies. The studies have also addressed critical inputs to fracture mechanics calculations such as the parameters that characterize the number and sizes of fabrication flaws in piping welds. Other calculations quantify uncertainties associated with the inputs to the calculations, the uncertainties in the fracture mechanics models, and the uncertainties in the resulting calculated failure probabilities. A final set of calculations address the effects of flaw-sizing errors on the effectiveness of inservice inspection programs.
\end{abstract}




\section{Foreword}

The goal of inservice inspection (ISI) of nuclear reactor piping and pressure vessels is to reliably detect service- related defects in a timely manner and thereby maintain the structural integrity of the inspected components. As nuclear power plants have aged and instances of unexpected materials degradation have been reported, a goal of the nuclear industry has increasingly been to predict component degradation before it occurs. In addition to assessing potential degradation mechanisms and identifying components and materials that are expected to experience degradation, a proactive approach must consider inspection. The U.S. Nuclear Regulatory Commission (NRC), in an effort to assess the effectiveness of ISI programs, has supported research at Pacific Northwest National Laboratory (PNNL) to evaluate the reliability and accuracy of ISI, and recommend improvements to nondestructive examination (NDE) methods and requirements. The results from another program at PNNL, a study to collect estimates of failure probability and their associated uncertainties for passive reactor components, were published in May 2007 in NUREG/CR-6936, entitled "Probabilities of Failure and Uncertainty Estimate Information for Passive Components - A Literature Review."

Probabilistic fracture mechanics (PFM) models have been used for reliability analyses. Performance demonstrations have been implemented to upgrade the quality of ultrasonic testing (UT) by addressing the key elements needed for effective inspections - namely, personnel, procedures, and equipment. This report presents the results of a study to (1) apply PFM models to predict component failure probabilities by modes ranging from leaks to rupture, and (2) evaluate the potential effectiveness of ISI to reduce such failure probabilities. A PFM model was used to simulate the effects of flaw detection and sizing errors that may occur during vessel and piping inspections. That model was then used to perform calculations for a range of representative values of flaw detection probabilities, flaw sizing errors, and flaw acceptance criteria.

The candidate inspection programs considered three different levels of NDE reliability and different inspection frequencies. A collection of curves was generated that describes the effects of inservice inspections on piping reliability. The curves can be used to identify optimum inspection strategies for specified conditions of cyclic stresses. The calculations show that high-quality inservice inspections can significantly reduce leak and break probabilities, particularly if the inspections are performed relatively frequently. It was also shown that preservice inspections can be effective in reducing leak probabilities and failures. Probability of detection (POD) capability appears to be the most limiting factor with regard to the overall capability of ISI to reduce leak probabilities.

Results from the research have been used to support development of NRC guidance for implementation of risk-informed ISI of piping. The results have also been used in the development of PFM tools to generate failure probabilities for vessels that has been used in regulatory decision making. 


\section{Contents}

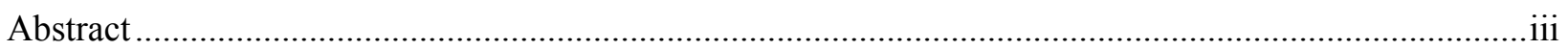

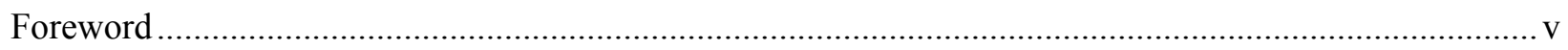

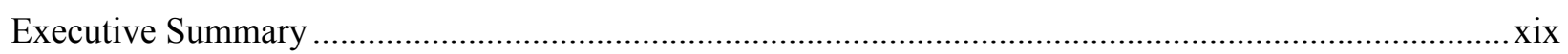

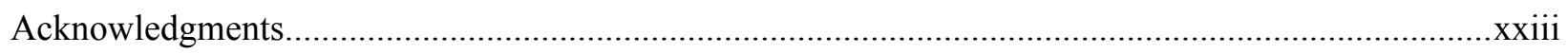

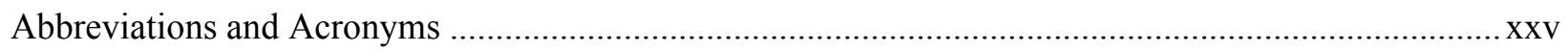

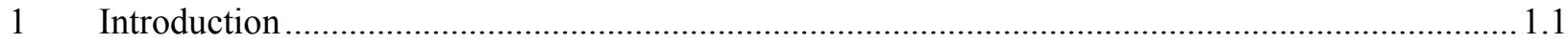

2 Flaw-Size Distribution and Flaw-Existence Frequencies in Nuclear Piping ...............................2.1

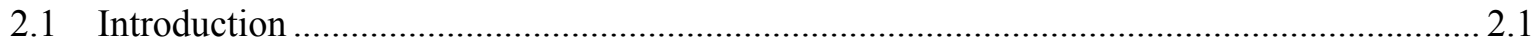

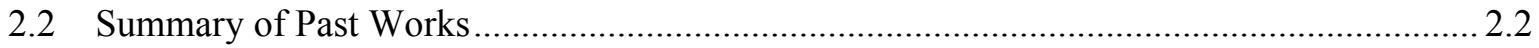

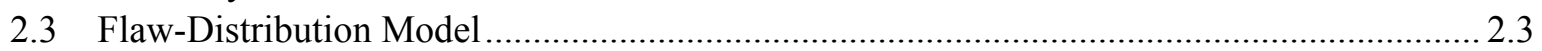

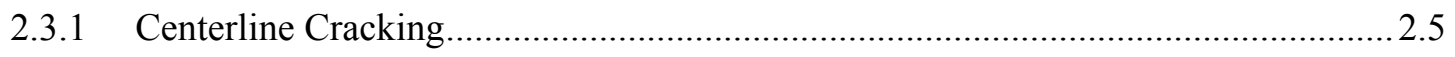

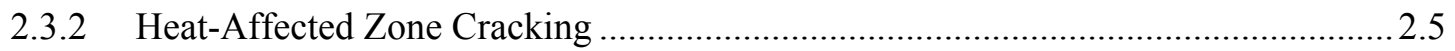

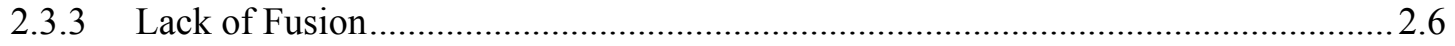

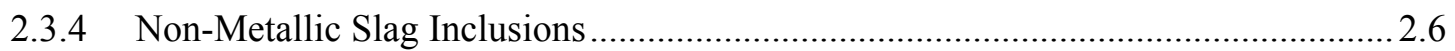

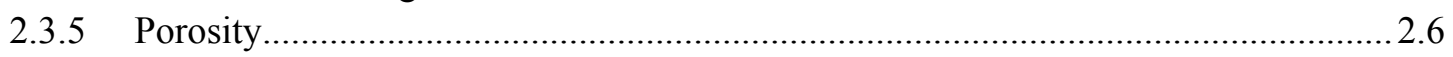

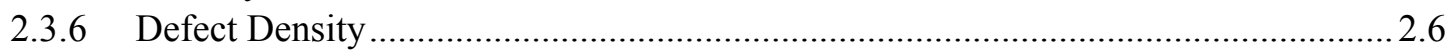

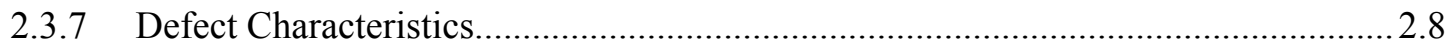

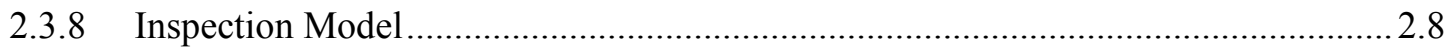

2.4 Computer-Based Implementation................................................................................ 2.9

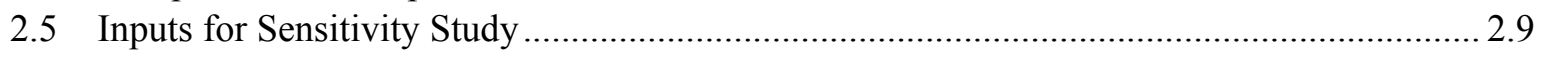

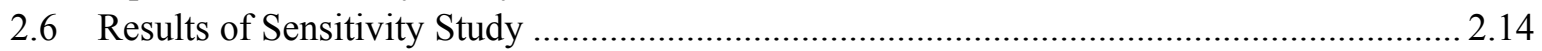

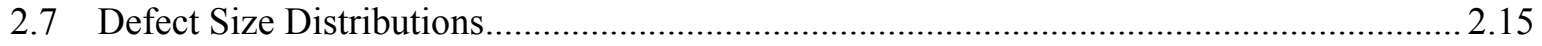

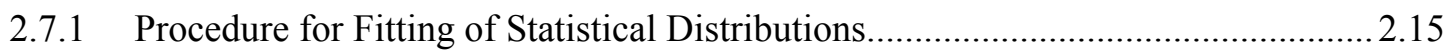

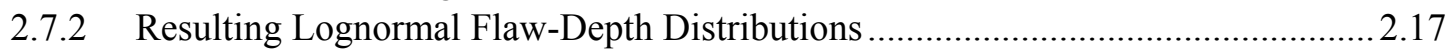

2.7.3 Effects of Weld Process on Depth Distribution ..................................................2.17

2.8 Flaw Depth Distribution Parameters versus Wall Thickness …......................................... 2.24

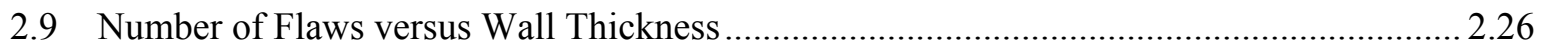

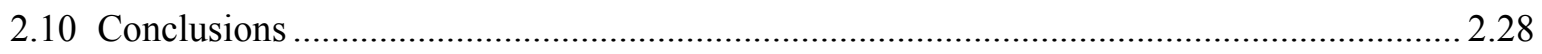

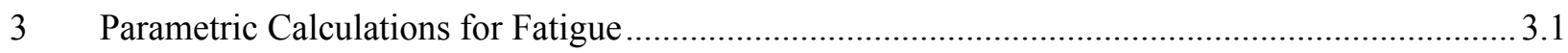

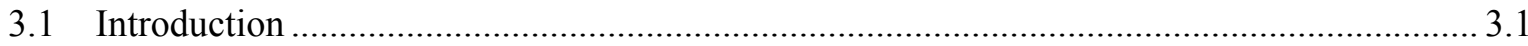

3.2 Piping Reliability Code pc-PRAISE ...................................................................... 3.1

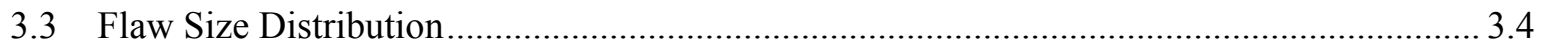

3.4 Parametric Treatment of Cyclic Stresses Using the Q-Factor ........................................... 3.5

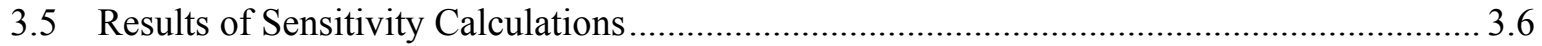

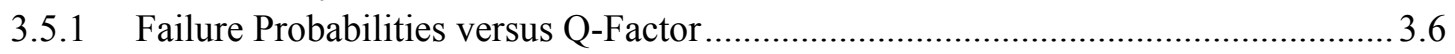

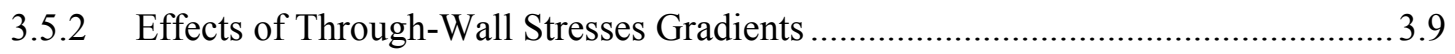

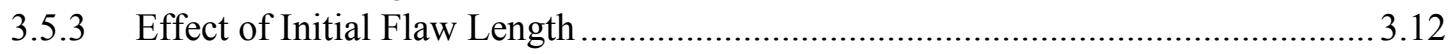

3.5.4 Effect of Sustained Primary Stress Level on Failure Probabilities ........................... 3.14

3.5.5 Effect of Abnormal/Seismic Stress Level on Failure Probabilities .............................. 3.14

3.5.6 Effect of Leak Detection on Failure Probabilities ..................................................... 3.17 


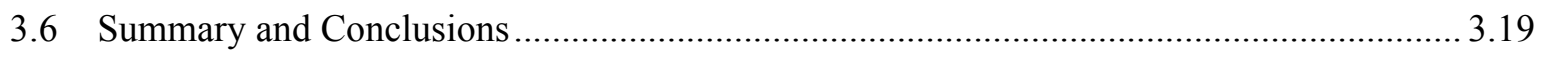

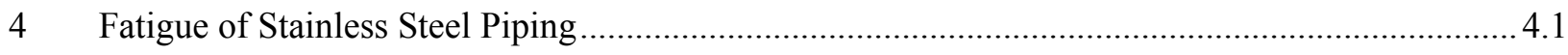

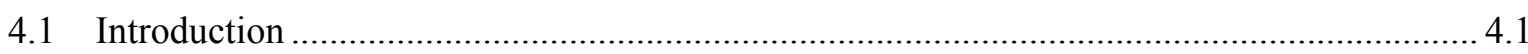

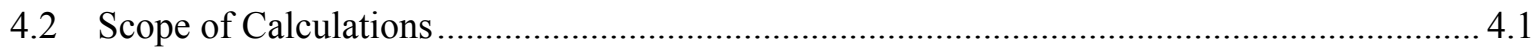

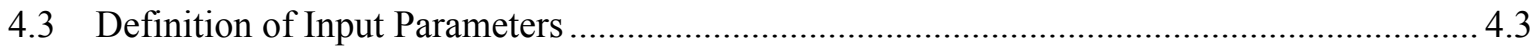

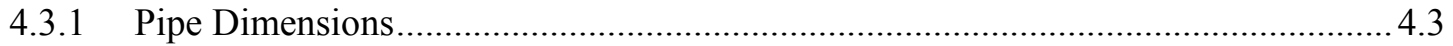

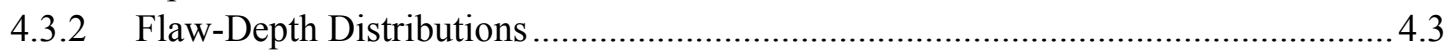

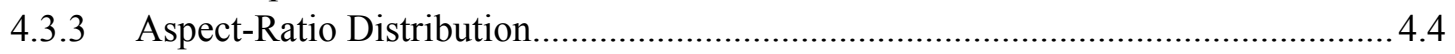

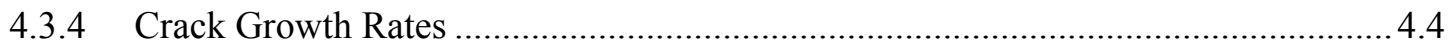

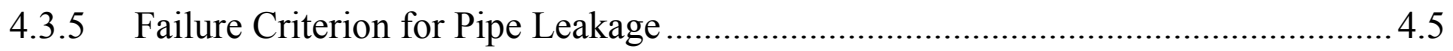

4.3.6 Failure Criterion for Pipe Break ................................................................................... 4.5

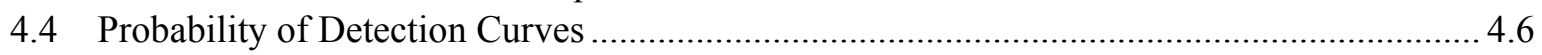

4.5 Sensitivity Calculations for Inspection Model ............................................................... 4.7

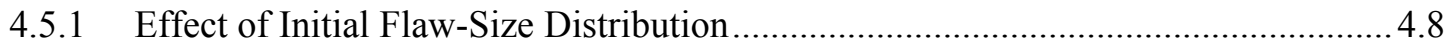

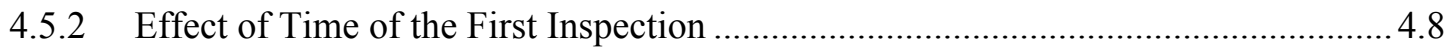

4.5.3 Independent versus Correlated Inspections .................................................. 4.9

4.5.4 Combined Preservice Inspections and Inservice Inspections ............................... 4.10

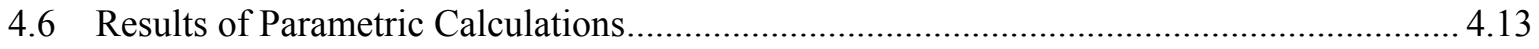

4.6.1 Effects of Pipe Wall Thickness on Failure Probabilities......................................... 4.13

4.6.2 Effects of Pipe-Wall Thickness on Improvement Factor ..........................................4.16

4.6.3 Improvement Factors for Various Pipe-Wall Thicknesses .................................... 4.18

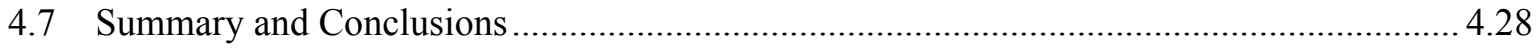

$5 \quad$ Intergranular Stress Corrosion of Stainless Steel Piping........................................................ 5.1

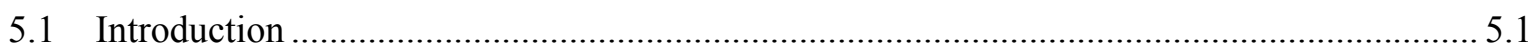

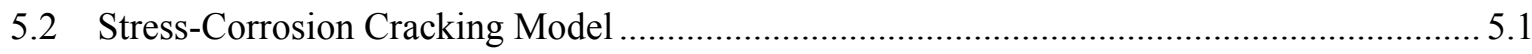

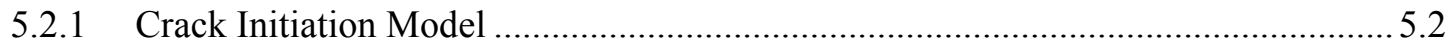

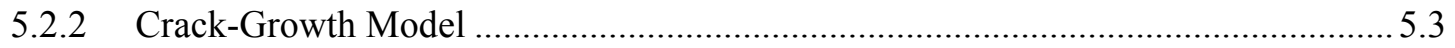

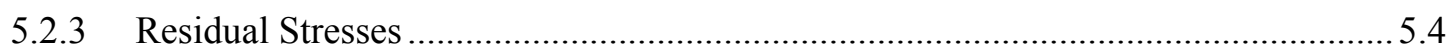

5.2.4 Failure Criterion for Pipe Breakage and Leakage ................................................. 5.4

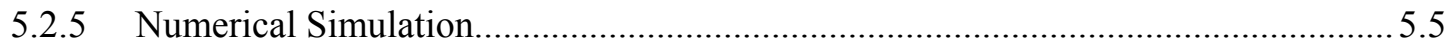

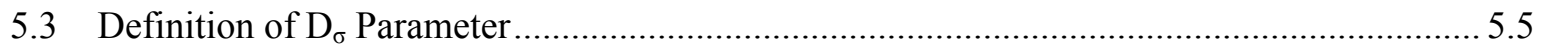

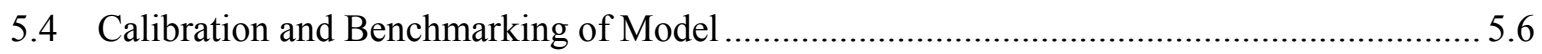

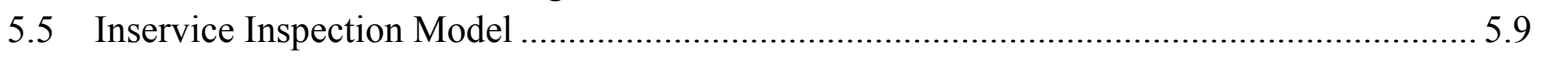

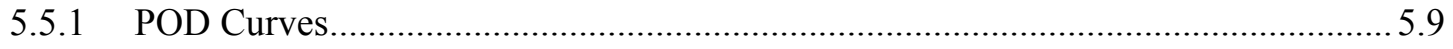

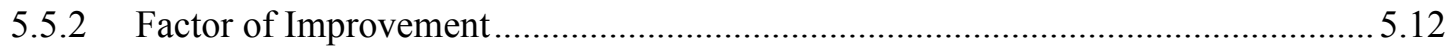

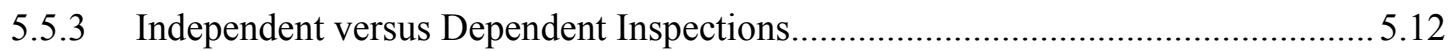

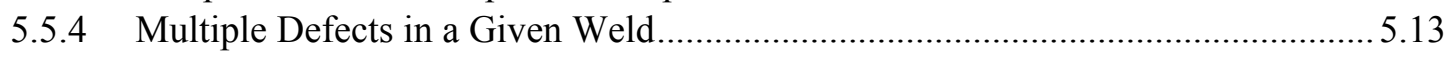

5.6 Input Parameters for Parametric Calculations ................................................................. 5.13

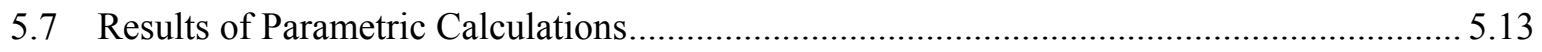

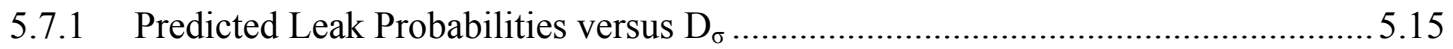

5.7.2 Effect of Pipe Size on Leak Probabilities................................................................. 5.15

5.7.3 Effect of POD Curve and ISI Frequency on Improvement Factor ......................... 5.15

5.7.4 Improvement Factors for Small Pipe Size............................................................... 5.18

5.7.5 Improvement Factors for Intermediate Pipe Size ................................................5.18

5.7.6 Improvement Factors for Large Pipe Size ............................................................. 5.19 
5.7.7 Effect of Pipe Size on Improvement Factor ......................................................... 5.19

5.7.8 Time Dependence of Calculated Failure Probabilities ...........................................5.19

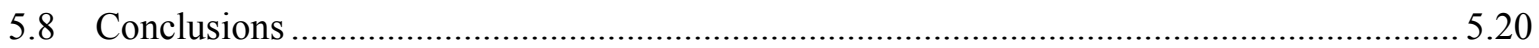

$6 \quad$ Uncertainty Analysis for Calculated Failure Probabilities of Piping Welds .................................. 6.1

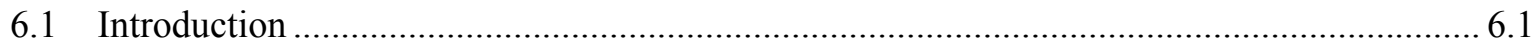

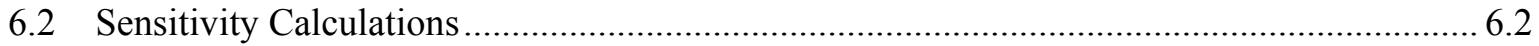

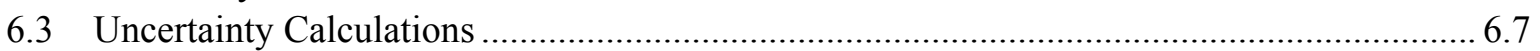

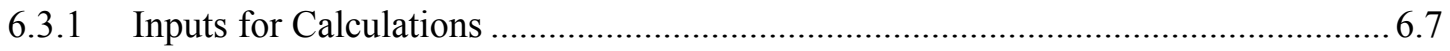

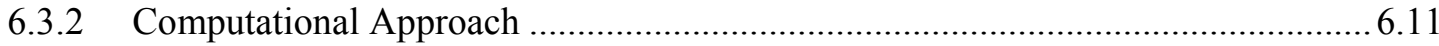

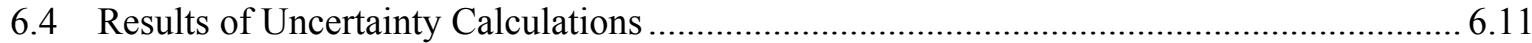

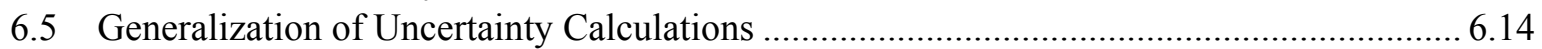

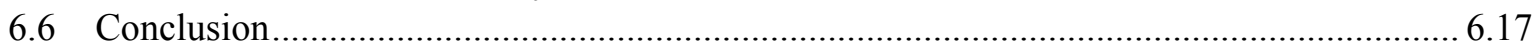

7 Effects of Flaw-Sizing Errors on the Reliability of Vessels and Piping ........................................ 7.1

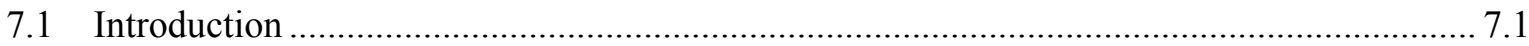

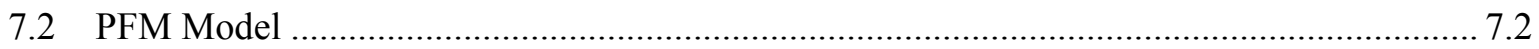

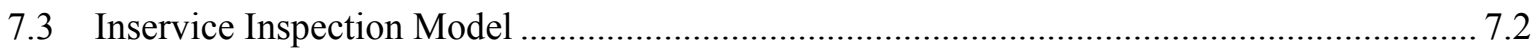

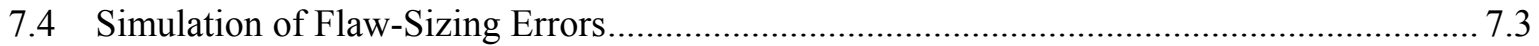

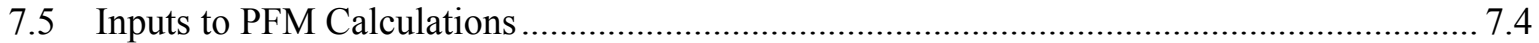

7.6 Results of Example Calculations......................................................................................... 7.6

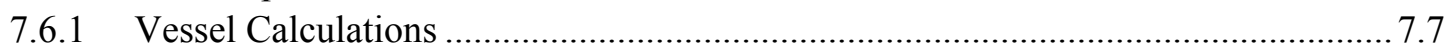

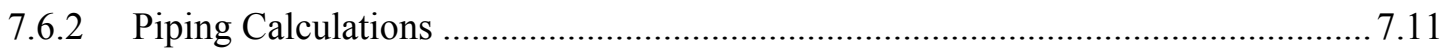

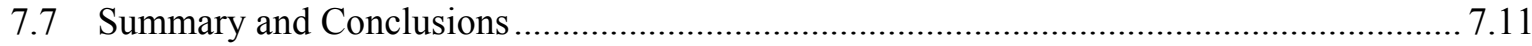

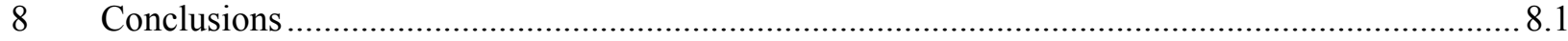

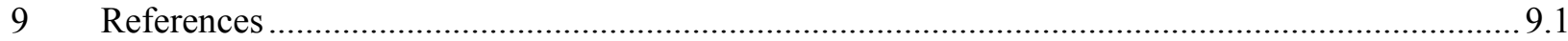




\section{Figures}

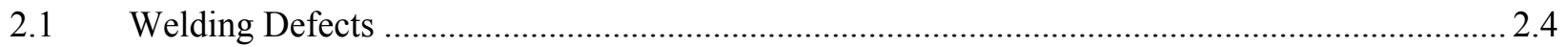

2.2 Schematic Representation of Weld Build Up and the Position of Different Types of

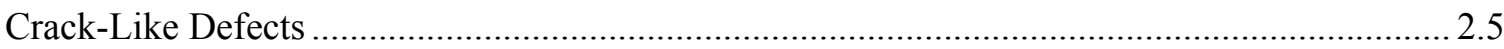

2.3 Complimentary Distribution of Flaw Depth on Normal Probability Paper for Case 1 ............. 2.16

2.4 Lognormal Complimentary Distribution of Flaw Depth for Case 1 ....................................... 2.18

2.5 Lognormal Complimentary Distribution of Flaw Depth for Case 2 ..................................... 2.18

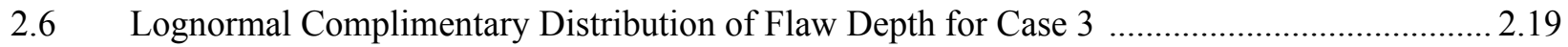

2.7 Lognormal Complimentary Distribution of Flaw Depth for Case 6 .......................................2.19

2.8 Lognormal Complimentary Distribution of Flaw Depth for Case 7 ......................................2.20

2.9 Lognormal Complimentary Distribution of Flaw Depth for Case 10 ..................................... 2.20

2.10 Comparison of Simulated Flaw Depth Distributions for 1-in. Wall Pipe Welds ..................... 2.22

2.11 Comparison of Simulated Flaw Depth Distributions for Three Pipe Wall Thicknesses ........... 2.23

2.12 Comparison of Simulated Flaw Depth Distributions for Three Pipe Wall Thicknesses

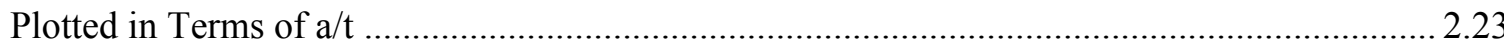

2.13 Median Flaw Size and Shape Parameter for MMAW ......................................................... 2.24

2.14 Median Flaw Size and Shape Parameters for TIG Weld .................................................... 2.25

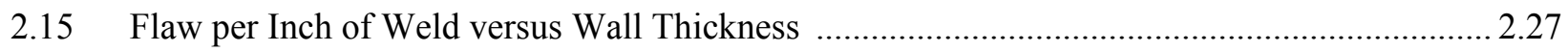

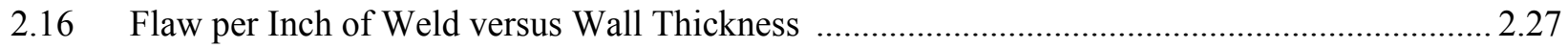

3.1 Schematic Diagram of Piping Failure Probability Calculations as Performed by pc

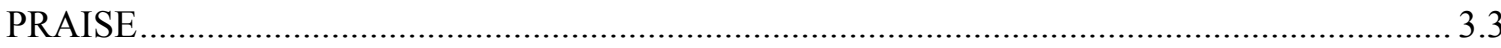

3.2 Probability of Leak as a Function of Crack Growth Parameter $\mathrm{Q}=\alpha \mathrm{N}(\Delta \sigma)^{4}$ for Two

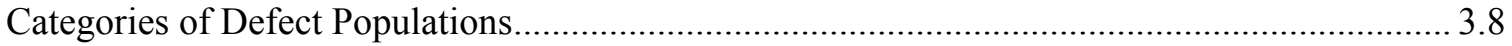

3.3 Probability of Break as a Function of the Leak Probability ...................................................... 3.9

3.4 Probability of Leak as a Function of Fatigue Crack Growth Parameter $\mathrm{Q}=\alpha \mathrm{N}(\Delta \sigma)^{4} \ldots \ldots \ldots \ldots . . .10$

3.5 Probability of Leak as a Function of Fatigue Crack Growth Parameter $\mathrm{Q}=\alpha \mathrm{N}(\Delta \sigma)^{4} \ldots \ldots \ldots \ldots . .10$

3.6 Probability of Leak as a Function of Fatigue Crack Growth Parameter $\mathrm{Q}=\alpha \mathrm{N}(\Delta \sigma)^{4} \ldots \ldots \ldots \ldots . . .11$

3.7 Probability of Leak as a Function of Fatigue Crack Growth Parameter $\mathrm{Q}=\alpha \mathrm{N}(\Delta \sigma)^{4} \ldots \ldots \ldots \ldots . . .11$

3.8 The Effect of Sustained Primary Stress on Leak Probability with Crack Growth Parameter $=\alpha \mathrm{N}(\Delta \sigma)^{4}$.

3.9 The Effect of Crack Growth Parameter $=\alpha \mathrm{N}(\Delta \sigma)^{4}$ and the Abnormal Stress on the Leak Probability 
3.10 Comparison Between the Peak Probability for a Sustained Primary Stress of 24 ksi and Abnormal Plus Primary Stress of 24 ksi with Crack Growth Parameter $=\alpha N(\Delta \sigma)^{4}$

3.11 Effect of Leak Detection Threshold on Leak and Break Probabilities 3.18

4.1 The Effect of Initial Flaw Distribution on the Improvement Factors with $Q=\alpha N(\Delta \sigma)^{4}$.

4.2 The Effect of Arrival Time of First Inservice Inspection on the Leak Probability of Failure

4.3 Improvement Factors for Independent and Correlated Inspections versus $Q=\alpha N(\Delta \sigma)^{4}$ for ISI Every 10 Years.

4.4 Improvement Factors for Independent and Correlated Inspections versus $Q=\alpha N(\Delta \sigma)^{4}$ for ISI Every 5 Years

4.5 Improvement Factors for Independent and Correlated Inspections versus $\mathrm{Q}=\alpha \mathrm{N}(\Delta \sigma)^{4}$ for ISI Every 2 Years

4.6 Improvement Factors for Independent and Correlated Inspections versus $Q=\alpha N(\Delta \sigma)^{4}$ for ISI Every Year.

4.7 Effect of Combined Preservice and Inservice Inspection on Improvement Factors $\mathrm{Q}=\alpha \mathrm{N}(\Delta \sigma)^{4}$.

4.8 Effect of Wall Thickness on Calculated Leak Probabilities for Stainless Steel Piping Assuming One Flaw per Weld and No Inservice Inspection with $\mathrm{Q}=\alpha \mathrm{N}(\Delta \sigma)^{4}$

4.9 Effect of Wall Thickness on Calculated Break Probabilities for Fatigue of Stainless Steel Piping Assuming One Flaw per Weld and No Inservice Inspection with Q = $\alpha \mathrm{N}(\Delta \sigma)^{4}$

4.10 Effect of Wall Thickness on Calculated Leak Probabilities for Stainless Steel Piping Assuming Number of Flaws per Weld from Flaw Simulation Model and No Inservice Inspection with $\mathrm{Q}=\alpha \mathrm{N}(\Delta \sigma)^{4}$

4.11 Effect of Wall Thickness on Calculated Break Probabilities for Fatigue of Stainless Steel Piping Assuming Number of Flaws per Weld from Flaw Simulation Model and No Inservice Inspection with $\mathrm{Q}=\alpha \mathrm{N}(\Delta \sigma)^{4}$.

4.12 Effect of Wall Thickness on Improvement Factor for Stainless Steel Fatigue Using Inservice Inspection with "Very Good" Probability of Detection Curve and Periodic Inspections Performed at 10 -Year Intervals with $\mathrm{Q}=\alpha \mathrm{N}(\Delta \sigma)^{4}$

4.13 Effect of Wall Thickness on Improvement Factor for Stainless Steel Fatigue Using Inservice Inspection with "Very Good" Probability of Detection Curve and Periodic Inspections Performed at 2-Year Intervals with $\mathrm{Q}=\alpha \mathrm{N}(\Delta \sigma)^{4}$

4.14a Improvement Factor for Fatigue of 0.25-in. Wall Stainless Steel Piping Using Inservice Inspection with "Marginal" Probability of Detection Curve and Periodic Inspections Performed at Various Intervals with $\mathrm{Q}=\alpha \mathrm{N}(\Delta \sigma)^{4}$.

4.14b Improvement Factor for Fatigue of 0.25-in. Wall Stainless Steel Piping Using Inservice Inspection with "Very Good" Probability of Detection Curve and Periodic Inspections Performed at Various Intervals with $\mathrm{Q}=\alpha \mathrm{N}(\Delta \sigma)^{4}$ 
4.14c Improvement Factor for Fatigue of 0.25-in. Wall Stainless Steel Piping Using Inservice Inspection with "Outstanding" Probability of Detection Curve and Periodic Inspections Performed at Various Intervals with $\mathrm{Q}=\alpha \mathrm{N}(\Delta \sigma)^{4}$

4.15a Improvement Factor for Fatigue of 0.562-in. Wall Stainless Steel Piping Using Inservice Inspection with "Marginal" Probability of Detection Curve and Periodic Inspections Performed at Various Intervals with $\mathrm{Q}=\alpha \mathrm{N}(\Delta \sigma)^{4}$.

4.15b Improvement Factor for Fatigue of 0.562-in. Wall Stainless Steel Piping Using Inservice Inspection with "Very Good" Probability of Detection Curve and Periodic Inspections Performed at Various Intervals with $\mathrm{Q}=\alpha \mathrm{N}(\Delta \sigma)^{4}$

4.15c Improvement Factor for Fatigue of 0.562-in. Wall Stainless Steel Piping Using Inservice Inspection with "Outstanding" Probability of Detection Curve and Periodic Inspections Performed at Various Intervals with $\mathrm{Q}=\alpha \mathrm{N}(\Delta \sigma)^{4}$

4.16a Improvement Factor for Fatigue of 1.0-in. Wall Stainless Steel Piping Using Inservice Inspection with "Marginal" Probability of Detection Curve and Periodic Inspections Performed at Various Intervals with $\mathrm{Q}=\alpha \mathrm{N}(\Delta \sigma)^{4}$

4.16b Improvement Factor for Fatigue of 1.0-in. Wall Stainless Steel Piping Using Inservice Inspection with "Very Good" Probability of Detection Curve and Periodic Inspections Performed at Various Intervals with $\mathrm{Q}=\alpha \mathrm{N}(\Delta \sigma)^{4}$

4.16c Improvement Factor for Fatigue of 1.0-in. Wall Stainless Steel Piping Using Inservice Inspection with "Outstanding" Probability of Detection Curve and Periodic Inspections Performed at Various Intervals with $\mathrm{Q}=\alpha \mathrm{N}(\Delta \sigma)^{4}$

4.17a Improvement Factor for Fatigue of 2.5-in. Wall Stainless Steel Piping Using Inservice Inspection with "Marginal" Probability of Detection Curve and Periodic Inspections Performed at Various Intervals with $\mathrm{Q}=\alpha \mathrm{N}(\Delta \sigma)^{4}$

4.17b Improvement Factor for Fatigue of 2.5-in. Wall Stainless Steel Piping Using Inservice Inspection with "Very Good" Probability of Detection Curve and Periodic Inspections Performed at Various Intervals with $\mathrm{Q}=\alpha \mathrm{N}(\Delta \sigma)^{4}$

4.17c Improvement Factor for Fatigue of 2.5-in. Wall Stainless Steel Piping Using Inservice Inspection with "Outstanding" Probability of Detection Curve and Periodic Inspections Performed at Various Intervals with $\mathrm{Q}=\alpha \mathrm{N}(\Delta \sigma)^{4}$

4.18a Improvement Factor for Fatigue of 1.0-in. Wall Stainless Steel Piping Using Inservice Inspection with Various Probability of Detection Curves and Periodic Inspections Performed at 10-Year Intervals with $\mathrm{Q}=\alpha \mathrm{N}(\Delta \sigma)^{4}$.

4.18b Improvement Factor for Fatigue of 1.0-in. Wall Stainless Steel Piping Using Inservice Inspection with Various Probability of Detection Curves and Periodic Inspections Performed at 4-Year Intervals with $\mathrm{Q}=\alpha \mathrm{N}(\Delta \sigma)^{4}$

4.18c Improvement Factor for Fatigue of 1.0-in. Wall Stainless Steel Piping Using Inservice Inspection with Various Probability of Detection Curves and Periodic Inspections Performed at 2-Year Intervals with $\mathrm{Q}=\alpha \mathrm{N}(\Delta \sigma)^{4}$ 
4.18d Improvement Factor for Fatigue of 1.0-in. Wall Stainless Steel Piping Using Inservice Inspection with Various Probability of Detection Curves and Periodic Inspections Performed at 1-Year Intervals with $\mathrm{Q}=\alpha \mathrm{N}(\Delta \sigma)^{4}$

5.1 Field Observations of Leak Probabilities Compared with pc-PRAISE Results for Various Values of the Residual Stress Adjustment Factors and Plant Cycles (Small Diameter Pipes)

5.2 Field Observations of Leak Probabilities Compared with pc-PRAISE Results for Various Values of the Residual Stress Adjustment Factors and Plant Cycles (Intermediate Diameter Pipes)

5.3 Field Observations of Leak Probabilities Compared with pc-PRAISE Results for Various Values of the Residual Stress Adjustment Factors and Plant Cycles (Large Diameter Pipes). 5.8

5.4 Predicted Leak Probabilities for Various Values of Yearly Plant Transients for Intermediate Diameter Pipes

5.5 Probability of Detection Curves for Intergranular Stress-Corrosion Cracking

5.6 Cumulative Leak Probability Over 40 Years as a Function of the Stress-Corrosion Damage Parameter; $\mathrm{D}_{\sigma}$ for Various Temperatures and Oxygen Contents

5.7 The Effect of Various Degrees of Sensitization on the Relationship Between the 40-Year Cumulative Leak Probability and $\mathrm{D}_{\sigma}$

5.8 Cumulative Leak Probability over 40 Years for Three Pipe Sizes as a Function of the Damage Parameter $\mathrm{D}_{\sigma}$

5.9 Cumulative Leak Probability as a Function of Time for Three Pipe Sizes and Comparable Values of the Damage Parameter $D_{\sigma}$

5.10a Improvement Factors versus $D_{\sigma}$ for "Marginal” POD Curve ................................................ 5.21

5.10b Improvement Factors versus Leak Probability for "Marginal" POD Curve ............................5.21

5.11a Improvement Factors versus $\mathrm{D}_{\sigma}$ for "Good” POD Curve …................................................... 5.22

5.11b Improvement Factors versus Leak Probability for "Good" POD Curve ................................... 5.22

5.12a Improvement Factors versus $D_{\sigma}$ for "Very Good" POD Curve ................................................. 5.23

5.12b Improvement Factors versus Leak Probability for "Very Good" POD Curve ........................... 5.23

5.13a Improvement Factors versus $D_{\sigma}$ for “Outstanding” POD Curve .............................................. 5.24

5.13b Improvement Factors versus Leak Probability for "Outstanding” POD Curve ......................... 5.24

5.14a Improvement Factors versus $D_{\sigma}$ for 10-Year ISI Interval ....................................................25

5.14b Improvement Factors versus Leak Probability for 10-Year ISI Interval .................................. 5.25

5.15a Improvement Factors versus $D_{\sigma}$ for 4 -Year ISI Interval ........................................................ 5.26

5.15b Improvement Factors versus Leak Probability for 4-Year ISI Interval ................................... 5.26

5.16a Improvement Factors versus $\mathrm{D}_{\sigma}$ for 2-Year ISI Interval ....................................................... 5.27 
5.16b Improvement Factors versus Leak Probability for 2-Year ISI Interval ................................... 5.27

5.17a Improvement Factors versus $\mathrm{D}_{\sigma}$ for 1 -Year ISI Interval .................................................... 5.28

5.17b Improvement Factors versus Leak Probability for 1-Year ISI Interval .................................. 5.28

5.18a Improvement Factors versus $D_{\sigma}$ for "Marginal" POD Curve ................................................. 5.29

5.18b Improvement Factors versus Leak Probability for "Marginal" POD Curve ............................. 5.29

5.19a Improvement Factors versus $D_{\sigma}$ for “Good” POD Curve ….................................................... 5.30

5.19b Improvement Factors versus Leak Probability for "Good" POD Curve.................................... 5.30

5.20a Improvement Factors versus $\mathrm{D}_{\sigma}$ for "Very Good" POD Curve …........................................... 5.31

5.20b Improvement Factors versus Leak Probability "Very Good" POD Curve ............................... 5.31

5.21a Improvement Factors versus $\mathrm{D}_{\sigma}$ for “Outstanding” POD Curve …............................................ 5.32

5.21b Improvement Factors versus Leak Probability for "Outstanding" POD Curve ......................... 5.32

$5.22 \mathrm{a}$ Improvement Factors versus $\mathrm{D}_{\sigma}$ for 10 -Year ISI Interval ...................................................5.33

5.22b Improvement Factors versus Leak Probability for 10-Year ISI Interval …............................. 5.33

5.23a Improvement Factors versus $D_{\sigma}$ for 4-Year ISI Interval ....................................................... 5.34

5.23b Improvement Factors versus Leak Probability for 4-Year ISI Interval .................................... 5.34

$5.24 \mathrm{a}$ Improvement Factors versus $\mathrm{D}_{\sigma}$ for 2-Year ISI Interval ....................................................... 5.35

5.24b Improvement Factors versus Leak Probability for 2-Year ISI Interval ................................ 5.35

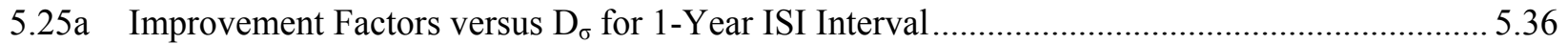

5.25b Improvement Factors versus Leak Probability for 1-Year ISI Interval .................................. 5.36

5.26a Improvement Factors versus $\mathrm{D}_{\sigma}$ for "Marginal" POD Curve ................................................ 5.37

5.26b Improvement Factors versus Leak Probability for "Marginal” POD Curve .............................. 5.37

5.27a Improvement Factors versus $\mathrm{D}_{\sigma}$ for “Good” POD Curve …..................................................... 5.38

5.27b Improvement Factors versus Leak Probability for "Good" POD Curve .................................... 5.38

5.28a Improvement Factors versus $D_{\sigma}$ for "Very Good" POD Curve …............................................ 5.39

5.28b Improvement Factors versus Leak Probability for "Very Good" POD Curve............................ 5.39

5.29a Improvement Factors versus $D_{\sigma}$ for 10-Year ISI Interval ........................................................ 5.40

$5.29 \mathrm{~b}$ Improvement Factors versus Leak Probability for 10-Year ISI Interval .................................. 5.40

5.30a Improvement Factors versus $\mathrm{D}_{\sigma}$ for 4 -Year ISI Interval..................................................... 5.41

5.30b Improvement Factors versus Leak Probability for 4-Year ISI Interval ..................................5.41

5.31a Improvement Factors versus $\mathrm{D}_{\sigma}$ for "Very Good" POD Curve and 4-Year ISI Interval

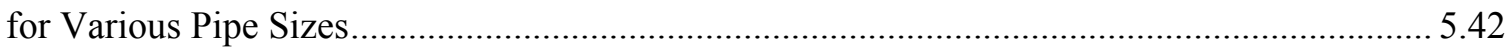

5.31b Improvement Factors versus Leak Probability for "Very Good" POD Curve and 4-Year ISI Interval for Various Pipe Sizes 
5.32a Improvement Factors versus Leak Probability for "Marginal" POD Curve and 10-Year ISI Interval for Various Pipe Sizes

5.32b Improvement Factors versus Leak Probability for "Marginal" POD Curve and 4-Year ISI Interval for Various Pipe Sizes

5.33a Improvement Factors versus Leak Probability for "Good" POD Curve and 10-Year ISI Interval for Various Pipe Sizes

5.33b Improvement Factors versus Leak Probability for "Good" POD Curve and 4-Year ISI Interval for Various Pipe Sizes

5.34a Improvement Factors versus Leak Probability for "Very Good" POD Curve and 10 -Year ISI Interval for Various Pipe Sizes

5.34b Improvement Factors versus Leak Probability for "Very Good" POD Curve and 4-Year ISI Interval for Various Pipe Sizes

5.35 Improvement Factors versus Leak Probability for "Outstanding” POD Curve and 4-Year ISI Interval for Various Pipe Sizes ......

5.36a Leak Probability as a Function of Time for Various Pipe Sizes Having Comparable Failure Probabilities

5.36b Leak Probability as a Function of Time for Various Pipe Sizes Having Comparable $\mathrm{D}_{\sigma}$ Values of about 5.0E-03

5.37a Leak Probability as a Function of Time for Small Pipe......................................................... 5.48

5.37b Leak Probability as a Function of Time for Intermediate Pipe .............................................. 5.48

6.1 Ratio of Calculated Leak Probabilities from Sensitivity Calculations for $\mathrm{Q}=100 \ldots \ldots \ldots \ldots \ldots \ldots \ldots . .6 .6$

6.2 Ratio of Calculated Break Probabilities from Sensitivity Calculations for $\mathrm{Q}=100$................ 6.6

6.3 Complementary Cumulative Flaw Depth Distribution Indicating Range for RRA Model .......... 6.9

6.4 Complementary Cumulative Flaw Aspect Ratio Distribution .............................................. 6.10

6.5 Complementary Cumulative Distribution for Fatigue Crack Growth Rate Parameter ............... 6.10

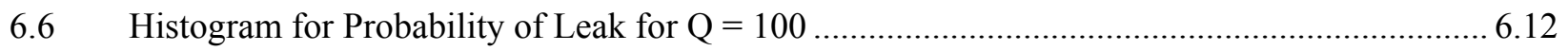

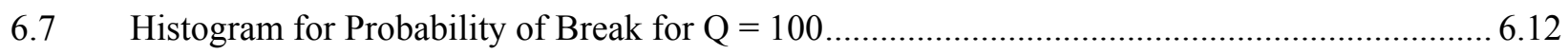

6.8 Comparisons of Probabilities from Uncertainty Analyses with Best Estimate

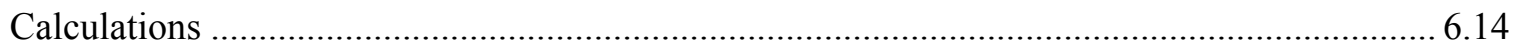

6.9 Complementary Cumulative Distributions of Calculated Leak Probabilities .......................... 6.15

6.10 Complementary Cumulative Distributions of Calculated Break Probabilities ....................... 6.15

6.11 Maximum of Leak and Break Probabilities from Sample of 100 Cases Evaluated by

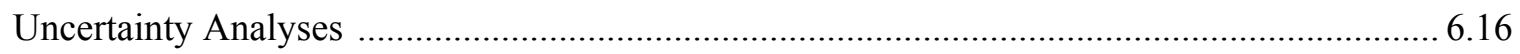

6.12 Upper and Lower Bounds of Leak and Break Probabilities from Uncertainty Analyses .......... 6.16

7.1 Vessel Leak Probability versus Cyclic Stress Frequency Assuming All Detected Flaws are Repaired..... 
7.2 Effect of Flaw-Sizing Errors for Vessel Inspected with "Very Good" POD ............................. 7.9

7.3 Effect of Flaw-Sizing Errors for Vessel Inspected with "Advanced” POD ................................ 7.9

7.4 Effect of Flaw-Sizing Errors for Vessel Inspected with “Advanced” POD.............................. 7.10

7.5 Factor of Improvement for Vessel Inspected with "Very Good" POD .................................... 7.10

7.6 Effect of Flaw-Sizing Errors for Low Fatigue of Piping Inspected with "Very Good"

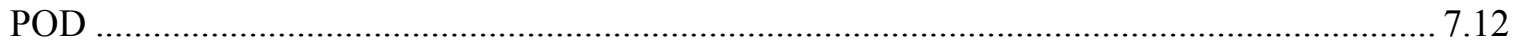

7.7 Effect of Flaw-Sizing Errors for High Fatigue of Piping Inspected with "Very Good" POD

7.8 Effect of Flaw-Sizing Errors for High Fatigue of Piping Inspected with "Advanced"

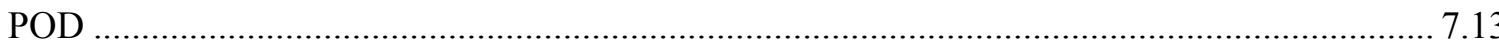

7.9 Effect of Flaw-Sizing Errors for Low Fatigue of Piping Inspected with "Very Good" POD 


\section{Tables}

2.1 Welding Factors that Affect the Probability of Defect Occurrence ......................................... 2.7

$2.2 \quad$ Piping Welds Considered in the Sensitivity Analysis............................................................ 2.10

2.3 Flaw Occurrence in Different Regions of the Wall .............................................................. 2.14

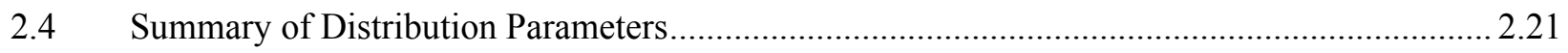

2.5 Parameter Extrapolation for Ferritic Pipes to 0.25 in. Wall Thickness .................................. 2.26

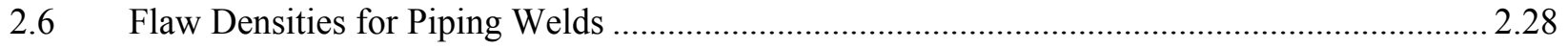

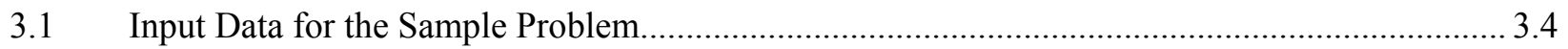

3.2 Fatigue Crack Growth Parameter $\mathrm{Q}=\alpha \mathrm{N}(\Delta \sigma)^{4}$ Used for Correlation of Leak

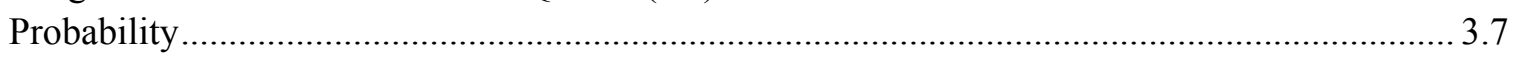

3.3 PRAISE Model for 6-in. Schedule 120 Stainless Steel Pipe: Baseline Case............................. 3.8

3.4 Results of Comparisons Between the pc-PRAISE and PARIS Codes..................................... 3.13

3.5 Fatigue Crack Growth Parameter $\mathrm{Q}=\alpha \mathrm{N}(\Delta \sigma)^{4}$ and Estimated Leak and Break

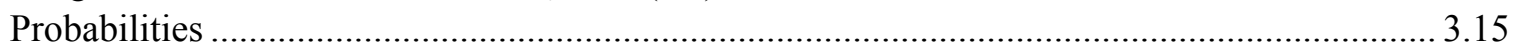

4.1 Pipe Size Inputs of Parametric Calculations for Stainless Steel Fatigue .................................. 4.3

4.2 Parameters for Lognormal Flaw Depth Distribution for Stainless Steel Piping ......................... 4.4

4.3 Primary Stress and Flow Stress Inputs for Stainless Steel Fatigue............................................ 4.5

4.4 Parameters of POD Curves for Three Performance Levels ....................................................... 4.7

5.1 Numerical Values of Constants Ci Used in the Equation for Predicting the Initiation of

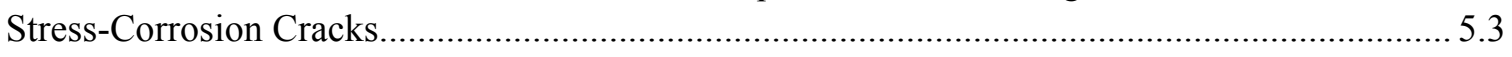

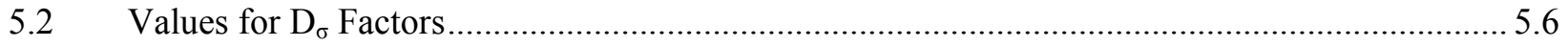

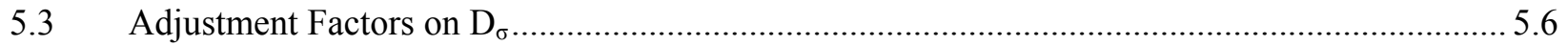

$5.4 \quad$ POD Curve Parameters for Four Performance Levels ......................................................... 5.11

5.5 Input Values for Parametric Calculations for Piping Subject to IGSCC Including the

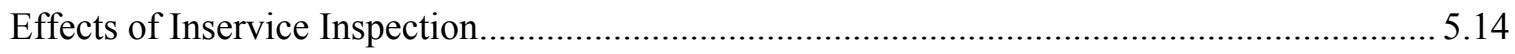

6.1 Assumed COV Values Used in Sensitivity Calculations ............................................................. 6.2

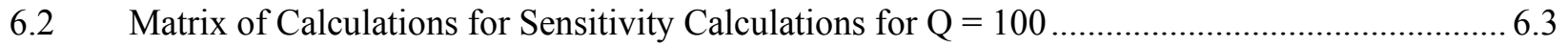

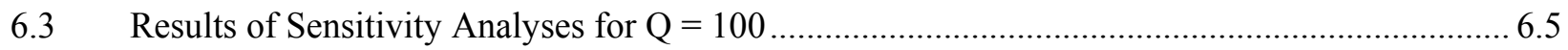

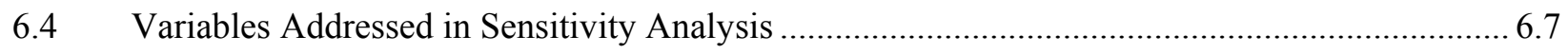

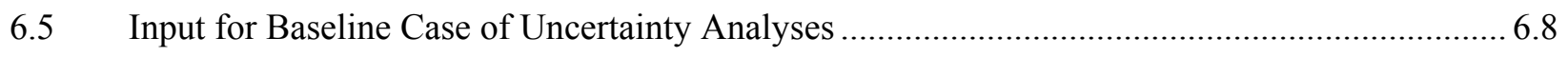

6.6 Parameters for Lognormal Distribution of Flaw Depth .............................................................. 6.9

6.7 Parameters for Lognormal Distribution of Flaw Aspect Ratio ..................................................... 6.9

6.8 Parameters for Lognormal Distribution of Fatigue Crack Growth Parameter C ...................... 6.11 
6.9 Descriptive Statistics for Leak and Break Probabilities........................................................ 6.13

7.1 Distribution Function for Fatigue Crack Growth Rate Factors................................................ 7.5

7.2 Flaw-Size Distribution for Reactor Pressure Vessel .............................................................. 7.6

7.3 Flaw-Size Distribution for 6-in. Schedule 120 Pipe ................................................................. 7.6

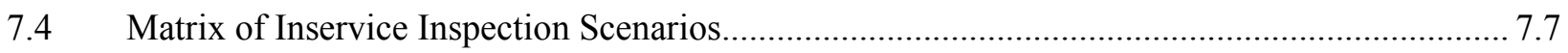




\section{Executive Summary}

The goal of inservice inspection (ISI) of nuclear reactor piping and pressure vessels is to detect servicerelated defects in a timely manner and thereby enhance the structural reliability of the inspected components. The U.S. Nuclear Regulatory Commission (NRC), in an effort to assess the effectiveness of inservice inspection programs, has supported a research project at Pacific Northwest National Laboratory (PNNL) titled "Assessment of the Reliability of Ultrasonic Testing (UT) and Improved Programs for Inservice Inspections." The objectives of this project have been to

- establish the accuracy and reliability of UT methods for ISI

- evaluate the impact of ISI reliability on system integrity

- provide technical bases for improving ISI programs for important reactor systems and components

- provide recommendations to the NRC staff for enhancements to the American Society of Mechanical Engineers (ASME) Code to improve the effectiveness, reliability, and adequacy of ISI methods and programs.

The work reported here first applied probabilistic structural mechanics models to predict the reliability of nuclear pressure boundary components. Second, and more importantly, it applied these models to evaluate the effectiveness of alternative programs for ISI to reduce these failure probabilities. Sections 3 and 4 discuss studies addressing the potential benefits of ultrasonic inspections to reduce failure probabilities associated with fatigue crack growth, and Section 5 addresses stress-corrosion cracking. The studies have addressed critical inputs to fracture mechanics calculations, such as the parameters that characterize the number and sizes of fabrication flaws in piping welds (Section 2). Results of the research could be used to support the development and implementation of risk-informed ISI of piping and vessels.

Section 2 describes an approach for estimating the numbers and sizes of fabrication flaws in piping welds and recommends inputs for these important parameters that are used in probabilistic fracture mechanics (PFM) calculations. The approach is based on calculations that applied an expert system model developed by Rolls Royce and Associates (RRA). The RRA methodology is summarized in Section 2 along with detailed results of calculations for flaw densities and depth distributions. Curve fitting was used to generalize the numerical results from the matrix of individual computer runs to derive a methodology to estimate the number and depths of welding-related flaws in piping components. The estimation methodology is the basis for flaw-related inputs used in the calculation described in Sections 3 and 4.

Section 3 describes calculations for the fatigue life of piping components performed in a structured parametric format. The approach addresses a wide range of pipe sizes, piping materials, operating stresses, and ISI programs. The section begins with a description of the pc-PRAISE (Piping Reliability Analysis Including Seismic Events) computer code that was used to perform most of the calculations of the present report. Results of example calculations are then described using curves and tables. 
Section 4 presents a large matrix of parametric calculations performed with the computer code pc-PRAISE that evaluated the effectiveness of alternative inspection strategies on reducing failure probabilities of stainless steel piping. It is assumed in these calculations that the failure mechanism of concern is that of fatigue crack growth from pre-existing fabrication flaws. Each strategy corresponds to a combination of a specific inspection frequency and an inspection method that provides a particular probability of flaw detection as a function of flaw depth. The results identify the ISI strategies that have the most effective combinations of flaw-detection capabilities and inspection frequencies. An extensive set of plots is presented that covers a wide range of pipe-wall thicknesses, cyclic operating stresses, and inspection strategies. These plots are intended to support the development of risk-informed ISI programs. Maximum reductions in failure probabilities are on the order of a factor of 100 for inspection programs with small inspection intervals (2 years) using UT methods with outstanding detection capabilities. Inspections at 10-year intervals and/or with marginal detection capabilities provide reductions in pipingfailure probabilities in the range of a factor of two or less.

Section 5 expands the scope of the calculations for stainless steel piping by addressing the failure mechanism of intergranular stress-corrosion cracking. A large matrix of calculations was again performed with the computer code pc-PRAISE. The resulting plots cover a wide range of pipe-wall thicknesses, susceptibility to stress-corrosion cracking, probability of detection curves, and inspection frequencies. The calculations show how various inspection strategies can reduce probabilities of piping failures due to the failure mechanism of stress-corrosion cracking. Maximum reductions are on the order of a factor of ten for inspection programs with small inspection intervals ( 2 years) using UT methods with outstanding detection capabilities. Inspections at 10-year intervals and/or with marginal detection capabilities provide little or no reduction in piping-failure probabilities.

Using the pc-PRAISE code, Section 6 quantifies uncertainties associated with the inputs to PFM calculations, the uncertainties in the PFM model itself, and the uncertainties in the resulting calculated piping-failure probabilities. Such uncertainties have been an important issue in the application of PFM models for implementing risk-informed procedures for developing improved ISI programs. The calculations apply the pc-PRAISE computer code to address both leak and break probabilities for piping components. A two-step process was used. The first step was a sensitivity study which identified those uncertainties that had the greatest effect on the results from pc-PRAISE. The second step was a quantitative uncertainty analysis that addressed the most critical parameters as identified by the sensitivity calculations. Results of the uncertainty calculations indicate that the largest sources of uncertainty are the number and sizes of fabrication-related defects. The distributions of calculated failure probabilities from the uncertainty analyses tend to be centered on the corresponding results of best-estimate calculations. Uncertainties are relatively small when calculated failure probabilities are large, whereas uncertainties are relatively high when the calculated failure probabilities are very small.

Section 7 describes PFM calculations that address the effects of flaw sizing errors on the effectiveness of ISI programs. Calculations evaluate some important interactions between sizing errors, probability of flaw-detection, and flaw-acceptance/repair criteria. Probability of detection capability appears to be the most limiting factor with regard to the overall capability of ISIs to reduce leak probabilities. The effects of flaw-sizing errors are relatively small when calculations are based on inputs for flaw-sizing capabilities and acceptance standards that are representative of current nondestructive examination (NDE) capabilities and code requirements. However, the calculations show that gross errors in flaw sizing or significant 
departures from current flaw-acceptance standards could negate the expected benefits of inspection methods that exhibit outstanding performance in the area of flaw detection.

Section 8 provides specific results and conclusions of the present report. 



\section{Acknowledgments}

The authors wish to acknowledge the contribution of Dr. David Harris and Dr. Delip Dedia of

Engineering Mechanics Technology, Inc. for their assistance in the application and enhancement of the pc-PRAISE computer code.

The authors wish to thank the U.S. Nuclear Regulatory Commission (NRC), Office of Nuclear Regulatory Research, for supporting this work, and, in particular, the NRC program managers, Ms. Deborah A. Jackson and Mr. Wallace E. Norris. 
xxiv 


\section{Abbreviations and Acronyms}

$\begin{array}{ll}\text { ASME } & \text { American Society of Mechanical Engineers } \\ \text { CERT } & \text { constant extension rate tests } \\ \text { COV } & \text { coefficient-of-variations } \\ \text { HAZ } & \text { heat-affected zones } \\ \text { HUCD } & \text { heatup and cooldown } \\ \text { ID } & \text { inside diameter } \\ \text { IGSCC } & \text { intergranular stress-corrosion cracking } \\ \text { ISI } & \text { inservice inspection } \\ \text { LOCA } & \text { loss-of-coolant accident } \\ \text { MMA } & \text { manual metal arc welding } \\ \text { NDE } & \text { nondestructive examination } \\ \text { NRC } & \text { U.S. Nuclear Regulatory Commission } \\ \text { pc-PRAISE } & \text { Piping Reliability Analysis Including Seismic Events } \\ \text { PFM } & \text { probabilistic fracture mechanics } \\ \text { PND } & \text { probability of non-detection } \\ \text { PNNL } & \text { Pacific Northwest National Laboratory } \\ \text { POD } & \text { probability of detection } \\ \text { PSI } & \text { preservice inspections } \\ \text { PTS } & \text { pressurized thermal shock } \\ \text { PWR } & \text { pressurized water reactor } \\ \text { RRA } & \text { Rolls Royce and Associates } \\ \text { RT } & \text { radiographic inspection } \\ \text { SAW } & \text { submerged arc welding } \\ \text { SRRA } & \text { Structural Reliability and Risk Assessment } \\ \text { TIG } & \text { ultrasonic testing } \\ \text { UT } & \text { mm } \\ 1 \text { inch (in.) } & \\ 1 \text { MPa }=145 \text { psi } & \\ 1 \text { gallon }(\text { gal.) } & \text { liters } \\ & \end{array}$


xxvi 


\section{Introduction}

Models for probabilistic structural mechanics are increasingly being used to predict the reliability of nuclear pressure boundary components such as welds in piping systems (Harris et al. 1981; Harris and Dedhia 1992) and reactor pressure vessels (Dickson 1994). The objectives of the work described in this report were to (1) develop and apply probabilistic fracture mechanics (PFM) models to predict component failure probabilities and (2) more importantly, to evaluate the potential effectiveness of inservice inspection (ISI) programs to reduce these failure probabilities.

This research work was supported by the U.S. Nuclear Regulatory Commission (NRC) at the Pacific Northwest National Laboratory (PNNL). Related studies have addressed the potential benefits of ultrasonic inspections to reduce failure probabilities associated with fatigue crack growth (Khaleel and Simonen 1994a) and stress-corrosion cracking (Khaleel and Simonen 1997). Work has also focused on improving PFM models, including the initiation of fatigue cracks (Khaleel and Simonen 1998), the effects of leak detection (Simonen et al. 1998), the significance of surface versus buried flaws (Simonen and Khaleel 1998a), and errors in measuring the sizes of detected flaws (Simonen and Khaleel 1997). Other studies have addressed critical inputs to fracture-mechanics calculations, such as the parameters that characterize the number and sizes of fabrication flaws in piping welds (Chapman and Simonen 1998; Khaleel et al. 1999; Simonen and Chapman 1999). Results of the research have supported the development of NRC guidance for implementation of risk-informed ISI of piping (USNRC 1997).

Section 2 describes an approach for estimating the numbers and sizes of fabrication-related flaws in piping welds and recommends inputs to be used for these important parameters to PFM calculations. The approach used trends from calculations that applied an expert system model developed by Rolls Royce and Associates (RRA). Section 2 summarizes the RRA methodology and presents results of the calculations for flaw density and depth distributions.

Section 3 describes an approach that performs calculations for the fatigue life of piping components by using a structured parametric format. The approach addresses a wide range of pipe sizes, piping materials, operating stresses, and ISI programs. The section begins with a summary of the pc-PRAISE (Piping Reliability Analysis Including Seismic Events) computer code that is extensively used for the calculations of the present report. Results of calculations are then described using curves and tables.

Section 4 presents parametric calculations that evaluate the relative effectiveness of alternative inspection strategies on reducing failure probabilities of stainless steel piping. It is assumed that the failure mechanism of concern is fatigue crack growth from preexisting fabrication flaws. The results were developed to identify ISI strategies that have optimal flaw-detection capabilities and inspection frequencies. An extensive set of plots is presented to cover a wide range of pipe-wall thicknesses, cyclic operating stresses, and inspection strategies.

Section 5 also addresses the inspection of stainless steel piping, but assumes that the failure mechanism of concern is that of intergranular stress-corrosion cracking. A collection of plots is presented to cover a wide range of wall thicknesses, susceptibility to stress-corrosion cracking, probability of detection curves, and inspection frequencies. 
Section 6 quantifies uncertainties associated with the inputs to PFM calculations, the uncertainties in the PFM model itself, and the uncertainties in the resulting calculated piping-failure probabilities. Such uncertainties are an important issue in applying PFM models (Bishop 1997) to the implementation of riskinformed procedures for developing improved ISI programs (ASME/CRTD 1992; Westinghouse Owners Group 1997). The calculations apply the pc-PRAISE computer code (Harris and Dedhia 1992) to address both leak and break probabilities for piping components. A two-step process was used. The first step was a sensitivity study that identified those uncertainties that had the greatest effect on the results from pc-PRAISE. The second step was a quantitative uncertainty analysis that addressed the most critical parameters as identified by the sensitivity calculations.

Section 7 describes PFM calculations that address the effects of flaw sizing errors on the effectiveness of ISI programs. Results of calculations are used to evaluate some important interactions of sizing errors, the probability of flaw detection, and flaw acceptance/repair criteria.

The conclusions of this research are discussed in Section 8. 


\section{Flaw-Size Distribution and Flaw-Existence Frequencies in Nuclear Piping}

\subsection{Introduction}

This section describes an approach for estimating the numbers and sizes of fabrication-related flaws in piping welds. The need for such an approach became evident during pilot applications of risk-informed inservice inspection (ISI) methods to the Surry Power Station (Shah et al. 1997). Efforts to benchmark failure-probability calculations revealed a lack of a uniform and consistent basis for establishing inputs to probabilistic structural-mechanics codes for the parameters that describe flaw densities and flaw-size distributions (Bishop 1997). It became clear that large uncertainties existed regarding flaws in piping welds such that different structural analysts performing independent estimates can produce divergent inputs, which in turn can result in calculated failure probabilities that can differ by several orders of magnitude.

RRA developed a flaw-estimation methodology (Chapman 1993; Chapman et al. 1996; Chapman and Simonen 1998) that offered a suitable approach to establish flaw-distribution inputs. This methodology was already being used on another NRC-funded project at PNNL in collaboration with RRA as a subcontractor, with the scope of this related work limited to flaw distributions in reactor pressure vessel welds rather than piping welds. However, it was known that RRA had another version of their weld simulation methodology that did address flaws in piping welds. A decision was made to subcontract with RRA to apply this methodology to generate representative data for flaws in piping welds.

Subsequent to the time that the calculations that are presented below were performed, there was a special effort at the request of the NRC staff to validate the predictions of RRA methodology (Simonen and Chapman 1999). This effort compared predicted flaw distributions with some available data from a study in the United Kingdom for flaws that were detected during examinations of a large population of nuclear piping welds. Another part of the validation used data on repair rates that covered several thousand girth welds in gas transmission piping. In both cases, there was a relatively good level of agreement between model predictions and the flaw rates indicated by the available data.

A summary of the RRA methodology for establishing flaw-distribution inputs and of the results generated by RRA for a matrix of piping-weld simulations is given below. These results filled an immediate need for a consistent basis to estimate the numbers and sizes of flaws for pipe thicknesses ranging from $0.25 \mathrm{in}$. $(6.35 \mathrm{~mm})$ to 2.5 in. $(63.5 \mathrm{~mm})$. PNNL has used these estimates as inputs to parametric calculations of piping reliability as described elsewhere in this report. In addition, the results from the weld simulations have been used in the pilot application of risk-informed ISI methods for the Surry Power Station (Shah et al. 1997) and have been incorporated into the most recent versions of the pc-PRAISE and Structural Reliability and Risk Assessment (SRRA) (Bishop 1997) computer codes.

The RRA model was applied to simulate flaws for a range of pipe-wall thicknesses, piping materials, welding processes, and post-weld inspection procedures. The simulated flaw densities and size distributions for this sample of welds were assumed to be representative of flaws in piping of a typical nuclear power plant. The data were used to establish trends as a function of pipe-wall thickness, type of piping material, welding procedure, and inspection practice. The resulting trends are believed to give a 
reasonable basis for inputs to be used for calculating piping-failure probabilities. More importantly, the formulation of the RRA model provides a physical basis for scaling flaw-distribution parameters between piping of different sizes and fabrication practices and thereby provides confidence that relative failure probabilities for different piping locations reflect correct trends for purposes of a risk-informed ranking of candidate locations for inservice inspections.

It should be stated that the present study draws generalized conclusions from a rather limited set of calculations performed by RRA. Additional combinations of parameters that expand the scope of the calculations could be considered that the present calculations do not address. The calculations could address additional values of pipe diameters and wall thicknesses, welding processes, number and sizes of weld passes, and post-weld inspection procedures.

\subsection{Summary of Past Works}

PFM codes, including pc-PRAISE (Harris and Dedhia 1992) for predicting the reliability of nuclear piping and VISA-II (Simonen et al. 1986) and FAVOR (Dickson 1994; Dickson et al. 1995) for predicting the reliability of reactor pressure vessels, require key inputs for the number and size distribution of crack-like flaws that are located within the pipe and/or vessel wall. Determining a suitable defect density and size distribution is one of the most difficult aspects of calculating probabilities of pipe/vessel failure. Various investigators have attempted to estimate these parameters for specific cases by examining cracked components or by using expert elicitation.

Wilson (1974) provided information on both flaw depth and aspect ratio based on judgment. The marginal distributions of the flaw depth and aspect ratio were found to be exponential. Harris (Harris and Fullwood 1976; Harris 1977, 1978) and Burns (Burns et al. 1978) applied the results from the Wilson study to the analyses of reactor piping reliability, but adjustments were made to include only cracks with initial surface lengths larger than 2 in. $(50 \mathrm{~mm})$. The Wilson data can be adequately fitted with an exponential distribution with a mean crack depth of 0.078 in. $(2 \mathrm{~mm})$.

Becher and Hanson (undated) found that flaw depth is lognormally distributed based on the data on the depths of 228 surface cracks found during successive removal of layers of steel weldment. In fact, the Becher and Hansen data are more accurately fitted with an exponential distribution with a mean crack depth of $0.067 \mathrm{in.}(1.7 \mathrm{~mm})$. The largest crack depth found by Becher and Hansen was $0.45 \mathrm{in}$. $(11.4 \mathrm{~mm})$.

The flaw distribution (for welds in reactor pressure vessels of approximately $8 \mathrm{in}$. [200 $\mathrm{mm}$ ] in thickness) suggested by the Marshall Committee $(1976,1982)$ is probably referenced more than any other. The Marshall Committee, which addressed one particular type of vessel weld, was able to call on the services of eminent experts representing the various relevant disciplines. The conclusions of the Marshall Committee were based on cracks found in the United Kingdom and the United States and on expert judgment related to both nuclear and non-nuclear vessels. The data were used to estimate a crack depth distribution, which was described as an exponential distribution with a mean crack depth of 0.24 in. $(6 \mathrm{~mm})$

Evaluations of reactor vessel integrity for pressurized thermal shock (PTS) transients have been largely based on the Marshall distribution with a conservative assumption that all flaws are surface-breaking cracks located on the inside of the vessel. Woo and Chou (1982) assumed semi-elliptical, interior surface cracks along the circumferential direction based on the results of sectioning a cracked pressurized water 
reactor (PWR) feedwater nozzle (Goldberg et al. 1980). Woo and Chou used the exponential distribution developed by Marshall (1976) to describe the crack depth. Philips et al. (1991) used an exponential distribution with a mean crack depth of $0.06 \mathrm{in} .(1.5 \mathrm{~mm})$ to study the reliability of passive components. Khaleel and Simonen (1994b) used a lognormal distribution to characterize the depth of fabrication flaws in nuclear piping.

Application of the Marshall Committee approach to address flaws in the various piping welds of interest would present practical problems due to the significant effort needed from the variety of experts. Use of the Marshall distribution based on vessel data is very conservative for piping and overestimates the probability of failure of nuclear piping. Many investigators, as cited above, have assumed a certain distribution (lognormal, exponential, gamma, Weibull) with assumed parameters and then have performed PFM calculations. The available information on crack size distributions is limited and does not adequately consider the effects of wall thickness on the values of the distribution parameter(s).

In most of the investigations cited above, the focus was on the flaw-depth distribution. This is because information on the flaw-aspect ratio is essentially non-existent and because the depth dimension has more influence on crack-tip stress-intensity factors than the length dimension. Cramond (1974) estimated a mean value for $\mathrm{b} / \mathrm{a}$ of 1.7 for the aspect ratio (where $2 \mathrm{~b}$ is the flaw length, and $\mathrm{a}$ is the flaw depth) and about $1 \%$ of the flaws having aspect ratios greater than 5. Frost and Denton (1967) predicted a mean value $\mathrm{b} / \mathrm{a}$ of 3.4 and about $20 \%$ of the initial cracks having $\mathrm{b} / \mathrm{a}>5$.

Information on crack-occurrence frequencies is limited. Cramond (1974) surveyed results from a number of sources and addressed crack frequencies in base plates, butt welds, multilayer circumferential arc welds, electroslag welds, and pressure-vessel butt welds. The bases for the data were examinations by radiography of $7073 \mathrm{ft}(2156 \mathrm{~m})$ of manual metal-arc butt welds and $4061 \mathrm{ft}(1238 \mathrm{~m})$ of automatic submerged-arc welds from ships under construction. Plates ranged from 0.6 to $1.4 \mathrm{in}$. (15 to $35 \mathrm{~mm}$ ) thick. Crack frequencies for welds varied from 1.1E-04 to 9.4E-04 per $1 \mathrm{in}$. (25.4 mm) of weld. The Marshall Committee (1976) presented relevant information on the crack frequencies. Harris et al. (1981) suggested that the crack frequencies based on the Marshall Report (1976) are between 5.4E-05 to 1.5E-03 per 1 in. $(25.4 \mathrm{~mm})$ of weld.

In this section, we first summarize research performed at RRA in the mid-eighties that developed an expert system that predicts defect size distributions and densities for multi-pass welds in piping up to approximately 4 in. (100 mm) in thickness (Chapman 1993). More recently, RRA collaborated with PNNL on an NRC-funded research program that extended the method to address welds in U.S. reactor pressure vessels (Chapman et al. 1996; Chapman and Simonen 1998). The discussion below describes the RRA model and then presents results from a matrix of calculations that predict distributions of flaws in piping welds made using specific welding and inspection processes.

\subsection{Flaw-Distribution Model}

The Chapman (1993) flaw-distribution model addresses the defects that occur during multi-pass welding and that may not be detected and repaired during or after the build up of the weld. The methodology is based on the concept that a weld is made of individual weld runs (beads) and layers. Most flaws are confined to a single weld layer such that the characteristic flaw sizes are directly related to the weld-bead 
dimensions. Larger flaws are associated with the forward propagation of defects from one layer to the next layer as the weld is built up.

The RRA approach uses expert elicitation and mathematical modeling to simulate the steps in manufacturing a weld and the errors that lead to different types of weld defects. The defects that may initiate in weld beads include centerline cracks, lack of fusion, slag, pores with tails, and cracks in heataffected zones (HAZs). Various welding processes are addressed, including submerged metal arc welding. The model simulates the effects of both radiographic and dye-penetrant inspections. Output from the simulation gives occurrence frequencies for defects as a function of both flaw size and flaw location (surface connected and buried flaws).

To address piping, a list of weld-defect types was identified based on discussions with experts involved with the welding and inspection of piping in use at operating nuclear power plants. The following defect types are addressed (see Figures 2.1 and 2.2).

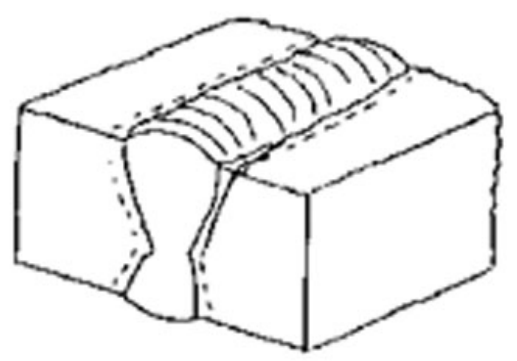

HAZ Crack

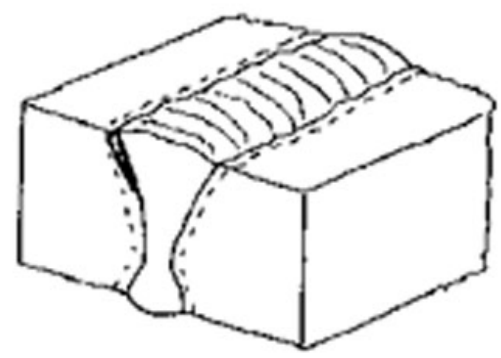

Lack of Sidewall Fusion Sidewall Slag

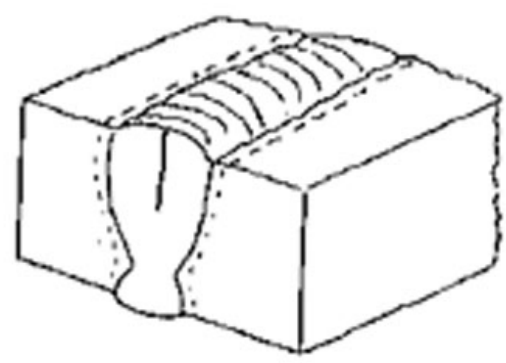

Centerline Crack
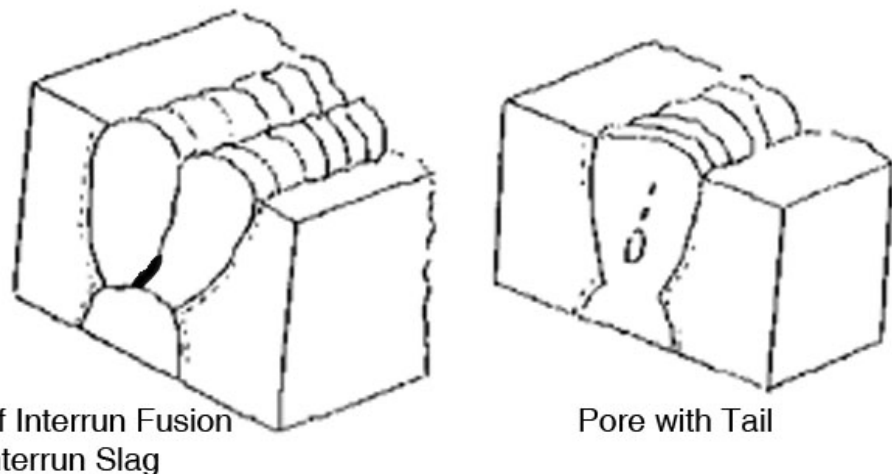

Pore with Tail

Figure 2.1 Welding Defects 


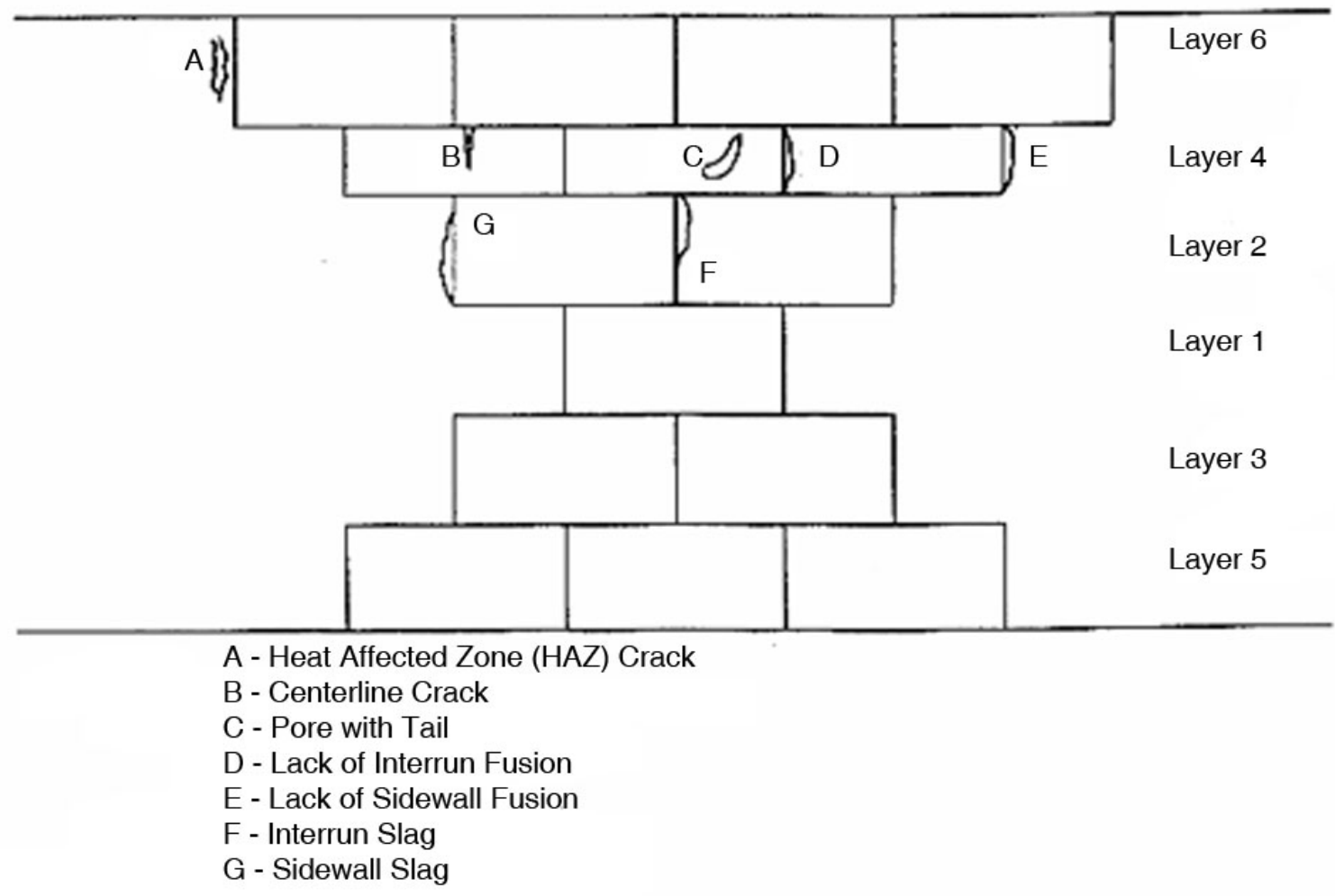

Figure 2.2 Schematic Representation of Weld Build Up and the Position of Different Types of Crack-Like Defects

\subsubsection{Centerline Cracking}

As a weld solidifies and contracts, any impurities tend to collect at the top center of the bead. The stresses present may then cause a centerline crack along the weld bead due to the presence of low-strength or low-melting-point phases. Automatic high-speed welding techniques, such as submerged arc welding (SAW), show a greater tendency for centerline segregation and cracking than do manual techniques. The fill runs of large multi-pass welds are generally less susceptible than are root runs.

\subsubsection{Heat-Affected Zone Cracking}

Hydrogen is usually absorbed on cooling while a weld is forming. The hydrogen may form hydrogen gas, which exerts a bursting pressure within the metal. If this effect is combined with the formation of a hardened structure, cracking may result. This usually occurs in the HAZ of the weld. The lower the heat input and the thicker the joint sections, the greater is the risk of HAZ cracking due to the lower level of diffusivity of any hydrogen gas present. Failure to ensure the correct level of preheat or postheat, which is highly welder dependent, leads to higher chances of HAZ cracking. 


\subsubsection{Lack of Fusion}

The lack-of-fusion defect results from a lack of union between the weld metal and the parent plate or (in multi-run welds) between successive weld runs. The narrower the weld preparation and/or the deeper the groove, the greater is the chance of lack of fusion in the weld root. There is a greater chance of lack of sidewall fusion for thicker sections as compared to thinner sections. Occurrence rates for lack-of-fusion defects do not depend on the material type being welded. Any access difficulty preventing the use of the correct electrode angle will increase the chances of lack of fusion. Welder skill is vital in helping to ensure sufficient fusion when using manual procedures.

\subsubsection{Non-Metallic Slag Inclusions}

Linear slag inclusions are normally due to incomplete slag removal between weld runs, but slag laminations within the parent plate may occasionally be the cause. Mill scale, rust on the plate, and damaged electrode coatings can cause isolated slag inclusions. Damaged electrode coatings denude the weld metal of slag-forming elements of adequate floatability; i.e., slag is left within the weld bead rather than floating to the top for removal. Slag inclusions are relatively common in manual metal arc (MMA) and submerged arc weld deposits. Any process resulting in an extremely concave weld bead will be prone to slag entrapment between welding runs. The tighter or more narrow the weld preparation and the thicker the joint, the greater is the likelihood of entrapped inclusions. A dirty base material or oxidized surfaces will lead to a greater likelihood of nonmetallic inclusions. Shop welds are less prone to slag defects than are welds produced in the field. Welder skill and environment are significant factors since the removal of slag between runs is important in avoiding non-metallic inclusions.

\subsubsection{Porosity}

A welded joint will usually contain gas-forming elements; these evolve into phases as the temperature decreases and result in formation of cavities or porosity. Porosity occurs uniformly along the weld. It may be caused by moisture, rust, or grease on the plate surface, oxygen or nitrogen contamination from the atmosphere, or oxygen contamination from the shielding gas. When porosity occurs in isolated groups, its most likely cause is the existence of unstable conditions as the arc is being struck. Weld metal may be deposited before the gas shield is established. Isolated porosity, which is strung out, is more likely to be caused by incorrect electrode angle or strikeout. Interdendritic porosity or shrinkage porosity may occur at weld stop-start positions and may be linked with solidification cracking. Fluxed processes, such as submerged arc or MMA welding, are less susceptible to porosity than fluxless processes. Cleanliness is extremely important. Damp or contaminated workpieces or consumables are a frequent cause of porosity.

\subsubsection{Defect Density}

Welding metallurgists and inspection engineers estimated the defect occurrence frequencies (per unit length of weld bead) for the set of "crack-like defect types" of the RRA flaw-simulation model. The RRA has not yet published this information for piping welds, which is encoded into the expert system computer code, or otherwise placed it into the public domain. However, the methodologies for piping and vessel welds are known to be very similar and the analogous parameters for vessel welds have been published (Chapman et al. 1996; Chapman and Simonen 1998). Table 2.1 indicates the various factors 
Table 2.1 Welding Factors that Affect the Probability of Defect Occurrence

\begin{tabular}{|c|c|}
\hline Process & $\begin{array}{l}\text { Manual Metal Arc } \\
\text { Submerged Arc } \\
\text { Autogenous Manual Tungsten Inert Gas (TIG) } \\
\text { Manual TIG with Filler } \\
\text { Autogenous Automatic TIG } \\
\text { Automatic TIG with Filler } \\
\text { Autogenous Electron Beam/Laser } \\
\text { Short Circuited Dip Metal Inert Gas }\end{array}$ \\
\hline Root Method & $\begin{array}{l}\text { EB Insert }{ }^{(a)} \\
\text { Single Pass } \\
\text { Open Root }\end{array}$ \\
\hline Restraint & $\begin{array}{l}\text { High } \\
\text { Meduim } \\
\text { Low }\end{array}$ \\
\hline Material & $\begin{array}{l}\text { Carbon or Carbon Manganese Steel } \\
\text { Low Alloy Steel } \\
\text { Austenitic Ferrite Uncontrolled } \\
\text { Austenitic Ferrite Controlled } \\
\text { Martensitic and Ferritic } \\
\text { Stainless } \\
\text { Monel } \\
\text { Cupronickel } \\
\text { Nickel Alloys }\end{array}$ \\
\hline Location & $\begin{array}{l}\text { Laboratory Weld } \\
\text { Vendor }\end{array}$ \\
\hline Position & $\begin{array}{l}\text { 1G (Down Hand) } \\
\text { 2G (Horizontal/Vertical) } \\
\text { 3G (Vertical Up) } \\
\text { 3G (Vertical Down) } \\
\text { 4G (Overhead) } \\
\text { 5G (Horizontal Fixed Pipe) } \\
\text { 6G (45 Fixed Pipe) }\end{array}$ \\
\hline Access & $\begin{array}{l}\text { Good } \\
\text { Average } \\
\text { Restricted }\end{array}$ \\
\hline
\end{tabular}

that the RRA model addresses in simulating defect densities in piping welds. The main difference between piping welds and vessel welds are the special procedures used to produce the root pass in the case of piping and the increased likelihood for defects to occur at the critical inner surface location of the weld joint. For vessels there is good access to the inner surface location for welding and inspections, and this assures that the material at the inner surface location is of high quality. 
As indicated in Table 2.1, the model explicitly accounts for the expected increases in defect occurrence rates for welding at field and vendor sites. The model also addresses difficult field welding conditions as they may be related to access to the weld (e.g., restricted access) and to the welding position (e.g., $4 \mathrm{G}$ Overhead welding).

\subsubsection{Defect Characteristics}

The Chapman (1993) model allows the expected number of each type of defect to be calculated, and also defines the various characteristics (e.g., size, location, etc.) of these defects. The defect parameters for piping welds are defect width, defect depth, length-to-depth ratio, defect angle, and probability of forward propagation. Through-wall and length dimensions are quantified for purposes of fracture mechanics calculations. The angle and separation between the surfaces of the defects are evaluated for purposes of simulating the effects of inspections. The weld-simulation model assigns defect locations within the pipe wall and identifies flaws that are surface connected with the assumption that defects occur randomly within the individual runs that make up the completed weld.

Experts believed that the sizes of the individual defects as they first initiate within a single weld run could be realistically estimated. The bead dimension served as a normalizing factor for the sizes of these initiated defects, although their sizes were not always truncated at the bead depth. A Weibull statistical distribution was selected to describe the depth dimensions of the initiated defects. The experts also estimated defect lengths that could be described by Weibull distributions for flaw-aspect ratios that related the lengths of the flaws to the corresponding flaw-depth dimensions.

The next question was the probability that a defect, once initiated, would propagate on to the next layer or additional layers of the weld. The model was therefore designed to predict the number of small defects initiated in the welding process that would grow to become larger defects before the weld was completed. The ability of the welder to observe and correct the errors was also considered for each type of propagating defect.

Probabilities to quantify the statistical distributions are encoded into the RRA expert system model. The probabilities for initial defect characteristics and for the propagation of these defects for piping welds have not yet been published, but are believed to be similar to those for the RRA model for vessel welds (Chapman et al. 1996; Chapman and Simonen 1998).

Interactions between the randomly located defects in a weld are also simulated using a flaw proximity rule described by Chapman (1993). One or more defects sufficiently close together are combined into a single larger defect. In general, the likelihood of flaw interaction is relatively low. The flaws that propagate from layer to layer during welding are the primary contributor to the simulated population of larger flaws.

\subsubsection{Inspection Model}

The data encoded within the expert system model describe the defects generated by the welding process. A second aspect of the model simulates the effects of the inspections that are performed in the shop both during and after the welding process is completed. The present report does not document details of the inspection models. The RRA model has implemented methods for calculating inspection efficiency 
curves for each type of defect in the weld of interest, with the inspection efficiency being a function of the defect size and its through-wall location. Both radiographic and surface (dye penetrant) inspection methods are addressed.

Radiography is simulated using the model of Halmshaw and Hunt (1975). Each defect is treated as a slot that produces a difference in image density that the inspector must interpret. Test data and experience of inspectors were used to convert calculated images to probabilities of detection. Inputs to the simulation model define details of the radiographic procedure, including specifying the isotope or x-ray source and film location. Image densities are predicted based on the defect location relative to the source and film, and the defect size, width, and angle. It should be noted that the defect width or separation between defect surfaces can be relatively large for welding defects as compared to the widths of typical serviceinduced cracks such, as fatigue cracks. As a result, the radiography inspections can be relatively effective for the larger welding-type defects.

For surface examinations, the inspection efficiencies for given defect lengths and widths were based on judgments with consideration of surface finish whether as welded or machined.

\subsection{Computer-Based Implementation}

The expert system model of weld buildup uses a Monte Carlo simulation procedure. A computer code (RR-PRODIGAL) has been written for application on a UNIX-based workstation with the parameters for the specific welds defined through interactive menu-driven inputs.

A weld is described as a series of activities. One type of activity consists of the stepwise process of constructing the weld as a series of weld beads and layers as a set of building blocks as indicated in Figure 2.2. Other activities are as follows.

Inspections. Either radiographic or surface inspections can be performed at any stage, either during the partial weld buildup or after all weld runs have been completed. It is assumed that all detected defects are repaired, and these defects are not counted as contributions to the simulated population of defects. Comparisons (Chapman and Simonen 1998) of the model predictions with data from vessel welds indicate that improved correlations should consider that governing repair criterion.

Machining. Post-weld machining is considered a factor that affects the surface finish, which in turn impacts the effectiveness of surface inspections. Machining can also expose near-surface buried defects and thereby increase the number of surface-breaking defects.

Post Weld Heat Treatment. The sizes of HAZ cracks can be extended by simulating the effects of postweld heat treatment.

\subsection{Inputs for Sensitivity Study}

RRA, under a subcontract arrangement with PNNL, used the RR-PRODIGAL computer code to perform a set of sensitivity calculations. These calculations studied the effects of various welding and inspection parameters on the predicted numbers and sizes of flaws in representative nuclear-piping welds. Table 2.2 lists the parameters for the sensitivity calculations. 
Table 2.2 Piping Welds Considered in the Sensitivity Analysis

\begin{tabular}{|c|c|c|c|c|c|c|}
\hline Case Number & 1 & 2 & $2 a$ & 3 & 4 & 5 \\
\hline Wall Thickness, in. (mm) & $1.00(25.4)$ & $0.25(6.35)$ & $0.25(6.35)$ & $2.5(63.5)$ & $1.00(25.4)$ & $1.00(25.4)$ \\
\hline Pipe Material & Stainless steel & Stainless steel & Stainless steel & Stainless steel & Stainless steel & Stainless steel \\
\hline Welding Process & Manual metal arc & Manual metal arc & Manual metal arc & Manual metal arc & Manual metal arc & Manual metal arc \\
\hline Inspection & RT; PT of OD & No RT; PT of OD & No RT; PT of OD & RT; PT of OD & No RT; no PT of OD & RT; PT of OD \\
\hline Welding Circumstances & $\begin{array}{l}\text { Controlled site; } \\
\text { average access } 6 \mathrm{G} \\
\left(45^{\circ} \text { fix pipe }\right)\end{array}$ & $\begin{array}{l}\text { Controlled site: } \\
\text { average access } 6 \mathrm{G} \\
\left(45^{\circ} \text { fix pipe }\right)\end{array}$ & $\begin{array}{l}\text { Controlled site; } \\
\text { average access } 6 \mathrm{G} \\
\left(45^{\circ} \text { fix pipe }\right)\end{array}$ & $\begin{array}{l}\text { Controlled site; } \\
\text { average access } 6 \mathrm{G} \\
\left(45^{\circ} \text { fix pipe }\right)\end{array}$ & $\begin{array}{l}\text { General site; } \\
\text { restricted access } 4 \mathrm{G} \\
\text { (overhead) ferrite } \\
\text { uncontrolled }\end{array}$ & $\begin{array}{l}\text { Controlled shop; } \\
\text { good access } 1 \mathrm{G} \\
\text { (down) }\end{array}$ \\
\hline Inner Diameter, in. (mm.) & $8.00(203)$ & $3.00(76)$ & $3.00(76)$ & $25.00(635)$ & $8.00(203)$ & $8.00(203)$ \\
\hline Weld Layers & 10 & 2 & 3 & 24 & 10 & 10 \\
\hline Weld Runs & 44 & 3 & 6 & 178 & 44 & 44 \\
\hline Total Defects/m (RRA) & 1.008 & 1.64 & 6.52 & 7.9 & 18.81 & 0.684 \\
\hline ID Surface Defects/m (RRA) & 0.0152 & 0.0563 & 0.0749 & 0.0174 & 0.0804 & 0.0051 \\
\hline Total Defects/in. (m) & $0.0277(1.091)$ & $0.044(1.732)$ & $0.175(6.890)$ & $0.214(8.425)$ & $0.517(20.354)$ & $0.0188(0.740)$ \\
\hline Total Defects/Weld & 0.697 & 0.414 & 1.649 & 16.810 & 13.009 & 0.473 \\
\hline $\begin{array}{l}\text { Weld Layer Thickness, } \\
\text { in. }(\mathrm{mm})\end{array}$ & $0.100(2.5)$ & $0.060(1.5)$ & $0.110(2.8)$ & $0.104(2.6)$ & $0.100(2.5)$ & $0.100(2.5)$ \\
\hline ID Surface Defects/Weld & $1.05 \mathrm{E}-02$ & $1.42 \mathrm{E}-02$ & $1.89 \mathrm{E}-02$ & $3.70 \mathrm{E}-02$ & $5.56 \mathrm{E}-02$ & $3.53 \mathrm{E}-03$ \\
\hline $\begin{array}{l}\text { ID Surface Defects/in. (m) of } \\
\text { Weld }\end{array}$ & $4.18 \mathrm{E}-04(1.65 \mathrm{E}-02)$ & $1.51 \mathrm{E}-03(5.94 \mathrm{E}-02)$ & $2.01 \mathrm{E}-03(7.91 \mathrm{E}-02)$ & 4.71E-04 (1.20E-02) & $2.21 \mathrm{E}-03(8.70 \mathrm{E}-02)$ & $1.40 \mathrm{E}-04(5.51 \mathrm{E}-03)$ \\
\hline $\begin{array}{l}\text { No. of Runs in First Two } \\
\text { Layers }\end{array}$ & 3 & 3 & 3 & 3 & 3 & 3 \\
\hline $\begin{array}{l}\text { Defects/in. (m) of Weld } \\
\text { Including Inner Surface \& } \\
\text { First Two Weld Runs }\end{array}$ & $1.62 \mathrm{E}-03(6.38 \mathrm{E}-04)$ & $3.96 \mathrm{E}-02(1.56 \mathrm{E}+00)$ & $7.87 \mathrm{E}-02(3.10 \mathrm{E}+00)$ & $3.18 \mathrm{E}-03(1.25 \mathrm{E}-01)$ & $3.03 \mathrm{E}-02(1.19 \mathrm{E}+00)$ & $1.10 \mathrm{E}-03(4.33 \mathrm{E}-02)$ \\
\hline All Flaws/in. ${ }^{3}\left(\mathrm{~m}^{3}\right)$ & $1.28 \mathrm{E}-02(780)$ & $8.47 \mathrm{E}-02(5170)$ & 3.37E-01 (2060) & $4.06 \mathrm{E}-02(2480)$ & $2.40 \mathrm{E}-01(14,650)$ & $8.72 \mathrm{E}-03(530)$ \\
\hline Inner Region Flaws/in. ${ }^{3}\left(\mathrm{~m}^{3}\right)$ & $1.94 \mathrm{E}-04(12)$ & $2.90 \mathrm{E}-03(180)$ & $3.86 \mathrm{E}-03(240)$ & $8.93 \mathrm{E}-05(5.4)$ & $1.02 \mathrm{E}-03(62)$ & $6.50 \mathrm{E}-05(4.0)$ \\
\hline
\end{tabular}


Table 2.2 Piping Welds Considered in the Sensitivity Analysis (cont'd)

\begin{tabular}{|c|c|c|c|c|c|c|}
\hline Case Number & 6 & $6 a$ & 7 & 8 & 9 & 10 \\
\hline Wall Thickness, in. (mm.) & $1.00(25.4)$ & $1.00(25.4)$ & $1.00(25.4)$ & $1.00(25.4)$ & $1.00(25.4)$ & $1.00(25.4)$ \\
\hline Pipe Material & Stainless steel & Stainless steel & Stainless steel & Stainless steel & Stainless steel & Stainless steel \\
\hline Welding Process & TIG & TIG & Manual metal arc & Manual metal arc & Manual metal arc & Manual metal arc \\
\hline Inspection & RT; PT of OD & RT; PT of OD & RT; PT of OD & RT; No PT of OD & No RT; PT of OD & No RT; No PT of OD \\
\hline Welding Circumstances & $\begin{array}{l}\text { Controlled site; } \\
\text { average access } 6 \mathrm{G} \\
\left(45^{\circ} \text { fix pipe }\right)\end{array}$ & $\begin{array}{l}\text { Controlled site: } \\
\text { average access } 6 \mathrm{G} \\
\left(45^{\circ} \text { fix pipe }\right)\end{array}$ & $\begin{array}{l}\text { Controlled site; } \\
\text { average access } 6 \mathrm{G} \\
\left(45^{\circ} \text { fix pipe }\right)\end{array}$ & $\begin{array}{l}\text { Controlled site; } \\
\text { average access } 6 \mathrm{G} \\
\left(45^{\circ} \text { fix pipe }\right)\end{array}$ & $\begin{array}{l}\text { Controlled site; } \\
\text { average access } 6 \mathrm{G} \\
\left(45^{\circ} \text { fix pipe }\right)\end{array}$ & $\begin{array}{l}\text { Controlled site; } \\
\text { average access } 6 \mathrm{G} \\
\left(45^{\circ} \text { fix pipe }\right)\end{array}$ \\
\hline Inner Diameter, in. (mm.) & $8.00(203)$ & $8.00(203)$ & $8.00(203)$ & $8.00(203)$ & $8.00(203)$ & $8.00(203)$ \\
\hline Weld Layers & 12 & 12 & 10 & 10 & 10 & 10 \\
\hline Weld Runs & 41 & 46 & 44 & 44 & 44 & 44 \\
\hline Total Defects/m (RRA) & 0.923 & 1.007 & 1.049 & 1.129 & 12.75 & 13.80 \\
\hline ID Surface Defects/m (RRA) & 0.0158 & 0.0156 & 0.0154 & 0.0152 & 0.047 & 0.047 \\
\hline Total Defects/ in. (m) & $0.0254(1.000)$ & $0.0277(1.091)$ & $0.0289(1.138)$ & $0.0311(1.224)$ & $0.3508(13.811)$ & $0.379(14.921)$ \\
\hline Total Defects/Weld & 0.638 & 0.696 & 0.725 & 0.781 & 8.817 & 9.546 \\
\hline $\begin{array}{l}\text { Weld Layer Thickness, } \\
\text { in. (mm.) }\end{array}$ & $0.083(2.1)$ & $(0.083(2.1)$ & $0.10(2.5)$ & $0.10(2.5)$ & $\begin{array}{l}0.25(6.35) \\
0.10(2.5)\end{array}$ & $\begin{array}{l}0.13(3.3) \\
0.10(2.5)\end{array}$ \\
\hline ID Surface Defects/Weld & $1.09 \mathrm{E}-02$ & $1.08 \mathrm{E}-02$ & $1.07 \mathrm{E}-02$ & $1.05 \mathrm{E}-02$ & $3.25 \mathrm{E}-02$ & $3.25 \mathrm{E}-02$ \\
\hline $\begin{array}{l}\text { ID Surface Defects/in. (m) of } \\
\text { Weld }\end{array}$ & 4.35E-04 (1.71E-02) & 4.29E-04 (1.69E-02) & 4.24E-04 (1.08E-02) & $4.18 \mathrm{E}-04(1.06 \mathrm{E}-02)$ & $1.29 \mathrm{E}-03(5.08 \mathrm{E}-02)$ & $1.29 \mathrm{E}-03(5.08 \mathrm{E}-02)$ \\
\hline $\begin{array}{l}\text { No. of Runs in First Two } \\
\text { Layers }\end{array}$ & 3 & 3 & 3 & 3 & 3 & 3 \\
\hline $\begin{array}{l}\text { Defects/in. (m) of Weld } \\
\text { including Inner Surface \& } \\
\text { First Two Weld Runs }\end{array}$ & $1.59 \mathrm{E}-03(6.26 \mathrm{E}-02)$ & $1.55 \mathrm{E}-03(6.10 \mathrm{E}-02)$ & $1.68 \mathrm{E}-03(6.61 \mathrm{E}-02)$ & $1.82 \mathrm{E}-03(7.16 \mathrm{E}-02)$ & $2.05 \mathrm{E}-02(8.07 \mathrm{E}-01)$ & $2.22 \mathrm{E}-03$ (8.74E-02) \\
\hline All Flaws per in. ${ }^{3}\left(\mathrm{~m}^{3}\right)$ & $1.18 \mathrm{E}-02(720)$ & $1.28 \mathrm{E}-02(780)$ & $1.34 \mathrm{E}-02(820)$ & $1.44 \mathrm{E}-02(880)$ & $1.63 \mathrm{E}-01(9,950)$ & $1.76 \mathrm{E}-01(10,740)$ \\
\hline $\begin{array}{l}\text { Inner Region Flaws per in. }{ }^{3} \\
\left(\mathrm{~m}^{3}\right)\end{array}$ & $2.01 \mathrm{E}-04(12)$ & 1.99E-04 (12) & $1.96 \mathrm{E}-04(12)$ & 1.94E-04 (12) & 5.99E-04 (36) & 5.99E-04 (36) \\
\hline
\end{tabular}


Table 2.2 Piping Welds Considered in the Sensitivity Analysis (cont'd)

\begin{tabular}{|c|c|c|c|c|}
\hline Case Number & 11 & 11a & 12 & $12 r$ \\
\hline Wall Thickness, in. (mm.) & $0.25(6.35)$ & $0.25(6.35)$ & $2.5(6.35)$ & $2.5(63.5)$ \\
\hline Pipe Material & Stainless steel & Stainless steel & Ferritic steel & Ferritic steel \\
\hline Welding Process & TIG & Manual metal arc & TIG & Manual metal arc \\
\hline Inspection & RT; PT of OD & RT; PT of OD & RT; PT of OD & RT; PT of OD \\
\hline Welding Circumstances & $\begin{array}{l}\text { Controlled site; } \\
\text { average access } 6 \mathrm{G} \\
\left(45^{\circ} \text { fix pipe }\right)\end{array}$ & $\begin{array}{l}\text { Controlled site: } \\
\text { average access } 6 \mathrm{G} \\
\left(45^{\circ} \text { fix pipe }\right)\end{array}$ & $\begin{array}{l}\text { Controlled site; } \\
\text { average access } 6 \mathrm{G} \\
\left(45^{\circ} \text { fix pipe }\right)\end{array}$ & $\begin{array}{l}\text { Controlled site; } \\
\text { average access } 6 \mathrm{G} \\
\left(45^{\circ} \text { fix pipe }\right)\end{array}$ \\
\hline Inner Diameter, in. (mm.) & $3.00(76)$ & $3.00(76)$ & $25.00(635)$ & $25.00(635)$ \\
\hline Weld Layers & 3 & 4 & 24 & 24 \\
\hline Weld Runs & 5 & 8 & 178 & 178 \\
\hline Total Defects/m (RRA) & 4.237 & 6.749 & 7.083 & 8.097 \\
\hline ID Surface Defects/m (RRA) & 0.1080 & 0.1080 & 0.0175 & 0.0175 \\
\hline Total Defects/in. (m) & $0.0029(0.1136)$ & $0.0046(0.1809)$ & $0.0049(0.1919)$ & $0.0056(0.2194)$ \\
\hline Total Defects/Weld & 1.071 & 1.705 & 15.07 & 17.22 \\
\hline Weld Layer Thickness, in. (mm.) & $0.0833(2.1)$ & $0.0625(1.6)$ & $0.1042(2.6)$ & $0.1042(2.6)$ \\
\hline ID Surface Defects/Weld & $2.73 \mathrm{E}-02$ & $2.73 \mathrm{E}-02$ & $3.72 \mathrm{E}-02$ & $3.72 \mathrm{E}-02$ \\
\hline $\begin{array}{l}\text { ID Surface Defects/in. (m) of } \\
\text { Weld }\end{array}$ & $2.90 \mathrm{E}-03(1.14 \mathrm{E}-01()$ & $2.90 \mathrm{E}-03(1.14 \mathrm{E}-01)$ & 4.74E-04 (1.87E-02) & 4.74E-04 (1.87E-02) \\
\hline No. of Runs in First Two layers & 3 & 3 & 3 & 3 \\
\hline $\begin{array}{l}\text { Defects/in. (m) of Weld including } \\
\text { Inner Surface \& First Two Weld } \\
\text { Runs }\end{array}$ & $6.13 \mathrm{E}-02(2.41 \mathrm{E}+00)$ & $6.11 \mathrm{E}-02(2.41 \mathrm{E}+00)$ & $2.85 \mathrm{E}-03(1.12 \mathrm{E}-01)$ & $3.26 \mathrm{E}-03(1.28 \mathrm{E}-01)$ \\
\hline All Flaws/in. ${ }^{3}\left(\mathrm{~m}^{3}\right)$ & $2.19 \mathrm{E}-01(13,360)$ & $3.48 \mathrm{E}-01(21,240)$ & $3.64 \mathrm{E}-02(2,220)$ & $4.16 \mathrm{E}-02(2,540)$ \\
\hline Inner Region Flaws/in. ${ }^{3}\left(\mathrm{~m}^{3}\right)$ & $5.57 \mathrm{E}-03(304)$ & $5.57 \mathrm{E}-03(340)$ & $8.98 \mathrm{E}-05(5.5)$ & $8.98 \mathrm{E}-05(5.5)$ \\
\hline
\end{tabular}


The selection of welds was guided in part by the needs of a pilot application of risk-informed ISI methods to the Surry Unit 1 Power Station (Shah et al. 1997; Bishop 1997). Discussions with the technical staff at Virginia Power defined an initial set of cases. This set of cases was then supplemented to include additional weld parameters to encompass a larger population of welds representative of nuclear-piping systems. Limiting cases were selected to bound the welds of interest and/or to enable trends of flawdistribution parameters to be established over a very wide range of welding conditions.

The cases of Table 2.2 cover the following ranges of parameters:

- pipe-wall thickness: 0.25 in. $(6.35 \mathrm{~mm}), 1.0$ in. $(25.4 \mathrm{~mm})$, and 2.5 in. $(63.5 \mathrm{~mm})$

- pipe material: ferritic and austenitic stainless steels

- weld process: MMA and TIG

- inspection: no inspection, radiography, and dye penetrant

- welding conditions: shop weld and field welds; difficult and easy conditions of access

The primary objective was not to address specific welds of the Surry-1 plant, but rather to cover the range of parameters relevant to Surry-1 and other plants of interest to risk-informed ISI. It should be noted that piping diameter was not included in the list of input parameters. This was because all results were normalized to the number of defects per inch of completed weld, which made the pipe wall thickness the critical dimensional parameter.

For each pipe-wall thickness and each welding process, the calculations required inputs for the number of weld layers and number of weld beads that make up the completed multi-pass weld. Based on discussions with Surry-1 technical staff and with the RRA welding specialist, values were selected to be typical of practices used for nuclear piping. For the thinner wall piping $(0.25$-in. [6.35 mm] wall), there were as few as 2 weld layers, whereas for the thicker wall piping $(2.5$-in. [63.5 mm] wall), there were as many as 24 weld layers.

It was assumed that TIG welding was always used for the root pass (located at the inner surface of the pipe), even for MMA welds. In general, TIG welding is used for thinner walled pipe, whereas MMA is practiced for thicker walled pipe. However, to establish parametric trends, the TIG and MMA processes were both addressed by calculations for all ranges of pipe-wall thickness.

In practice, radiography is usually performed only for welds in thicker walled piping. However, the present calculations addressed cases of radiography for very thin-walled piping, again for purposes of establishing generic trends. It is recommended that the effects of radiography should generally be discounted for smaller pipe sizes. 


\subsection{Results of Sensitivity Study}

Outputs from the RR-PRODIGAL simulation model included information on simulated flaw depths and flaw locations within the pipe wall. To create histograms, the simulated flaw population is decomposed into a set of bins that is dispatched as tables to a printer for hardcopy output. PNNL used this output file in post processing. All outputs were in the form of frequency distributions that indicated the number of flaws in each category per finished weld (after inspections and weld repairs).

Flaw categories were described first by their through-wall locations (i.e., location of the inner flaw tips relative to pipe inner surface). The first two categories were inner-surface breaking defects and nearsurface flaws that are located within the inner $1 / 4$ of the wall thickness. The other categories were embedded flaws corresponding to the middle 50 percent of the wall and the outer $1 / 4$ of the wall. The final category was that of outer surface-breaking flaws. For each category of flaw location, the number of flaws of given categories of depth dimensions defined by 1.0 percent increments of the pipe wall thickness were listed. Output files from the weld-flaw simulations as performed by RRA were provided to PNNL. The results are summarized in Table 2.3 in terms of flaws per meter of weld, both for innersurface breaking flaws and for all flaws, regardless for their location within the wall thickness.

Table 2.3 Flaw Occurrence in Different Regions of the Wall (Flaws per Meter of Weld)

\begin{tabular}{|c|c|c|c|c|c|c|}
\hline \multirow{2}{*}{$\begin{array}{c}\text { Case } \\
\text { Number }\end{array}$} & $\begin{array}{c}\text { Inner } \\
\text { Surface }\end{array}$ & $\begin{array}{c}\text { Embedded } \\
\text { Inner 1/4 }\end{array}$ & $\begin{array}{c}\text { Embedded } \\
\text { Center } \mathbf{1} / \mathbf{2}\end{array}$ & $\begin{array}{c}\text { Embedded } \\
\text { Outer 1/4 }\end{array}$ & $\begin{array}{c}\text { Outer } \\
\text { Surface }\end{array}$ & Total \\
\hline 1 & 0.0153 & 0.1028 & 0.5252 & 0.3605 & 0.0040 & 1.0078 \\
\hline 2 & 0.1897 & 0.1522 & 0.0332 & 1.2472 & 0.0247 & 1.6406 \\
\hline $2 \mathrm{a}$ & 0.1821 & 0.1996 & 2.0802 & 1.9014 & 0.0351 & 4.398 \\
\hline 3 & 0.0174 & 0.8572 & 4.1551 & 2.8582 & 0.0104 & 7.8983 \\
\hline 4 & 0.0850 & 2.0528 & 8.4715 & 7.0304 & 1.1741 & 18.8093 \\
\hline 5 & 0.0051 & 0.0704 & 0.3703 & 0.2347 & 0.0034 & 0.6839 \\
\hline 6 & 0.0159 & 0.104 & 0.5206 & 0.2799 & 0.0033 & 0.9236 \\
\hline $6 \mathrm{a}$ & 0.0157 & 0.1009 & 0.5840 & 0.3022 & 0.0038 & 1.0066 \\
\hline 7 & 0.0154 & 0.1097 & 0.5427 & 0.3768 & 0.0040 & 1.0066 \\
\hline 8 & 0.0152 & 0.1018 & 0.5237 & 0.3624 & 0.1256 & 1.1288 \\
\hline 9 & 0.047 & 0.1476 & 6.2614 & 4.9407 & 0.0277 & 12.7528 \\
\hline 10 & 0.0471 & 1.4768 & 6.2582 & 4.937 & 1.0834 & 13.8025 \\
\hline 11 & 0.1080 & 0.1397 & 2.3923 & 1.5725 & 0.0236 & 4.2361 \\
\hline $11 \mathrm{a}$ & 0.1080 & 0.2421 & 4.2286 & 2.1314 & 0.0393 & 6.7496 \\
\hline 12 & 0.01759 & 0.8818 & 4.2439 & 2.9426 & 0.0103 & 7.0830 \\
\hline $12 \mathrm{r}$ & 0.01759 & 0.7735 & 3.7100 & 2.5733 & 0.0094 & 8.0970 \\
\hline $1 \mathrm{~m}=39.4$ inches & & & & \\
\hline
\end{tabular}




\subsection{Defect Size Distributions}

The output of the RRA simulations of weld defects provided detailed data on flaw-occurrence frequencies and size distributions, which could be used as inputs for calculations of piping-failure probabilities. While the simulations uniquely size and position every defect, this information is too detailed for most PFM codes. In this regard, the flaws from RRA simulations are categorized into five types: surfacebreaking inner, surface-breaking outer, buried near inner surface, buried near outer surface, and buried middle. However, many PFM codes, such as pc-PRAISE and SRRA (Bishop 1997) need only a single statistical distribution for crack depths because all flaws are assumed to be inner-surface flaws.

It was originally desired to use statistical fitting to characterize the cracks that are inner-surface breaking along with the cracks whose inner tips are within the first two weld layers of the inner surface. However, the RRA outputs did not provide sufficient detail to allow this, since the crack locations are lumped into the five broad categories. It was therefore decided to characterize the crack-depth distributions by combining flaws from all regions and to estimate the number of flaws located within the inner two weld layers. It was assumed that the calculation by the PFM code would treat all of these flaws as inner surface-breaking cracks.

\subsubsection{Procedure for Fitting of Statistical Distributions}

The outputs from the RRA code were used directly to tabulate and plot complementary cumulative distributions of crack depths. Several statistical distributions were then considered for fitting this complementary distribution, such as the normal, Weibull, and lognormal. Figure 2.3 shows an example complementary distribution of flaw depths plotted on normal distribution probability paper, which indicates, by a lack of a linear trend, that a normal distribution is a poor approximation of the data. The Weibull distribution was also tried. The fit resulted in a shape parameter of 6 , which results in too small a variation in crack size. Since the normal and Weibull distributions failed to fit the data very well, the lognormal distribution was considered. The following alternate methods can be used to evaluate parameters of the lognormal distribution:

- Calculate the means and standard deviations directly from the data and then calculate the lognormal parameters, $\mathrm{a}_{50}$ (median) and $\mu$, from the characteristics of a lognormal distribution, where $\mu$ is the standard deviation of the natural logarithm of the crack depth.

- Calculate the mean and standard deviation of the logarithm of the data, from which the parameters of the distribution can be obtained.

- Calculate the values of $\mathrm{a}_{50}$ and $\mu$ by a least-squares fit to the logarithm of the complementary cumulative distribution.

Each of these methods was applied, and they each gave somewhat different results. Since the tail of the distribution for deep flaws has a very large influence on calculated piping-failure probabilities, the desired fitting procedure had to be accurate for deep cracks. This led PNNL to select the third method (i.e., least squares). 


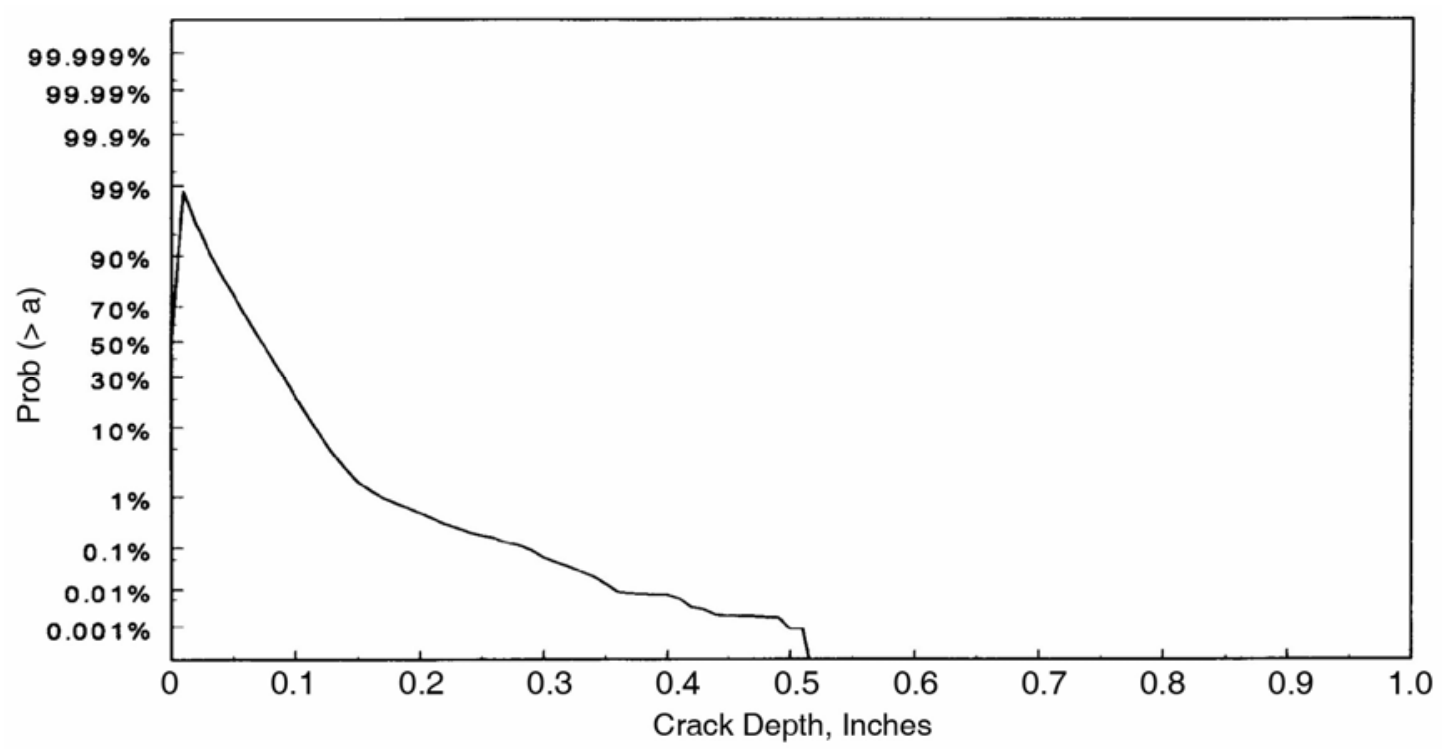

Figure 2.3 Complimentary Distribution of Flaw Depth on Normal Probability Paper for Case 1 (1.0-in. [25.4 mm] wall stainless steel, manual metal arc weld, with inspection) $(1$ inch $=25.4 \mathrm{~mm})$

The details of the selected least-squares method are as follows, beginning with the definition and the probability density of the lognormal distribution.

$$
p(a)=\frac{1}{\mu a \sqrt{2 \pi}} e^{\frac{-\left[\ln \left(a / a_{50}\right)\right]^{2}}{2 \mu^{2}}}
$$

where $\mathrm{a}_{50}$ is the median crack depth. The average crack depth $\left(\mathrm{a}_{\mathrm{ave}}\right)$ and standard deviation of the crack depth $\left(\mathrm{a}_{\mathrm{sd}}\right)$ are given in terms of $\mu$ and $\mathrm{a}_{50}$ by the following expressions:

$$
\begin{gathered}
\mu=\sqrt{\ln \left(1+\operatorname{cov}^{2}\right)} \\
\operatorname{cov}=\frac{a_{s d}}{a_{a v e}} \\
a_{50}=a_{\text {ave }} e^{-\frac{\mu^{2}}{2}}
\end{gathered}
$$

These relations follow from the properties of the lognormal distribution. The parameter $\mu$ is also the standard deviation of $\ln (a)$, and the median can be calculated as exp [mean of $\ln (a)$ ]. Hence, there are two ways to estimate the parameters directly from the data. However, the preferred method is to do a leastsquares fit to a cumulative plot of the data on lognormal probability paper. 
Before selecting the least-squares approach as the preferred method, the parameters of the log-normal distribution were calculated using, as possible alternatives, the method of moments and the maximumlikelihood method, along with the least-squares-fit method. The parameters obtained using the maximum-likelihood method provided a poor fit to the data. The method of moments consistently provided a lower shape parameter than the maximum-likelihood method and gave a better fit than the maximum-likelihood method. The method of moments gives equal weight to each data point. It provided undesirably high probabilities in the upper tail of the data. Therefore, the least-squares method was used to solve the following:

Find $\mu$ and $\mathrm{a}_{50}$ such that

$$
\sum_{i=1}^{100}\left\{\log \left(\frac{\frac{1}{2} \operatorname{erfc}\left[\frac{1}{\mu \sqrt{2}} \ln \left\langle\frac{a}{a_{50}}\right\rangle\right]}{P_{\text {actual }}[>0]}\right)\right\}^{2}=\text { minimum }
$$

The idea in the least-square method is to minimize the error and obtain the global minimum. In practice, the results appeared to be sensitive to the choice of the initial values of $\mu$ and $a_{50}$ used for the numerical search process, which indicated that there are several local minimum values to the above functions.

\subsubsection{Resulting Lognormal Flaw-Depth Distributions}

The outputs from the RRA code were first used directly to determine the complementary distribution of crack depths. Figure 2.4 was obtained by summing up the contents of the bins in the output tables for Case 1. This data distribution is not smooth, which is due to modeling assumptions and the finite number of Monte Carlo trails used in the computation. Figure 2.4 also provides fitting results that were obtained using the least-square procedure. The fit is relatively good given the lack of smoothness of the data. The parameters of the distribution are shown in the upper right corner of the plot.

The complementary distribution of the raw data and the lognormal distribution fit for several representative cases are shown in the plots of Figures 2.4 through 2.9. The results of the data fitting are summarized in Table 2.4. This table lists the pipe-wall thickness, the inner pipe diameter, the weld-layer thickness, and the mean, the standard deviation, the median, and the shape parameter of flaw-depth distribution for all cases addressed by the RRA calculations.

\subsubsection{Effects of Weld Process on Depth Distribution}

Figure 2.10 addresses the wall thickness of 1 in. $(25.4 \mathrm{~mm})$, and compares results for the baseline Case \#1 (stainless steel MMA weld with inspection) with other results obtained by taking variations for the welding parameters relative to those of the baseline case. The depth distributions are relatively insensitive to the welding parameters for small flaws, but become increasingly sensitive to welding parameters as the flaw depths become larger. The following trends are evident: 


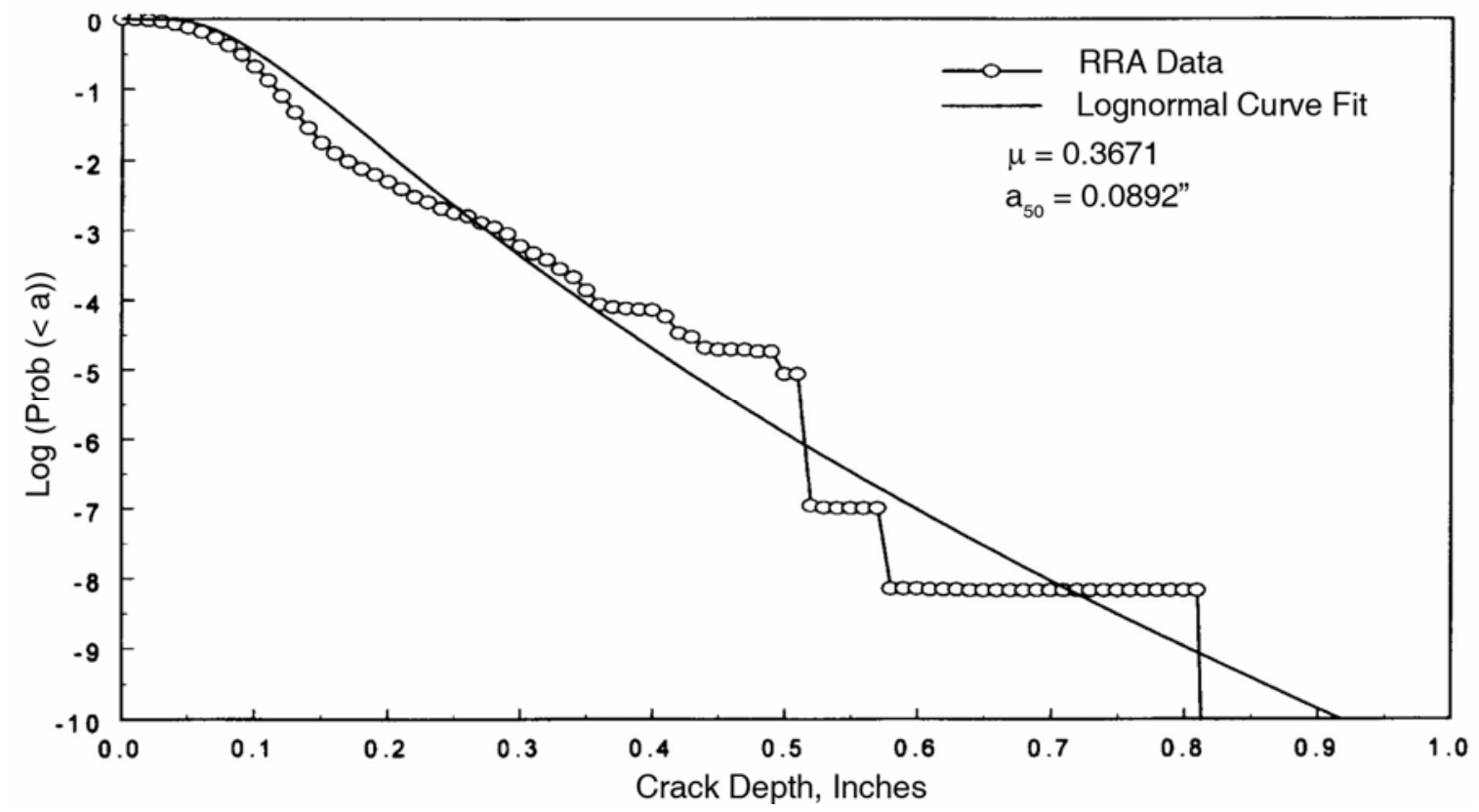

Figure 2.4 Lognormal Complimentary Distribution of Flaw Depth for Case 1 (1.0-in. [25.4 mm] wall stainless steel, manual metal arc weld, with inspection) $(1 \mathrm{inch}=25.4 \mathrm{~mm})$

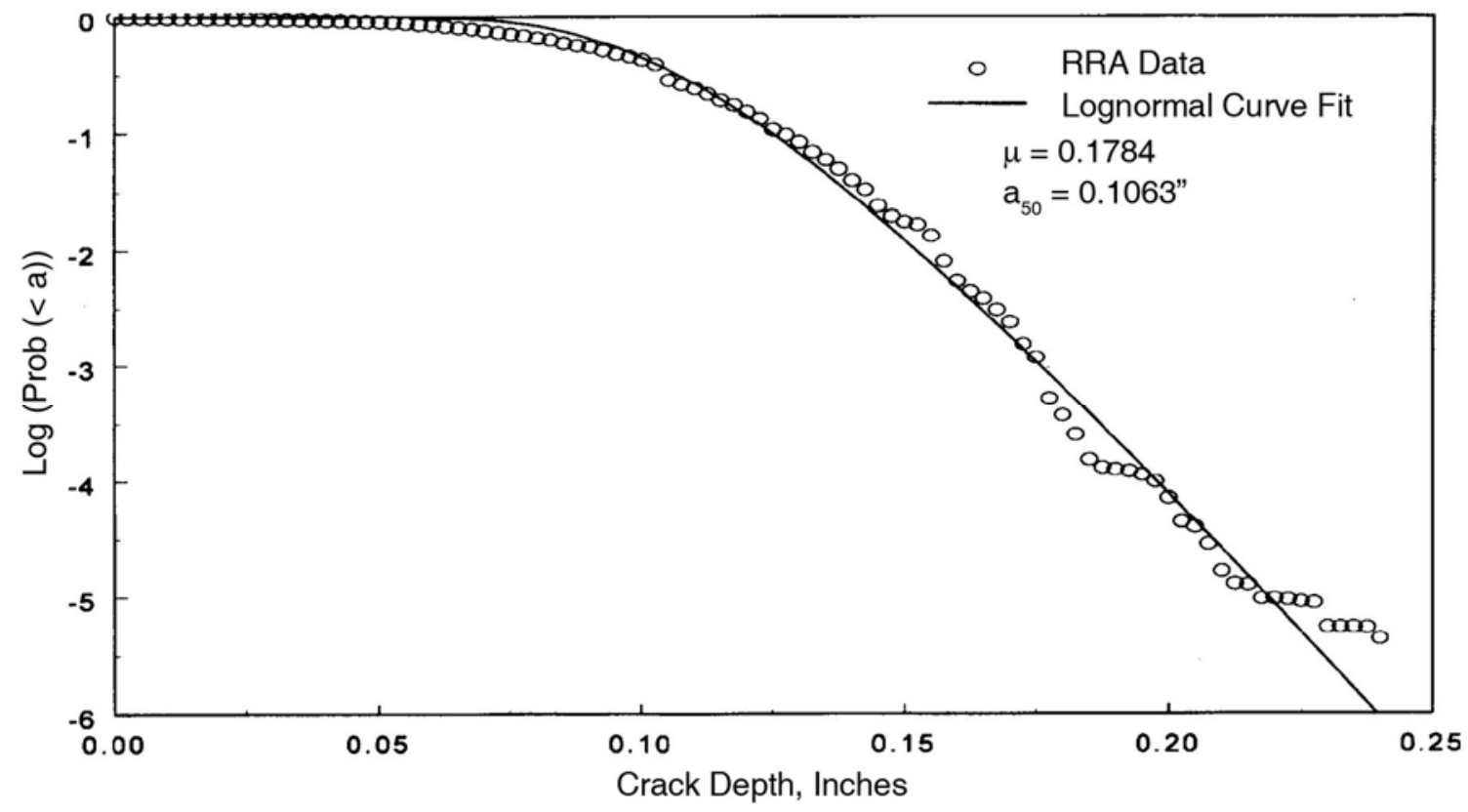

Figure 2.5 Lognormal Complimentary Distribution of Flaw Depth for Case 2 (0.25-in. [6.35 mm] wall stainless steel, manual metal arc weld, with inspection) $(1 \mathrm{inch}=25.4 \mathrm{~mm})$ 


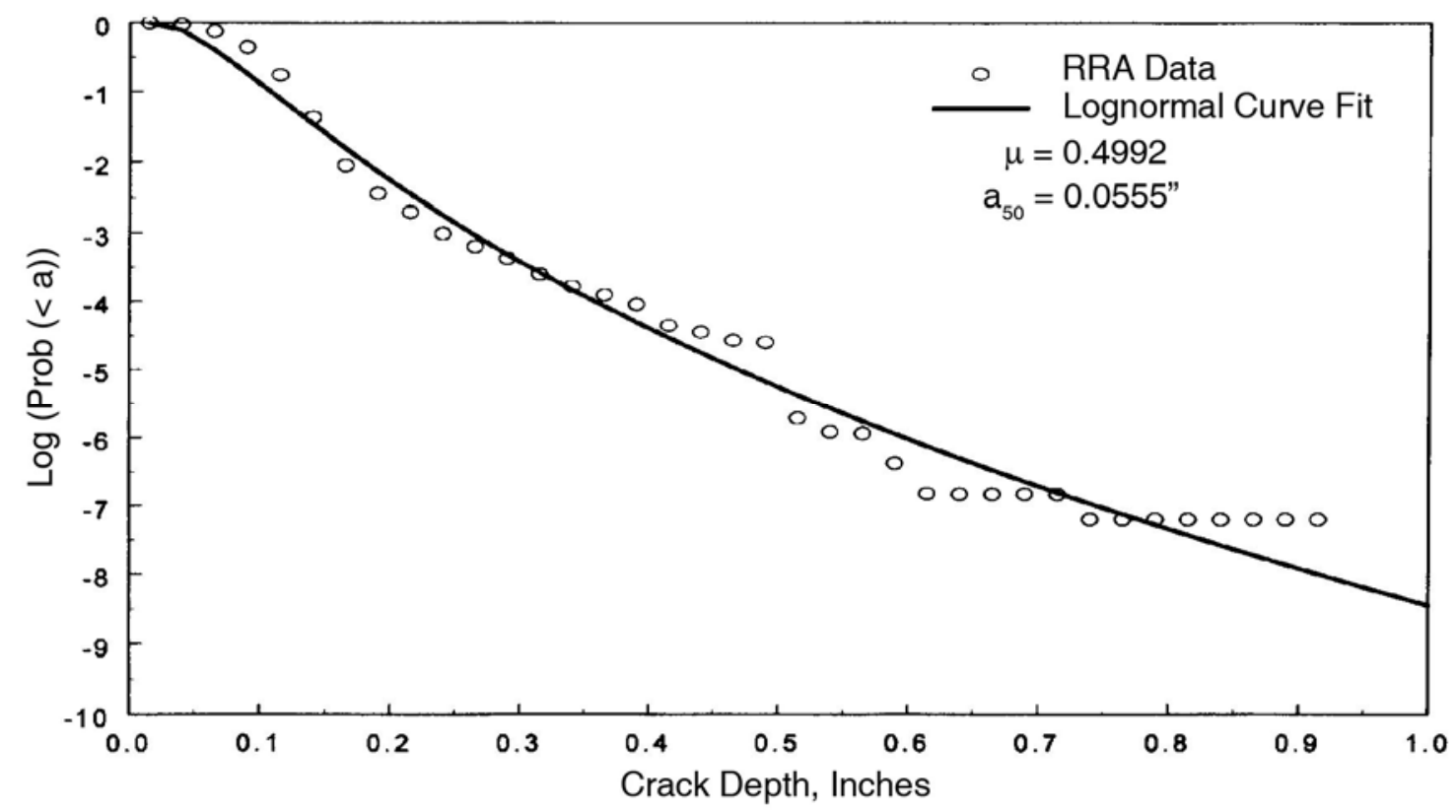

Figure 2.6 Lognormal Complimentary Distribution of Flaw Depth for Case 3 (2.5-in. [63.5 mm] wall stainless steel, manual metal arc weld, with inspection) $(1$ inch $=25.4 \mathrm{~mm}$ )

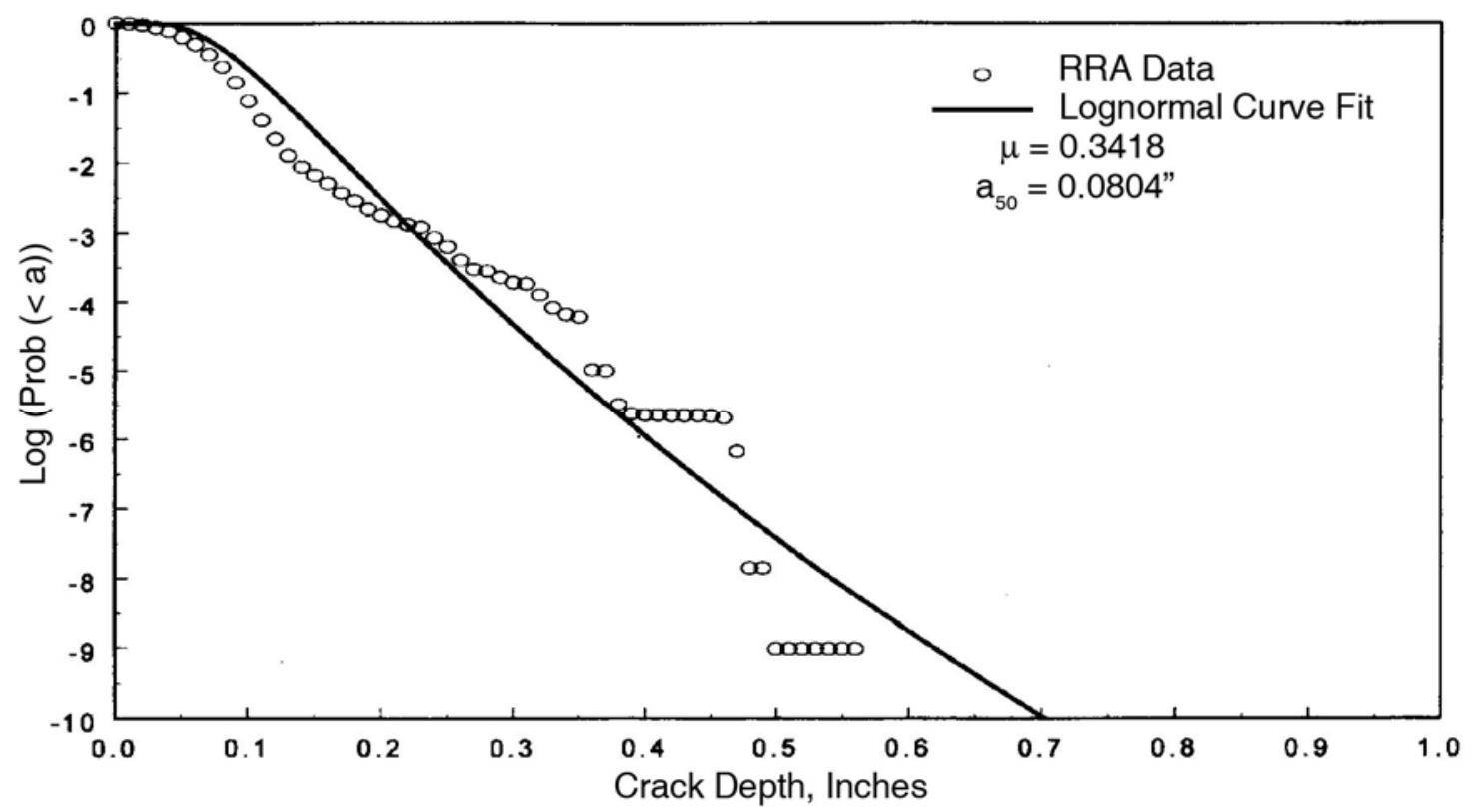

Figure 2.7 Lognormal Complimentary Distribution of Flaw Depth for Case 6 (1.0-in. [25.4 mm] wall stainless steel, tungsten inert gas weld, with inspection) $(1 \mathrm{inch}=25.4 \mathrm{~mm})$ 


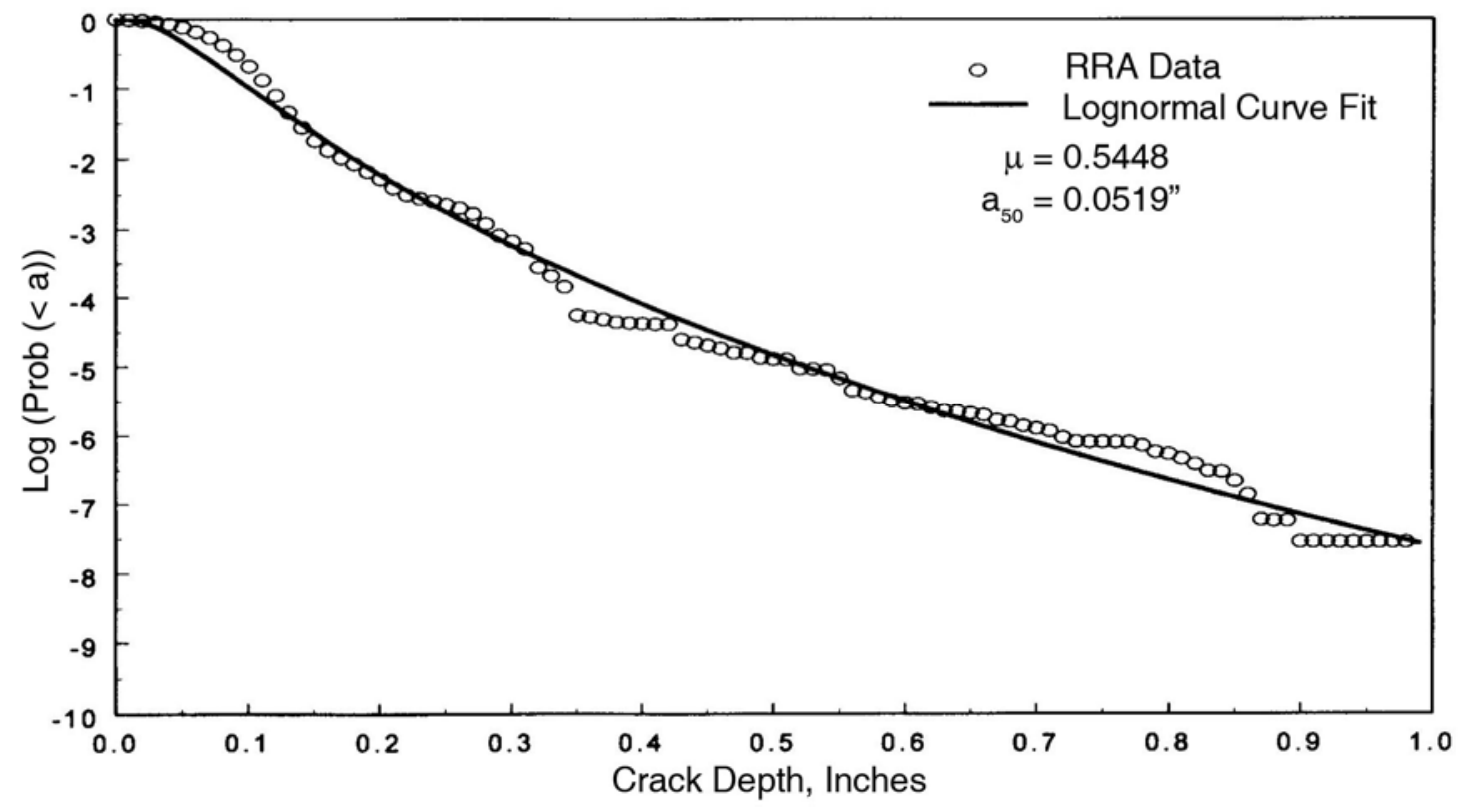

Figure 2.8 Lognormal Complimentary Distribution of Flaw Depth for Case 7 (1.0-in. [25.4 mm] wall stainless steel, manual metal arc weld, with inspection) $(1 \mathrm{inch}=\mathbf{2 5 . 4} \mathbf{~ m m})$

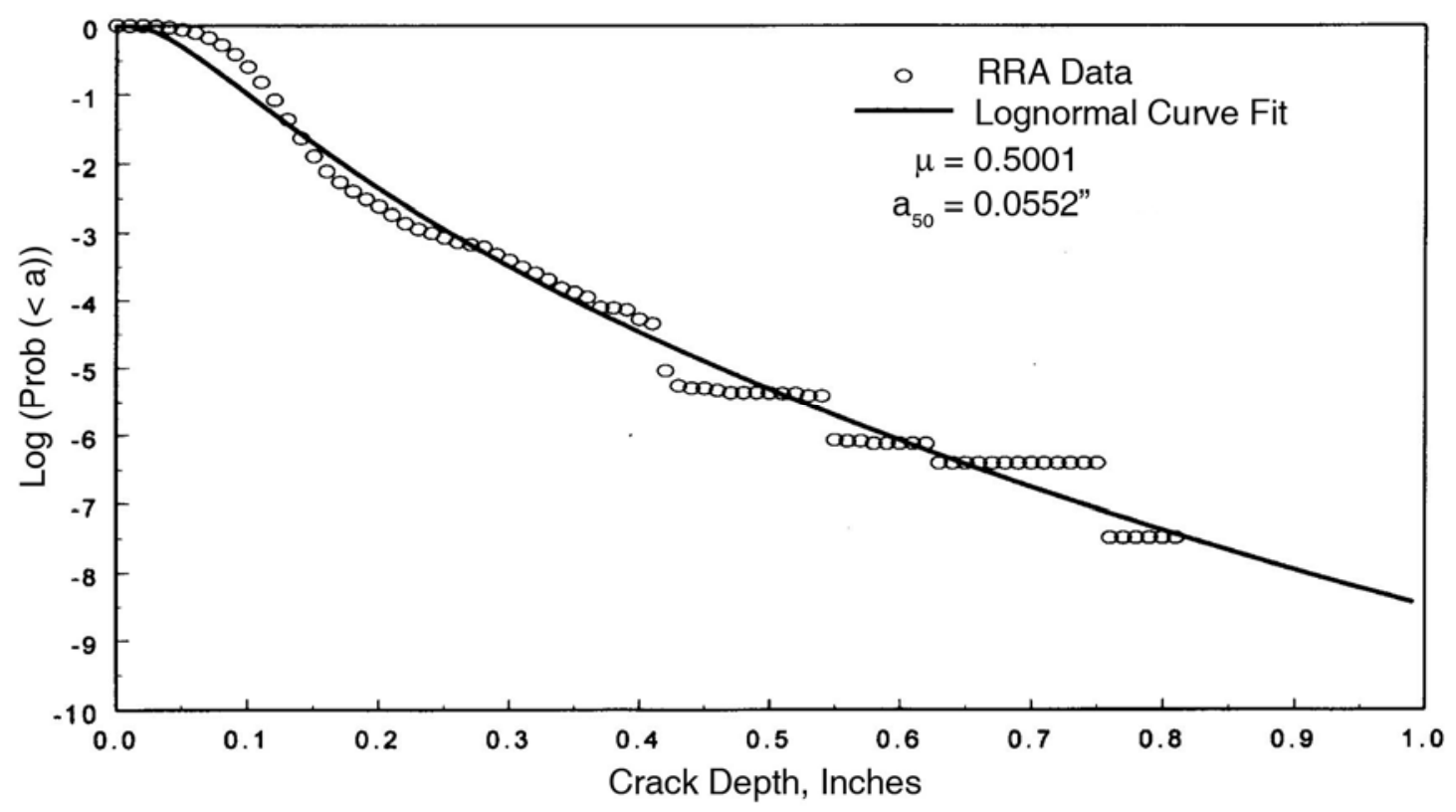

Figure 2.9 Lognormal Complimentary Distribution of Flaw Depth for Case 10 (1.0-in. [25.4 mm] wall stainless steel, manual metal arc weld, with inspection) $(1$ inch $=25.4 \mathrm{~mm})$ 
Table 2.4 Summary of Distribution Parameters

\begin{tabular}{|c|c|c|c|c|c|c|c|}
\hline $\begin{array}{c}\text { Case } \\
\text { Number }\end{array}$ & $\begin{array}{c}\text { Wall } \\
\text { Thickness, } \\
\text { in. (mm) }\end{array}$ & $\begin{array}{c}\text { Inner } \\
\text { Diameter, } \\
\text { in. }(\mathrm{mm})\end{array}$ & $\begin{array}{c}\text { Weld Layer } \\
\text { Thickness, } \\
\text { in. }(\mathrm{mm})\end{array}$ & $\begin{array}{l}\text { Mean Flaw } \\
\text { Depth, } \\
\text { in. }(\mathrm{mm})\end{array}$ & $\begin{array}{c}\text { Standard } \\
\text { Deviation, } \\
\text { in. }(\mathrm{mm})\end{array}$ & $\begin{array}{c}\text { Median } \\
\left(\mathbf{a}_{50}\right), \\
\text { in. }(\mathbf{m m})\end{array}$ & $\begin{array}{c}\text { Shape } \\
\text { Parameter } \\
(\mu)\end{array}$ \\
\hline 1 & $\begin{array}{c}1.00 \\
(25.4)\end{array}$ & $\begin{array}{c}8.00 \\
(203)\end{array}$ & $\begin{array}{l}0.100 \\
(2.54)\end{array}$ & $\begin{array}{l}0.095 \\
(2.42)\end{array}$ & $\begin{array}{c}0.036 \\
(0.92)\end{array}$ & $\begin{array}{l}0.089 \\
(2.26)\end{array}$ & 0.3671 \\
\hline 2 & $\begin{array}{c}0.25 \\
(6.35)\end{array}$ & $\begin{array}{l}3.00 \\
(76)\end{array}$ & $\begin{array}{l}0.125 \\
(3.17)\end{array}$ & $\begin{array}{l}0.109 \\
(2.77)\end{array}$ & $\begin{array}{l}0.022 \\
(0.58)\end{array}$ & $\begin{array}{l}0.106 \\
(2.70)\end{array}$ & 0.1784 \\
\hline $2 a$ & $\begin{array}{c}0.25 \\
(6.35) \\
\end{array}$ & $\begin{array}{l}3.00 \\
(76) \\
\end{array}$ & $\begin{array}{l}0.083 \\
(2.12)\end{array}$ & $\begin{array}{l}0.071 \\
(1.82)\end{array}$ & $\begin{array}{l}0.016 \\
(0.43)\end{array}$ & $\begin{array}{l}0.069 \\
(1.77) \\
\end{array}$ & 0.2593 \\
\hline 3 & $\begin{array}{c}2.50 \\
(63.5) \\
\end{array}$ & $\begin{array}{c}25.0 \\
(635) \\
\end{array}$ & $\begin{array}{l}0.104 \\
(2.65) \\
\end{array}$ & $\begin{array}{l}0.062 \\
(1.60) \\
\end{array}$ & $\begin{array}{l}0.033 \\
(0.85) \\
\end{array}$ & $\begin{array}{l}0.055 \\
(1.41) \\
\end{array}$ & 0.4992 \\
\hline 4 & $\begin{array}{c}1.00 \\
(25.4)\end{array}$ & $\begin{array}{l}8.00 \\
(203)\end{array}$ & $\begin{array}{l}0.100 \\
(2.54)\end{array}$ & $\begin{array}{l}0.084 \\
(2.15)\end{array}$ & $\begin{array}{l}0.035 \\
(0.89)\end{array}$ & $\begin{array}{l}0.078 \\
(1.99)\end{array}$ & 0.3977 \\
\hline 5 & $\begin{array}{c}1.00 \\
(25.4)\end{array}$ & $\begin{array}{l}8.00 \\
(203)\end{array}$ & $\begin{array}{l}0.100 \\
(2.54)\end{array}$ & $\begin{array}{l}0.072 \\
(1.85)\end{array}$ & $\begin{array}{l}0.035 \\
(0.90)\end{array}$ & $\begin{array}{l}0.065 \\
(1.66)\end{array}$ & 0.4606 \\
\hline 6 & $\begin{array}{c}1.00 \\
(25.4)\end{array}$ & $\begin{array}{l}8.00 \\
(203)\end{array}$ & $\begin{array}{l}0.083 \\
(2.12)\end{array}$ & $\begin{array}{l}0.085 \\
(2.16)\end{array}$ & $\begin{array}{l}0.030 \\
(0.76)\end{array}$ & $\begin{array}{l}0.080 \\
(2.04)\end{array}$ & 0.3418 \\
\hline $6 a$ & $\begin{array}{c}1.00 \\
(25.4)\end{array}$ & $\begin{array}{l}8.00 \\
(203)\end{array}$ & $\begin{array}{l}0.083 \\
(2.12)\end{array}$ & $\begin{array}{l}0.081 \\
(2.06)\end{array}$ & $\begin{array}{l}0.029 \\
(0.74)\end{array}$ & $\begin{array}{l}0.076 \\
(1.94)\end{array}$ & 0.3503 \\
\hline 7 & $\begin{array}{c}1.00 \\
(25.4)\end{array}$ & $\begin{array}{l}8.00 \\
(203)\end{array}$ & $\begin{array}{l}0.100 \\
(2.54)\end{array}$ & $\begin{array}{l}0.060 \\
(1.53)\end{array}$ & $\begin{array}{l}0.035 \\
(0.90)\end{array}$ & $\begin{array}{l}0.052 \\
(1.32)\end{array}$ & 0.5448 \\
\hline 8 & $\begin{array}{c}1.00 \\
(25.4)\end{array}$ & $\begin{array}{l}8.00 \\
(203)\end{array}$ & $\begin{array}{l}0.100 \\
(2.54)\end{array}$ & $\begin{array}{l}0.125 \\
(3.20)\end{array}$ & $\begin{array}{l}0.037 \\
(0.95)\end{array}$ & $\begin{array}{l}0.052 \\
(1.32)\end{array}$ & 0.2902 \\
\hline 9 & $\begin{array}{c}1.00 \\
(25.4)\end{array}$ & $\begin{array}{l}8.00 \\
(203)\end{array}$ & $\begin{array}{l}0.100 \\
(2.54)\end{array}$ & $\begin{array}{l}0.114 \\
(2.90)\end{array}$ & $\begin{array}{l}0.034 \\
(0.88)\end{array}$ & $\begin{array}{l}0.106 \\
(2.71)\end{array}$ & 0.3021 \\
\hline 10 & $\begin{array}{c}1.00 \\
(25.4)\end{array}$ & $\begin{array}{l}8.00 \\
(203)\end{array}$ & $\begin{array}{l}0.100 \\
(2.54)\end{array}$ & $\begin{array}{l}0.062 \\
(1.59)\end{array}$ & $\begin{array}{l}0.033 \\
(0.85)\end{array}$ & $\begin{array}{l}0.055 \\
(1.40)\end{array}$ & 0.5001 \\
\hline 11 & $\begin{array}{c}0.25 \\
(6.35)\end{array}$ & $\begin{array}{l}3.00 \\
(76)\end{array}$ & $\begin{array}{l}0.083 \\
(2.12)\end{array}$ & $\begin{array}{l}0.066 \\
(1.70)\end{array}$ & $\begin{array}{l}0.021 \\
(0.54)\end{array}$ & $\begin{array}{l}0.063 \\
(1.61)\end{array}$ & 0.3113 \\
\hline $11 \mathrm{a}$ & $\begin{array}{c}0.25 \\
(6.35) \\
\end{array}$ & $\begin{array}{l}3.00 \\
(76) \\
\end{array}$ & $\begin{array}{l}0.062 \\
(1.59) \\
\end{array}$ & $\begin{array}{l}0.043 \\
(1.10) \\
\end{array}$ & $\begin{array}{l}0.019 \\
(0.48) \\
\end{array}$ & $\begin{array}{l}0.039 \\
(1.01) \\
\end{array}$ & 0.4199 \\
\hline 12 & $\begin{array}{c}2.50 \\
(63.5)\end{array}$ & $\begin{array}{c}25.0 \\
(635)\end{array}$ & $\begin{array}{l}0.104 \\
(2.65)\end{array}$ & $\begin{array}{l}0.045 \\
(1.15)\end{array}$ & $\begin{array}{l}0.035 \\
(0.90)\end{array}$ & $\begin{array}{l}0.038 \\
(0.98)\end{array}$ & 0.6996 \\
\hline $12 \mathrm{r}$ & $\begin{array}{c}2.50 \\
(63.5)\end{array}$ & $\begin{array}{c}25.0 \\
(635)\end{array}$ & $\begin{array}{l}0.104 \\
(2.65)\end{array}$ & $\begin{array}{l}0.027 \\
(0.69)\end{array}$ & $\begin{array}{l}0.027 \\
(0.71)\end{array}$ & $\begin{array}{l}0.019 \\
(0.49)\end{array}$ & 0.8346 \\
\hline
\end{tabular}




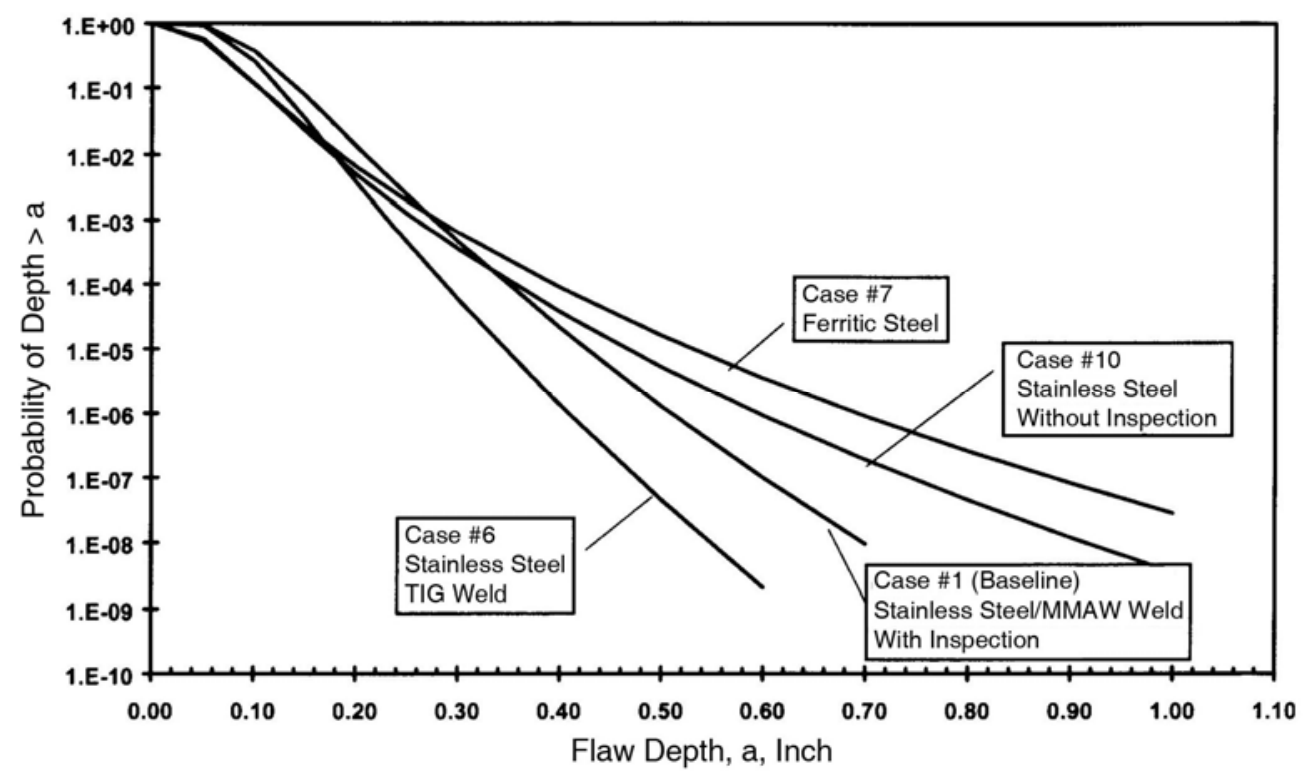

Figure 2.10 Comparison of Simulated Flaw Depth Distributions for 1-in. (25.4 mm) Wall Pipe Welds $(1$ inch $=25.4 \mathrm{~mm})$

- Ferritic steel welds have a greater potential than stainless steel welds to have flaws with very large depths. This trend may be because ferritic steels are susceptible to the mechanism of HAZ cracking. While the occurrence rate for this mechanism is relatively small, it has the potential to produce very deep cracks if the proper conditions are present to cause its occurrence.

- Inservice inspections are most effective in reducing the number of very deep flaws with a much smaller effect on reducing the number of shallow flaws.

- TIG welds tend to have fewer flaws of large depths compared to welds made by the MMA welding process. This effect is due in part to the inherent occurrence rates for flaws from the TIG welding process. In addition, there are typically more weld layers for TIG welds, which creates more opportunities for the arrest of flaws as they grow from layer to layer during the welding.

Figure 2.11 addresses welds made with the same set of process parameters, but for piping of different wall thicknesses, which range from 0.25 to $2.5 \mathrm{in}$. (6.35 to $63.5 \mathrm{~mm})$. It is seen that there is a greater likelihood for a given flaw to have a larger depth as the pipe-wall thickness becomes greater. It should also be noted that Figure 2.11 addresses only conditional depth distributions with the flaw density not being a factor. Thicker welds in larger diameter piping will have more weld metal, which in turn implies more flaws per weld for larger piping. As a result, the expected number of large flaws per weld will be significantly greater for larger sizes of piping, both from the standpoint of flaw-density considerations and from the trends of the conditional flaw-depth distribution curves.

Figure 2.12 presents a somewhat different perspective on the relationship of pipe-wall thickness to the simulated distributions of flaw depths. The flaw depths are shown here as a function of the flaw depth as a fraction of the wall thickness (i.e., as a function of a/t) as opposed to Figure 2.11, which gives flaw 


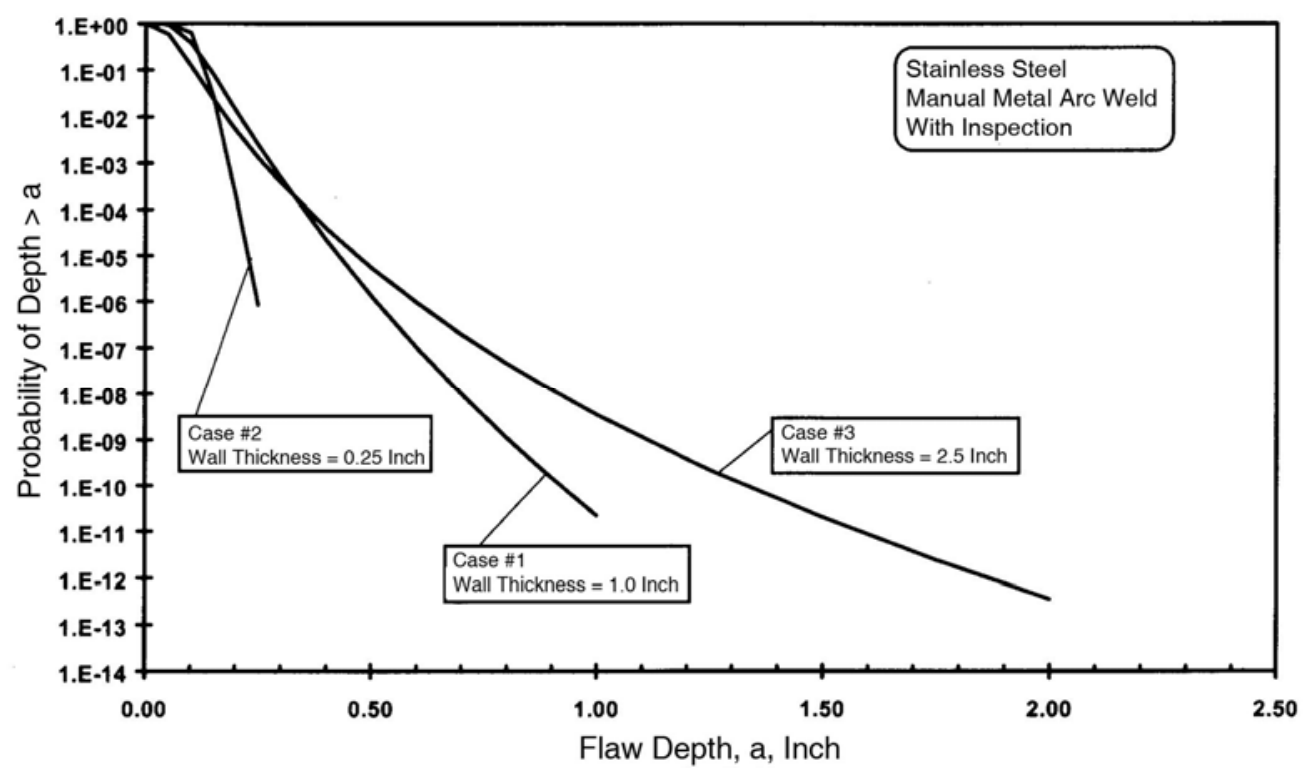

Figure 2.11 Comparison of Simulated Flaw Depth Distributions for Three Pipe Wall Thicknesses $(1 \mathrm{inch}=25.4 \mathrm{~mm})$

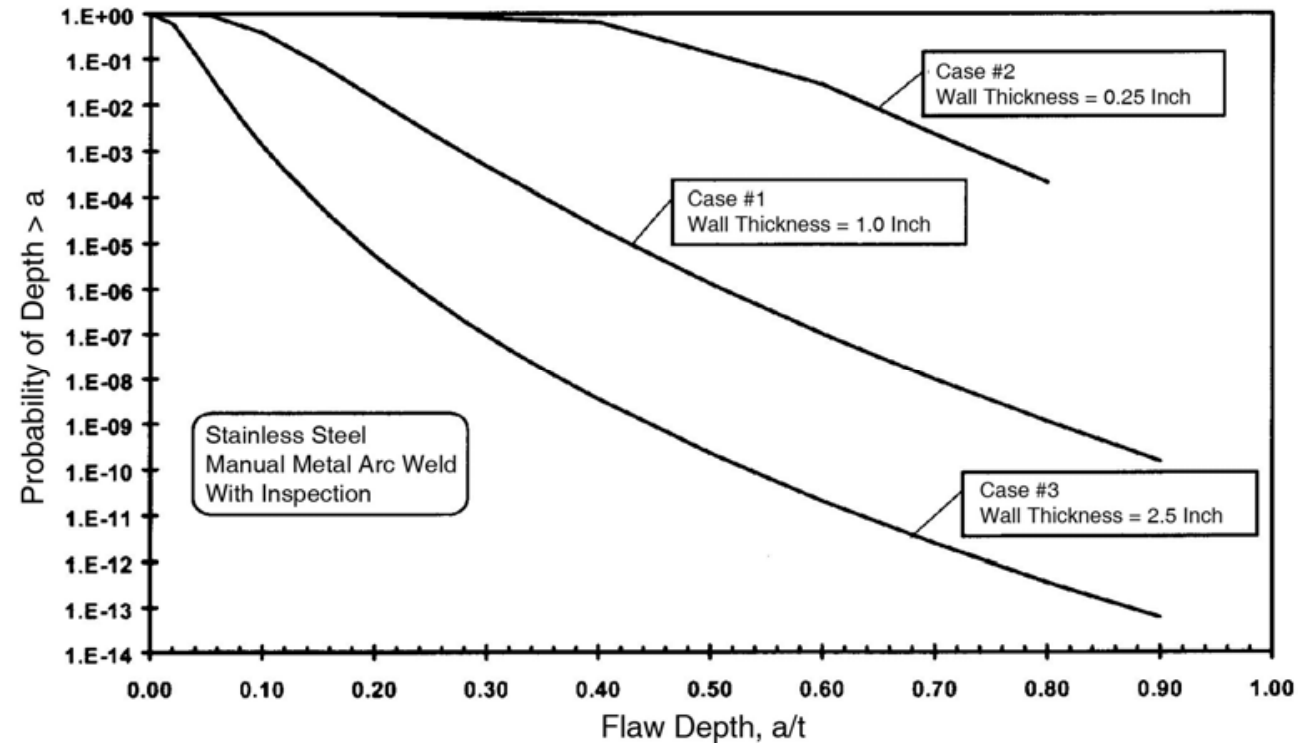

Figure 2.12 Comparison of Simulated Flaw Depth Distributions for Three Pipe Wall Thicknesses Plotted in Terms of a/t $(1$ inch $=25.4 \mathrm{~mm})$

depths in terms of absolute dimensions (inches). It is seen in Figure 2.12 that the very thin 0.25 -in. (6.35-mm) wall pipe now appears to have much deeper flaws than the thicker wall piping. In this regard, welds in thin wall piping will consist of only 2 or 3 layers, which means that a flaw must penetrate only one or two layers before it achieves a depth approaching through-wall dimensions. Figure 2.12 shows that it is inappropriate to assume that flaw-depth distributions are a common function of $a / t$ independent 
of pipe-wall thickness. Such an assumption could result in a very significant error (underestimation) of failure probabilities for small pipe sizes.

\subsection{Flaw Depth Distribution Parameters versus Wall Thickness}

The calculations with the RRA code provided PNNL with data on flaw-size distributions in the format of tables. While these tables could be used directly as inputs to a PFM code, existing codes such as pcPRAISE or SRRA (Bishop 1997) are designed to accept inputs for flaw-size distributions in the form of parameters of standard statistical distributions (e.g., lognormal or exponential distributions). To meet this need, curve fits of the RRA data were developed (as described above) with these curve fits providing the needed parameters for the standard lognormal distribution. This approach also provided a convenient basis to estimate flaw distributions for pipe sizes and welding parameters not covered by the limited number of cases addressed by the RRA calculations. The discussion below describes the methods and results from the effort to seek general trends for the parameters of the lognormal distributions of flaw depths.

The data points for median crack depths $\left(\mathrm{a}_{50}\right)$ and the shape parameters $(\mu)$ are plotted as functions of pipe-wall thickness in Figures 2.13 and 2.14, along with least-square fits of the data points. Each figure provides separate plots for stainless steel and ferritic steel, with Figure 2.13 addressing MMA welding and Figure 2.14 addressing the TIG welding. The figures also give the equations corresponding to the least-squares fitting of the data points.
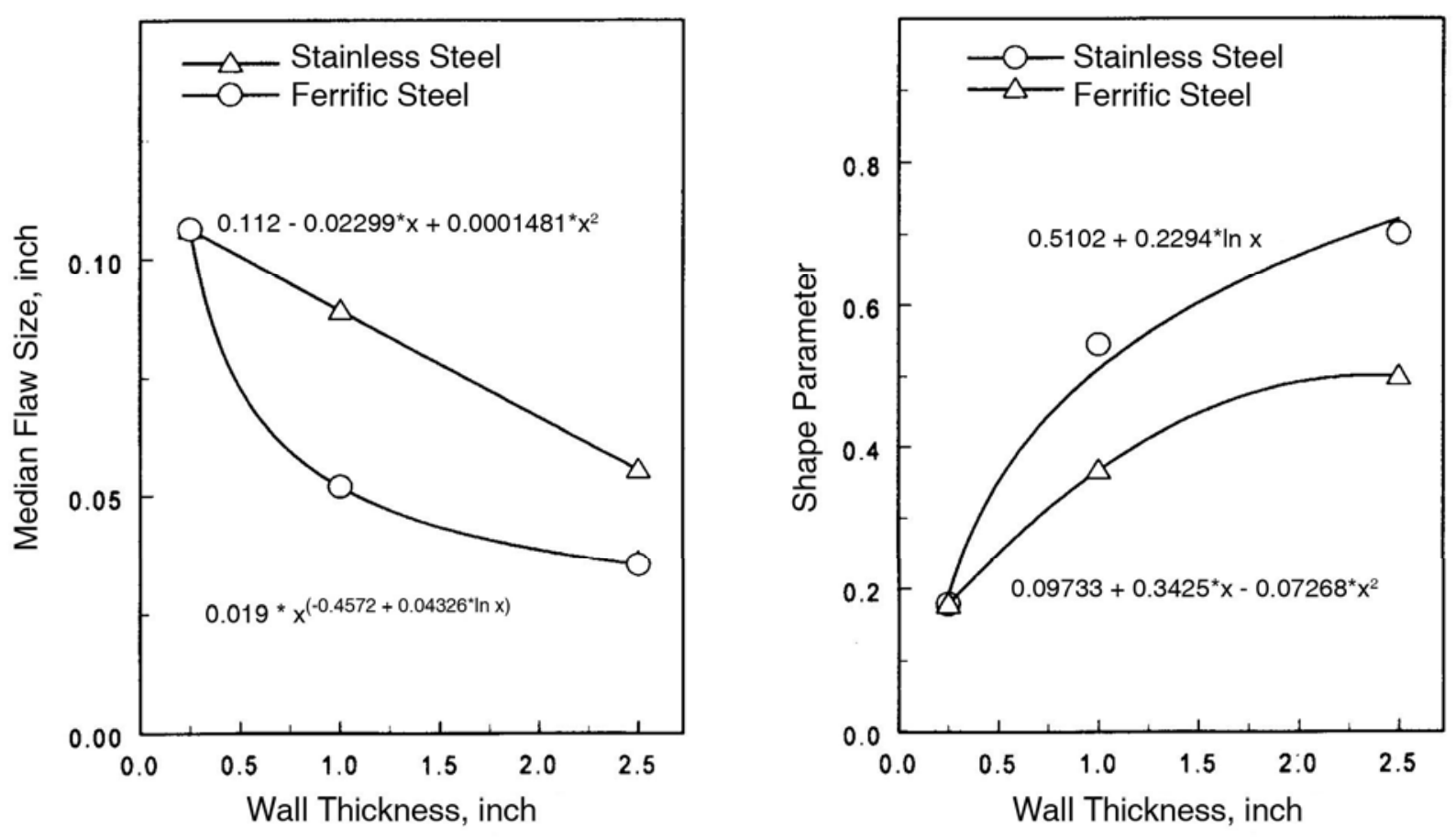

Figure 2.13 Median Flaw Size and Shape Parameter for MMAW $(1$ inch $=25.4 \mathrm{~mm})$ 

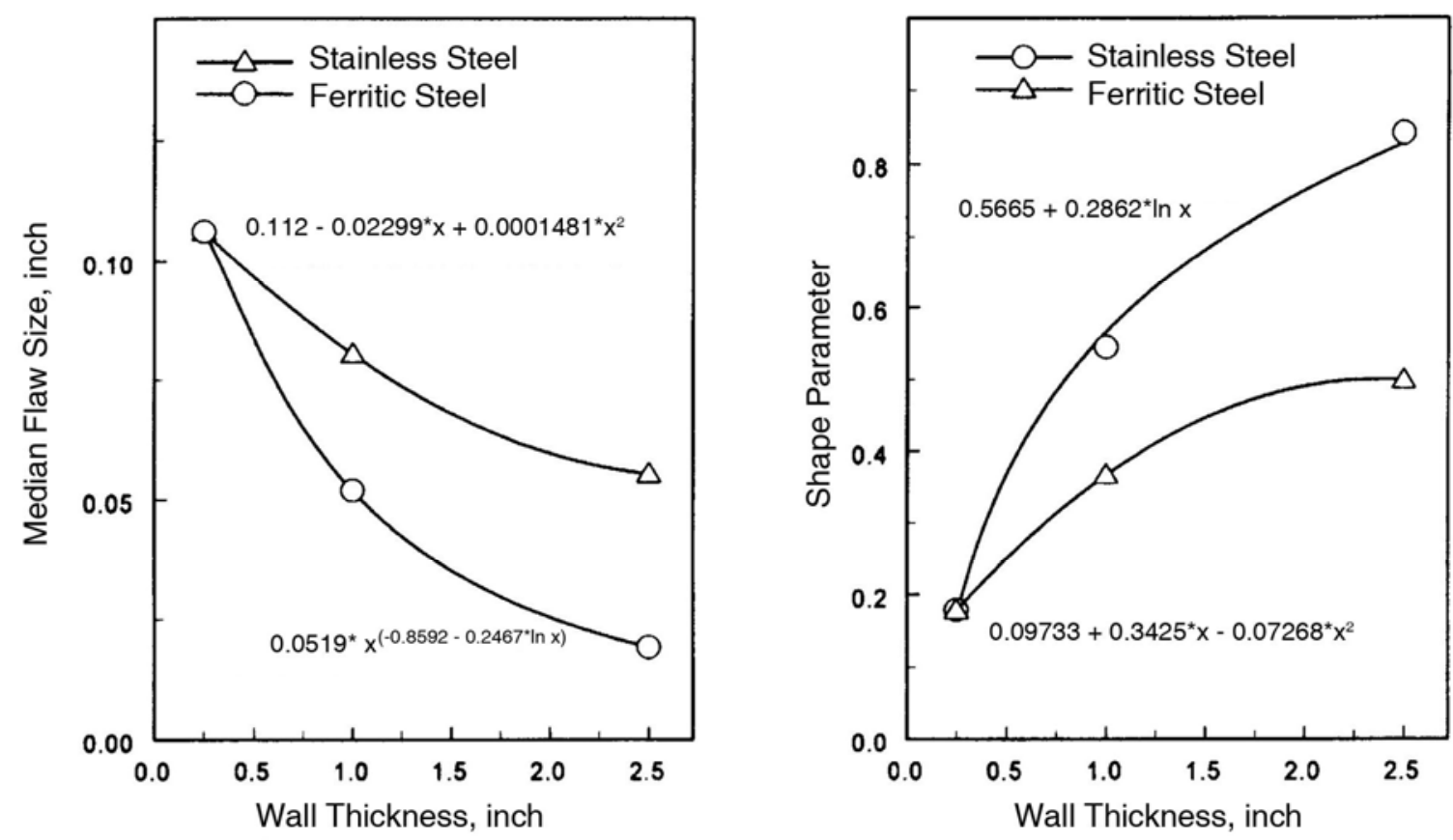

Figure 2.14 Median Flaw Size and Shape Parameters for TIG Weld (1 inch $=25.4 \mathrm{~mm})$

Extrapolation of the curves of Figures 2.13 and 2.14 to wall thicknesses less than $0.25 \mathrm{in}$. $(6.35 \mathrm{~mm})$ or greater than 2.5 in. $(63.5 \mathrm{~mm})$ has been found to give unrealistic results. In these two cases, it is recommended that the flaw depths first be calculated for the lognormal distributions corresponding to the wall thickness of $0.25 \mathrm{in}$. $(6.35 \mathrm{~mm})$ or $2.5 \mathrm{in}$. $(63.5 \mathrm{~mm})$, respectively. For extrapolation, it is then recommended that these simulated flaw depths be used to calculate the corresponding a/t values (depth to thickness ratios) based on values of wall thickness ( $\mathrm{t}$ ) corresponding to 0.25 or $2.5 \mathrm{in}$. (6.35 to $63.5 \mathrm{~mm}$ ). These $a / t$ values should then be assumed to apply for actual wall thicknesses of interest. For the example of a pipe with a wall thickness of $0.15 \mathrm{in}$. $(3.81 \mathrm{~mm})$, one would simulate a flaw depth of (say) $0.06 \mathrm{in}$. $(1.52 \mathrm{~mm})$, based on an assumed wall thickness of $0.25 \mathrm{in} .(6.35 \mathrm{~mm})$. The simulated flaw depth for the 0.15 -in. $(3.81-\mathrm{mm})$ wall pipe would then be assigned as " $\mathrm{a}=(0.06 / 0.25)(0.15)=0.036$ in. $(0.91 \mathrm{~mm}) . "$

The expert system calculations failed to provide some important data points needed to generate the curves of Figures 2.13 and 2.14 because of the limited number of calculations for ferritic piping and for TIG welds. To enable curves to be generated, parameters of the lognormal distribution for MMA welds were used also for TIG welds. The difference in the flaw-depth distributions due to the effects of inspections were neglected, with this factor being addressed only through the effects of inspections of flaw density. All curves for flaw-depth distributions were developed on the basis of the calculations with the RRA code, which included the effects of inspection. The matrix of calculations for thin wall ( $0.25 \mathrm{in}$. [6.35 mm]) piping unfortunately did not address ferritic steels. As described in the next paragraph, it was decided to use the parameters for the 0.25 -in. $(6.35 \mathrm{~mm})$ stainless steel piping also for ferritic steel piping.

Cases 7 and 12r address ferritic steel and MMA for 1.0- and 2.5-in. (25.4- and 63.5-mm) wall thicknesses with no corresponding case for the 0.25 -in. $(6.35-\mathrm{mm})$ wall pipe. Three methods (i.e., methods A, B, and C) to estimate the parameters for ferritic pipes of 0.25 -in. $(6.35-\mathrm{mm})$ wall thickness were considered: 
(1) a straight line extrapolation of the ferritic steel data, (2) an extrapolation based on translation of the stainless steel curves, and (3) assigning the same values for the ferritic steel as those of stainless steel (Method A). Unreasonably high probabilities of a through-wall defect after welding was the basis for discarding the translation and linear extrapolation methods. Table 2.5 summarizes the results of applying the three alternative methods. The probability of a through-wall defect for method A is on the same order as those for the 1.0-in. (25.4- $\mathrm{mm})$ and 2.5-in. $(63.5-\mathrm{mm})$ pipes. Therefore, it was decided to adopt method A for estimating the parameters for ferritic pipes of 0.25-in. thickness. As seen in Figures 2.13 and 2.14, the resulting curves for ferritic steels have smooth trends over the flaw depth range of 0.25 to 2.5 in. (6.35 to $63.5 \mathrm{~mm})$ consistent with the curves for stainless steels.

Table 2.5 Parameter Extrapolation for Ferritic Pipes to 0.25 in. (6.35 mm) Wall Thickness

\begin{tabular}{|c|c|c|c|c|c|c|c|c|}
\hline \multirow[b]{2}{*}{$\begin{array}{l}\text { h, in. } \\
\text { (mm) }\end{array}$} & \multicolumn{4}{|c|}{ "Austenitic } & \multicolumn{4}{|c|}{ Ferritic } \\
\hline & Case & $a_{50}$ in. $(\mathrm{mm})$ & $\mu$ & $\mathbf{P}(>\mathbf{h})$ & Case & $a_{50}$ in. $(\mathrm{mm})$ & $\boldsymbol{\mu}$ & $\mathbf{P}(>\mathbf{h})$ \\
\hline $1 / 4(6.35)$ & & $0.1(2.54)$ & 0.18 & $1.8 \mathrm{E}-07$ & $\begin{array}{l}\text { A } \\
\text { B } \\
\text { C }\end{array}$ & $\begin{array}{l}0.10(2.54) \\
0.06(1.52) \\
0.06(1.52)\end{array}$ & $\begin{array}{l}0.18 \\
0.36 \\
0.48\end{array}$ & $\begin{array}{l}1.8 \mathrm{E}-07 \\
3.7 \mathrm{E}-05 \\
1.5 \mathrm{E}-03\end{array}$ \\
\hline $1(25.4)$ & 1 & $0.089(2.26)$ & 0.376 & $2.2 \mathrm{E}-11$ & 7 & $0.052(1.32)$ & 0.545 & $2.8 \mathrm{E}-08$ \\
\hline $2^{1 / 2}(63.5)$ & 3 & $0.056(1.42)$ & 0.50 & $3.1 \mathrm{E}-14$ & 12 & $0.035(0.89)$ & 0.70 & $1.2 \mathrm{E}-09$ \\
\hline
\end{tabular}

\subsection{Number of Flaws versus Wall Thickness}

The results of the calculations for defect densities are described by Figures 2.15 and 2.16 and Table 2.6, which provide a basis for estimating the number of defects in a weld as a function of (1) the pipe wall thickness and (2) whether a radiographic inspection (RT) was performed on the completed weld. If the wall thickness of interest is greater than $2.5 \mathrm{in} .(63.5 \mathrm{~mm})$ or less than $0.25 \mathrm{in}$. $(6.35 \mathrm{~mm})$ the flaw densities can be estimated from the values given in Table 2.6 by an extrapolation based on the trends of the data in terms of the semi-log scales as displayed by Figures 2.15 and 2.16.

The defect density is expressed as the number of defects per inch of weld, with the weld length measured along the inner surface of the pipe circumference. The total number of defects within a given weld is determined by multiplying the defect density by the inner circumference of the pipe. The results of Figures 2.15 and 2.16 are based on the calculations with the RRA flaw distribution model. Defect densities for other wall thicknesses can be estimated by interpolation in Table 2.6, or by use of the plots of Figures 2.15 and 2.16.

The calculations with the RRA code did not indicate significant differences in flaw densities for (1) stainless and ferritic steels, and (2) MMA versus TIG welds. Therefore the flaw densities were based on the calculations of stainless steel and MMA welds. The flaw densities of Table 2.6 assume that the fracture mechanics model will conservatively place all flaws at the inner surface of the pipe. As such, the indicated densities were derived to account for the effect of taking all flaws to be at the inner surface and assume that only a fraction of the buried flaws contribute to piping-failure probabilities. 


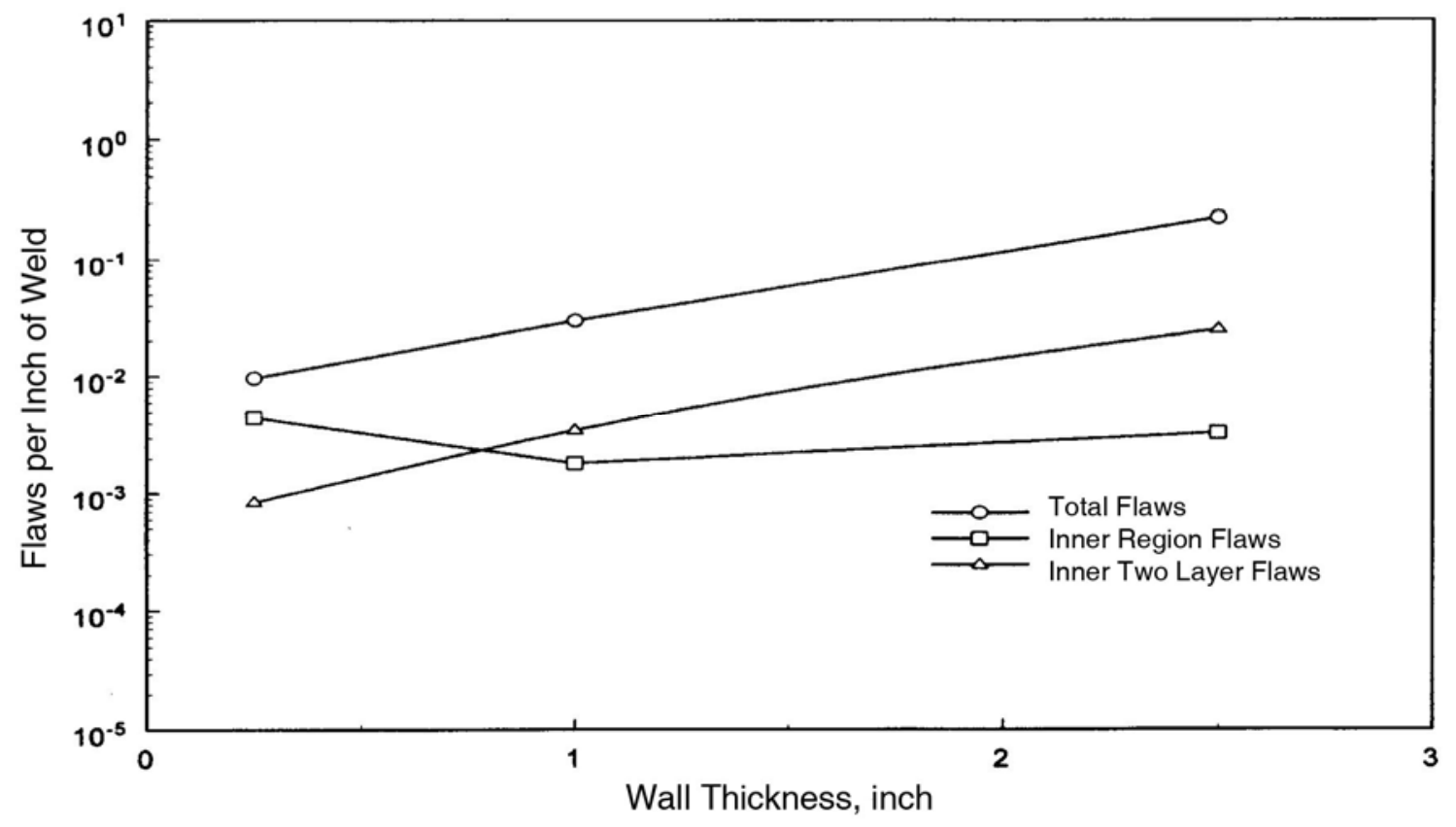

Figure 2.15 Flaw per Inch of Weld versus Wall Thickness (with RT) $(1$ inch $=25.4 \mathbf{~ m m})$

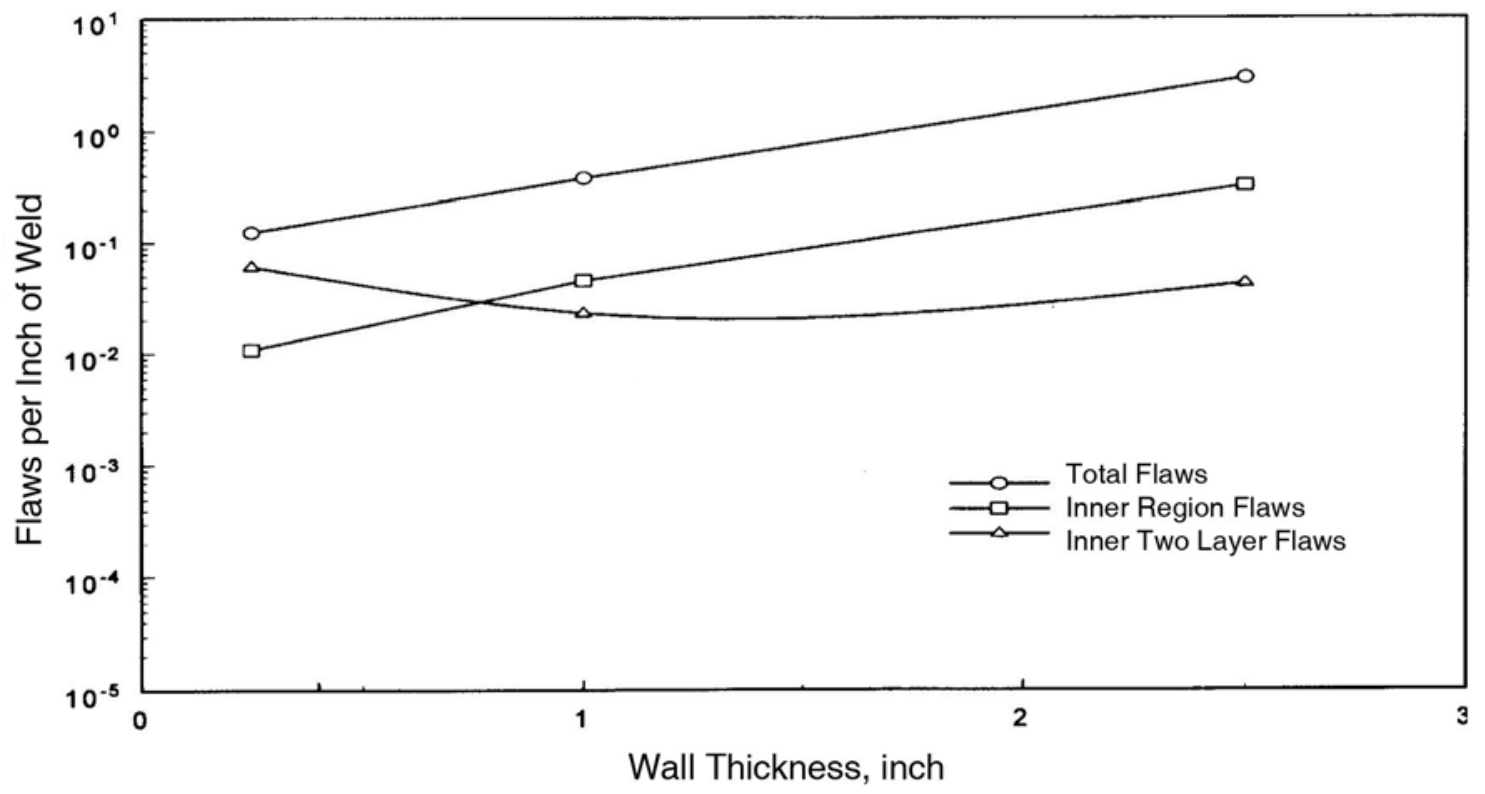

Figure 2.16 Flaw per Inch of Weld versus Wall Thickness (without RT) $(1$ inch $=25.4 \mathrm{~mm})$ 
Table 2.6 Flaw Densities for Piping Welds

\begin{tabular}{|c|c|c|}
\hline & \multicolumn{2}{|c|}{$\begin{array}{l}\text { Flaw Density, Flaws/in. (m) } \\
\end{array}$} \\
\hline Pipe Wall Thickness, in. (mm) ${ }^{(\mathrm{a})}$ & With RT Inspection & Without RT Inspection \\
\hline $0.25^{(\mathrm{a})}(6.35)$ & $0.0047(0.185)$ & $0.0602(2.37)$ \\
\hline $0.50(12.7)$ & $0.0030(0.118)$ & $0.0384(1.51)$ \\
\hline $0.75(19.1)$ & $0.0020(0.079)$ & $0.0256(1.01)$ \\
\hline $1.00(25.4)$ & $0.0035(0.138)$ & $0.0448(1.76)$ \\
\hline $1.50(38.1)$ & $0.0067(0.264)$ & $0.0858(3.38)$ \\
\hline $2.00(50.8)$ & $0.0120(0.472)$ & $0.1536(6.05)$ \\
\hline $2.50^{(\mathrm{a})}(63.5)$ & $0.0256(1.008)$ & $0.3277(12.90)$ \\
\hline $\begin{array}{l}\text { (a) See text for guidance in estima } \\
(6.35 \mathrm{~mm}) \text { or greater than } 2.5 \mathrm{i}\end{array}$ & $\begin{array}{l}\text { flaw densities for wall } \\
3.5 \mathrm{~mm}) \text {. }\end{array}$ & nesses less than 0.25 in. \\
\hline
\end{tabular}

Two methods were used to estimate the fraction of buried defects (for each wall thickness) that would contribute to piping failure. One method considered the sum of the RRA categories of "SURF_I" (inner surface breaking) and "EMBED_I" (region extending from ID surface to the quarter wall thickness location) defects. The other method considered flaws with inner tips located within the first two layers of the weld passes. The results in Table 2.6 are based on the method for each pipe wall thickness that gave the larger fraction of flaws.

Figures 2.15 and 2.16 show the results of calculations to predict the number of flaws per inch of weld, both with RT and without RT. These figures show that without inspection, the predicted number of flaws increases by a factor equal to 12.8. This factor of 12.8 , which accounts for the effect of inspection by RT, was based on the calculations for MMA welds of 1.0-in. (25.4-mm)-thick piping. It was assumed that this factor is approximately correct for other wall thicknesses, materials, and welding processes. In general, the factor of 12.8 should not be applied to smaller piping (e.g., less than 3-in. [76.2-mm] diameter) because such piping is typically not inspected by RT.

\subsection{Conclusions}

A simulation model for estimating flaw densities and size distributions for welds in piping has been described. Application of the simulation model has provided a basis for estimating flaw densities and size distributions as a function of pipe-wall thickness, type of piping material, welding procedure, and inspection practice.

Results from the calculations described in this section are believed to give reasonable inputs for calculating piping-failure probabilities. In addition, the flaw simulation model provides a physically based method for scaling flaw-distribution parameters between piping of different sizes and fabrication practices and thereby provides confidence that calculated values of failure probabilities reflect correct trends for use in risk-informed ranking processes for selecting locations for inservice inspection. 


\section{Parametric Calculations for Fatigue}

\subsection{Introduction}

Piping systems experience cyclic stresses caused by anticipated plant transients (e.g., rapid cooling of the piping during auxiliary feedwater initiation following a scram) and unanticipated transients (e.g., checkvalve leakage). This section describes the probabilistic fracture mechanics (PFM) computer code and the structural mechanics modeling approach that PNNL has used to simulate the effects of these cyclic fatigue stresses on the reliability of reactor piping. Also described are sensitivity calculations that evaluate the effects of modeling assumptions. The overall objective of the study was to develop a piping reliability model that could be used to simulate the effects of alternative inservice inspection strategies so that inspection methods (flaw-detection capability) and inspection intervals can be established in a manner consistent with goals for the reliability of the piping systems.

The calculations of this section (and Sections 4 and 5) were performed in a structured parametric format, with the parameters selected to cover the range of pipe sizes, piping materials, and operating stresses relevant to reactor piping systems. This permitted estimates of piping reliability (including the effects of alternative inspection strategies) to be made without incurring the high costs of individual calculations for the hundreds of welds and fittings that make up the piping systems of interest.

Probabilistic fracture mechanics calculations often assume that the stress state in the pipe wall is uniform through the thickness of the pipe wall. This approach is appropriate for the stresses caused by internal pressure. It is a good approximation for the bending of piping caused by thermal expansion of pipe systems, but it does not address through-wall stress gradients caused by radial thermal gradients, residual stresses, or geometric discontinuities. This section includes calculations that evaluate the effects of such through-wall stress variations by applying the capability of pc-PRAISE to simulate radial gradient stresses with the so-called "g-function" approach for calculating crack-tip stress intensity factors (Harris et al. 1992).

The first part of this section describes the pc-PRAISE code, compares the results of the pc-PRAISE and PARIS codes, and describes details of the fracture mechanics model used for predicting piping reliability. Parametric calculations are then performed using the model, and the results are presented as sets of curves and tables.

\subsection{Piping Reliability Code pc-PRAISE}

The use of PFM models to simulate the growth of the cracks in welds that pass undetected during piping fabrication has become a common practice in recent years. Probabilistic fracture mechanics evaluations combine the methods of reliability theory and fracture mechanics to assess the structural reliability of components containing defects. Lawrence Livermore National Laboratory developed the PFM computer code pc-PRAISE (Harris et al. 1992) for the NRC to support probabilistic analyses of piping containing circumferential surface-breaking cracks. This code has been used extensively for the calculations described in the present report. 
The pc-PRAISE code calculates failure probabilities caused by the growth of pre-existing cracks caused by cyclic loads, and by the initiation and growth of stress-corrosion cracks. The model simulates the growth of two-dimensional cracks in both their length and depth directions with the initial crack sizes described by statistical distributions. The crack distributions are modified as a result of preservice and inservice inspections, with the probability of flaw detection (POD) expressed as a function of crack size. Limit states for piping loads and crack sizes are used to calculate the probabilities of leak and break. The criterion for pipe break is that of the net section stress becoming equal to the material flow stress. A small leak occurs if the stable crack growth results in a through-wall crack. A big leak occurs if the crack opening area (related to the through-wall crack length and applied loads) is such that the leakage exceeds the prescribed flow rate corresponding to the operating definition of a large leak. Because of typically low probabilities of failure and the large number of input parameters, a Monte Carlo simulation with variance reduction by stratified sampling is applied to calculate failure probabilities.

The schematic diagram of the steps in the pipe reliability calculations with pc-PRAISE are presented in Figure 3.1. Starting at the upper left-hand corner of the diagram, a new weld in a pipe is assumed to begin its life with a distribution of welding flaws. This distribution is immediately modified as a result of post-weld inspections and repairs. Once the plant is placed into operation, there are cyclic stresses that cause the remaining cracks to grow. Some cracks can be sufficiently large and/or will grow at sufficiently high rates such that their depths could penetrate the pipe wall. In some cases, there will be inservice inspections that detect some of the growing cracks in a timely manner. In these cases, the damaged pipe will be repaired. In other cases, the growing crack will penetrate the wall of the pipe and thereby cause a leak. In many cases, this leakage will be small and the leakage will be detected. In these cases, the pipe will be repaired before a big leak or pipe break (loss of coolant accident [LOCA]) occurs. The pcPRAISE model predicts the rate of leakage for through-wall cracks in pipes and calculates the probability that the leakage rates are sufficiently large to cause the LOCA type of event. In summary, the Monte Carlo simulation as performed by pc-PRAISE involves a large number of trials. The outcome of each trial is (1) there is no through-wall crack and the pipe does not fail, (2) a small leak occurs, (3) a large leak occurs, or 4) the pipe fails in the mode of a break or LOCA.

One objective of PNNL's studies was to compare the numerical results and procedures used in the pcPRAISE code with other similar computer codes. One of these efforts consisted of a set of benchmarking calculations that compared predictions of pc-PRAISE with predictions made by a similar code that Westinghouse Electric Corporation developed (Bishop 1997).

Burckner-Foit et al. (1989) compared results from the PARIS code and the pc-PRAISE code in a second benchmarking effort. The PARIS code was developed at the Nuclear Research Center Karlsruhe (Germany) for predicting the reliability of components containing through-wall or surface cracks. Both the pc-PRAISE and PARIS codes use the Monte Carlo simulation techniques to determine failure probabilities, but use different variance-reduction techniques to reduce the computational effort needed to perform the Monte Carlo simulation. The PARIS code uses importance sampling (as used also by Bishop 1997) whereas the pc-PRAISE code uses stratified sampling (Harris et al. 1992). 


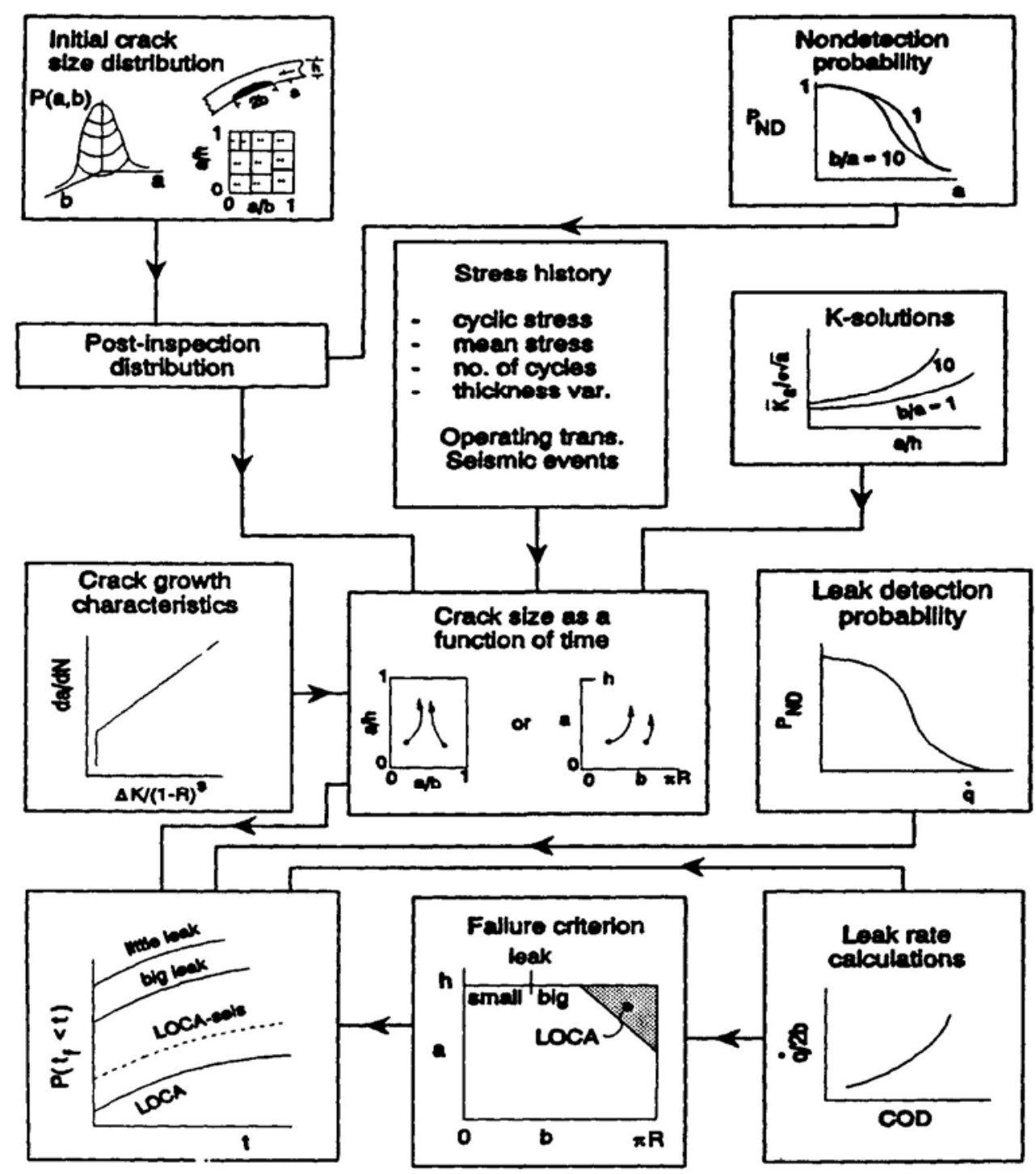

Figure 3.1 Schematic Diagram of Piping Failure Probability Calculations as Performed by pc-PRAISE

The sample problems chosen to compare pc-PRAISE and PARIS addressed results from piping containing circumferential surface cracks. The input data for the sample problem are given in Table 3.1. Permission to use this copyrighted material is granted by Elsevier. This example addressed a relatively thick-wall pipe ( $2 \mathrm{in}$. [51 mm]) subject to a low level of cyclic stresses ( 5 cycles per year). The selected inputs resulted in relatively low values of calculated failure probabilities (leak probability of less than $1.0 \mathrm{E}-07)$ and thereby provided a good test of both the fracture-mechanics models and the convergence characteristics of the Monte Carlo sampling approaches. The mean and standard errors for failure probabilities calculated with both codes. The differences between the results are very small, which indicates the reproducibility of calculated failure probabilities given common input parameters and modeling assumptions. 
Table 3.1 Input Data for the Sample Problem (Bruckner-Foit et al. 1989)

(Permission to use this copyrighted material is granted by Elsevier.)

\begin{tabular}{|c|c|}
\hline Input Quantity & Value \\
\hline Component Geometry & Pipe with mean radius 15 in. $(380 \mathrm{~mm})$, wall thickness 2 in. $(51 \mathrm{~mm})$ \\
\hline Crack Model & Semi-elliptical internal surface crack subjected to tension \\
\hline Distribution of Crack Depth & $\begin{array}{l}\text { Lognormal with mean value } 0.065 \text { in. }(1.65 \mathrm{~mm}) \text {, standard deviation } \\
0.085 \text { in. }(2.16 \mathrm{~mm})\end{array}$ \\
\hline $\begin{array}{l}\text { Distribution of Crack Aspect } \\
\text { Ratio }\end{array}$ & $\begin{array}{l}\text { Lognormal with mean value of } 2.51 \text {, standard deviation } 1.66 \text {, lower bound } \\
\text { at } 1\end{array}$ \\
\hline Crack Growth Law & Stainless Steel - Paris Law \\
\hline Fatigue Loads & $\Delta \sigma=23.2 \mathrm{ksi}(160 \mathrm{MPa}), \sigma_{\min } / \sigma_{\max }=-0.1188$, frequency $=5$ cycles $/$ year \\
\hline Failure Criterion & Plastic limit load \\
\hline Load Controlled Stress & $11.2 \mathrm{ksi}(77 \mathrm{MPa})$ \\
\hline Flow Stress & $\begin{array}{l}\text { Normal distribution with mean value } 53 \mathrm{ksi}(365 \mathrm{MPa}) \text { and standard } \\
\text { deviation } 2 \mathrm{ksi}(14 \mathrm{MPa})\end{array}$ \\
\hline Nondetection Probability & $\mathrm{a}^{*}=0.35$ in. $(8.9 \mathrm{~mm}), \mathrm{D}=1.0$ in. $^{2}\left(645 \mathrm{~mm}^{2}\right), v=1.6, \varepsilon=0.005$ \\
\hline Inspection & Preservice \\
\hline Proof Stress & $14.8 \mathrm{ksi}(102 \mathrm{MPa})$ \\
\hline Critical Leak & $\begin{array}{l}1 \mathrm{gal} / \mathrm{min}(3.8 \mathrm{liter} / \mathrm{min}) \text { for small leaks, } 100 \mathrm{gal} / \mathrm{min}(378 \mathrm{liter} / \mathrm{min}) \text { for big } \\
\text { leaks }\end{array}$ \\
\hline
\end{tabular}

Other calculations with PRAISE (Woo and Chou 1982) have been performed to address relatively high values of calculated failure probabilities. In these studies PRAISE was applied to components that had failed at operating nuclear power plants. The calculated failure probabilities were shown to approach 100 percent in agreement with the operating experience.

\subsection{Flaw Size Distribution}

It is generally recognized that the inputs for distributions of initial flaws in welds are perhaps the most uncertain aspect of PFM calculations. Inputs and assumptions for flaw-size distributions used in the pc-PRAISE calculations are summarized here because of their importance to the calculations described throughout this report.

Section 2 of this report describes calculations by RRA with a weld simulation model. These results, which are recommended as a basis for estimating flaw densities and size distributions for piping welds, became available to PNNL after many of the calculations of this report were performed. Although many of the present calculations were based on the RRA methodology, other calculations were based on prior estimates of flaw-depth distributions. These early results are still of interest as sensitivity calculations, although their application for estimating absolute values of piping-failure probabilities should be avoided. The inputs for flaw distributions will be documented for each set of calculations as they are presented in the various sections of this report. 
Two broad categories of flaws are addressed as follows:

- Larger flaws whose depths approach or exceed the thickness of the weld beads (or layers) that make up the weld. These are fabrication flaws associated with the welding process that could be detected by preservice inspection and that may be repaired if detected. As noted above, the numbers and sizes of these larger flaws were estimated either using the RRA expert system model as described in Section 2, or, in the case of other calculations, were based on earlier estimates of flaw-distribution parameters.

- Small flaws below the detection and repair thresholds that the RRA model does not address. These flaws can represent very small fabrication defects below the usual threshold flaw sizes of concern, or they can represent flaws that initiate by fatigue under service conditions. Only a few calculations of this report address this category of small flaws because the category of larger fabrication flaws is usually the primary contributor to calculated piping-failure probabilities. All calculations for the small-flaw category were based on a uniform distribution of flaw depths ranging from 0.002 to 0.010 in. (0.05 to $0.25 \mathrm{~mm})$, with the assumption that each pipe weld had one such flaw at the inner surface of the pipe.

It should be noted that the RRA weld simulation model provides tables or numerical histograms of the sizes and locations of the defects within a weld. For the purposes of the calculations with pc-PRAISE, these histograms were approximated by standard lognormal distributions using the approach described in Section 2 of this report.

\subsection{Parametric Treatment of Cyclic Stresses Using the Q-Factor}

Piping stresses along with the operational transients that govern the cyclic nature of these stresses are unique and plant specific for each location within the piping systems of a given plant. An evaluation of piping reliability in complete detail might involve thousands of calculations to address all locations of interest for even a single plant. Therefore, the evaluations of this report were performed using a parametric approach to minimize the number of calculations needed to establish trends for piping-failure probabilities and to establish the effects of inservice inspections on these failure probabilities. With the parametric approach, it was possible to cover the full range of stresses and cyclic conditions of interest with a limited number of calculations.

For fatigue of stainless steel piping, it was possible to further reduce the number of calculations by using a single numerical parameter (Q-factor) that captured the combined effect of cyclic stress level and number of stress cycles. The Q-factor approach was made possible by the simple Paris Law crack growth equation $\left(\mathrm{da} / \mathrm{dN}=\left(\mathrm{C} \Delta \sigma^{4}\right)\right)$ that applies to stainless steel. Attempts to identify a similar factor for fatigue of ferritic steels (also for stress-corrosion cracking of stainless steels) were unsuccessful because of the more complex mathematical form of the crack growth rate equations.

For fatigue of stainless steels, the parameter Q was defined as

$$
Q=\alpha N(\Delta \sigma)^{4}
$$


where $\mathrm{N}$ is the cumulative number of stress cycles and $\Delta \sigma$ is the cyclic stress range. The exponent of 4 corresponds to the parameter in the fatigue crack growth law for stainless steels. The constant $\alpha$ is determined to be $9.87 \mathrm{E}-08$ by setting $\mathrm{Q}=1$ for $\mathrm{N}=$ number of cycles accumulated at five cycles per year over 40 years with $\Delta \sigma=15 \mathrm{ksi}$ (103 MPa). Five cycles per year is representative of operating heatup/cooldown cycles at a nuclear power plant, and $\Delta \sigma=15 \mathrm{ksi}(103 \mathrm{MPa})$ is a typical level of cyclic stress caused by internal pressure and piping thermal expansion. Table 3.2 gives values for the fatigue crack growth parameter Q. The listed values correspond to the wide spectrum of cyclic stress levels and cyclic frequencies of potential interest to reactor piping.

\subsection{Results of Sensitivity Calculations}

Sensitivity calculations were performed to gain insight into the effect of cyclic stress level on the failure probabilities calculated by the pc-PRAISE code. These fatigue crack growth calculations were limited to a single size of stainless steel piping and did not address the effects of inservice inspections on the failure probabilities. Sections 4 and 5 provide more comprehensive results for a range of inservice inspection scenarios and cover other pipe materials and pipe sizes, along with the failure mechanism of stresscorrosion cracking of stainless steel piping.

\subsubsection{Failure Probabilities versus Q-Factor}

Figure 3.2 provides example results of pc-PRAISE calculations that predicted piping-failure probabilities as a function of the Q-factor. The failure mode is that of a small leak from a through-wall crack. The calculations addressed a stainless steel pipe with an outer diameter $(6.625 \mathrm{in}$. [168 mm]) and wall thickness (0.562 in. [14.3 mm]) corresponding to a 6-in. (152-mm) Schedule 120 pipe. The other input parameters (as given by Table 3.3) included a lognormal distribution for the crack depths that had a median crack depth of 0.0959 in. $(2.44 \mathrm{~mm})$ or about 20 percent of the wall thickness.

Figure 3.2 was constructed by considering the actual number of flaws that might be present in the weld rather than by assuming exactly one flaw per weld. In these sensitivity calculations, the estimated probability of having an inner surface flaw of the category as described by the lognormal distribution of crack depths was 1.0E-02. A second category of flaws was also addressed, which corresponded to small surface flaws at the inner surface with depths uniformly distributed between 0.002 and 0.010 in. ( 0.05 to $0.25 \mathrm{~mm}$ ) with each weld having one flaw of this category. The estimated probability of leak (cumulative probability of a leak over 40 years of operation) caused by the combination of these two flaw populations corresponds to the sum of the probabilities for the two individual curves of Figure 3.2. It is seen that the calculated failure probability is only about 1.0E-07 for a small Q-factor of 1.0. On the other hand, the calculated leak probabilities approach 100 percent as the Q-factor becomes very large (i.e., 1.0E+05$1.0 \mathrm{E}+06)$. This cyclic stress is predicted because the fracture mechanics model calculates crack growth rates sufficient to grow a very small initial crack to a through-wall depth over the 40 -year operating life of the piping system.

Other sensitivity calculations focused on pipe breaks and on the effects of through-wall stress gradients on calculated break probabilities. The pc-PRAISE calculations of critical crack sizes for unstable crack propagation were based on the net section collapse criteria. Predicted break probabilities are presented in Figure 3.3 as a ratio of the break probability to the corresponding leak probability. Low values of this ratio imply that conditions are favorable for achieving leak-before-break. As indicated, the calculated 
Table 3.2 Fatigue Crack Growth Parameter $Q=\alpha \mathbf{N}(\Delta \sigma)^{4}$ Used for Correlation of Leak Probability

\begin{tabular}{|c|c|c|c|c|c|c|c|c|c|c|}
\hline \multirow[b]{2}{*}{$\begin{array}{c}\text { Cyclic } \\
\text { Frequency }\end{array}$} & \multicolumn{10}{|c|}{ Cyclic Stress Range, ksi (MPa) } \\
\hline & $\begin{array}{c}5 \\
(34)\end{array}$ & $\begin{array}{c}10 \\
(69)\end{array}$ & $\begin{array}{c}15 \\
(103)\end{array}$ & $\begin{array}{c}20 \\
(138)\end{array}$ & $\begin{array}{c}30 \\
(207)\end{array}$ & $\begin{array}{c}40 \\
(276)\end{array}$ & $\begin{array}{c}60 \\
(414)\end{array}$ & $\begin{array}{c}80 \\
(552)\end{array}$ & $\begin{array}{c}100 \\
(690)\end{array}$ & $\begin{array}{c}120 \\
(828)\end{array}$ \\
\hline 1 Cycle per Year & $2.5 \mathrm{E}-03$ & $3.9 \mathrm{E}-02$ & $2.0 \mathrm{E}-01$ & $6.3 \mathrm{E}-01$ & $3.2 \mathrm{E}+00$ & $1.0 \mathrm{E}+01$ & $5.1 \mathrm{E}+01$ & $1.6 \mathrm{E}+02$ & $3.9 \mathrm{E}+02$ & $8.2 \mathrm{E}+02$ \\
\hline 5 Cycles per Year & $1.2 \mathrm{E}-02$ & $2.0 \mathrm{E}-01$ & $1.0 \mathrm{E}+00$ & $3.2 \mathrm{E}+00$ & $1.6 \mathrm{E}+01$ & $5.1 \mathrm{E}+01$ & $2.6 \mathrm{E}+02$ & $8.1 \mathrm{E}+02$ & $2.0 \mathrm{E}+03$ & $4.1 \mathrm{E}+03$ \\
\hline 1 Cycle per Month & $3.0 \mathrm{E}-02$ & 4.7E-01 & $2.4 \mathrm{E}+00$ & $7.6 \mathrm{E}+00$ & $3.8 \mathrm{E}+01$ & $1.2 \mathrm{E}+02$ & $6.1 \mathrm{E}+02$ & $1.9 \mathrm{E}+03$ & $4.7 \mathrm{E}+03$ & $9.8 \mathrm{E}+03$ \\
\hline 1 Cycle per Week & $1.3 \mathrm{E}-01$ & $2.0 \mathrm{E}+00$ & $1.0 \mathrm{E}+01$ & $3.3 \mathrm{E}+01$ & $1.7 \mathrm{E}+02$ & $5.3 \mathrm{E}+02$ & $2.7 \mathrm{E}+03$ & $8.4 \mathrm{E}+03$ & $2.0 \mathrm{E}+04$ & $4.3 \mathrm{E}+04$ \\
\hline 1 Cycle per Day & $9.0 \mathrm{E}-01$ & $1.4 \mathrm{E}+01$ & $7.3 \mathrm{E}+01$ & $2.3 \mathrm{E}+02$ & $1.2 \mathrm{E}+03$ & $3.7 \mathrm{E}+03$ & $1.9 \mathrm{E}+04$ & $5.9 \mathrm{E}+04$ & $1.4 \mathrm{E}+05$ & $3.0 \mathrm{E}+05$ \\
\hline 5 Cycles per Day & $4.5 \mathrm{E}+00$ & $7.2 \mathrm{E}+01$ & $3.6 \mathrm{E}+02$ & $1.1 \mathrm{E}+03$ & $5.8 \mathrm{E}+03$ & $1.8 \mathrm{E}+04$ & $9.3 \mathrm{E}+04$ & $2.9 \mathrm{E}+05$ & $7.2 \mathrm{E}+05$ & $1.5 \mathrm{E}+06$ \\
\hline 1 Cycle per Hour & $2.2 \mathrm{E}+01$ & $3.5 \mathrm{E}+02$ & $1.7 \mathrm{E}+03$ & $5.5 \mathrm{E}+03$ & $2.8 \mathrm{E}+04$ & $8.9 \mathrm{E}+04$ & $4.5 \mathrm{E}+05$ & $1.4 \mathrm{E}+06$ & $3.5 \mathrm{E}+06$ & $7.2 \mathrm{E}+06$ \\
\hline 5 Cycles per Hour & $1.1 \mathrm{E}+02$ & $1.7 \mathrm{E}+03$ & $8.8 \mathrm{E}+03$ & $2.8 \mathrm{E}+04$ & $1.4 \mathrm{E}+05$ & $4.4 \mathrm{E}+05$ & $2.2 \mathrm{E}+06$ & $7.1 \mathrm{E}+06$ & $1.7 \mathrm{E}+07$ & $3.6 \mathrm{E}+07$ \\
\hline 1 Cycle per Minute & $1.3 \mathrm{E}+03$ & $2.1 \mathrm{E}+04$ & $1.0 \mathrm{E}+05$ & $3.3 \mathrm{E}+05$ & $1.7 \mathrm{E}+06$ & $5.3 \mathrm{E}+06$ & $2.7 \mathrm{E}+07$ & $8.5 \mathrm{E}+07$ & $2.1 \mathrm{E}+08$ & $4.3 \mathrm{E}+08$ \\
\hline 5 Cycles per Minute & $6.5 \mathrm{E}+03$ & $1.0 \mathrm{E}+05$ & $5.3 \mathrm{E}+05$ & $1.7 \mathrm{E}+06$ & $8.4 \mathrm{E}+06$ & $2.7 \mathrm{E}+07$ & $1.3 \mathrm{E}+08$ & $4.2 \mathrm{E}+08$ & $1.0 \mathrm{E}+09$ & $2.1 \mathrm{E}+09$ \\
\hline 1 Cycle per Second & $7.8 \mathrm{E}+04$ & $1.2 \mathrm{E}+06$ & $6.3 \mathrm{E}+06$ & $2.0 \mathrm{E}+07$ & $1.0 \mathrm{E}+08$ & $3.2 \mathrm{E}+08$ & $1.6 \mathrm{E}+09$ & $5.1 \mathrm{E}+09$ & $1.2 \mathrm{E}+10$ & $2.6 \mathrm{E}+10$ \\
\hline 10 Cycles per Second & $7.8 \mathrm{E}+05$ & $1.2 \mathrm{E}+07$ & $6.3 \mathrm{E}+07$ & $2.0 \mathrm{E}+08$ & $1.0 \mathrm{E}+09$ & $3.2 \mathrm{E}+09$ & $1.6 \mathrm{E}+10$ & $5.1 \mathrm{E}+10$ & $1.2 \mathrm{E}+11$ & $2.6 \mathrm{E}+11$ \\
\hline 100 Cycles per Second & $7.8 \mathrm{E}+06$ & $1.2 \mathrm{E}+08$ & $6.3 \mathrm{E}+08$ & $2.0 \mathrm{E}+09$ & $1.0 \mathrm{E}+10$ & $3.2 \mathrm{E}+10$ & $1.6 \mathrm{E}+11$ & $5.1 \mathrm{E}+11$ & $1.2 \mathrm{E}+12$ & $2.6 \mathrm{E}+12$ \\
\hline
\end{tabular}




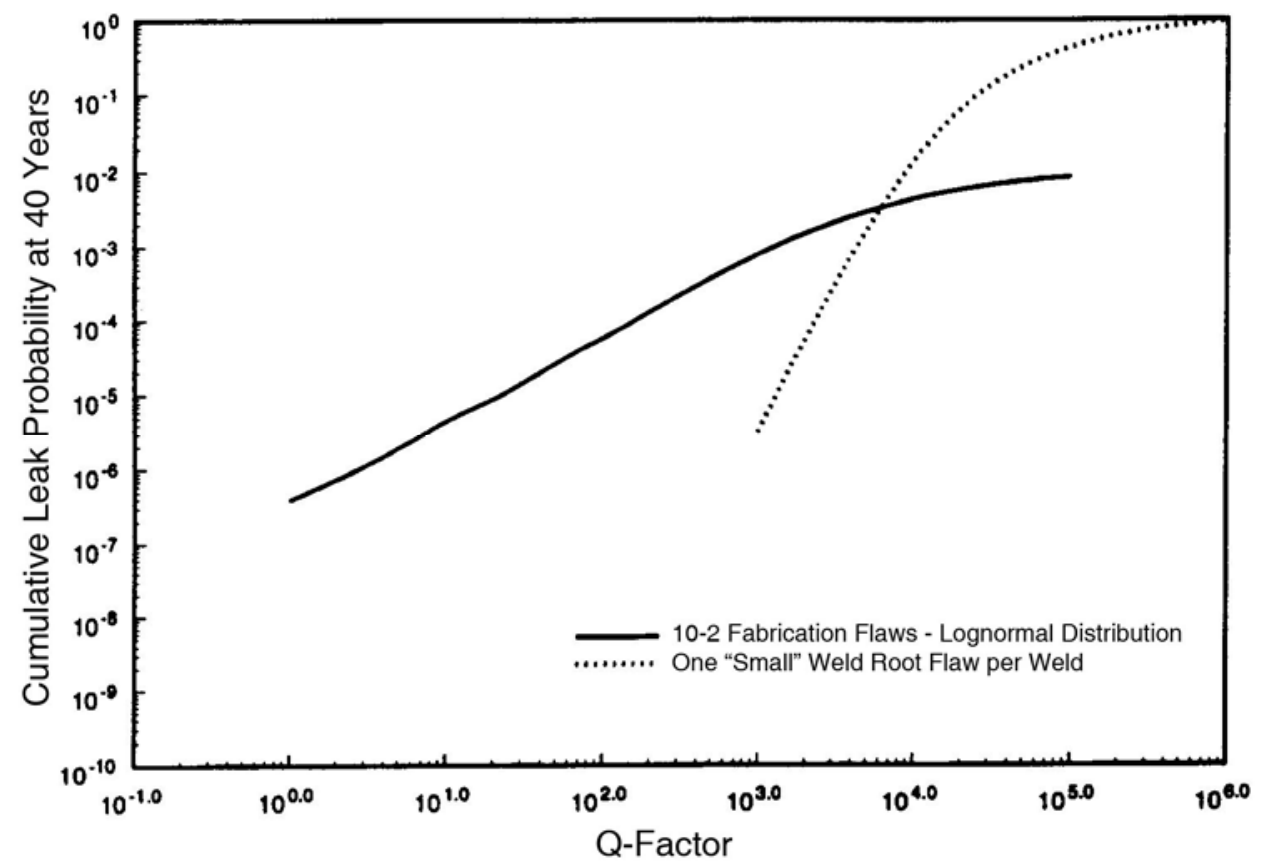

Figure 3.2 Probability of Leak as a Function of Crack Growth Parameter $Q=\alpha N(\Delta \sigma)^{4}$ for Two Categories of Defect Populations

Table 3.3 PRAISE Model for 6-in. (152-mm) Schedule 120 Stainless Steel Pipe: Baseline Case

\begin{tabular}{||l|l||}
\hline Flaw Depth Distribution & $\begin{array}{l}\text { Lognormal (Median Depth }=0.0959 \mathrm{in} . \\
\text { Parameter }=0.262)\end{array}$ \\
\hline Flaw Aspect Ratio & Lognormal $($ Parameter $=0.689)$ \\
\hline Stress Through Wall Thickness & Uniform Tension \\
\hline Cyclic Stress Amplitude & $15 \mathrm{ksi}(103 \mathrm{MPa}) / 5$ cycles per year \\
\hline da/dN Curves & See Khaleel and Simonen $(1994 \mathrm{a})$ \\
\hline Threshold $\Delta \mathrm{K}$ for da/dN & 0.00 \\
\hline Flow Stress & Normal $\left(\mathrm{Mean}=43 \mathrm{ksi}[296 \mathrm{MPa}], \mathrm{C} . \mathrm{O} . \mathrm{V} .^{(\mathrm{a})}=0.0977\right)$ \\
\hline Pipe Inner Radius & $2.75 \mathrm{in} .(69.8 \mathrm{~mm})$ \\
\hline Pipe Wall Thickness & $0.562 \mathrm{in} .(14.3 \mathrm{~mm})$ \\
\hline Pressure & $2.250 \mathrm{ksi}(15.5 \mathrm{MPa})$ \\
\hline Dead Weight Stress & $3 \mathrm{ksi}(20.7 \mathrm{MPa})$ \\
\hline Thermal Expansion Stress & $10 \mathrm{ksi}(69.0 \mathrm{MPa})$ \\
\hline (a) C.O.V. = Coefficient of variation & $=$ standard deviation/mean. \\
\hline \hline
\end{tabular}




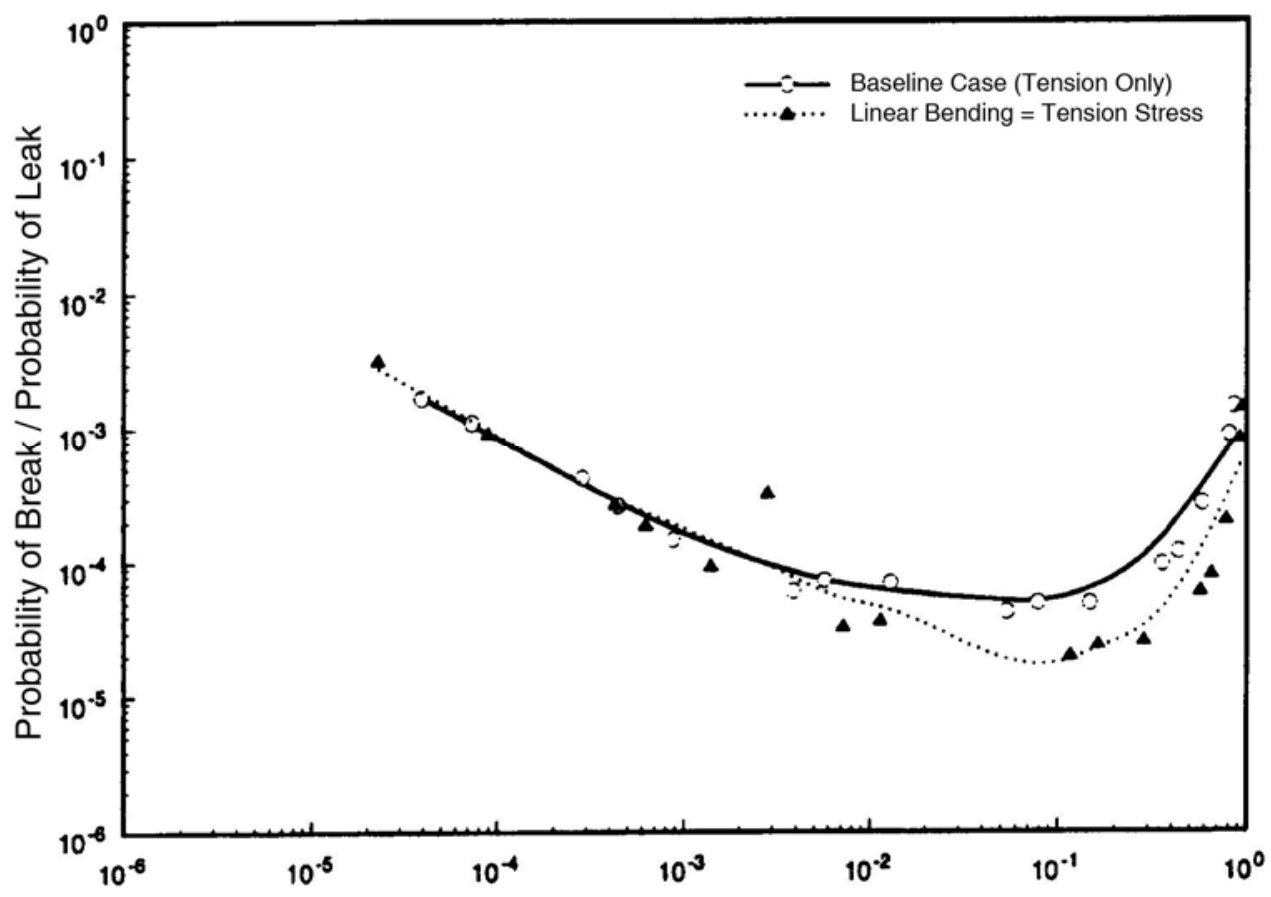

Figure 3.3 Probability of Break as a Function of the Leak Probability

pipe failures are most likely to be in the mode of breaks when the corresponding leak probabilities are relatively small. These breaks are caused by large fabrication flaws that are both very deep and very long. High values of calculated leak probabilities corresponded to fabrication flaws which have small initial depths that are associated with high levels of cyclic stress. The fracture mechanics model predicts that this combination of parameters will usually result in pipe failures as leaks rather than as breaks. This predicted trend occurs because the fatigue crack growth process gives much larger increases in flaw depths as a fraction of wall thickness as opposed to the corresponding increases in flaw lengths as a fraction of the pipe circumference.

\subsubsection{Effects of Through-Wall Stresses Gradients}

The calculations of this report generally assume a simple state of uniform tensile stress through the thickness of the pipe wall, thereby neglecting the effects of more complex stress distributions associated with through-wall stress gradients due to (1) discontinuity bending stresses, (2) radial thermal gradients, and (3) stress concentrations at geometric discontinuities located at the pipe inner surface. Sensitivity calculations have been performed with pc-PRAISE to evaluate the effect of the more complex stress states on calculated failure probabilities. The calculations provide a basis for estimating the effects of these stress contributions by using a modification of the calculated Q-factor.

Figures 3.4 through 3.7 provide results of calculations that show the sensitivity of calculated leak probabilities to the contributions of uniform tension stress, linear stress gradients, and weld-root stress concentrations. In these calculations, exactly one flaw was assumed to exist in the weld of interest. All cases were based on the dimensions of a 6-in. (152-mm) Schedule 120 stainless steel pipe and the other 


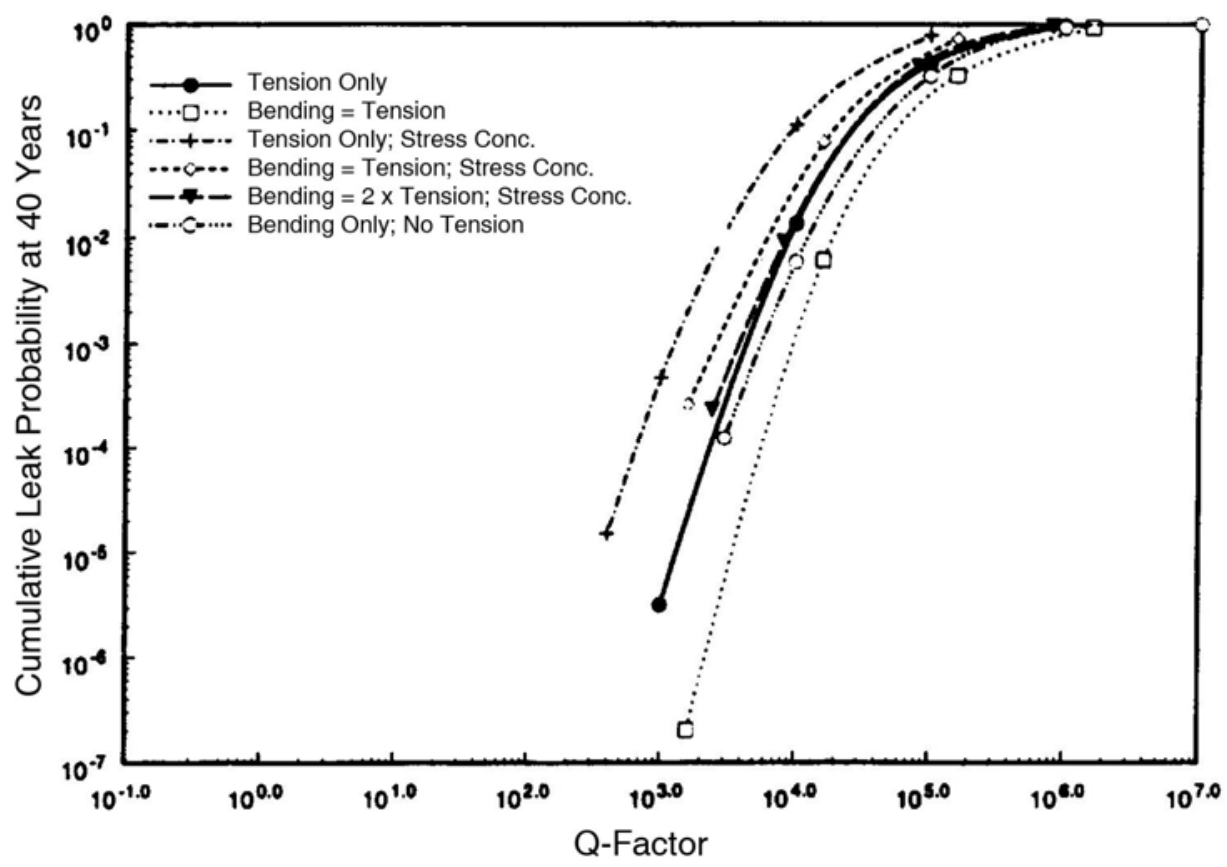

Figure 3.4 Probability of Leak as a Function of Fatigue Crack Growth Parameter $Q=\alpha N(\Delta \sigma)^{4}$ (small flaws with depths $=0.002$ to 0.01 in. $[0.051$ to $0.25 \mathrm{~mm}], Q$ based on maximum stress)

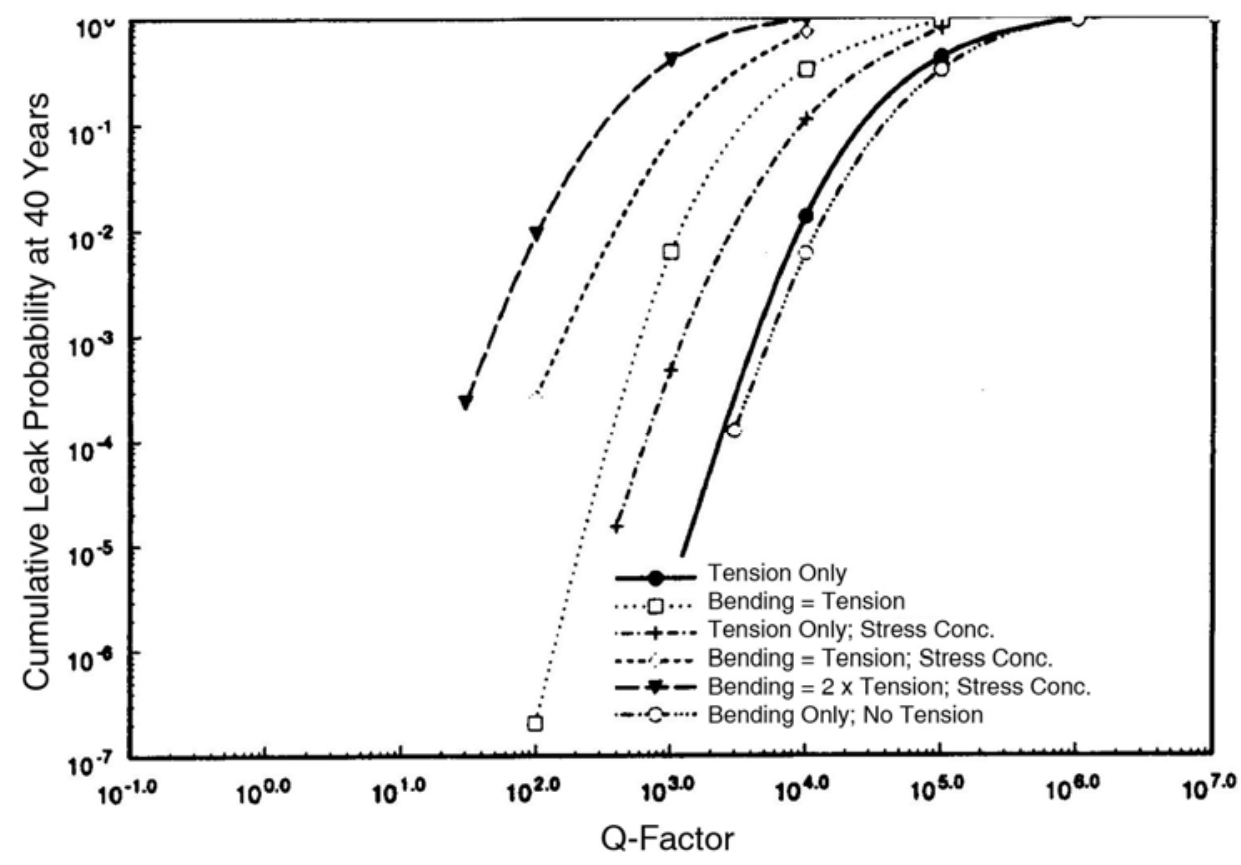

Figure 3.5 Probability of Leak as a Function of Fatigue Crack Growth Parameter $Q=\alpha \mathrm{N}(\Delta \sigma)^{4}$ (small flaws with depths $=0.002$ to 0.01 in. $[0.051$ to $0.25 \mathrm{~mm}], \mathrm{Q}$ based on averaged through-thickness stress) 


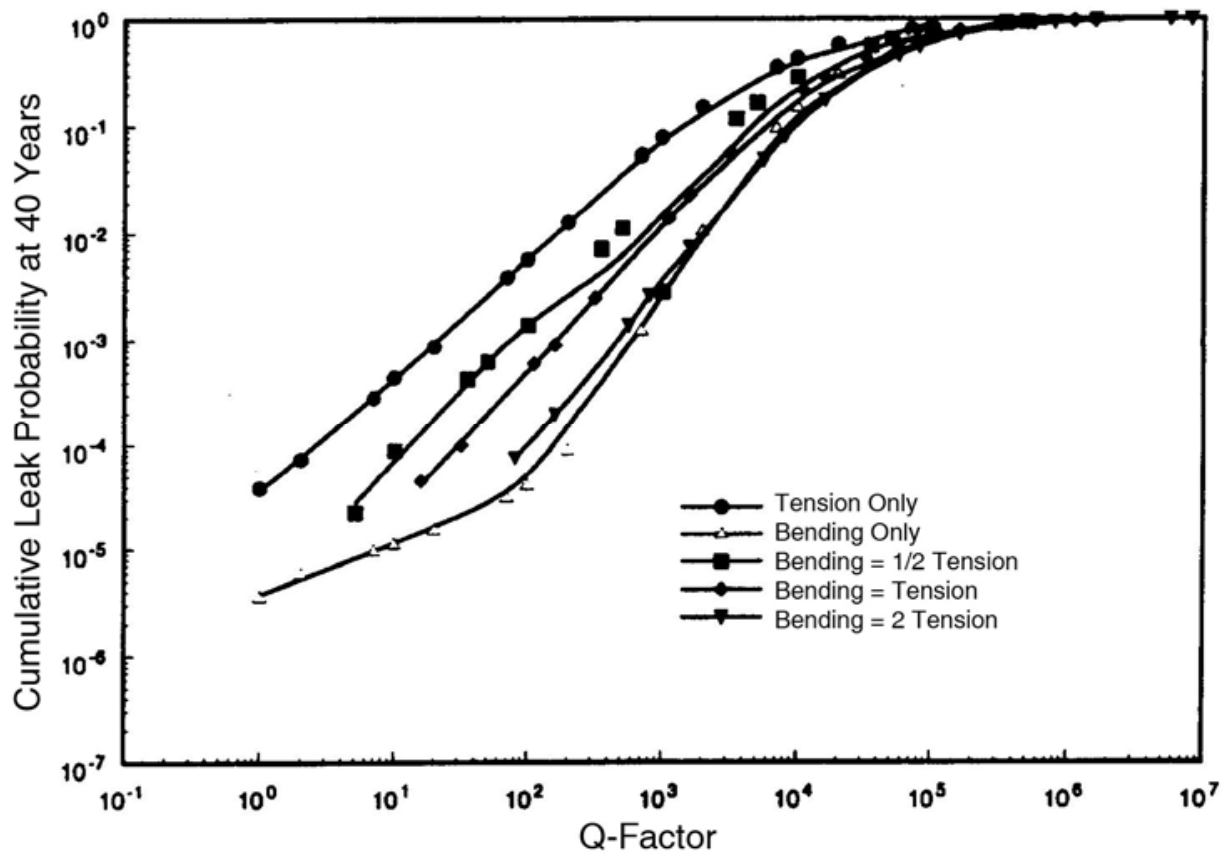

Figure 3.6 Probability of Leak as a Function of Fatigue Crack Growth Parameter $Q=\alpha \mathbf{N}(\Delta \sigma)^{4}$ (flaws depths based on lognormal distribution, $Q$ based on maximum stress)

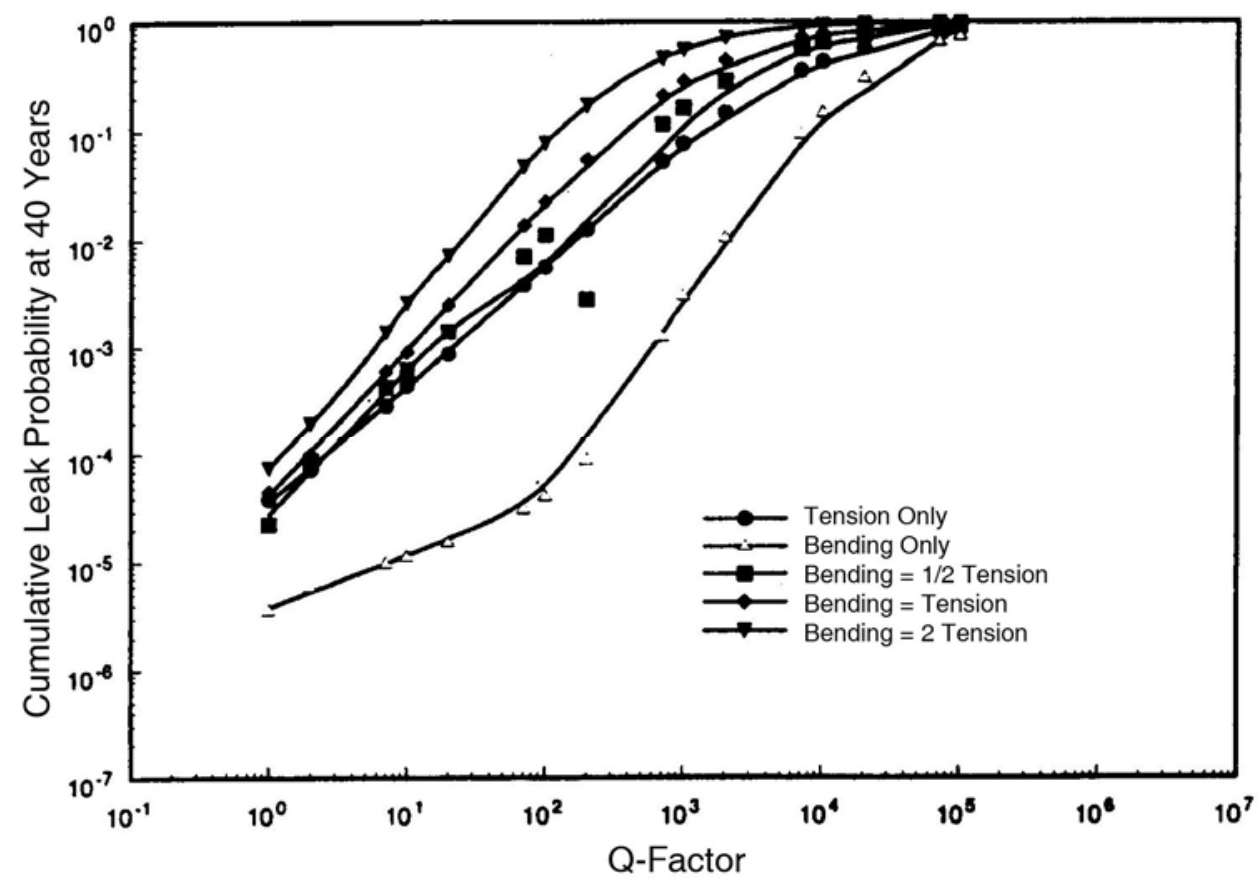

Figure 3.7 Probability of Leak as a Function of Fatigue Crack Growth Parameter $Q=\alpha \mathbf{N}(\Delta \sigma)^{4}$ (flaws depths based on lognormal distribution, $Q$ based on averaged through-thickness stress) 
baseline parameters listed in Table 3.4. Two categories of flaw-depth distributions were considered, the first being a lognormal distribution with a median of 0.0959 in. $(2.4 \mathrm{~mm})$ and a shape parameter of 0.262 . The second distribution represented much smaller and more likely flaws as described by a uniform distribution of depths ranging from 0.002 to 0.010 in. $(0.05$ to $0.25 \mathrm{~mm})$ All defects were assumed to be circumferential cracks at the inside surface of the pipe.

Two definitions for the calculated fatigue crack growth parameter Q were used. In the first method, the maximum or inner surface value of the tensile cyclic stress was used to calculate the $Q$ parameter (see Figures 3.4 and 3.6). This maximum value represented the peak stress at the location of the flaw and included the combined effects of uniform tension, through-wall linear stress gradient, and the weld-root stress concentration.

In the second method (see Figures 3.5 and 3.7), the fatigue crack growth parameter Q was calculated using a spatially averaged stress value (i.e., the uniform tension component of cyclic stress). Various cases of non-uniform through-wall stress distributions are indicated in Figures 3.4 through 3.7. In each case, the average stress had a superimposed component of stress that represented cyclic linear gradient stresses (tension on the inner surface and compression on the outer surface) and/or local stress concentrations (factor of 3.0 at the ID of the pipe) due to geometric irregularities such as at a weld root.

Comparisons of Figures 3.4 and 3.6 and Figures 3.5 and 3.7 show that the use of the through thickness averaged stress for calculating the Q-factor results in a very wide band of curves for leak probabilities. Also, Figures 3.4 and 3.6 show that the uniform-tension case based on the maximum inner-surface stress provides an upper bound for all other cases of superimposed through-thickness stress gradients.

Figure 3.4 versus 3.6 and Figure 3.5 versus 3.7 show that the calculated leak probabilities differ significantly for the "small" weld-root flaws and the "large" lognormally distributed weld-root flaws. However, both categories of depth distributions result in leak probabilities that approach 100 percent for high values of the fatigue crack growth parameter Q (e.g., $\mathrm{Q}=1.0 \mathrm{E}+05$ ).

The calculations clearly show that leak probabilities are sensitive to superimposed linear gradient stresses and local stress concentrations. Other calculations focused on pipe breaks, for which critical crack sizes that give unstable crack propagation were based on the net section collapse criteria. In Figure 3.3, the predicted rupture probabilities are presented as a ratio of the break probability to the corresponding leak probability. Low values of this ratio imply that conditions are favorable to achieving leak-before-break. As indicated, the calculated leak-versus-break trends are relatively insensitive to through-wall stress gradients.

\subsubsection{Effect of Initial Flaw Length}

The flaw lengths in the baseline calculations were sampled from an aspect ratio distribution, which in most cases have flaw aspect ratios of $6: 1$ or less. In sensitivity calculation, the length distribution was modified to give longer flaws whereby there was an equal probability of any given flaw having a length up to a maximum length equal to the full circumference of the pipe. While this assumption regarding the initial flaw length had little effect on calculated leak probabilities, the assumption of longer flaws significantly increased the calculated break probabilities. 
Table 3.4 Results of Comparisons Between the pc-PRAISE and PARIS Codes

\begin{tabular}{|c|c|c|c|c|c|c|c|c|c|c|c|c|}
\hline \multirow{2}{*}{$\begin{array}{c}\text { Number } \\
\text { of Load } \\
\text { Cycles }\end{array}$} & \multicolumn{2}{|c|}{ pc-PRAISE } & \multicolumn{2}{|c|}{ PARIS } & \multicolumn{2}{|c|}{ pc-PRAISE } & \multicolumn{2}{|c|}{ PARIS } & \multicolumn{2}{|c|}{ pc-PRAISE } & \multicolumn{2}{|c|}{ PARIS } \\
\hline & Qleak & $\begin{array}{c}\sigma \\
\%\end{array}$ & Q leak & $\begin{array}{l}\sigma \\
\%\end{array}$ & $Q_{\text {big leak }}$ & $\begin{array}{l}\sigma \\
\%\end{array}$ & $Q_{\text {big leak }}$ & $\begin{array}{l}\sigma \\
\%\end{array}$ & $Q_{\text {break }}$ & $\begin{array}{c}\sigma \\
\%\end{array}$ & $Q_{\text {break }}$ & $\begin{array}{l}\sigma \\
\%\end{array}$ \\
\hline 1 & $1.88 \mathrm{E}-09$ & 8.6 & $2.01 \mathrm{E}-09$ & 3.3 & $8.6 \mathrm{E}-10$ & 13 & $9.0 \mathrm{E}-10$ & 4.1 & 0 & 0 & 0 & \\
\hline 50 & $2.34 \mathrm{E}-08$ & 1.9 & $2.33 \mathrm{E}-08$ & 0.6 & $1.20 \mathrm{E}-08$ & 2.8 & $1.10 \mathrm{E}-08$ & 1.0 & 0 & 0 & 0 & \\
\hline 100 & $3.66 \mathrm{E}-08$ & 1.2 & $3.61 \mathrm{E}-08$ & 0.5 & $1.85 \mathrm{E}-08$ & 1.7 & $1.71 \mathrm{E}-08$ & 1.0 & 3.3E-14 & 9 & 8.7E-14 & 9 \\
\hline 150 & 4.73E-08 & 0.9 & 4.68E-08 & 0.8 & $2.43 \mathrm{E}-08$ & 1.4 & $2.23 \mathrm{E}-08$ & 1.6 & $5.2 \mathrm{E}-13$ & 3 & $5.9 \mathrm{E}-13$ & 3 \\
\hline 200 & $5.75 \mathrm{E}-08$ & 0.7 & $5.73 \mathrm{E}-08$ & 1.4 & $3.05 \mathrm{E}-08$ & 1.4 & $2.71 \mathrm{E}-08$ & 2.9 & $1.3 \mathrm{E}-12$ & 2 & $1.4 \mathrm{E}-13$ & 3 \\
\hline
\end{tabular}




\subsubsection{Effect of Sustained Primary Stress Level on Failure Probabilities}

Parametric calculations of this report focus on pipe leak probabilities, with the objective to relate these leak probabilities to the cyclic stresses that cause preexisting cracks in a weld to grow to through-wall depths. The level of primary stress acting on the cracked pipe (used as input to the net section collapse calculation) was held constant in the parametric calculations. In all cases, the assigned primary stress levels were sufficiently low to have only a secondary effect on calculated leak probabilities. Nevertheless, in some cases (very deep flaws), the sustained primary stresses can induce unstable crack growth for part-through flaws resulting in the simulation of sudden leaks.

In this discussion, the primary stress is defined as the sum of the axial stresses caused by internal pressure, dead weight, and seismic bending moments. The stress caused by thermal-expansion bending moments is not included as part of the primary stress.

Sensitivity calculations were performed to determine the effect of relatively high levels of primary stress on calculated leak probabilities. These calculations were for the same 6-in. (152-mm) Schedule 120 stainless pipe addressed by other sensitivity calculations.

Two loading conditions were modeled in the pc-PRAISE calculations. The first simulated an essentially constant level of primary stress that was sustained over the simulated operating life of the piping system. The second transient simulated a fatigue stress at a rate of 5 cycles per year with the stress level adjusted to provide a range of Q-factors. The input to pc-PRAISE for the pipe dead-weight stress was adjusted to simulate the desired levels of primary stress.

Table 3.5 relates leak and rupture probabilities to the primary stress levels and to the cyclic stress levels as characterized by the fatigue crack growth parameter Q. Typical examples of the results are shown in Figure 3.8 for five levels of primary stress $(8,16,24,32$, and $43 \mathrm{ksi}[55,110,165,220$, and $296 \mathrm{MPa}])$. For the baseline case ( $8 \mathrm{ksi}$ [55 MPa] primary stress) the break probability is 3 to 4 orders of magnitude less than the leak probability. Increasing the primary stress increases both the leak and break probabilities, but with a much larger effect on the break probabilities. The net effect is to diminish the difference between the two probabilities as shown in Table 3.5.

For the very high primary stress of $43 \mathrm{ksi}(296 \mathrm{MPa})$, the leak and break probabilities have virtually the same magnitude. This limiting level of applied primary stress approaches the average flow stress of the pipe material, with the result being that even relatively small cracks can cause limit load failures of the pipe. In this situation, the pipe fails because of unstable crack extension such that a leak and a break occur at the same time.

\subsubsection{Effect of Abnormal/Seismic Stress Level on Failure Probabilities}

The above calculations assumed that the cracked pipe experiences the specified primary stress as a continuous or sustained load. In many cases (as for seismic events), the maximum value of primary stress is not a sustained stress. A potential pipe failure must await the actual occurrence of the event. The above calculations would therefore overestimate leak and break probabilities for two reasons: 
Table 3.5 Fatigue Crack Growth Parameter $Q=\alpha \mathrm{N}(\Delta \sigma)^{4}$ and Estimated Leak and Break Probabilities

\begin{tabular}{|c|c|c|c|c|c|}
\hline \multirow[b]{2}{*}{$\mathbf{Q}$} & \multicolumn{5}{|c|}{ Total Axial Stress, MPa (ksi) } \\
\hline & $8(55)$ & $16(110)$ & $24(165)$ & $32(220)$ & 43 (296) \\
\hline 1 & $\begin{array}{l}P_{L}=1.51 \mathrm{E}-05 \\
P_{B}=2.67 \mathrm{E}-08\end{array}$ & $\begin{array}{l}\mathrm{P}_{\mathrm{L}}=2.38 \mathrm{E}-05 \\
\mathrm{P}_{\mathrm{B}}=3.04 \mathrm{E}-07\end{array}$ & $\begin{array}{l}P_{L}=3.94 \mathrm{E}-05 \\
P_{B}=5.49 \mathrm{E}-06\end{array}$ & $\begin{array}{l}P_{L}=4.67 E-03 \\
P_{B}=4.64 E-04\end{array}$ & $\begin{array}{l}\mathrm{P}_{\mathrm{L}}=5.15 \mathrm{E}-01 \\
\mathrm{P}_{\mathrm{B}}=5.15 \mathrm{E}-01\end{array}$ \\
\hline 10 & $\begin{array}{l}P_{L}=1.21 \mathrm{E}-04 \\
P_{B}=9.43 \mathrm{E}-08\end{array}$ & $\begin{array}{l}\mathrm{P}_{\mathrm{L}}=2.02 \mathrm{E}-04 \\
\mathrm{P}_{\mathrm{B}}=8.15 \mathrm{E}-07\end{array}$ & $\begin{array}{l}P_{L}=4.01 E-4 \\
P_{B}=9.46 E-06\end{array}$ & $\begin{array}{l}\mathrm{P}_{\mathrm{L}}=5.40 \mathrm{E}-03 \\
\mathrm{P}_{\mathrm{B}}=4.82 \mathrm{E}-3\end{array}$ & $\begin{array}{l}\mathrm{P}_{\mathrm{L}}=5.17 \mathrm{E}-01 \\
\mathrm{P}_{\mathrm{B}}=5.16 \mathrm{E}-01\end{array}$ \\
\hline 100 & $\begin{array}{l}P_{L}=3.97 \mathrm{E}-03 \\
P_{B}=4.48 \mathrm{E}-07\end{array}$ & $\begin{array}{l}\mathrm{P}_{\mathrm{L}}=8.01 \mathrm{E}-03 \\
\mathrm{P}_{\mathrm{B}}=3.84 \mathrm{E}-06\end{array}$ & $\begin{array}{l}P_{L}=1.46 \mathrm{E}-02 \\
P_{B}=3.37 \mathrm{E}-05\end{array}$ & $\begin{array}{l}P_{L}=2.79 \mathrm{E}-02 \\
P_{B}=5.69 \mathrm{E}-03\end{array}$ & $\begin{array}{l}\mathrm{P}_{\mathrm{L}}=5.36 \mathrm{E}-01 \\
\mathrm{P}_{\mathrm{B}}=5.29 \mathrm{E}-01\end{array}$ \\
\hline 1000 & $\begin{array}{l}P_{L}=1.06 \mathrm{E}-01 \\
P_{B}=3.96 \mathrm{E}-06\end{array}$ & $\begin{array}{l}\mathrm{P}_{\mathrm{L}}=1.62 \mathrm{E}-01 \\
\mathrm{P}_{\mathrm{B}}=2.87 \mathrm{E}-05\end{array}$ & $\begin{array}{l}P_{L}=2.19 \mathrm{E}-01 \\
P_{B}=2.11 \mathrm{E}-04\end{array}$ & $\begin{array}{l}P_{L}=2.78 E-01 \\
P_{B}=1.02 E-02\end{array}$ & $\begin{array}{l}\mathrm{P}_{\mathrm{L}}=6.85 \mathrm{E}-01 \\
\mathrm{P}_{\mathrm{B}}=6.08 \mathrm{E}-01\end{array}$ \\
\hline 10,000 & $\begin{array}{l}\mathrm{P}_{\mathrm{L}}=5.53 \mathrm{E}-01 \\
\mathrm{P}_{\mathrm{B}}=5.30 \mathrm{E}-05\end{array}$ & $\begin{array}{l}\mathrm{P}_{\mathrm{L}}=6.40 \mathrm{E}-01 \\
\mathrm{P}_{\mathrm{B}}=5.72 \mathrm{E}-04\end{array}$ & $\begin{array}{l}P_{L}=7.07 E-01 \\
P_{B}=5.17 E-03\end{array}$ & $\begin{array}{l}P_{L}=7.59 \mathrm{E}-01 \\
P_{B}=9.75 \mathrm{E}-02\end{array}$ & $\begin{array}{l}\mathrm{P}_{\mathrm{L}}=9.08 \mathrm{E}-01 \\
\mathrm{P}_{\mathrm{B}}=8.22 \mathrm{E}-01\end{array}$ \\
\hline
\end{tabular}

(1) The calculations do not account for the low probability that the event will actually occur even once over the life span of the component

(2) The event is unlikely to occur at the very end of the component life span, at which time the cracked pipe is in its most severely degraded state.

The results of Figure 3.8 could be applied to abnormal/seismic events in an approximate manner. The 40-year cumulative failure probabilities (treated now as conditional failure probabilities given that the primary stress occurs) could be multiplied by the probability that the event occurs at least one time over the 40-year period. Such a calculation would still be conservative. It would account for the first of the two issues, but would not address the second issue related to exact time at which the event occurs. The sensitivity calculations described below address this second issue.

To evaluate the effects of abnormal loadings, an extensive set of PFM calculations was performed with pc-PRAISE. As part of these calculations, the abnormal stress (e.g., from a seismic event) was also included in the stress history that pc-PRAISE considered along with the fatigue crack growth analysis. Consistent with the methodology of pc-PRAISE, the abnormal stress history was converted to an equivalent constant amplitude stress history of a selected number of cycles. After the simulated occurrence of the postulated abnormal stress, the crack size was returned to its size before the event. Figure 3.9 can be interpreted as cumulative leak probabilities versus time over 40 years versus time (i.e., it is implied that the Q parameter increases with time) for various abnormal stress levels. The probabilities are conditional that the particular abnormal event occurs at the given value of $\mathrm{Q}$ (or time). The sustained contribution to the primary stress was held constant at $8 \mathrm{ksi}(55 \mathrm{MPa})$. The leak probabilities of Figure 3.9 consider levels of sustained primary stress plus abnormal primary stress equal to 16 and $24 \mathrm{ksi}(110$ and $165 \mathrm{MPa}$ ) for which the results are very close to those for the same level of purely sustained primary stress. 


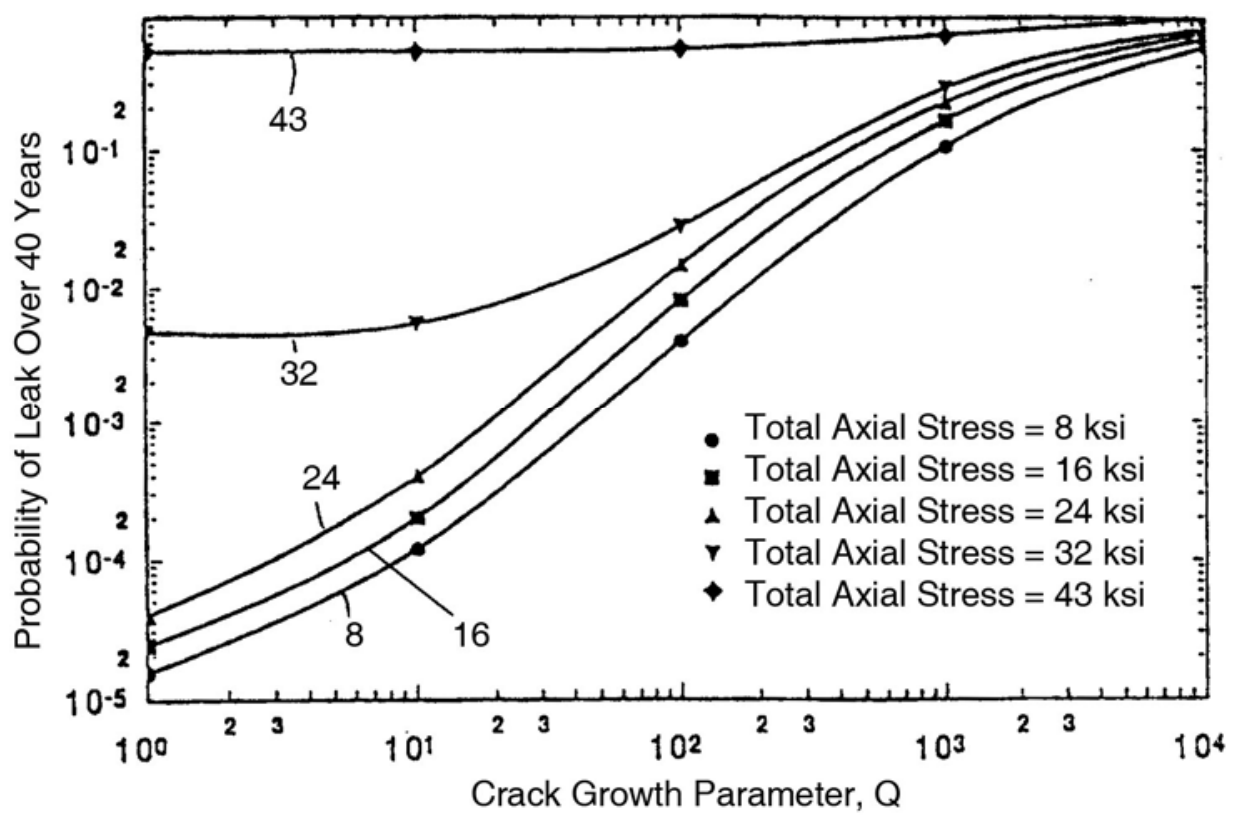

Figure 3.8 The Effect of Sustained Primary (Axial) Stress on Leak Probability with Crack Growth Parameter $=\alpha \mathrm{N}(\Delta \sigma)^{4}(1 \mathrm{ksi}=6.9 \mathrm{MPa})$

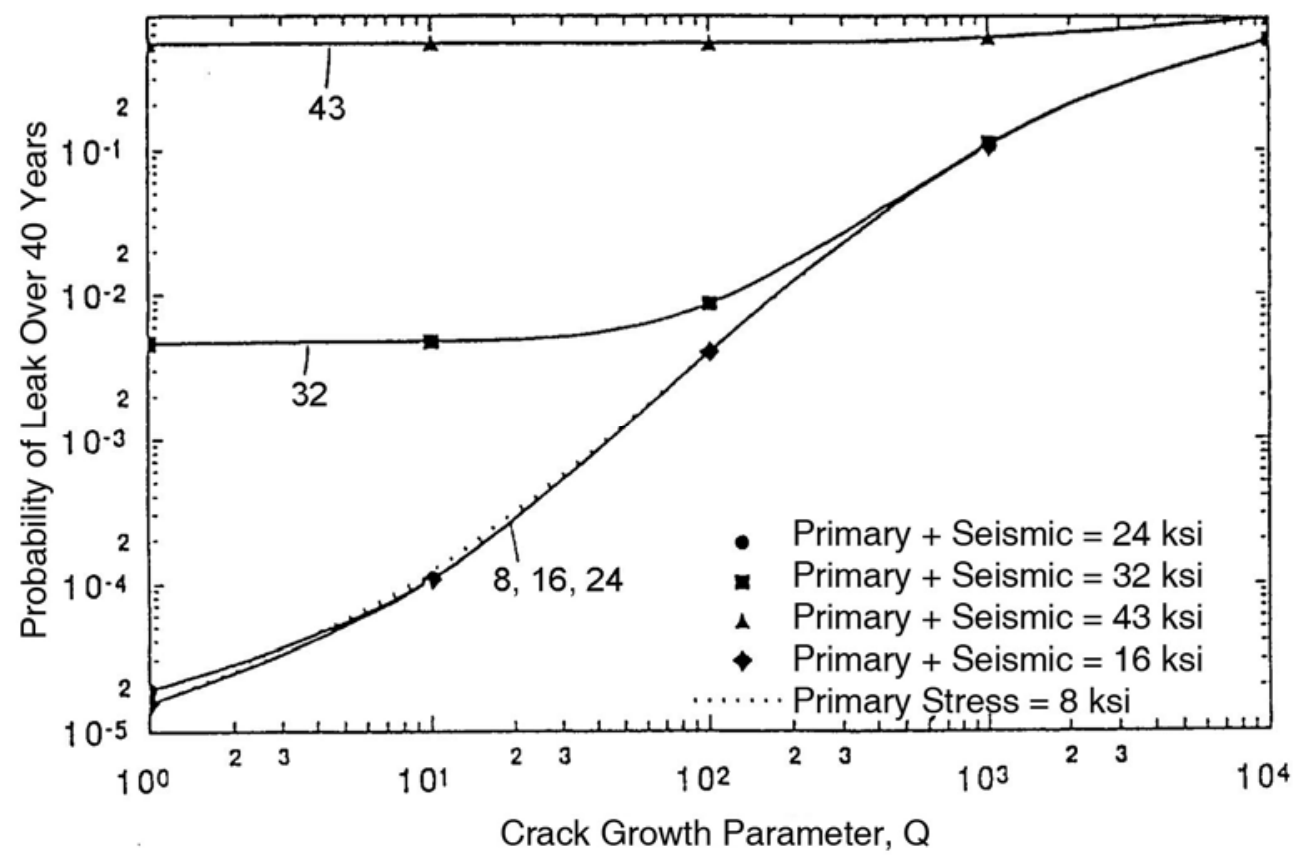

Figure 3.9 The Effect of Crack Growth Parameter $=\alpha \mathrm{N}(\Delta \sigma)^{4}$ and the Abnormal Stress on the Leak Probability (1 ksi = 6.9 MPa) 
Figure 3.10 compares the results for a sustained primary stress of $24 \mathrm{ksi}(165 \mathrm{MPa})$ with the results for an abnormal stress plus the sustained primary stress of $24 \mathrm{ksi}(165 \mathrm{MPa}$ ) (with only $8 \mathrm{ksi}$ [55 MPa] of this total being of the sustained category). The leak probabilities for a sustained axial stress of $24 \mathrm{ksi}$ $(165 \mathrm{MPa})$ are somewhat higher than those for an abnormal stress of $16 \mathrm{ksi}$ plus $8 \mathrm{ksi}$ (110 MPa plus $55 \mathrm{MPa}$ ) of sustained primary stress. The maximum ratio of these leak probabilities is about 3 . In conclusion, it appears that the results of the parametric calculations can be applied in an approximate manner to address the effects of low probability abnormal/seismic stresses. The sensitivity calculations show that it is conservative to use the results for the relevant level of primary stress (treated as a sustained stress), provided that these failure probabilities are multiplied by the probability that the event corresponding to the abnormal stress will occur over the life span of the component.

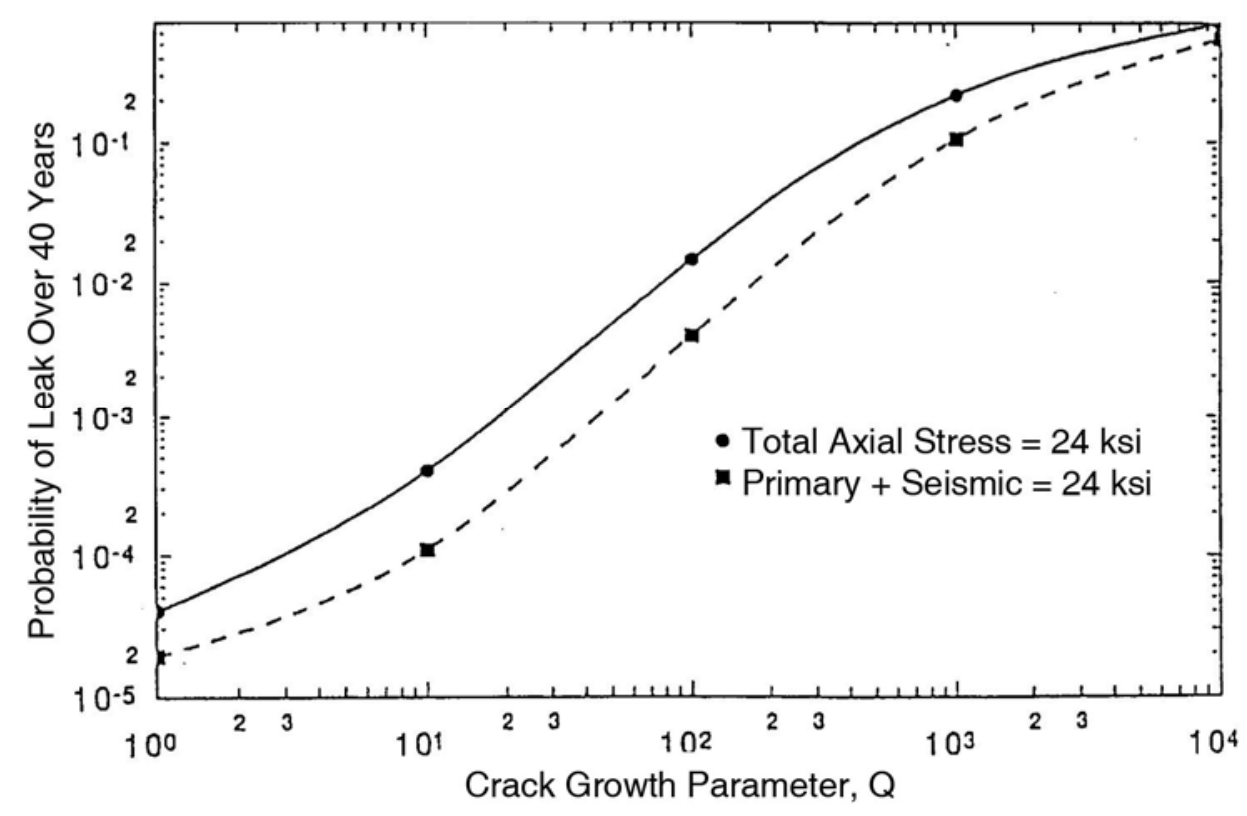

Figure 3.10 Comparison Between the Peak Probability for a Sustained Primary Stress of 24 ksi (165 MPa) and Abnormal Plus Primary Stress of 24 ksi (165 MPa) with Crack Growth Parameter $=\alpha \mathrm{N}(\Delta \sigma)^{4}(1 \mathrm{ksi}=6.9 \mathrm{MPa})$

\subsubsection{Effect of Leak Detection on Failure Probabilities}

The parametric calculations of this report have simulated and thereby taken credit for the effects of leak detection (assuming relatively sensitive levels of leak detection) as a measure that can reduce the probabilities of both pipe breaks and of large leaks. However, it is important to note that leak detection can have no effect on the calculated "small leak" probabilities, because pc-PRAISE defines a "small leak" as any through-wall crack, even if the leakage from the crack is essentially zero. This section describes sensitivity calculations that evaluate the effects of a wide range of leak-detection thresholds on calculated pipe break probabilities. 
The leak rate model in pc-PRAISE calculates leak rates for through-wall cracks by using the leak-rate model of the NRC/EPRI "SQUIRT" code (Paul et al. 1991). This model is based on two-phase flow through the crack geometry and on elastic-plastic crack opening area calculations. Several pc-PRAISE runs were performed to investigate the effect of the leak-detection threshold on the small leak, big leak, and break probabilities.

Figure 3.11 shows that (as expected) the calculated small leak probability from pc-PRAISE for the 6-in. (152-mm) Schedule 120 pipe is not affected by the leak-detection threshold. The break probabilities are constant up to a leak-detection threshold of about $300 \mathrm{gal} / \mathrm{min}(1,135 \mathrm{liter} / \mathrm{min})$ and is much less (by a factor of $\left.10^{-4}\right)$ than the corresponding small leak probabilities. Beyond $300 \mathrm{gal} / \mathrm{min}(1,135 \mathrm{liter} / \mathrm{min})$, the break probabilities become very sensitive to the leak-detection threshold as indicated by Figure 3.11. For thresholds above $1000 \mathrm{gal} / \mathrm{min}(3,785 \mathrm{liter} / \mathrm{min})$, the difference between the calculated leak and break probabilities become relatively small (a factor of 10 or less). This trend can be explained because a leaking through-wall fatigue crack will continue to grow in length at an accelerating rate under the action of the same cyclic stresses that originally grew the small fabrication crack to a through-wall depth. Without leak detection, all leaking cracks will eventually grow (with increasing high leak rates) to the critical size needed for a pipe break. For this scenario, the final pipe break will be prevented only if the end of the operating life span occurs before the crack grows to critical size.

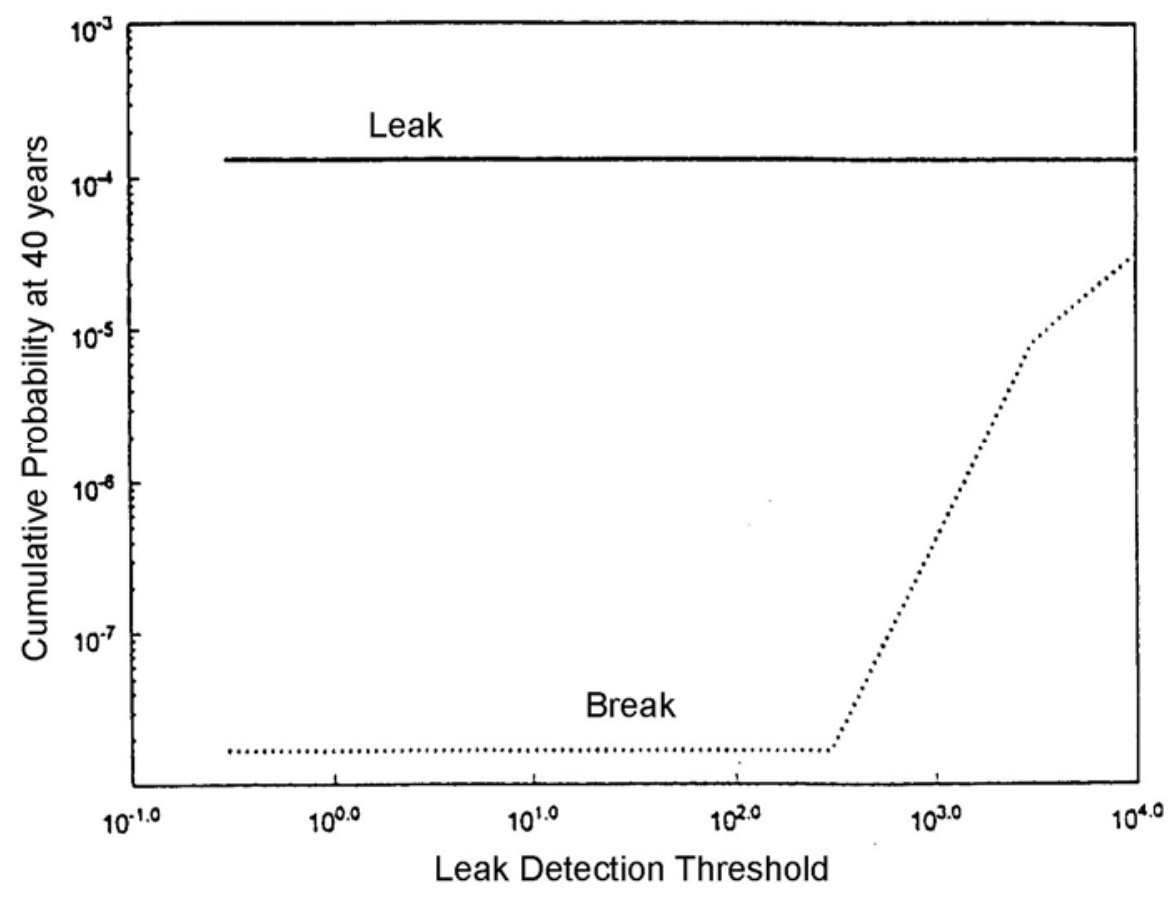

Figure 3.11 Effect of Leak Detection Threshold (gallons per minute) on Leak and Break Probabilities $(1$ gallon $/$ minute $=3.8$ liters $/$ minute $)$ 


\subsection{Summary and Conclusions}

This section has described a piping-reliability model and a method for predicting leak and break probabilities in a structured parametric format. The approach permits piping reliability to be estimated for a large number of welds and fittings without performing detailed Monte Carlo simulations for each component of interest. The model uses a fatigue crack growth model to grow pre-existing, semi-elliptical, fabrication defects. Critical flaw sizes for pipe breaks are based on a net section collapse criteria of fracture.

Sensitivity studies have been performed for various combinations of uniform tension and through-wall stress gradients caused by (1) discontinuity bending stresses, (2) radial thermal gradients, and (3) stress concentrations. Calculated leak probabilities were found to be sensitive to the different types of superimposed through-wall stress gradients and local stress concentrations. The parametric curves corresponding to uniform tension can be used as an upper bound to estimate leak probabilities for given Q-factor values. The lengths of initial flaws have little effect on small leak probabilities, whereas other calculations have shown that longer flaws significantly increase pipe-break probabilities. Pipe failures are more likely to be in the form of breaks when the corresponding leak probabilities and these break probabilities are relatively small because the initial flaws that result in pipe failures are both very deep and very long.

The sensitivity calculations indicate that pipe leak probabilities can be sensitive to the sustained primary stress (as opposed to cyclic stresses), but only when the primary stresses are a large fraction of the flow stress of the pipe material. These calculations also show that the effects of potentially high primary stresses caused by low probability abnormal/seismic events can be estimated using the results of the parametric calculations.

Leak detection can significantly reduce probabilities of pipe breaks and large leaks. The potential benefits of leak detection are relatively insensitive to the leak-detection threshold for typical leakdetection capabilities. The threshold is important only if the leak-detection capability can detect only very large rates of leakage. 


\section{Fatigue of Stainless Steel Piping}

\subsection{Introduction}

This section presents results of parametric calculations that predict the effects of alternative inspection strategies on reducing failure probabilities for stainless steel piping. It is assumed in these calculations that the failure mechanism of concern is fatigue crack growth from pre-existing fabrication flaws located at or near the inner surface of circumferential welds. The results were developed to guide the selection of inservice strategies with suitable flaw-detection capabilities and inspection frequencies. The selected strategies should ensure reliable detection of degradation before through-wall cracks result in leaks, and thereby provide desired reductions in piping-failure probabilities. The overall objective was to generate a broad base of data suitable for use in developing risk-informed inservice inspection plans. Section 5 of this report presents results of calculations that predict stress-corrosion cracking of stainless steel piping.

Calculations were performed with the pc-PRAISE computer code using the technical approach and procedures described in Section 3. A large range of parameters was addressed to cover a broad sample of pipe sizes and cyclic stress levels. In each case, the probabilistic model simulated fatigue crack growth from fabrication cracks in circumferential welds of as-built piping systems. The calculations addressed the full operating life of the components and simulated the detection of the growing cracks along with the resulting prevention of piping failures due to timely repairs.

\subsection{Scope of Calculations}

The calculations of this section were based on a number of modeling assumptions and addressed a range of parameters as follows:

It was assumed that all failures were associated with weld fabrication flaws, which were conservatively treated as surface breaking flaws at the inner surface of the pipe. Fatigue crack initiation caused by cyclic stresses was not addressed.

- The pipe sizes ranged in wall thickness from 0.25 to 2.5 in. (6.35 to $63.5 \mathrm{~mm})$

- The cyclic fatigue stresses corresponded to Q-factors that ranged from 1.0 to values as large as 106. For low values of Q-factors, the calculations predicted very little fatigue crack growth even for very large initial flaws (50 percent of wall or greater). For the highest values of Q-factors, even relatively small flaws (10 percent of wall or less) could grow to become through-wall cracks before the end of the 40-year service life. The levels of cyclic stresses for the very high Q-factors were clearly outside the range of the cyclic stresses acceptable to piping design codes (ASME Section III). These cases are nevertheless of interest to the development of inservice inspection programs in that the stresses correspond to unanticipated loadings, such as thermal fatigue, that have often occurred during plant operation and have been the cause of pipe-cracking incidents. 
- All parametric calculations in this section assumed a simple uniform distribution of tensile stress through the thickness of the pipe wall. The effects of through-wall stress gradients can be estimated by using an effective value of uniform tension. Sensitivity calculations described in Section 3 provide a basis for selecting suitable values for this effective value of uniform tensile stress.

- The simulations of inspection strategies focused on pipe leakage (i.e., through-wall cracks) as the failure mode of most concern to inspection rather than pipe breaks. The implied assumption was that the primary goal of inspection programs is to prevent leaks, with leak prevention considered to be an important element that is part of a defense-in-depth approach to piping integrity. In this regard, calculations of leak probabilities involve fewer uncertainties than calculations of pipe-break probabilities and are less sensitive to certain poorly defined inputs, such as flaw-length distributions, leak-detection thresholds, and occurrence frequencies for design-limiting loads. However, it should be recognized that the growth of cracks to through-wall depths is a precursor event to pipe breaks. Therefore, the prevention of pipe leaks can also serve as a useful surrogate measure for the effectiveness of given inservice inspections to prevent pipe breaks.

- The calculations of leak probabilities were not intended to study the effects of primary stresses in piping systems, which are a major factor for pipe-break probabilities, but are a secondary factor for leak probabilities. Primary stresses were included in the calculations, but were assigned relatively low values consistent with the relative low-stress primary stress levels for most locations within piping systems.

- Results of calculations are reported in terms of the cumulative failure probabilities at 40 years (endof-life). While the pc-PRAISE code calculates failure probabilities corresponded to each year throughout the plant operating life, it was not the intent to address aging effects or time-dependent failure rates in detail. Nevertheless, the presentations of results (i.e., cumulative failure probability versus Q-factor) can provide insight into time-dependent effects because the Q-factor itself can be interpreted as a surrogate for time.

- The calculations address inservice inspections by ultrasonic NDE performed on a periodic basis. Each inspection strategy corresponds to a given probability of detection (POD) curve associated with a given schedule of inspection intervals. It was assumed that all detected flaws are repaired. As such, the calculations did not address the effects of flaw-sizing errors and flaw-acceptance criteria.

- All calculations take credit for leak detection and assume a relatively sensitive leak-detection threshold of $3 \mathrm{gal} / \mathrm{min}$ (11.3 liter/min). This assumption had an effect only on calculated probabilities of large leaks and pipe breaks, but had no effect on small-leak probabilities.

- The calculations also address some important features of the inspection model, such as the effect of correlated versus independent probabilities of flaw detection during a sequence of periodic inspections.

- The calculations characterize the benefits of each inspection strategy in terms of a single parameter "improvement factor." The improvement factor is the ratio of failure probability without inspection to the failure probability with inspection. No inspection (or a totally ineffective inspection) corresponds to an improvement factor of 1.0. In this report, we will assume that a relatively effective 
inspection strategy is one that provides improvement factors of 10 or greater. An important objective of the probabilistic calculations was to identify those inspection strategies that provide high values of improvement factors for a wide range of conditions, such as pipe size and operating stresses.

\subsection{Definition of Input Parameters}

In this section, we document the values for the input parameters used in the parametric calculations and define both the inputs that were held constant and the inputs that were varied in a parametric manner.

\subsubsection{Pipe Dimensions}

Four pipe sizes with wall thicknesses ranging from 0.25 to 2.5 in. (6.35 to $63.5 \mathrm{~mm})$ were addressed with the dimensions as listed in Table 4.1.

Table 4.1 Pipe Size Inputs of Parametric Calculations for Stainless Steel Fatigue

\begin{tabular}{||l|c|c||}
\hline \multicolumn{1}{|c|}{ Pipe Size } & \multicolumn{1}{|c|}{$\begin{array}{c}\text { Inner Diameter, } \\
\text { in. }(\mathbf{m m})\end{array}$} & $\begin{array}{c}\text { Wall Thickness, } \\
\text { in. }(\mathbf{m m})\end{array}$ \\
\hline 3-in. Nominal Diameter & $3.00(76)$ & $0.250(6.35)$ \\
\hline 6-in. Schedule 120 & $5.50(140)$ & $0.562(14.3)$ \\
\hline 10-in. Nominal Diameter & $8.76(222)$ & $1.000(25.4)$ \\
\hline 27-in. Nominal Diameter & $25.5(648)$ & $2.500(63.5)$ \\
\hline 1 inch =25.4 mm & \multicolumn{3}{|l}{} \\
\hline
\end{tabular}

\subsubsection{Flaw-Depth Distributions}

The calculations were based on flaw-depth distributions that were developed in Section 2 by applying results from an expert system model (Chapman 1993). Inputs to the expert system were the pipe dimensions, material type, welding practices, configuration of weld passes, and the inspection methods. It was assumed that the welds for all pipe sizes used the manual metal arc process and that the welds (except for the 0.25 -in. [6.35-mm] wall thickness) were inspected using radiographic inspection. The initial flaws were circumferential and were conservatively placed at the inner pipe surface. The semielliptical surface cracks had depths between 0 and (with a low probability) the full wall thickness of the pipe wall. Surface lengths were such to give flaws ranging from a semi-circular shape and (with a low probability) to flaws extending the full pipe circumference. The lognormal distribution was used to characterize the defect depths. The parameters of the lognormal distributions are listed in Table 4.2.

Table 4.2 also lists the values of flaw densities and the corresponding number of flaws per weld for each of the four pipe sizes. These values were derived from the results of Section 2. In all cases, the flaws were treated in the fracture-mechanics calculations as inner-surface breaking cracks with a circumferential orientation. It should be noted that most of the results of this section are reported in terms of a ratio of failure probabilities (leak probabilities with inspection versus leak probabilities without inspection), which means that conclusions based on the calculations are insensitive to the estimated values of flaw densities. 
Table 4.2 Parameters for Lognormal Flaw Depth Distribution for Stainless Steel Piping

\begin{tabular}{||c|c|c|c|c|}
\hline \multicolumn{5}{|c|}{ Parameters } \\
\hline $\begin{array}{c}\text { Pipe Wall } \\
\text { Thickness, } \\
\text { in. (mm) }\end{array}$ & $\begin{array}{c}\text { Median Flaw } \\
\text { Depth, } \\
\text { in. (mm) }\end{array}$ & $\begin{array}{c}\text { Shape } \\
\text { Parameter }\end{array}$ & $\begin{array}{c}\text { Flaw Density Flaws per } \\
\text { Inch of Weld (Flaws per } \\
\text { Meter of Weld) }\end{array}$ & Flaws per Weld \\
\hline $0.250(6.35)$ & $0.1063(2.7)$ & 0.1784 & $0.0047(0.185)$ & 0.044 \\
\hline $0.562(14.3)$ & $0.0991(2.5)$ & 0.2669 & $0.0028(0.110)$ & 0.048 \\
\hline $1.000(25.4)$ & $0.0892(2.3)$ & 0.3672 & $0.0035(0.138)$ & 0.096 \\
\hline $2.500(63.5)$ & $0.0555(1.4)$ & 0.4993 & $0.0256(1.008)$ & 2.051 \\
\hline
\end{tabular}

\subsubsection{Aspect-Ratio Distribution}

The flaw-aspect ratio for the pc-PRAISE calculations is defined as $\beta=b / a$, where $2 b$ is the total length of the surface flaw and a is the depth of the flaw. The aspect ratio $\beta$ distribution recommended in the documentation for the pc-PRAISE code (Harris and Dedhia 1992) is given by the lognormal distribution

$$
f_{0}(\beta)=\left\{\begin{array}{cc}
0 & \beta<1 \\
\frac{C_{\beta}}{\lambda \beta(2 \pi)^{1 / 2}} e^{-\frac{1}{2 \lambda^{2}\left(\ln \beta / \beta_{m}\right)^{2}}} \beta \geq 1
\end{array}\right.
$$

where in the present calculations, $\lambda=0.5382, \mathrm{C}_{\beta}=1.419$, and $\beta_{\mathrm{m}}=1.136$. This aspect-ratio distribution was assumed to be independent of the flaw depth. A common distribution was used for all of the pipe sizes addressed in the present calculations. The data from the expert system model of Chapman (1993) also provided detailed information on simulated flaw-aspect ratios for each pipe size and for each category of flaw depth. This information was not used because the pc-PRAISE code would have required significant changes to the stratified sampling logic that was used by the code to simulate flaw dimensions.

\subsubsection{Crack Growth Rates}

Fatigue crack growth rates were calculated for stainless steels using an existing crack growth law of the pc-PRAISE code. The crack growth rate was given by

$$
\frac{d a}{d N}=C\left[\frac{\Delta K}{(1-R)^{1 / 2}}\right]^{m}
$$

$$
\text { where } \begin{aligned}
\Delta \mathrm{K} & =\mathrm{K}_{\max }-\mathrm{K}_{\min } \\
\mathrm{R} & =\mathrm{K}_{\min } / \mathrm{K}_{\max } \\
\mathrm{C} & =\text { lognormally distributed constant } \\
\mathrm{K}_{\max } & =\text { maximum stress intensity factor during transient } \\
\mathrm{K}_{\min } & =\text { minimum stress intensity factor during transient } \\
\mathrm{a} & =\text { crack depth } \\
\mathrm{N} & =\text { number of fatigue cycles } \\
\mathrm{m} & =\text { crack growth rate constant. }
\end{aligned}
$$


This equation approximates the ASME Section XI stainless steel crack growth rate equation for air environment $(\mathrm{R}=0)$, but does not address certain conditions that can accelerate crack growth rates. The literature shows crack growth rates in water (for low rates of cycling) to be up to 10 times greater than the air curve. For $\Delta \mathrm{K}$ in units of $\mathrm{MPa} \cdot \mathrm{m}^{1 / 2}$ and da/dN in units of meter/cycle, the median value of $\mathrm{C}$ and $90^{\text {th }}$ percentile become 62.6E-12 in./cycle (1.59E-13 m/cycle) and 2.39E-11 in./cycle (6.09E-13 m/cycle). For piping systems, the exponent $\mathrm{m}$ used by Harris and Lim (1983) was 4.0. A zero threshold $\Delta \mathrm{K}$ for the fatigue crackgrowth threshold was assumed. With $\Delta \mathrm{K}$ in units of $\mathrm{ksi} \cdot$ in $^{1 / 2}$, the median value of the $\mathrm{C}$ and the $90^{\text {th }}$ percentile were $9.14 \mathrm{E}-12 \mathrm{in} . /$ cycle $(2.32 \mathrm{E}-13 \mathrm{~m} /$ cycle) and 3.5E-11 in./cycle $(8.89 \mathrm{E}-13 \mathrm{~m} /$ cycle), respectively.

The results of parametric calculations are presented in this section using the parameter Q that accounts for the severity of the cyclic stresses, where Q is defined as follows:

$$
Q=\alpha N(\Delta \sigma)^{4}
$$

In this expression

$$
\begin{aligned}
\mathrm{N}= & \text { number of fatigue cycles } \\
\Delta \sigma= & \text { stress range }\left(\mathrm{ksi} \cdot \mathrm{in}^{1 / 2}\right) \\
\alpha= & \text { a constant, which is determined by setting } \mathrm{Q}=1 \text { for } \mathrm{N}=\text { number of accumulated cycles } \\
& \text { occurring at } 5 \text { cycles per year over } 40 \text { years, with } \Delta \sigma=15 \mathrm{ksi}(103.5 \mathrm{MPa}) .
\end{aligned}
$$

The rationale and the derivation for the Q-factor (Khaleel and Simonen 1994b) are based on the form of the above crack growth rate law with particular reference to the value of 4.0 for the exponent $\mathrm{m}$.

\subsubsection{Failure Criterion for Pipe Leakage}

The failure criterion for pipe leakage used in the pc-PRAISE code was $a_{n}=h$, where $h$ is the wall thickness and $a_{n}$ is the crack depth. It is to be noted that every simulated pipe break was also considered to be a leak with the Monte Carlo counting procedure used by the pc-PRAISE code.

\subsubsection{Failure Criterion for Pipe Break}

The failure criterion for pipe break in the pc-PRAISE code is net section collapse. The inputs to pcPRAISE specified the values for the applied primary stress and the material flow stress, with the values used in the present calculations to simulate the occurrence of net section collapse given in Table 4.3.

Table 4.3 Primary Stress and Flow Stress Inputs for Stainless Steel Fatigue

\begin{tabular}{||c|c|c|c||}
\hline $\begin{array}{c}\text { Pipe Wall } \\
\text { Thickness, } \\
\text { in. (mm) }\end{array}$ & $\begin{array}{c}\text { Primary Stress, } \\
\text { ksi (MPa) }\end{array}$ & $\begin{array}{c}\text { Flow Stress } \\
\text { Mean Value, } \\
\text { ksi (MPa) }\end{array}$ & $\begin{array}{c}\text { Flow Stress } \\
\text { Standard Deviation, } \\
\text { ksi (MPa) }\end{array}$ \\
\hline $0.250(6.35)$ & $7.98(55.0)$ & $43.0(296)$ & $4.2(28)$ \\
\hline $0.562(14.3)$ & $7.99(55.1)$ & $43.0(296)$ & $4.2(28)$ \\
\hline $1.000(25.4)$ & $8.00(55.2)$ & $43.0(296)$ & $4.2(28)$ \\
\hline $2.500(63.5)$ & $8.03(55.4)$ & $43.0(296)$ & $4.2(28)$ \\
\hline
\end{tabular}




\subsection{Probability of Detection Curves}

The evaluations of alternative inspection strategies addressed a range of probability of detection (POD) curves for ultrasonic examination of stainless steel piping. The approach was to establish POD curves that represented widely differing levels of NDE performance. Individual curves were intended to bound the probability of detection levels expected from inspection teams performing inservice inspections of piping at plants in the field. To establish POD curves, an informal expert judgment elicitation was performed using staff at PNNL with specialized knowledge of NDE performance data from pipinginspection round robins and from recent industry efforts in the area of NDE performance demonstrations.

It was recognized that a population of inspection teams operating under field conditions can exhibit a considerable range of POD performance, even though all such teams meet minimum code requirements for inspecting nuclear piping systems. The basic premise in estimating POD curves for the present calculations was that the better teams had passed the ASME Section XI Appendix VIII performance demonstration. ASME Section XI requires a minimum of 10 flaws and 20 blanks to be examined and requires a failing grade for either of the following: (1) more than two false calls or (2) the team fails to detect at least 8 of the 10 flaws. The informal expert judgment also considered information and trends observed in the PNNL round robin studies on UT inspection of wrought stainless steel piping with wall thicknesses of 0.60 to 1.0 in. (15.2 to $25.4 \mathrm{~mm}$ ) (Bates et al. 1987; Heasler et al. 1990).

The NDE experts at PNNL were asked to define POD curves by estimating parameters for the POD function used in the pc-PRAISE code:

$$
\begin{aligned}
\mathrm{P}_{\mathrm{ND}}(A) & =\varepsilon+\frac{1}{2}(1-\varepsilon) \\
& =\operatorname{erfc}\left[v\left(A / A^{*}\right)\right]
\end{aligned}
$$

where $\mathrm{P}_{\mathrm{ND}}=$ probability of nondetection $=1-\mathrm{POD}$

$\mathrm{A}=$ area of the crack

$\mathrm{A}^{*}=$ area of crack for $50 \% \mathrm{P}_{\mathrm{ND}}$

$\varepsilon=$ smallest possible $\mathrm{P}_{\mathrm{ND}}$ for very large cracks

$v=$ "slope" of the $\mathrm{P}_{\mathrm{ND}}$ curve.

Based on measured performance levels for PNNL's mini round-robin teams (Heasler et al. 1990; Heasler and Doctor 1996), a range of estimates for A* (crack area for 50\% POD) was provided by the NDE experts. Harris, Dedhia, and Lu (1992) assumed that the "slope" parameter $v$ is 1.6. Several POD curves from PNNL studies were reviewed, and it was determined that a value of $v=1.6$ was consistent with published curves. While the assigned value of the slope parameter $v$ was held constant, the actual slope of the plotted curves becomes steeper for better POD curves. Thus, the slope was implicitly correlated to the detection threshold parameter $A^{*}$. The value of $\varepsilon$ was assigned to ensure that smaller values of $\varepsilon$ also correspond to smaller values of $A^{*}$. Three POD curves were selected:

- Marginal Performance: A team with a POD performance that is described by this curve would have only a small chance of passing an Appendix VIII performance demonstration, and its performance would not be representative of current day capabilities. 
- Very Good Performance: This curve corresponds to the very best teams. Such teams significantly exceed the minimum level of performance needed to pass an Appendix VIII performance demonstration test.

- Outstanding Performance: This curve corresponds to a team that has a level of performance significantly better than expected from most teams that have passed an Appendix VIII-type of performance demonstration. Such a team would need to apply advanced technologies and/or improved procedures that might be available in the future.

Table 4.4 summarizes the data used as input to pc-PRAISE to describe the above three POD curves. The same POD curves with the POD function expressed in terms of the normalized flaw depth $(\mathrm{a} / \mathrm{t})$ were used for all pipe sizes. The parameter $\mathrm{a}^{*}$ is the crack depth for $50 \%$ probability of non-detection $\left(\mathrm{P}_{\mathrm{ND}}\right)$ and is related to $A^{*}$ (i.e., $A^{*}=\pi / 4 D_{B} a^{*}$, where $D_{B}$ is the beam diameter of the ultrasonic probe). The slope parameter is assumed to be constant for the selected range of POD curves. The smallest possible $\mathrm{P}_{\mathrm{ND}}$ for large cracks is the largest for the marginal POD (i.e., 10\%) and is the smallest for the outstanding POD curve (i.e., $0.5 \%$ ). The parameter $\varepsilon$ varies in the same manner as $\mathrm{a}^{*}$.

Table 4.4 Parameters of POD Curves for Three Performance Levels

\begin{tabular}{|c|c|c|c|}
\hline $\begin{array}{c}\text { Inspection } \\
\text { Performance }\end{array}$ & $a *(\% a / t)$ & $\varepsilon$ & $v$ \\
\hline Marginal & 40 & 0.1 & 1.6 \\
\hline Very Good & 15 & 0.02 & 1.6 \\
\hline Outstanding & 5 & 0 & 1.6 \\
\hline
\end{tabular}

\subsection{Sensitivity Calculations for Inspection Model}

Sensitivity calculations were performed to evaluate the effect of inspection modeling assumptions on the predicted benefits of inservice inspections relative to a set of baseline calculations. The baseline calculations used the following inputs for modeling inservice inspections:

1. lognormal distribution of flaw depth

2. the results of successive inspections to detect a particular flaw in a given weld (assumed to be correlated rather than independent events, meaning that a flaw not detected in one inspection is likely to go undetected in a later inspection)

3. the time corresponding to the first (arrival time) of the series of periodic inspections (assumed to be $50 \%$ of the time interval between inspections)

4. only inservice inspections (no preservice inspections). 


\subsubsection{Effect of Initial Flaw-Size Distribution}

Figure 4.1 addresses the 6-in. (152-mm) Schedule 120 pipe size and shows the calculated improvement factors for three reference POD curves (i.e., marginal, very good, and outstanding). It was assumed that ISI is performed every 10 years (assuming no preservice inspection) with the first inspection occurring at 5 years. The sensitivity calculations evaluated the effect of the prescribed initial crack-depth distribution (lognormal versus exponential) on calculated improvement factors. Figure 4.1 indicates that the selected functional form used to fit the initial crack-depth distribution to the flaw sizes (see Section 2) has little influence on the values of the improvement factors. For low values of Q, the difference between the results obtained by using the lognormal versus the exponential distribution decreases as the POD performance level becomes less effective. Harris and Lim (1983) also noted this lack of sensitivity of such results to the initial crack-depth distribution.

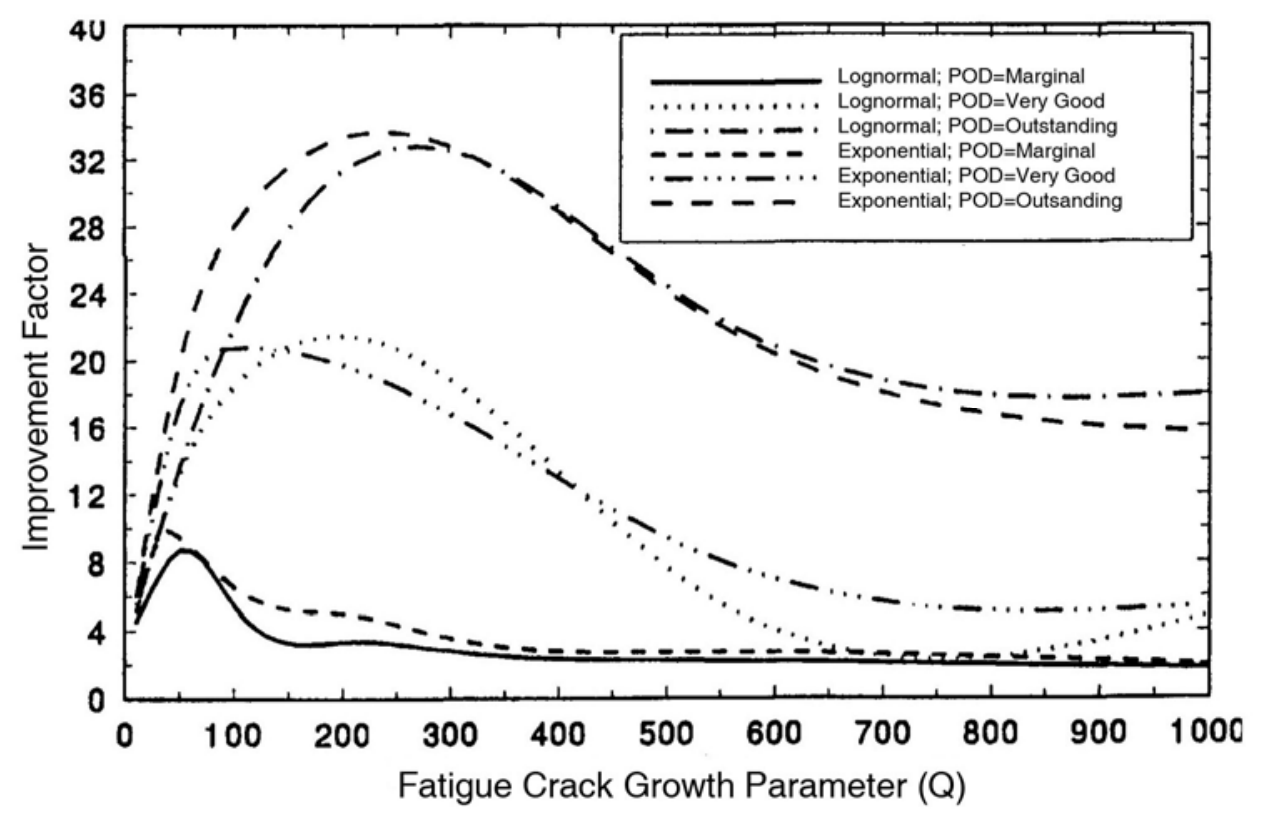

\section{Figure 4.1 The Effect of Initial Flaw Distribution on the Improvement Factors (ISI does not include PSI) with $Q=\alpha \mathrm{N}(\Delta \sigma)^{4}$}

\subsubsection{Effect of Time of the First Inspection}

To investigate the effect of the time of the first inspection on the calculated leak probability, several arrival times were considered. Typical results of these calculations are shown in Figure 4.2 for the four different arrival times for the first inspection, with subsequent inspections performed every 10 years thereafter. From time zero to the earliest of the various arrival times, the probabilities of failure for each POD curve are identical (as expected). In this example calculation, after the first inspection, the probabilities of failure diverge as a consequence of detecting and eliminating defects.

Figure 4.2 shows that the cumulative leak probability for a first arrival at 2 years is 3 times lower than that for the same strategy, but with an arrival time at 10 years. This trend is typical for cases with 
relatively low leak probabilities. The results support the selection of strategies that include a high quality inspection early in life. The results of Figure 4.2 show that such early inspections can detect a significant number of the unusually large fabrication flaws that can eventually result in pipe failure very early in the operating life of the plant.

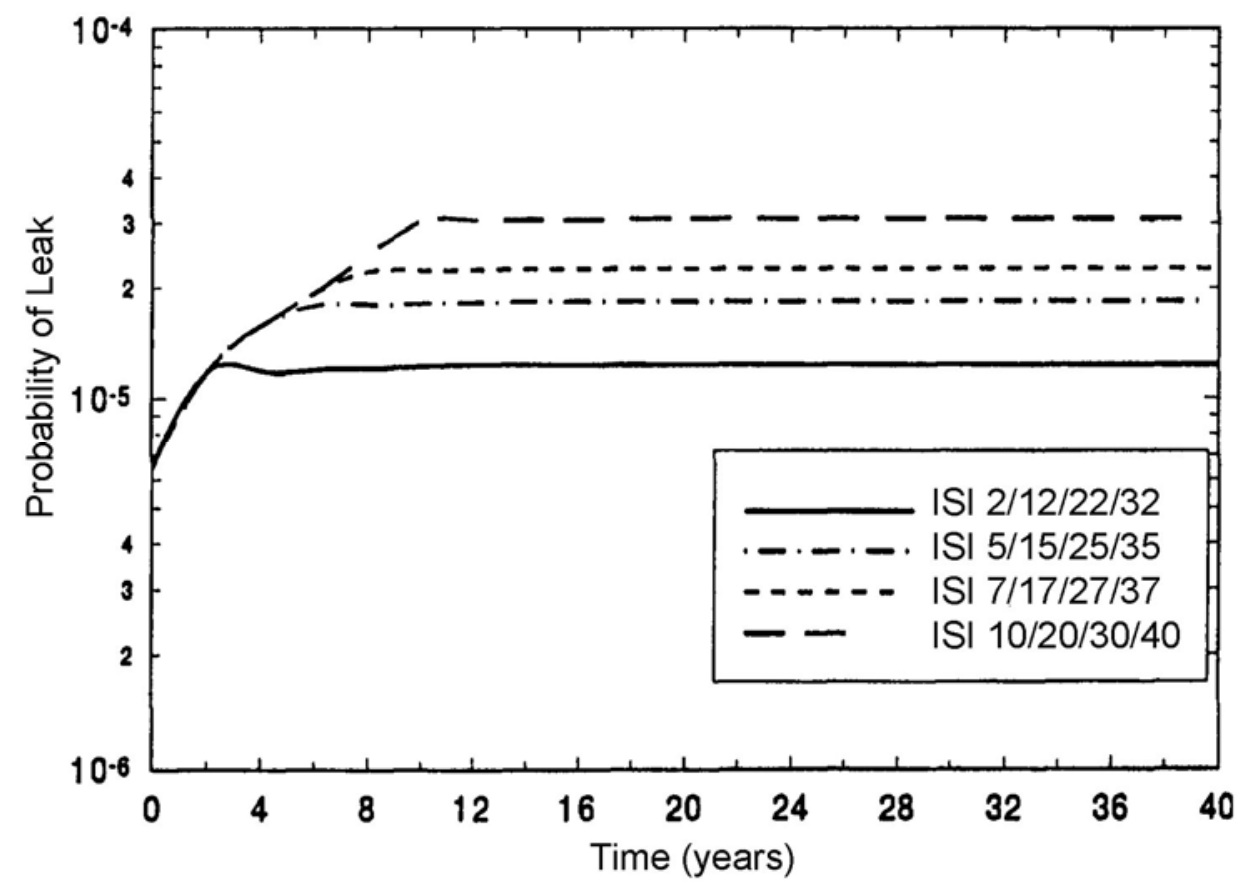

Figure 4.2 The Effect of Arrival Time of First Inservice Inspection on the Leak Probability of Failure

\subsubsection{Independent versus Correlated Inspections}

The concept of independent versus correlated inspections accounts for the fact that failure to detect a particular crack during one inspection often means that the crack has characteristics that will make it difficult to detect during subsequent inspections.

When the results of a series of inservice inspections are correlated, the ISI simulations of pc-PRAISE take credit only for one of the sequence of periodic inspections, namely the last of the inspections performed just before the crack would become through-wall. For independent inspections, credit is taken for all inspections. Figures 4.3 through 4.6 show the calculated improvement factors for both correlated (solid lines) and independent (dashed lines) inspections. The independent inspection assumption yields higher improvement factors than those calculated for the assumption of correlated inspections. The difference between the improvement factors for correlated versus independent inspection assumptions becomes particularly large for closely spaced inspections in combination with the POD curves that have relatively low performance levels. 


\subsubsection{Combined Preservice Inspections and Inservice Inspections}

Figure 4.7 shows calculated improvement factors for inspection strategies that include both preservice (PSI) and inservice inspections (ISI). The ISI schedules were every 10, 5, 2, and 1 years. These results reinforce the importance of high-quality preservice inspections in reducing leak probabilities. For a given POD curve and for Q-factors less than about 100, all inservice inspection schedules yielded the same improvement factor. Figure 4.7 also includes a limiting case that has the outstanding POD combined with ISI performed every year. In this case, the constant high value of the improvement factor extends significantly beyond the Q-factor of 100 out to a value of about 10,000 .

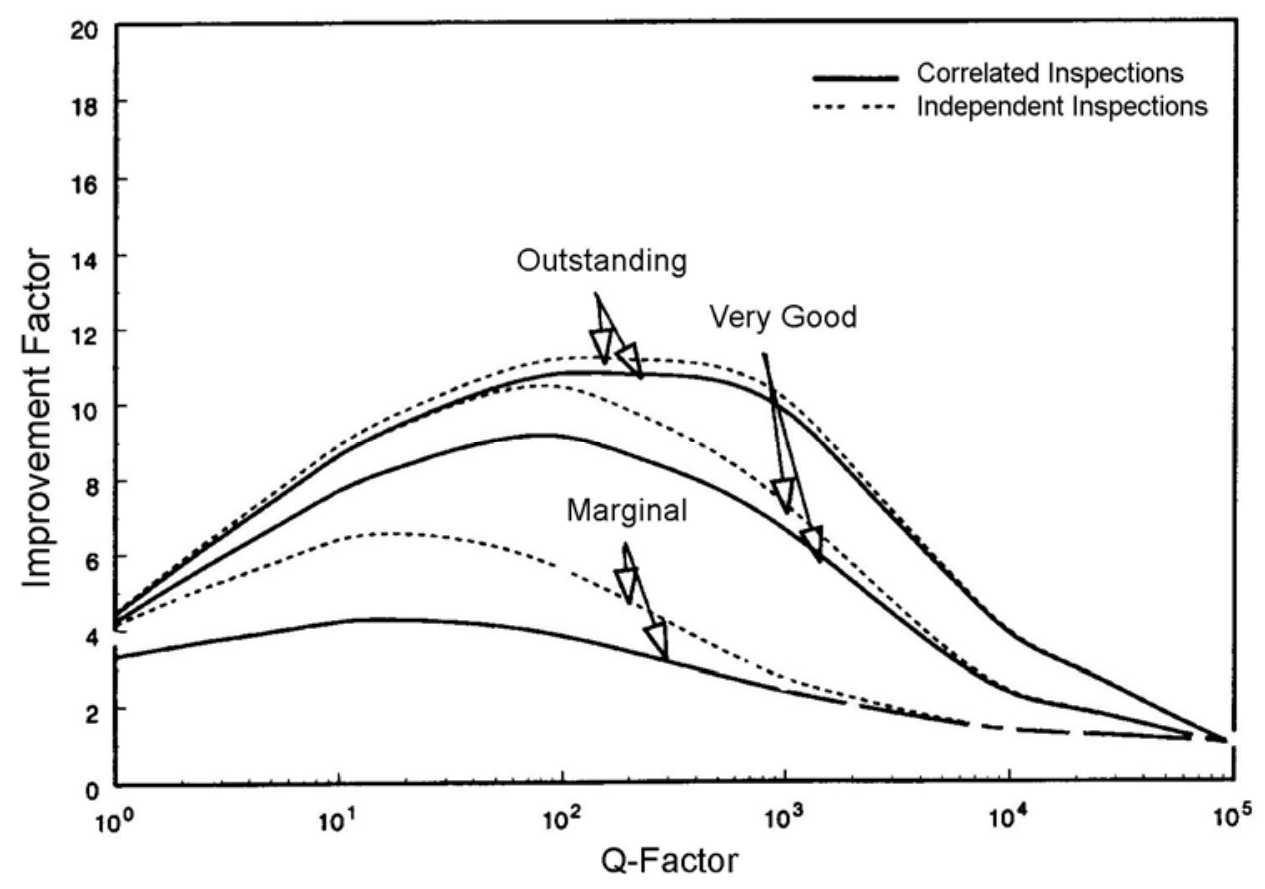

Figure 4.3 Improvement Factors for Independent and Correlated Inspections versus $Q=\alpha N(\Delta \sigma)^{4}$ for ISI Every 10 Years 


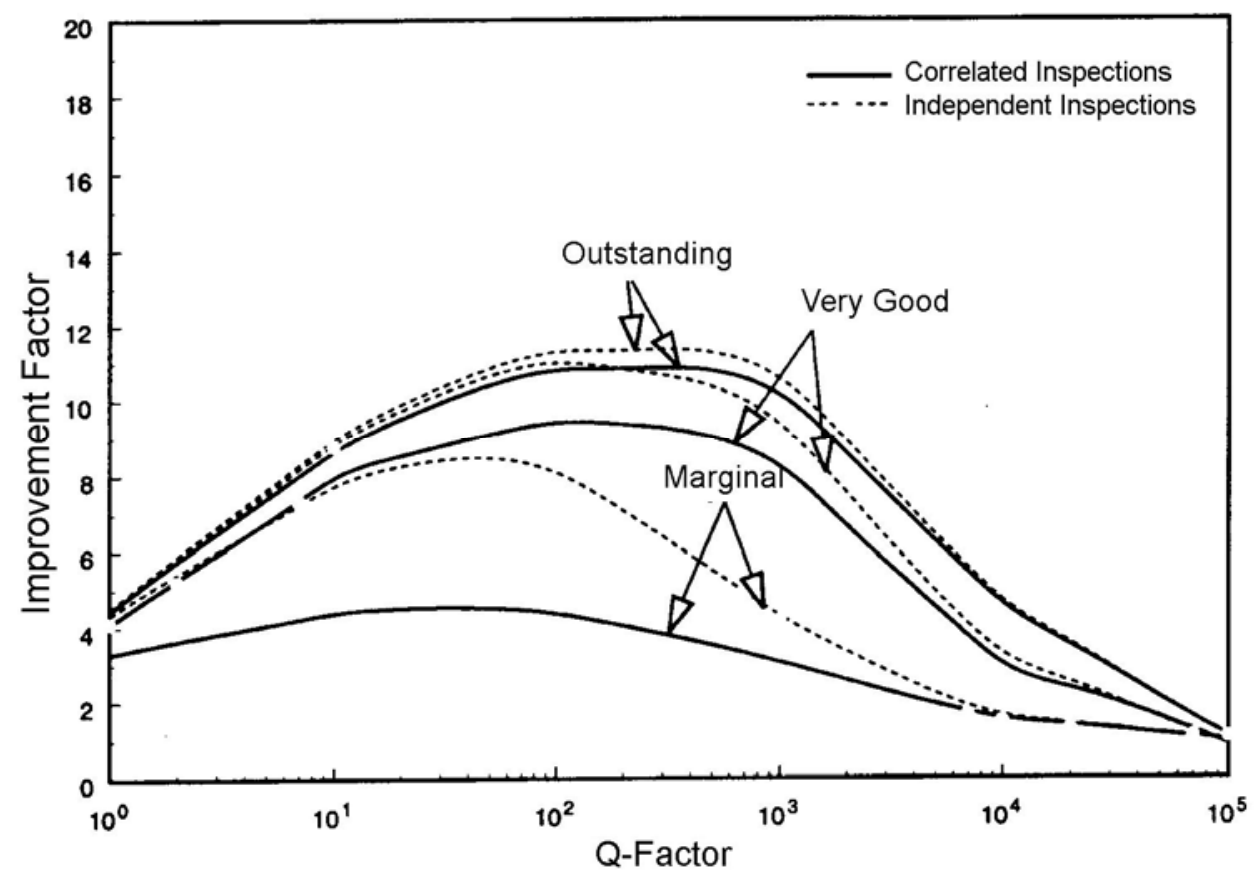

Figure 4.4 Improvement Factors for Independent and Correlated Inspections versus $Q=\alpha N(\Delta \sigma)^{4}$ for ISI Every 5 Years

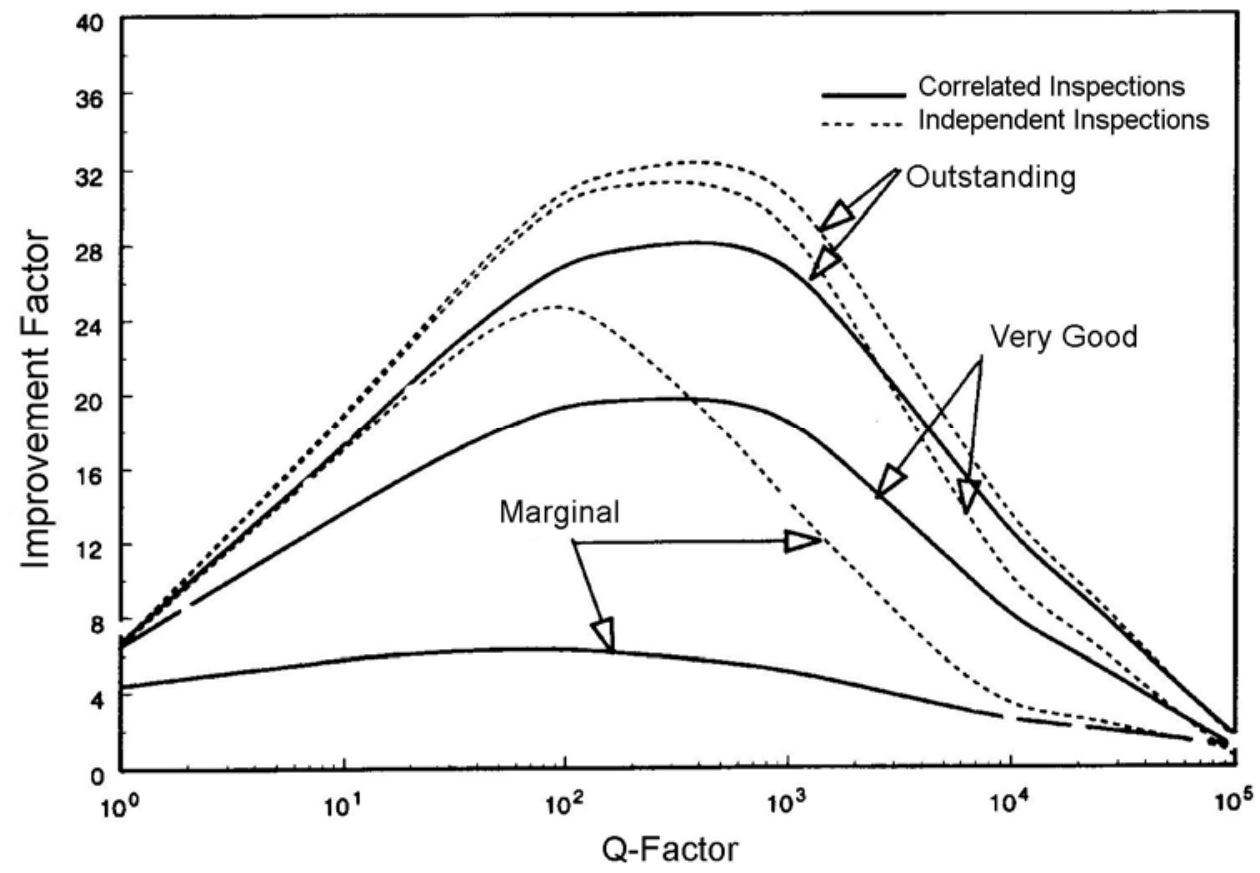

Figure 4.5 Improvement Factors for Independent and Correlated Inspections versus $Q=\alpha \mathrm{N}(\Delta \sigma)^{4}$ for ISI Every 2 Years 


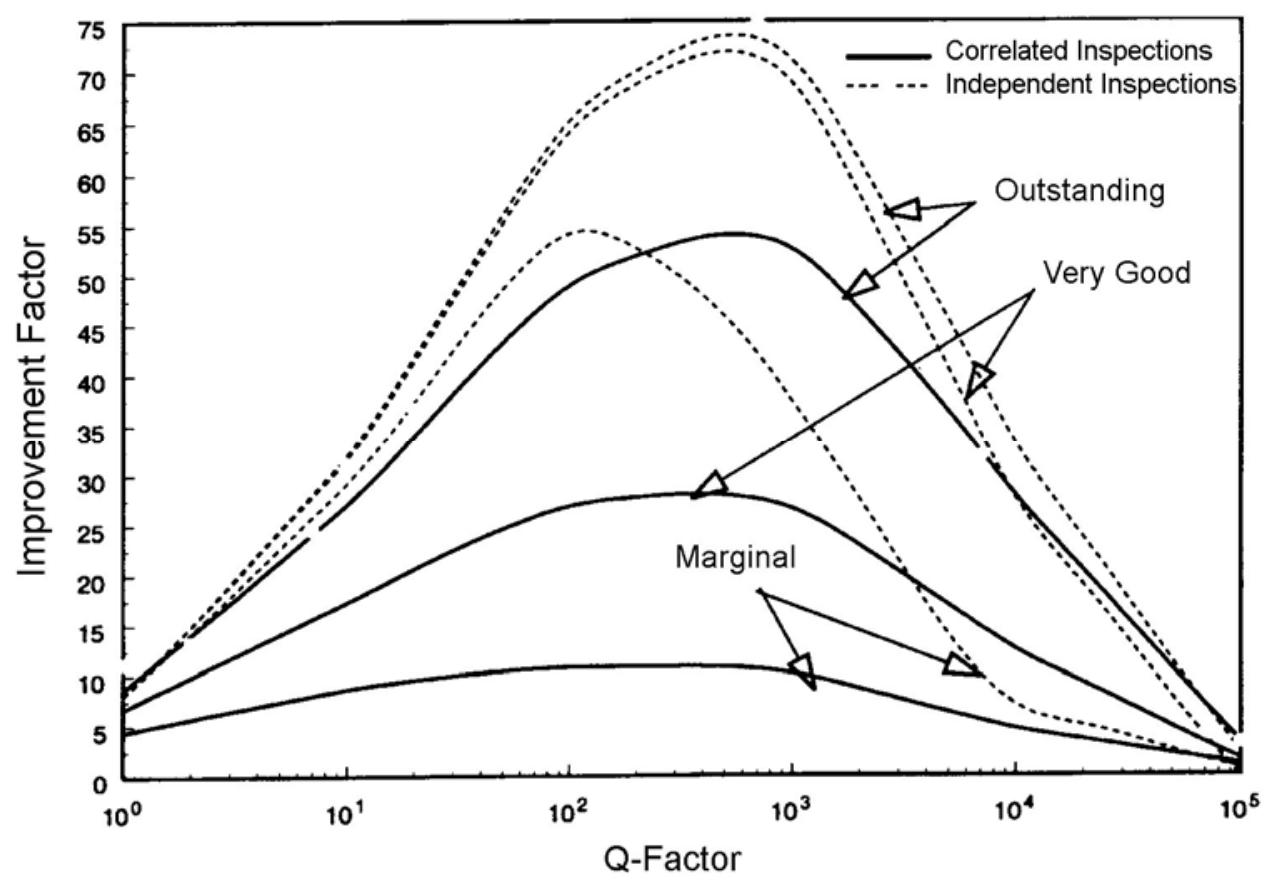

Figure 4.6 Improvement Factors for Independent and Correlated Inspections versus $Q=\alpha N(\Delta \sigma)^{4}$ for ISI Every Year

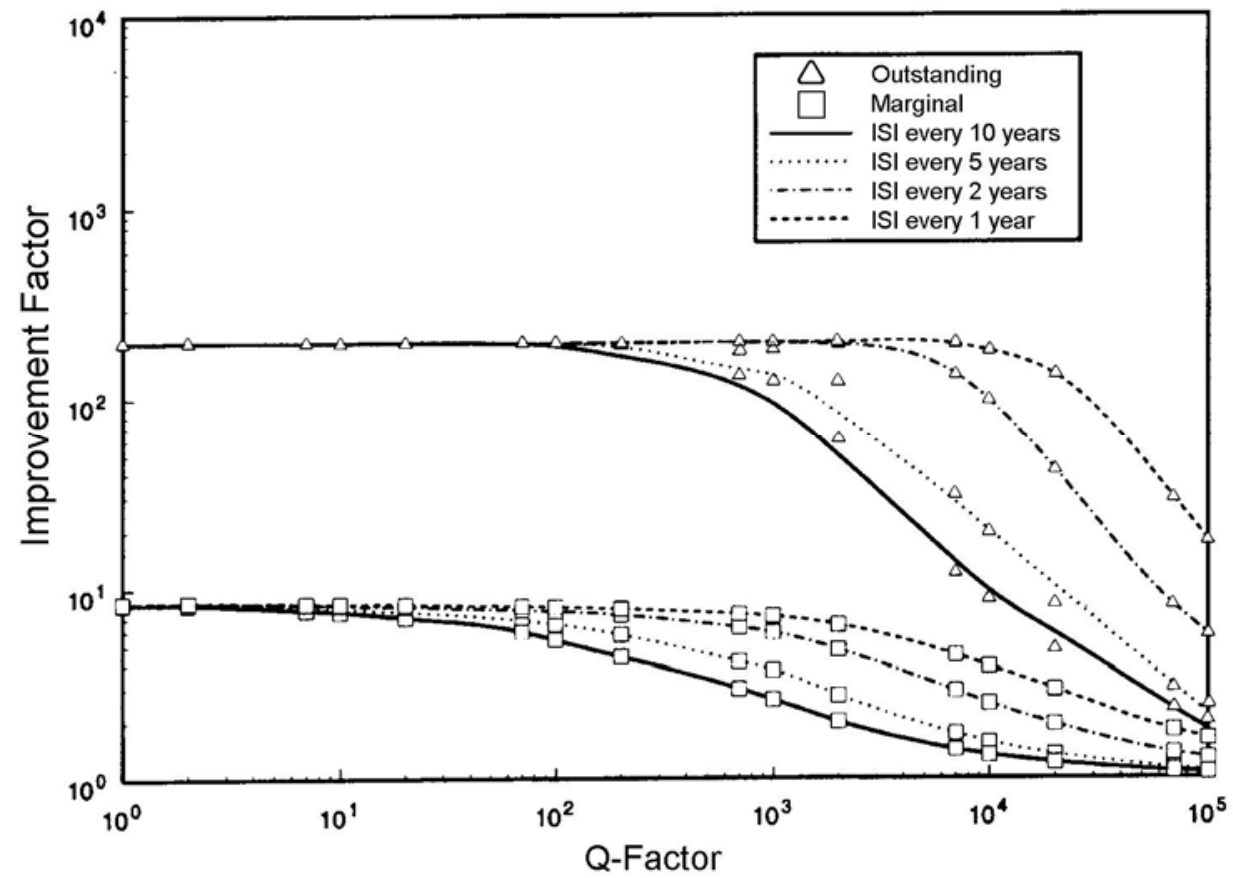

Figure 4.7 Effect of Combined Preservice and Inservice Inspection on Improvement Factors $Q=\alpha N(\Delta \sigma)^{4}$ 


\subsection{Results of Parametric Calculations}

Leak probabilities for stainless steel piping were predicted for a matrix of fatigue crack-growth scenarios using the inputs described above. The pc-PRAISE calculations simulated fatigue crack growth for preexisting defects in circumferential welds, but did not consider the initiation of new fatigue cracks. Results of the calculations are presented here in a structured parametric format making use of the Q-factor approach. This format permits failure probabilities, including the effects of inservice inspections, to be evaluated for a large number of welds without performing detailed Monte Carlo simulation for each location of interest (see Khaleel and Simonen 1994b). The benefits of inspections were evaluated using the inservice inspection option of the pc-PRAISE code. Inspections for specified POD curves were simulated in the Monte Carlo calculation by checking crack sizes at the prescribed inspection intervals to determine if the crack size at the time of the inspection was detectable.

The following discussion presents a large collection of plots for future reference and does not discuss each plot in detail. These plots can be applied to establish suitable combinations of flaw-detection capabilities and inservice inspection intervals that can provide desired reductions in piping-failure probabilities. Tradeoffs can be made between high-detection capabilities versus high-inspection frequencies. The discussion of the data will be limited to broad trends in the calculated failure probabilities, taking particular note of potential strengths and limitations of alternate inspection strategies.

\subsubsection{Effects of Pipe Wall Thickness on Failure Probabilities}

Figures 4.8 through 4.11 show cumulative 40 -year leak and break probabilities as a function of the Q-factor. Results are given for each of the four pipe sizes having wall thicknesses ranging from 0.25 to 2.5 in. (6.35 to $63.5 \mathrm{~mm})$. The number and sizes of initial fabrication flaws were assigned for each pipe size using the recommendations of Section 2. The calculated leak and break probabilities were found to be dependent on the pipe-wall thickness, with thicker pipes having somewhat lower failure probabilities for a given Q-factor than thinner wall piping. The calculated leak probabilities are generally at least three orders of magnitude greater than the corresponding break probabilities.

The calculated leak and break probabilities for low values of the $\mathrm{Q}$-factor $(\mathrm{Q}=1$ to 10$)$ are very small, with cumulative leak probabilities (over 40 years) being less than about $1.0 \mathrm{E}-06$ for all pipe sizes. The corresponding break probabilities are all less than 1.0E-10. On the other hand, large Q values (say greater than 10,000) give leak probabilities that approach 1.0. The corresponding break probabilities remain less than 1.0E-04, which is consistent with the assumptions of relatively low levels of primary stress and high material toughness levels sufficient to ensure limit-load failure of the cracked pipe. The results from the pipe-break calculations are consistent with leak-before-break behavior.

Figures 4.8 and 4.9 present conditional leak and break probabilities, which assume exactly one flaw at the pipe inner surface, whereas Figures 4.10 and 4.11 take into account the estimated flaw densities for each of the pipe wall thicknesses as listed in Table 4.2. These flaw densities were estimated using the flawdistribution methodology of Section 2. Except for the 0.25 -in. $(6.35-\mathrm{mm})$ piping, all estimates for the number of fabrication flaws took credit for preservice X-ray examinations performed after the welds were completed. This assumption regarding $\mathrm{x}$-ray examinations is consistent with construction codes, which require radiography of all welds except for those in smaller diameter piping. 


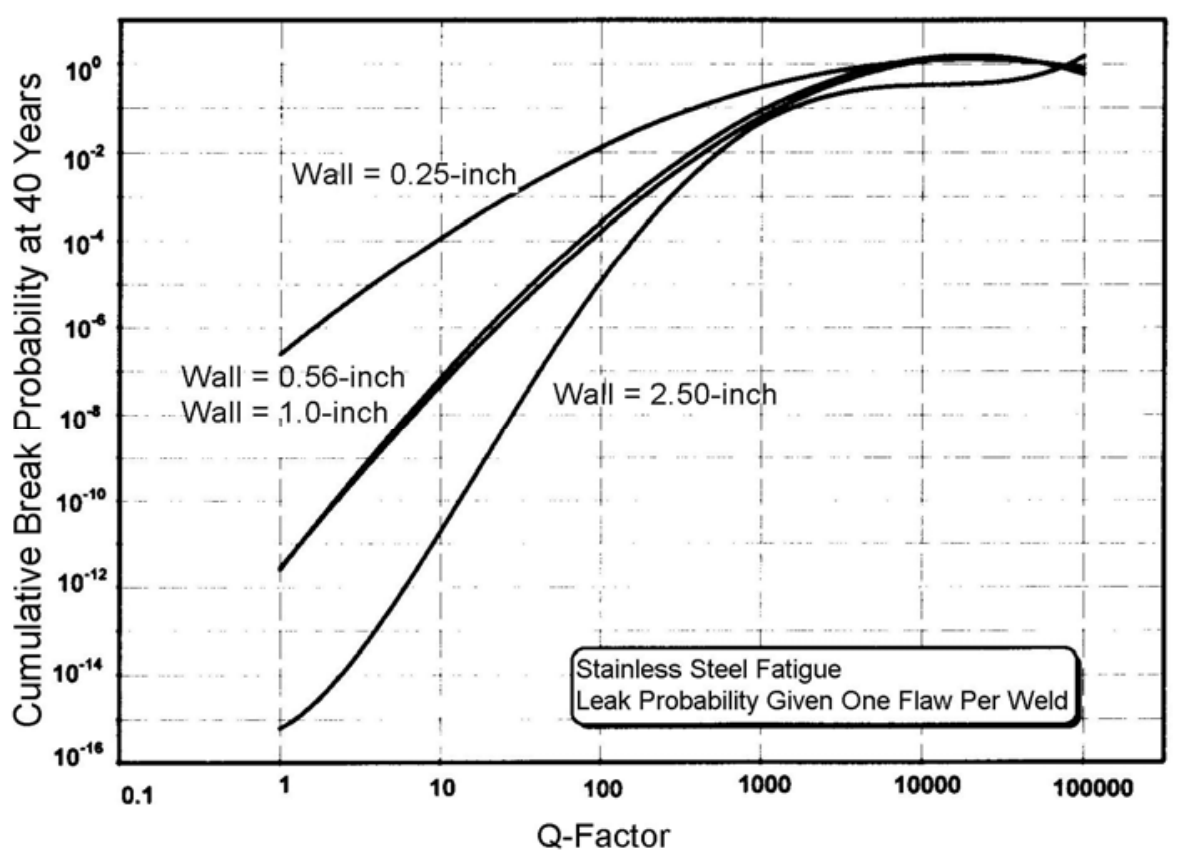

Figure 4.8 Effect of Wall Thickness on Calculated Leak Probabilities for Stainless Steel Piping Assuming One Flaw per Weld and No Inservice Inspection with $Q=\alpha N(\Delta \sigma)^{4}$ $(1$ inch $=25.4 \mathrm{~mm})$

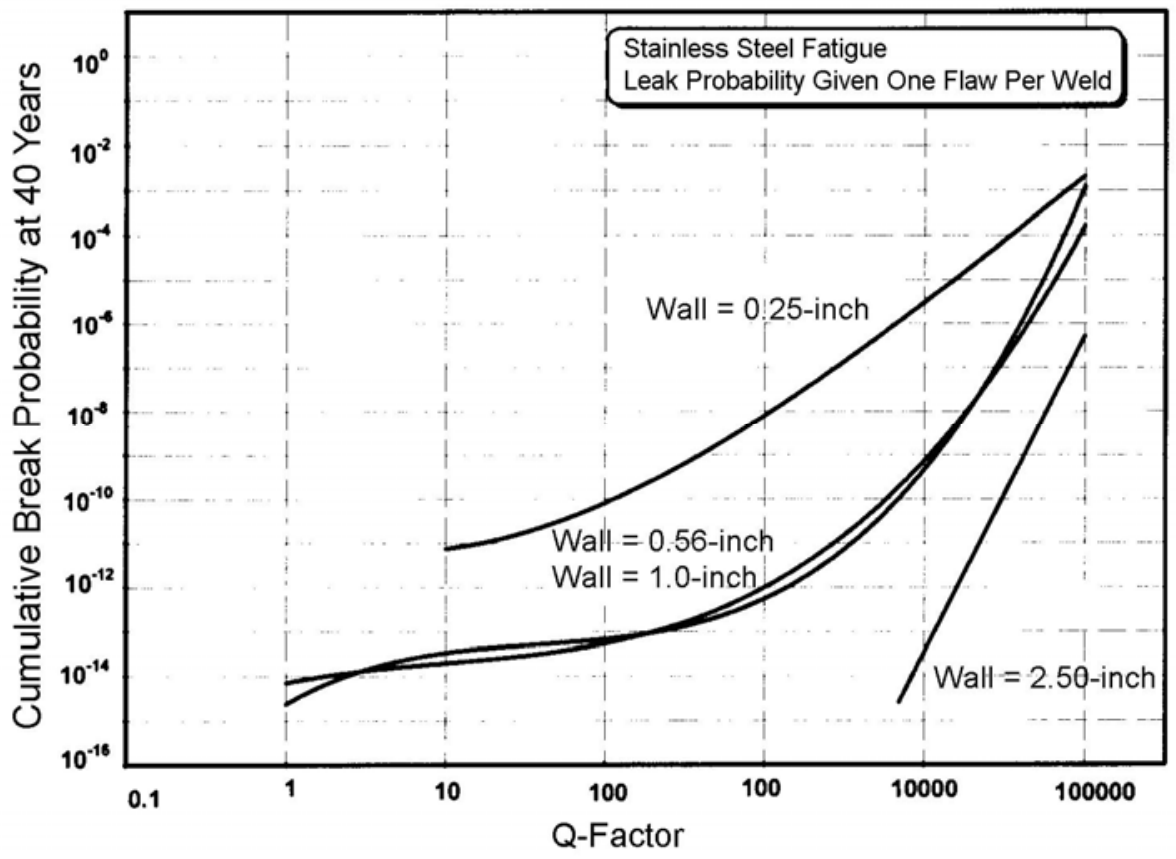

Figure 4.9 Effect of Wall Thickness on Calculated Break Probabilities for Fatigue of Stainless Steel Piping Assuming One Flaw per Weld and No Inservice Inspection with $Q=\alpha N(\Delta \sigma)^{4}(1$ inch $=25.4 \mathrm{~mm})$ 


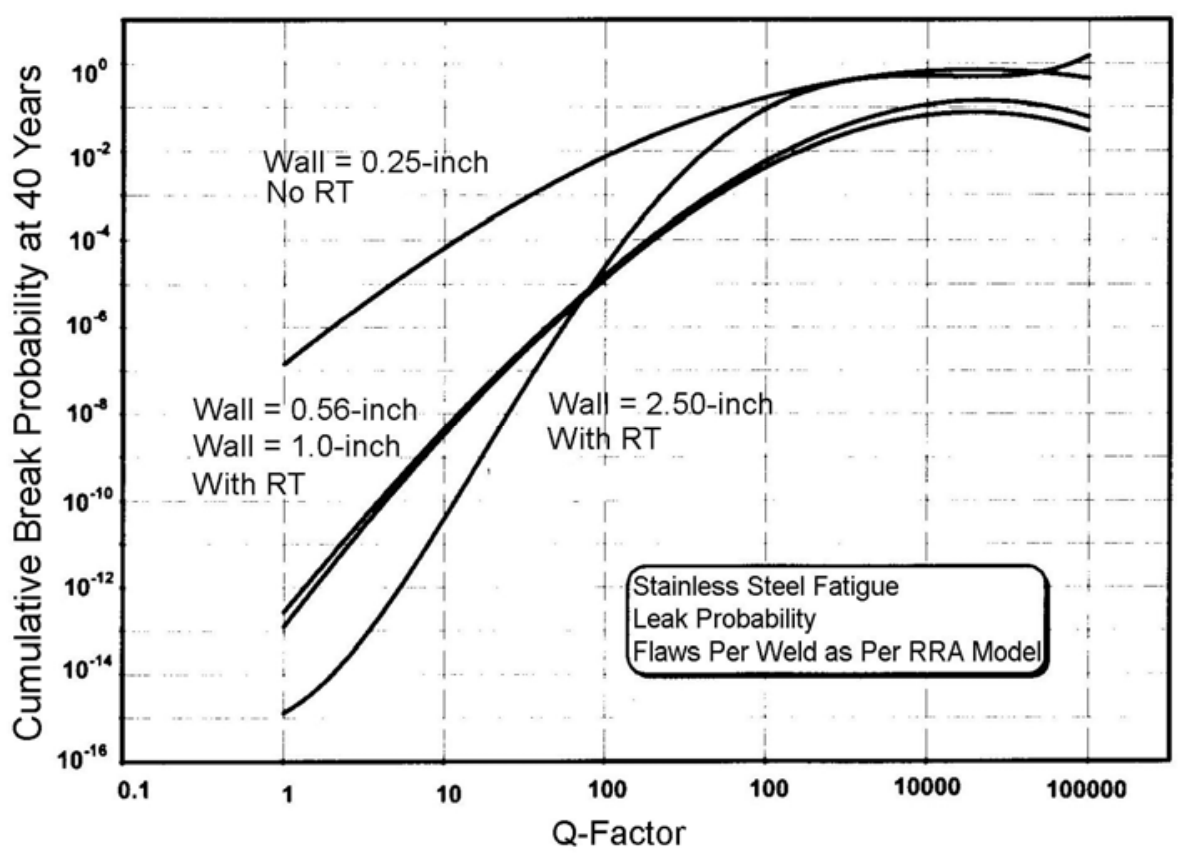

Figure 4.10 Effect of Wall Thickness on Calculated Leak Probabilities for Stainless Steel Piping Assuming Number of Flaws per Weld from Flaw Simulation Model and No Inservice Inspection with $Q=\alpha \mathrm{N}(\Delta \sigma)^{4}(1$ inch $=25.4 \mathrm{~mm})$

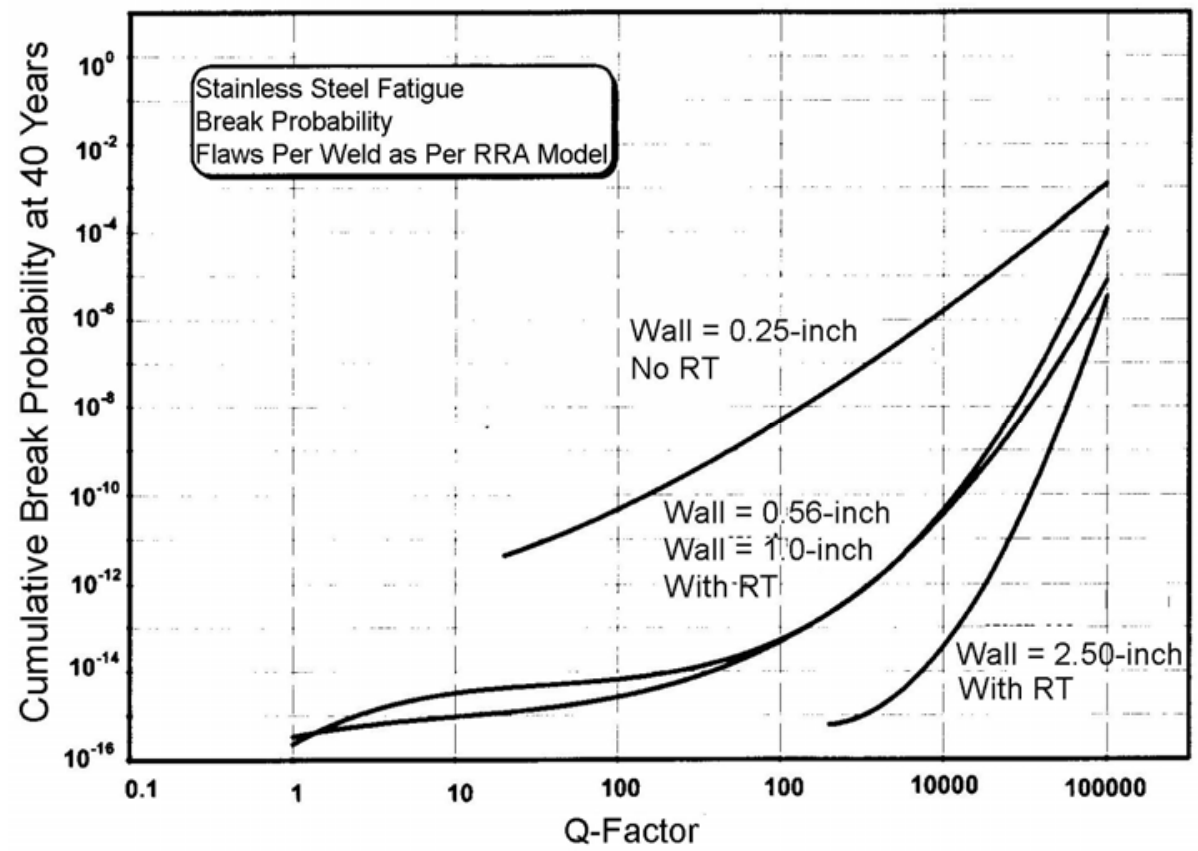

Figure 4.11 Effect of Wall Thickness on Calculated Break Probabilities for Fatigue of Stainless Steel Piping Assuming Number of Flaws per Weld from Flaw Simulation Model and No Inservice Inspection with $Q=\alpha \mathrm{N}(\Delta \sigma)^{4}(1$ inch $=25.4 \mathrm{~mm})$ 
The calculations predict that thinner pipes will have higher conditional failure probabilities than thicker wall pipes. These calculations assume the presence of exactly one inner surface flaw and the same conditions of cyclic stress for all pipe sizes. As indicated by Figures 4.8 and 4.9, the conditional failure probabilities (given one flaw per weld) are significantly greater for the smaller pipe sizes. This trend comes from the interaction of several factors that enter into the estimation of flaw-depth distributions. Whereas flaw depths for thin pipes (absolute flaw depth expressed as mm or inches) will be somewhat less than the flaw depths for thicker pipes, these depths will be significantly larger for the thinner pipes if measured as a fraction of the pipe-wall thickness. These alternative measures of flaw depth explain why the calculated failure probabilities for 0.562 - and 1.0 -in. (14.3- and $25.4-\mathrm{mm}$ ) wall pipes are essentially the same.

Although the flaws for the 1.0-in. (25.4-mm) wall pipe have somewhat greater depths than those for the 0.562-in. (14.3-mm) pipe, the flaw depth distributions are nearly identical when expressed in terms of crack tip stress intensity factors.

Trends shown by Figures 4.10 and 4.11 indicate that failure probabilities are also governed by the fact that larger pipes are likely to have more flaws because welds in large pipes have a greater amount of weld metal. However, the differences in the number of flaws are not directly proportional to the volume of weld metal. Rather, the number of flaws is related to the total length of the weld beads within the first few weld layers at the inner surface of the pipe. As such, the number of flaws is more nearly proportional to the pipe diameter, rather than the cube of the pipe diameter, as would be the case if the number of flaws were truly proportional to the volume of weld metal.

The estimated number of flaws per weld for the 2.5-in. (63.5-mm) wall thickness (as given by Table 4.2) is believed to be quite conservative. All flaws located within the inner quarter wall thickness were treated as inner surface-breaking flaws. This resulted in a somewhat more conservative basis for estimating the flaw density for the thick pipe than that used for the thinner wall pipes (for which only flaws within the first two weld layers were included). In this regard, uncertainty analyses conclude that inputs for flaw distributions are the greatest source of uncertainty in calculations of piping-failure probabilities. Flaw densities for the 2.5-in. $(63.5-\mathrm{mm})$ wall pipe were taken for the present calculations at the high end of an uncertainty band, which may bias the calculated leak probabilities for large piping upwards by a factor of perhaps ten.

\subsubsection{Effects of Pipe-Wall Thickness on Improvement Factor}

A subset of the parametric calculations serves to illustrate how inspection effectiveness is related to pipe size, with the pipe size being expressed here in terms of wall thickness. Figures 4.12 and 4.13 show the effects of inservice inspections on reducing leak probabilities for the range of wall thicknesses. It was assumed that the inspections are performed at either 10-year (Figure 4.12) or 2-year (Figure 4.13) intervals. Both cases assume the "very good" POD curve. The calculated improvement factors are shown for the wide range of Q-factors of interest.

The results of Figures 4.12 and 4.13 show that a specific combination of POD curve and inspection frequency gives reductions in pipe-leak probabilities that appear to be relatively insensitive to the pipewall thickness. However, more detailed evaluations indicate that somewhat more sensitive POD curves (i.e., POD expressed as a function of a/t) are needed for the larger wall thicknesses to ensure that inspections provide desired factors of improvement in piping reliability. 
Cumulative Leak Probability at 40 Years

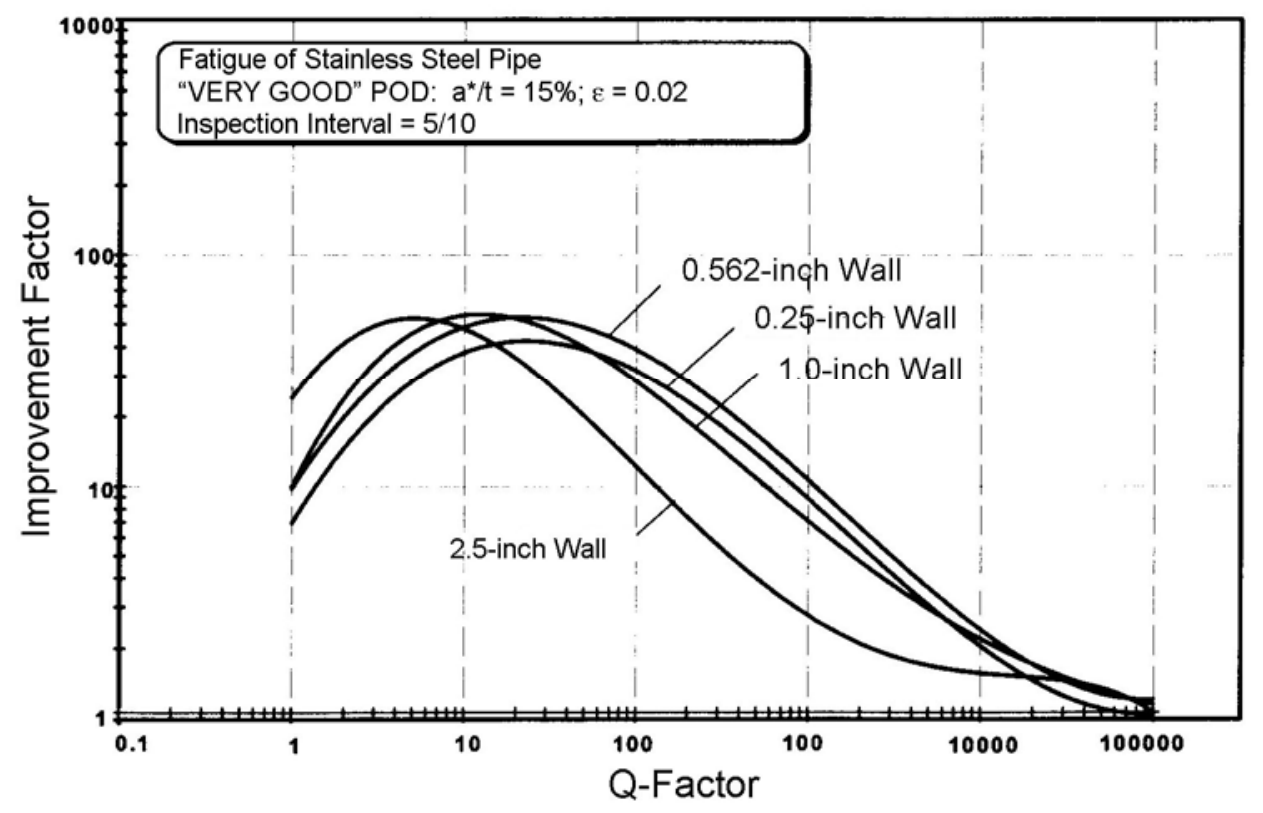

Figure 4.12 Effect of Wall Thickness on Improvement Factor for Stainless Steel Fatigue Using Inservice Inspection with "Very Good" Probability of Detection Curve and Periodic Inspections Performed at 10-Year Intervals (5/10) with $Q=\alpha \mathrm{N}(\Delta \sigma)^{4}(1 \mathrm{inch}=25.4 \mathrm{~mm})$

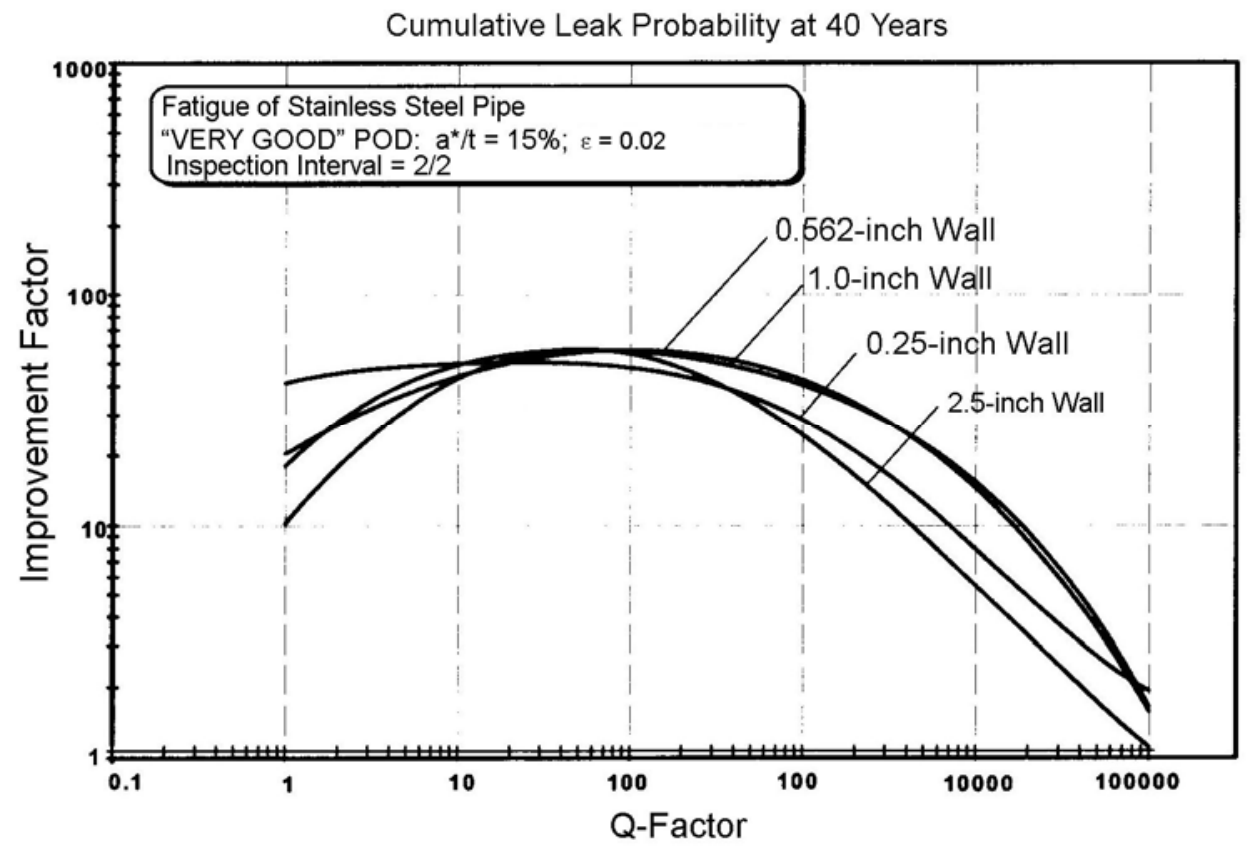

Figure 4.13 Effect of Wall Thickness on Improvement Factor for Stainless Steel Fatigue Using Inservice Inspection with "Very Good" Probability of Detection Curve and Periodic Inspections Performed at 2-Year Intervals (2/2) with $Q=\alpha \mathrm{N}(\Delta \sigma)^{4}(1 \mathrm{inch}=25.4 \mathrm{~mm})$ 


\subsubsection{Improvement Factors for Various Pipe-Wall Thicknesses}

Figures 4.14 through 4.18 give improvement factors for the four pipe-wall thicknesses ranging from 0.25 to 2.5 in. (6.35 to $63.5 \mathrm{~mm}$ ) and give calculated factors for the "marginal," "very good," and "outstanding" POD curves. The inspection intervals range from 1 year to 10 years. The collection of plots is organized in the following manner:

- Figures $4.14 \mathrm{a}, 4.14 \mathrm{~b}$, and $4.14 \mathrm{c}$ are for the stainless steel piping with a wall thickness of 0.25 in. $(6.35 \mathrm{~mm})$. Factors of improvement in piping reliability for the "marginal," "very good," and "outstanding" POD curves are addressed by Figures 4.14a, 4.14b, and 4.14c respectively. Each of the individual curves on these figures corresponds to a different ISI interval (1, 2, 4, and 10 years).

- Figures 4.15a, 4.15b, and 4.15c are for the 0.562-in. (14.3-mm) wall stainless steel piping and cover the same range of POD curves and inspection intervals as for the 0.25 -in. $(6.35-\mathrm{mm})$ wall piping.

- Figures 4.16a, 4.16b, and 4.16c are for the 1.0-in. (25.4-mm) wall stainless steel piping and cover the same range of POD curves and inspection intervals as for the other pipe sizes.

- Figures $4.17 \mathrm{a}, 4.17 \mathrm{~b}$, and $4.17 \mathrm{c}$ are for the 2.5 -in. $(63.5-\mathrm{mm})$ wall stainless steel piping and cover the same range of POD curves and inspection intervals as for the other pipe sizes.

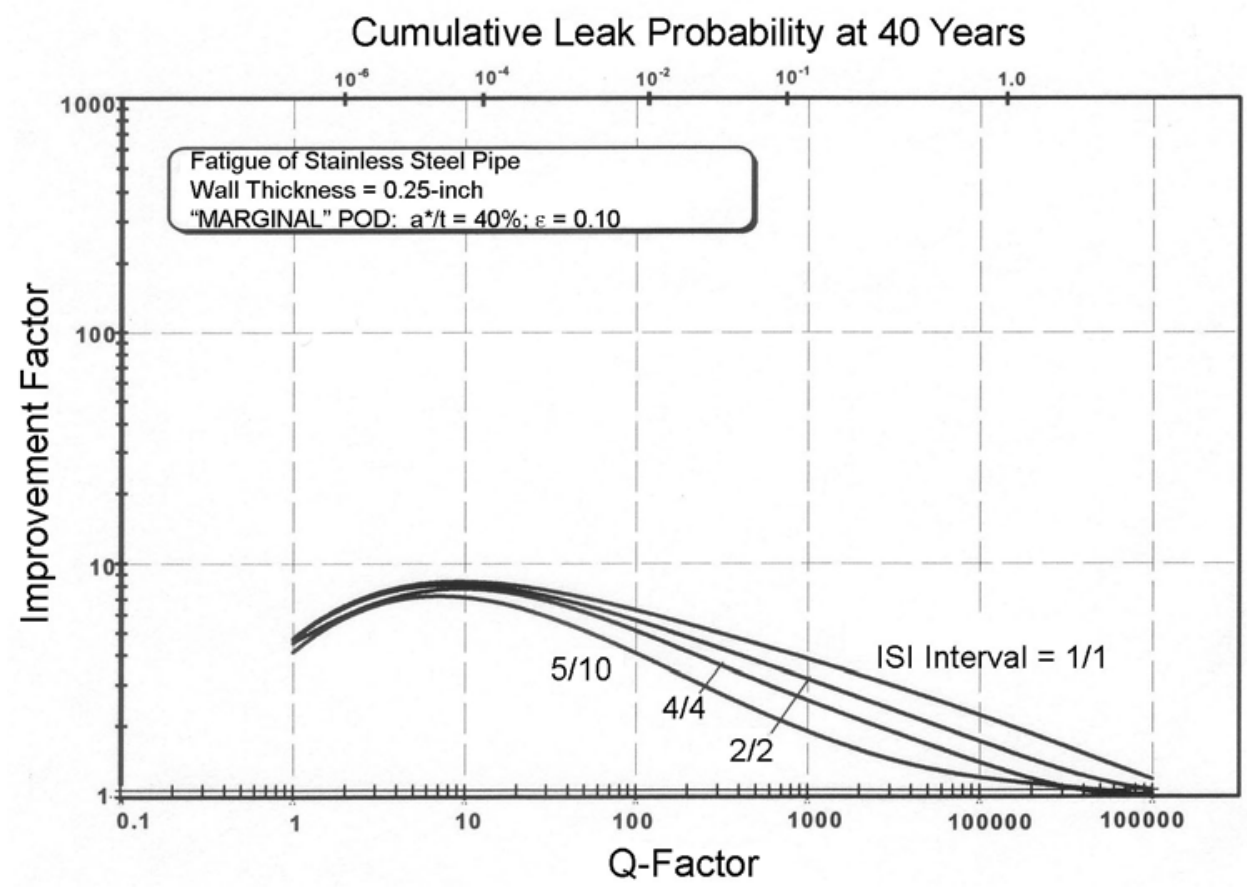

Figure 4.14a Improvement Factor for Fatigue of 0.25-in. (6.35-mm) Wall Stainless Steel Piping Using Inservice Inspection with "Marginal" Probability of Detection Curve and Periodic Inspections Performed at Various Intervals with $Q=\alpha \mathrm{N}(\Delta \sigma)^{4}$ 
Cumulative Leak Probability at 40 Years

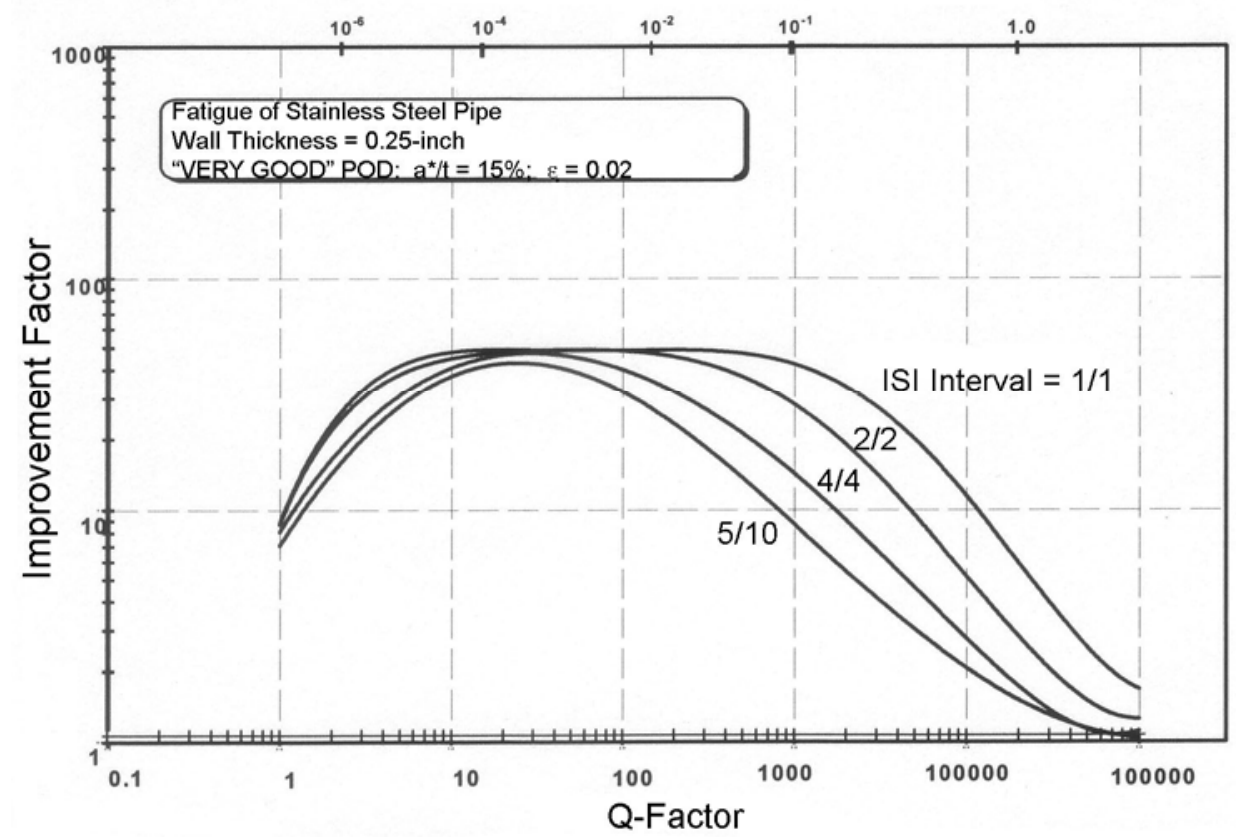

Figure 4.14b Improvement Factor for Fatigue of 0.25-in. (6.35-mm) Wall Stainless Steel Piping Using Inservice Inspection with "Very Good" Probability of Detection Curve and Periodic Inspections Performed at Various Intervals with $Q=\alpha \mathrm{N}(\Delta \sigma)^{4}$

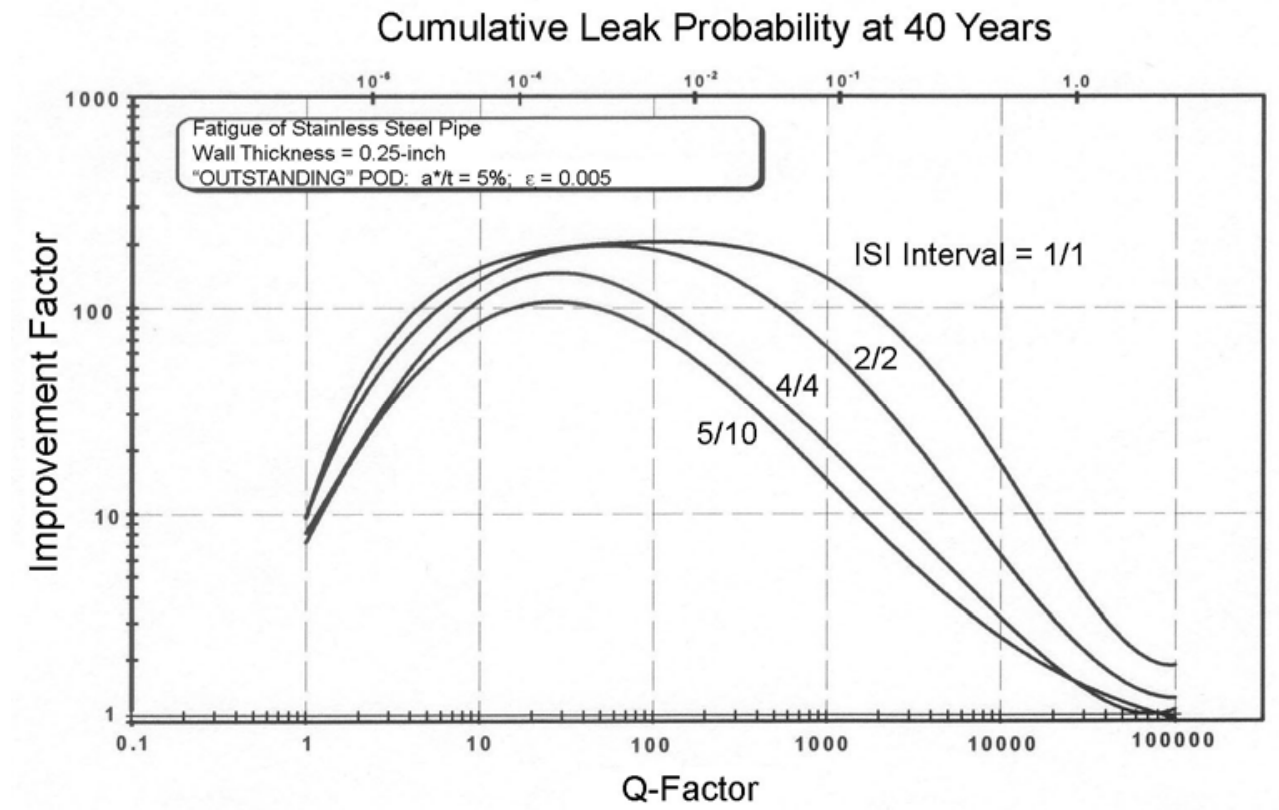

Figure 4.14c Improvement Factor for Fatigue of 0.25-in. (6.35-mm) Wall Stainless Steel Piping Using Inservice Inspection with "Outstanding" Probability of Detection Curve and Periodic Inspections Performed at Various Intervals with $Q=\alpha \mathrm{N}(\Delta \sigma)^{4}$ 


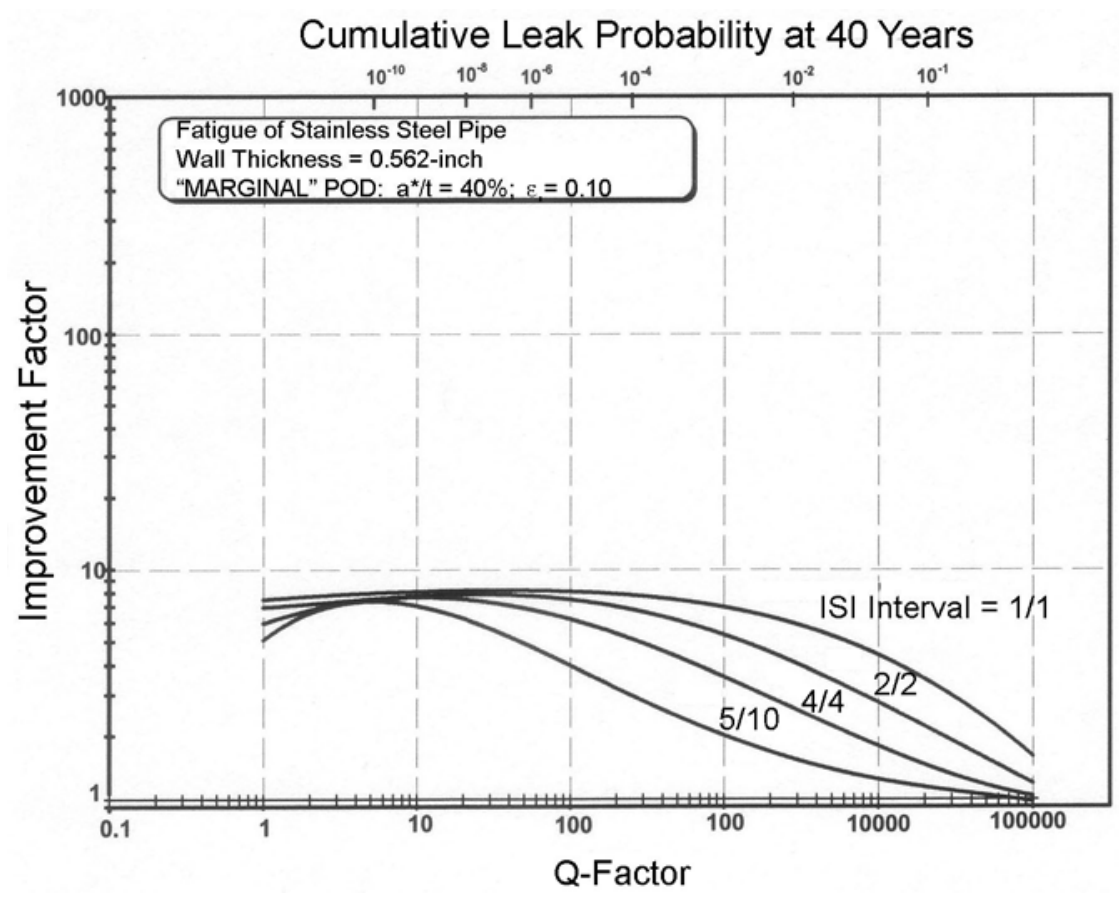

Figure 4.15a Improvement Factor for Fatigue of 0.562-in. (14.3-mm) Wall Stainless Steel Piping Using Inservice Inspection with "Marginal" Probability of Detection Curve and Periodic Inspections Performed at Various Intervals with $Q=\alpha \mathrm{N}(\Delta \sigma)^{4}$

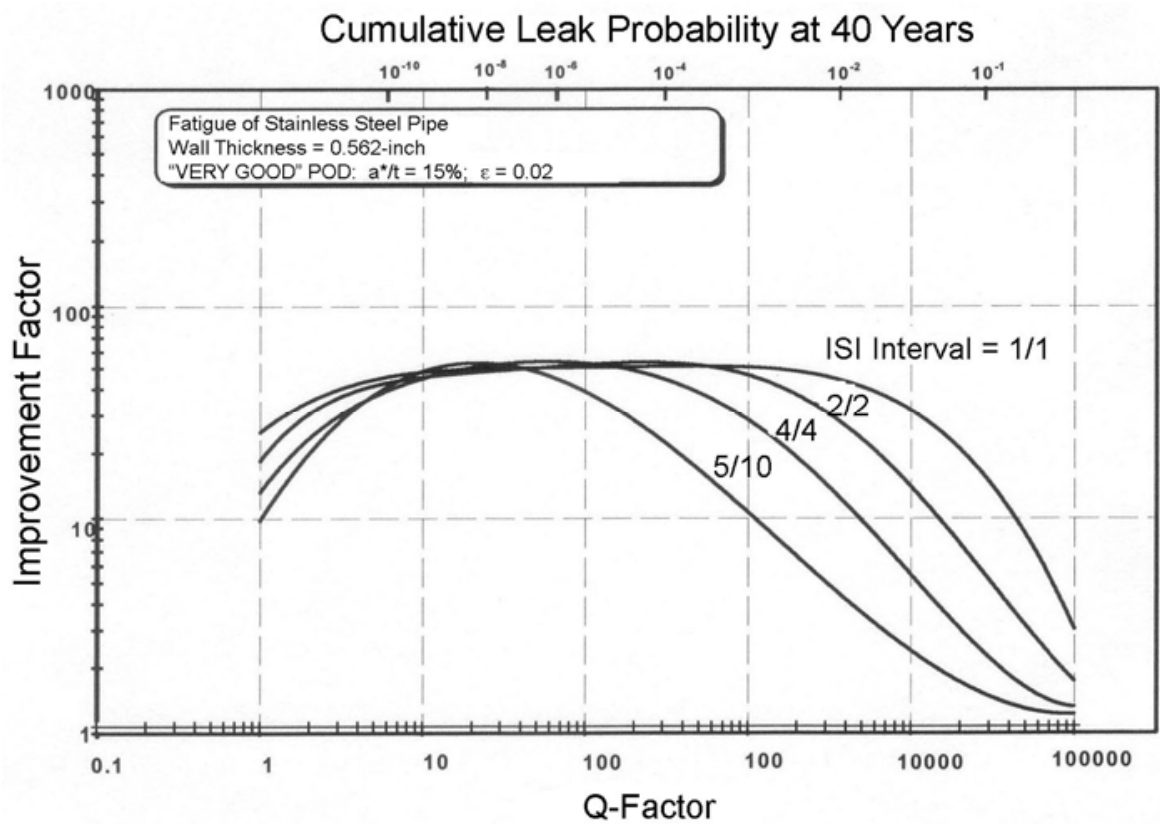

Figure 4.15b Improvement Factor for Fatigue of 0.562-in. (14.3-mm) Wall Stainless Steel Piping Using Inservice Inspection with "Very Good" Probability of Detection Curve and Periodic Inspections Performed at Various Intervals with $Q=\alpha \mathrm{N}(\Delta \sigma)^{4}$ 


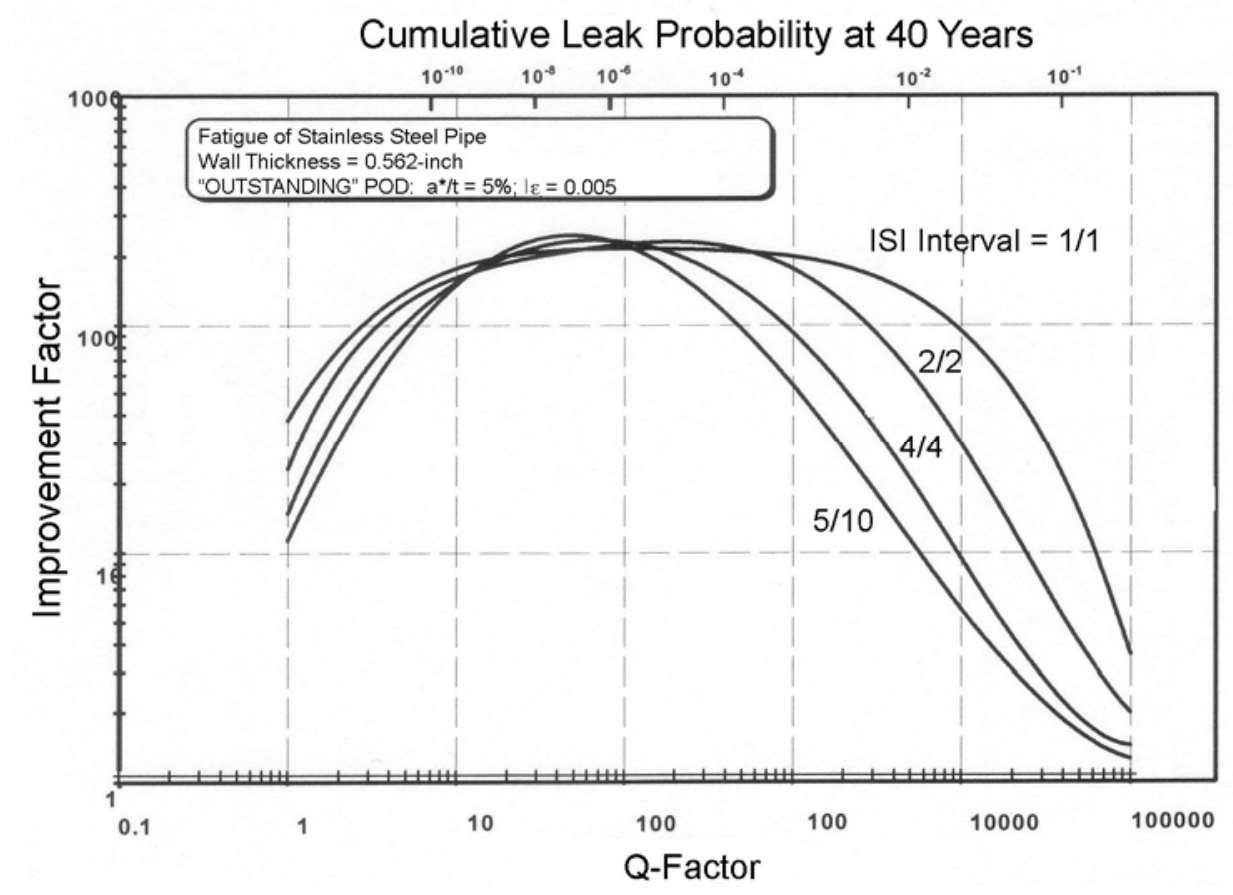

Figure 4.15c Improvement Factor for Fatigue of 0.562-in. (14.3-mm) Wall Stainless Steel Piping Using Inservice Inspection with "Outstanding” Probability of Detection Curve and Periodic Inspections Performed at Various Intervals with $Q=\alpha \mathrm{N}(\Delta \sigma)^{4}$

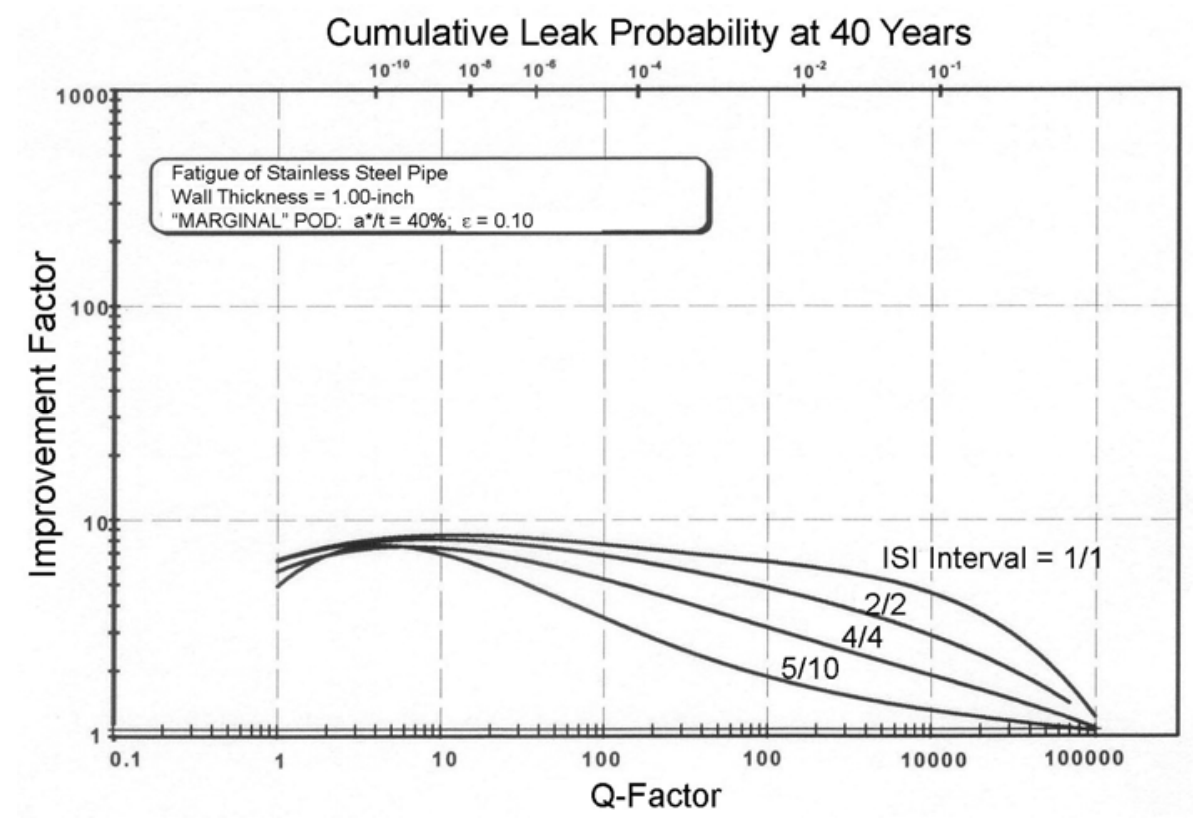

Figure 4.16a Improvement Factor for Fatigue of 1.0-in. (25.4-mm) Wall Stainless Steel Piping Using Inservice Inspection with "Marginal" Probability of Detection Curve and Periodic Inspections Performed at Various Intervals with $Q=\alpha \mathrm{N}(\Delta \sigma)^{4}$ 
Cumulative Leak Probability at 40 Years

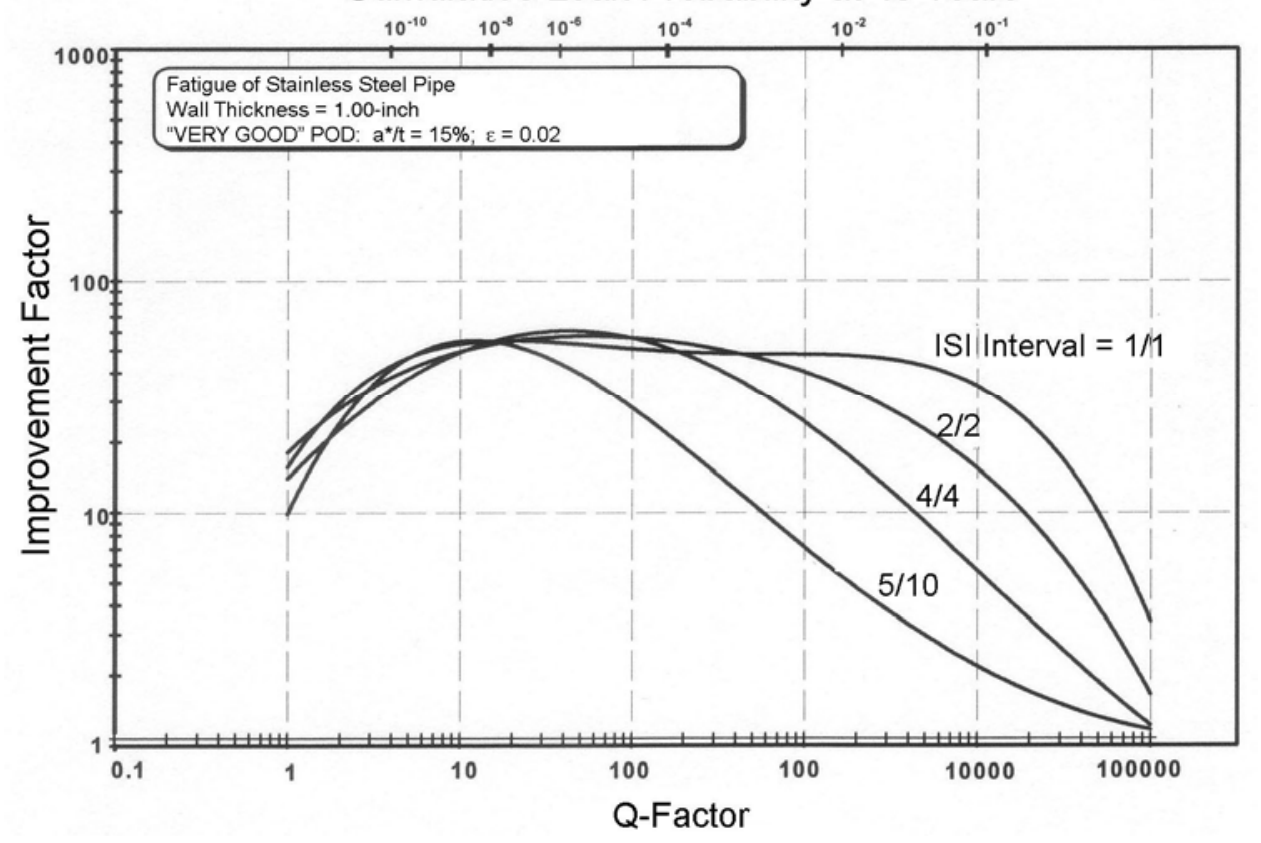

Figure 4.16b Improvement Factor for Fatigue of 1.0-in. (25.4-mm) Wall Stainless Steel Piping Using Inservice Inspection with "Very Good" Probability of Detection Curve and Periodic Inspections Performed at Various Intervals with $Q=\alpha \mathrm{N}(\Delta \sigma)^{4}$

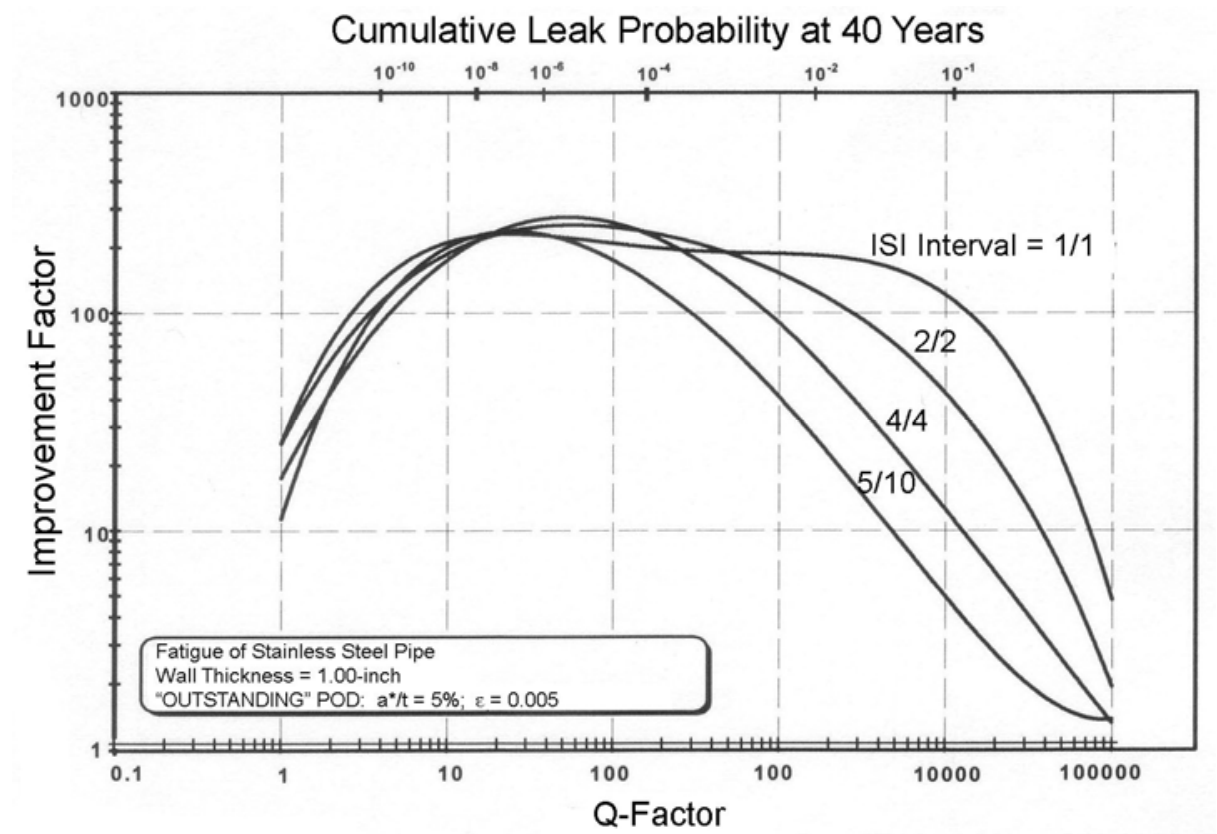

Figure 4.16c Improvement Factor for Fatigue of 1.0-in. (25.4-mm) Wall Stainless Steel Piping Using Inservice Inspection with "Outstanding" Probability of Detection Curve and Periodic Inspections Performed at Various Intervals with $Q=\alpha \mathrm{N}(\Delta \sigma)^{4}$ 


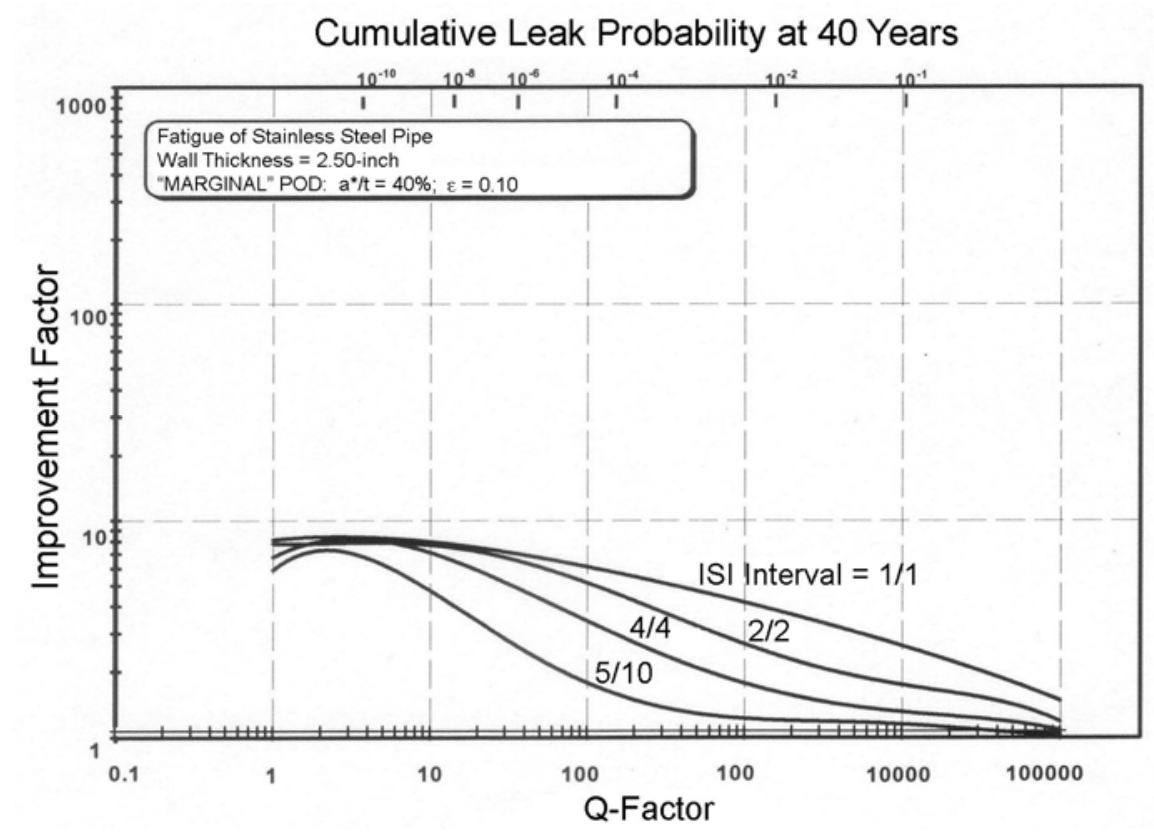

Figure 4.17a Improvement Factor for Fatigue of 2.5-in. (63.5-mm) Wall Stainless Steel Piping Using Inservice Inspection with "Marginal" Probability of Detection Curve and Periodic Inspections Performed at Various Intervals with $Q=\alpha \mathrm{N}(\Delta \sigma)^{4}$

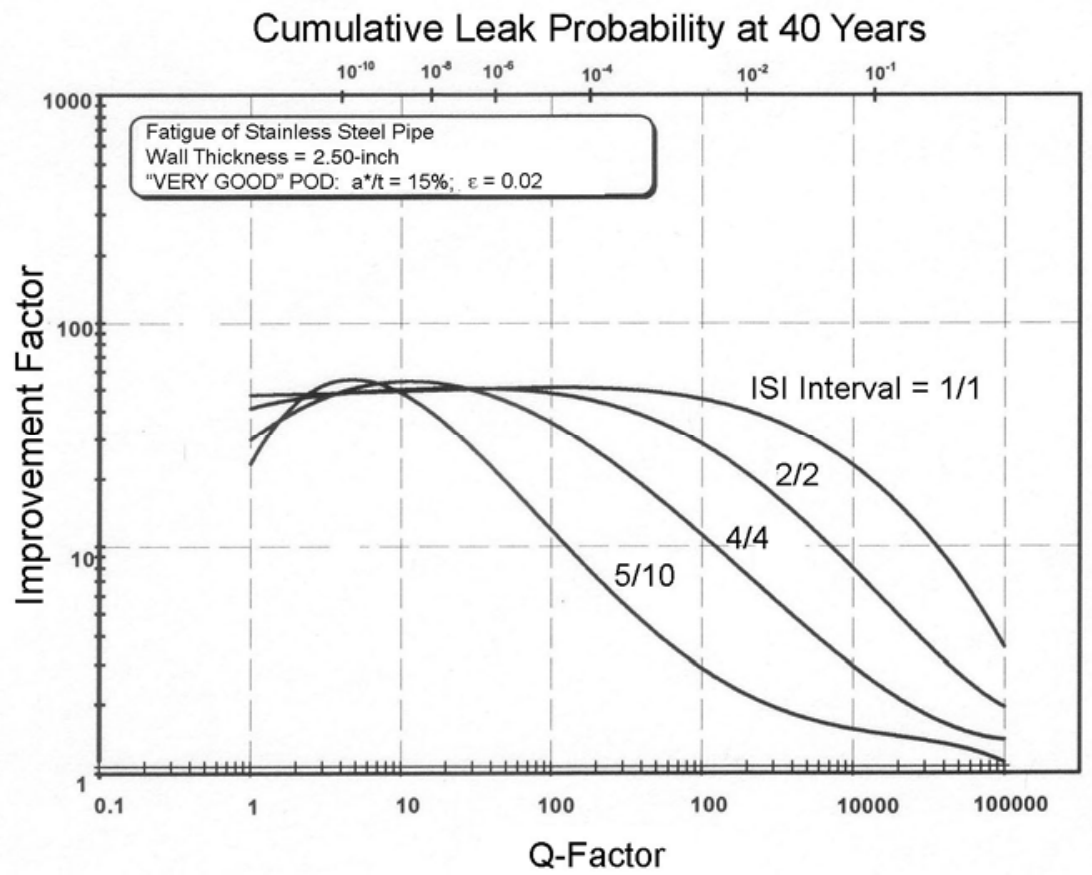

Figure 4.17b Improvement Factor for Fatigue of 2.5-in. (63.5-mm) Wall Stainless Steel Piping Using Inservice Inspection with "Very Good" Probability of Detection Curve and Periodic Inspections Performed at Various Intervals with $Q=\alpha \mathrm{N}(\Delta \sigma)^{4}$ 


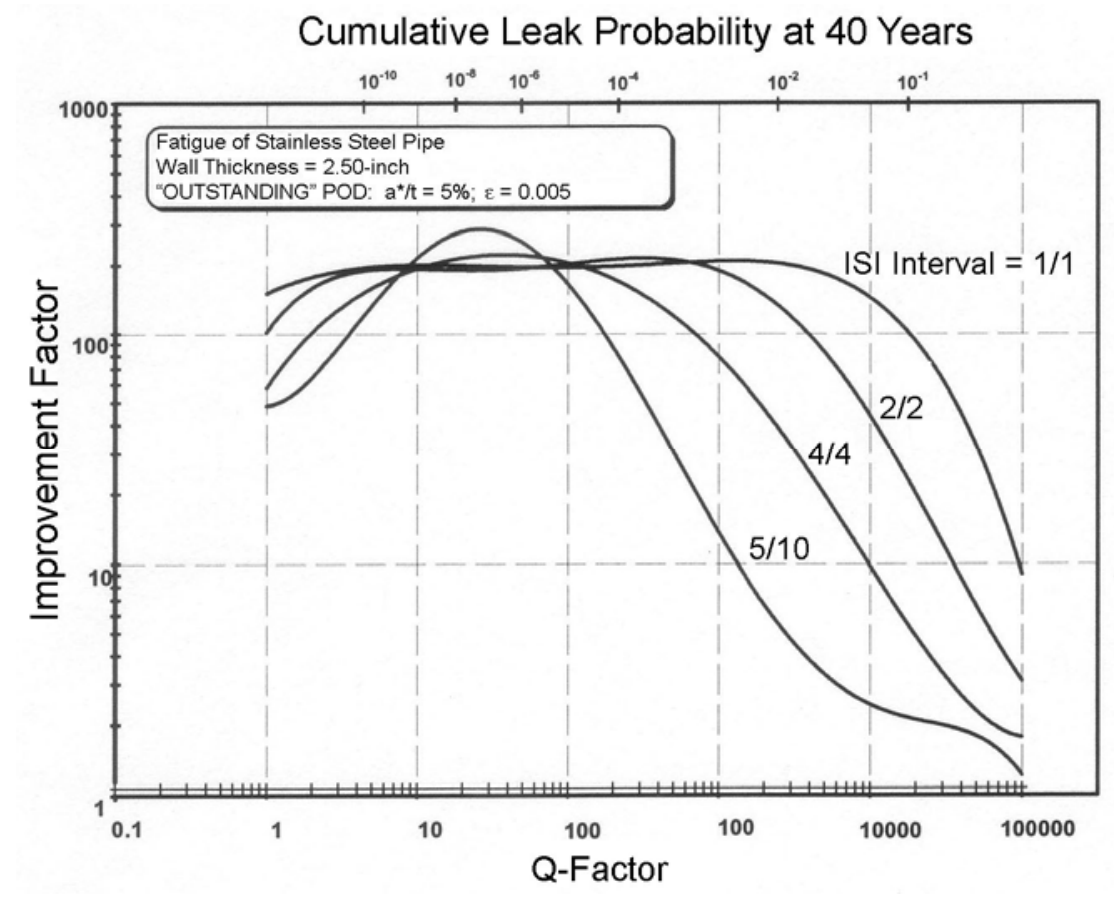

Figure 4.17c Improvement Factor for Fatigue of 2.5-in. (63.5-mm) Wall Stainless Steel Piping Using Inservice Inspection with "Outstanding” Probability of Detection Curve and Periodic Inspections Performed at Various Intervals with $Q=\alpha \mathrm{N}(\Delta \sigma)^{4}$

- Figures 4.18a-d are for the 1.0-in. (25.4-mm) wall piping and present already covered by Figure 4.16 but group the curves to better show the effect of POD curves rather than inspection intervals. Intervals of 10, 4, 2, and 1 year are addressed by Figures $4.18 \mathrm{a}, \mathrm{b}, \mathrm{c}$, and $\mathrm{d}$, respectively. The large differences between the effectiveness of these POD levels are quite apparent on each of the plots.

The following general comments apply to the results for all four pipe sizes:

- The curves of Figures 4.14 through 4.18 show maximum improvement factors for the mid-range of Q-factors. The decreasing effectiveness of the inspections at very low Q-factors is because these failures occur very early in life before the end of the first inspection interval. The decreasing effectiveness of inspections for very high Q-factors is because these cases correspond to high levels of cyclic stresses and associated high crack-growth rates, such that very frequent inspections are needed.

- The curves of Figures 4.14 through 4.18 remain flat at a maximum level over a mid-range of Q-factors. This maximum level is directly related the value of $\varepsilon$ in the equation for the POD curve, where $\varepsilon$ describes the maximum achievable level (or saturation level) of the POD curve corresponding to relatively deep cracks. 


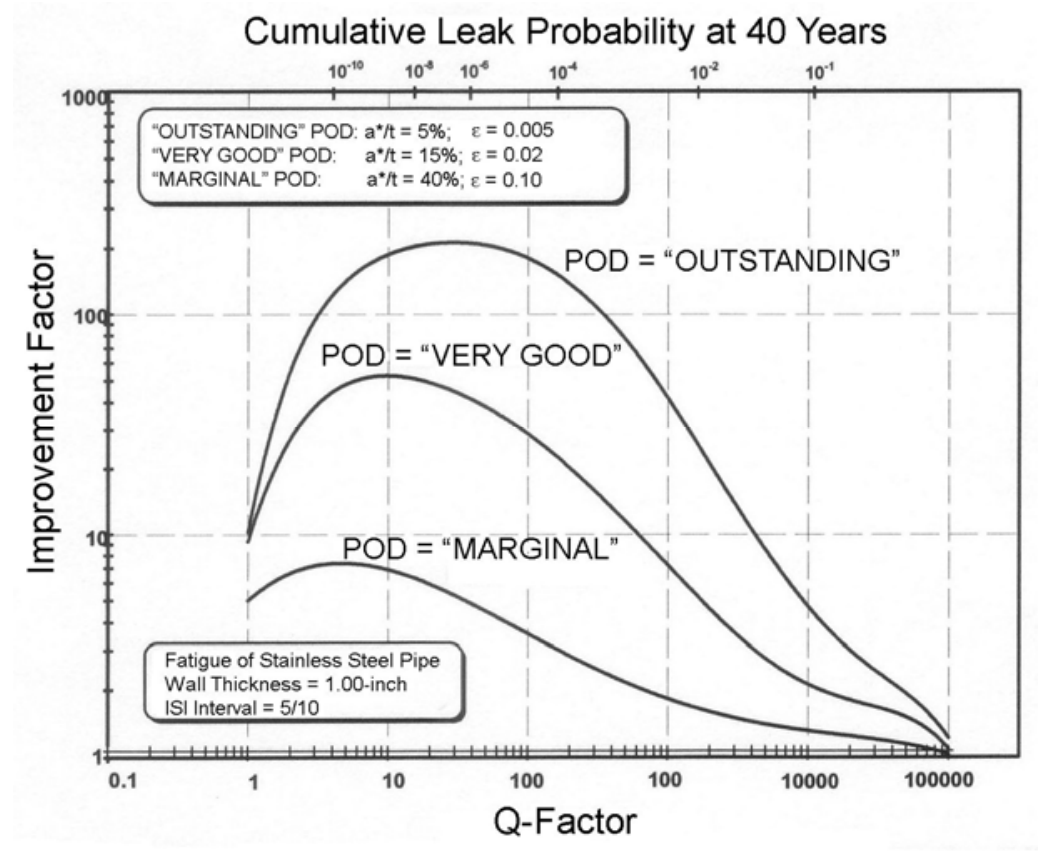

Figure 4.18a Improvement Factor for Fatigue of 1.0-in. (25.4-mm) Wall Stainless Steel Piping Using Inservice Inspection with Various Probability of Detection Curves and Periodic Inspections Performed at 10-Year Intervals with $Q=\alpha \mathrm{N}(\Delta \sigma)^{4}$

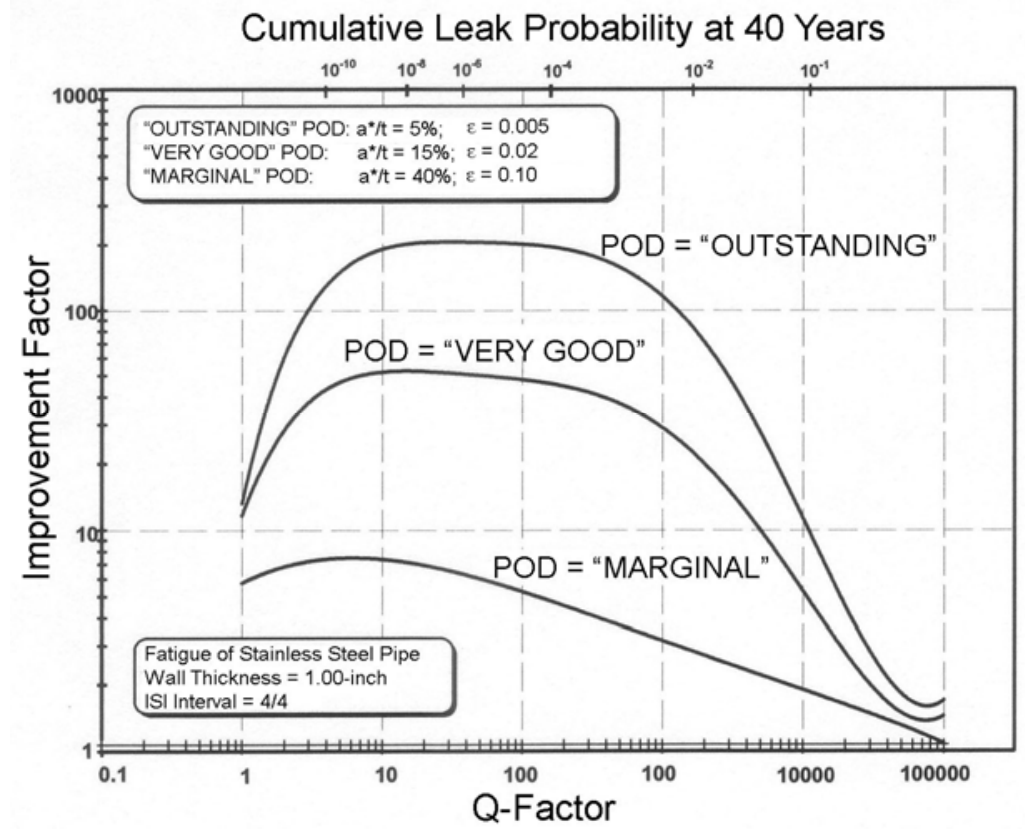

Figure 4.18b Improvement Factor for Fatigue of 1.0-in. (25.4-mm) Wall Stainless Steel Piping Using Inservice Inspection with Various Probability of Detection Curves and Periodic Inspections Performed at 4-Year Intervals with $Q=\alpha \mathrm{N}(\Delta \sigma)^{4}$ 
Cumulative Leak Probability at 40 Years

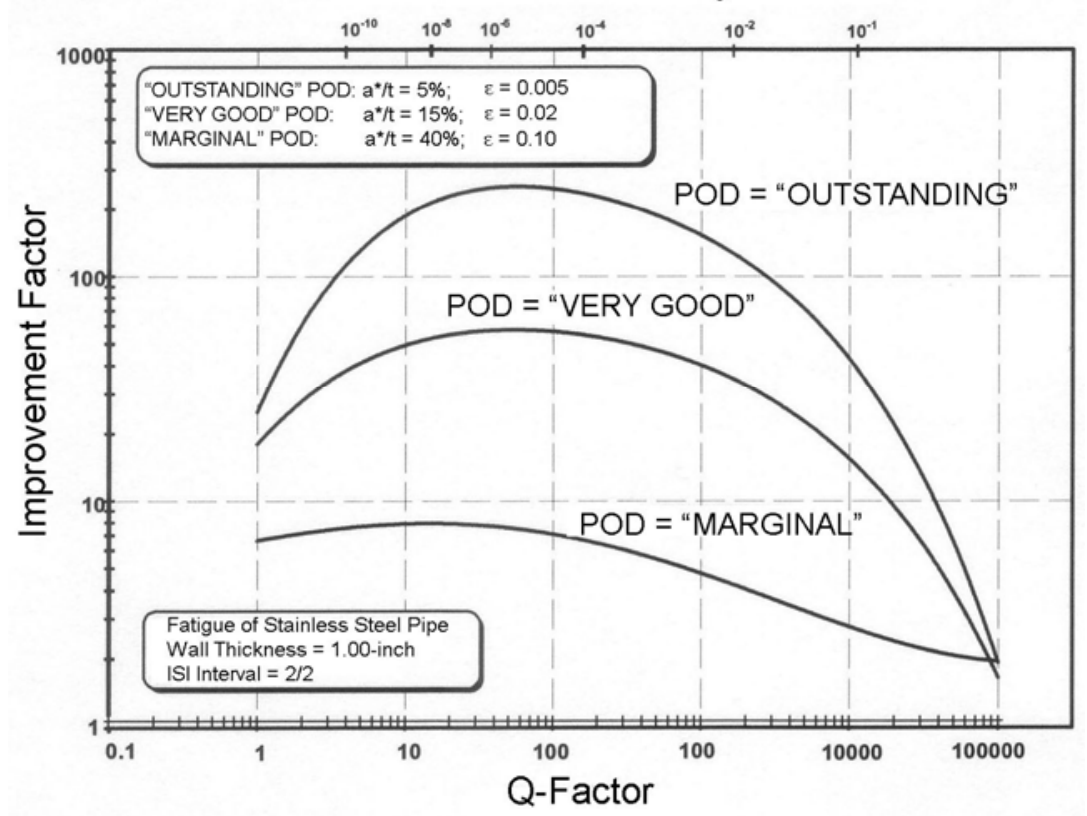

Figure 4.18c Improvement Factor for Fatigue of 1.0-in. (25.4-mm) Wall Stainless Steel Piping Using Inservice Inspection with Various Probability of Detection Curves and Periodic Inspections Performed at 2-Year Intervals with $Q=\alpha \mathrm{N}(\Delta \sigma)^{4}$

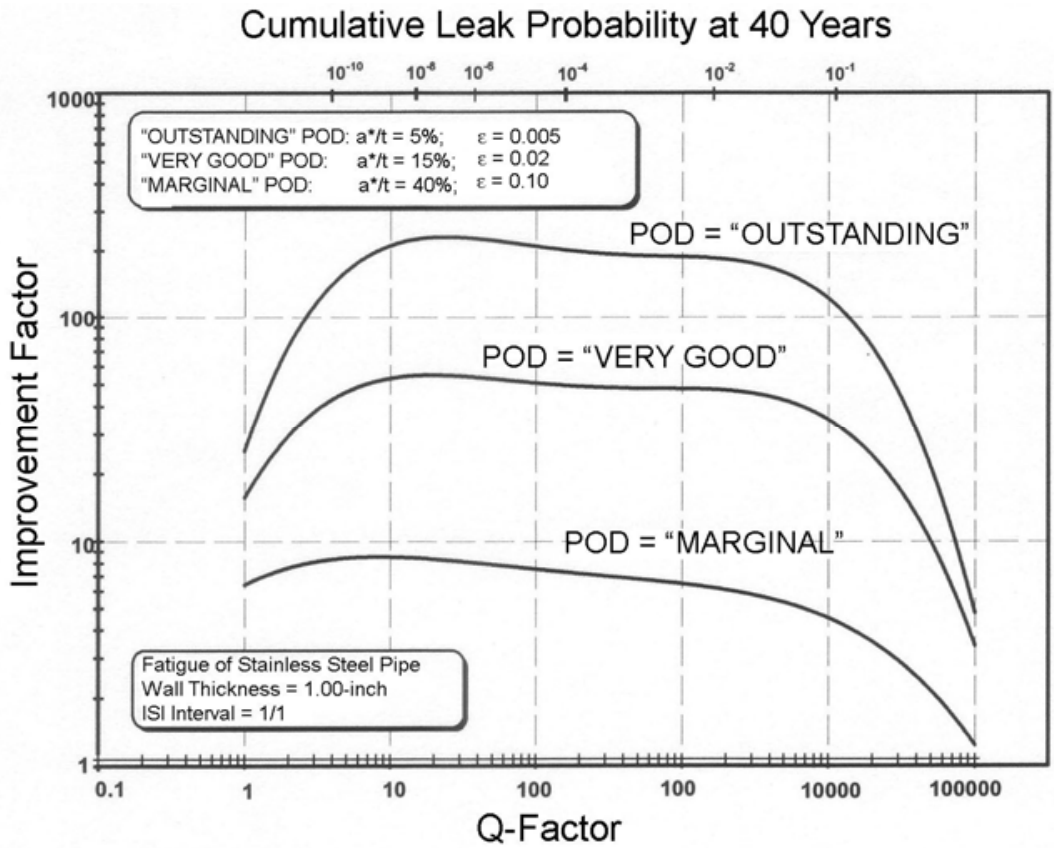

Figure 4.18d Improvement Factor for Fatigue of 1.0-in. (25.4-mm) Wall Stainless Steel Piping Using Inservice Inspection with Various Probability of Detection Curves and Periodic Inspections Performed at 1-Year Intervals with $Q=\alpha \mathbf{N}(\Delta \sigma)^{4}$ 
- Each curve of Figures 4.14 through 4.18 comes from a polynomial regression analysis of 16 data points for each curve. Each data point represents a ratio of two calculated failure probabilities (one with inspection and the other without inspection) from two pc-PRAISE calculations. Each curve therefore required 32 pc-PRAISE runs. Although there was some data scatter from the numerical approximations in the Monte-Carlo calculations, the regression analysis of the data points produced a consistent set of trend curves.

- The inspection frequencies are described by a notation that gives both the number of years between the inservice inspections and the time at which the first of the inspections is performed. For example, an inspection schedule of 5/10 has the first inspection performed after 5 years of operation and the subsequent inspections performed at 10-year intervals.

- The lower horizontal scale for the Q-factor is supplemented with an upper scale that indicates calculated values of leak probabilities corresponding to the Q-factors. These leak probabilities include the effects of the flaw density corresponding to the given combinations of wall thickness and pipe diameter. The calculated results have leak probabilities (cumulative over 40 years) ranging from $10^{-6}$ to probabilities that approach 1.0.

- Curves for the calculated improvement factors are presented using two formats to assist the reader to better identify trends. The format of Figures 4.14 through 4.17 focuses on the effects of inspection intervals by restricting each plot to one pipe size and one probability of detection curve. Figures 4.18a through $4.18 \mathrm{~d}$ focus on the effects of parameters of the detection curves, and restrict each plot to one pipe size and one inspection interval.

- The effect of the upper bound on flaw-detection probability for very large flaws is evident in Figures 4.14 through 4.18. For very frequent inspections (e.g., 1/1), it is seen that the improvement factors remain essentially constant (at a value of $1 / \varepsilon$ ) over a wide range of Q-factors. In these situations, there is no predicted benefit to increasing the frequency of inspections. On the other hand, inspections with enhanced POD curves would increase the improvement factors.

- Improvement factors are relatively small for the lower values of the Q-factor (e.g., Q = 1.0). These situations correspond to the very low levels of cyclic stress whereby failures can occur only if large fabrication flaws are present. A large fraction of such failures would occur early in life and before the first inservice inspection is performed. A preservice inspection of high quality is the most effective strategy to prevent such failures.

- For higher values of the Q-factor (e.g., $\mathrm{Q}=1000$ or greater), the calculated leak probabilities become relatively large. The cyclic stress conditions are sufficiently severe that even small fabrication flaws can grow to become through-wall cracks. Because the crack growth rates are quite high, an effective inservice inspection program (even with an enhanced POD curve) requires frequent inspections. The plots of Figures 4.14 through 4.18 show that improvement factors of 10 or greater can be achieved for these situations given a suitable combination of POD curve and inspection interval. However, inspection strategies having a 10-year inspection interval are inherently ineffective, even if they utilize the "outstanding" POD curve. Frequent inspections are essential. 
- The calculated improvement-factor curves for given inspection strategies are quite similar for the various wall thicknesses (as also indicated in Section 4.6.2). However, the calculations have assumed that the POD curve, when expressed as a function of normalized flaw depth (ratio of flaw depth to wall thickness), are independent of pipe-wall thickness. This assumption may not be a realistic description of performance levels for typical inspection practices. Nevertheless, the present results support ISI programs that seek a consistent POD level as function of a/t independent of the pipe-wall thickness.

\subsection{Summary and Conclusions}

A piping reliability model has been developed for predicting leak probabilities caused by fatigue crack growth. An initial defect size distribution is combined with a model of fatigue crack growth to evaluate the ability of inservice inspections to reduce failure probabilities. The candidate inspection programs have considered three different levels of NDE reliability and different inspection frequencies. A collection of curves was generated that describes the effects of inservice inspections on piping reliability.

The curves of this section can identify optimum inspection strategies for specified conditions of cyclic stresses. One potential application is the development of risk-informed inservice inspection programs. The factor of improvement curves can support a risk-informed approach for an inspection strategy by showing that a proposed combination of NDE reliability and inspection intervals will lead to a desired reduction (e.g., by a factor of ten) in failure probabilities. It is recognized that large uncertainties exist in estimated failure probabilities. The present results show that inspection programs can address such uncertainties because a select strategy can reduce failure probabilities for a wide range of cyclic stress conditions.

For most piping locations with relatively low cyclic stresses, it was found that the time at which the first inspection is performed was a significant factor. For these locations, the optimum strategy should include a high-quality preservice inspection to ensure early detection and repair of fabrication defects. In other cases, where the cyclic stresses are much higher, small fabrication flaws can contribute to failure probabilities. Because such flaws are undetectable at the time of the preservice inspection, an optimum strategy is that of an ongoing program of inservice inspections. The present calculations indicate that high-quality inservice inspections can significantly reduce leak and break probabilities, particularly if the inspections were performed relatively frequently. Preservice inspection was also effective in reducing the leak probabilities, but only for low Q-values (i.e., failures associated with low stresses and deeper flaws). High quality, closely spaced, inservice inspections were the most effective means to prevent failures caused by shallow flaws and high levels of cyclic stress (large Q-values).

Finally, it should be emphasized that this section has addressed one particular material (stainless steel) and one failure mechanism (crack growth from fabrication defects driven by mechanical and thermal fatigue). Section 5 addresses stainless steel piping subject to the mechanism of stress-corrosion cracking. Additional work could be performed to evaluate the effects of inservice inspections for additional materials and degradation mechanisms, including fatigue of ferritic steel piping with fabrication flaws and failures caused by the initiation of fatigue cracks in both ferritic and stainless steels. 


\section{Intergranular Stress Corrosion of Stainless Steel Piping}

\subsection{Introduction}

This section describes probabilistic calculations that address intergranular stress-corrosion cracking (IGSCC) of stainless steel piping, a degradation mechanism of major concern to nuclear pressure boundary integrity. The objective was to simulate the cracking of stainless steel piping under IGSCC conditions and to evaluate the benefits of alternate inspection strategies as a means to reduce failure probabilities. A range of pipe sizes and operating conditions was addressed by a matrix of parametric calculations. The results permit the development of effective inservice inspection programs that are based on tradeoffs between inspection frequencies and NDE sensitivities. The objective is to identify the most effective approaches to improving piping reliability.

The present calculations build on past studies by the nuclear power industry (General Electric Company 1982a, 1982b) and NRC (Hazelton and Koo 1988), which have addressed both IGSCC causes and preventive actions. A critical issue has been the difficulty of using ultrasonic testing (UT) to detect IGSCC. PNNL's past research on NDE reliability has included systematic studies to quantify NDE effectiveness. The first statistically based data for the probability of detection (POD) of IGSCC in welds of stainless steel piping were generated based on a piping inspection round robin (Doctor et al. 1983). The resulting data related POD to crack size and other important variables and covered the performance of several inspection teams participating in the round robin. Later work (Taylor et al. 1989; Heasler et al. 1990) further evaluated effects of personnel, equipment, crack characteristics, and human factors. Trends of data from these studies were used in the work described below to develop input data for pc-PRAISE for characterizing the effectiveness of UT inspections in detecting IGSCC.

The first part of this section describes the stress-corrosion cracking model used in the pc-PRAISE computer program (Harris 1981; Harris et al. 1986a; Harris et al. 1986b; Harris and Dedhia 1992) for simulating the initiation and growth of IGSCC cracks. This model is based on laboratory data from IGSCC tests in combination with calibration of the model using field data from pipe-cracking experience. PNNL has improved on the prior calibrations (Harris et al. 1986b) by making adjustments to the modeling of plant loading/unloading cycles in addition to adjustments to residual stress levels. The crack detection data (POD curves) by use of the pc-PRAISE model are also described.

A parametric approach was adopted in the present calculations to characterize IGSCC by a single damage parameter. This parameter $\left(D_{\sigma}\right)$ depends on residual stresses, environment conditions, and degree of sensitization. A matrix of calculations to address a wide range of pipe sizes, materials, and service conditions was developed. The results of these calculations quantify the reductions in failure probabilities that can be achieved with various ISI strategies. The final subsection of this section presents conclusions regarding ISI effectiveness as a mitigation action to enhance the reliability of BWR piping.

\subsection{Stress-Corrosion Cracking Model}

The probabilistic model of the pc-PRAISE code for stress-corrosion cracking includes a prediction of crack initiation, a simulation of the growth of very small cracks, and a treatment of the time and 
environment-dependent growth of larger cracks using a fracture mechanics model. The model accounts for material variabilities, statistical distributions, and through-thickness variations of stresses.

The model developed by Harris et al. (1986a, 1986b) separates the overall time-to-pipe-leak into three phases:

(1) time-to-initiate a small crack

(2) time spent growing the small crack at an "initiation velocity" or crack growth rate expressed as in./day

(3) time spent growing a larger fracture mechanics crack to become a through-wall crack.

The following describes the probabilistic model for each of these three aspects of stress-corrosion cracking.

\subsubsection{Crack Initiation Model}

Three conditions are required for stress-corrosion cracks to initiate in stainless steel: material sensitization, adverse environmental conditions, and high levels of tensile stresses. Therefore, a damage parameter $\left(\mathrm{D}_{\sigma}\right)$, which includes three multiplicative terms, has been developed (Harris et al. 1986a, 1986b):

$$
\begin{aligned}
D_{\sigma}= & f_{1}(\text { material }) \times f_{2}(\text { environmental }) \\
& \times f_{3}(\text { loading })
\end{aligned}
$$

where $f_{1}, f_{2}$, and $f_{3}$ are

$$
\begin{gathered}
f_{1}=C_{1}(P a)^{C_{2}} \\
f_{2}=O_{2}^{C_{3}} e^{\frac{C_{4}}{(T+273)}} \log \left(C_{5} \gamma^{C_{6}}\right) \\
f_{3}=\left(C_{8} \sigma^{C_{9}}\right)^{C_{1}}
\end{gathered}
$$

where $\mathrm{Pa}$ is a measure of the degree of material sensitization $\left(\mathrm{C} / \mathrm{cm}^{2}\right), \mathrm{O}_{2}$ is the oxygen concentration in parts per million, $T$ is the temperature in degrees centigrade, $\gamma$ is the water conductivity in $\mu \mathrm{s} / \mathrm{cm}$, and $\sigma$ is the stress in ksi. In the above equations, $\mathrm{C}_{\mathrm{i}}$ are constants evaluated by curve-fitting of laboratory and field data (Harris et al. 1986a, 1986b). Values for these constants are presented in Table 5.1 for 304 stainless steel. Similar constants developed by Harris and colleagues for $316 \mathrm{NG}$ stainless steel are not described here because the scope of the calculations of this section is limited to the more cracking-prone 304 grade of stainless steel. 
Table 5.1 Numerical Values of Constants $C_{i}$ Used in the Equation for Predicting the Initiation of Stress-Corrosion Cracks

\begin{tabular}{|c|c|c|c|c|c|c|c|c|c|c|c|c|}
\hline \multirow[b]{2}{*}{$\mathbf{C}_{1}$} & \multirow[b]{2}{*}{$\mathrm{C}_{2}$} & \multirow[b]{2}{*}{$\mathrm{C}_{3}$} & \multirow[b]{2}{*}{$\mathrm{C}_{4}$} & \multirow[b]{2}{*}{$\mathrm{C}_{5}$} & \multirow[b]{2}{*}{$C_{6}$} & \multirow[b]{2}{*}{$\mathrm{C}_{7}$} & \multirow[b]{2}{*}{$\mathrm{C}_{8}$} & \multirow[b]{2}{*}{$\mathrm{C}_{9}$} & \multicolumn{2}{|c|}{$\mathbf{A}^{1(\mathbf{a})}$} & \multirow[b]{2}{*}{ B } & \multirow[b]{2}{*}{ C } \\
\hline & & & & & & & & & $\boldsymbol{\mu}$ & $\sigma$ & & \\
\hline 23 & 0.51 & 0.18 & -1123 & 8.7096 & 0.35 & 0.55 & $2.21 \times 10-15$ & 6 & -3.1671 & 0.7260 & 1.4692 & 0.0694 \\
\hline
\end{tabular}

(a) $\mathrm{A}^{1}$ is normally distributed.

The time to crack initiation under static load conditions has been found to be a function of the damage parameter of Eq. (5.1), although the observed crack-initiation times for a given $\mathrm{D}_{\sigma}$ value exhibit considerable scatter. Therefore, the crack-initiation time for a given $\mathrm{D}_{\sigma}$ value is taken in pc-PRAISE to be log-normally distributed, with the distribution based on the available test data. The mean value of the $\log 10 t_{I}$ was estimated to be (Harris and Dedhia 1992)

$$
\text { mean } \log _{10}\left(\mathrm{t}_{\mathrm{I}}\right)=-3.10-4.21 \log _{10} \mathrm{D}_{\sigma}
$$

The standard deviation of $\log _{10}\left(\mathrm{t}_{\mathrm{I}}\right)$ is constant with a value of 0.3081 . The surface lengths of initiated cracks are assumed to be log-normally distributed, with a median value of $1 / 8 \mathrm{in}$. $(3.175 \mathrm{~mm})$ and a shape parameter of 0.85 . The depth of initiated cracks is taken to be $0.001 \mathrm{in} .(0.0254 \mathrm{~mm})$.

\subsubsection{Crack-Growth Model}

The growth of the very small cracks that have just initiated cannot be treated from a fracture mechanics standpoint (Andresen and Ford 1994). Therefore, an initiation velocity is assigned to newly initiated cracks

$$
\log \dot{a}=F+1.34 \log \left(D_{\sigma}\right)
$$

where the velocity is in in./day, $\mathrm{D}$ is the damage parameter, and $\mathrm{F}$ is normally distributed with a mean of 2.551 and standard deviation of 0.427 . The fracture mechanics velocity is

$$
\log (\dot{a})=A+B \log \left(O_{2}^{C_{3}} \exp \left[\frac{C_{4}}{(T+273)}\right] \log \left(C_{5^{\gamma}}{ }^{C_{6}}\right)\right)+C K
$$

where $\mathrm{K}$ is the stress intensity factor. The values of the constants are provided by Harris and Dedhia (1992), with A taken to be log-normally distributed to describe the scatter in the data. If the fracture mechanics velocity is greater than the initiation velocity, or if the crack depth is greater than $0.1 \mathrm{in}$. $(2.54 \mathrm{~mm})$, then the crack growth is treated by fracture mechanics. 


\subsubsection{Residual Stresses}

Residual stresses influence both crack initiation and propagation. The damage parameter $\mathrm{D}_{\sigma}$ is a function of the stress, which consists of both the applied (service-induced pressure and thermal) and residual stresses. The crack-tip stress-intensity factor is

$$
\mathrm{K}=\mathrm{K}_{\mathrm{ap}}+\mathrm{K}_{\mathrm{res}}
$$

where $\mathrm{K}_{\mathrm{ap}}$ and $\mathrm{K}_{\mathrm{res}}$ are the stress-intensity factors attributable to the applied stress and residual stresses respectively.

Data on welding residual stresses display large scatter (Harris et al. 1986b; Harris and Dedhia 1992) and differ significantly, depending on pipe size. The calculations reported here are concerned with the stresscorrosion cracking behavior of small pipes ( $\mathrm{OD}=4$ to $10 \mathrm{in}$. [102 to $254 \mathrm{~mm}$ ]), intermediate pipes $(\mathrm{OD}=10$ to $20 \mathrm{in}$. [254 to $508 \mathrm{~mm}$ ), and large pipes (OD $>20$ in. [508 mm]). The present calculations are based on the pc-PRAISE default inputs for residual stresses, which are the values originally estimated by Harris et al. (1986b).

The local residual stresses at the inside surface of small and intermediate pipes are treated as being normally distributed. The through-thickness distributions of stress are assumed to be linear variations between local values sampled for the inner and outer surfaces. For small pipes, the mean value of residual stress at the inner surface was $24.4 \mathrm{ksi}(168 \mathrm{MPa})$ with a standard deviation of $14.58 \mathrm{ksi}(100 \mathrm{MPa})$. The independently sampled stress at the outside surface was $24.4 \mathrm{ksi}(168 \mathrm{MPa})$ with a standard deviation of $14.47 \mathrm{ksi}$ (98 MPa). For the intermediate pipe, the inner surface stress had a mean value of $9.3 \mathrm{ksi}(64 \mathrm{MPa})$ and a standard deviation of $14.47 \mathrm{ksi}(98 \mathrm{MPa})$. The independently sampled stress at the outer surface of the intermediate pipe had a mean value of $9.3 \mathrm{ksi}(64 \mathrm{MPa})$ with a standard deviation of $14.47 \mathrm{ksi}(98 \mathrm{MPa})$.

Harris and Dedhia (1992) document the default pc-PRAISE inputs in detail for the complex pattern of residual stresses in large pipes. In summary, the inner surface had a mean tensile stress of $38 \mathrm{ksi}$ $(262 \mathrm{MPa})$. The through-thickness variation in stress had compressive stresses developing within the inner quarter-wall thickness and changing again to tension stress at greater depths.

\subsubsection{Failure Criterion for Pipe Breakage and Leakage}

In this study, flaws can fail the pipe by either breakage (pipe rupture) or leakage. The failure criterion for pipe break was that of the net section stress becoming equal to the material-flow stress. This criterion is applicable to high-toughness materials where failure occurs because of insufficient remaining crosssectional area to support the applied loads. The criterion is expressed as

$$
\sigma_{\mathrm{Lc}} \mathrm{A}_{\mathrm{p}}>\sigma_{\text {flo }}\left(\mathrm{A}_{\mathrm{p}}-\mathrm{A}_{\text {crack }}\right)
$$

In Equation (5.9), $A_{p}$ is the pipe cross-sectional area, $A_{\text {crack }}$ is the area of the crack, $\sigma_{\text {flo }}$ is the material flow stress, and $\sigma_{\mathrm{Lc}}$ is the axial component of load-controlled stress, which in pc-PRAISE is due to internal pressure and dead weight. The flow stress of the material $\sigma_{\mathrm{Lc}}$ used in Equation (5.9) was taken to 
be normally distributed, with an expected value of $43 \mathrm{ksi}(296 \mathrm{MPa})$ and a standard deviation of $4.2 \mathrm{ksi}$ (29 MPa). For leak failure, the criterion was that of a crack depth equal to the pipe-wall thickness.

\subsubsection{Numerical Simulation}

The pc-PRAISE code calculates leak and break probabilities caused by the initiation and growth of stresscorrosion cracks in stainless steel weldments. The model also addresses the growth of pre-existing fabrication cracks. All cracks are two-dimensional circumferential ID surface cracks. The numbers and sizes of cracks change with time because of the stress-corrosion cracking process, and they also change as a result of inservice inspections. A Monte Carlo simulation method is used for statistical sampling the several random variables in the model.

The pc-PRAISE code subdivides the circumferential welds into 2-in.- $(5.08-\mathrm{cm})$ long segments, and the initiation time-of-stress-corrosion cracks in each segment is assumed to be independent. Therefore, multiple cracks may exist and coalesce to become longer cracks. The crack linking is treated using the procedures described by Article IWA-3000 of the ASME Section XI Boiler and Pressure Vessel Code.

\subsection{Definition of $\mathrm{D}_{\sigma}$ Parameter}

The reliability for a large number of welds and fittings in a piping system can be estimated quickly if the results of detailed Monte Carlo simulations are provided in a structured parametric format. Such an approach was applied in Section 4 for estimating leak and rupture probabilities for stainless steel piping subject to mechanical and thermal fatigue.

The stress-corrosion damage parameter $\mathrm{D}_{\sigma}$ presented in Eqs. (5.1) through (5.4) is used in this section to generalize the results of PFM calculations. Values for $\mathrm{D}_{\sigma}$ can be calculated using these equations or can be read from the tables. Table 5.2 gives values for various degrees of sensitization, different levels of applied stress, a steady-state temperature equal to $550^{\circ} \mathrm{F}\left(288^{\circ} \mathrm{C}\right)$, an $\mathrm{O}_{2}$ content equal to $8 \mathrm{ppm}$, and a conductivity equal to $0.51 \mu \mathrm{S} /$ in. $(0.2 \mu \mathrm{S} / \mathrm{cm})$. For other levels of $\mathrm{O}_{2}$ and steady-state temperatures, adjustment factors are presented in Table 5.3. To obtain the value of $\mathrm{D}_{\sigma}$ that corresponds with conditions other than those for Table 5.2, multiply the $\mathrm{D}_{\sigma}$ values from Table 5.3 by the adjustment factors from Table 5.3.

The parametric calculations as presented below consisted of many pc-PRAISE runs that covered a range of leak probabilities from 1.0E-04 to 1.0. It was believed that $\mathrm{D}_{\sigma}$ could serve as a suitable parameter to summarize results for calculated failure probabilities in a manner similar to that used with the Q-Factor for the case of fatigue of stainless steel piping. Results presented later in this section show a good correlation between 40 -year cumulative leak probabilities and $D_{\sigma}$. However, the technical basis for $D_{\sigma}$ is not as strong as the basis for the Q-Factor. Although the correlation tends to break down for smaller values of $D_{\sigma}$, the parameter does provide a useful basis to generalize results for piping-leak probabilities. 
Table 5.2 Values for $D_{\sigma}$ Factors

\begin{tabular}{||l|c|c|c|c|c|c||}
\hline \multirow{2}{*}{ Total Stress } & \multicolumn{7}{|c||}{ Degree of Sensitization } \\
\cline { 2 - 7 } & $\mathbf{0 . 1}$ & $\mathbf{1 . 0}$ & $\mathbf{1 0}$ & $\mathbf{2 5}$ & $\mathbf{5 0}$ & $\mathbf{1 0 0}$ \\
\hline $40 \mathrm{ksi}(276 \mathrm{MPa})$ & 0.001633 & 0.005284 & 0.017100 & 0.027287 & 0.038857 & 0.055335 \\
\hline $42 \mathrm{ksi}(290 \mathrm{MPa})$ & 0.001918 & 0.006208 & 0.020087 & 0.032053 & 0.045646 & 0.065002 \\
\hline $43 \mathrm{ksi}(297 \mathrm{MPa})$ & 0.002073 & 0.006709 & 0.021709 & 0.034641 & 0.049331 & 0.070250 \\
\hline $43.4 \mathrm{ksi}(299 \mathrm{MPa})$ & 0.002138 & 0.006917 & 0.022383 & 0.035716 & 0.050862 & 0.072430 \\
\hline $45 \mathrm{ksi}(310 \mathrm{MPa})$ & 0.002409 & 0.007795 & 0.025223 & 0.040249 & 0.057316 & 0.081621 \\
\hline $47.5 \mathrm{ksi}(328 \mathrm{MPa})$ & 0.002879 & 0.009317 & 0.030150 & 0.048110 & 0.068512 & 0.097564 \\
\hline $50 \mathrm{ksi}(345 \mathrm{MPa})$ & 0.003410 & 0.011036 & 0.035711 & 0.056984 & 0.081148 & 0.115559 \\
\hline $52.5 \mathrm{ksi}(362 \mathrm{MPa})$ & 0.004006 & 0.012964 & 0.041949 & 0.066939 & 0.095324 & 0.135746 \\
\hline $55 \mathrm{ksi}(375 \mathrm{MPa})$ & 0.004671 & 0.015115 & 0.048910 & 0.078045 & 0.111141 & 0.158270 \\
\hline
\end{tabular}

Table 5.3 Adjustment Factors on $D_{\sigma}$

\begin{tabular}{|l|c|c|c|c|c|}
\hline $\mathrm{O}_{2}$ Level, ppm & 0.2 & 1.0 & 2.0 & 8.0 & 16.0 \\
\hline $\mathrm{O}_{2}$ Adjustment Factor & 0.5148 & 0.6878 & 0.7792 & 1.0 & 1.1329 \\
\hline Temperature, ${ }^{\circ} \mathrm{F}\left({ }^{\circ} \mathrm{C}\right)$ & $200(93)$ & $300(140)$ & $400(204)$ & $500(260)$ & $600(315)$ \\
\hline $\begin{array}{l}\text { Temperature } \\
\text { Adjustment Factor }\end{array}$ & 0.36433 & 0.55137 & 0.73776 & 0.91552 & 1.08128 \\
\hline
\end{tabular}

\subsection{Calibration and Benchmarking of Model}

The pc-PRAISE model for stress-corrosion cracking is based on laboratory data from IGSCC tests. However, unlike the fatigue failures (which have rarely occurred), there is extensive experience with pipe cracking to provide data to calibrate models and to benchmark the results of calculations. A calibration was part of the original development of the pc-PRAISE model for IGSCC. The approach was to identify an input (i.e., residual stress) to the model with a high level of uncertainty. This input was then adjusted to achieve a better correlation between the calculations and field experience.

The original development of pc-PRAISE (Harris et al. 1986b) limited the calibration to an adjustment to the residual stress level. To achieve agreement with field experience, it was necessary to reduce the residual stresses to 15 percent of measured levels. However, this large adjustment exceeded the estimated bounds of the uncertainties in residual stress levels. Therefore, PNNL revisited the prior calibrations by adjusting the effects of plant loading/unloading cycles in addition to adjusting the residual stress.

Materials science staff at PNNL reviewed pc-PRAISE assumptions (Harris et al. 1986b) relative to current knowledge of IGSCC. It was concluded that data from the constant extension rate tests (CERT) significantly overestimated the IGSCC damage produced by plant transients. The previous calibrations assumed five cycles of loading/unloading per year (Harris et al. 1986b).

Tests used to generate CERT data subject specimens to very slow strain rates, which continue for a long time until the specimens eventually fracture. IGSCC can cause cracking to initiate at accumulated strain 
levels much less than the corresponding elongations exhibited by short-term tensile tests. The PNNL materials specialists concluded that strains to failure from CERT tests are an overly conservative basis to estimate limits on the small levels of elastic tensile strains applied in a cyclic manner.

Therefore, the original calibration of the pc-PRAISE (Harris et al. 1986) was modified. The frequency of plant loading/unloading transients was decreased as a surrogate for a calibration factor on the calculated material damage associated with the five cycles per year of loading/unloading transients. This adjustment was combined with an adjustment to the residual stress level. One cycle of loading/unloading per 40 years was selected as the limiting case, which effectively removed all effects of cyclic loading on the IGSCC. This calibration considered various frequencies of the plant loading/unloading cycles.

Figures 5.1 through 5.3 shows results of pc-PRAISE calculations for the three pipe sizes along with some data points from field experience as used by Harris et al. (1986a). The pc-PRAISE results are for various values of the factor $f$ (where $f$ is a multiplier on the estimated residual stresses, with $f=1$ corresponding to no adjustment). The dotted lines are for the plant loading/unloading frequency held at five per year, but with various adjustment factors on residual stresses. The dashed lines are for the plant loading frequency set at $1 / 40$ per year ( $1 / 40$ in the plots) and various adjustment factors applied on residual stresses (i.e., 1.0, 0.9, 0.85, 0.80, and 0.75).

Figure 5.4 shows the effects of various plant-loading frequencies with the factor on residual stress held constant at $f=0.75$. The leak probability is only slightly influenced by the number of transients if the number of transients is less than one per year. Hence, the calibration of the model was based on assuming one or fewer transients per year with the factor $\mathrm{f}$ selected to provide agreement with the service experience. The results of Figures 5.1 through 5.3 (dashed lines) for the three pipe sizes indicate that a value of $f=0.75$ gives good agreement between predicted and observed leak probabilities. The remaining parametric calculations of this section were based on this calibration.

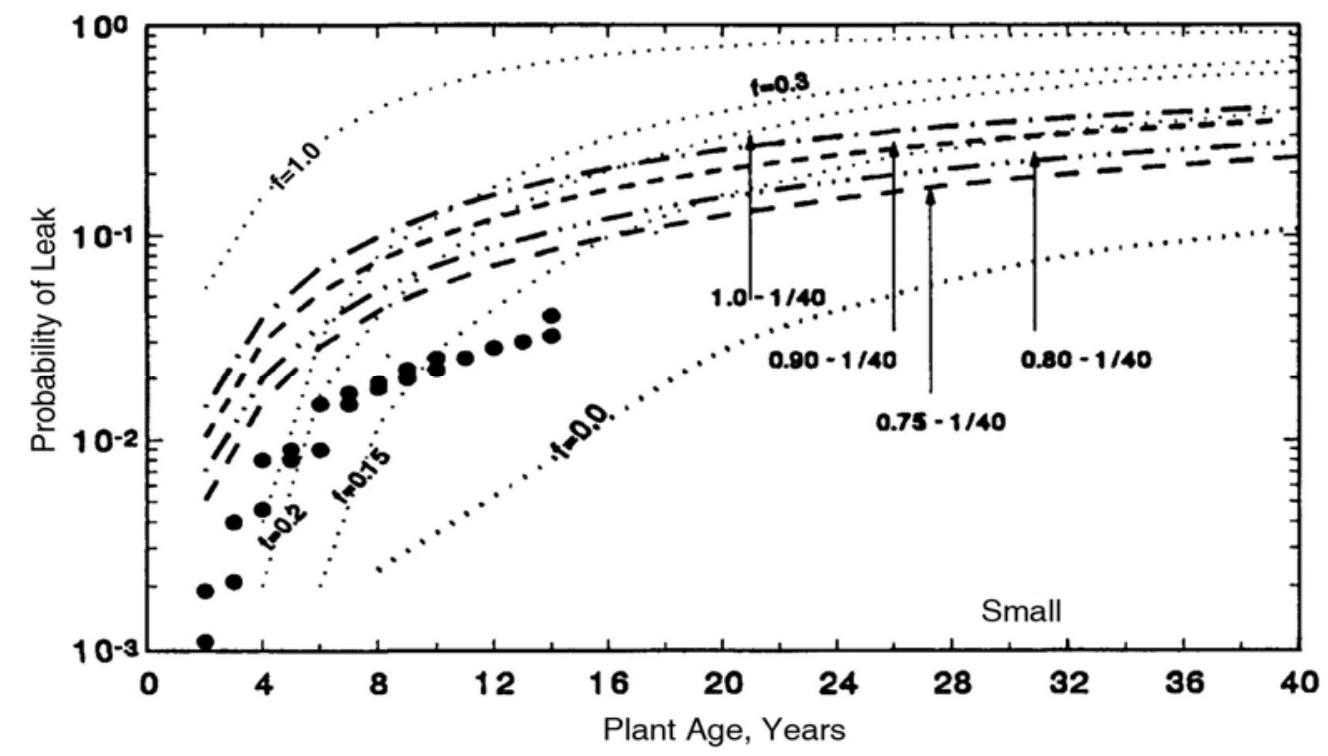

Figure 5.1 Field Observations of Leak Probabilities Compared with pc-PRAISE Results for Various Values of the Residual Stress Adjustment Factors and Plant Cycles (Small Diameter Pipes) 


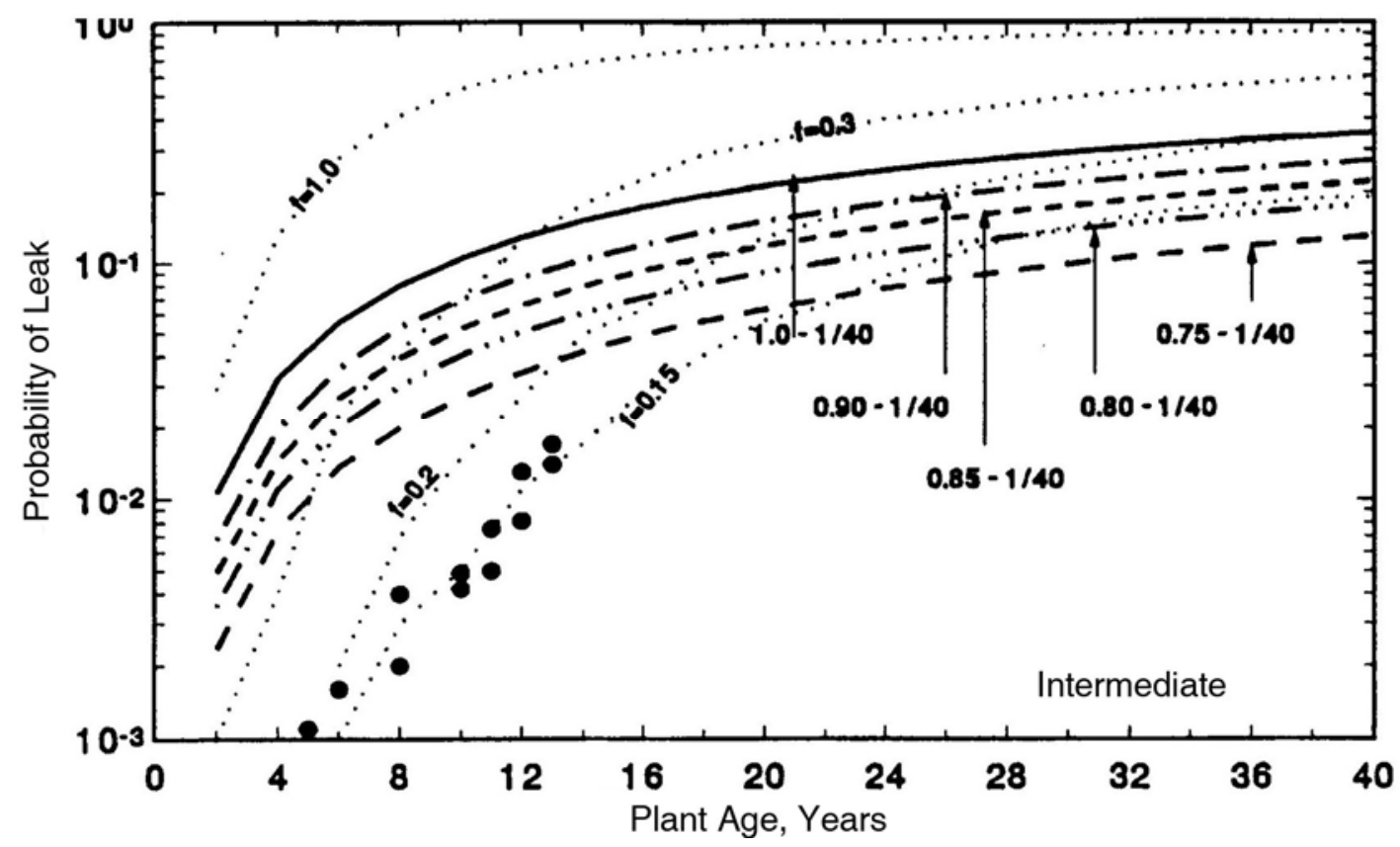

Figure 5.2 Field Observations of Leak Probabilities Compared with pc-PRAISE Results for Various Values of the Residual Stress Adjustment Factors and Plant Cycles (Intermediate Diameter Pipes)

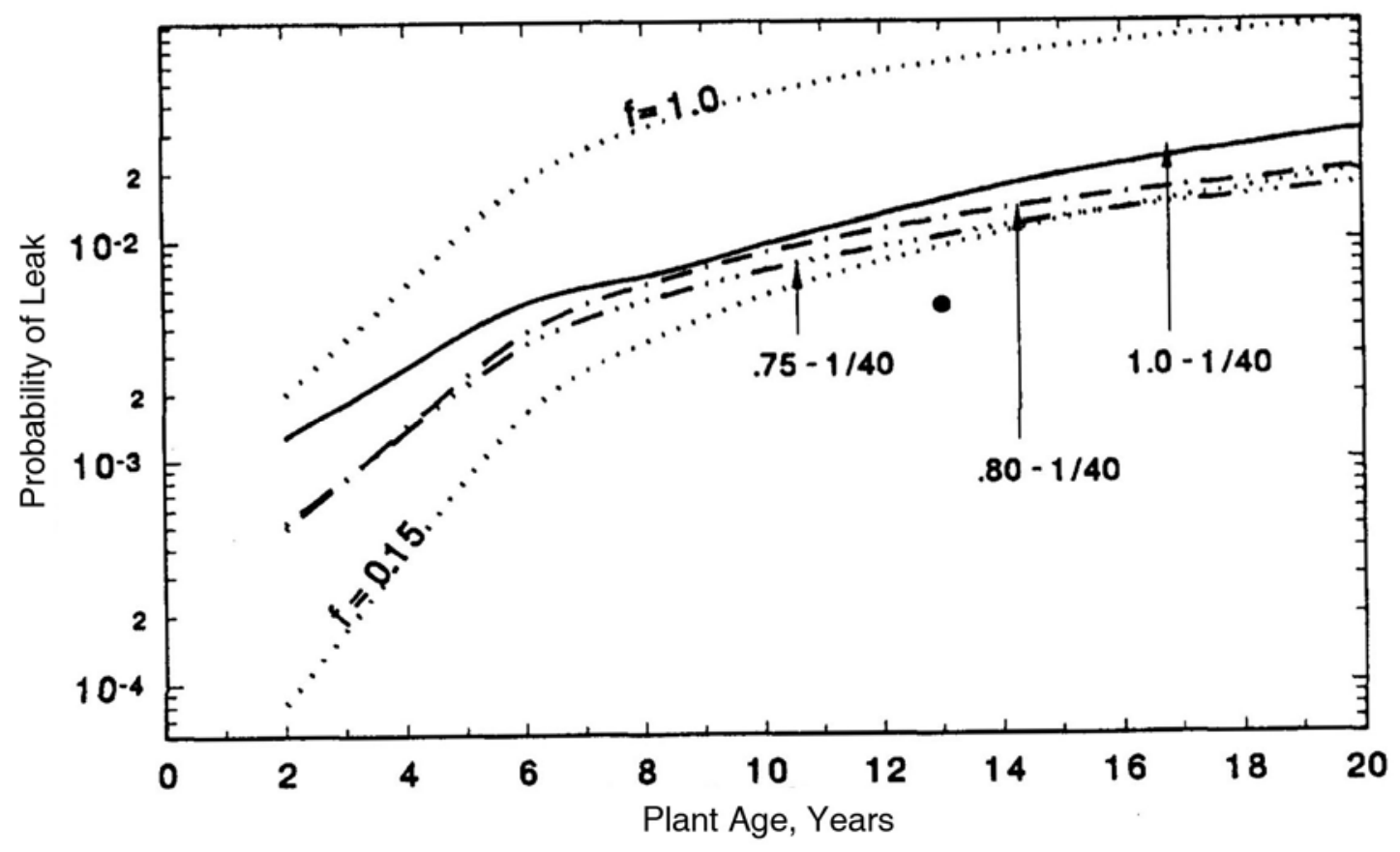

Figure 5.3 Field Observations of Leak Probabilities Compared with pc-PRAISE Results for Various Values of the Residual Stress Adjustment Factors and Plant Cycles (Large Diameter Pipes) 


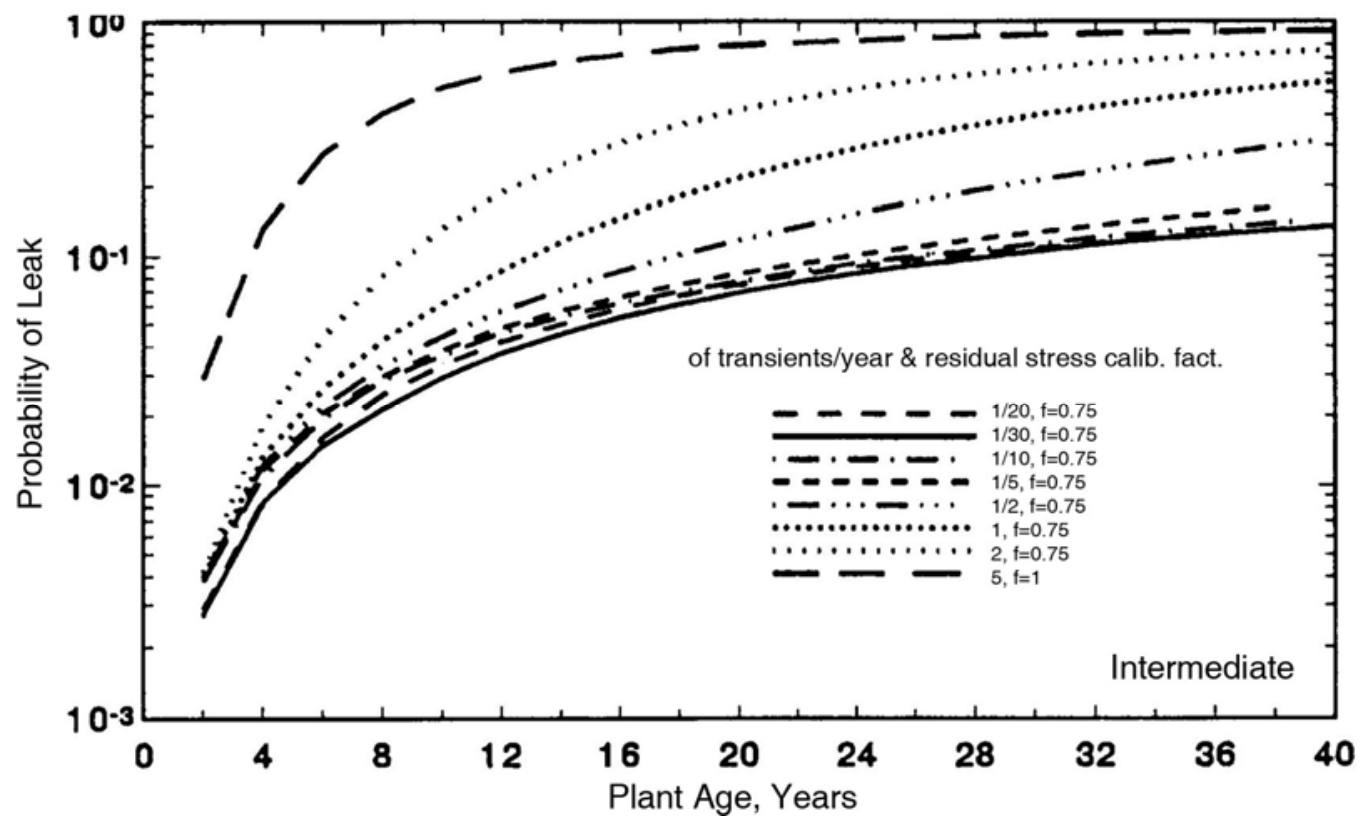

Figure 5.4 Predicted Leak Probabilities for Various Values of Yearly Plant Transients for Intermediate Diameter Pipes (f $=$ Residual Stress Adjustment Factor)

\subsection{Inservice Inspection Model}

The flaw-detection model of the pc-PRAISE computer code was applied in the calculations of this section. The required inputs to pc-PRAISE are (1) the POD curve and (2) the times at which the inspections are performed. Another input (as described below) specified whether the individual detection results from a sequence of periodic inspections were assumed to be independent or dependent outcomes. The calculations assumed that all detected flaws were repaired, which meant that flaw-sizing accuracy and flaw-acceptance criteria were not addressed.

\subsubsection{POD Curves}

In the literature, POD curves have been used to characterize the capability of a specific NDE technique and inspection team (Packman et al. 1976; Berens and Hovey 1984; Chang et al. 1994). The probability of detecting a crack depends on many factors, including the degradation mechanism; flaw location (e.g., surface or buried), crack depth, length, orientation, and shape; material (e.g., stainless and ferritic steel); inspector training and experience; and inspection procedures. Usually POD curves are expressed as a function of only the defect size (depth or area) with a given POD curve corresponding to a given material type, degradation mechanism, wall thickness, NDE technique, inspection procedure, etc. In general, the POD curve for a certain NDE method, procedure, and inspection team is estimated based on a statistical methodology using either experimental data, expert elicitation, or both data and elicitation.

Alternate inspection strategies for IGSCC were evaluated based on a set of POD curves for the ultrasonic examination of welds in stainless steel piping of various sizes. The approach was to postulate four POD curves that represented widely differing levels of NDE performance. These curves were intended to 
bound the performance expected from inspection teams operating in the field. To establish parameters for the POD curves, an informal expert judgment elicitation was made using staff at PNNL with knowledge of NDE performance data from inspection round robins and of recent industry efforts in the area of NDE performance demonstrations.

It was recognized that a population of inspection teams operating under field conditions can exhibit a considerable range of POD performance, even though all such teams have successfully completed the same performance demonstration process. The basic premise in estimating POD curves was that all teams had passed the ASME Section XI, Appendix VIII, Supplement 2 performance demonstration. The typical performance demonstration test in ASME Section XI requires a minimum of 10 flaws and 20 blank areas to be examined. A team that does not detect at least 8 of the 10 flaws or makes more than two false calls does not demonstrate acceptable performance and does not pass.

The informal expert judgment also considered information and trends observed in the PNNL mini-round robin and piping inspection round robin on UT inspection of wrought stainless steel (Heasler et al. 1990; Taylor et al. 1989; Doctor et al. 1983; Heasler and Doctor 1996). The NDE experts at PNNL were asked to define POD curves by estimating parameters for the following form of a POD function used in the pc-PRAISE code:

$$
\mathrm{P}_{\mathrm{ND}}(\mathrm{A})=\varepsilon+\frac{1}{2}(1-\varepsilon) \operatorname{erfc}\left(v \ln \left[\frac{\mathrm{A}}{\mathrm{A}^{*}}\right]\right)
$$

where $\mathrm{P}_{\mathrm{ND}}(\mathrm{A})=1-\mathrm{POD}(\mathrm{A})$ is the probability of nondetection, $\mathrm{A}$ is the area of the crack, $\mathrm{A}^{*}$ is the area of crack for $50 \% \mathrm{P}_{\mathrm{ND}}, \varepsilon$ is the smallest possible $\mathrm{P}_{\mathrm{ND}}$ for very large cracks, and $v$ is the "slope" of the $\mathrm{P}_{\mathrm{ND}}$ curve. The NDE experts provided a range of estimates for $A^{*}$ (crack area for 50\% POD) based on measured performance for PNNL's mini-round robin teams. Harris, Lim, and Dedhia (1981) assumed that the "slope" parameter $v$ is 1.6. Several POD curves from PNNL studies (Simonen and Woo 1984) were reviewed; a value of $v=1.6$ was determined to be consistent with published curves. For the various curves, the assigned value of this parameter was held constant, whereas the actual "slope" on a linear scale of the plotted curves becomes steeper for better POD curves. Thus, the "slope" on a linear scale is correlated to the detection threshold parameter $A^{*}$. The value of $\varepsilon$ was assigned such that a smaller value of A* also implies a smaller value of $\varepsilon$. Four POD curves were selected for the calculations of this section to characterize the levels of NDE reliability of interest:

- Marginal Performance. In the judgment of PNNL experts, a POD performance described by this curve would represent a team using given equipment and procedures that would have only a small chance of passing an Appendix VIII performance demonstration.

- Good Performance. A POD described by this curve corresponds to the better performance levels in the PNNL round robins.

- Very Good Performance. In the opinion of PNNL experts, this curve corresponds to a team (with given equipment and procedures) that significantly exceeds the minimum level of performance needed to pass an Appendix VIII performance demonstration test. 
- Advanced Performance. This curve describes a level of performance significantly better than expected from present-day teams, equipment, and procedures that have passed an Appendix VIII-type of performance demonstration. This performance level implies advanced technologies and/or improved procedures that could be developed in the future.

Table 5.4 summarizes the input data for the above four POD curves, which are shown in Figure 5.5. These particular curves assume that POD is a function of the crack depth as a fraction of pipe-wall thickness, independent of the actual wall thickness. Parameters indicated in Table 5.4 are considered appropriate to wall thicknesses of $1.0 \mathrm{in} .(2.54 \mathrm{~cm})$ and greater. Lower levels of POD as a function of a/h should be assumed for thinner-walled pipe. The parameter $a^{*}$ is the crack depth for $50 \%$ probability of nondetection and is related to $\mathrm{A}^{*}$ (i.e., $\mathrm{A}^{*}=\pi / 4 \mathrm{DB} \mathrm{a}^{*}$, where $\mathrm{DB}$ is the beam diameter of the ultrasonic probe).

Table 5.4 POD Curve Parameters for Four Performance Levels

\begin{tabular}{||l|c|c|c||}
\hline $\begin{array}{c}\text { Inspection } \\
\text { Performance Level }\end{array}$ & $\mathbf{a * / \mathbf { t } ^ { ( \mathbf { a } ) }}$ & $\boldsymbol{\varepsilon}$ & $\mathbf{v}$ \\
\hline Marginal & 0.65 & 0.25 & 1.4 \\
\hline Good & 0.40 & 0.10 & 1.6 \\
\hline Very Good & 0.15 & 0.02 & 1.6 \\
\hline Advanced & 0.05 & 0.005 & 1.6 \\
\hline (a) $\mathrm{t}$ is the wall thickness of the pipe. \\
\hline
\end{tabular}

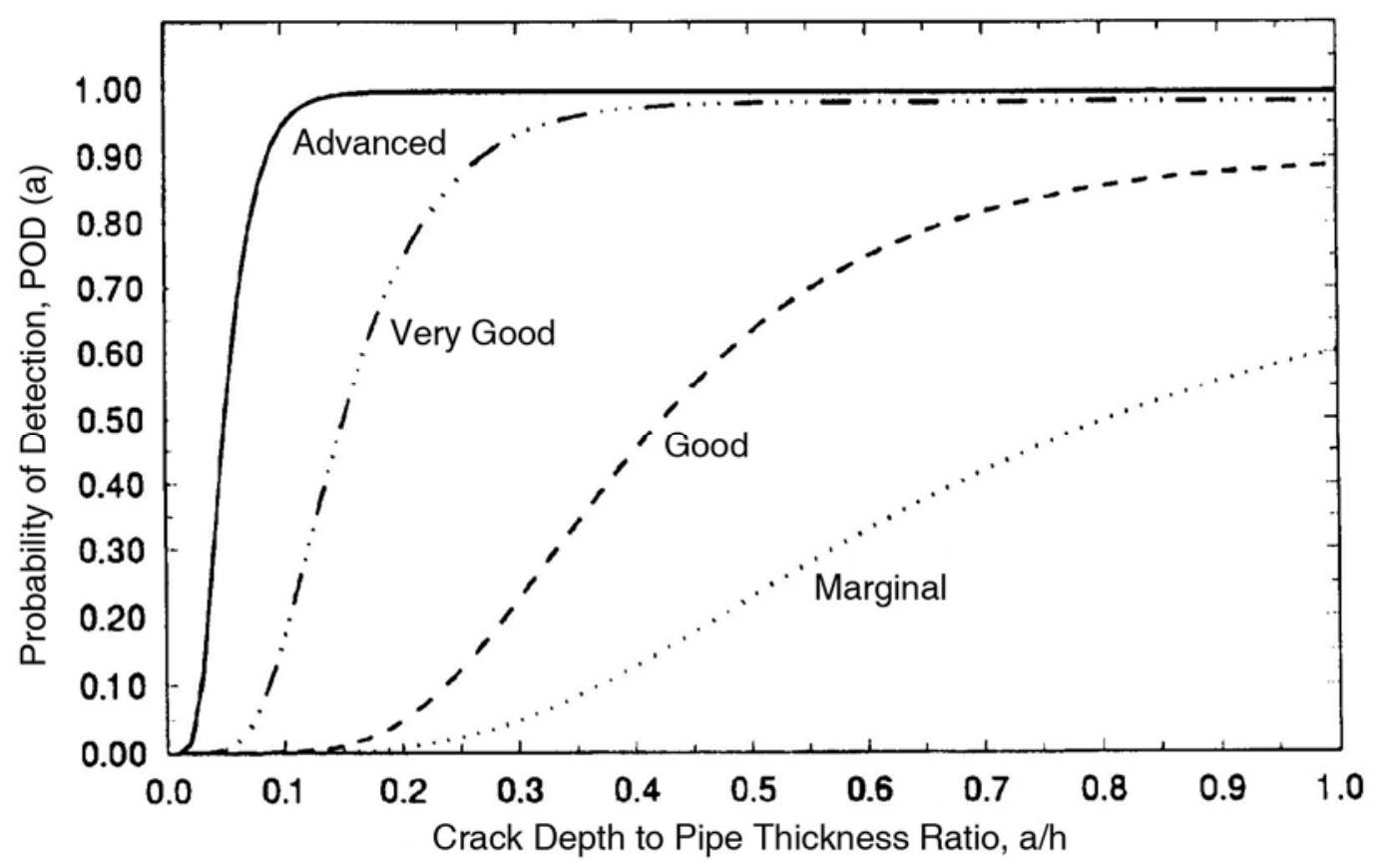

Figure 5.5 Probability of Detection Curves for Intergranular Stress-Corrosion Cracking 
In this study, the value of $a^{*}$ is taken to vary linearly with wall thickness, $t$. This may or may not be the case in a given situation. The assigned value of $v$ is 1.4 for the marginal performance level and 1.6 for all the other performance levels. The $v$ values were obtained by examining the POD data for 10 -in. (25.4-cm) stainless steel piping with stress-corrosion cracks generated by PNNL (Doctor et al. 1983; Simonen and Woo 1984). The smallest possible $\mathrm{P}_{\mathrm{ND}}$ for a large crack is the largest for the marginal POD (i.e., 25\%) and is the smallest for the advanced POD curve (i.e., $0.5 \%$ ).

\subsubsection{Factor of Improvement}

The effectiveness of inservice inspection programs is quantified by a parameter called "factor of improvement" (Thomas 1979; Simonen 1990; Khaleel and Simonen 1994a, 1994b). In this section, the factor of improvement is based on the cumulative leak probabilities that occur over the 40-year design life of the plant, and the "factor of improvement" is defined as the ratio

$$
\text { Factor of Improvement }=\frac{\text { leak probability at } 40 \text { years without NDE }}{\text { leak probability at } 40 \text { years with NDE }}
$$

\subsubsection{Independent versus Dependent Inspections}

Inspection unreliability comes mainly from two sources of variation: (1) factors that change from inspection to inspection, including inspection personnel, equipment, procedures, and other random factors; and (2) systematic factors related to the flaw location, component geometry, etc. The four POD curves presented earlier account for the degradation mechanism and the variations due to the flaw location, geometry, orientation, etc. In addressing the NDE reliability factors, which may change from inspection to inspection, the calculations of this section were based in most cases on the assumption that the series of inspections of a given weld over time are "dependent."

The original version of pc-PRAISE (Harris and Dedhia 1992) addressed only the independent option for modeling a sequence of inspections. To permit calculation for the dependent assumption, PNNL subcontracted with Drs. Harris and Dedhia at Engineering Mechanics Technology to modify pc-PRAISE to include the option of dependent inspections.

When the probability of failure after $\mathrm{n}$ inspections is "independent"

$$
\mathrm{P}_{\mathrm{f}}^{\mathrm{n}}(\mathrm{t})
$$

is equal to the product of the probability of failure without inspection and the product of the values of the POD curve evaluated at each previous inspection. If the inspections are "dependent," credit is only taken for the last inspection (i.e., the probability of detecting the flaw over the course of all of the inspections equals the probability corresponding to only the last inspection). It is believed that field performance can be bounded by the "dependent" and "independent" cases. Section 4 presents results that compare the results of pc-PRAISE calculations for fatigue that compare the "dependent" case with results for the "independent" case. These results indicate significant differences when the inspection frequency is large (e.g., ISI at 1-year intervals). Sensitivity calculations for IGSCC have shown a similar trend, but with the IGSCC results being less sensitive to the assumption of dependent versus independent inspections. 


\subsubsection{Multiple Defects in a Given Weld}

Welds are divided by pc-PRAISE into 2 -in. $(5.08-\mathrm{cm})$ segments. Each segment has only one corrosioncritical location where a crack can initiate. Cracks in neighboring segments may link to form larger cracks. The entire weld is assumed to be inspected at the time of each scheduled inspection. If a crack is detected in the weld, the weld is repaired, and the entire weld regains its integrity (perfect repair.)

\subsection{Input Parameters for Parametric Calculations}

The parametric calculations concentrated on welds in 304 stainless steel piping and on the cumulative leak probabilities over a 40-year time frame of plant operation. Consideration of remedial actions for IGSCC was limited to inservice inspections. Other mitigative measures, such as hydrogen injection, weld overlays, or induction heating stress improvement (IHSI), were not addressed. The calculations focused on leak probabilities because pc-PRAISE calculates probabilities of leaks more accurately and economically than probabilities of double-ended pipe breaks.

Table 5.5 summarizes the matrix of calculations along with the input parameters for the calculations. Base-case pc-PRAISE runs were first made for each of the three pipe sizes. These calculations assumed realistic ranges for the various input variables that govern the initiation and growth of IGSCC cracks. The following variables were addressed: $\mathrm{O}_{2}$ content, temperature, coolant conductivity, applied stress, and frequency of heatup and cooldown (HUCD). The resulting calculations therefore covered the relevant range of the $D_{\sigma}$ parameter used to present the results in a generalized manner. This initial set of pc-PRAISE runs assumed no inservice inspection and gave calculated 40-year cumulative leak probabilities ranging from $1.0 \mathrm{E}-04$ to 1.0 .

The second phase of the calculations included simulations of inservice inspections for a range of POD curves and inspection frequencies as indicated in Table 5.5. These calculations addressed only a selected subset of the base-case calculations, but were sufficient to cover the full range of the $\mathrm{D}_{\sigma}$ parameter. Ratios of leak probabilities from the inspection simulation runs and the corresponding base-case runs gave values for improvement factors. The establishment of these improvement factors for alternative inspection strategies was the primary objective of the calculations.

\subsection{Results of Parametric Calculations}

A large volume of numerical data was produced by completion of the parametric calculations for IGSCC of piping as described by Table 5.5. This section presents a collection of plots that show trends for pipeleak probabilities and for the effectiveness of various inservice inspection strategies in reducing leak probabilities. 
Table 5.5 Input Values for Parametric Calculations for Piping Subject to IGSCC Including the Effects of Inservice Inspection

\begin{tabular}{|c|c|c|c|}
\hline \multirow[b]{2}{*}{ Input Parameter } & \multicolumn{3}{|c|}{ Pipe Size } \\
\hline & Small & Medium & Large \\
\hline Outside Diameter, in. (mm) & $3.46(88)$ & $14.32(364)$ & $21.60(549)$ \\
\hline $\begin{array}{l}\text { Welding Residual Stress, } \\
\text { ksi (MPa) }\end{array}$ & $\begin{array}{l}\text { Randomized pc-PRAISE input values for } \\
\text { small lines with adjustment of } \\
\mathrm{f}=0.75 \\
\text { Stress at ID } \\
\quad \text { Mean }=24.40(168) \\
\quad \mathrm{SD}=14.58(100)\end{array}$ & $\begin{array}{l}\text { Randomized pc-PRAISE input values for } \\
\text { medium lines with adjustment of } \\
\mathrm{f}=0.75 \\
\text { Stress at ID } \\
\quad \text { Mean }=9.3(64) \\
\quad \mathrm{SD}=14.47(98)\end{array}$ & $\begin{array}{l}\text { Randomized pc-PRAISE input values } \\
\text { for medium lines with adjustment of } \\
\mathrm{f}=0.75 \\
\text { Stress at ID } \\
\quad \text { Mean }=38.0(262)\end{array}$ \\
\hline $\begin{array}{l}\text { Axial Component of } \\
\text { Primary Stress, ksi (MPa) }\end{array}$ & 2 to 15 (13.8 to 103$)$ & 2 to 15 (13.8 to 103$)$ & 2 to 12.5 (13.8 to 86$)$ \\
\hline $\begin{array}{l}\text { Flow Stress of Piping } \\
\text { Material, ksi (MPa) }\end{array}$ & $\begin{array}{l}\text { Normal Distribution } \\
\text { Mean }=43.0(296) \\
\text { Standard Deviation }=4.2(29)\end{array}$ & $\begin{array}{l}\text { Normal Distribution } \\
\text { Mean }=43.0(296) \\
\text { Standard Deviation }=4.2(29)\end{array}$ & $\begin{array}{l}\text { Normal Distribution } \\
\text { Mean }=43.0(296) \\
\text { Standard Deviation }=4.2(29)\end{array}$ \\
\hline $\begin{array}{l}\text { Probability of Detection } \\
\text { Curve }\end{array}$ & Four POD Curves as per Table 5.4 & Four POD Curves as per Table 5.4 & Four POD Curves as per Table 5.4 \\
\hline $\begin{array}{l}\text { Frequency of Inspection, } \\
\text { (Yr) (Time of Initial } \\
\text { ISI/Frequency) }\end{array}$ & $5 / 10 ; 5 / 5 ; 2 / 2 ; 1 / 1$ & $5 / 10 ; 5 / 5 ; 2 / 2 ; 1 / 1$ & $5 / 10 ; 5 / 5 ; 2 / 2 ; 1 / 1$ \\
\hline Range of $D_{\sigma}$ Parameter & $2.39 \mathrm{E}-03$ to $7.28 \mathrm{E}-03$ & $2.94 \mathrm{E}-03$ to $1.77 \mathrm{E}-02$ & $1.07 \mathrm{E}-02$ to $2.09 \mathrm{E}-02$ \\
\hline $\begin{array}{l}\text { Crack Initiation and } \\
\text { Growth }\end{array}$ & As per Eqs. (5.1)-(5.7) of Section 5.2 & As per Eqs. (5.1)-(5.7) of Section 5.2 & As per Eqs. (5.1)-(5.7) of Section 5.2 \\
\hline
\end{tabular}




\subsubsection{Predicted Leak Probabilities versus $D_{\sigma}$}

Figure 5.6 shows the cumulative leak probability at 40 years versus $D_{\sigma}$ for large pipes. In the parametric calculations, many pc-PRAISE runs were performed to cover a range of leak probabilities from 1E-4 to 1.0. In these calculations for the large pipe size, the residual stress and degree of sensitization were kept constant ( $\mathrm{f}=0.75, \mathrm{~Pa}=8$ ). On the other hand, Figure 5.7 shows the 40 -year cumulative leak probability versus $D_{\sigma}$ for various degrees of sensitization, temperature, and oxygen content, but with the level of residual stress $(f=0.75)$ held constant. As shown by both figures, a strong correlation between the 40 -year cumulative leak probabilities and $\mathrm{D}_{\sigma}$ exists.

\subsubsection{Effect of Pipe Size on Leak Probabilities}

Calculated leak probabilities are shown in Figure 5.8 for each of three pipe sizes for the assumption of no inservice inspection. For all pipe sizes, the leak probabilities increase as the $\mathrm{D}_{\sigma}$ value increases. However, each pipe size gives a separate trend curve. It is believed that different levels and distributions in welding residual stress for the various pipe sizes is a major contributing factor to the size dependence of the curves relating pipe leak probability to the $D_{\sigma}$ parameter. It is seen that the two smaller pipe sizes have higher calculated leak probabilities than large pipe sizes. Figure 5.9 also shows the effect of pipe size on the calculated probabilities of a leak, but now as a function of time for a fixed value of $D_{\sigma}$ $\left(\mathrm{D}_{\sigma}=5.0 \mathrm{E}-03\right)$. Each curve shows a similar trend of increasing leak probability as a function of time with the large pipe size having the lowest probabilities and the intermediate pipe size having the highest probabilities.

\subsubsection{Effect of POD Curve and ISI Frequency on Improvement Factor}

The matrix of calculations addressed three pipe sizes (small, intermediate, and large), four POD curves (marginal, good, very good, and outstanding) and four inservice inspection intervals (10, 4, 2, and 1 year) giving $4 \times 4 \times 3=48$ ISI scenarios. Detailed results of the calculated improvement factors associated with the inservice inspection cases are presented for reference purposes by Figures 5.10 through 5.30 below (see end of section for Figures 5.10 through 5.30). To maximize the usefulness of the plots, each set of results is presented both as a function of the $D_{\sigma}$ parameter and of the corresponding leak probability (cumulative probability of through-wall crack at 40 years). The individual data points for improvement factors exhibited some data scatter, which was addressed by using a regression analysis to establish bestfit curves (second order polynomial) in terms of improvement factors versus the logarithm (base 10) of $\mathrm{D}_{\sigma}$ and the corresponding leak probabilities. For clarity, the plots below show only the best-fit curves rather than the individual data points. 


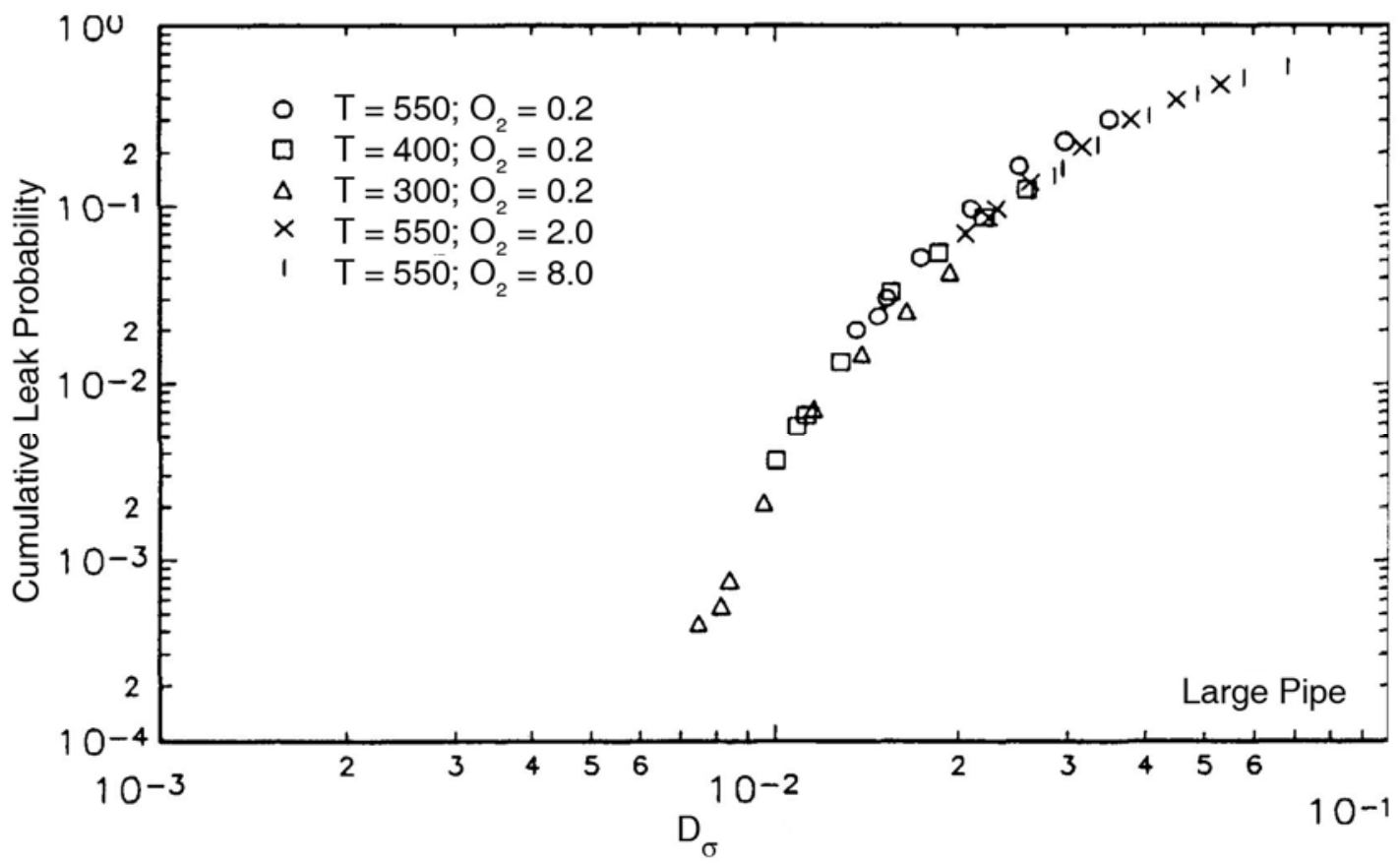

Figure 5.6 Cumulative Leak Probability Over 40 Years as a Function of the Stress-Corrosion Damage Parameter; $D_{\sigma}$ for Various Temperatures $\left({ }^{\circ} F\right)$ and Oxygen Contents $\left({ }^{\circ} \mathrm{C}=\left[{ }^{\circ} \mathbf{F}-32\right] / 1.8\right)$

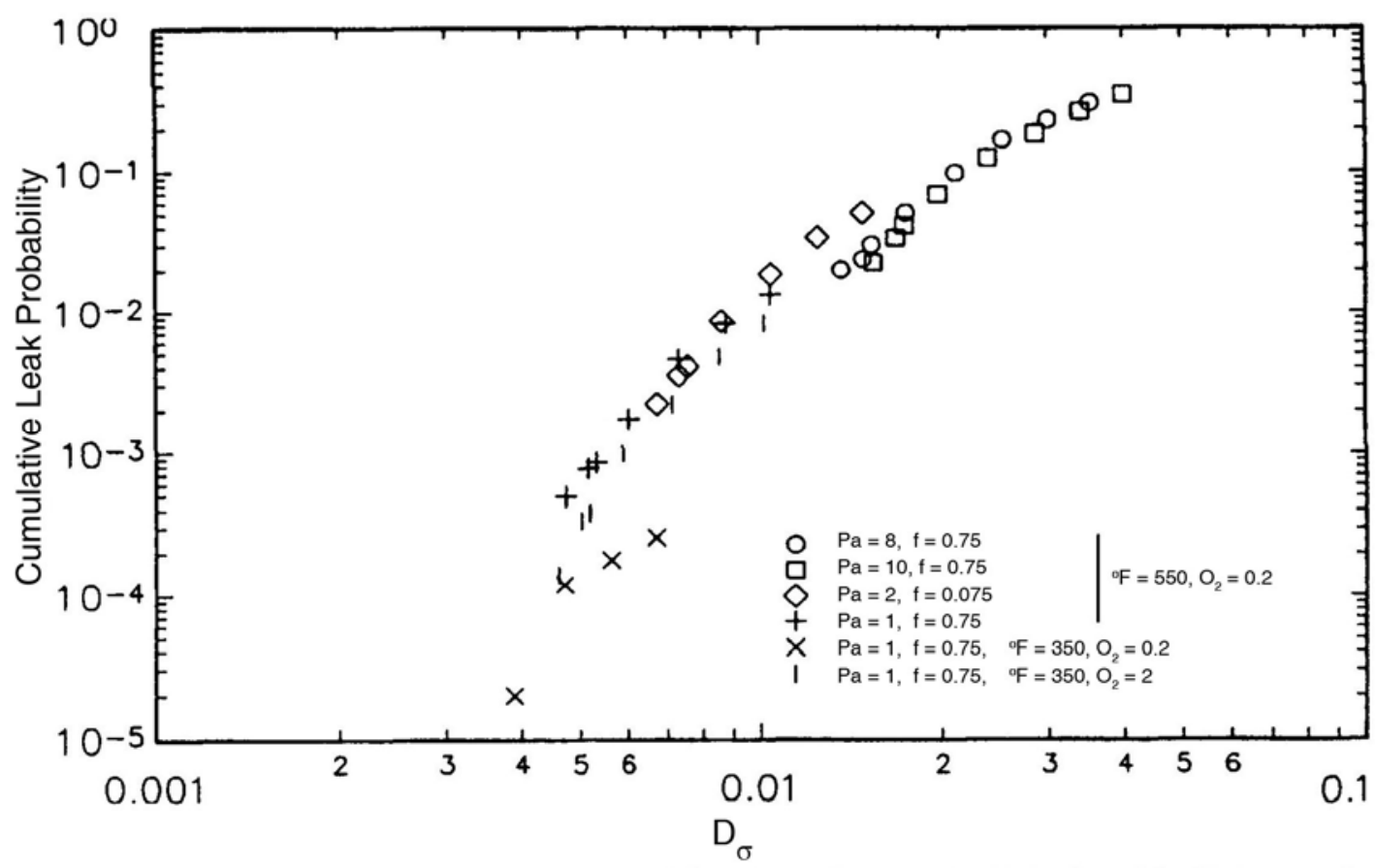

Figure 5.7 The Effect of Various Degrees of Sensitization on the Relationship Between the 40-Year Cumulative Leak Probability and $D_{\sigma}\left({ }^{\circ} \mathrm{C}=\left[{ }^{\circ} \mathrm{F}-32\right] / 1.8\right)$ 


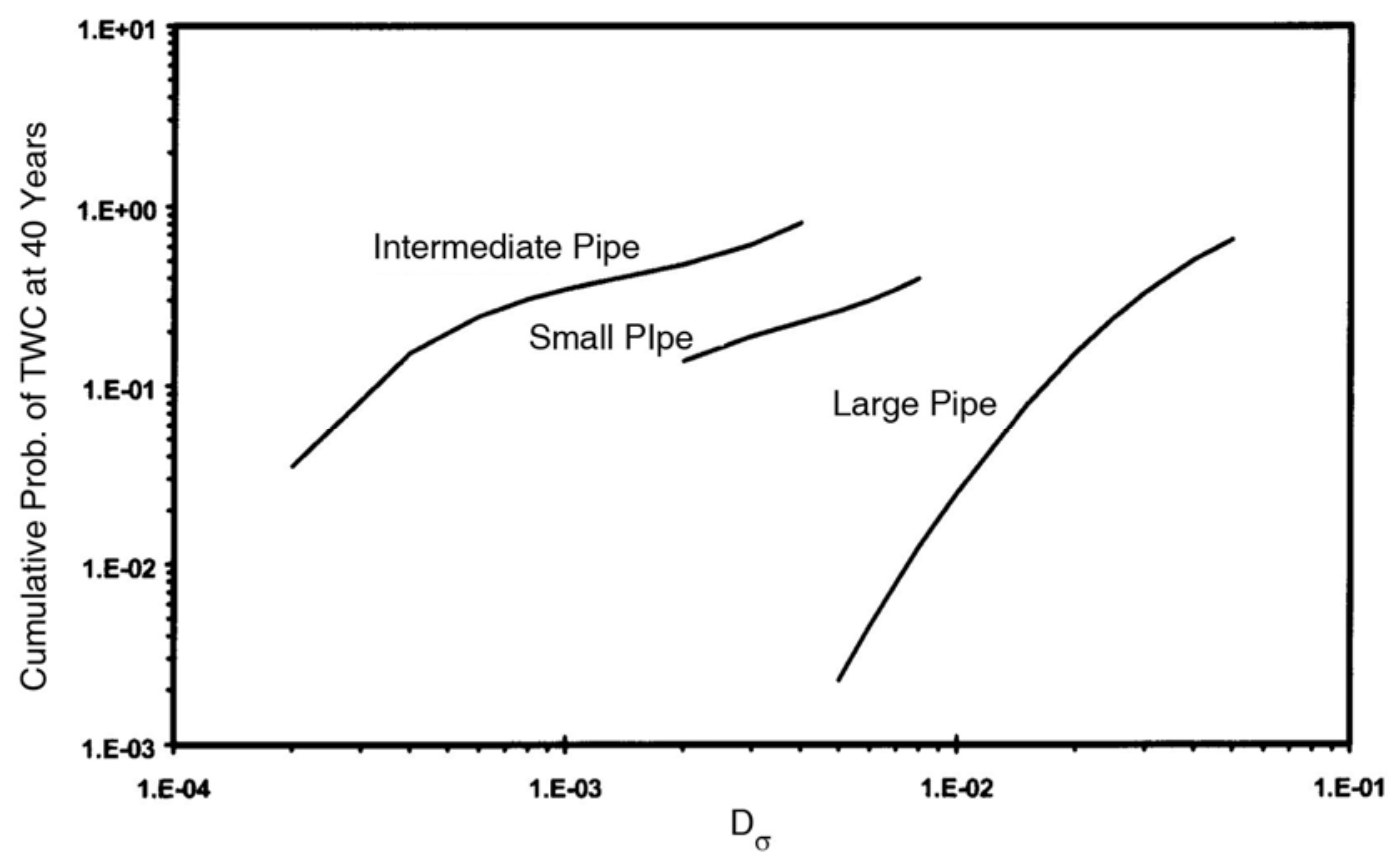

Figure 5.8 Cumulative Leak Probability over 40 Years for Three Pipe Sizes as a Function of the Damage Parameter $\mathbf{D}_{\sigma}$

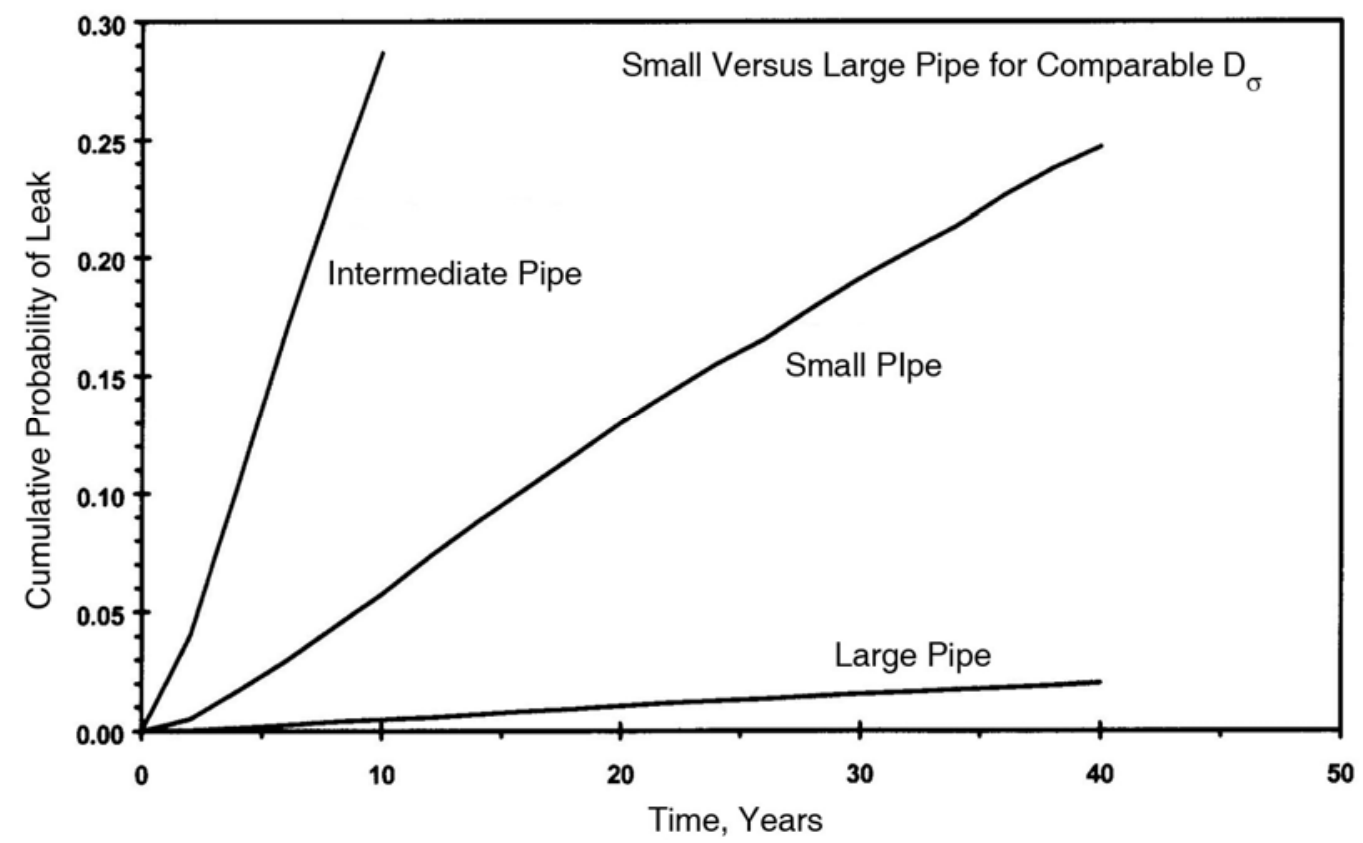

Figure 5.9 Cumulative Leak Probability as a Function of Time for Three Pipe Sizes and Comparable Values of the Damage Parameter $D_{\sigma}\left(D_{\sigma}=5.0 E-03\right)$ 


\subsubsection{Improvement Factors for Small Pipe Size}

Figures 5.10 through 5.17 show predicted improvements in reliability over a 40-year design life for the small pipe size that results from inservice inspections performed over the 40 -year operating period. Four different levels of NDE performance are addressed. In Figures 5.10 through 5.13, the inspection method (POD curve) was held constant, and the inspection intervals ranged from 1 to 10 years. Figures 5.14 through 5.17 show the same set of results, but with the curves rearranged to maintain a common inspection interval for each plot, with the individual curves corresponding to different POD curves.

Figure 5.10 (and successive figures) describes inspections performed at a 10-year interval and with the first inspection at the $10^{\text {th }}$ year using the notation 10/10. Similarly, $4 / 4$ indicates inspections at 4 -year intervals with the first inspection at the $4^{\text {th }}$ year.

It is seen that POD curves with the better detection probabilities always increase the factor improvement, no matter what inspection interval is used. However, the results for the small pipe size show that even the "outstanding" POD curve requires frequent inspection to achieve meaningful reductions in failure probabilities.

From Figure 5.10, it is seen that the "marginal" POD curve provides little benefit in reducing the probability of failure, even when the inspection interval is reduced to one inspection per year. The inspections with the "good" and "very good" POD curves (Figures 5.11 and 5.12) give more meaningful enhancement in piping reliability. The greatest benefits are predicted for the "outstanding" NDE technology and procedures, for which an order-of-magnitude improvement on the leak probability can be achieved for an inspection frequency of once per year. This improvement factor of 10 is still significantly less than the theoretical limit of $200(1 / \varepsilon=1 / 0.005=200)$ for the "outstanding" POD curve. In contrast, comparable results given in Section 4 on fatigue crack growth indicate more encouraging improvement factors of about 100 for inspections using the "outstanding" POD curve performed at a frequency of once per year. The lower benefits of ISI for IGSCC compared to the benefits for fatigue crack growth can be explained in terms of long incubation periods for stress-corrosion cracking followed by a period of rapid crack growth.

\subsubsection{Improvement Factors for Intermediate Pipe Size}

Figures 5.18 through 5.25 show the calculated improvement factors in the reliability (over a 40 -year design life) for the intermediate pipe size. The simulated inservice inspections performed over the 40-year operating period were the same as those for the small pipe size. In the case of Figures 5.18 through 5.21, the inspection method (POD curve) was held constant for each figure, and the time intervals between inspections were varied from 1 to 10 years. Figures 5.22 through 5.25 show the same set of results, but with the curves rearranged to maintain a common inspection interval for each plot, with the individual curves corresponding to different POD capabilities. As for the small pipe size, the POD curves with the better detection probabilities always produce greater improvement factors. Nevertheless, even the "outstanding" POD curve requires frequent inspections to achieve meaningful reductions in failure probabilities. 


\subsubsection{Improvement Factors for Large Pipe Size}

Figures 5.26 through 5.30 show calculated improvement factors (over a 40-year design life) for the large pipe size. The number of simulated inservice inspection scenarios was somewhat more limited than for the small and intermediate pipe sizes. Figures 5.26 through 5.28 each address a specific inspection method (POD curve) with the individual curves corresponding to different inspection intervals ranging from 1 to 10 years. Figures 5.29 and 5.30 have rearranged the curves to maintain a common inspection interval for each plot, with the individual curves corresponding to different POD capabilities. As for the smaller pipe sizes, the POD curves with the better detection probabilities give greater improvement factors. In general, it appears that a given inspection strategy (combination of POD curve and inspection interval) gives somewhat greater improvement factors for the large pipe than predicted for the smaller sizes of pipe.

\subsubsection{Effect of Pipe Size on Improvement Factor}

Differences in calculated improvement factors for the three pipe sizes are indicated in Figures 5.31 through 5.35. As indicated by Figure 5.31, a better alignment of the improvement factor curves results when the data are plotted against leak probability (Figure 5.31b) rather than against $\mathrm{D}_{\sigma}$ (Figure 5.31a). For this reason, the remaining plots (Figures 5.33 through 5.35) for several combinations of POD curves and inspection intervals have used leak probability as the independent variable.

The differences in the calculated improvement factors for the three pipe sizes are believed to be mainly due to the different through-wall distributions of the welding residual stresses than to an effect of pipe size per se. There is a consistent trend that shows greater benefits from inspections for the large pipe as compared to the small or intermediate pipe size. This can be explained in terms of differences in the through-wall distributions of residual stresses. The default stress distribution for the large pipe shows relatively steep stress gradients near the inner surface of the pipe and becomes compressive at subsurface locations relatively close to the inner surface. It would therefore be expected that cracks initiated in the large pipe would exhibit relatively uniform growth rates, making such cracks easier to detect by periodic examinations. In contrast, initiated cracks in the smaller pipes are believed to grow very slowly for extended time periods, followed by a short period of rapidly accelerated crack growth until a leak results. Detection of such a rapidly growing crack would require relatively small time intervals between the periodic inspections.

\subsubsection{Time Dependence of Calculated Failure Probabilities}

A final set of plots (Figures 5.36 and 5.37) show leak probability as a function of time, in contrast to all previous plots that only indicate the cumulative leak probability at the single time of 40 years.

Figure 5.36 shows how the calculated leak probabilities for the three pipe sizes increase with time. It also shows that the failure rate (slope of the curves) remains relatively constant of the 40-year time span covered by the plots. In one case (Figure 5.36a), the $\mathrm{D}_{\sigma}$ for each pipe size was selected to give a leak probability at 40 years of about 20 percent. In the other case (Figure 5.36b), a common value of $\mathrm{D}_{\sigma}$ is equal to about 5.0E-03, which results in a large difference in the calculated leak probabilities for the three pipe sizes. 
Figure 5.37 compares leak probabilities for various inspection strategies with the baseline of no inspection. In each case, the benefits of the inspection become evident soon after the first inspection is performed, and the curve of failure probability as a function of time then experiences a change in slope. It is noted that the families of curves shown by Figure 5.37 are consistent and well behaved, which justifies the assumption implied in the prior collection of plots that one can use the cumulative failure probability as a representative measure to evaluate the effectiveness of inspections over the entire 40-year operating period of a plant.

\subsection{Conclusions}

A probabilistic stress-corrosion cracking model was applied to assess the effect of various inspection scenarios on leak probabilities. This section has also discussed probability of detection curves and the benefits of inservice inspection in the framework of reductions in the leak probabilities for nuclear piping systems subjected to IGSCC.

The results for typical NDE performance levels indicate that low inspection frequencies (e.g., one inspection every 10 years) can provide only modest reductions in failure probabilities. More frequent inspections appear to be even more effective. However an "Outstanding" NDE reliability can achieve a factor of 10 improvement in preventing IGSCC leaks at typical operating conditions even when inspections occur approximately every $10 \mathrm{yr}$; this can be increased to a factor even greater than 10 with the inspection interval decreased sufficiently (e.g., one inspection every one or two years). 


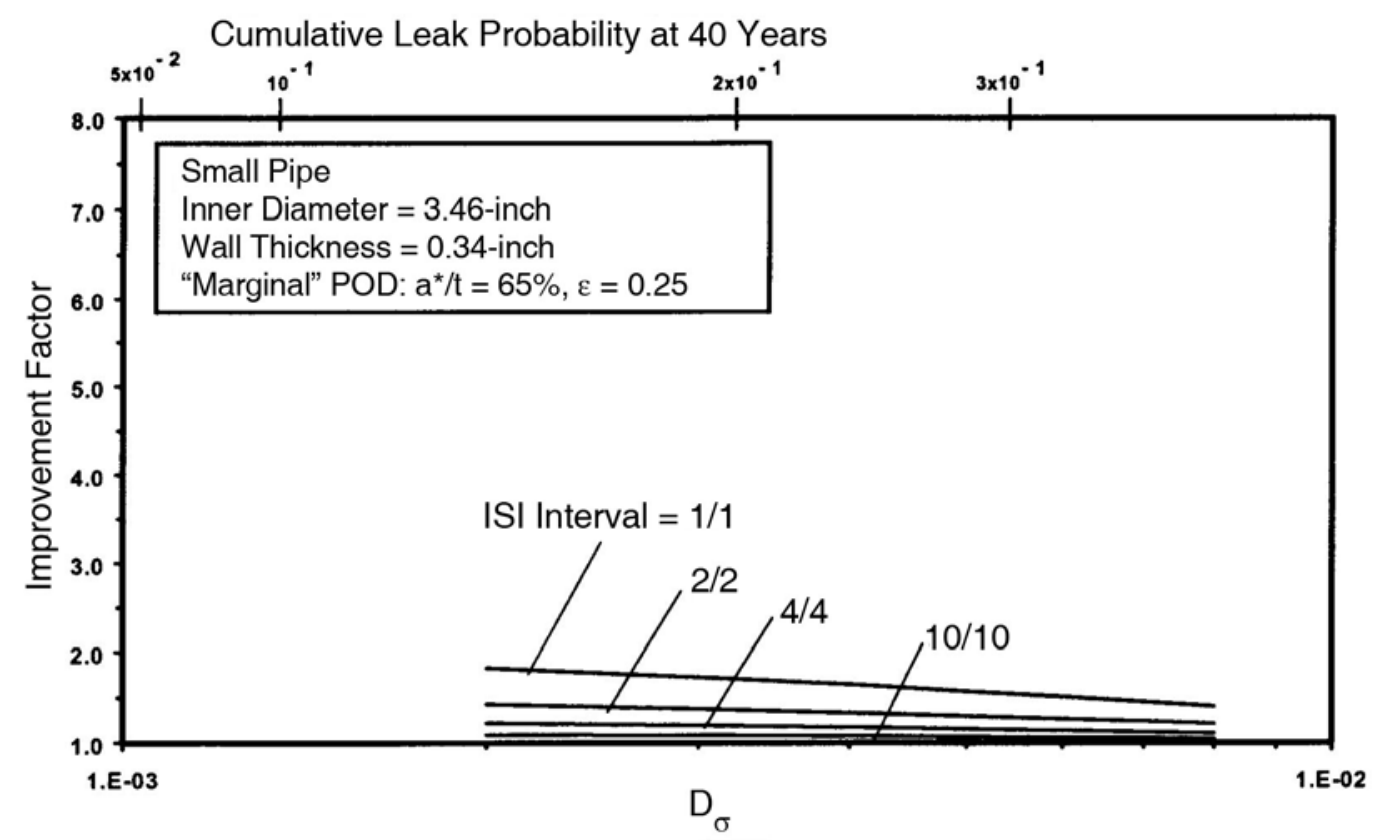

Figure 5.10a Improvement Factors versus $D_{\sigma}$ for "Marginal" POD Curve (Small Pipe Size and Various Inspection Intervals) $(1$ inch $=25.4 \mathrm{~mm})$

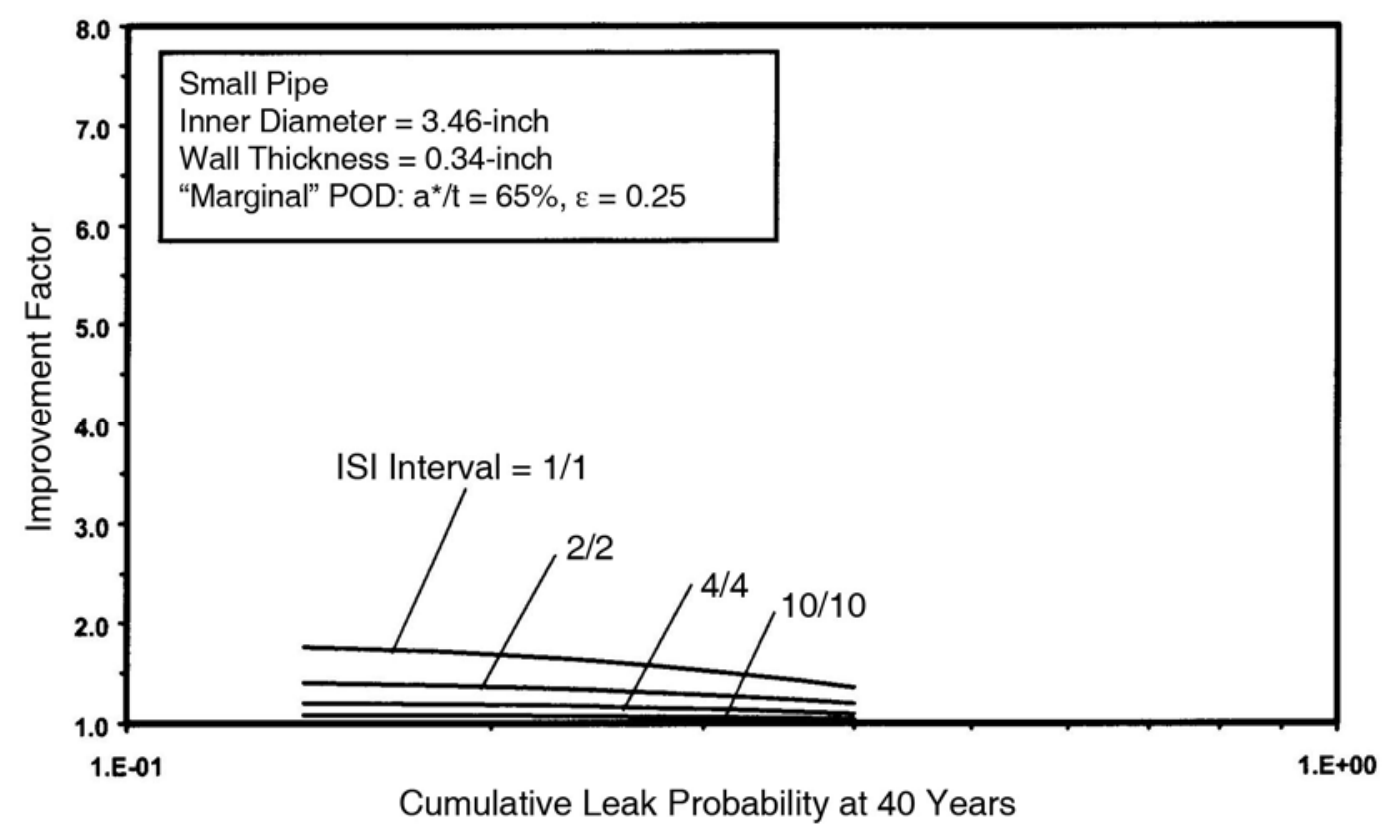

Figure 5.10b Improvement Factors versus Leak Probability for "Marginal" POD Curve (Small Pipe Size and Various Inspection Intervals) $(1$ inch $=25.4 \mathrm{~mm})$ 


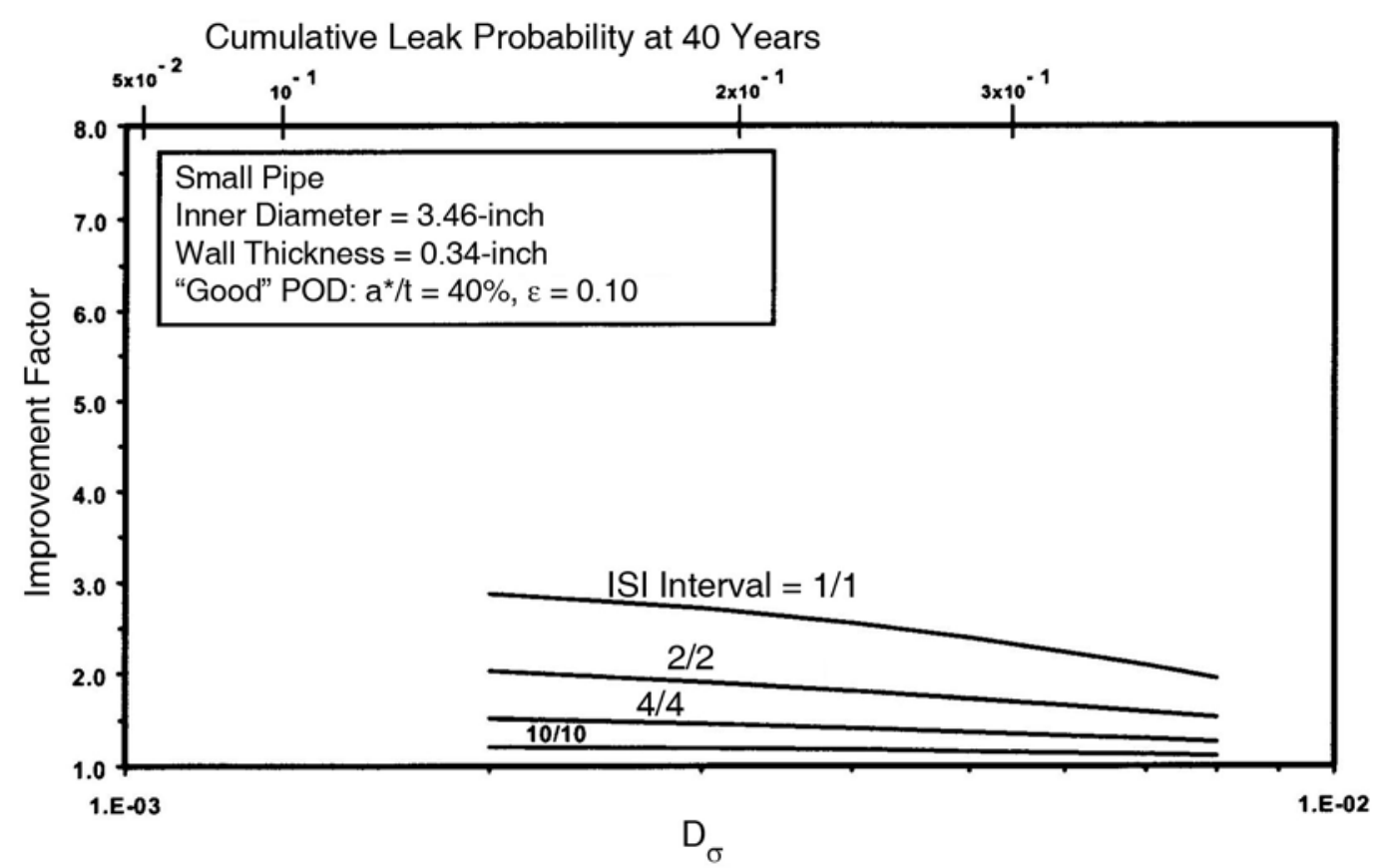

Figure 5.11a Improvement Factors versus $D_{\sigma}$ for "Good" POD Curve (Small Pipe Size and Various Inspection Intervals) $(1$ inch $=25.4 \mathrm{~mm})$

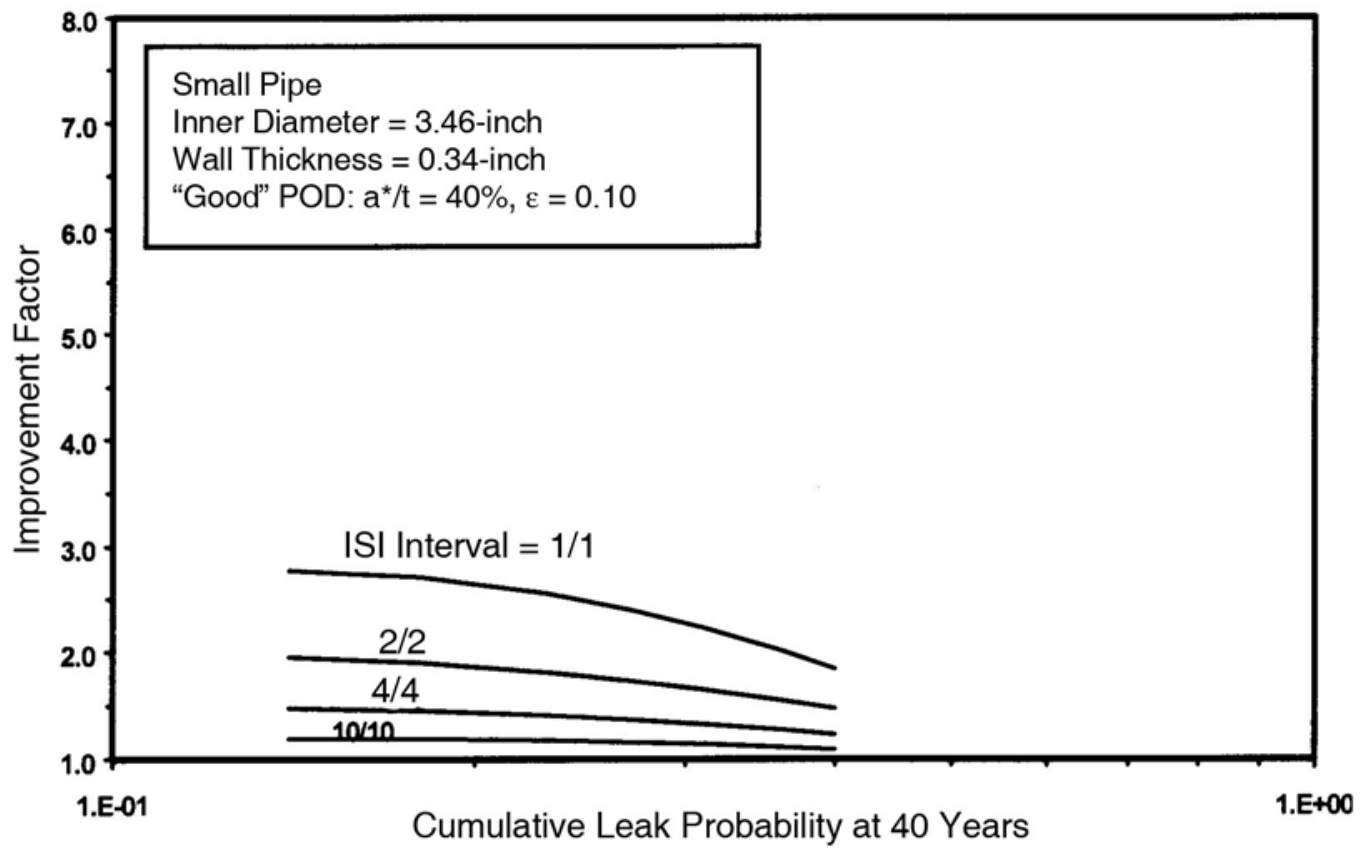

Figure 5.11b Improvement Factors versus Leak Probability for "Good" POD Curve (Small Pipe Size and Various Inspection Intervals $)(1$ inch $=25.4 \mathrm{~mm})$ 


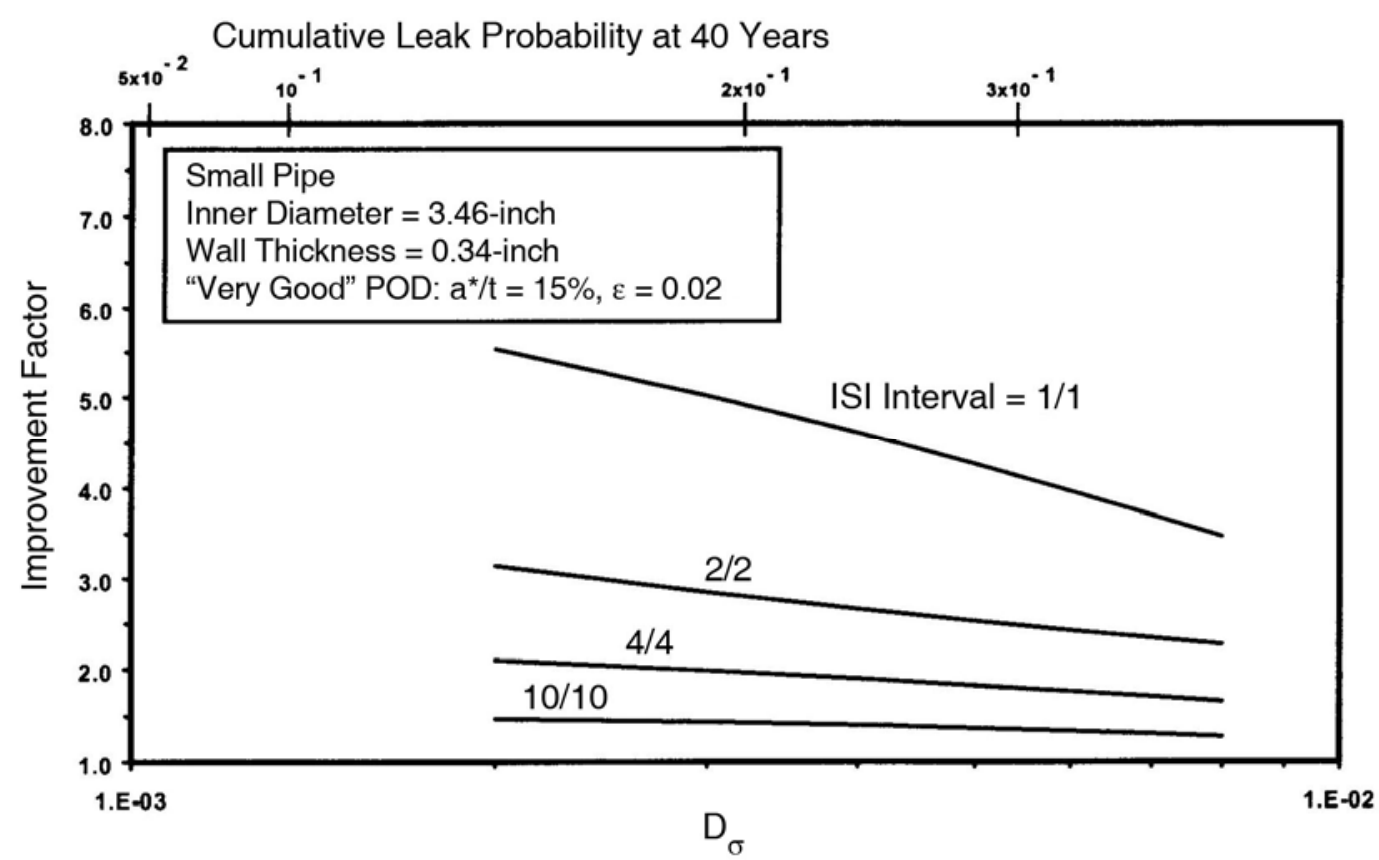

Figure 5.12a Improvement Factors versus $D_{\sigma}$ for "Very Good" POD Curve (Small Pipe Size and Various Inspection Intervals) $(1$ inch $=\mathbf{2 5 . 4} \mathbf{~ m m})$

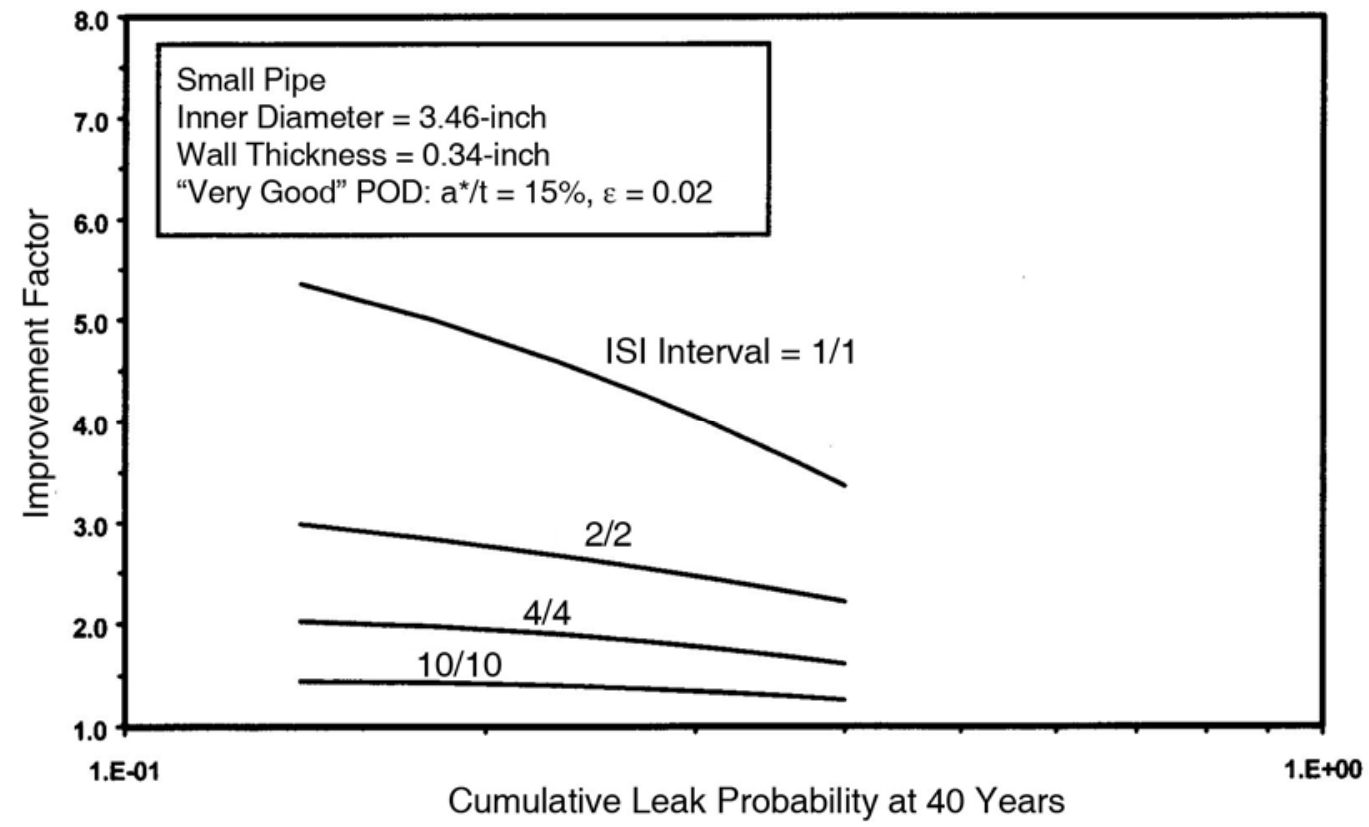

Figure 5.12b Improvement Factors versus Leak Probability for "Very Good" POD Curve (Small Pipe Size and Various Inspection Intervals) $(1$ inch $=25.4 \mathrm{~mm})$ 


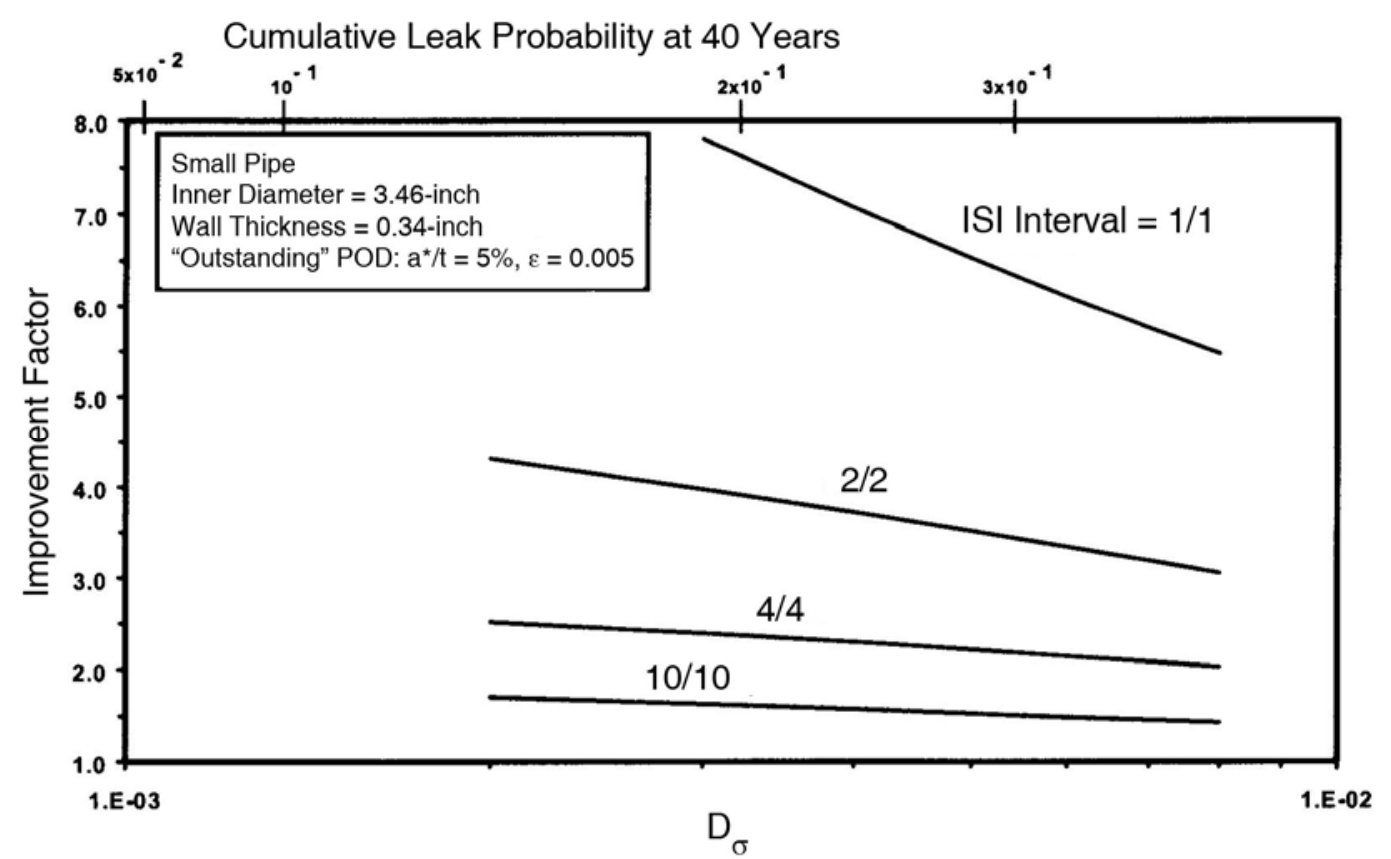

Figure 5.13a Improvement Factors versus $D_{\sigma}$ for "Outstanding” POD Curve (Small Pipe Size and Various Inspection Intervals) $(1$ inch $=25.4 \mathrm{~mm})$

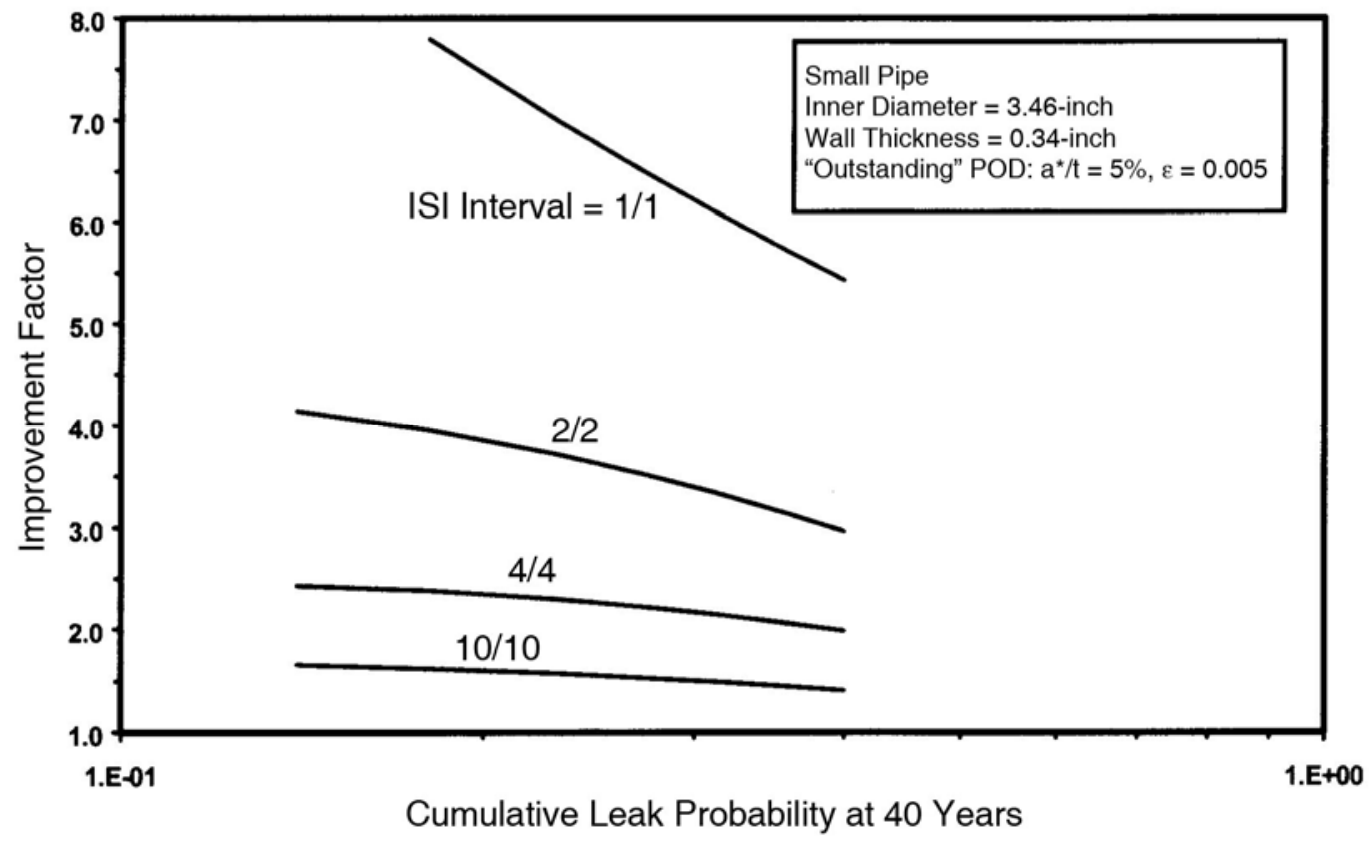

Figure 5.13b Improvement Factors versus Leak Probability for "Outstanding" POD Curve (Small Pipe Size and Various Inspection Intervals) $(1 \mathrm{inch}=\mathbf{2 5 . 4} \mathbf{~ m m})$ 


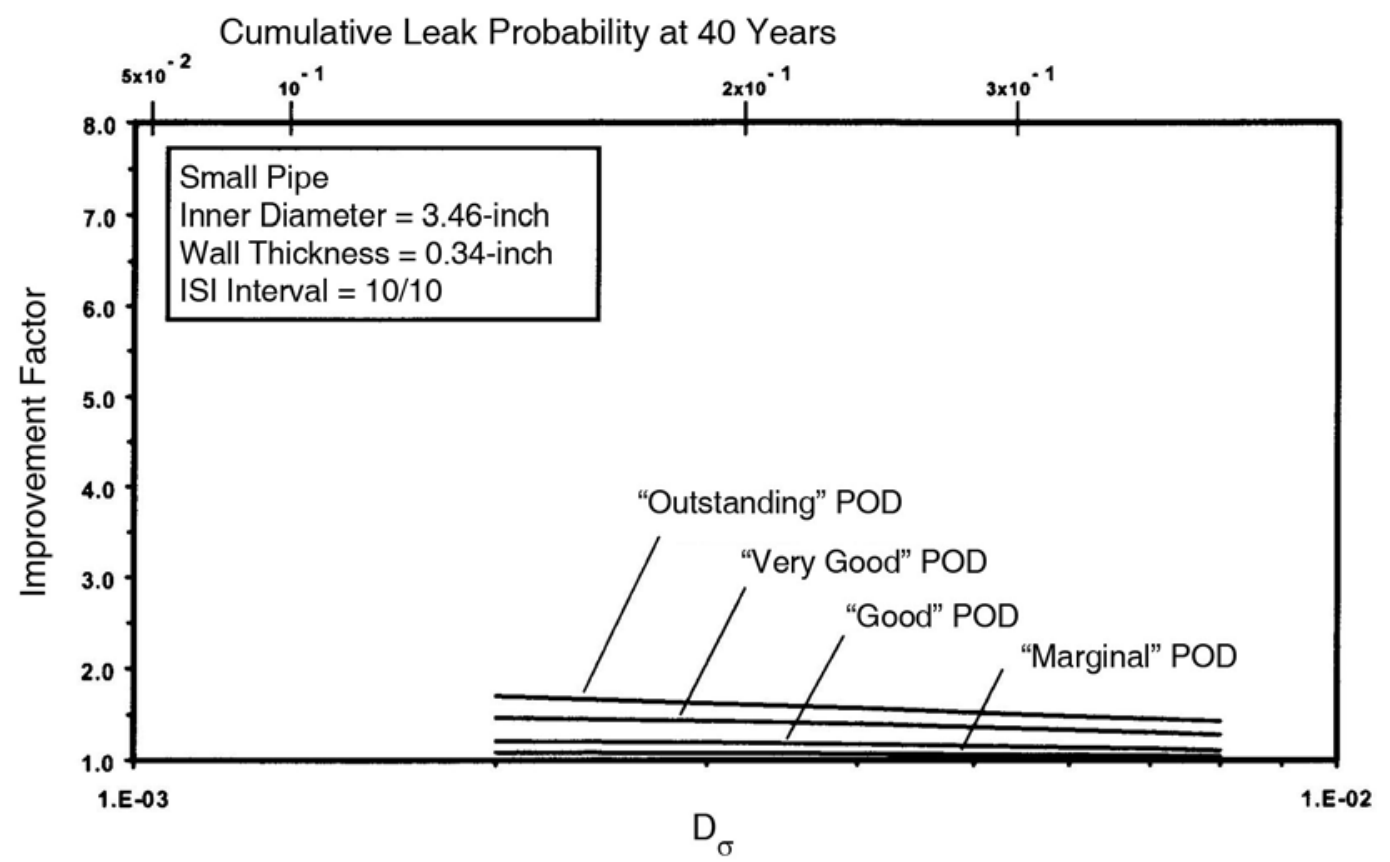

Figure 5.14a Improvement Factors versus $D_{\sigma}$ for 10-Year ISI Interval (Small Pipe Size and Various POD Curves) $(1$ inch $=25.4 \mathrm{~mm})$

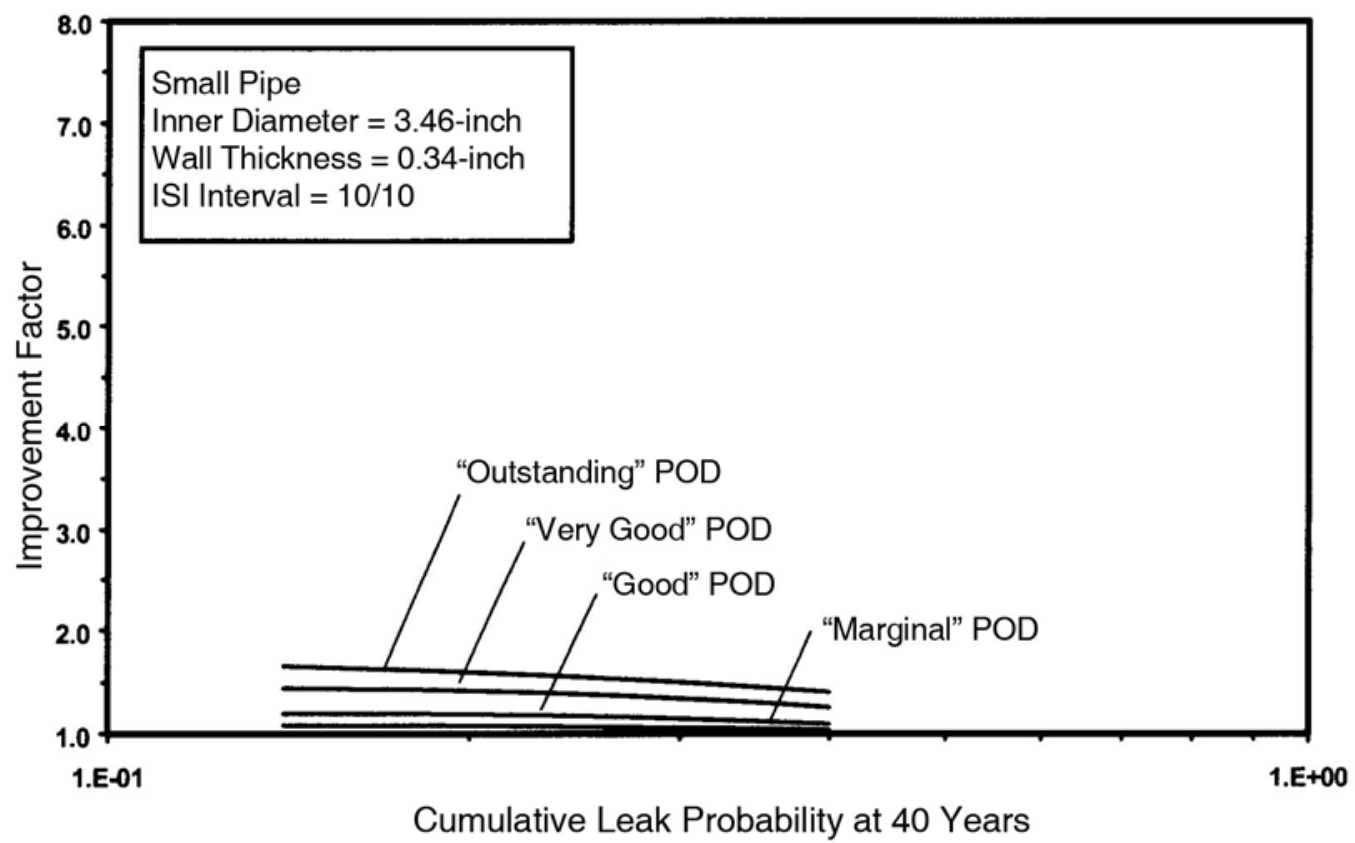

Figure 5.14b Improvement Factors versus Leak Probability for 10-Year ISI Interval (Small Pipe Size and Various POD Curves $)(1$ inch $=25.4 \mathrm{~mm})$ 


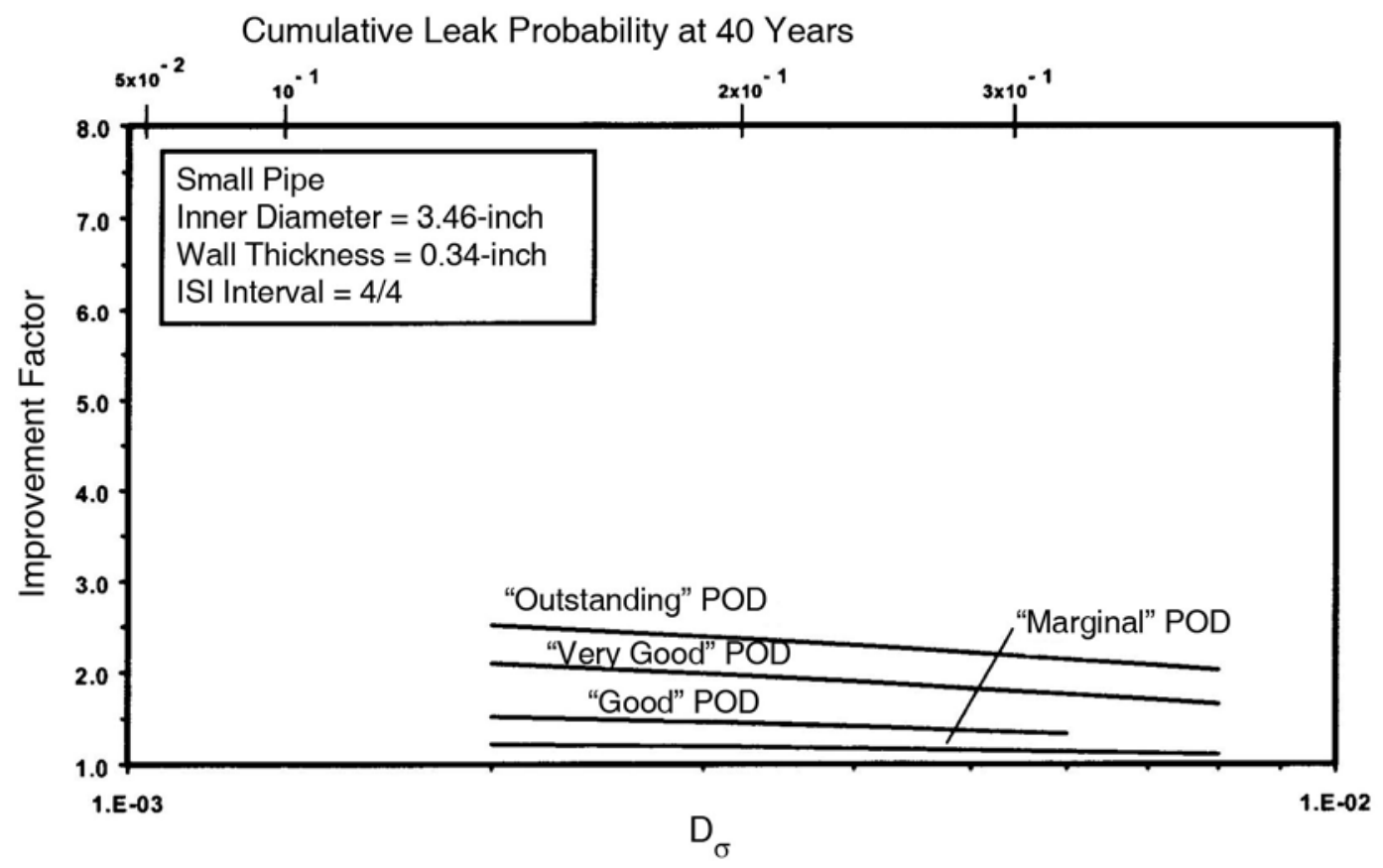

Figure 5.15a Improvement Factors versus $D_{\sigma}$ for 4-Year ISI Interval (Small Pipe Size and Various POD Curves) $(1$ inch $=\mathbf{2 5 . 4} \mathbf{~ m m})$

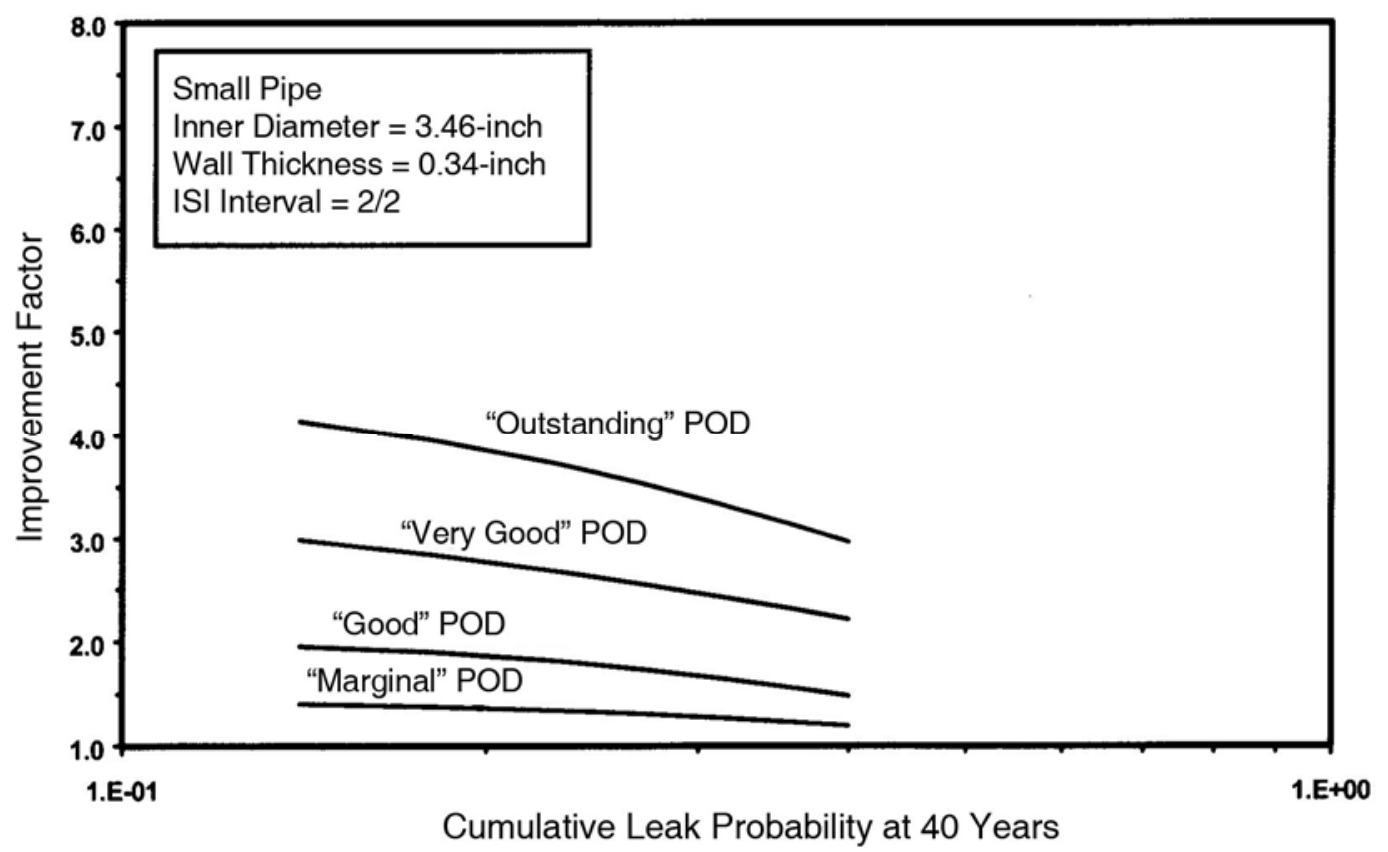

Figure 5.15b Improvement Factors versus Leak Probability for 4-Year ISI Interval (Small Pipe Size and Various POD Curves) $(1$ inch $=25.4 \mathrm{~mm})$ 


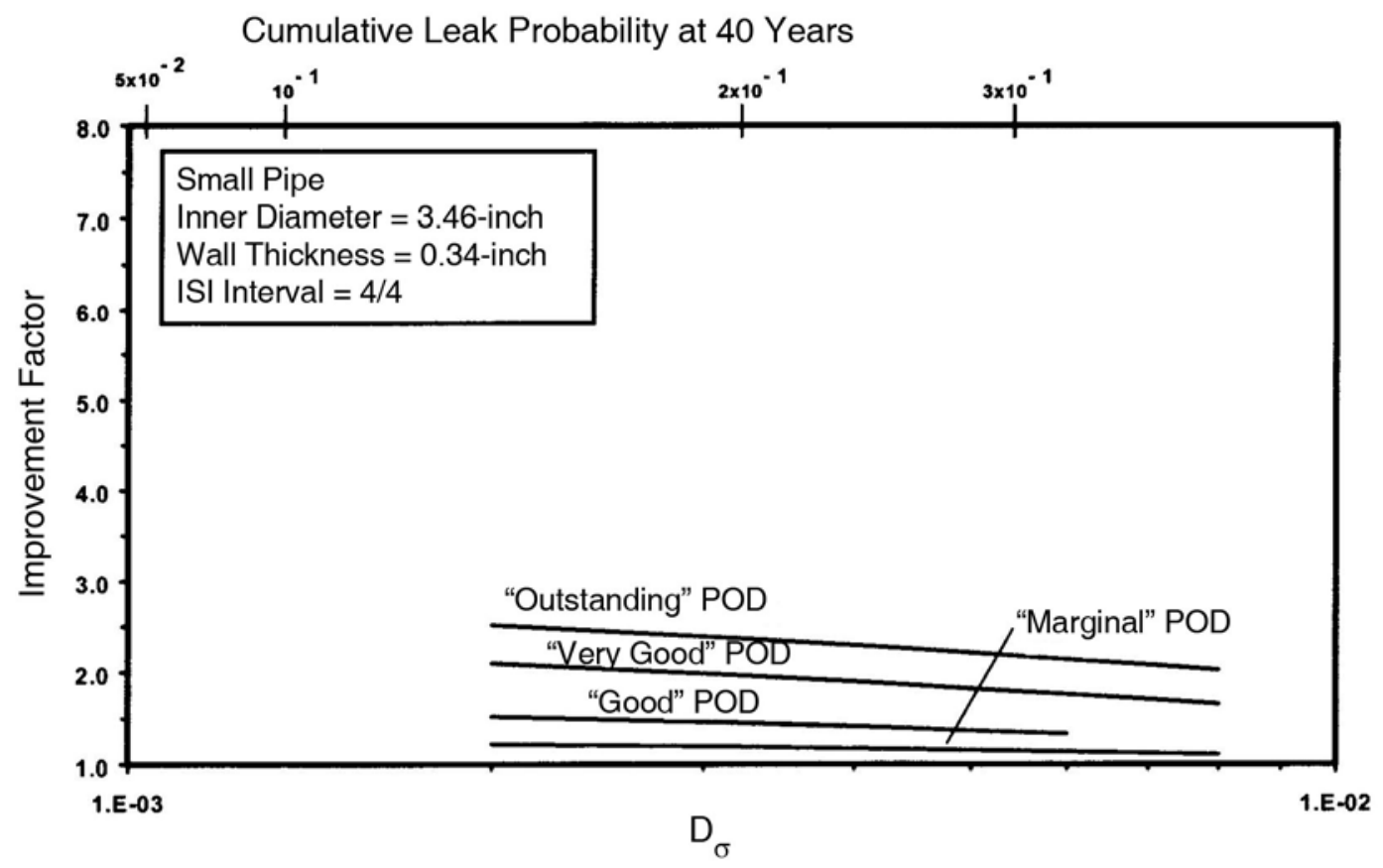

Figure 5.16a Improvement Factors versus $D_{\sigma}$ for 2-Year ISI Interval (Small Pipe Size and Various POD Curves) $(1$ inch $=\mathbf{2 5 . 4} \mathbf{~ m m})$

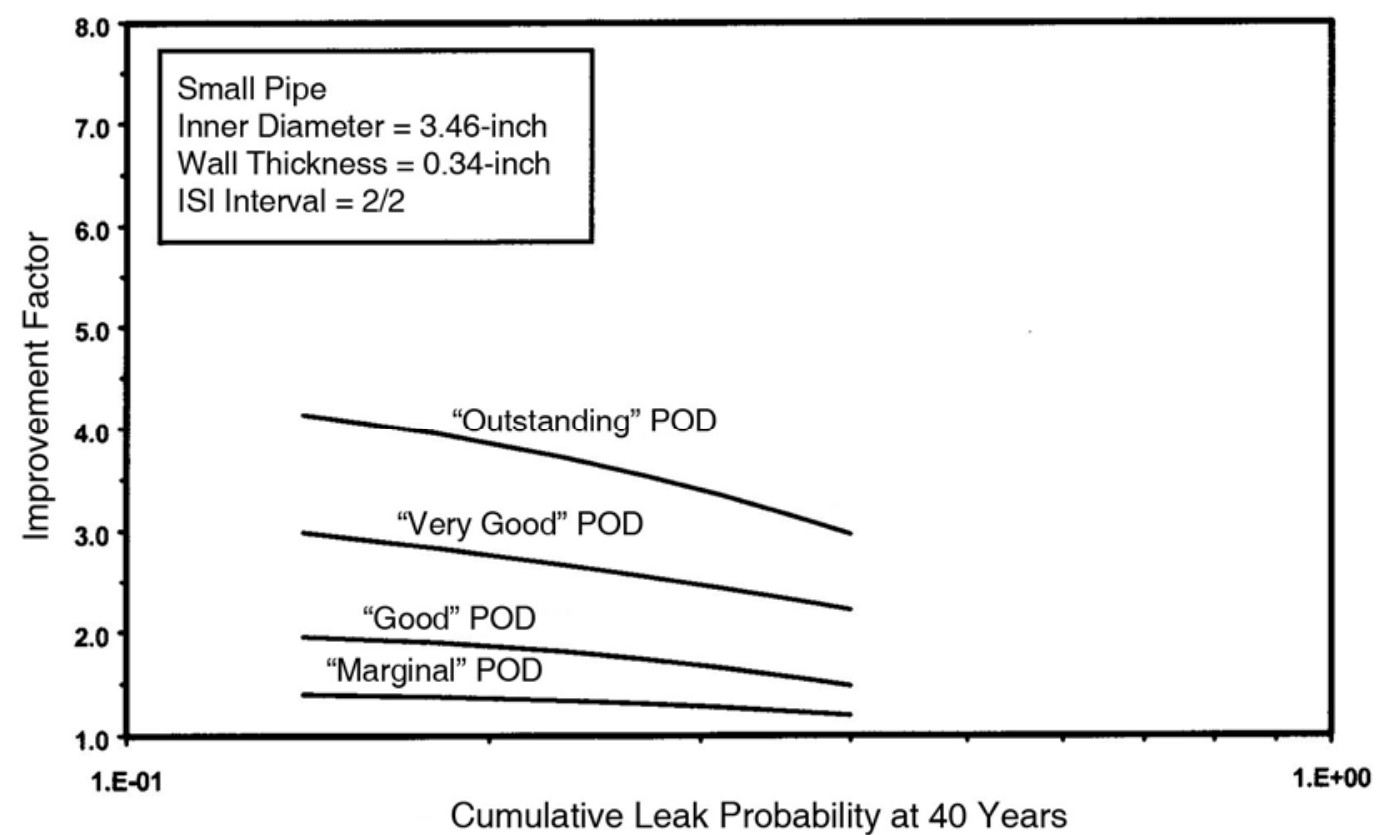

Figure 5.16b Improvement Factors versus Leak Probability for 2-Year ISI Interval (Small Pipe Size and Various POD Curves $)(1$ inch $=25.4 \mathrm{~mm})$ 


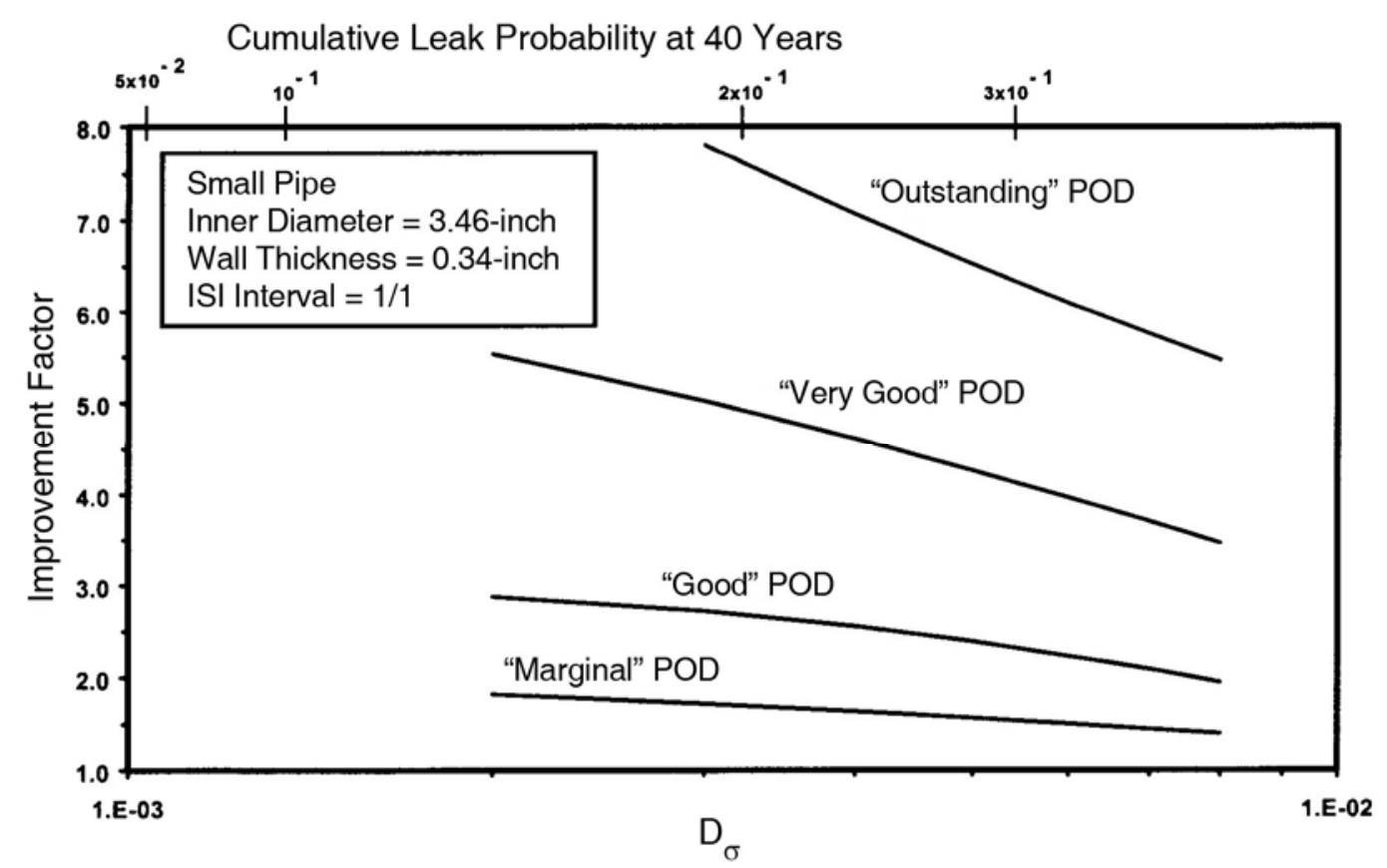

Figure 5.17a Improvement Factors versus $D_{\sigma}$ for 1-Year ISI Interval (Small Pipe Size and Various POD Curves) $(1$ inch $=25.4 \mathrm{~mm})$

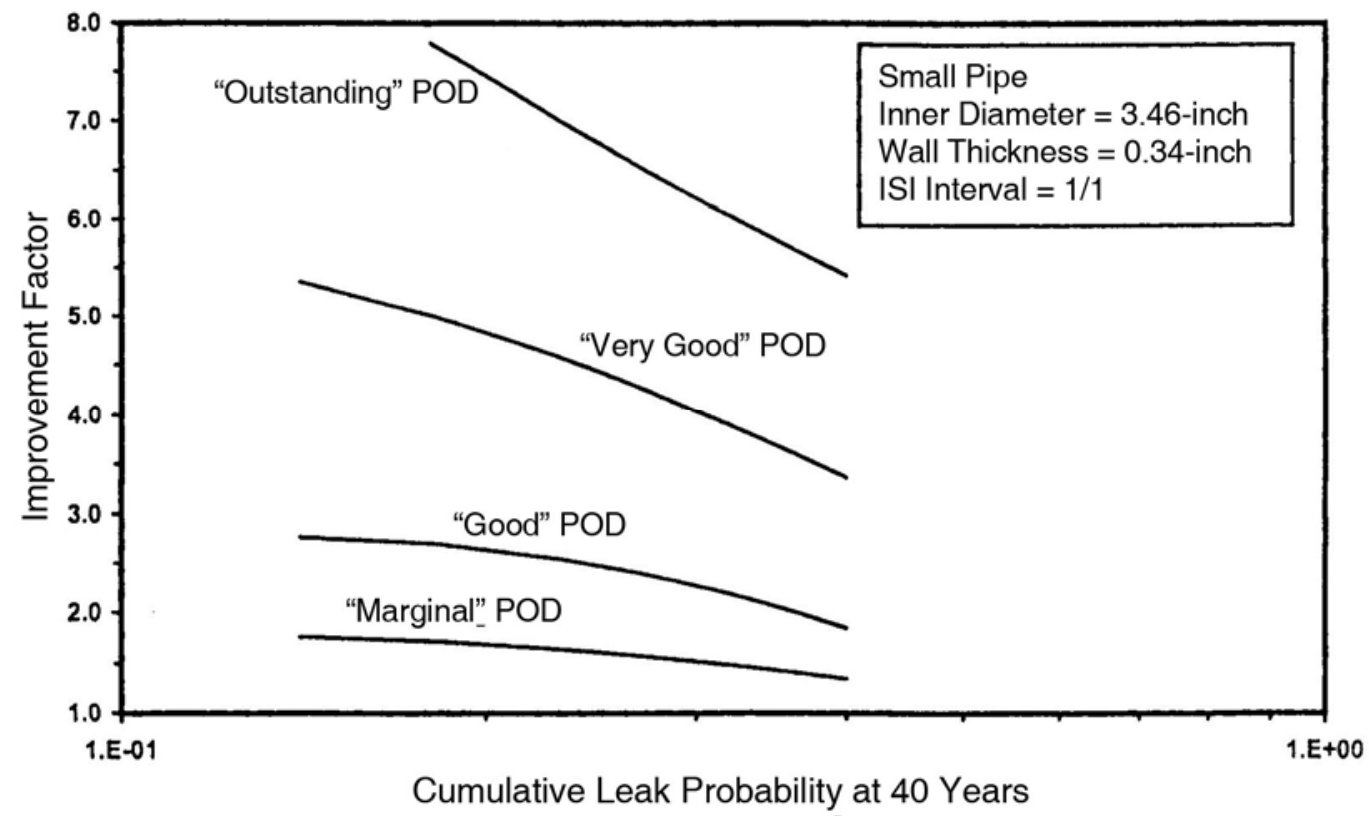

Figure 5.17b Improvement Factors versus Leak Probability for 1-Year ISI Interval (Small Pipe Size and Various POD Curves) $(1$ inch $=25.4 \mathrm{~mm})$ 


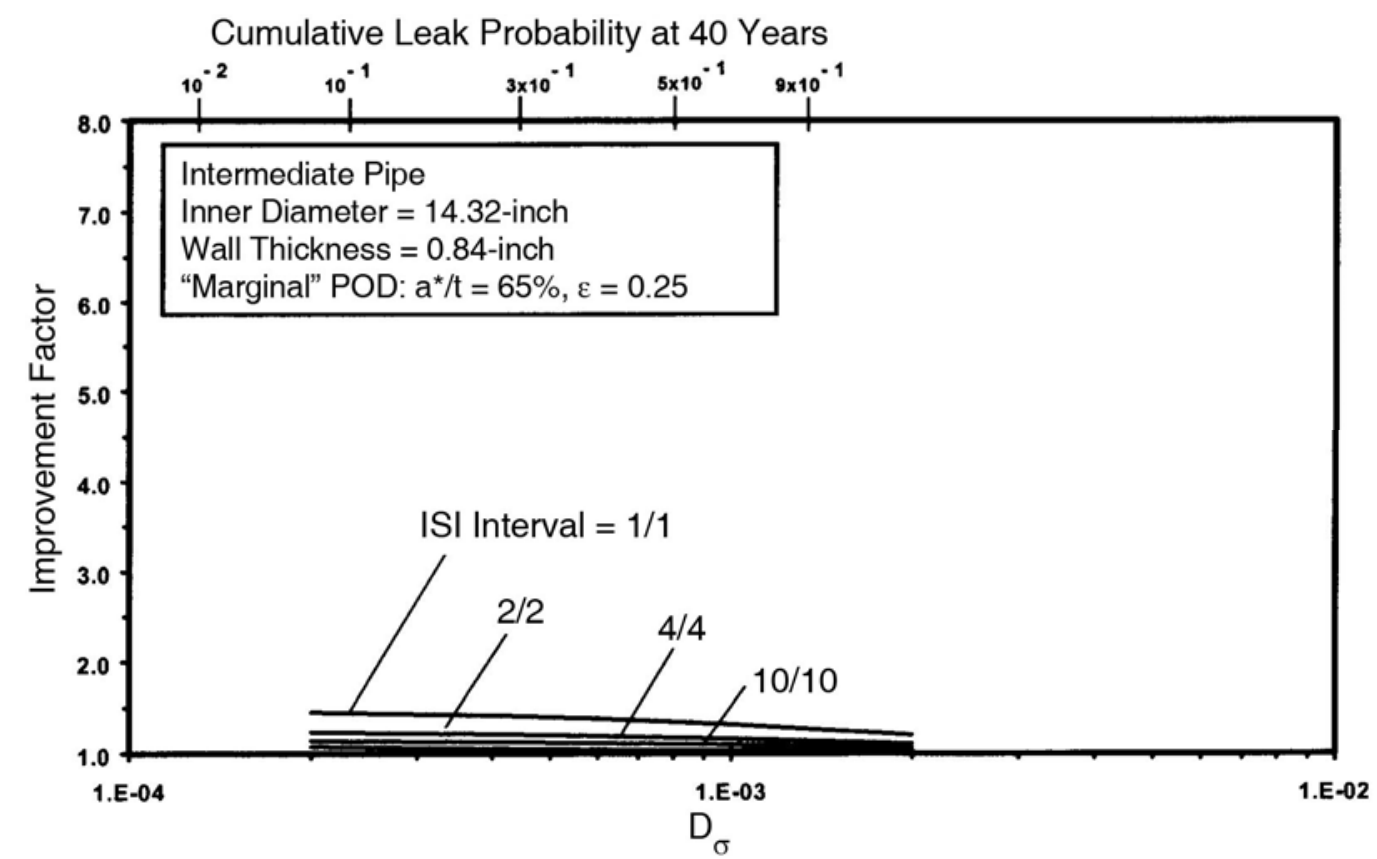

Figure 5.18a Improvement Factors versus $D_{\sigma}$ for "Marginal" POD Curve (Intermediate Pipe Size and Various Inspection Intervals) $(1$ inch $=25.4 \mathrm{~mm})$

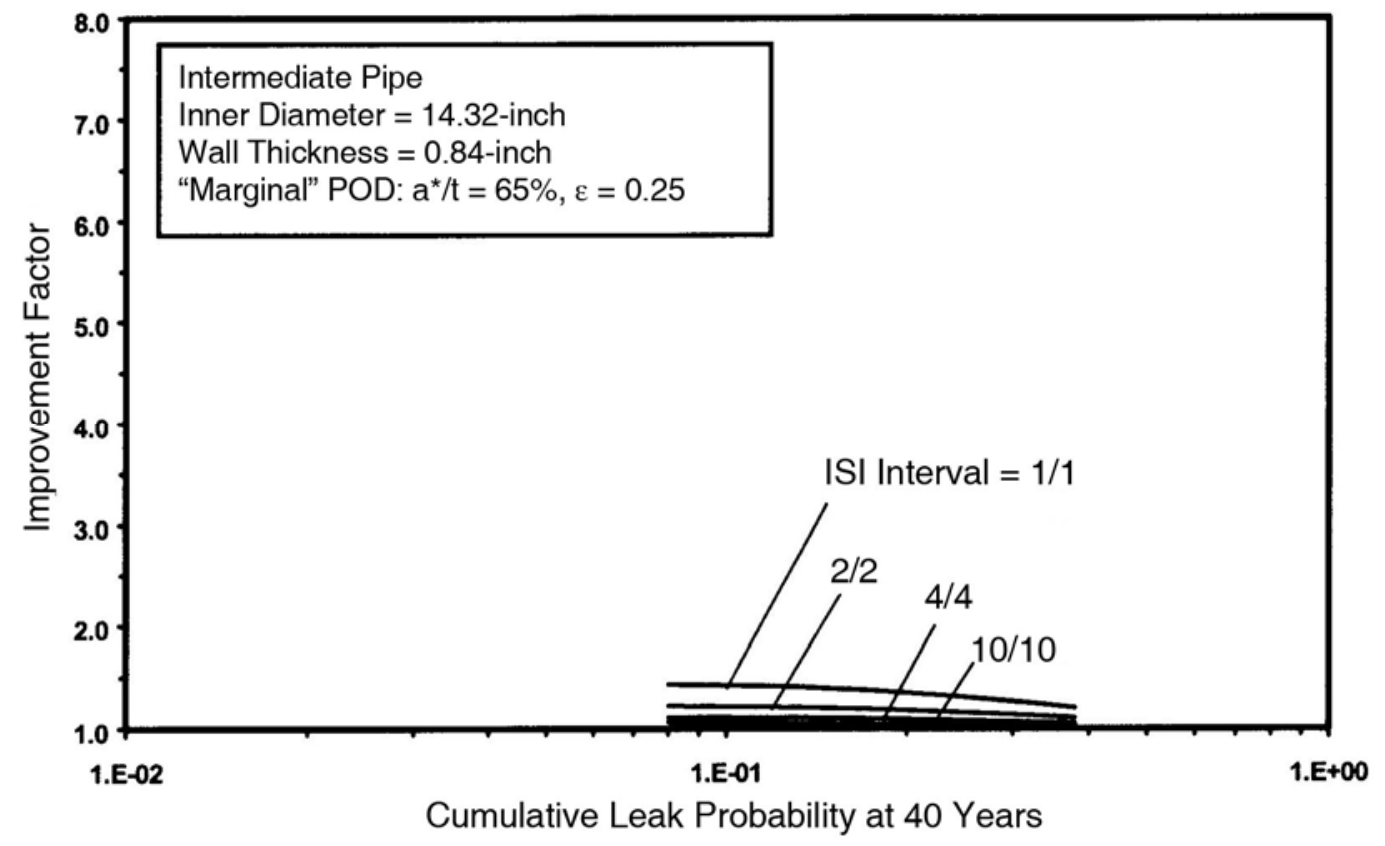

Figure 5.18b Improvement Factors versus Leak Probability for "Marginal" POD Curve (Intermediate Pipe Size and Various Inspection Intervals) $(1$ inch $=25.4 \mathbf{~ m m})$ 


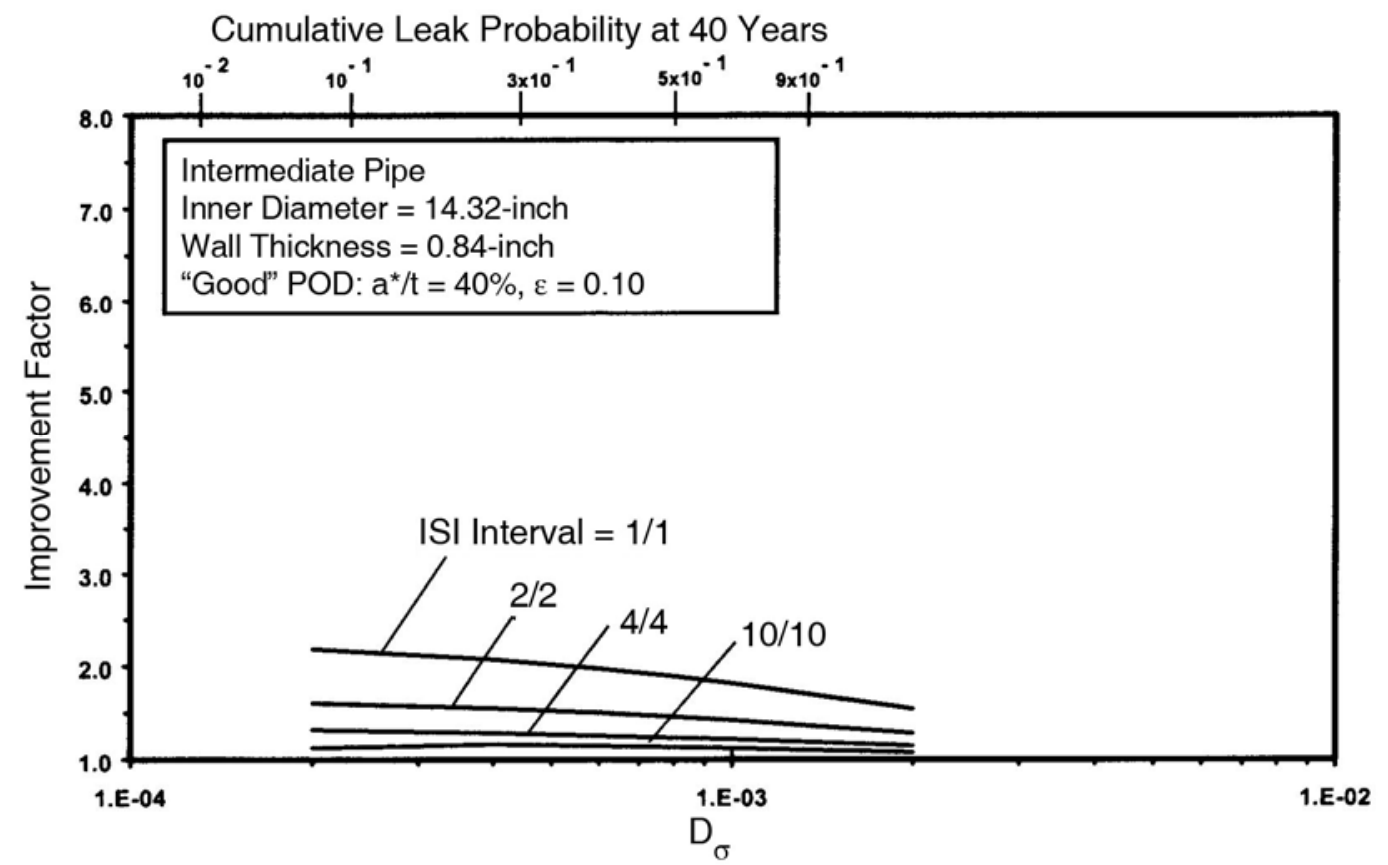

Figure 5.19a Improvement Factors versus $D_{\sigma}$ for "Good" POD Curve (Intermediate Pipe Size and Various Inspection Intervals) $(1$ inch $=25.4 \mathrm{~mm})$

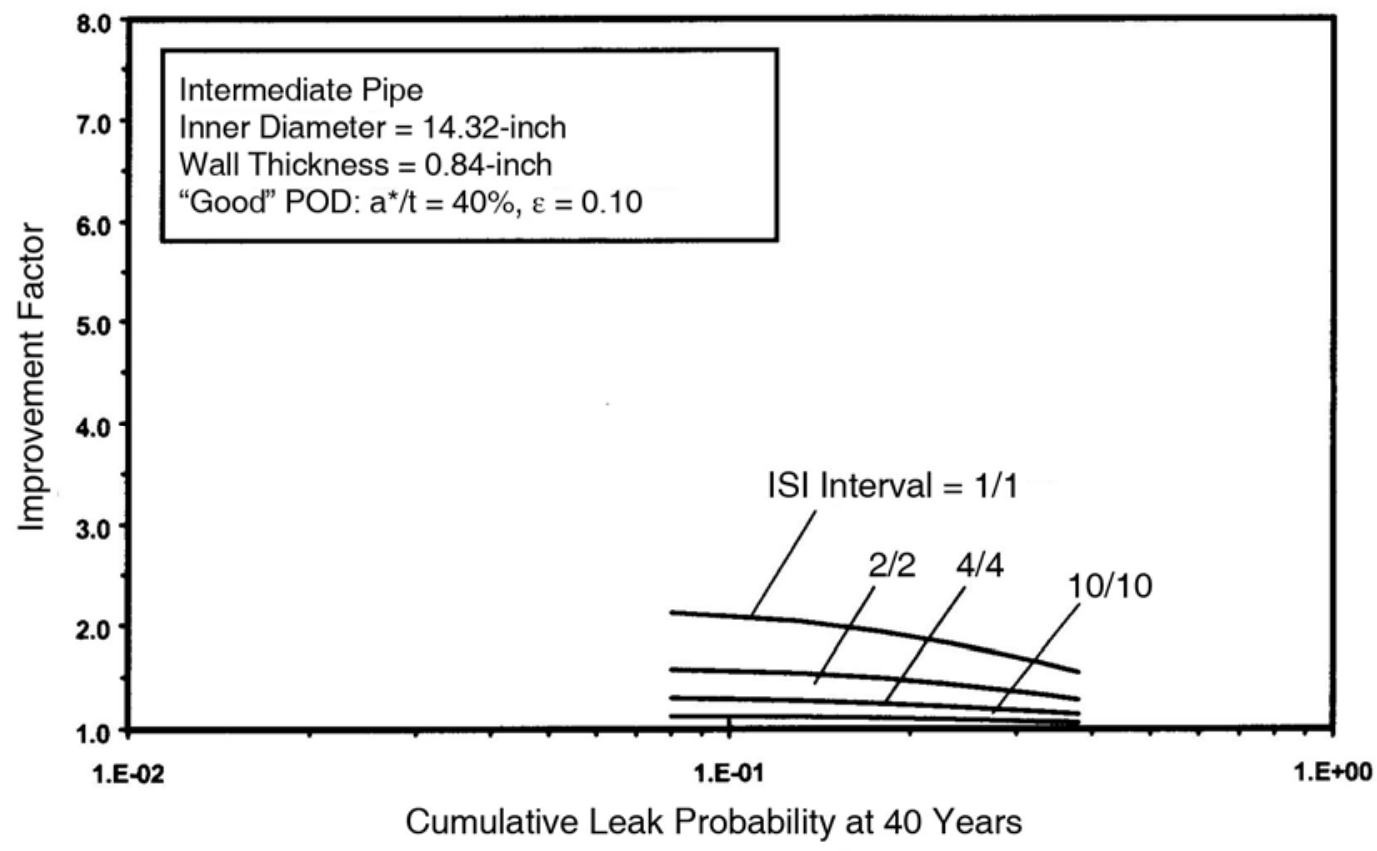

Figure 5.19b Improvement Factors versus Leak Probability for "Good" POD Curve (Intermediate Pipe Size and Various Inspection Intervals) $(1$ inch $=25.4 \mathrm{~mm}$ ) 


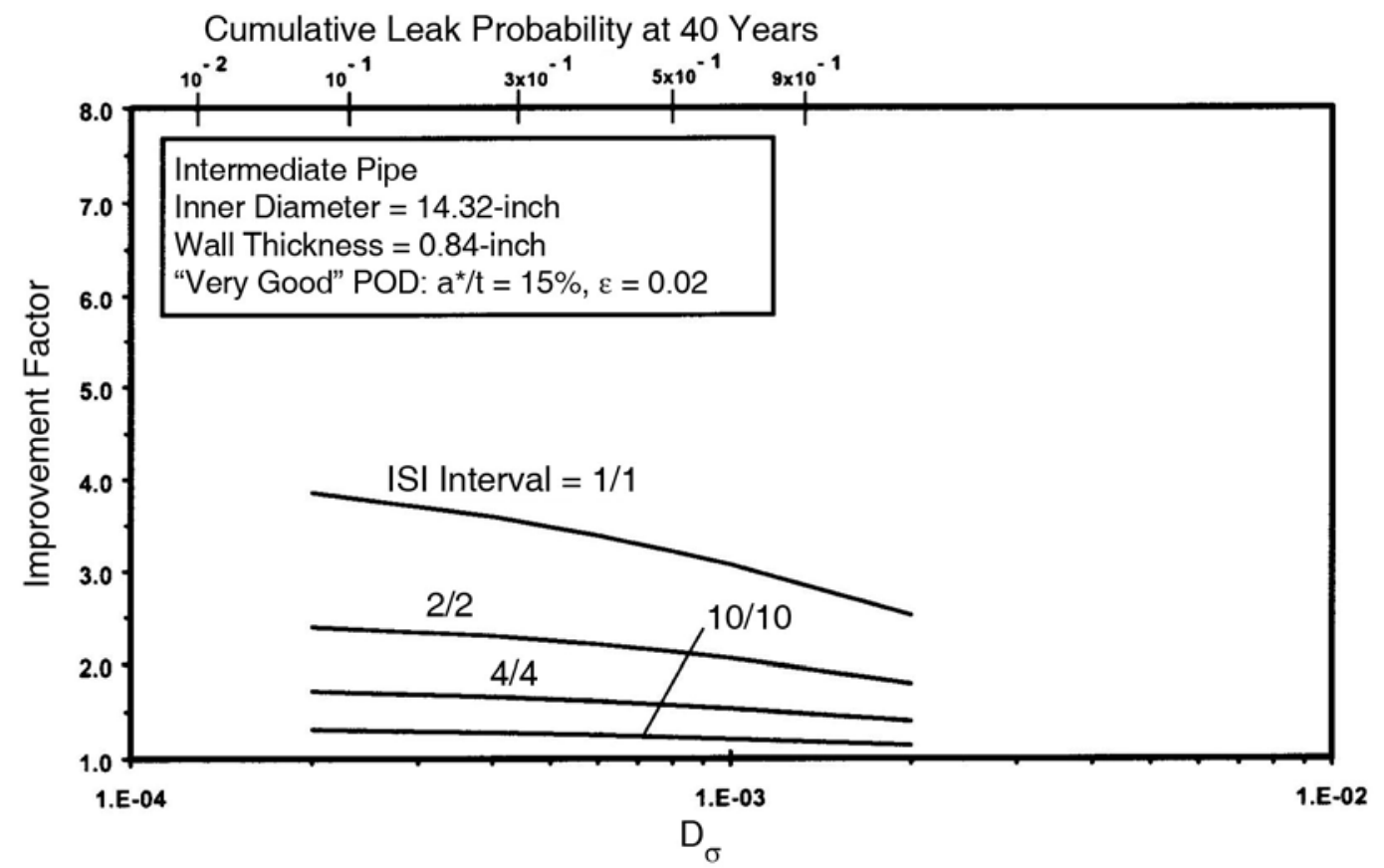

Figure 5.20a Improvement Factors versus $D_{\sigma}$ for "Very Good" POD Curve (Intermediate Pipe Size and Various Inspection Intervals) $(1$ inch $=25.4 \mathrm{~mm})$

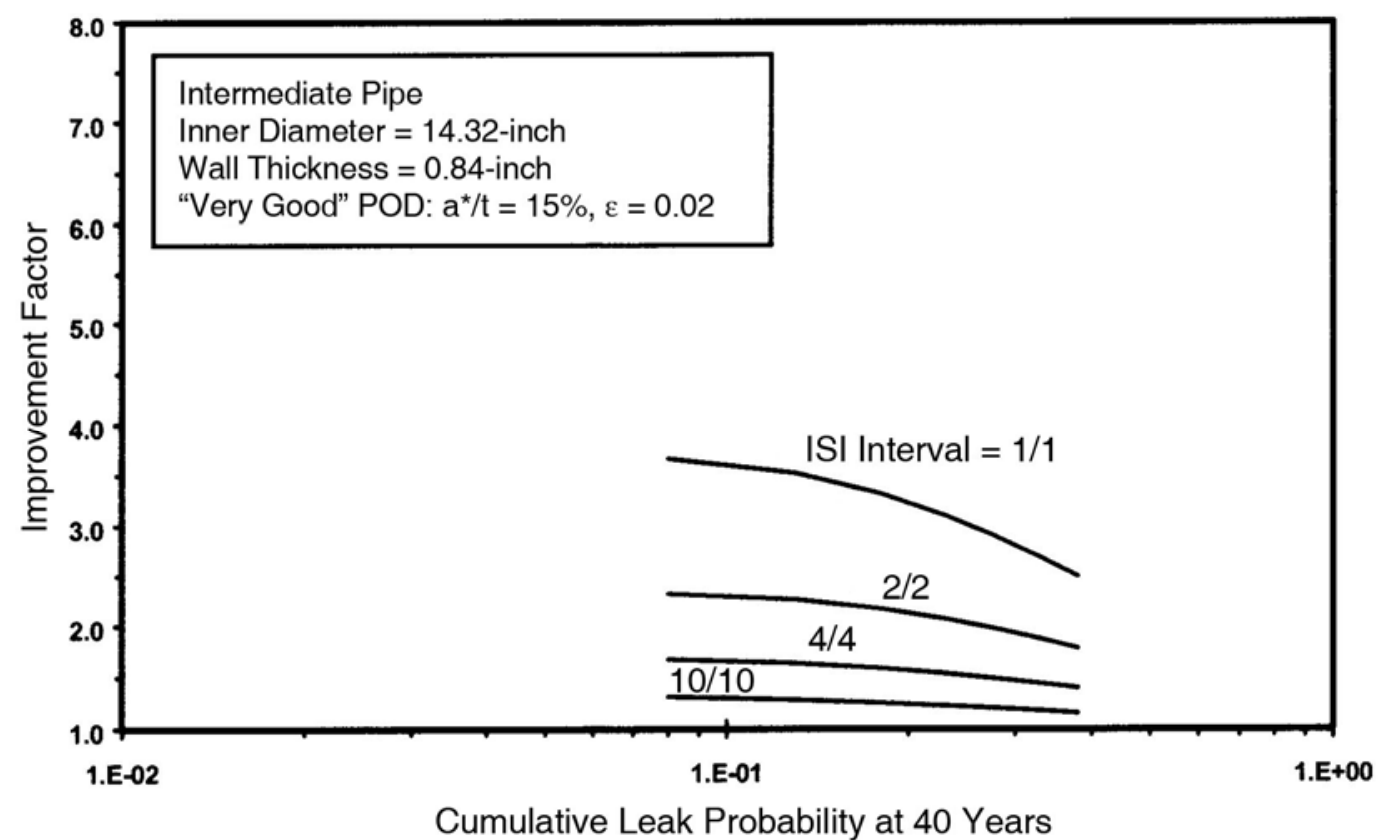

Figure 5.20b Improvement Factors versus Leak Probability for "Very Good" POD Curve (Intermediate Pipe Size and Various Inspection Intervals) $(1 \mathrm{inch}=25.4 \mathrm{~mm})$ 


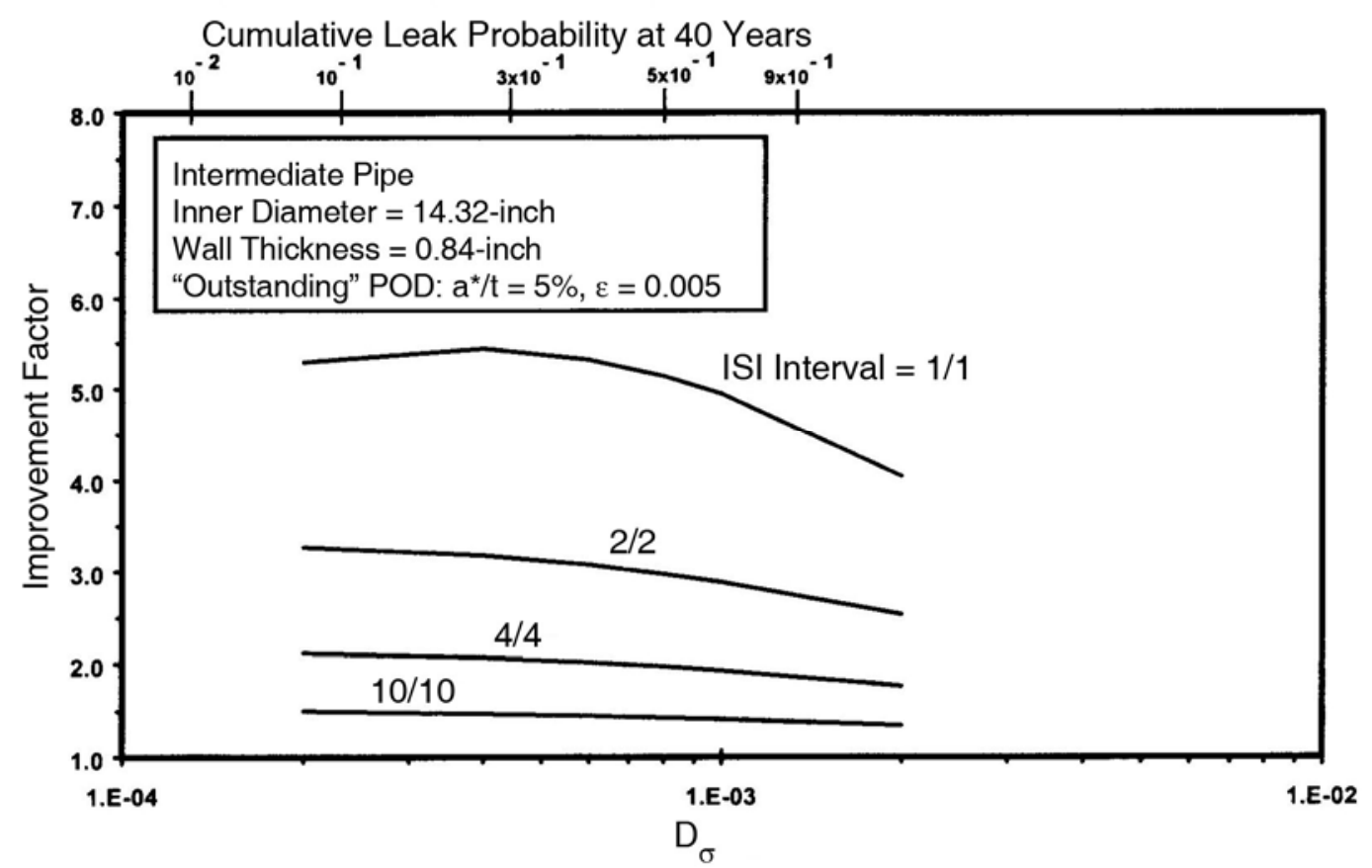

Figure 5.21a Improvement Factors versus $D_{\sigma}$ for "Outstanding" POD Curve (Intermediate Pipe Size and Various Inspection Intervals) $(1$ inch $=25.4 \mathbf{~ m m})$

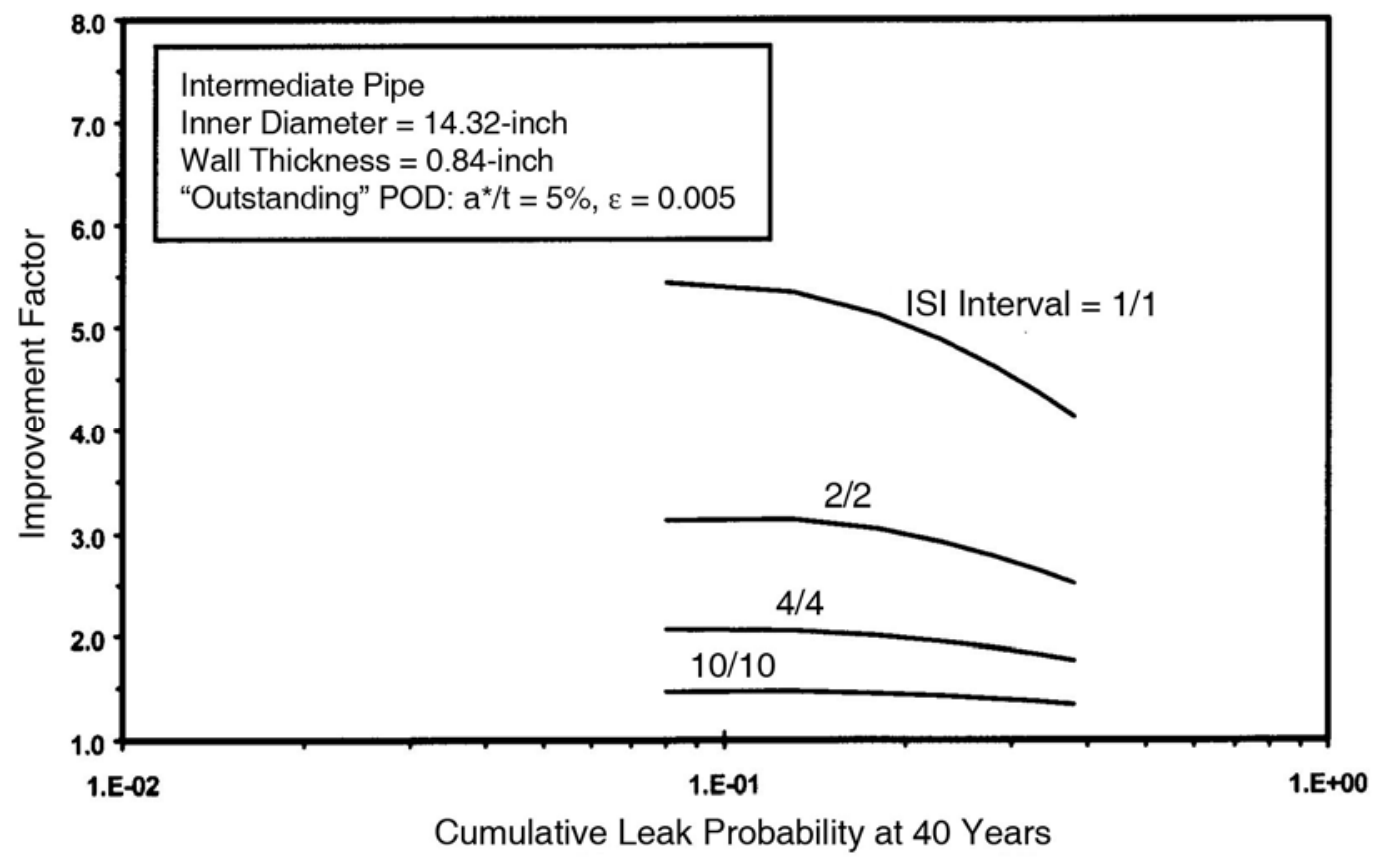

Figure 5.21b Improvement Factors versus Leak Probability for "Outstanding” POD Curve (Intermediate Pipe Size and Various Inspection Intervals) $(1$ inch $=25.4 \mathrm{~mm})$ 


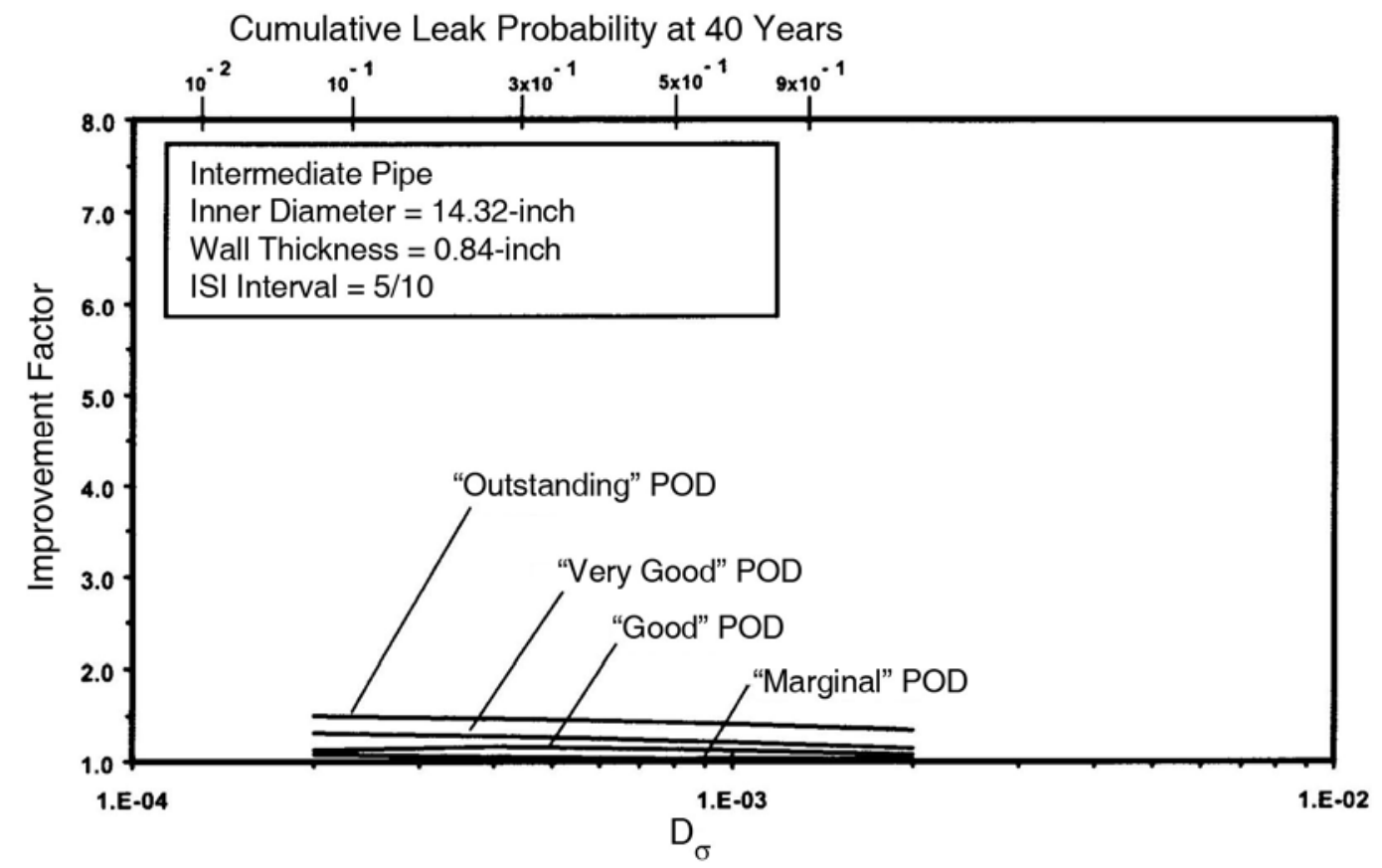

Figure 5.22a Improvement Factors versus $D_{\sigma}$ for 10 -Year ISI Interval (Intermediate Pipe Size and Various POD Curves) $(1$ inch $=25.4 \mathrm{~mm})$

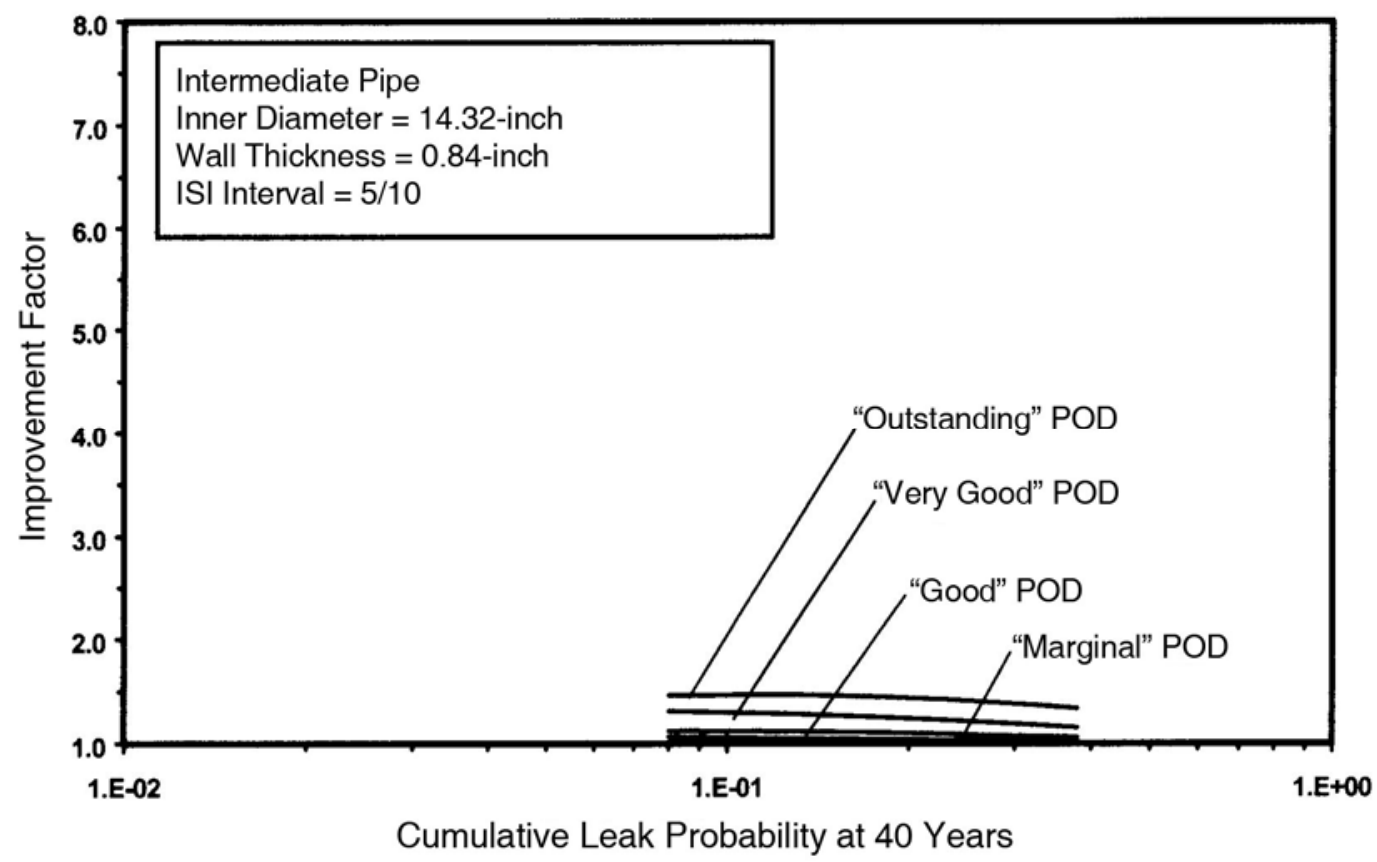

Figure 5.22b Improvement Factors versus Leak Probability for 10-Year ISI Interval (Intermediate Pipe Size and Various POD Curves) $(1$ inch $=25.4$ mm) 


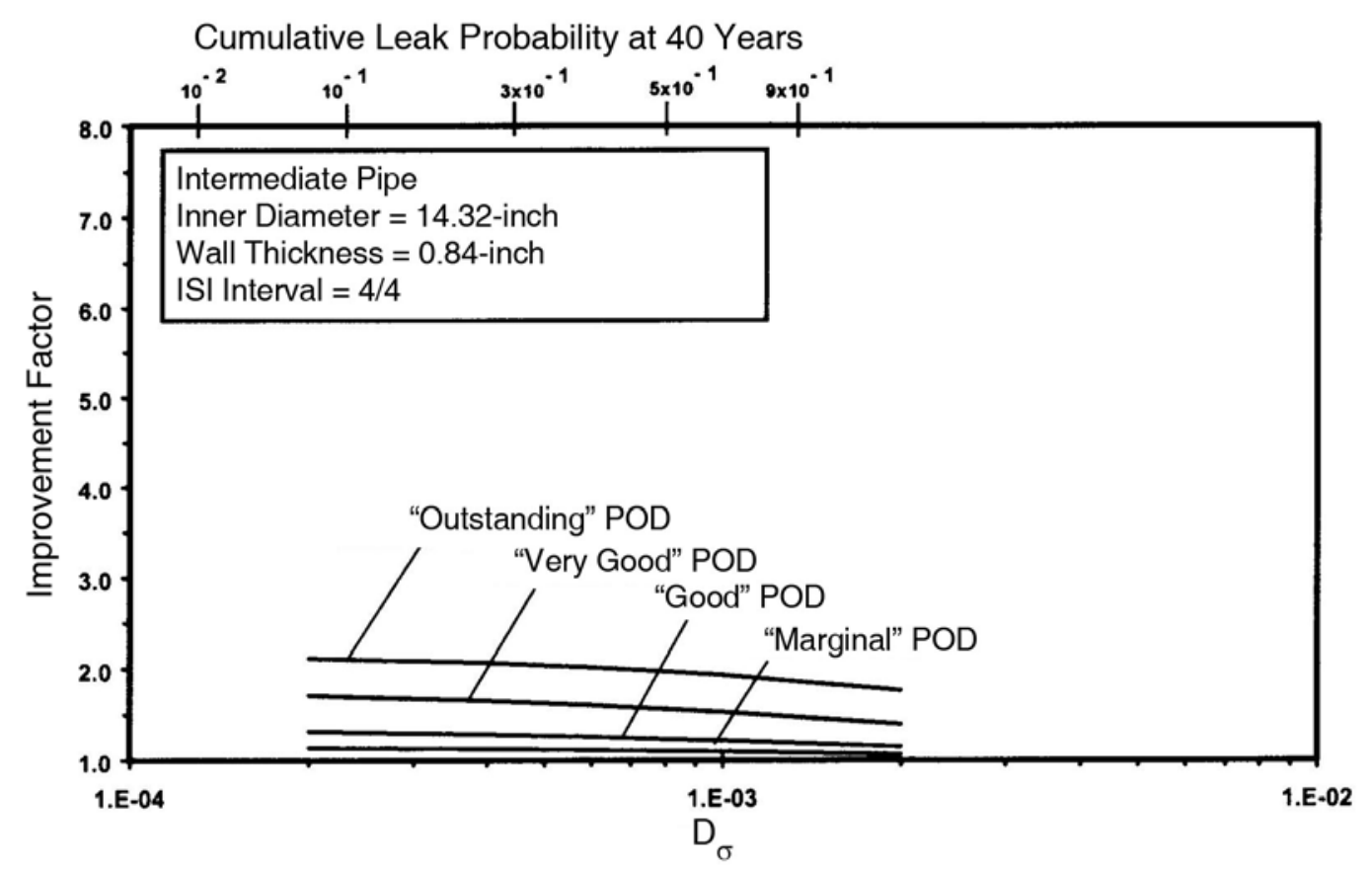

Figure 5.23a Improvement Factors versus $D_{\sigma}$ for 4-Year ISI Interval (Intermediate Pipe Size and Various POD Curves) $(1$ inch $=25.4 \mathrm{~mm})$

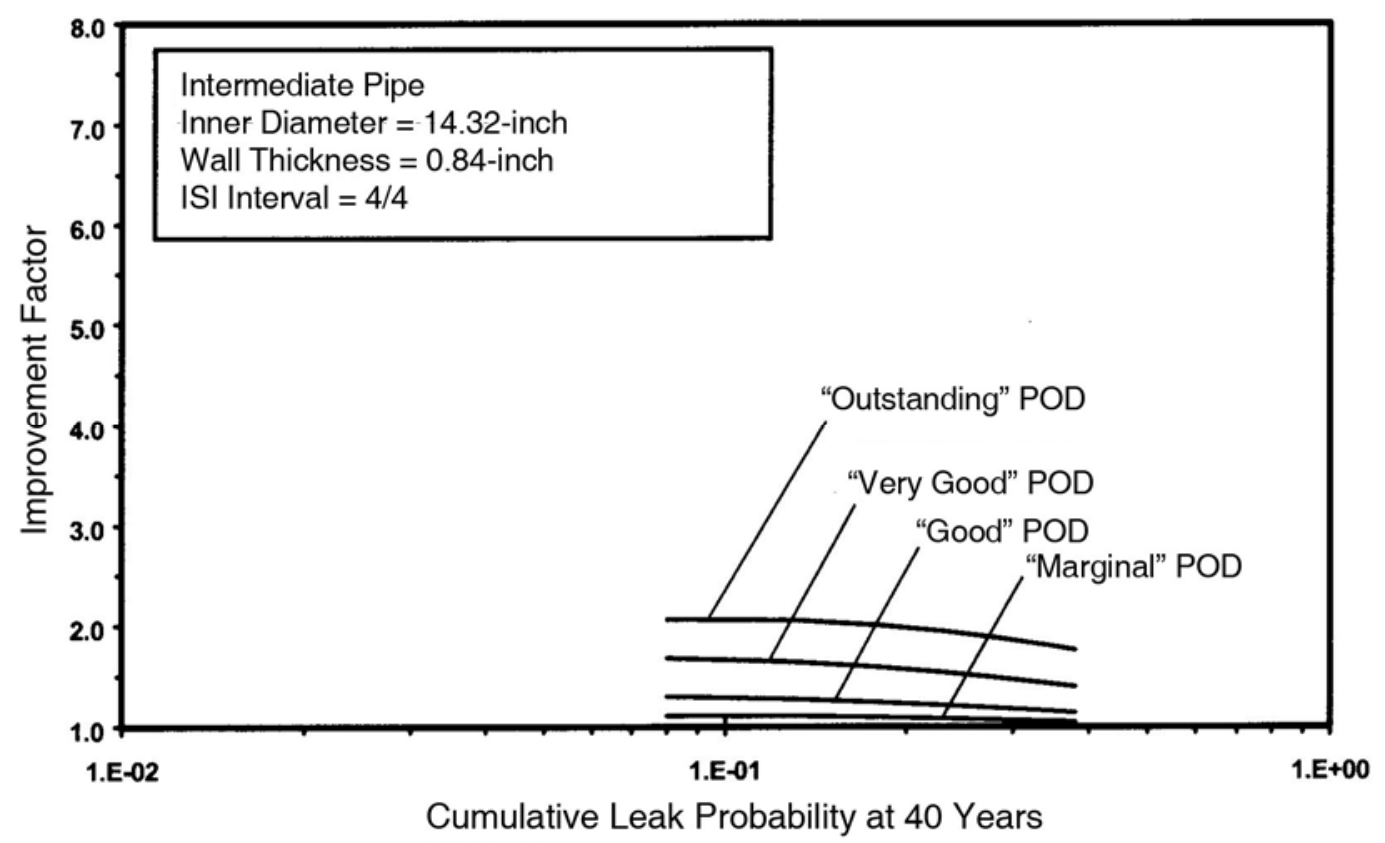

Figure 5.23b Improvement Factors versus Leak Probability for 4-Year ISI Interval (Intermediate Pipe Size and Various POD Curves) $(1 \mathrm{inch}=25.4 \mathrm{~mm})$ 


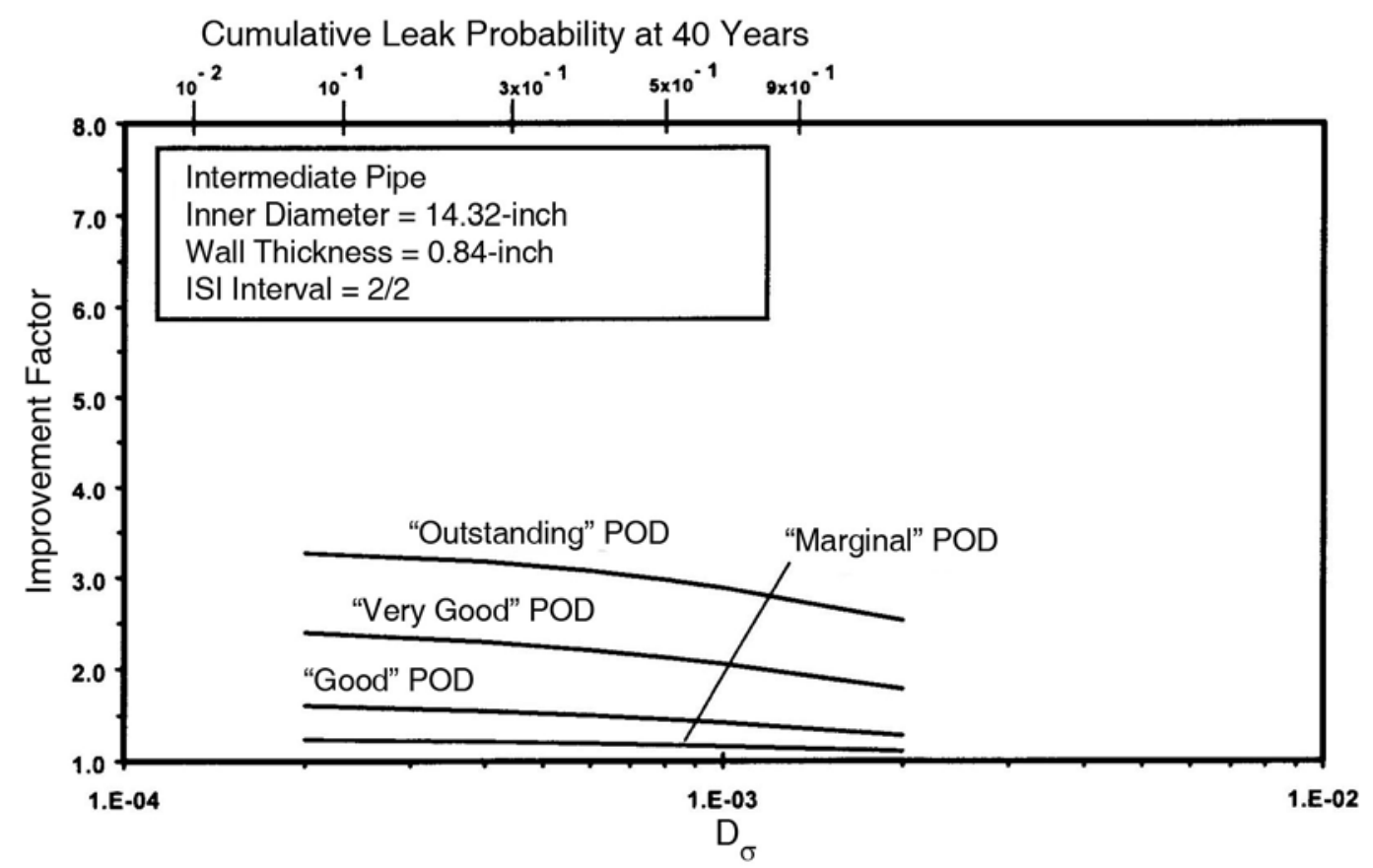

Figure 5.24a Improvement Factors versus $D_{\sigma}$ for 2-Year ISI Interval (Intermediate Pipe Size and Various POD Curves) $(1$ inch $=25.4 \mathrm{~mm})$

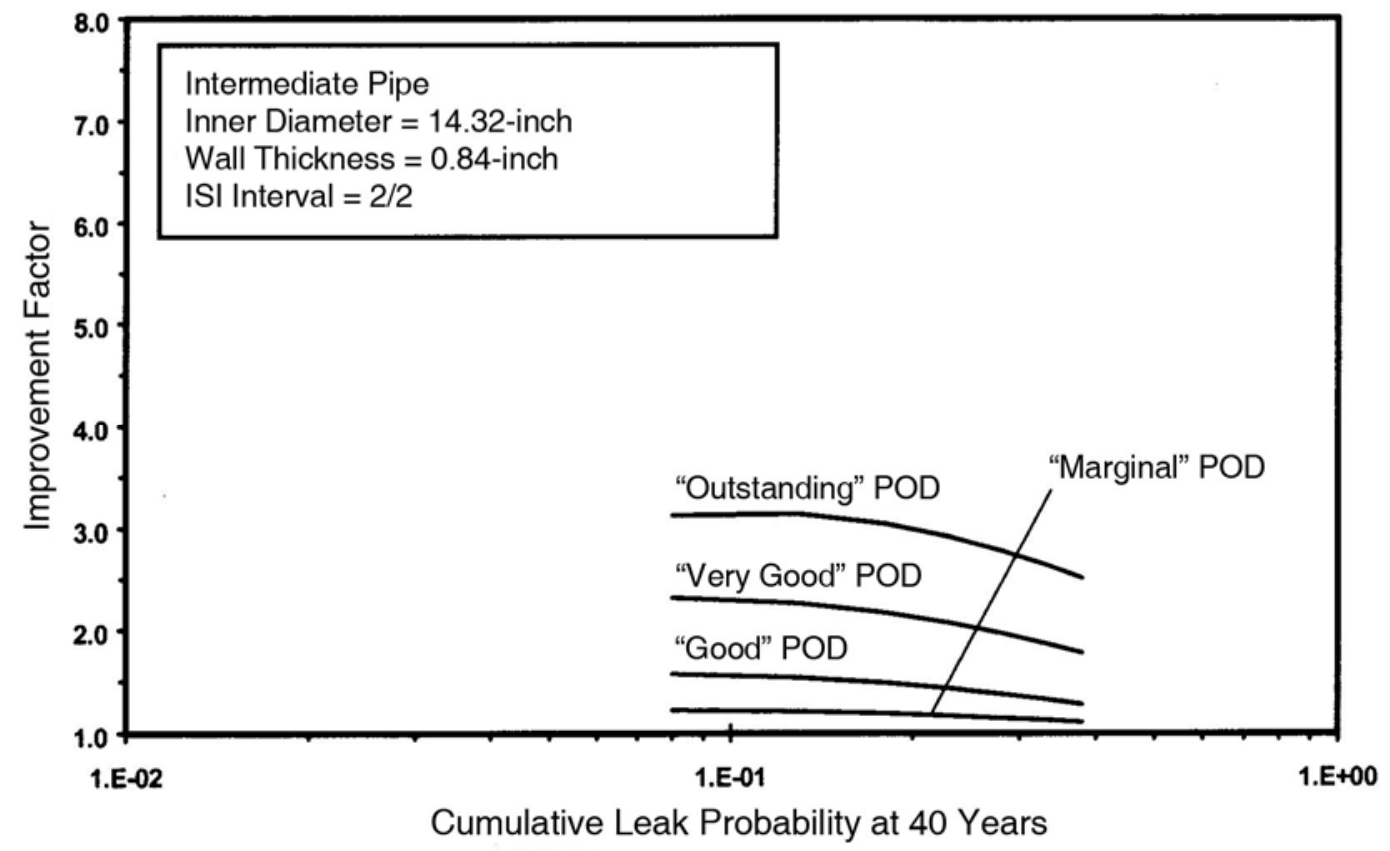

Figure 5.24b Improvement Factors versus Leak Probability for 2-Year ISI Interval (Intermediate Pipe Size and Various POD Curves) $(1$ inch $=25.4 \mathrm{~mm})$ 


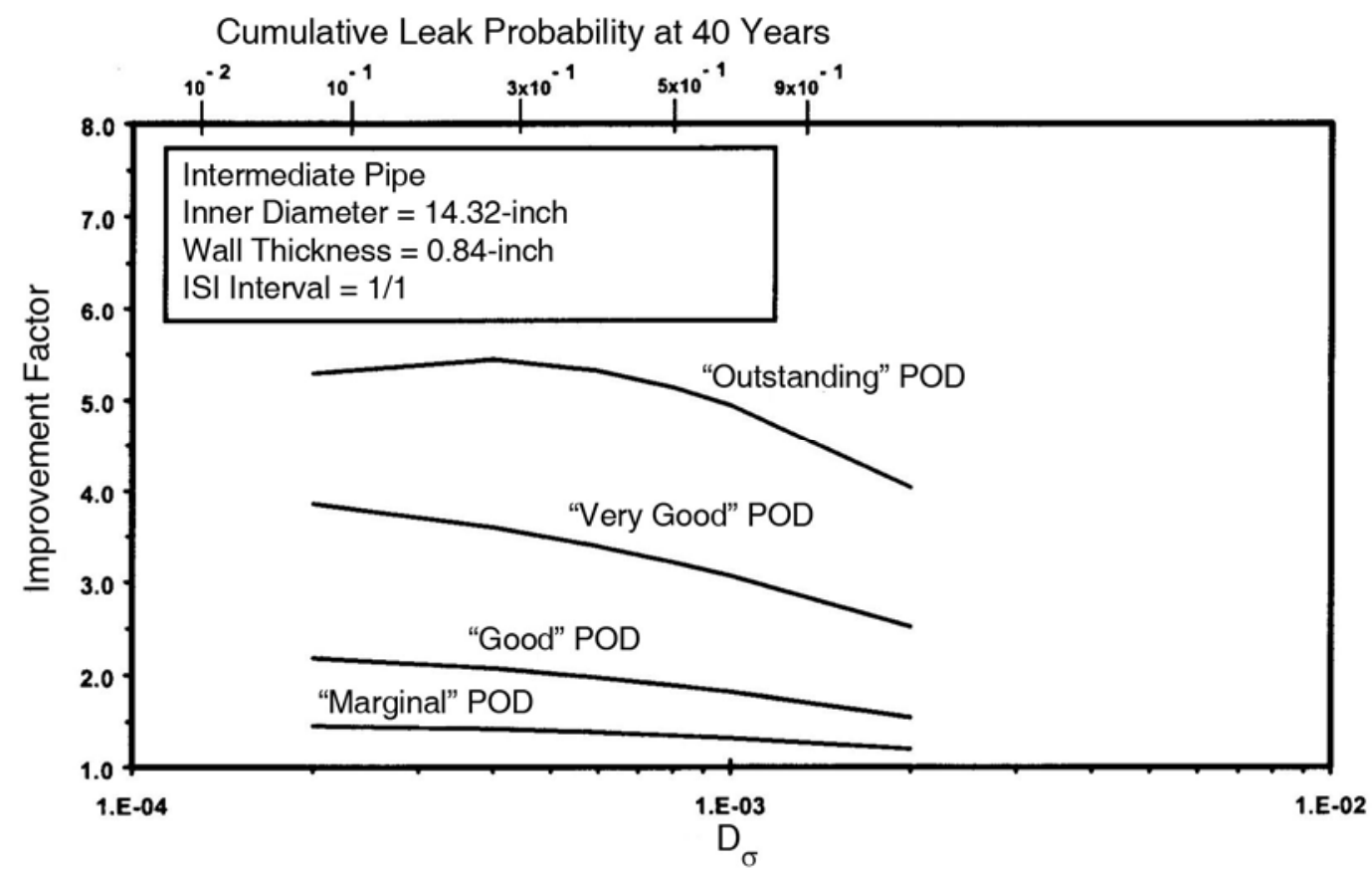

Figure 5.25a Improvement Factors versus $D_{\sigma}$ for 1-Year ISI Interval (Intermediate Pipe Size and Various POD Curves) $(1$ inch $=25.4 \mathrm{~mm})$

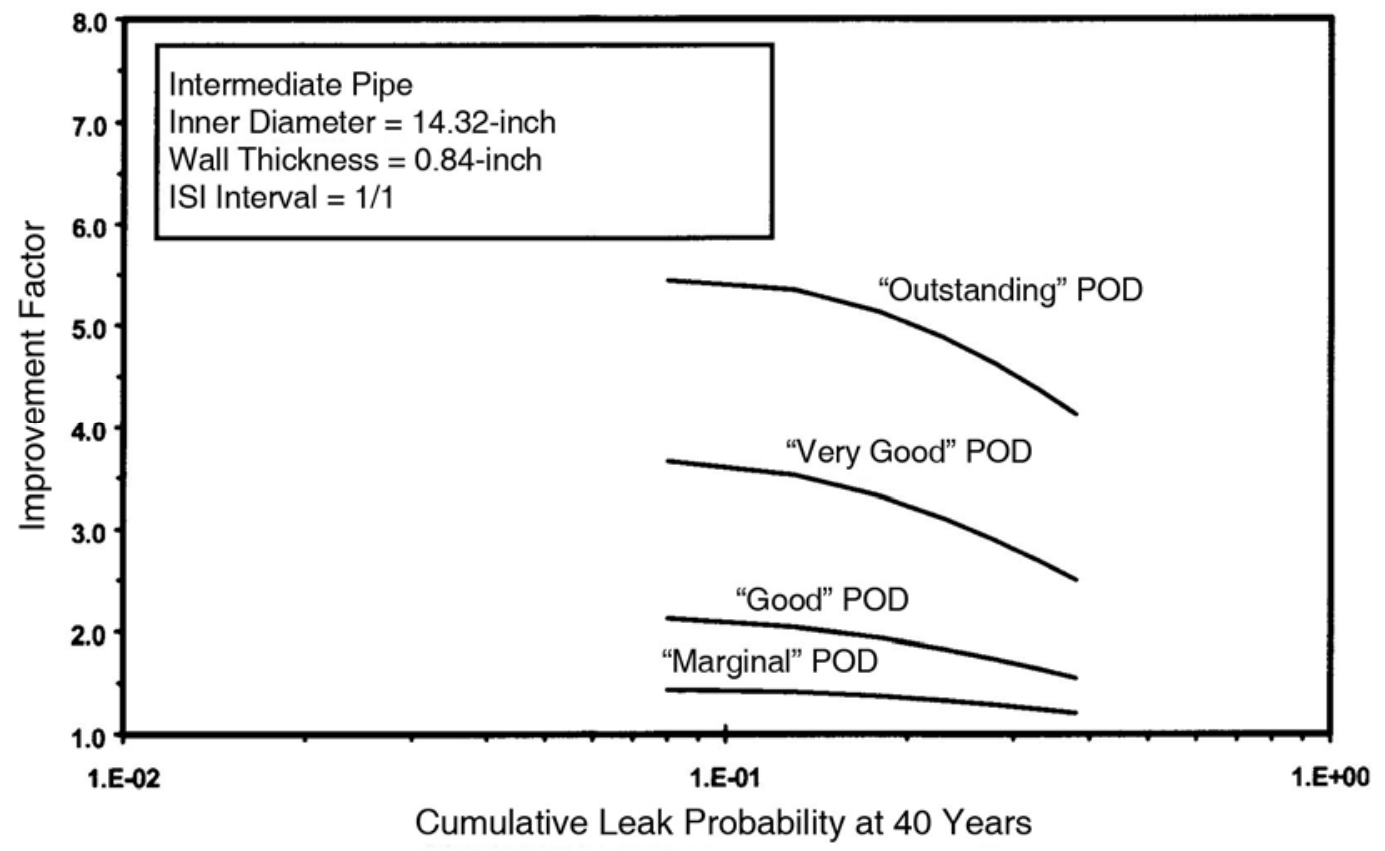

Figure 5.25b Improvement Factors versus Leak Probability for 1-Year ISI Interval (Intermediate Pipe Size and Various POD Curves) $(1$ inch $=25.4$ mm) 


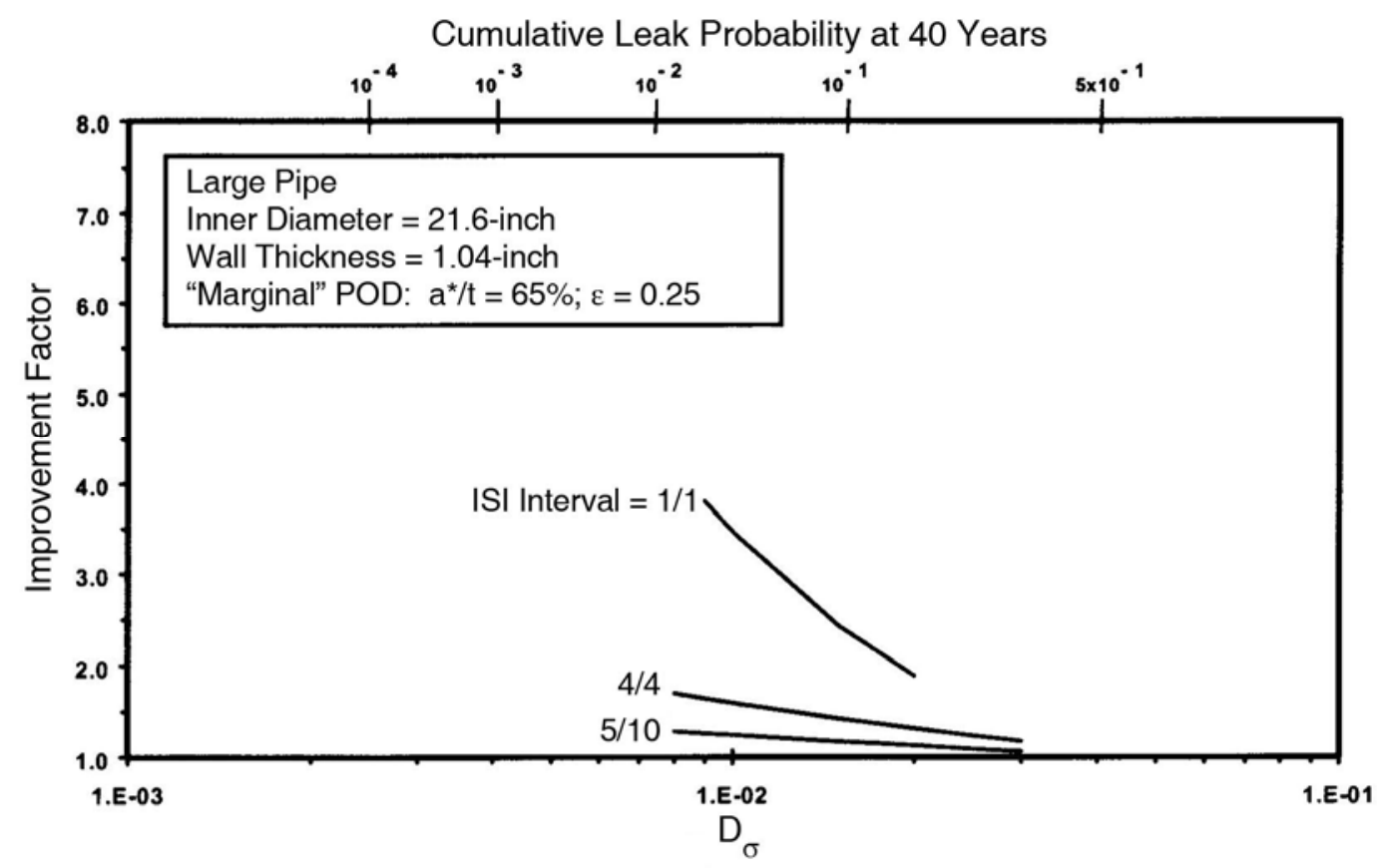

Figure 5.26a Improvement Factors versus $D_{\sigma}$ for "Marginal” POD Curve (Large Pipe Size and Various Inspection Intervals) $(1$ inch $=25.4 \mathrm{~mm})$

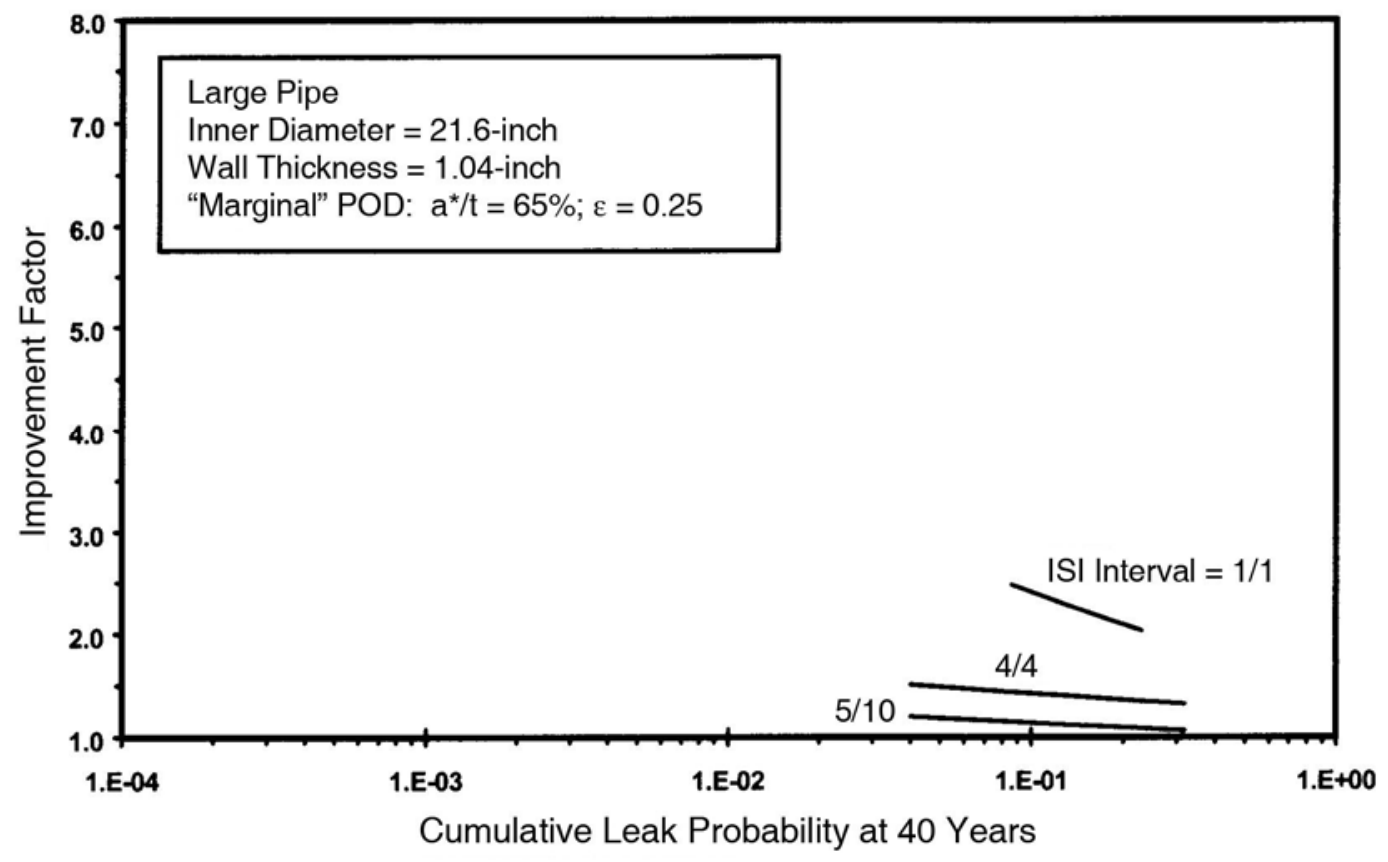

Figure 5.26b Improvement Factors versus Leak Probability for "Marginal" POD Curve (Large Pipe Size and Various Inspection Intervals) $(1$ inch $=25.4 \mathrm{~mm})$ 


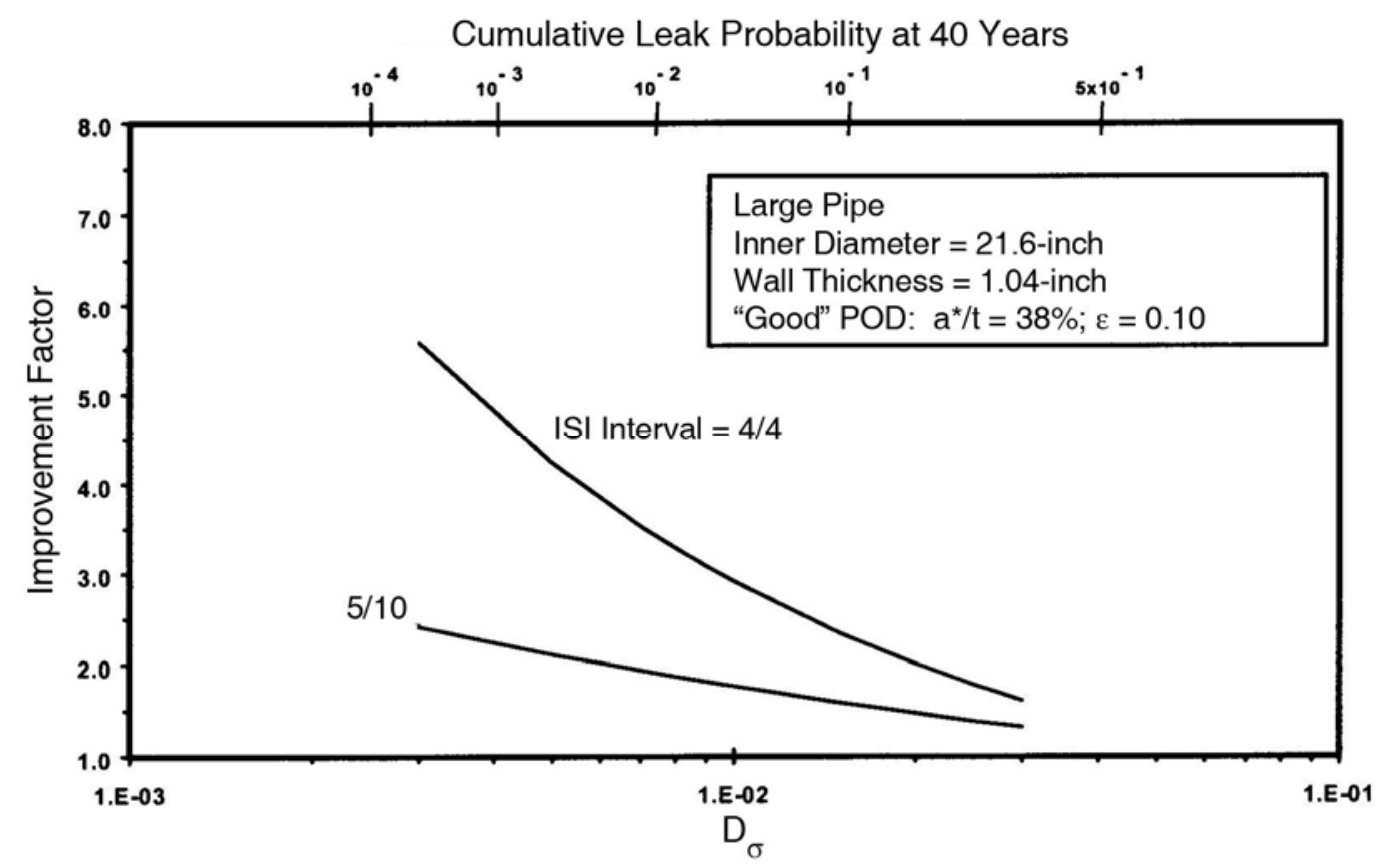

Figure 5.27a Improvement Factors versus $D_{\sigma}$ for "Good" POD Curve (Large Pipe Size and Various Inspection Intervals) $(1$ inch $=25.4 \mathrm{~mm})$

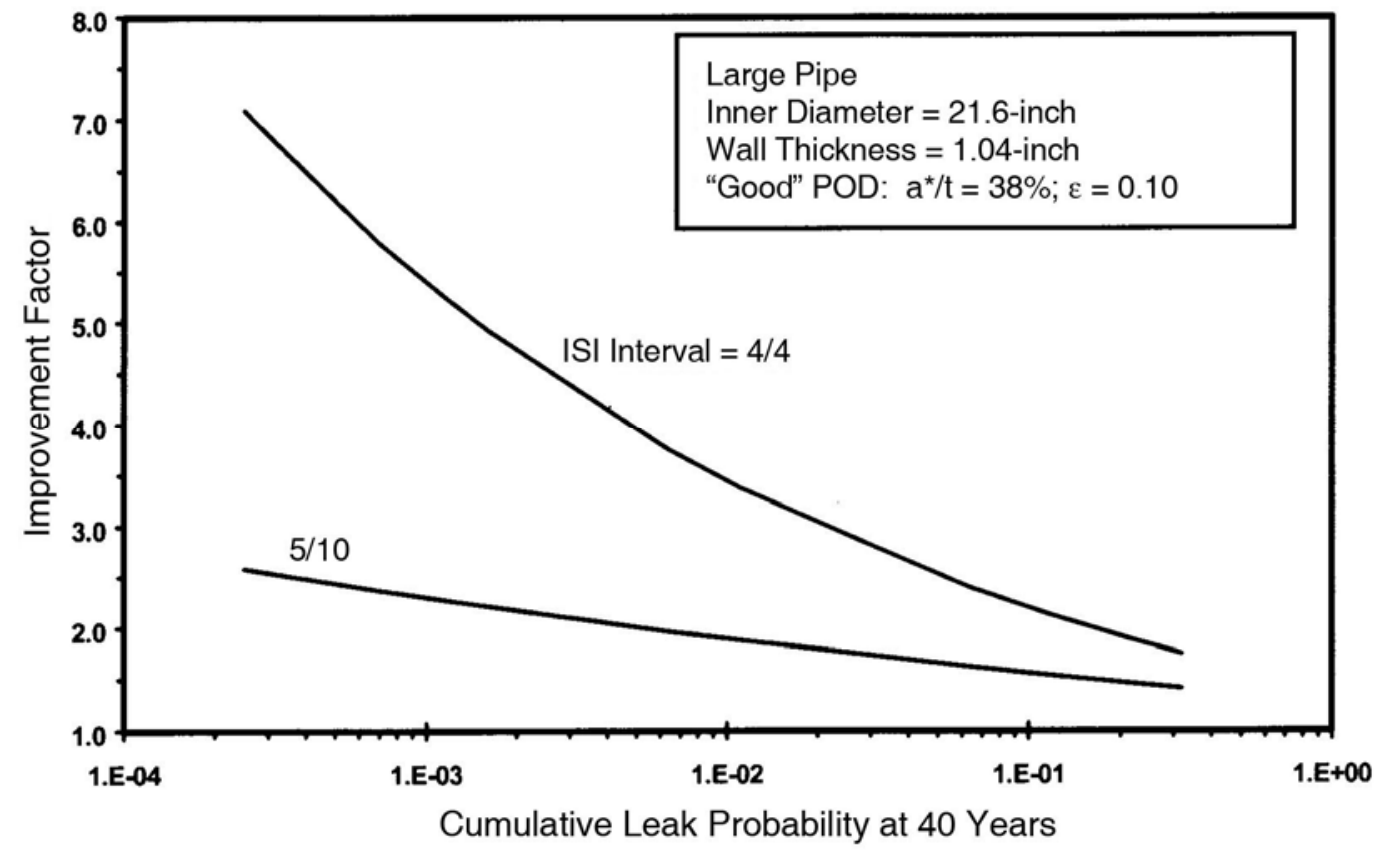

Figure 5.27b Improvement Factors versus Leak Probability for "Good" POD Curve (Large Pipe Size and Various Inspection Intervals $)(1 \mathrm{inch}=\mathbf{2 5 . 4} \mathbf{~ m m})$ 


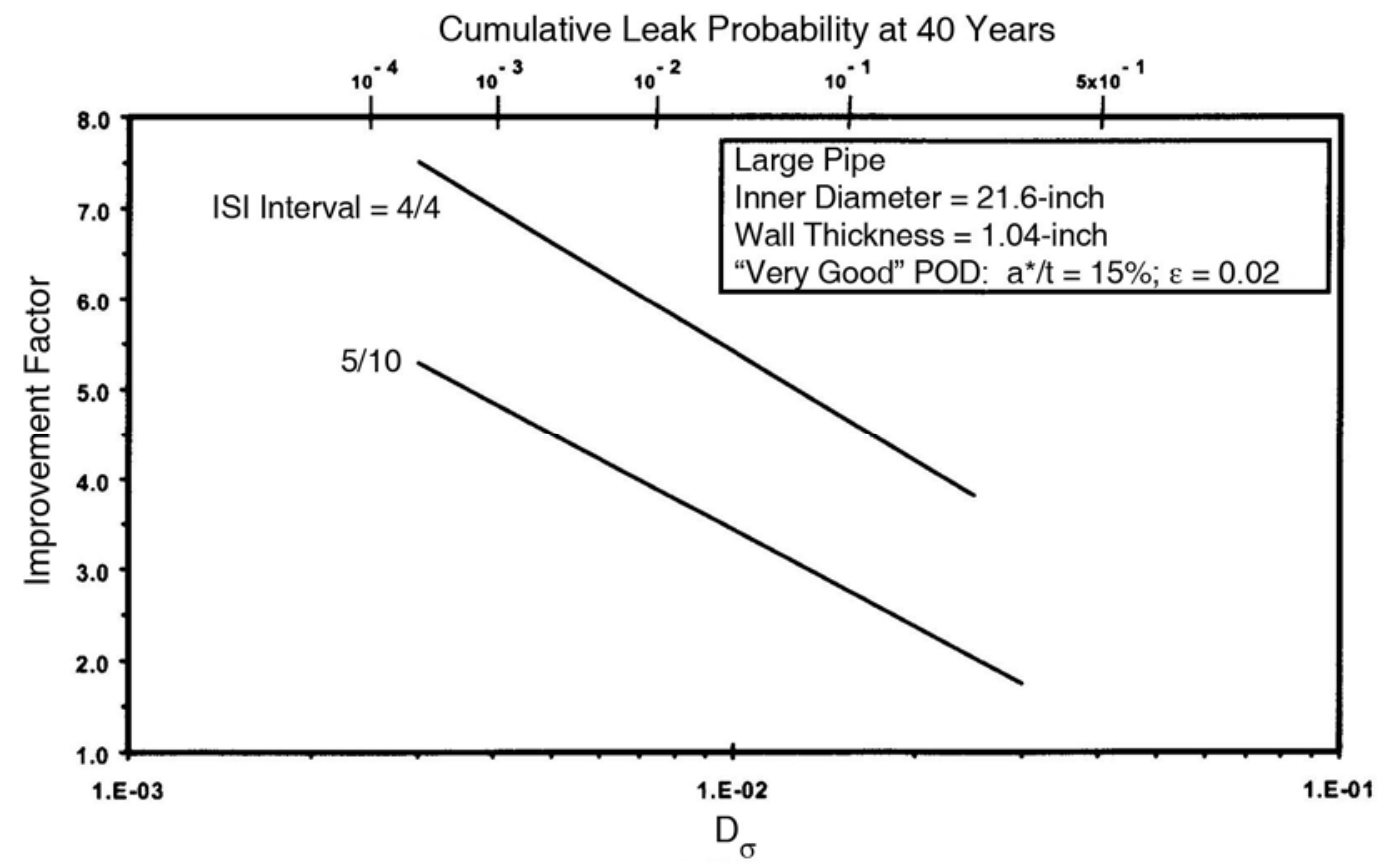

Figure 5.28a Improvement Factors versus $D_{\sigma}$ for "Very Good" POD Curve (Large Pipe Size and Various Inspection Intervals) $(1$ inch $=25.4 \mathrm{~mm})$

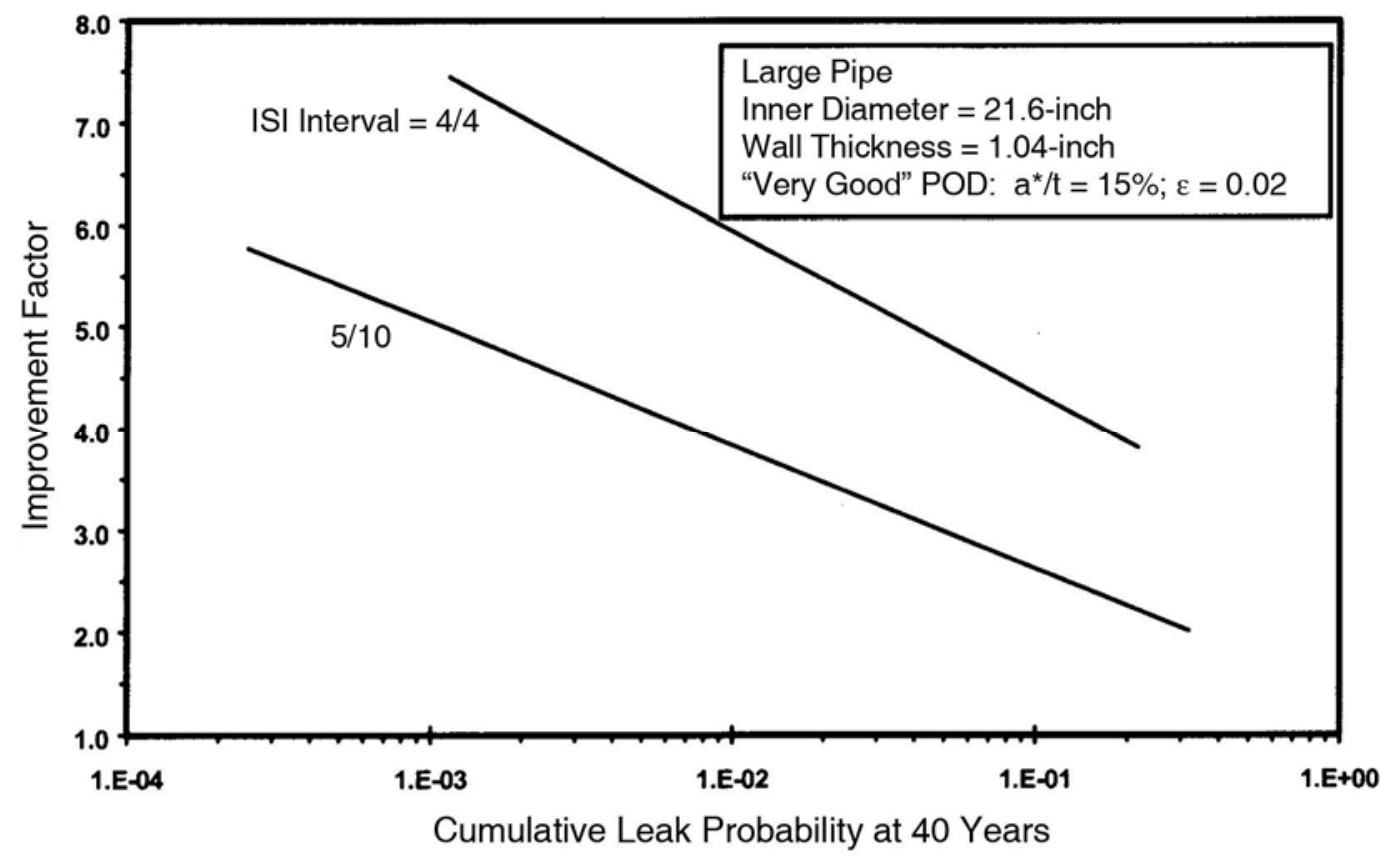

Figure 5.28b Improvement Factors versus Leak Probability for "Very Good" POD Curve (Large Pipe Size and Various Inspection Intervals) $(1$ inch $=25.4 \mathrm{~mm})$ 


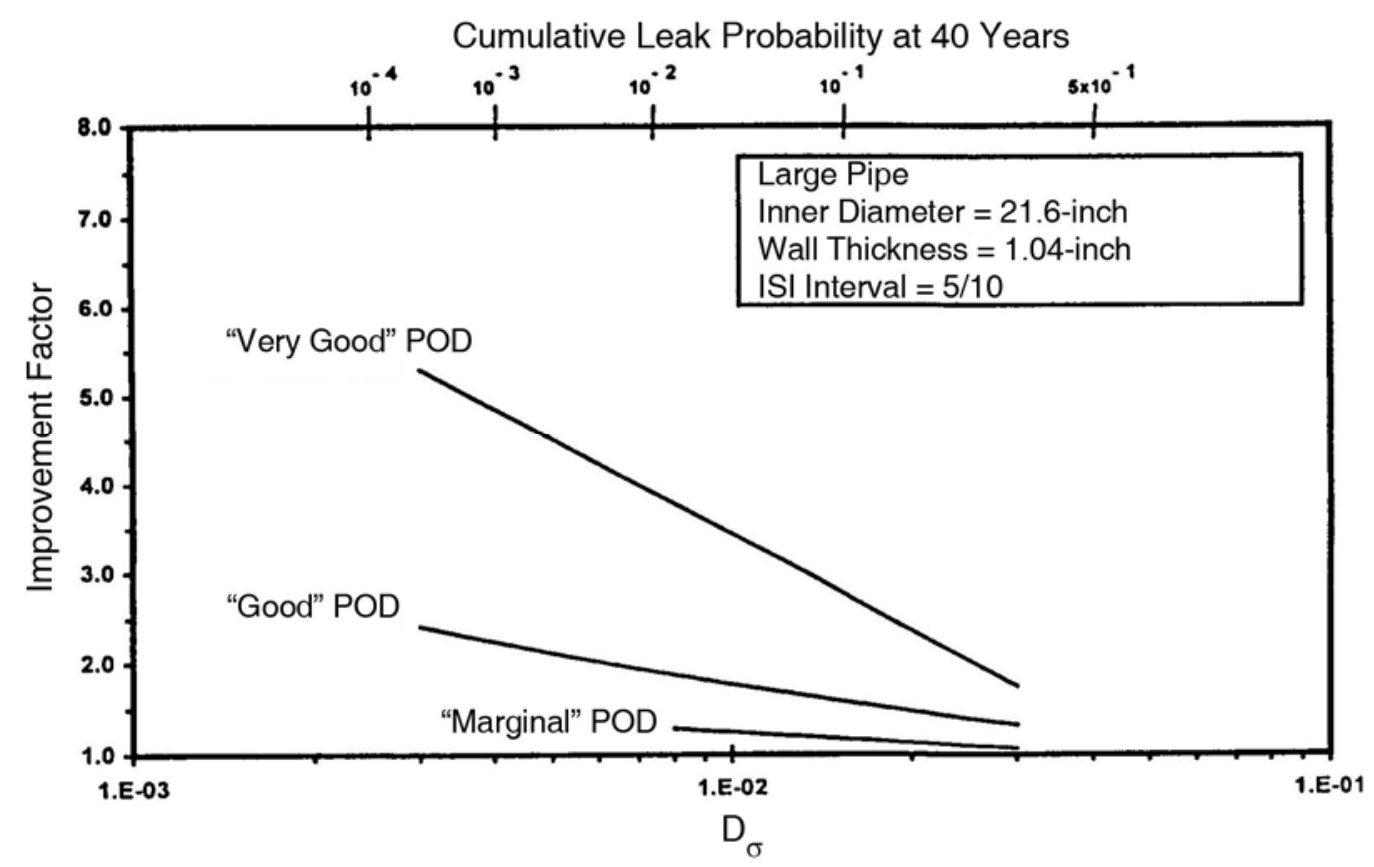

Figure 5.29a Improvement Factors versus $D_{\sigma}$ for 10-Year ISI Interval (Large Pipe Size and Various POD Curves) $(1$ inch $=25.4 \mathrm{~mm})$

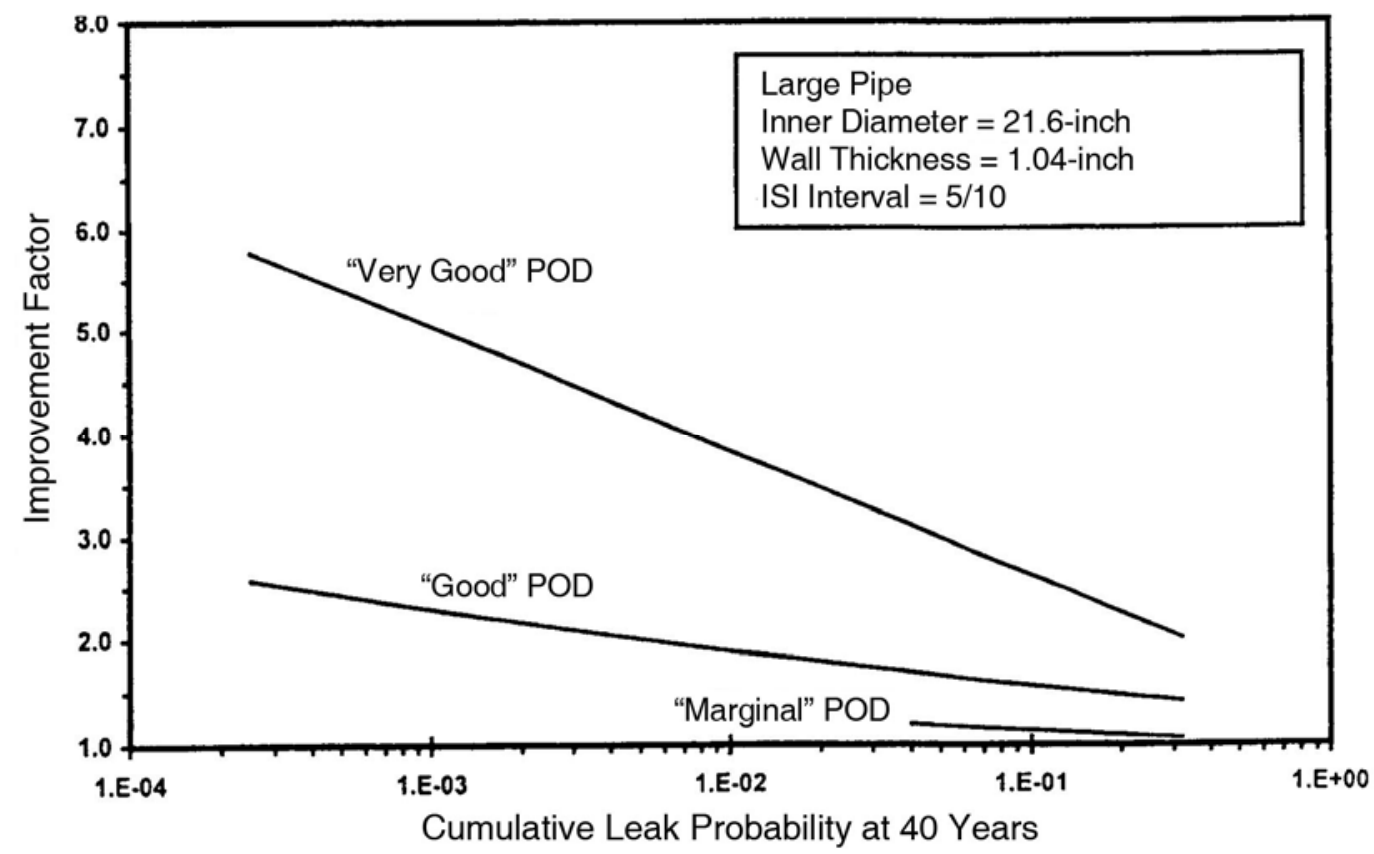

Figure 5.29b Improvement Factors versus Leak Probability for 10-Year ISI Interval (Large Pipe Size and Various POD Curves) $(1$ inch $=25.4 \mathrm{~mm})$ 


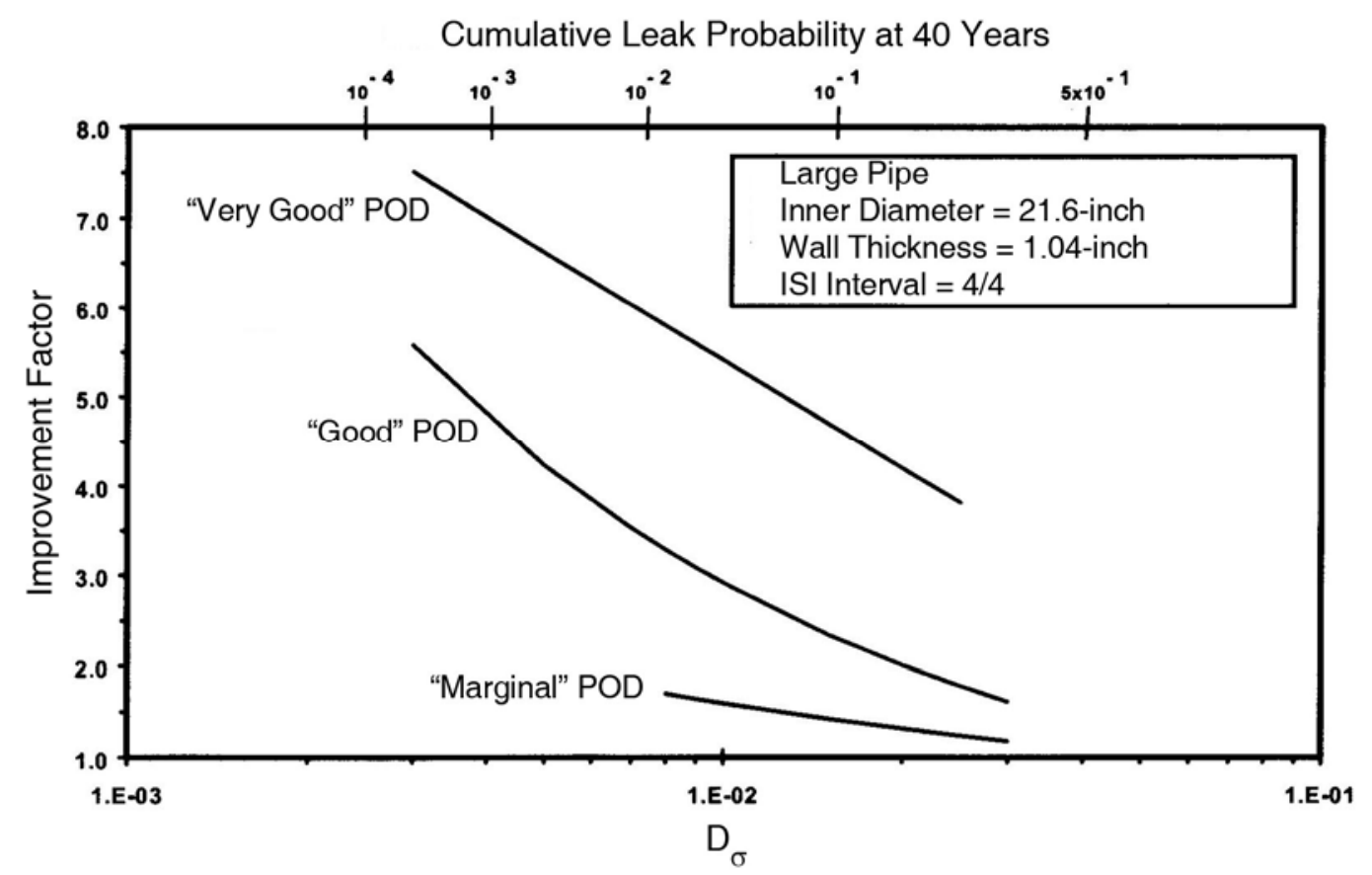

Figure 5.30a Improvement Factors versus $D_{\sigma}$ for 4-Year ISI Interval (Large Pipe Size and Various POD Curves) $(1$ inch $=25.4 \mathrm{~mm})$

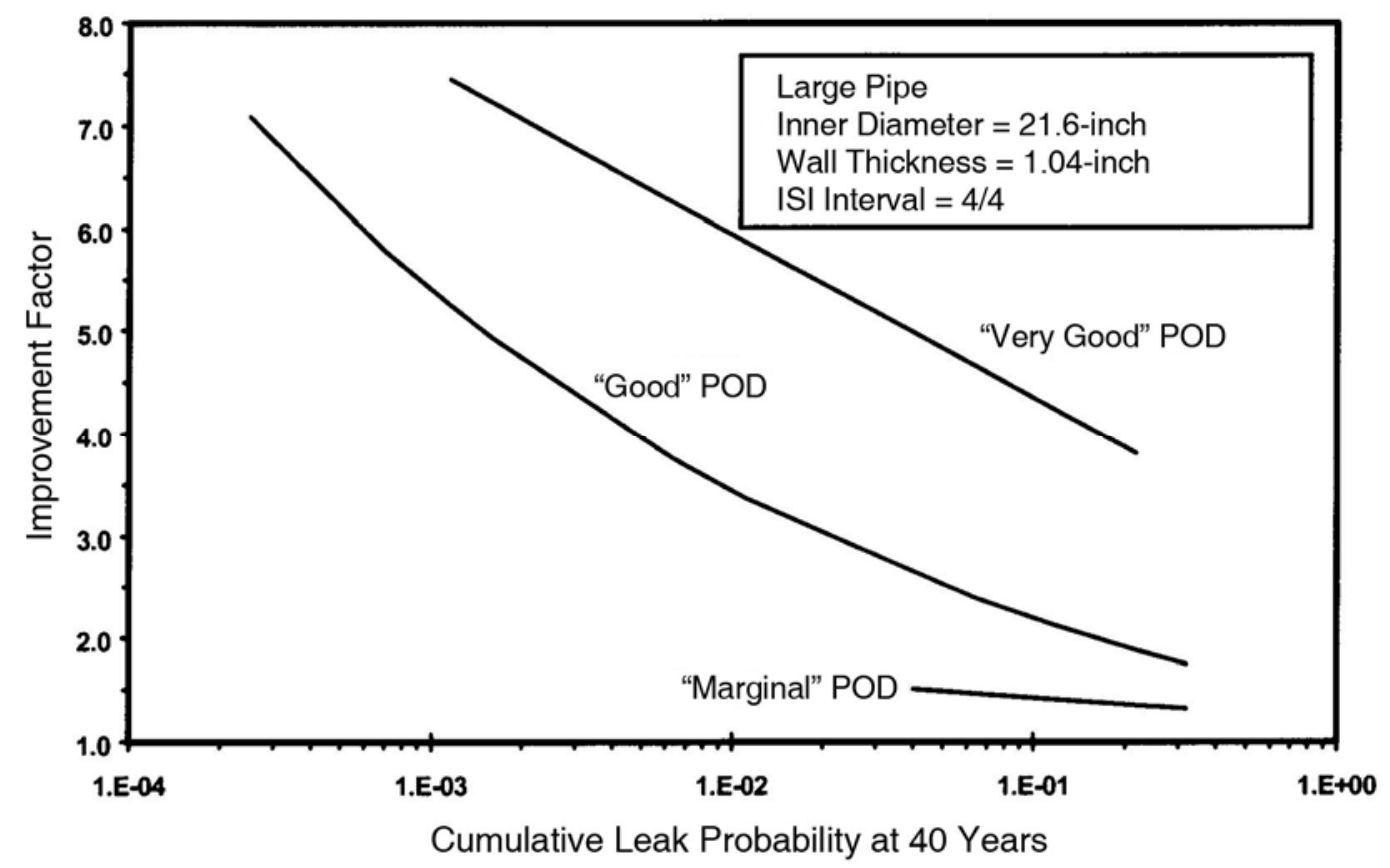

Figure 5.30b Improvement Factors versus Leak Probability for 4-Year ISI Interval (Large Pipe Size and Various POD Curves) $(1$ inch $=25.4 \mathrm{~mm})$ 


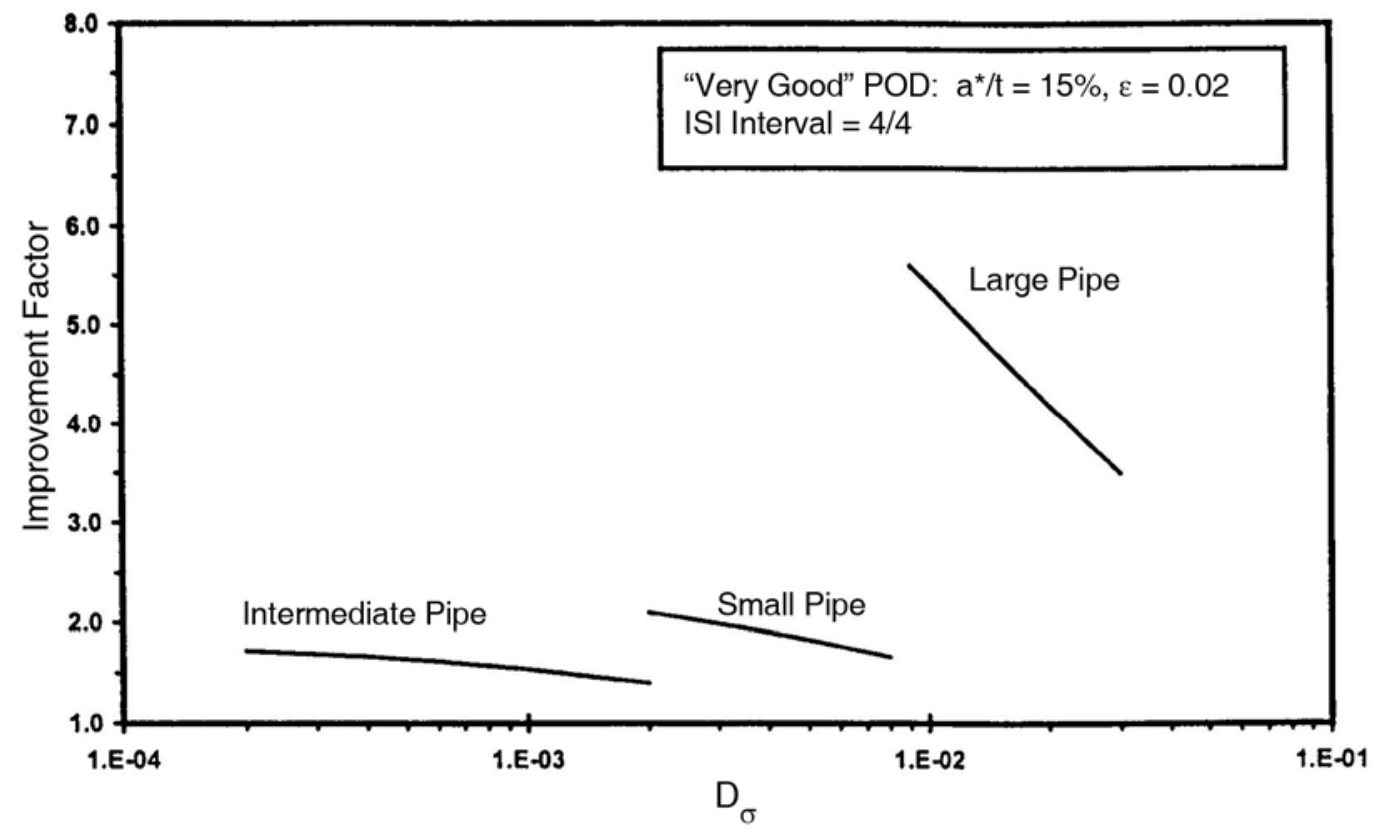

Figure 5.31a Improvement Factors versus $D_{\sigma}$ for "Very Good" POD Curve and 4-Year ISI Interval for Various Pipe Sizes

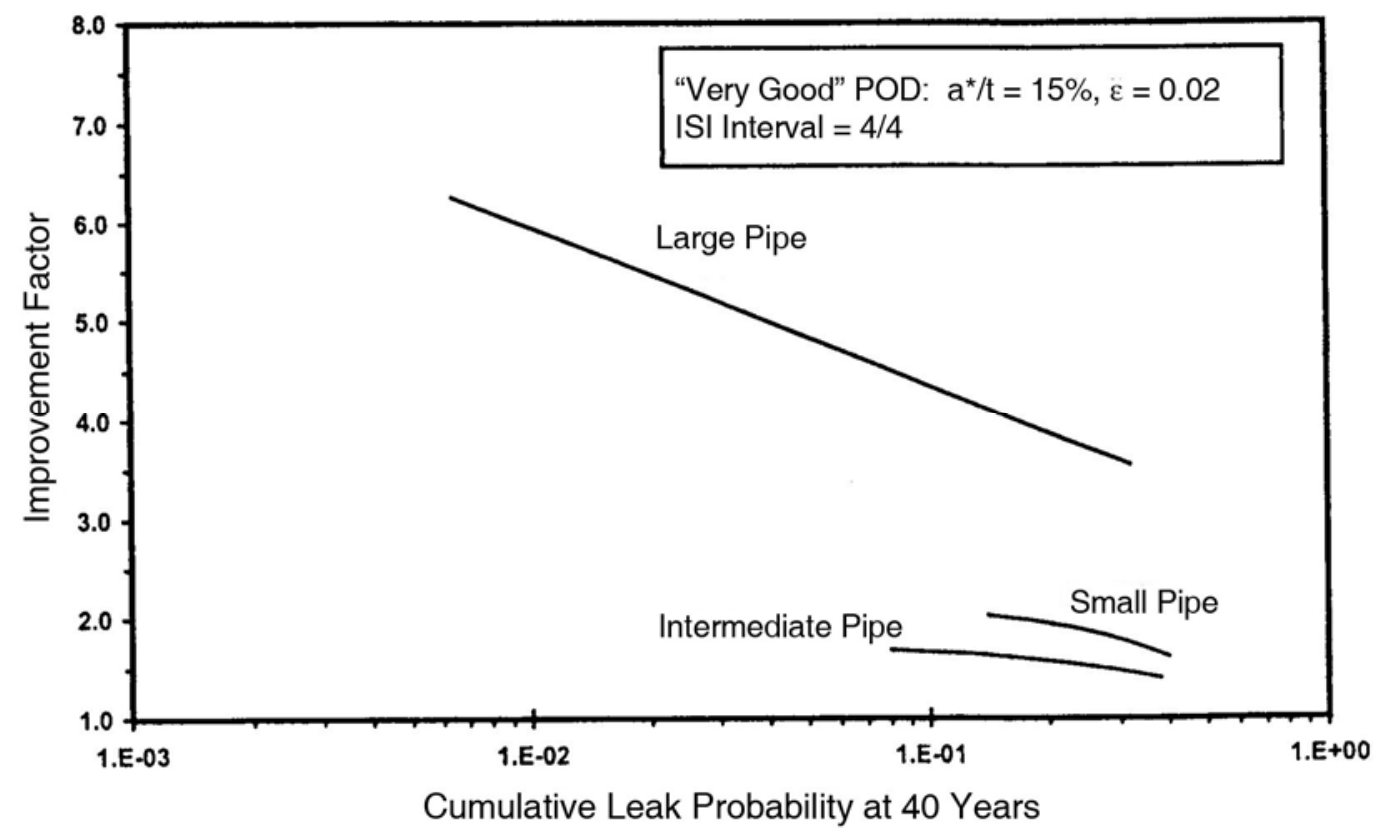

Figure 5.31b Improvement Factors versus Leak Probability for "Very Good" POD Curve and 4-Year ISI Interval for Various Pipe Sizes 


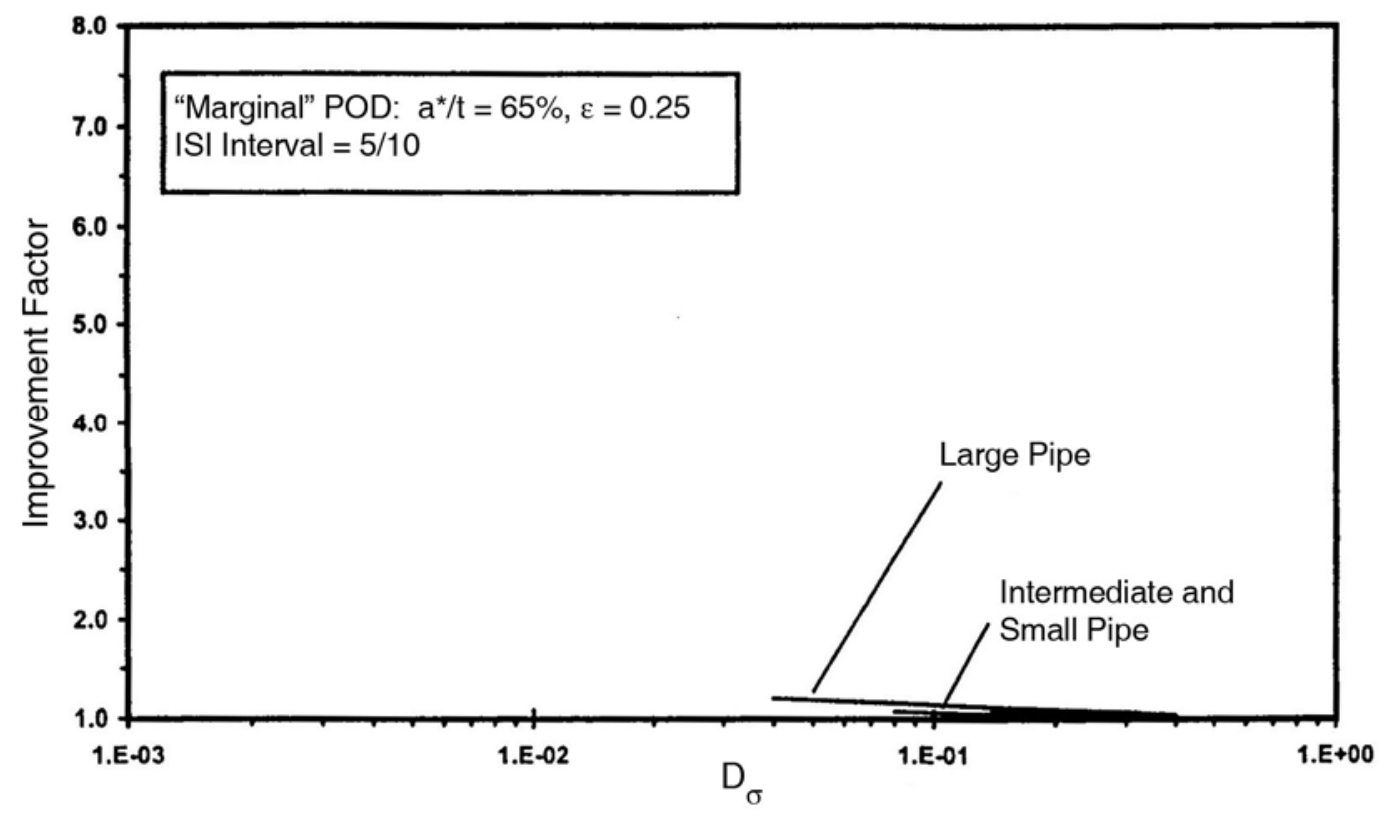

Figure 5.32a Improvement Factors versus Leak Probability for "Marginal" POD Curve and 10-Year ISI Interval for Various Pipe Sizes

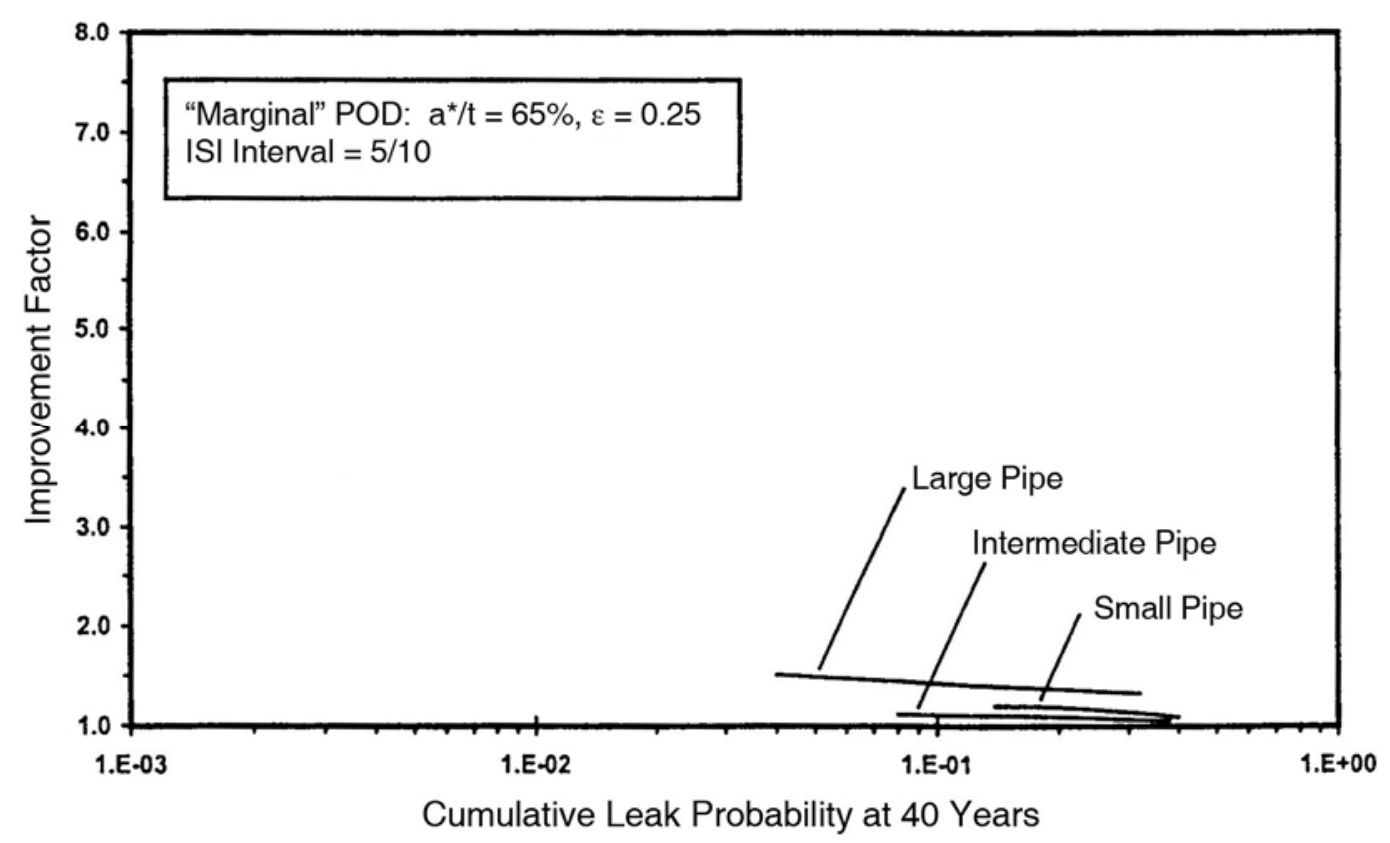

Figure 5.32b Improvement Factors versus Leak Probability for "Marginal" POD Curve and 4-Year ISI Interval for Various Pipe Sizes 


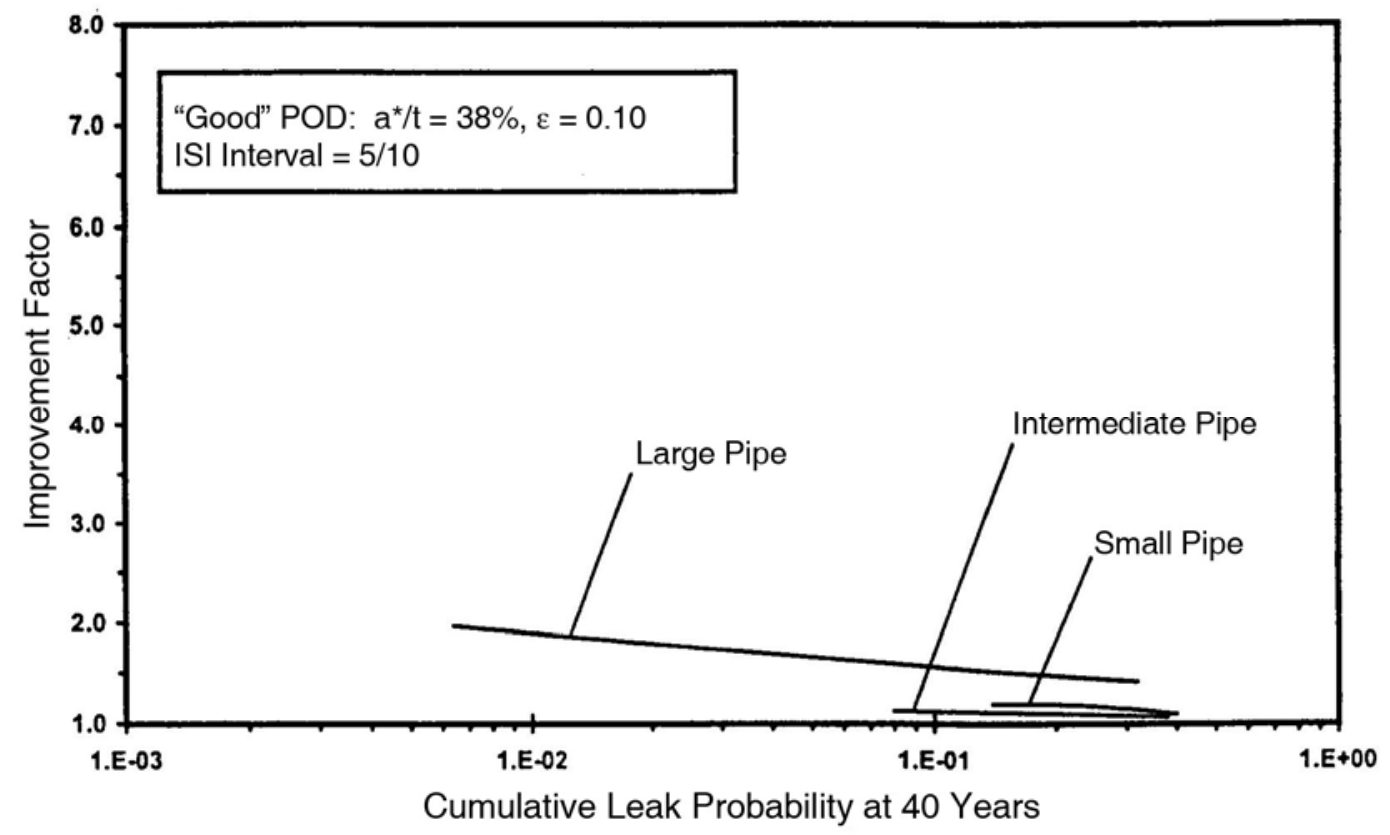

Figure 5.33a Improvement Factors versus Leak Probability for "Good" POD Curve and 10-Year ISI Interval for Various Pipe Sizes

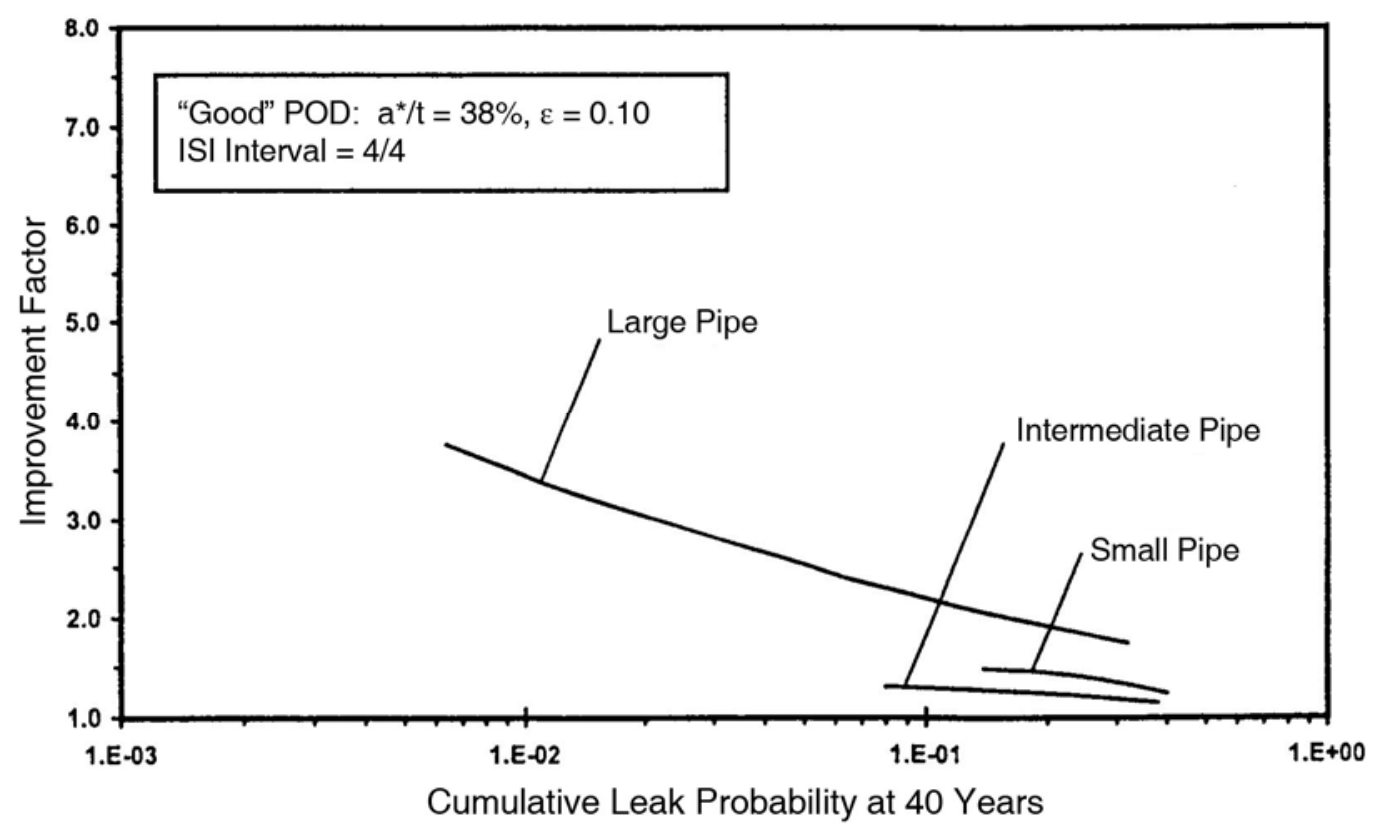

Figure 5.33b Improvement Factors versus Leak Probability for "Good" POD Curve and 4-Year ISI Interval for Various Pipe Sizes 


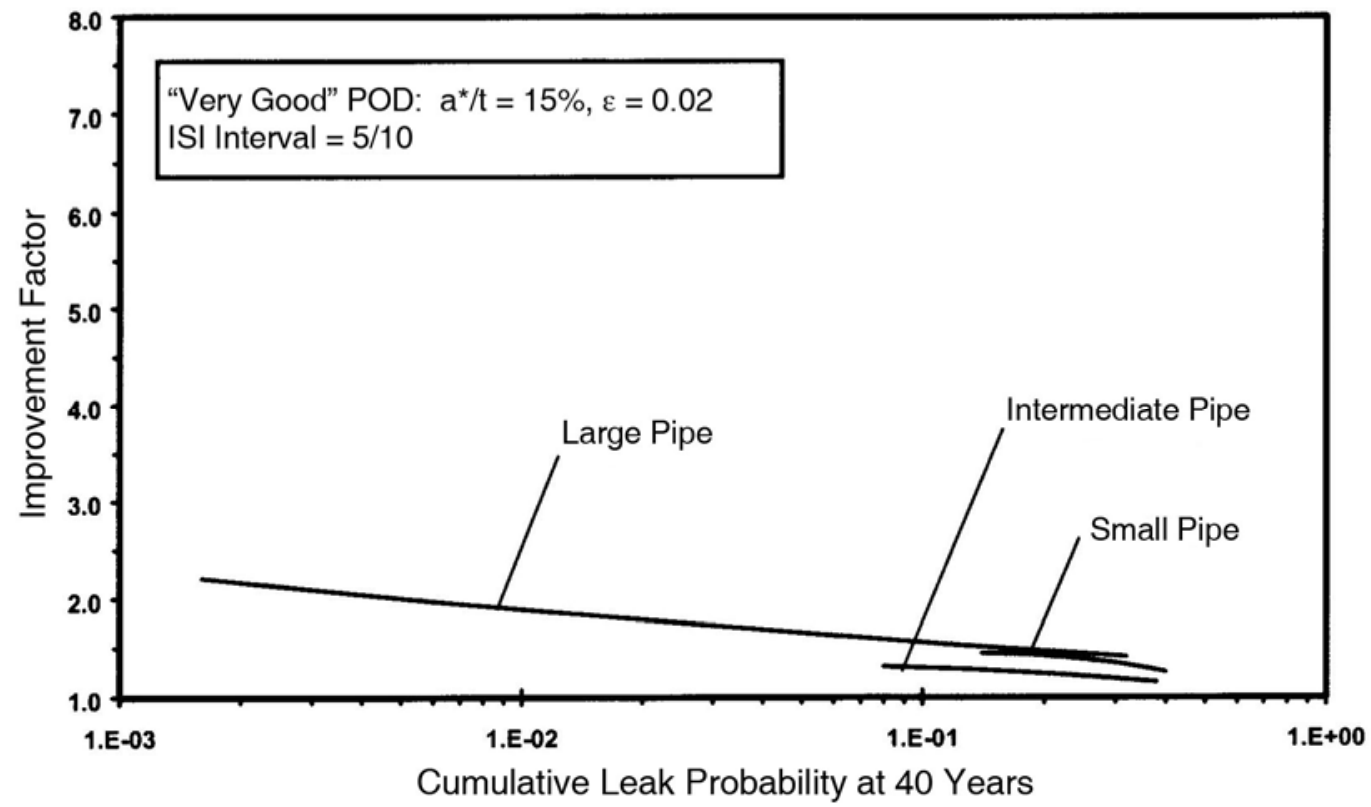

Figure 5.34a Improvement Factors versus Leak Probability for "Very Good" POD Curve and 10-Year ISI Interval for Various Pipe Sizes

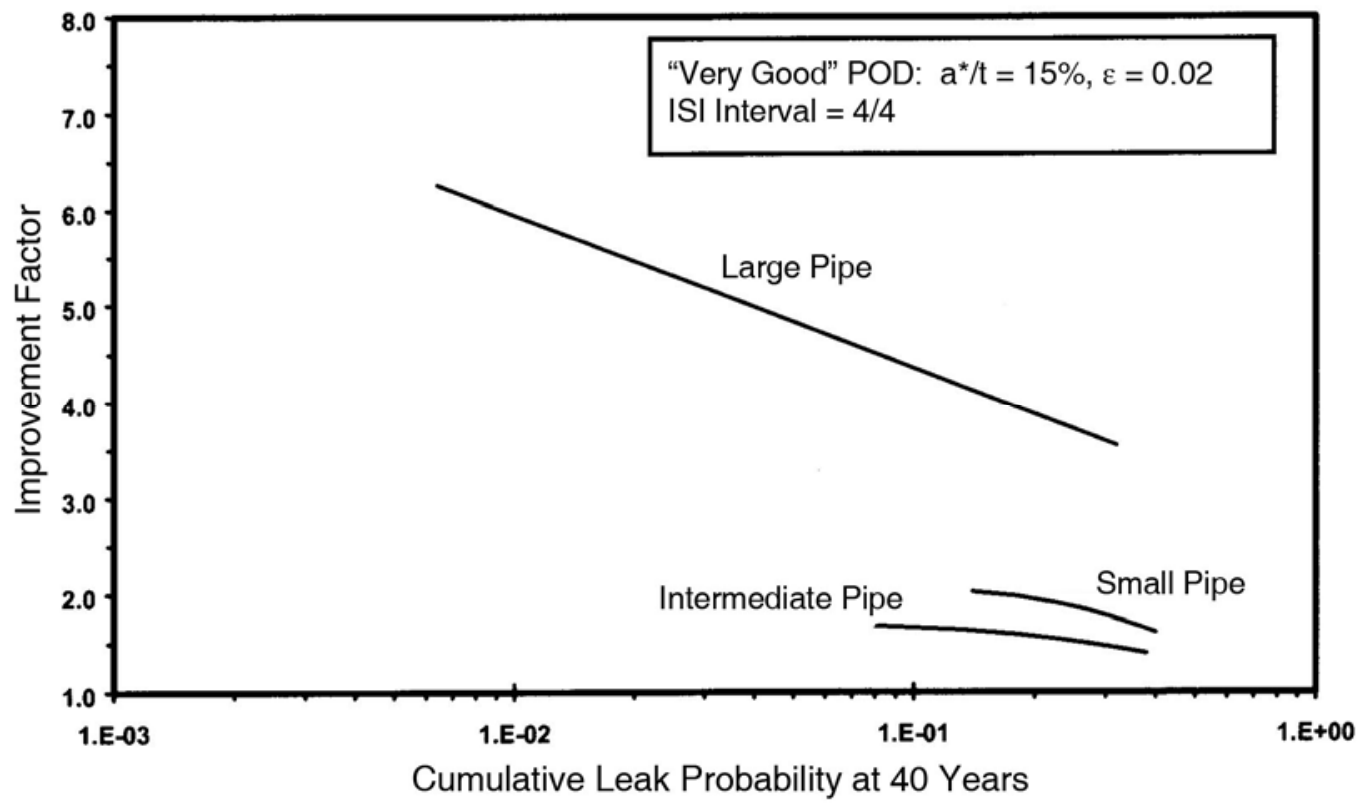

Figure 5.34b Improvement Factors versus Leak Probability for "Very Good" POD Curve and 4-Year ISI Interval for Various Pipe Sizes 


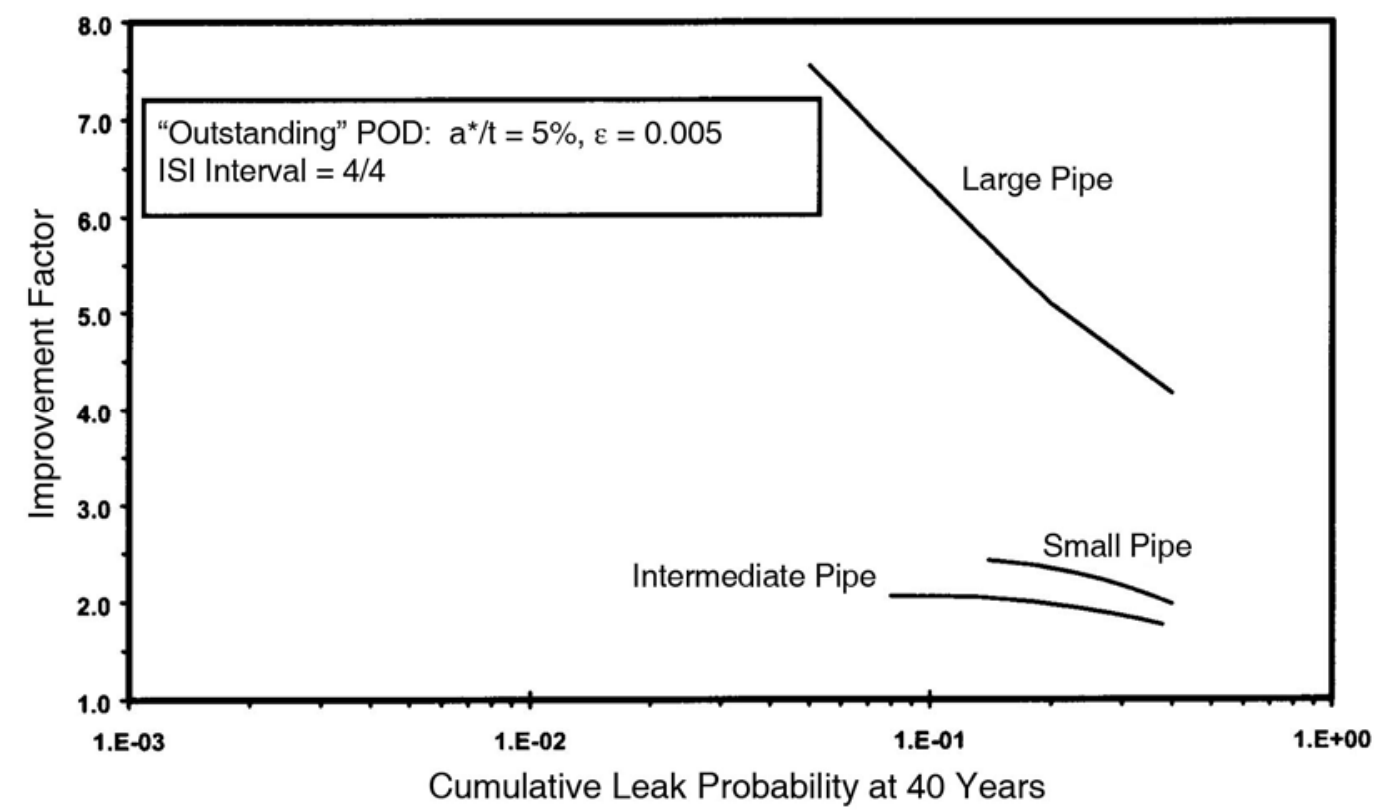

Figure 5.35 Improvement Factors versus Leak Probability for "Outstanding" POD Curve and 4-Year ISI Interval for Various Pipe Sizes 


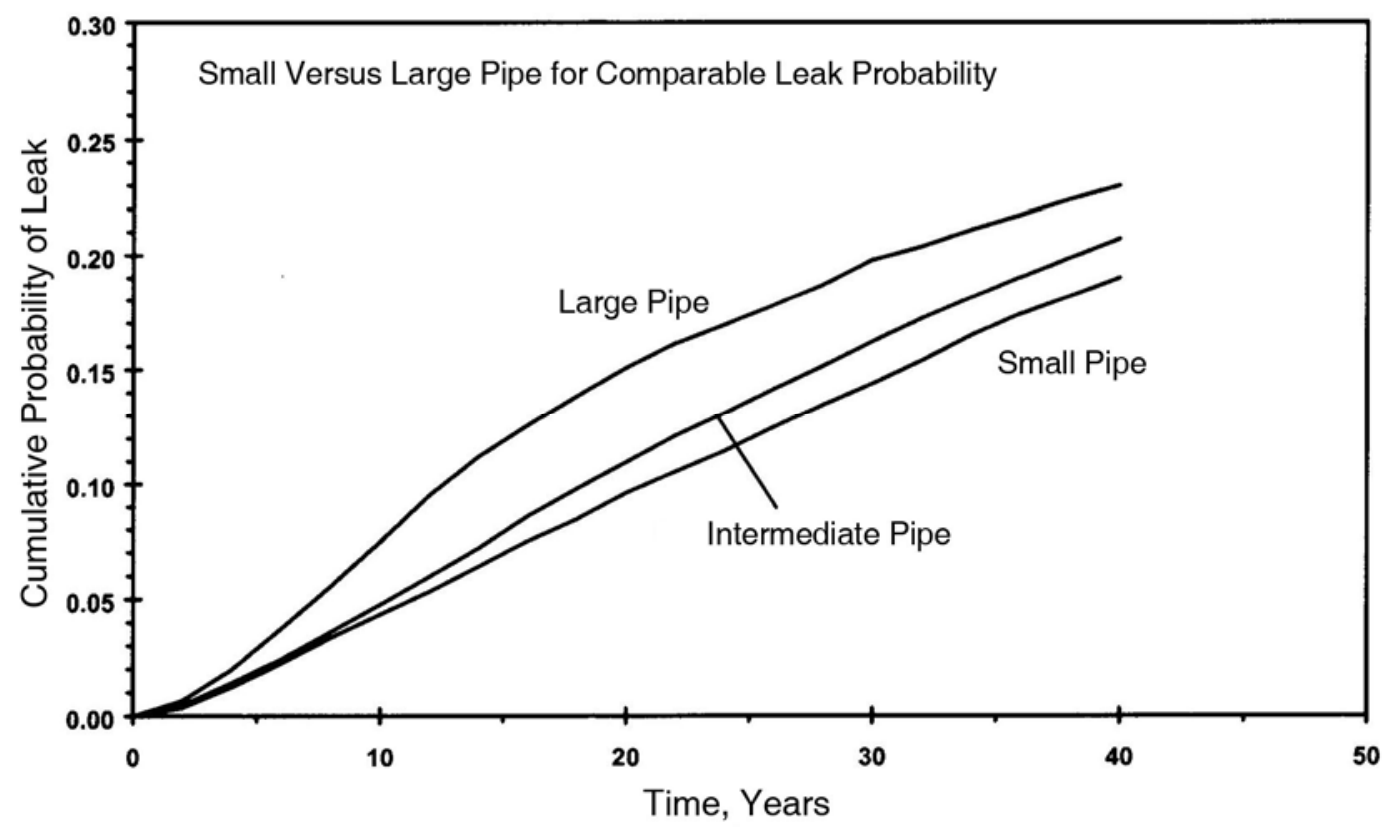

Figure 5.36a Leak Probability as a Function of Time for Various Pipe Sizes Having Comparable Failure Probabilities (No Inservice Inspection)

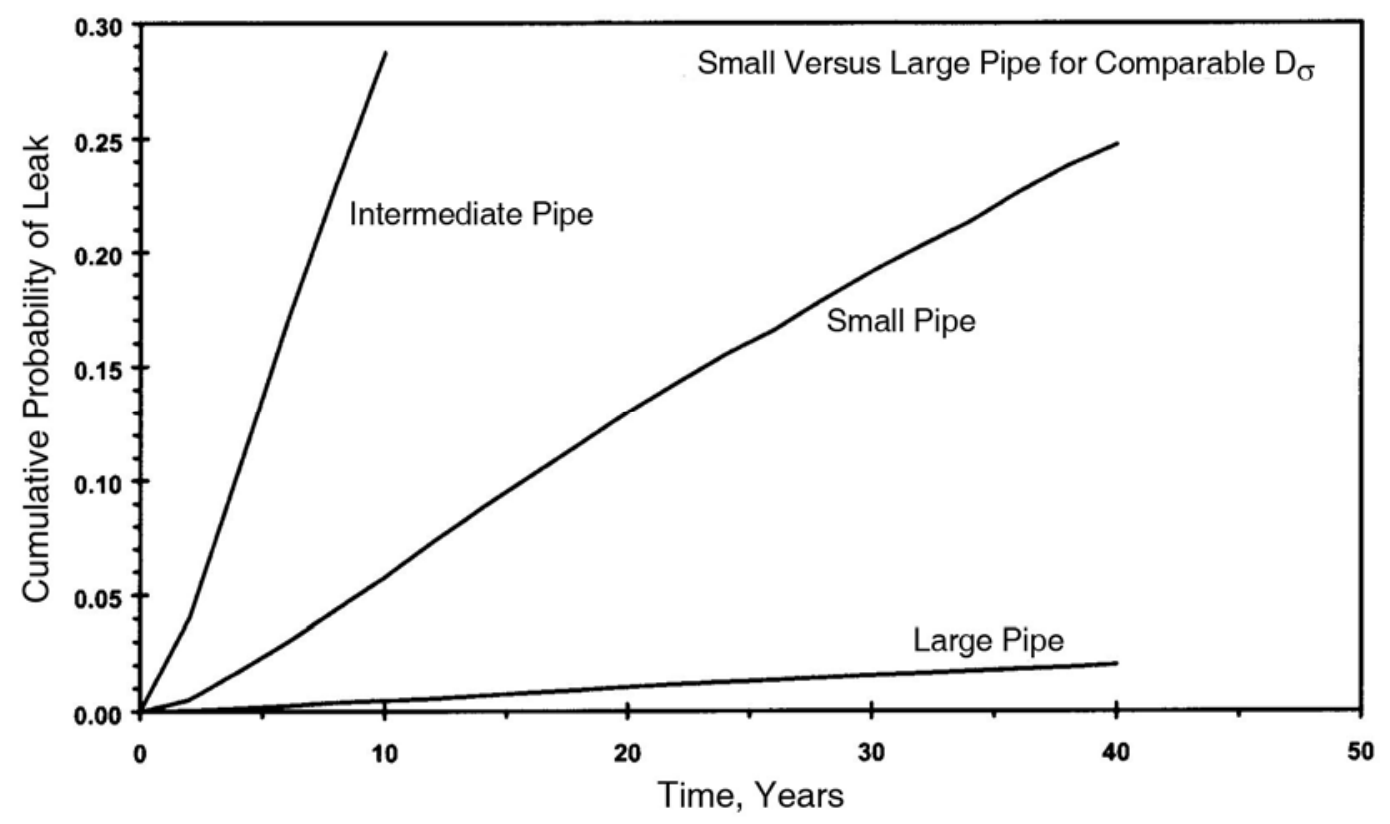

Figure 5.36b Leak Probability as a Function of Time for Various Pipe Sizes Having Comparable $D_{\sigma}$ Values of about 5.0E-03 (No Inservice Inspection) 


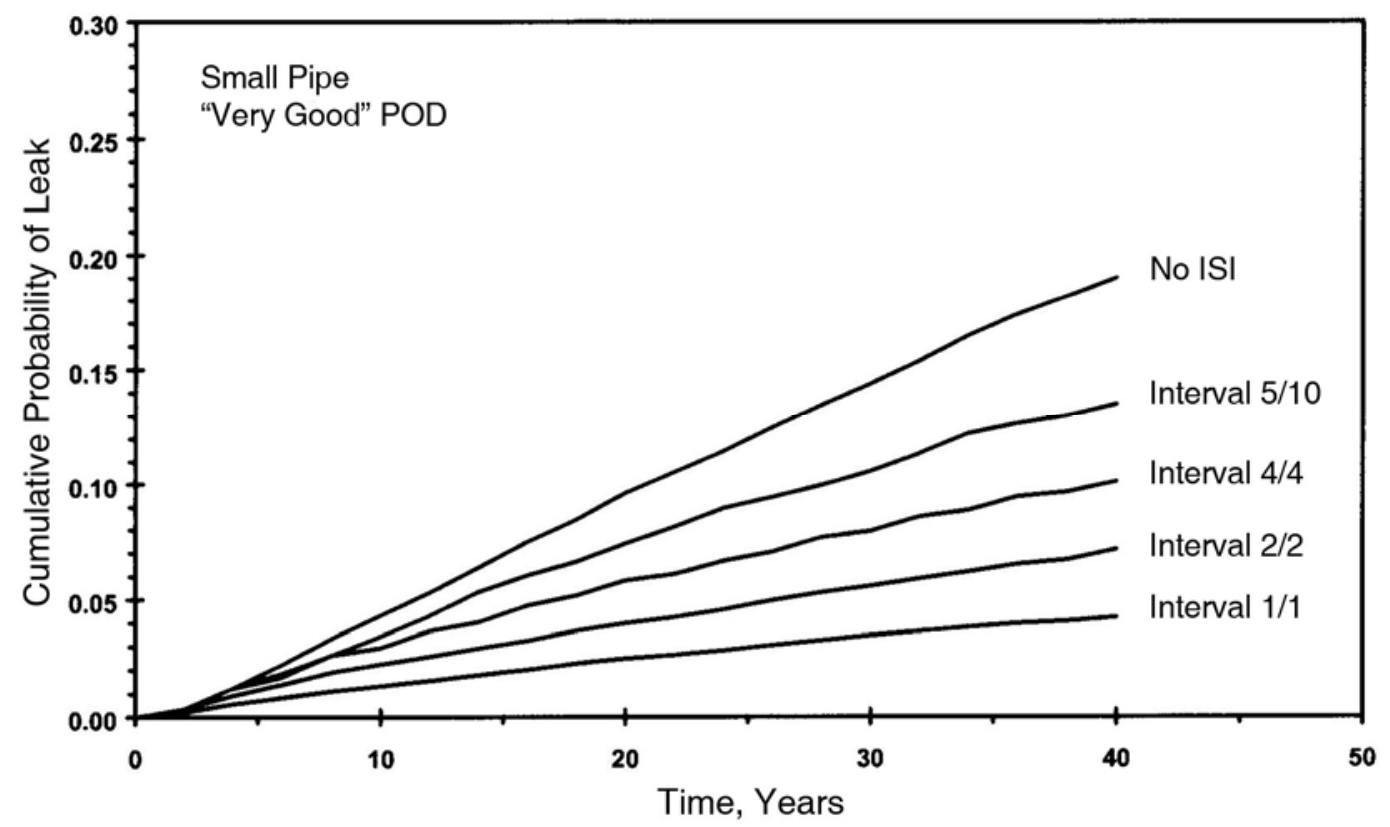

Figure 5.37a Leak Probability as a Function of Time for Small Pipe ("Very Good" POD and Various Inspection Intervals)

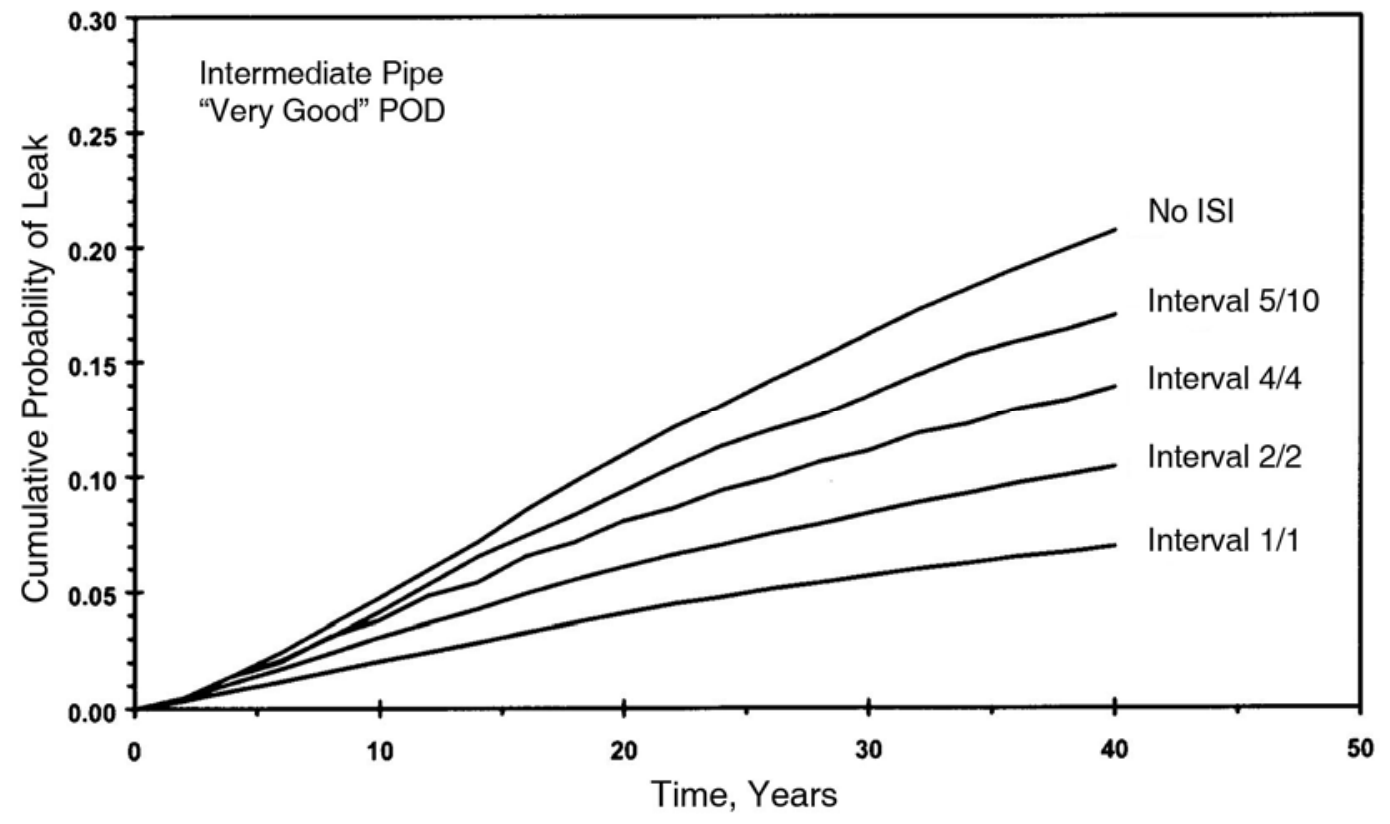

Figure 5.37b Leak Probability as a Function of Time for Intermediate Pipe ("Very Good" POD and Various Inspection Intervals) 


\section{Uncertainty Analysis for Calculated Failure Probabilities of Piping Welds}

\subsection{Introduction}

Probabilistic structural mechanics models are increasingly being used to predict the reliability of nuclear pressure-boundary components such as pressure vessels (Dickson 1994) and welds in piping systems (Harris et al. 1981; Harris and Dedhia 1992). The objective of the work described in this section was to quantify the uncertainties associated with the inputs to PFM calculations and the uncertainties in the PFM model itself, as well as the uncertainties in the resulting calculated piping-failure probabilities. Such uncertainties are an important issue in applying PFM models (Bishop 1997) to the implementation of riskinformed procedures for developing improved inservice inspection programs (ASME/CRTD 1992; Westinghouse Owners Group 1997).

The work presented here was an important part of the research that was intended to develop improved methods for predicting the reliability of nuclear piping systems and the effect of inservice inspections on reducing piping-failure probabilities. All calculations presented below used the pc-PRAISE computer code (Harris and Dedhia 1992) to address both leak and break probabilities for piping components. A two-step process was used to quantify the uncertainties in calculated failure probabilities (Simonen and Khaleel 1998b). The first step was a sensitivity study that identified those uncertainties having the greatest effect on the results from pc-PRAISE. The second step was a quantitative uncertainty analysis that addressed the most critical parameters as identified by the sensitivity calculations.

While the selected methodology is generally applicable to PFM calculations for piping, the calculations described within this section address specific examples as follows:

- Mechanical and thermal fatigue of stainless steel piping due to the presence of fabrication defects (the initiation of fatigue cracks is not addressed).

- A single pipe size (6-in. [152-mm] Schedule 120) with an inner radius of 2.75-in. (70 mm) and a wall thickness of 0.562 in. $(14.3 \mathrm{~mm})$.

- A wide range of cyclic stresses are addressed using the Q-factor approach of Khaleel and Simonen (1994a,b) with Q factors of 1, 100, and 10,000. The lowest value corresponds to typical locations in piping systems for which fatigue failures are not expected, whereas the highest value corresponds to locations exposed to thermal fatigue by which the pipe experiences unexpected and excessive levels of cyclic stress.

- While probabilities of both leak (through-wall crack) and pipe break were calculated, the main interest was in the uncertainties for leak probabilities. Uncertainties in leak probabilities were of particular interest to the development of a Regulatory Guide on Risk-Informed Inservice Inspection (Parry 1997) and to the application of the "Perdue Model" for weld inspection sampling plans (Balkey et al. 1997). 
The methodology of the present section and the resulting estimated uncertainties in the calculated probabilities were found to be similar to those for PFM calculations for reactor pressure vessels subject to pressurized thermal shock (Bozarth et al. 1985). Additional uncertainty evaluations could address other pipe sizes and failure mechanisms in addition to fatigue. Nevertheless, the uncertainties as characterized by the results of this chapter are believed to be representative of results expected for piping components in general. It is noted that the present study focuses on the probabilistic modeling approach of the pcPRAISE code. Other approaches (Bishop 1997) simulate uncertainties in additional parameters (e.g., applied stresses and loads), and as such, an uncertainty analysis for these codes could show a lower level of uncertainty in calculated failure probabilities.

\subsection{Sensitivity Calculations}

Inputs to pc-PRAISE consist of both probabilistic and deterministic parameters. Flaw depth, flaw-aspect ratio, crack-growth rate, and flow and ultimate stresses are probabilistic, while the pipe radius and wall thickness, internal pressure, dead-weight load, thermal stresses, and the number and level of cyclic stresses are deterministic.

In the sensitivity analyses, baseline failure probabilities were first calculated using best-estimate values for all input parameters. In a series of calculations, these inputs were changed one-by-one, with all other values unchanged from the baseline calculation. The numerical change for each input corresponded to the estimated uncertainty associated with that input parameter. The sensitivity calculations represented a simplified version of the subsequent detailed uncertainty analyses. Unlike the sensitivity calculations, the more complex uncertainty calculations assigned distributions for all input parameters rather than assigning a single incremental change. In addition, all inputs were allowed to vary at once in a series of pc-PRAISE runs to address the effects of interactions between variables.

The sensitivity study varied the scale parameter (i.e., the mean for a normally distributed variable, and the median for a lognormally distributed variable) while keeping the shape parameter (i.e., the standard deviation for normal variables) constant. Incremental changes for the deterministic inputs were established by assuming that they were normally distributed. Standard deviations for the distributions were based on the uncertainties in the deterministic input parameters. Table 6.1 shows the coefficient-ofvariations $(\mathrm{COV})$ for the pc-PRAISE deterministic inputs that were used in the sensitivity analyses. The $\mathrm{COV}$ is the ratio of the standard deviation to the mean value and, thus, is dimensionless.

Table 6.2 shows the matrix of calculations and input data used in the pc-PRAISE runs for the case of $\mathrm{Q}=100$, with similar tables applicable to the other values of the Q-factor. Identification numbers are indicated for each of the 44 cases in Table 6.2 as footnotes with "Run Number 1" being the baseline case.

Table 6.1 Assumed COV Values Used in Sensitivity Calculations

\begin{tabular}{||c|c|c|c|c|c|c||}
\hline $\begin{array}{c}\text { Wall } \\
\text { Thickness }\end{array}$ & Pipe Radius & Pressure & Dead Load & $\begin{array}{c}\text { Thermal } \\
\text { Stress }\end{array}$ & $\begin{array}{c}\text { Number of } \\
\text { Cycles }\end{array}$ & $\begin{array}{c}\text { Cyclic } \\
\text { Stress }\end{array}$ \\
\hline 0.3 & 0.005 & 0.02 & 0.10 & 0.10 & 1.0 & 0.25 \\
\hline
\end{tabular}


Table 6.2 Matrix of Calculations for Sensitivity Calculations for $Q=100$

\begin{tabular}{|c|c|c|c|c|c|c|c|c|}
\hline \multirow[b]{2}{*}{ Variable } & \multirow[b]{2}{*}{ Distribution } & \multicolumn{7}{|c|}{ Case } \\
\hline & & $-2 \sigma$ & $-\sigma$ & $+\sigma$ & & $+2 \sigma$ & & Baseline \\
\hline $\begin{array}{l}\text { Flaw Depth, } \\
\text { in. }(\mathrm{mm})\end{array}$ & Lognormal & & $\begin{array}{ll}0.026(0.66) & 2 \\
0.47(11.9) & \end{array}$ & $\begin{array}{l}0.105(2.7) \\
0.47(11.9)\end{array}$ & 3 & $\begin{array}{l}0.143(3.6) \\
0.47(11.9)\end{array}$ & 4 & $\begin{array}{l}0.063(1.6) \\
0.47(11.9)\end{array}$ \\
\hline Flaw Aspect Ratio & Lognormal & $\begin{array}{ll}0.033 & 5 \\
0.433 & \end{array}$ & $\begin{array}{ll}0.787 & 6 \\
0.433 & \end{array}$ & $\begin{array}{l}.620 \\
0.433\end{array}$ & 7 & $\begin{array}{l}3.500 \\
0.433\end{array}$ & 8 & $\begin{array}{l}1.710 \\
0.433\end{array}$ \\
\hline $\begin{array}{l}\text { Wall Thickness, } \\
\text { in. }(\mathrm{mm})\end{array}$ & Normal & $0.528(13.4) \quad 9$ & $0.545(13.8) \quad 10$ & $0.579(14.7)$ & 11 & $0.596(15.1)$ & 12 & $\begin{array}{l}0.562(14.3) \\
0.017(0.43)\end{array}$ \\
\hline $\begin{array}{l}\text { Pipe Radius, } \\
\text { in. }(\mathrm{mm})\end{array}$ & Normal & $2.72(69.1) \quad 13$ & $2.74(69.6) \quad 14$ & $2.76(70.1)$ & 15 & $2.78(70.6)$ & 16 & $\begin{array}{l}2.75(69.8) \\
0.014(0.36)\end{array}$ \\
\hline $\begin{array}{l}\text { Crack Growth, } \\
\text { in./cycle } \\
\text { (mm/cycle) }\end{array}$ & Lognormal & & & $\begin{array}{l}3.25 \mathrm{E}-7(8.26 \mathrm{E}-6) \\
1.24 \mathrm{E}-6(3.15 \mathrm{E}-5)\end{array}$ & 17 & $\begin{array}{l}6.36 \mathrm{E}-7(1.62 \mathrm{E}-5) \\
2.43 \mathrm{E}-6(6.17 \mathrm{E}-5)\end{array}$ & 18 & $\begin{array}{l}9.14 \mathrm{E}-8(2.32 \mathrm{E}-6) \\
3.50 \mathrm{E}-7(8.89 \mathrm{E}-6)\end{array}$ \\
\hline $\begin{array}{l}\text { Flow Stress, } \\
\text { ksi (MPa) }\end{array}$ & Normal & $34.6(239) \quad 19$ & $38.8(268) \quad 20$ & $47.2(326)$ & 21 & $51.4(354)$ & 22 & $\begin{array}{l}43(296) \\
4.2(29) \\
\end{array}$ \\
\hline $\begin{array}{l}\text { Ultimate Stress, } \\
\text { ksi (MPa) }\end{array}$ & Normal & $48(331)$ & $54(372)$ & $66(455)$ & 25 & $72(496)$ & 26 & $\begin{array}{l}60(414) \\
6(41)\end{array}$ \\
\hline $\begin{array}{l}\text { Pressure, } \\
\text { ksi (MPa) }\end{array}$ & Normal & $2.16(14.9) \quad 27$ & $2.205(15.2) \quad 28$ & $2.295(15.8)$ & 29 & $2.340(16.1)$ & 30 & $\begin{array}{l}2.25(15.5) \\
0.045(0.31) \\
\end{array}$ \\
\hline $\begin{array}{l}\text { Dead Load, } \\
\text { ksi (MPa) }\end{array}$ & Normal & $2.4(16.5)$ & $2.7(18.6) \quad 32$ & $3.3(22.7)$ & 33 & $3.6(24.8)$ & 34 & $\begin{array}{l}3.0(21) \\
0.3(2.1)\end{array}$ \\
\hline $\begin{array}{l}\text { Thermal Stress, } \\
\text { ksi (MPa) }\end{array}$ & Normal & $8.0(55)$ & $9.0(62)$ & $11.0(76)$ & 37 & $12.0(83)$ & 38 & $\begin{array}{l}10.0(69) \\
1.0(7)\end{array}$ \\
\hline Number of Cycles & Normal & & & 10.0 & 39 & 15 & 40 & 55 \\
\hline $\begin{array}{l}\text { Cyclic Stress } \\
\text { Level, ksi (MPa) }\end{array}$ & Normal & $7.5(52)$ & $11.25(78)$ & $18.75(129)$ & 43 & $22.5(155)$ & 44 & $\begin{array}{l}15.0(103 \\
3.75(26)\end{array}$ \\
\hline
\end{tabular}


A total of 44 cases was run for each of the three $Q$-factor values $(Q=1,100$, and 10,000). These three values addressed a wide range of cyclic stresses and resulting failure probabilities. In the sensitivity studies, the interest was in leak (with big leaks being $>100 \mathrm{gal} / \mathrm{min}$ [378 liters/min]) and break probabilities relative to the corresponding probabilities for the baseline case. These relative probabilities are listed in the columns of Table 6.3 under the headings "Leak Ratio" and "LOCA Ratio," with these same ratios shown plotted in Figures 6.1 and 6.2. The probabilities are insensitive to changes in the parameter if the ratio of probabilities is close to 1 .

Calculations indicated that leak probabilities were most sensitive to changes in the flaw depth, flawaspect ratio, crack-growth rate, and level and number of cyclic stresses. The prescribed changes in some of these parameters resulted in leak probabilities higher/lower than the baseline by as much as 2.5 orders of magnitude. 
Table 6.3 Results of Sensitivity Analyses for $Q=100$

\begin{tabular}{|c|c|c|c|c|c|c|c|}
\hline Case & Leak & Big Leak & LOCA & Leak Ratio & $\begin{array}{c}\text { LOCA } \\
\text { Ratio }\end{array}$ & $\begin{array}{c}\text { Sorted Case } \\
\text { by Leak } \\
\text { Ratio } \\
\end{array}$ & $\begin{array}{c}\text { Sorted } \\
\text { Case by } \\
\text { LOCA }\end{array}$ \\
\hline 1 & $6.84 \mathrm{E}-04$ & $3.36 \mathrm{E}-11$ & $3.36 \mathrm{E}-11$ & 1.000 & 1.000 & 4 & 8 \\
\hline 2 & $2.01 \mathrm{E}-06$ & $1.24 \mathrm{E}-15$ & $1.24 \mathrm{E}-15$ & 0.003 & 0.000 & 18 & 4 \\
\hline 3 & $8.45 \mathrm{E}-03$ & $2.65 \mathrm{E}-09$ & $2.65 \mathrm{E}-09$ & 12.334 & 79.001 & 17 & 18 \\
\hline 4 & $3.03 \mathrm{E}-02$ & $2.15 \mathrm{E}-08$ & $2.15 \mathrm{E}-08$ & 44.417 & 640.632 & 3 & 3 \\
\hline 5 & $1.87 \mathrm{E}-04$ & $0.00 \mathrm{E}+00$ & $0.00 \mathrm{E}+00$ & 0.273 & 0.000 & 44 & 7 \\
\hline 6 & $1.88 \mathrm{E}-04$ & $8.57 \mathrm{E}-15$ & $8.57 \mathrm{E}-15$ & 0.275 & 0.000 & 40 & 17 \\
\hline 7 & $1.49 \mathrm{E}-03$ & $2.10 \mathrm{E}-09$ & $2.10 \mathrm{E}-09$ & 2.189 & 62.620 & 39 & 38 \\
\hline 8 & $2.53 \mathrm{E}-03$ & 4.47E-08 & $4.47 \mathrm{E}-08$ & 3.706 & 1329.860 & 43 & 37 \\
\hline 9 & $9.03 \mathrm{E}-04$ & $6.04 \mathrm{E}-11$ & $6.04 \mathrm{E}-11$ & 1.320 & 1.794 & 8 & 9 \\
\hline 10 & 7.94E-04 & $4.29 \mathrm{E}-11$ & $4.29 \mathrm{E}-11$ & 1.161 & 1.275 & 7 & 19 \\
\hline 11 & $5.91 \mathrm{E}-04$ & $3.21 \mathrm{E}-11$ & $3.21 \mathrm{E}-11$ & 0.864 & 0.954 & 9 & 34 \\
\hline 12 & $5.22 \mathrm{E}-04$ & $3.09 \mathrm{E}-11$ & $3.09 \mathrm{E}-11$ & 0.763 & 0.920 & 38 & 10 \\
\hline 13 & $6.84 \mathrm{E}-04$ & $3.86 \mathrm{E}-11$ & $3.86 \mathrm{E}-11$ & 0.999 & 1.147 & 10 & 20 \\
\hline 14 & $6.84 \mathrm{E}-04$ & $3.61 \mathrm{E}-11$ & $3.61 \mathrm{E}-11$ & 0.999 & 1.073 & 37 & 30 \\
\hline 15 & $6.84 \mathrm{E}-04$ & $3.36 \mathrm{E}-11$ & $3.36 \mathrm{E}-11$ & 1.000 & 1.000 & 34 & 33 \\
\hline 16 & $6.89 \mathrm{E}-04$ & $3.36 \mathrm{E}-11$ & $3.36 \mathrm{E}-11$ & 1.007 & 1.000 & 33 & 13 \\
\hline 17 & $8.74 \mathrm{E}-03$ & $1.47 \mathrm{E}-09$ & $1.47 \mathrm{E}-09$ & 12.782 & 43.713 & 30 & 29 \\
\hline 18 & $3.01 \mathrm{E}-02$ & $3.85 \mathrm{E}-09$ & $3.85 \mathrm{E}-09$ & 44.069 & 114.672 & 29 & 14 \\
\hline 19 & $6.84 \mathrm{E}-04$ & $5.84 \mathrm{E}-11$ & $5.84 \mathrm{E}-11$ & 1.000 & 1.736 & 16 & 1 \\
\hline 20 & $6.84 \mathrm{E}-04$ & $4.26 \mathrm{E}-11$ & $4.26 \mathrm{E}-11$ & 1.000 & 1.267 & 15 & 15 \\
\hline 21 & $6.84 \mathrm{E}-04$ & $3.28 \mathrm{E}-11$ & $3.28 \mathrm{E}-11$ & 1.000 & 0.977 & 1 & 16 \\
\hline 22 & $6.84 \mathrm{E}-04$ & $2.88 \mathrm{E}-11$ & $2.88 \mathrm{E}-11$ & 1.00 & 0.857 & 19 & 23 \\
\hline 23 & $6.84 \mathrm{E}-04$ & $3.36 \mathrm{E}-11$ & $3.36 \mathrm{E}-11$ & 1.000 & 1.000 & 20 & 24 \\
\hline 24 & $6.84 \mathrm{E}-04$ & $3.36 \mathrm{E}-11$ & $3.36 \mathrm{E}-11$ & 1.000 & 1.000 & 21 & 25 \\
\hline 25 & $6.84 \mathrm{E}-04$ & $3.36 \mathrm{E}-11$ & $3.36 \mathrm{E}-11$ & 1.000 & 1.000 & 22 & 26 \\
\hline 26 & $6.84 \mathrm{E}-04$ & $3.36 \mathrm{E}-11$ & $3.36 \mathrm{E}-11$ & 1.000 & 1.000 & 23 & 28 \\
\hline 27 & $6.77 \mathrm{E}-04$ & $3.28 \mathrm{E}-11$ & $3.28 \mathrm{E}-11$ & 0.989 & 0.977 & 24 & 39 \\
\hline 28 & $6.84 \mathrm{E}-04$ & $3.36 \mathrm{E}-11$ & $3.36 \mathrm{E}-11$ & 0.999 & 1.000 & 25 & 40 \\
\hline 29 & $6.90 \mathrm{E}-04$ & $3.86 \mathrm{E}-11$ & $3.86 \mathrm{E}-11$ & 1.009 & 1.147 & 26 & 41 \\
\hline 30 & $6.91 \mathrm{E}-04$ & $4.09 \mathrm{E}-11$ & $4.09 \mathrm{E}-11$ & 1.011 & 1.216 & 14 & 42 \\
\hline 31 & $6.50 \mathrm{E}-04$ & $3.28 \mathrm{E}-11$ & $3.28 \mathrm{E}-11$ & 0.950 & 0.977 & 13 & 43 \\
\hline 32 & $6.75 \mathrm{E}-04$ & $3.28 \mathrm{E}-11$ & $3.28 \mathrm{E}-11$ & 0.987 & 0.977 & 28 & 44 \\
\hline 33 & $6.97 \mathrm{E}-04$ & $3.94 \mathrm{E}-11$ & $3.94 \mathrm{E}-11$ & 1.019 & 1.170 & 27 & 21 \\
\hline 34 & $7.20 \mathrm{E}-04$ & $4.42 \mathrm{E}-11$ & $4.42 \mathrm{E}-11$ & 1.052 & 1.313 & 32 & 27 \\
\hline 35 & $5.63 \mathrm{E}-04$ & $2.67 \mathrm{E}-11$ & $2.67 \mathrm{E}-11$ & 0.822 & 0.794 & 31 & 31 \\
\hline 36 & $6.14 \mathrm{E}-04$ & $3.13 \mathrm{E}-11$ & $3.13 \mathrm{E}-11$ & 0.897 & 0.931 & 36 & 32 \\
\hline 37 & $7.25 \mathrm{E}-04$ & $7.65 \mathrm{E}-10$ & $7.65 \mathrm{E}-10$ & 1.060 & 22.731 & 11 & 11 \\
\hline 38 & $8.34 \mathrm{E}-04$ & $7.69 \mathrm{E}-10$ & $7.69 \mathrm{E}-10$ & 1.219 & 22.874 & 35 & 36 \\
\hline 39 & $3.08 \mathrm{E}-03$ & $3.36 \mathrm{E}-11$ & $3.36 \mathrm{E}-11$ & 4.508 & 1.000 & 12 & 12 \\
\hline 40 & $6.50 \mathrm{E}-03$ & $3.36 \mathrm{E}-11$ & $3.36 \mathrm{E}-11$ & 9.508 & 1.000 & 6 & 22 \\
\hline 41 & $1.82 \mathrm{E}-05$ & $3.36 \mathrm{E}-11$ & $3.36 \mathrm{E}-11$ & 0.026 & 1.000 & 5 & 35 \\
\hline 42 & $1.24 \mathrm{E}-04$ & $3.36 \mathrm{E}-11$ & $3.36 \mathrm{E}-11$ & 0.182 & 1.000 & 42 & 6 \\
\hline 43 & $2.99 \mathrm{E}-03$ & $3.36 \mathrm{E}-11$ & $3.36 \mathrm{E}-11$ & 4.371 & 1.000 & 41 & 2 \\
\hline 44 & $8.06 \mathrm{E}-03$ & $3.36 \mathrm{E}-11$ & $3.36 \mathrm{E}-11$ & 11.785 & 1.000 & 2 & 5 \\
\hline
\end{tabular}




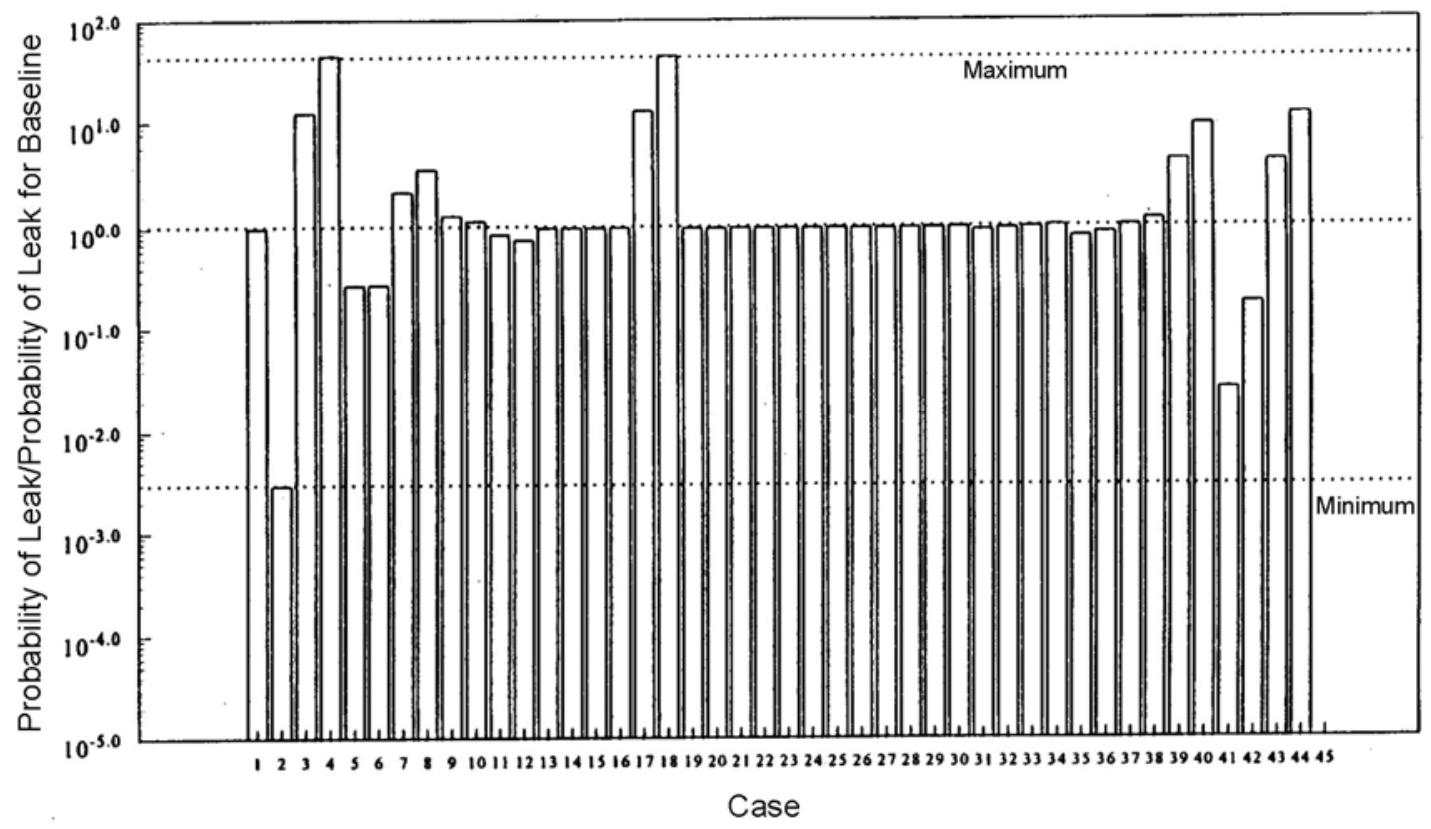

Figure 6.1 Ratio of Calculated Leak Probabilities from Sensitivity Calculations for $Q=100$

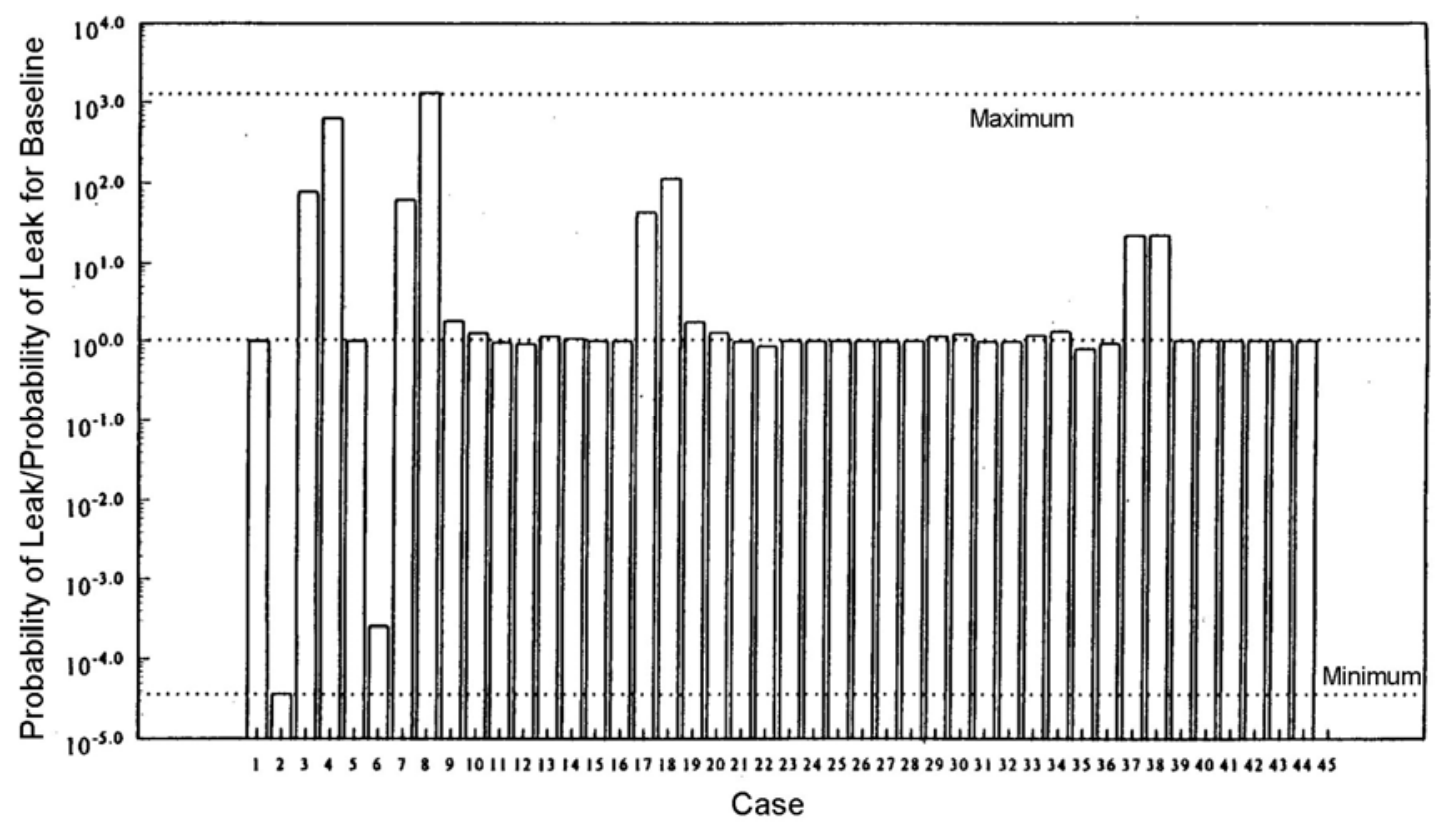

Figure 6.2 Ratio of Calculated Break Probabilities from Sensitivity Calculations for $Q=100$

Table 6.4 presents the conclusions of the sensitivity study in terms of the variables that have larger effects versus the variables that have smaller effects on calculated failure probabilities. Uncertainties in pipe inner radius, pipe-wall thickness, internal pressure, dead-weight load, and flow stress were determined to have relatively small effects on calculated failure probabilities. The flow stress of the pipe material was 
nevertheless included as one of the more important input parameters due to large uncertainties in the simplified fracture-mechanics model used to predict pipe breaks (Wilkowski et al. 1994). In this regard, the flow stress was a surrogate for uncertainties in the pc-PRAISE fracture-mechanics model.

Table 6.4 Variables Addressed in Sensitivity Analysis

\begin{tabular}{|l|l|}
\hline \multicolumn{1}{|c|}{$\begin{array}{c}\text { Variables with Larger Effects on } \\
\text { Calculated Failure Probabilities }\end{array}$} & \multicolumn{1}{|c|}{$\begin{array}{c}\text { Variables with Smaller Effects on } \\
\text { Calculated Failure Probabilities }\end{array}$} \\
\hline Flaw Depth & Pipe Wall Thickness \\
\hline Flaw Aspect Ratio & Pipe Inner Radius \\
\hline Fatigues Crack Growth Rate Constant & Internal Pressure \\
\hline Flow Stress for Pipe Material & Dead Weight Load \\
\hline Ultimate Strength for Pipe Material & \\
\hline Thermal Stress & \\
\hline Number of Stress Cycles & \\
\hline Cyclic Stress Level & \\
\hline
\end{tabular}

\subsection{Uncertainty Calculations}

The objective of the uncertainty analysis was to estimate the mean, median, and standard deviation of the calculated leak and break probabilities. We estimated these statistical parameters using Monte Carlo simulations on the variables described above. Many of the eight critical parameters of Table 6.4 are related to probabilistic inputs, whereas others (thermal stress, cyclic-stress level, and number of stress cycles) are deterministic inputs to pc-PRAISE. The uncertainty analyses required that both probabilistic and deterministic inputs be randomized as described below.

Calculations were performed for different sets of inputs obtained by sampling from triangular distributions that described the estimated uncertainties in the pc-PRAISE input parameters. Each Monte Carlo trial was a pc-PRAISE run that gave values of the failure probabilities. There were 100 trials for each of the three $Q$ values, which provided a sample of 100 failure probabilities from which means, medians, and standard deviations for the calculated probabilities were established.

Triangular distributions were appropriate because quantifying the uncertainties was based only on limited data in combination with engineering judgment. The triangular distribution assumes zero probability for values outside the bounding range of values and is not influenced by assumptions regarding tails of distributions. This distribution also accounts for the best-estimate values for the variables without implying detailed knowledge of the shape of the distribution function.

\subsubsection{Inputs for Calculations}

Table 6.5 summarizes the input data used for the baseline case. The following describes the uncertainties associated with each of the influential input parameters. 
Table 6.5 Input for Baseline Case of Uncertainty Analyses

\begin{tabular}{||l|c|l|c|c|l||}
\hline \multicolumn{1}{|c|}{ Name } & Symbol & $\begin{array}{c}\text { Distribution } \\
\text { Type }\end{array}$ & Median & $\begin{array}{c}\text { Shape } \\
\text { Parameter }\end{array}$ & \multicolumn{1}{c||}{ Basis } \\
\hline $\begin{array}{l}\text { Initial Flaw } \\
\text { Depth }\end{array}$ & $\mathrm{a}$ & $\mathrm{LN}$ & $0.09 \mathrm{in.}(2.29 \mathrm{~mm})$ & 0.24 & RRA weld simulation \\
\hline $\begin{array}{l}\text { Initial Aspect } \\
\text { Ratio }\end{array}$ & $\mathrm{b} / \mathrm{a}$ & $\mathrm{LN}(\beta>1)$ & 1.336 & 0.5382 & $\begin{array}{l}\text { Judgment; applications } \\
\text { of pc-PRAISE; RRA } \\
\text { weld simulations }\end{array}$ \\
\hline $\begin{array}{l}\text { Fatigue } \\
\text { Coefficient }\end{array}$ & $\mathrm{C}$ & $\mathrm{LN}$ & $\begin{array}{c}(9.14 \mathrm{E}-12 \mathrm{in} . / \mathrm{cycle}) \\
1.59 \mathrm{E}-13 \mathrm{~m} / \mathrm{cycle}\end{array}$ & 1.042 & $\begin{array}{l}\text { Data; prior applications } \\
\text { of pc-PRAISE }\end{array}$ \\
\hline Thermal Stress & $\sigma_{\text {th }}$ & Deterministic & $10 \mathrm{ksi}(69 \mathrm{MPa})$ & -- & Judgment \\
\hline $\begin{array}{l}\text { Number of } \\
\text { Cycles }\end{array}$ & $\mathrm{n} \mathrm{n}$ & Deterministic & $5 \mathrm{cycles} / \mathrm{yr}$ & -- & Judgment \\
\hline Cyclic Stress & $\Delta \sigma$ & Deterministic & $15 \mathrm{ksi}(103 \mathrm{MPa})$ & -- & Judgment \\
\hline $\begin{array}{l}\text { Fabrication } \\
\text { Flaws per Weld }\end{array}$ & $\mathrm{p}$ & Deterministic & $1.0 \mathrm{E}-02$ & -- & RRA weld simulation \\
\hline LN indicates lognormal. & & & \\
\hline
\end{tabular}

Flaw Depth (a): The uncertainty in the flaw-depth distribution was based on data from the RRA flaw distribution model (Chapman 1993; Chapman et al. 1996; Chapman and Simonen 1998; Khaleel et al. 1999) as indicated by the complementary lognormal distribution functions of Figure 6.3. These data based on different modeling assumptions showed that $\mathrm{P}(\mathrm{a}>90 \%$ of the wall thickness) can vary from $1.0 \mathrm{E}-08$ to $1.0 \mathrm{E}-15$, with the baseline case having $\mathrm{P}(\mathrm{a}>90 \% \mathrm{t})=1.0 \mathrm{E}-13$. The parameter " $\mathrm{a}$ " is the flaw depth and " $\mathrm{t}$ " is the thickness of the pipe wall.

As indicated in Figure 6.3, the RRA data showed that the median flaw depth was about the same for three cases, whereas the shape parameter $(\lambda)$ of the lognormal distribution had very different values. In modeling the uncertainty in the flaw depth, the calculations assumed that the shape parameter $(\lambda)$ was a randomly distributed variable with the baseline case being the mode of the triangular distribution. Table 6.6 gives parameters for the lognormal flaw-depth distribution for the three cases corresponding to the lower bound, best estimate, and upper bound of the parameter $\lambda$, which are Cases 1,2 , and 3 , respectively.

Flaw-Aspect Ratio $\boldsymbol{\beta}=\mathbf{b} / \mathbf{a}$ : The uncertainty in the distribution for $\beta$ was described by estimating a value for the probability that the flaw length-to-depth ratio exceeded 10. In pc-PRAISE, the $\operatorname{Prob}(\beta>5)$ is 1.0E-02. Data (Harris et al. 1981) have indicated that about $20 \%$ of the initial cracks have b/a $>5$. Therefore, the largest percentage of initial cracks with $b / a>5$ was estimated to be $20 \%$ and the smallest would be $1.0 \mathrm{E}-04$. The values of the median and shape parameter for $\mathrm{P}(\mathrm{b} / \mathrm{a}>5)$ equal to $1.0 \mathrm{E}-04$, 1.0E-02, and 0.20 are given in Table 6.7. Cases 1, 2, and 3 correspond to the lower bound, best estimate, and upper bound of the parameters $\beta$ and $\lambda$. The complementary distributions of the aspect ratio of the three cases are shown by Figure 6.4.

Fatigue Crack Growth: The fatigue crack-growth rate in the pc-PRAISE code is characterized by the following empirical relation: 


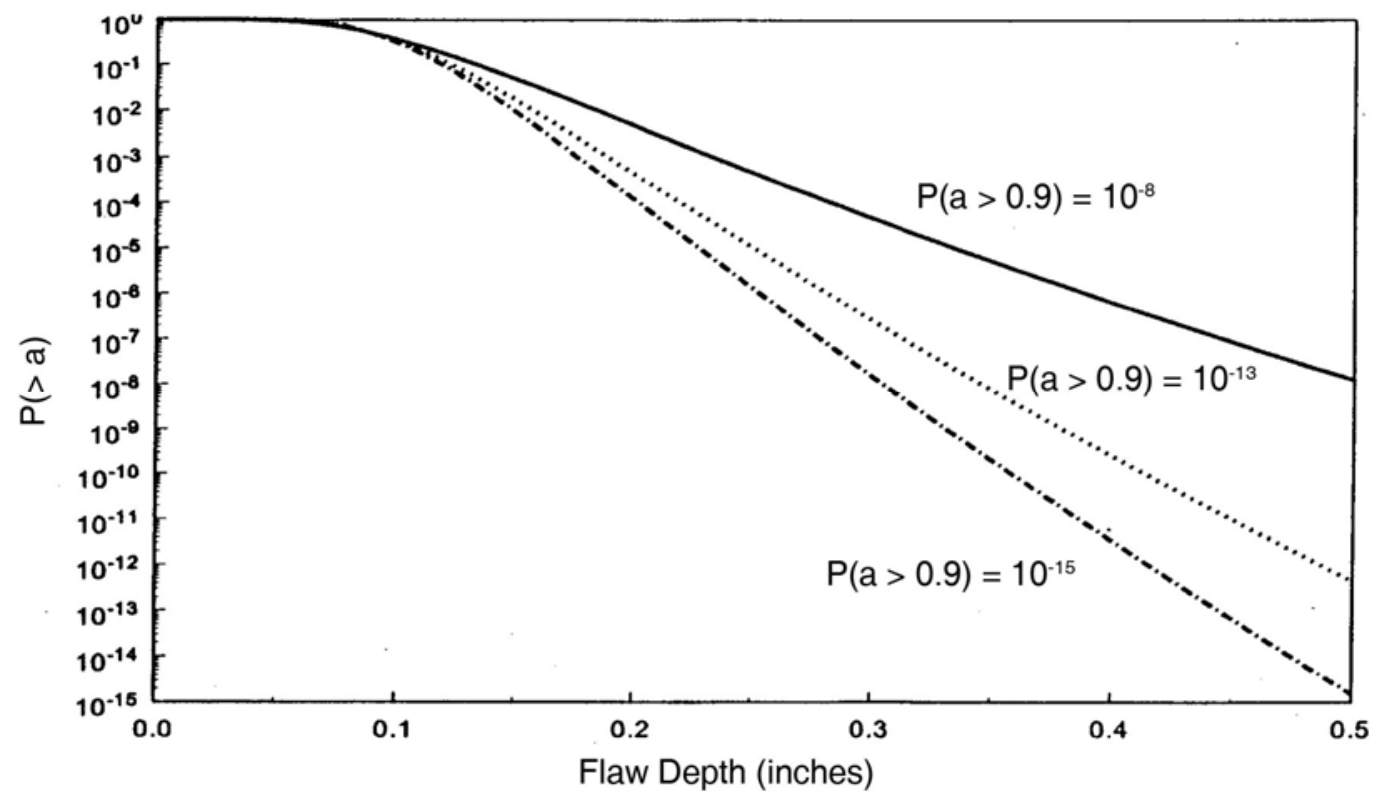

Figure 6.3 Complementary Cumulative Flaw Depth Distribution Indicating Range for RRA Model $(1 \mathrm{inch}=25.4 \mathrm{~mm})$

Table 6.6 Parameters for Lognormal Distribution of Flaw Depth

\begin{tabular}{|c|c|c|c||}
\hline Case Number & $\mathbf{P}(\mathbf{a}>\mathbf{9 0} \% \mathbf{t})$ & $\mathbf{a}_{\mathbf{5 0}}$ in. $\left.\mathbf{( m m}\right)$ & $\boldsymbol{\lambda}$ \\
\hline 1 & $1.0 \mathrm{E}-15$ & $0.09(2.29)$ & 0.218 \\
\hline 2 & $1.0 \mathrm{E}-13$ & $0.09(2.29)$ & 0.24 \\
\hline 3 & $1.0 \mathrm{E}-08$ & $0.09(2.29)$ & 0.308 \\
\hline
\end{tabular}

Table 6.7 Parameters for Lognormal Distribution of Flaw Aspect Ratio

\begin{tabular}{|c|c|c|c||}
\hline Case Number & $\mathbf{P}(\mathbf{b} / \mathbf{a}>\mathbf{5})$ & $\beta$ & $\lambda$ \\
\hline 1 & $1.0 \mathrm{E}-04$ & 1.162 & 0.387 \\
\hline 2 & $1.0 \mathrm{E}-02$ & 1.337 & 0.539 \\
\hline 3 & 0.2 & 2.124 & 0.868 \\
\hline
\end{tabular}

The parameters $\mathrm{C}$ and $\mathrm{m}$ are empirical constants, and $\mathrm{K}$ ' is the effective crack-tip stress-intensity factor. Scatter in the fatigue data is accounted for by considering the coefficient $\mathrm{C}$ to be randomly distributed in accordance with a lognormal distribution. The scatter in $\mathrm{da} / \mathrm{dN}$ is relatively well characterized from extensive test data. For the baseline case, the probability that $\mathrm{C}>3.5 \mathrm{E}-11$ is $10 \%$. To describe the uncertainty in $\mathrm{C}$, it was assumed that $\mathrm{P}(\mathrm{C}>3.5 \mathrm{E}-11)$ equals $5 \%, 10 \%$, and $15 \%$. Figure 6.5 shows the 
complementary cumulative distribution for these three cases. Numerical values for the lognormal distribution (median values and shape parameters) are indicated in Table 6.8 along with 90th and 95th percentile of the constant C. Cases 1, 2, and 3 correspond to the lower bound, best estimate, and upper bound of the parameter $\lambda$.

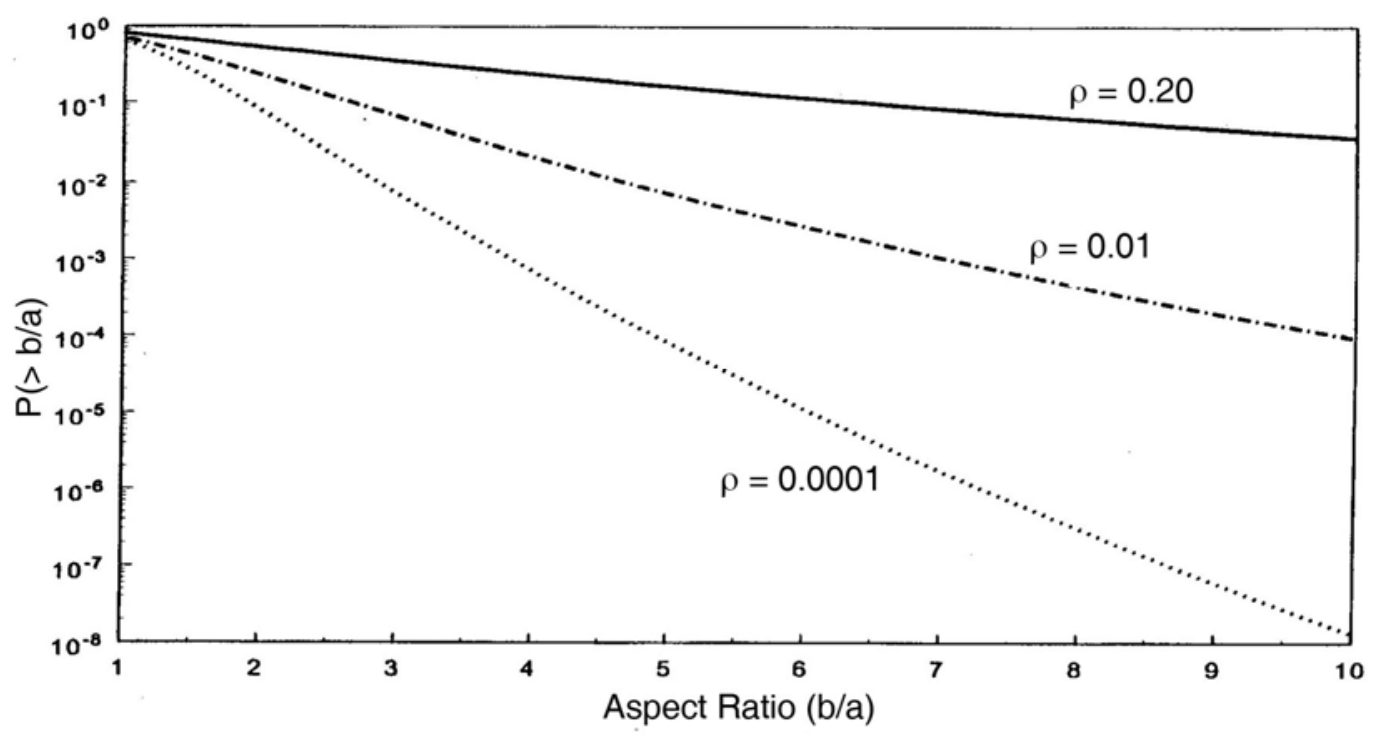

Figure 6.4 Complementary Cumulative Flaw Aspect Ratio Distribution $(a=$ flaw depth, $2 \mathrm{~b}=$ flaw length)

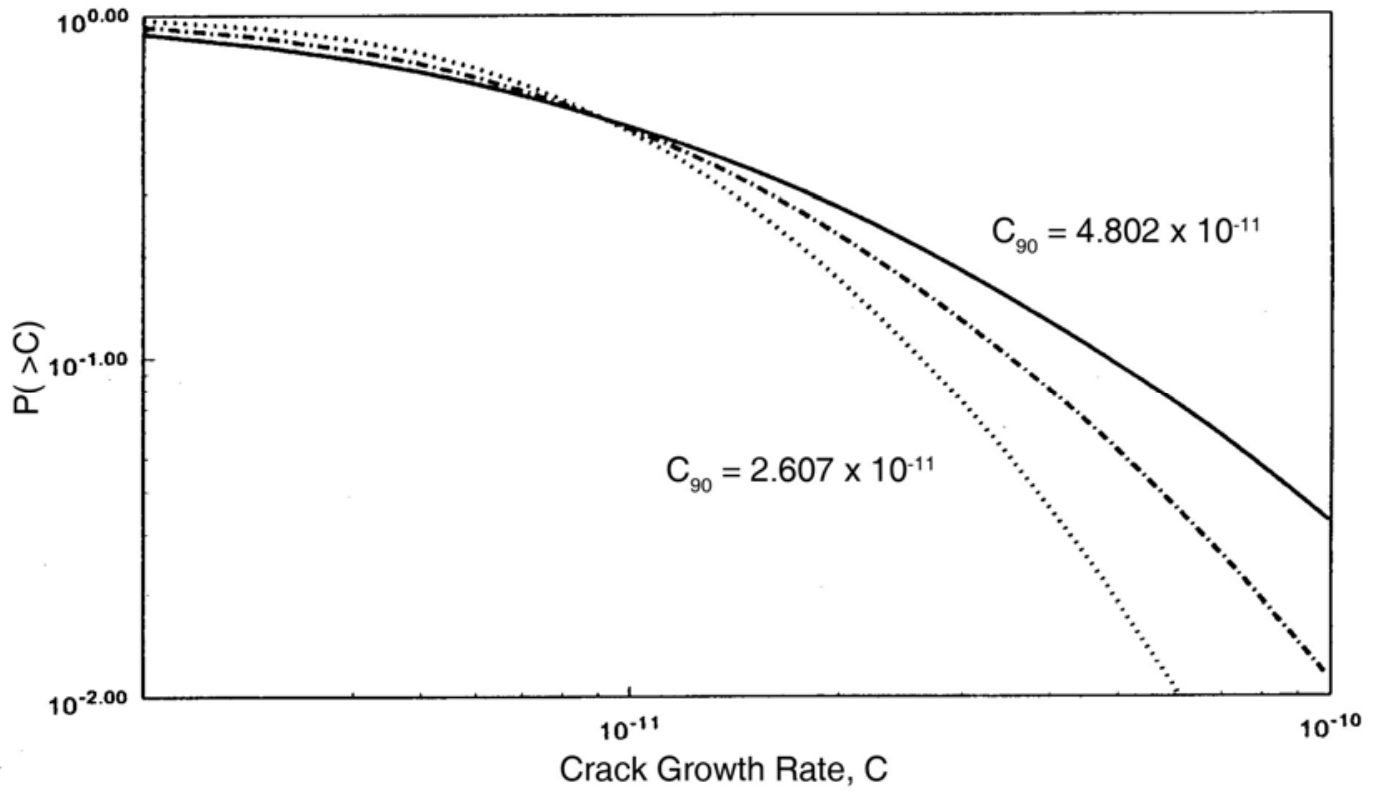

Figure 6.5 Complementary Cumulative Distribution for Fatigue Crack Growth Rate Parameter 
Table 6.8 Parameters for Lognormal Distribution of Fatigue Crack Growth Parameter C

\begin{tabular}{|c|c|c|c|c|c|}
\hline $\begin{array}{c}\text { Case } \\
\text { Number }\end{array}$ & $\begin{array}{c}P>3.5 \mathrm{E}-11 \text { in./cycle } \\
\text { or } P>6.1 \mathrm{E}-13 \mathrm{~m} / \text { cycle } \\
(\%)\end{array}$ & $\lambda$ & $\begin{array}{c}\mathrm{C}_{50}, \text { in./cycle } \\
\text { (m/cycle) }\end{array}$ & $\begin{array}{c}\mathrm{C}_{90}, \text { in./cycle } \\
(\mathrm{m} / \text { cycle })\end{array}$ & $\begin{array}{c}\mathrm{C}_{95}, \text { in./cycle } \\
\text { (m/cycle) }\end{array}$ \\
\hline 1 & 0.05 & 0.82 & $\begin{array}{c}9.14 \mathrm{E}-12 \\
(1.59 \mathrm{E}-13)\end{array}$ & $\begin{array}{l}2.607 \mathrm{E}-11 \\
(4.53 \mathrm{E}-13)\end{array}$ & $\begin{array}{l}3.507 \mathrm{E}-11 \\
(6.10 \mathrm{E}-13)\end{array}$ \\
\hline 2 & 0.1 & 1.05 & $\begin{array}{c}9.14 \mathrm{E}-12 \\
(1.59 \mathrm{E}-13)\end{array}$ & $\begin{array}{l}3.501 \mathrm{E}-11 \\
(6.09 \mathrm{E}-13)\end{array}$ & $\begin{array}{l}5.118 \mathrm{E}-11 \\
(8.90 \mathrm{E}-13)\end{array}$ \\
\hline 3 & 0.15 & 1.29 & $\begin{array}{c}9.14 \mathrm{E}-12 \\
(1.59 \mathrm{E}-13)\end{array}$ & $\begin{array}{l}4.802 \mathrm{E}-11 \\
(8.35 \mathrm{E}-13)\end{array}$ & $\begin{array}{l}7.677 \mathrm{E}-11 \\
(1.33 \mathrm{E}-12)\end{array}$ \\
\hline
\end{tabular}

Thermal and Cyclic Stresses: The thermal stress was assumed to be lognormally distributed with a median of $10 \mathrm{ksi}(69 \mathrm{MPa})$ and a 0.2 shape parameter. The median and shape parameters for the cyclic stress were $15 \mathrm{ksi}(103 \mathrm{MPa})$ and 0.2 .

Number of Cycles: The number of cycles for every year of the 40-year life was 5 cycles/year and was assumed to follow Poisson's distribution.

Number of Flaws/Weld: The number of flaws per weld was represented by a triangular distribution having a mode, minimum, and maximum of 1.0E-02, 1.0E-03, and 1.0E-01.

Flow Stress: The flow stress was described by a triangular distribution. The mode, minimum, and maximum were 60,45 , and $75 \mathrm{ksi}(414,310$, and $517 \mathrm{MPa})$, respectively.

\subsubsection{Computational Approach}

Monte Carlo simulations sampled from the uncertainty distributions and generated 100 sets of random inputs for the pc-PRAISE runs. A separate Monte Carlo computer code was written to sample from the uncertainty distributions.

Each pc-PRAISE run assumed one flaw per weld and provided conditional leak and break probabilities. The uncertainty associated with the best estimate flaw density ( 0.01 flaws per weld) was then evaluated to obtain a distribution of unconditional leak and break probabilities. The final step was to evaluate and interpret the distribution of 100 failure probabilities. Mean and median values of the distributions of 100 failure probabilities were calculated for comparison with the corresponding probabilities from bestestimate calculations.

\subsection{Results of Uncertainty Calculations}

There were 100 pc-PRAISE runs for each of the three $Q$ values. This gave a total of 300 sets of pipingfailure probabilities (leaks and breaks) as a function time, with the time span going from the start of plant operation to the end-of-life at 40 years. To keep the amount of data at a manageable level, the focus was limited to cumulative failure probabilities at 40 years. The results of the computer runs for conditional probabilities, corresponding to the assumption that one pre-existing crack exists in the weld, were then tabulated. 
Figures 6.6 and 6.7 show example histograms (leak and break probabilities for $\mathrm{Q}=100$ ) along with the best-estimate probabilities. From the 100 calculated values of leak and break probabilities, it was possible to calculate mean and median values for comparison with the corresponding values from the best-estimate calculation from pc-PRAISE. Table 6.9 gives the results from these evaluations.

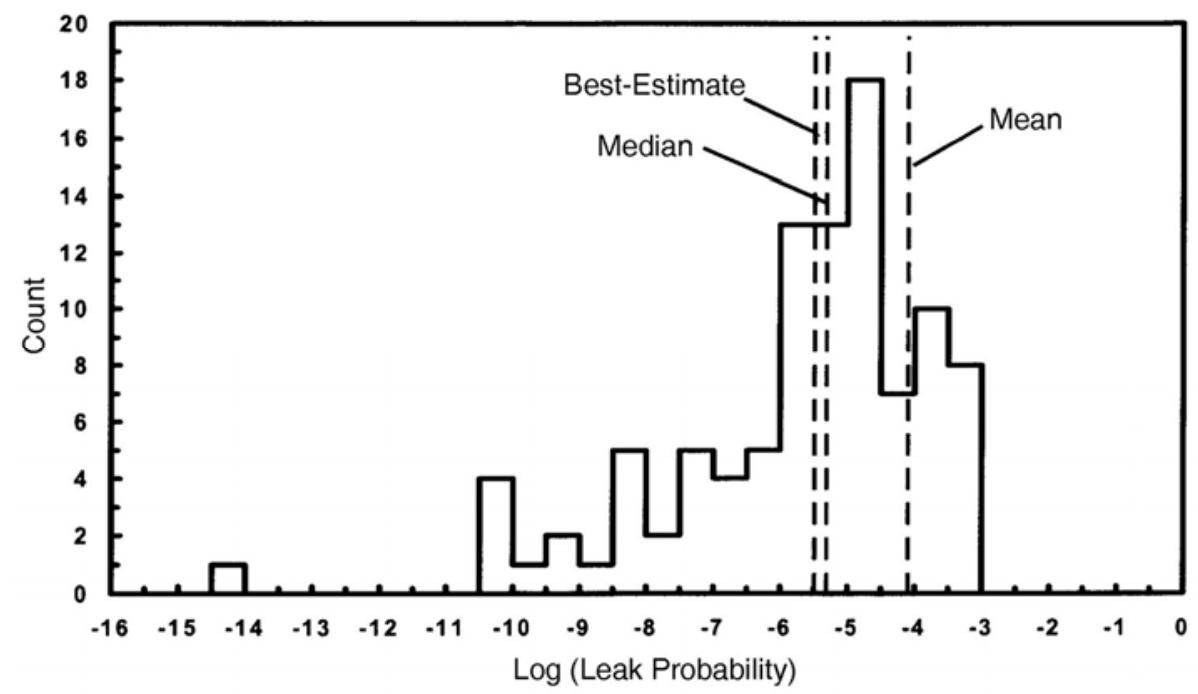

Figure 6.6 Histogram for Probability of Leak for $Q=100$

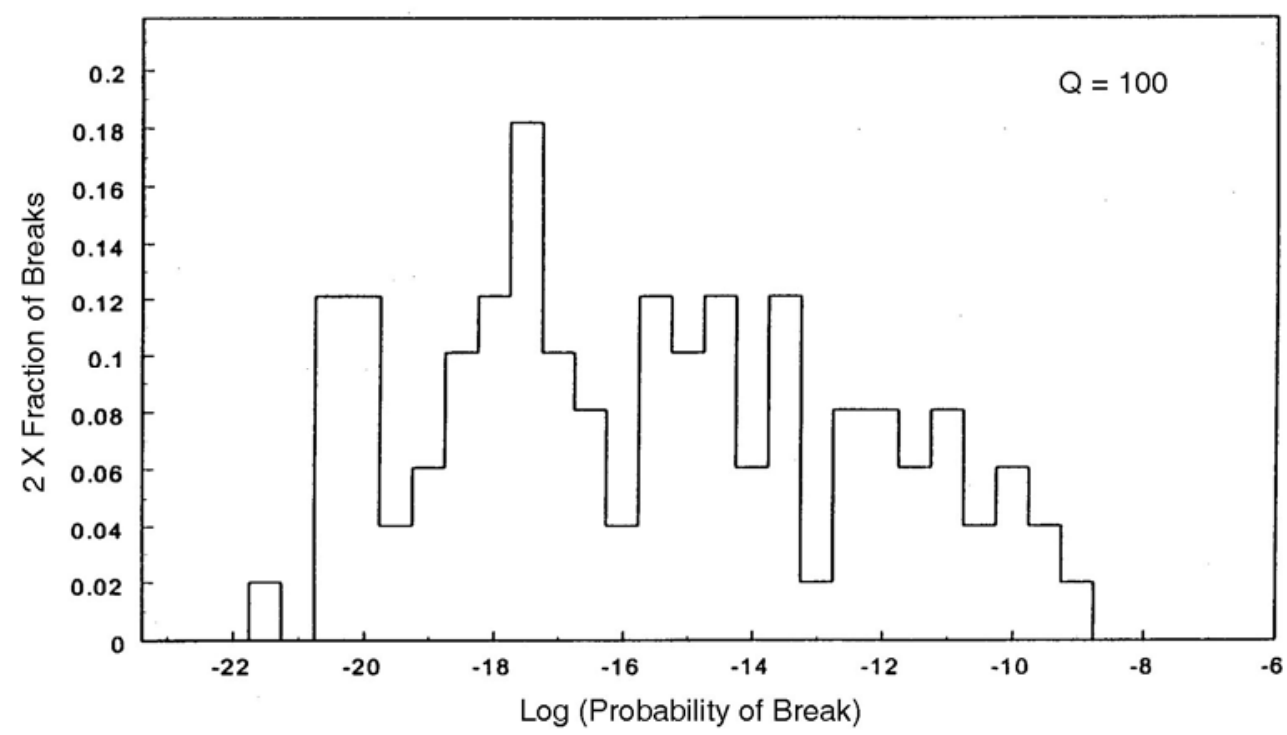

Figure 6.7 Histogram for Probability of Break for $Q=100$ 
Table 6.9 Descriptive Statistics for Leak and Break Probabilities

\begin{tabular}{|c|l|c|c|c|c||}
\hline Q-Value & \multicolumn{1}{|c|}{ Probability } & $\begin{array}{c}\text { Best } \\
\text { Estimate }\end{array}$ & Mean & Median & $\begin{array}{c}\text { Standard } \\
\text { Deviation }\end{array}$ \\
\hline 1 & Conditional Leak & $7.20 \mathrm{E}-13$ & $8.85 \mathrm{E}-10$ & $4.49 \mathrm{E}-12$ & $3.99 \mathrm{E}-09$ \\
\hline & Conditional Break & $4.11 \mathrm{E}-16$ & $2.53 \mathrm{E}-12$ & $1.49 \mathrm{E}-16$ & $1.49 \mathrm{E}-11$ \\
\hline & Leak & $7.20 \mathrm{E}-15$ & $2.46 \mathrm{E}-11$ & $1.47 \mathrm{E}-13$ & $1.07 \mathrm{E}-10$ \\
\hline & Break & $4.11 \mathrm{E}-18$ & $7.34 \mathrm{E}-14$ & $7.84 \mathrm{E}-18$ & $4.11 \mathrm{E}-13$ \\
\hline 100 & Conditional Leak & $3.24 \mathrm{E}-04$ & $1.87 \mathrm{E}-03$ & $1.33 \mathrm{E}-04$ & $4.39 \mathrm{E}-03$ \\
\hline & Conditional Break & $2.89 \mathrm{E}-15$ & $8.81 \mathrm{E}-10$ & $3.92 \mathrm{E}-15$ & $4.79 \mathrm{E}-09$ \\
\hline & Leak & $3.24 \mathrm{E}-06$ & $7.90 \mathrm{E}-05$ & $4.93 \mathrm{E}-06$ & $1.79 \mathrm{E}-04$ \\
\hline & Break & $2.89 \mathrm{E}-17$ & $3.04 \mathrm{E}-11$ & $2.52 \mathrm{E}-16$ & $1.47 \mathrm{E}-10$ \\
\hline 10000 & Conditional Leak & $7.17 \mathrm{E}-01$ & $6.10 \mathrm{E}-01$ & $6.43 \mathrm{E}-01$ & $2.33 \mathrm{E}-01$ \\
\hline & Conditional Break & $4.83 \mathrm{E}-11$ & $4.00 \mathrm{E}-07$ & $4.10 \mathrm{E}-10$ & $3.02 \mathrm{E}-06$ \\
\hline & Leak & $7.17 \mathrm{E}-03$ & $3.03 \mathrm{E}-02$ & $2.50 \mathrm{E}-02$ & $2.15 \mathrm{E}-02$ \\
\hline & Break & $4.83 \mathrm{E}-13$ & $1.86 \mathrm{E}-08$ & $1.02 \mathrm{E}-11$ & $1.39 \mathrm{E}-07$ \\
\hline
\end{tabular}

Figure 6.8 summarizes the results from all of the uncertainty analyses. Whereas there are relatively small differences between the best-estimate and median values of leak probabilities, Figure 6.8 shows more significant differences for mean values. The mean values for leak probabilities are about a factor of ten greater than the best estimate. The mean values for break probabilities are several orders of magnitude greater than the best estimate. It is noted that

- Median values of probabilities correlate relatively well with the best estimates.

- Differences between best-estimate and mean values for leak probabilities are 1 to 3 orders of magnitude, and 4 to 6 orders of magnitude for break probabilities.

- The greater uncertainties for pipe-break probabilities compared to leak probabilities are largely due to the sensitivity of calculations to inputs for flaw depths and aspect ratios. There are little data to support the estimates for the low occurrence rates for very deep and long flaws. Estimates of probabilities for these rare defects are based on extrapolations having high levels of uncertainty.

- The uncertainties in calculated leak probabilities become relatively small for leak probabilities greater than 1.0E-04. Given a 40-year operating life for a reactor piping system, this 1.0E-04 cumulative probability corresponds to a failure frequency of 2.5E-06 leaks per weld per year. The high levels of uncertainty for calculated leak probabilities less than 1.0E-04 is not of great importance because piping with such low failure rates would generally fall below levels that would contribute significantly to plant risk. 


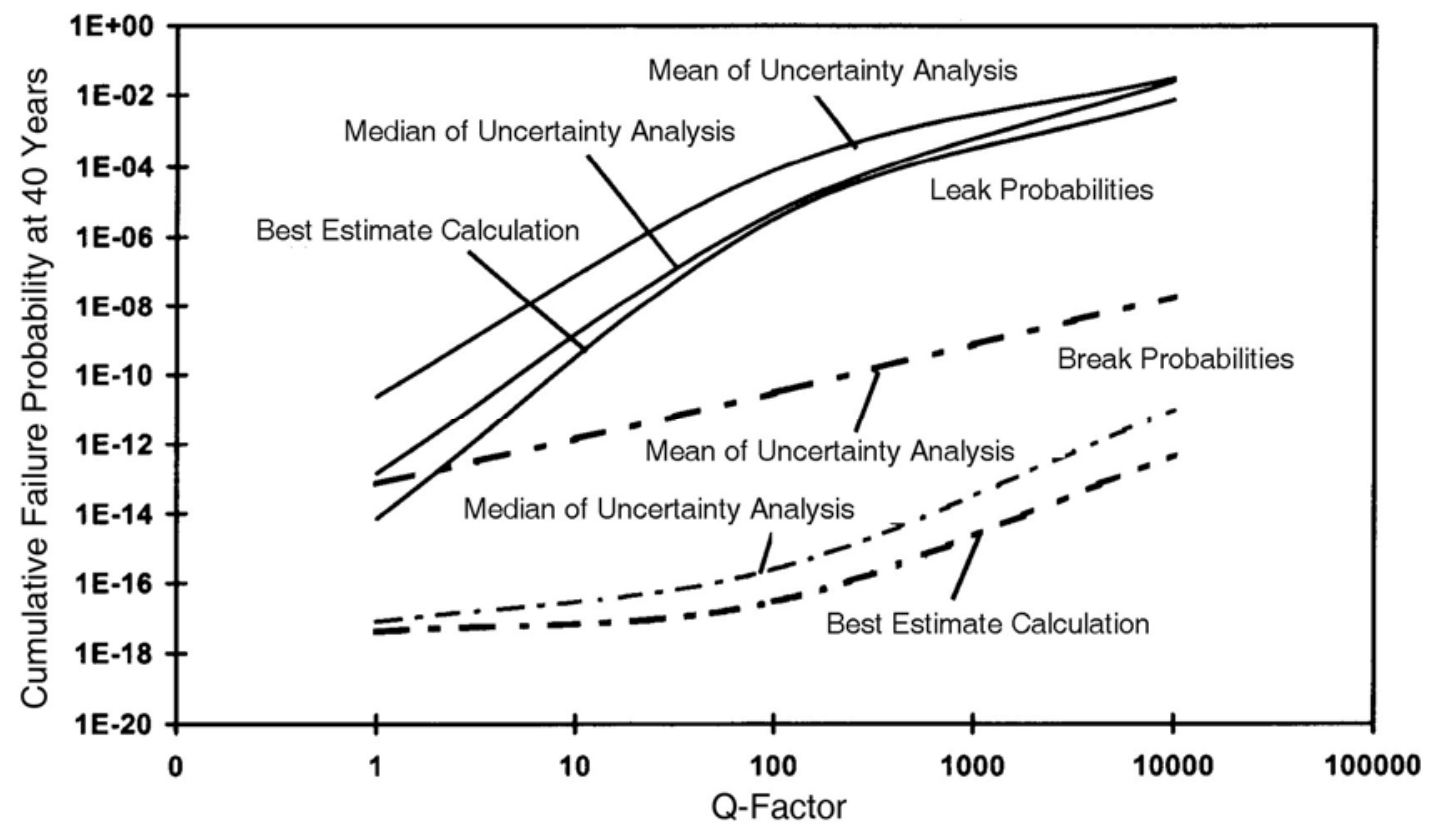

Figure 6.8 Comparisons of Probabilities from Uncertainty Analyses with Best Estimate Calculations

\subsection{Generalization of Uncertainty Calculations}

The present calculations of piping-failure probabilities were intended for use as inputs to a much larger uncertainty analysis that involved contributions of piping failures to core-damage frequencies. As such, the uncertainties of the present paper were to be used as inputs to a probabilistic risk assessment, which was to address all the uncertainties in the risk evaluation. Therefore, it was desirable to identify some broad trends from the present calculations that could be extrapolated to other piping sizes and service conditions.

Figures 6.9 and 6.10 show the results from the 300 pc-PRAISE runs plotted in a normalized format. These plots give cumulative distributions of leak and break probabilities, with all 100 probabilities for each case normalized to the median probability. These data can be further summarized (Figure 6.11) as a ratio of the maximum probability (i.e., largest of the 100 calculated values) to the median probability, and this ratio can be plotted as a function of the median probability.

Figure 6.11 shows that the scatter in leak probabilities and break probabilities follows similar trends. When this common trend is described by a linear fit of the six data points of Figure 6.11, a high-level summary of the uncertainty calculations can be developed as indicated in Figure 6.12. In developing this plot, it was assumed that the median and best-estimate failure probabilities are equal. It is noted that 


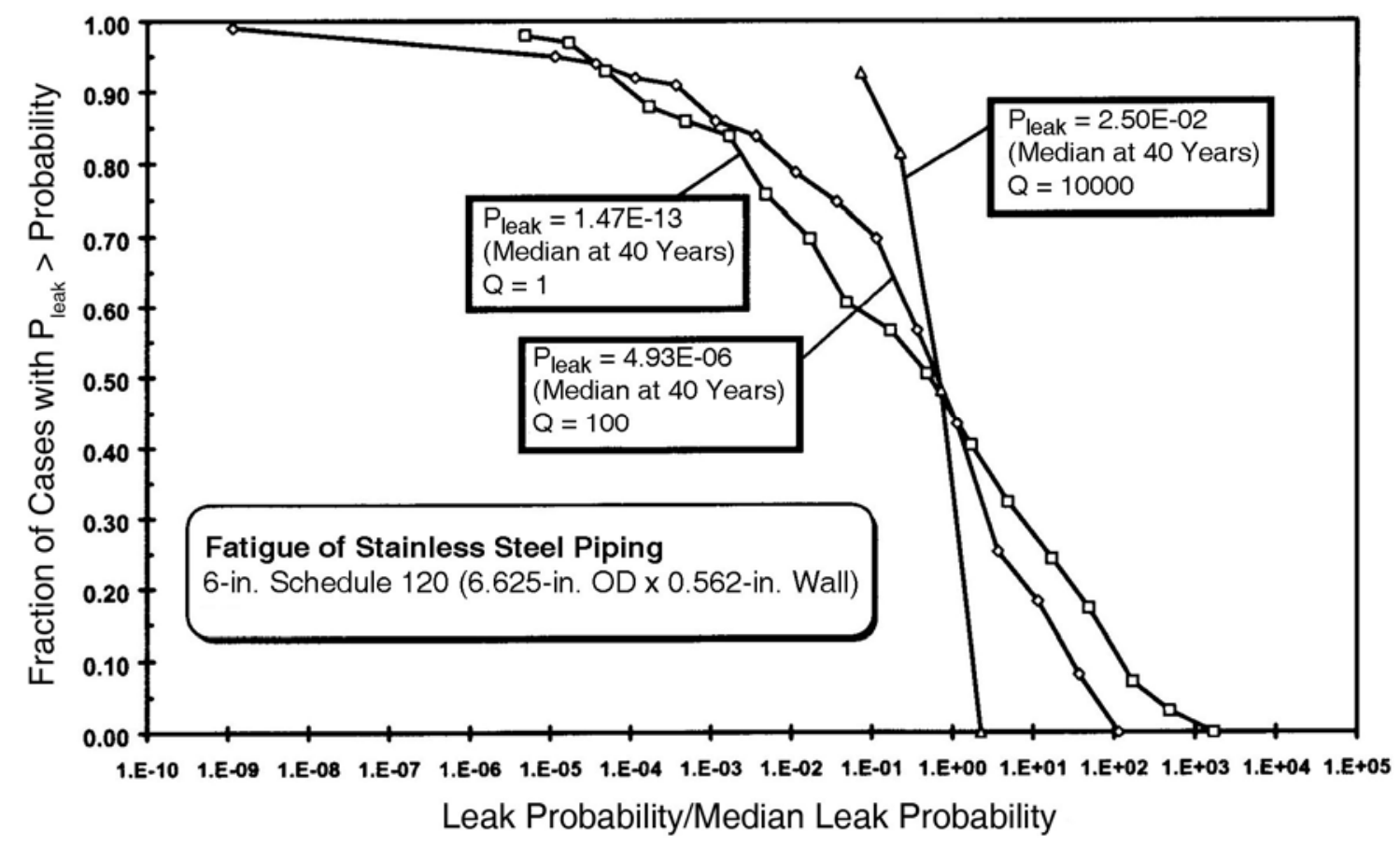

Figure 6.9 Complementary Cumulative Distributions of Calculated Leak Probabilities $(\mathrm{Q}=1$, 100, 10,000) $(1$ inch $=25.4 \mathrm{~mm})$

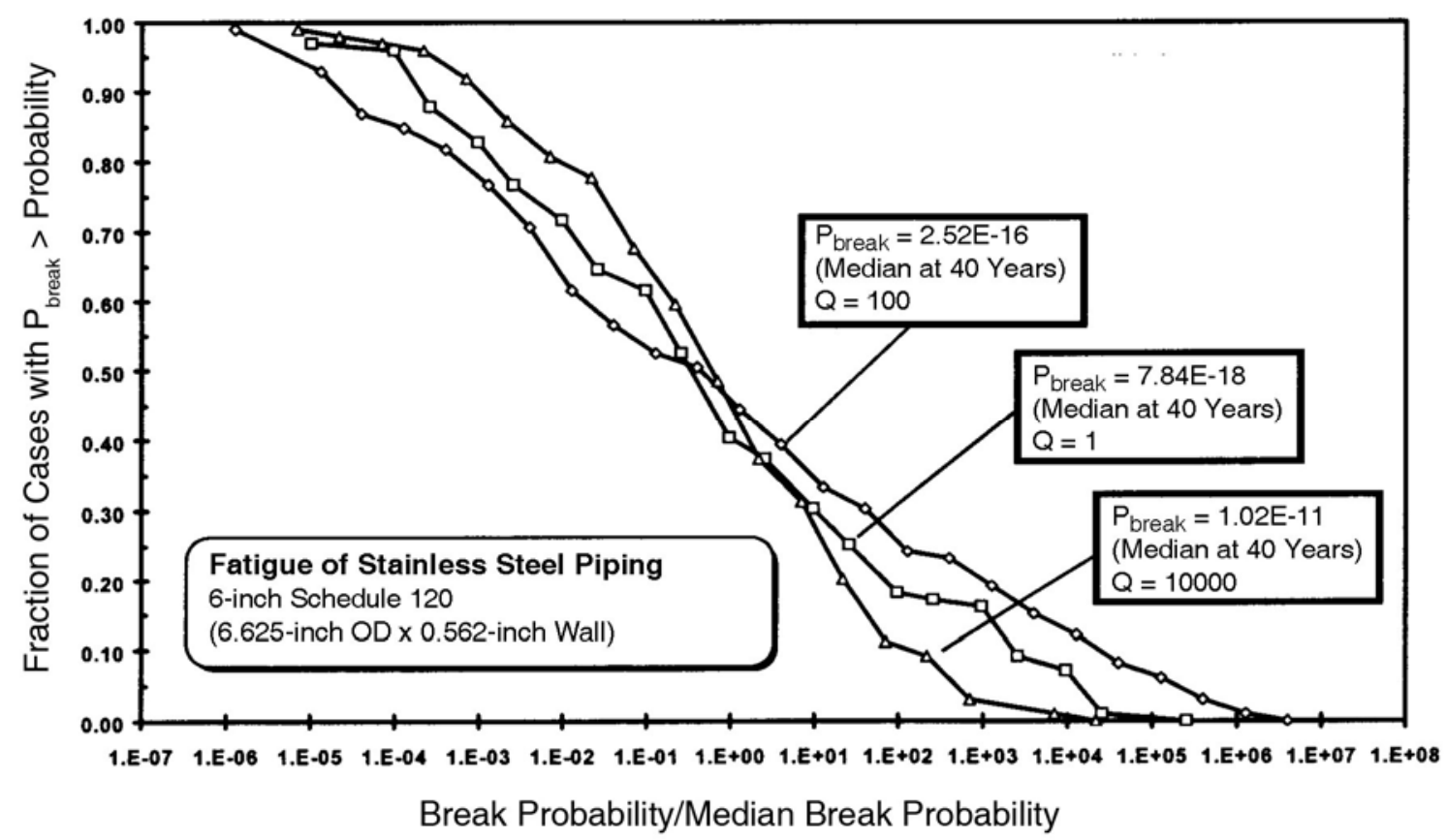

Figure 6.10 Complementary Cumulative Distributions of Calculated Break Probabilities $(\mathrm{Q}=1$, $100,10,000)(1$ inch $=25.4 \mathrm{~mm})$ 


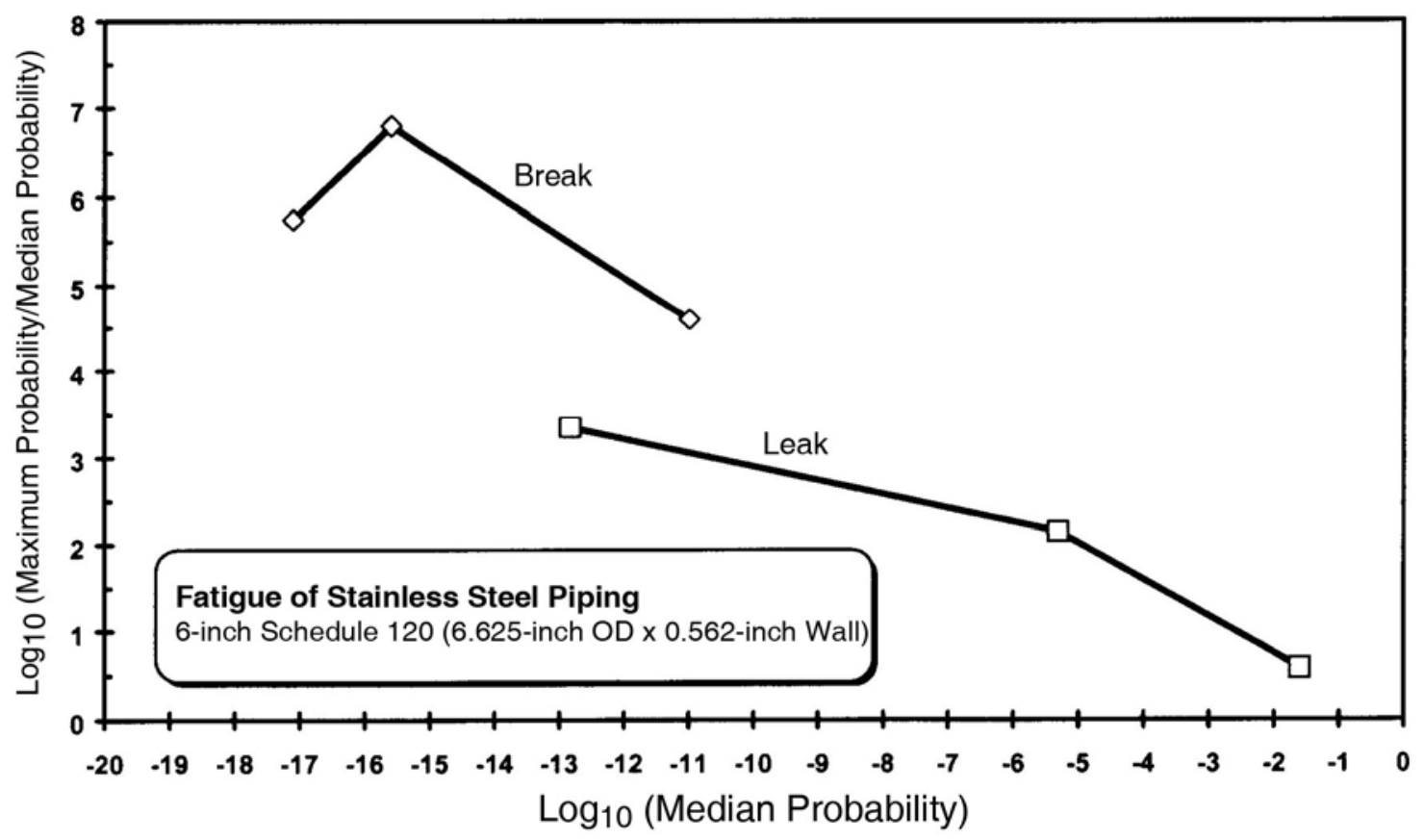

Figure 6.11 Maximum of Leak and Break Probabilities from Sample of 100 Cases Evaluated by Uncertainty Analyses (1 inch $=25.4 \mathrm{~mm}$ )

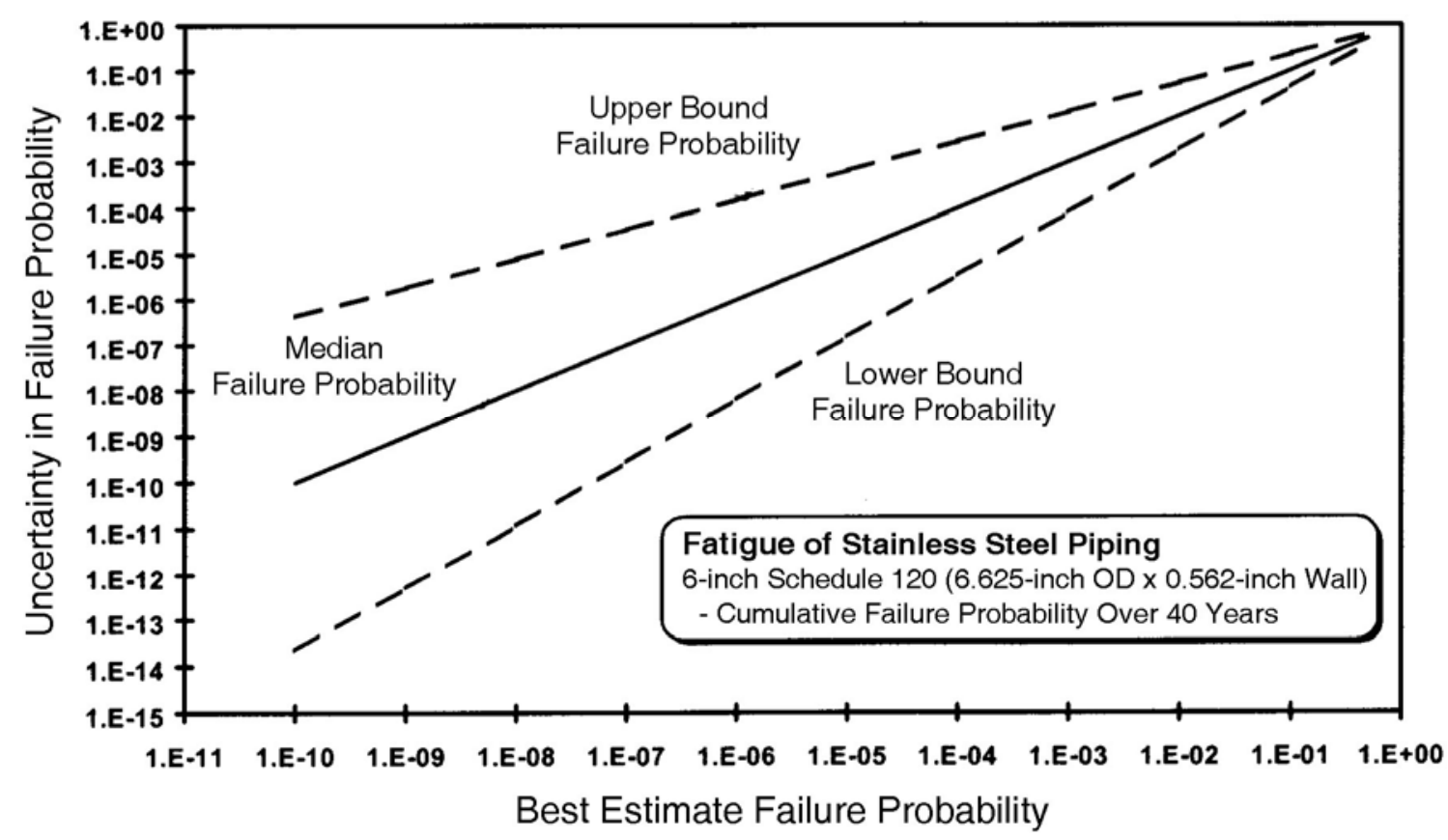

Figure 6.12 Upper and Lower Bounds of Leak and Break Probabilities from Uncertainty Analyses $(1$ inch $=25.4 \mathrm{~mm})$ 
(1) the uncertainties in calculated failure probabilities are highest when the probabilities themselves are very low (e.g., 1.0E-08 per weld per 40-year life) and are much smaller when the failure probabilities become larger (e.g., 1.0E-02)

(2) large uncertainties for components with low failure probabilities should have relatively small impacts on conclusions of risk-based assessments, because risk-based decisions are focused on components with the higher values of failure probabilities

(3) failure probabilities for components with higher calculated failure probabilities can be more readily checked for consistency with plant operating experience; the ability to make such comparisons helps to minimize the uncertainties in these calculated probabilities.

\subsection{Conclusion}

A methodology has been presented for quantifying the level of uncertainty associated with calculated failure probabilities for piping components. The results clearly show (as expected) large uncertainties in calculated failure probabilities, with the uncertainties being particularly large for break probabilities. The median values of leak probabilities from the uncertainty calculations generally agree (within an order of magnitude) with the best-estimate calculations. This correlation supports the viability of using bestestimate calculations for risk-informed decision making. Initial flaw-size distributions were the greatest source of uncertainty in calculated failure probabilities because of the unavoidable difficulty in estimating the very low probabilities for the large fabrication flaws, which (if present) have a major impact on piping integrity. Finally, it should be noted that any future uncertainty analyses should address other pipe sizes, piping materials, and failure mechanisms. 


\section{Effects of Flaw-Sizing Errors on the Reliability of Vessels and Piping}

\subsection{Introduction}

The nuclear power industry has continued to upgrade the quality of UT inspections by addressing the key elements needed for effective inspections, namely personnel, procedures, and equipment. Central to these efforts has been the qualification of inspection teams before field inspections, whereby the teams must demonstrate acceptable performance in detecting and sizing defects during trial inspections of degraded piping samples. These efforts have resulted in significant enhancement of inservice inspection (ISI) effectiveness through changes to ASME Section XI Code, Appendix VIII (Cowfer 1989).

This section describes PFM calculations that simulate fatigue crack growth, flaw detection, flaw-sizing accuracy, and the impacts of flaw-acceptance criteria. A numerical implementation based on a Latin Hypercube approach was applied in calculations, which have been performed for a range of parameters. These were used to obtain representative values of flaw-detection probability, flaw-sizing errors, and flaw-acceptance criteria.

Performance demonstrations have addressed two measures of NDE reliability-POD and flaw-sizing accuracy. Both measures impact the ability of an inspection program to enhance the reliability of piping. Previous papers by the present authors (Woo and Simonen 1984; Simonen 1990; Khaleel and Simonen 1994a, 1994b, 1995; Khaleel et al. 1995) have described PFM calculations that have evaluated the impact inspections with various POD levels (i.e., POD as a function of flaw size) on the ability of inservice and preservice inspections to reduce leak and rupture probabilities of reactor piping.

Flaw detection with a high level of reliability is an important step to ensure that ISIs will reduce the failure probabilities of the inspected piping locations. However, inspections can impact piping reliability only if the detected flaws are repaired or other corrective actions are performed. Significant undersizing of flaws could cause incorrect repair decisions. In some cases flaws could be incorrectly classified as benign if their measured sizes are less than the sizes of the governing flaw-acceptance criteria.

This section describes calculations that address flaw-sizing errors in numerical simulations of alternative inspection scenarios. These calculations are applicable to mechanical and thermal fatigue of stainless steel piping. In addition to flaw-sizing errors, the uncertainties addressed in the PFM model are

- the numbers of initial flaws

- the distribution of flaw sizes

- the crack growth rates for the flaws

- POD curves

- frequency of the inspection

- flaw-acceptance criteria. 
The calculations predict inspection effectiveness for a range of cyclic operating stresses that give crackgrowth rates ranging from high to low. Inspection strategies address a selection of reference POD curves, a range of flaw-sizing accuracies, and a standard inspection interval of 10 years.

\subsection{PFM Model}

A computer code (Khaleel and Simonen 1995) for predicting vessel and piping-failure probabilities due to fatigue crack growth was modified in the present study to include the simulation of flaw-sizing errors as part of the existing model for flaw detection. Features of this PFM code are summarized as follows.

The fracture mechanics model assumes that piping failures are due to initial fabrication flaws that can grow by fatigue to become through-wall leaking cracks. In the numerical implementation of the model, the initial flaw sizes, the flaw locations within the pipe wall, and the parameters governing fatigue crackgrowth rates are treated as stochastic variables.

Buried flaws are assumed to grow by fatigue in accordance with air data, whereas the growth of surface flaws is governed by data for water environments. Buried flaws are taken to be distributed randomly within the wall thickness with only those flaws having inner tips located within the inner half of the wall thickness assumed to contribute to the failure probability. Once the inner tip of a buried flaw grows and penetrates the inner surface of the pipe, the model treats the flaw as a surface-breaking flaw that grows in accordance with crack growth rates for a water environment.

A Latin Hypercube approach (Khaleel and Simonen 1995) is used to permit efficient numerical calculations, with the stochastic variables described by histograms that represent the statistical distributions of the variables. This numerical approach permits calculations of very small failure probabilities, which would otherwise require a large computational effort using conventional Monte Carlo approaches.

\subsection{Inservice Inspection Model}

The parameters of the ISI model are as follows:

- POD Curve

- Flaw-Sizing Error

- ISI Interval

- Flaw-Acceptance Criteria.

The probability of flaw detection is assumed to be a function of the flaw depth as given by the following POD function (Harris and Dedhia 1992):

$$
\mathrm{P}_{\mathrm{ND}}=\varepsilon+\frac{1}{2}(1-\varepsilon) \operatorname{erfc}\left[v \ln \left(a / a^{*}\right)\right]
$$

where $\mathrm{P}_{\mathrm{ND}}$ is the probability of nondetection, $\mathrm{a}$ is the depth of the crack, $\mathrm{a}^{*}$ is the depth of the crack for $50 \% \mathrm{P}_{\mathrm{ND}}, \varepsilon$ is the smallest possible $\mathrm{P}_{\mathrm{ND}}$ for very large crack depths, and $v$ is the "slope" of the $\mathrm{P}_{\mathrm{ND}}$ curve. 
Based on the work of Harris and Dedhia (1992), a value of $v=1.6$ was used for all the calculations of the present paper. The values of $\varepsilon$ were correlated with the values of $a^{*}$ such that smaller values of a* imply smaller values of $\varepsilon$.

Data on flaw-sizing errors are available from a number of studies involving inspection trials, including the PISC-II trials (Nichols and Crutzen 1988) and piping trials for stress corrosion cracks (EPRI 1989). For the present calculations, the sizing errors (i.e., the difference between measured crack depth and the true crack depth) were described by uniform distributions. Two independent input parameters to the model permit a sizing error to be prescribed in terms of a maximum undersizing error and a maximum oversizing error.

All calculations of this paper assume equal errors for undersizing and oversizing. The calculations also assumed that the sizing errors were independent of the true flaw depths, although the model also has an option to allow the sizing errors to be prescribed as a fraction of the true flaw depths. The sizing errors were also constrained such that flaws are never sized at (physically impossible) negative depths.

The calculations assumed that inspections were performed at time intervals consistent with current ASME Section XI requirements (i.e., a 10-year inspection interval with the first inspection performed during the fifth year of operation). The effects of performing inspections at time intervals other than 10 years have been evaluated in prior studies as reported, for example, in Khaleel and Simonen (1994a,b).

The detections (or nondetections) of a growing flaw for a series of periodic inspections were assumed to be dependent (or correlated) events. In the numerical simulations, this assumption of dependent outcomes was implemented by taking credit only for one of the series of periodic inspections (i.e., the final inspection just before the flaw would become through-wall).

The final parameter described by the inspection model was the flaw-acceptance criteria. In this regard, meaningful calculations for sizing errors must also address flaw-acceptance criteria. In the present model, it was assumed that flaws with depths less than the acceptance criteria will not be repaired, whereas all flaws with greater measured depths will be repaired. The limiting case of an acceptance criteria of zero flaw depth (i.e., all flaws are repaired) means that flaw-sizing errors have no impact on piping reliability, whereas a criteria with a very large acceptable flaw depth (no repairs even for relatively large flaws) would preclude any benefits of ISIs.

\subsection{Simulation of Flaw-Sizing Errors}

An existing computer code was modified to include the simulation of flaw-sizing errors. The overall logic and computational details of the original code has been described in a prior paper (Khaleel and Simonen 1995). The original code assumed that all welds having detected flaws were repaired with defect-free material. The modified code addresses cases for which repairs are not made. These cases correspond to flaws that are undersized to such an extent that weld repairs are not performed.

The computational approach simulates flaw-sizing errors through an adjustment of the curve that gives the POD as a function of flaw depth. The POD value for each flaw depth is multiplied by a correction factor that is a function of the flaw-sizing error and the maximum acceptable flaw depth. This factor has the following characteristics: 
(1) The correction factor is assigned a value of 1.0 if the maximum possible error (in the direction of undersizing) will still result in a measured flaw size greater than the flaw-acceptance criteria.

(2) The correction factor is assigned a value of 0.0 if the maximum possible error (in the direction of oversizing) will result in a measured flaw size less than the flaw-acceptance criteria.

(3) The correction factor can have values between 0.0 and 1.0 for cases not covered by the special conditions of 1 and 2, with this factor being calculated as follows:

$$
\text { FACTOR }=\frac{\text { DMAX }- \text { AMAX }}{\text { SSMAX }+ \text { SSMIN }}
$$

where

$$
\begin{aligned}
\text { DMIN } & =\text { DEPTH }- \text { SSMIN } \\
\text { DMAX } & =\text { DEPTH }- \text { SSMAX } \\
\text { DEPTH } & =\text { True Flaw Depth } \\
\text { AMAX } & =\text { Maximum Allowable Crack Depth (Repair Criteria) } \\
\text { SSMIN } & =\text { Maximum Undersizing Error (from Uniform Distribution) } \\
\text { SSMAX } & =\text { Maximum Oversizing Error (from Uniform Distribution). }
\end{aligned}
$$

Lacking a sufficient source of detailed experimental data on flaw-sizing errors to develop a statistically based distribution function, a uniform distribution of sizing errors was assumed. That is, the measured depth of a given flaw with a true depth of "DEPTH" had possible measured depths ranging from "DMIN" to "DMAX" as indicated by the above equations.

It is interesting to note that flaw-sizing errors can (in theory) improve the effectiveness of a particular inspection for cases of small flaws that are smaller than the flaw-acceptance criteria. Such flaws may be oversized to the extent that a repair is performed for flaws that would otherwise be accepted by ASME Section XI. However, such occurrences will seldom impact piping reliability because these small flaws will usually be too small to contribute significantly to piping-failure probabilities.

\subsection{Inputs to PFM Calculations}

Calculations have simulated inspections for both piping and vessel components. These components include a ferritic steel reactor pressure vessel (100-in. [2540-mm] inner radius and 10.0-in. [254-mm] wall thickness) and a 6-in. (152.4-mm) Schedule 120 stainless steel pipe (2.75-in. [69.8-mm] inner radius and 0.562 -in. [14.3-mm] wall thickness).

The mean values of fatigue crack-growth rates were assumed to be as described by the ASME Section XI curves for ferritic steels (air and reactor water environments). As in the pc-PRAISE code (Harris and Dedhia 1992), a lognormal distribution described the uncertainties in fatigue crack growth rates. Table 7.1 gives the discrete representation of the lognormal distribution in terms of a factor applied to the mean crack growth rates of the ASME code. 
Table 7.1 Distribution Function for Fatigue Crack Growth Rate Factors

\begin{tabular}{|c|c|c|c|c|c|}
\hline $\begin{array}{c}\text { Factor on } \\
\text { Crack Growth } \\
\text { Rate }\end{array}$ & Probability & $\begin{array}{c}\text { Factor on } \\
\text { Crack Growth } \\
\text { Rate }\end{array}$ & Probability & $\begin{array}{c}\text { Factor on } \\
\text { Crack Growth } \\
\text { Rate }\end{array}$ & Probability \\
\hline 0.0032 & $3.20 \mathrm{E}-14$ & 0.2480 & $1.73 \mathrm{E}-02$ & 10.2000 & $7.73 \mathrm{E}-04$ \\
\hline 0.0046 & $1.24 \mathrm{E}-12$ & 0.2990 & $2.67 \mathrm{E}-02$ & 12.2000 & $3.44 \mathrm{E}-04$ \\
\hline 0.0067 & $3.38 \mathrm{E}-11$ & 0.3600 & $3.88 \mathrm{E}-02$ & 14.7200 & $1.45 \mathrm{E}-04$ \\
\hline 0.0097 & $9.46 \mathrm{E}-10$ & 0.4330 & $5.30 \mathrm{E}-02$ & 17.7000 & $5.63 \mathrm{E}-05$ \\
\hline 0.0127 & $3.66 \mathrm{E}-09$ & 0.5220 & $6.79 \mathrm{E}-02$ & 21.3000 & $2.09 \mathrm{E}-05$ \\
\hline 0.0153 & $1.43 \mathrm{E}-08$ & 0.6280 & $8.19 \mathrm{E}-02$ & 25.7000 & $7.28 \mathrm{E}-06$ \\
\hline 0.0185 & $5.97 \mathrm{E}-08$ & 0.7570 & $9.27 \mathrm{E}-02$ & 30.9000 & $2.38 \mathrm{E}-06$ \\
\hline 0.0223 & $2.08 \mathrm{E}-07$ & 0.9110 & $9.87 \mathrm{E}-02$ & 37.2000 & $7.30 \mathrm{E}-07$ \\
\hline 0.0268 & $7.30 \mathrm{E}-07$ & 1.0970 & $9.87 \mathrm{E}-02$ & 44.8000 & $2.08 \mathrm{E}-07$ \\
\hline 0.0343 & $2.38 \mathrm{E}-06$ & 1.3200 & $9.27 \mathrm{E}-02$ & 53.9000 & $5.97 \mathrm{E}-08$ \\
\hline 0.0389 & $7.28 \mathrm{E}-06$ & 1.5900 & $8.19 \mathrm{E}-02$ & 64.9000 & $1.43 \mathrm{E}-08$ \\
\hline 0.0468 & $2.09 \mathrm{E}-05$ & 1.9140 & $6.79 \mathrm{E}-02$ & 78.2000 & $3.66 \mathrm{E}-09$ \\
\hline 0.0564 & $5.63 \mathrm{E}-05$ & 2.3000 & $5.30 \mathrm{E}-02$ & 103.3000 & $9.46 \mathrm{E}-10$ \\
\hline 0.0678 & $1.45 \mathrm{E}-04$ & 2.7700 & $3.88 \mathrm{E}-02$ & 150.0000 & $3.38 \mathrm{E}-11$ \\
\hline 0.0817 & $3.44 \mathrm{E}-04$ & 3.3300 & $2.67 \mathrm{E}-02$ & 217.0000 & $1.24 \mathrm{E}-12$ \\
\hline 0.0983 & $7.73 \mathrm{E}-04$ & 4.0200 & $1.73 \mathrm{E}-02$ & 314.0000 & $3.20 \mathrm{E}-14$ \\
\hline 0.1180 & $1.63 \mathrm{E}-03$ & 4.8400 & $1.05 \mathrm{E}-02$ & & \\
\hline 0.1420 & $3.23 \mathrm{E}-03$ & 5.8200 & $6.19 \mathrm{E}-03$ & & \\
\hline 0.1710 & $6.19 \mathrm{E}-03$ & 7.0100 & $3.23 \mathrm{E}-03$ & & \\
\hline 0.2060 & $1.05 \mathrm{E}-02$ & 8.4400 & $1.63 \mathrm{E}-03$ & & \\
\hline
\end{tabular}

Tables 7.2 and 7.3 give the discrete distributions of flaw depth for the vessel and pipe examples. Flaws in the vessel had a longitudinal orientation, whereas the pipe flaws had a circumferential orientation. All calculations assumed that the flaws were "long," which implied a full $360^{\circ}$ circumferential flaw for the piping calculations. The flaw density was one flaw per weld for all the calculations. Stresses for both the vessel and piping examples correspond to a simple situation of pressure cycling from zero to $2250 \mathrm{psi}$ $(15.5 \mathrm{MPa})$ at rates of 5, 20, and 200 cycles per year.

Inspection scenarios (as indicated in Table 7.4) were limited to a single ISI interval of 10 years, but addressed a wide range of assumed flaw-detection capabilities, sizing errors, and flaw-acceptance criteria. This involved a rather large matrix of calculations. This section presents only sample results from the full matrix. 
Table 7.2 Flaw-Size Distribution for Reactor Pressure Vessel

\begin{tabular}{|c|c|c||}
\hline Crack Depth (in.) & Crack Depth (mm) & Probability \\
\hline 0.125 & 3.2 & $8.336 \mathrm{E}-01$ \\
\hline 0.250 & 6.3 & $1.617 \mathrm{E}-01$ \\
\hline 0.500 & 12.7 & $4.167 \mathrm{E}-03$ \\
\hline 1.000 & 25.4 & $4.167 \mathrm{E}-03$ \\
\hline 1.500 & 38.1 & $1.333 \mathrm{E}-04$ \\
\hline 2.000 & 50.8 & $4.167 \mathrm{E}-05$ \\
\hline 2.500 & 63.5 & $1.333 \mathrm{E}-05$ \\
\hline 3.000 & 76.2 & $5.330 \mathrm{E}-06$ \\
\hline 3.500 & 88.9 & $3.330 \mathrm{E}-06$ \\
\hline
\end{tabular}

Table 7.3 Flaw-Size Distribution for 6-in. (152-mm) Schedule 120 Pipe

\begin{tabular}{|c|c|c|}
\hline Crack Depth (in.) & Crack Depth (mm) & Probability \\
\hline 0.005 & 0.13 & $1.540 \mathrm{E}-01$ \\
\hline 0.015 & 0.38 & $1.280 \mathrm{E}-01$ \\
\hline 0.025 & 0.63 & $1.100 \mathrm{E}-01$ \\
\hline 0.035 & 0.89 & $9.400 \mathrm{E}-02$ \\
\hline 0.045 & 1.14 & $7.800 \mathrm{E}-02$ \\
\hline 0.055 & 1.40 & $6.700 \mathrm{E}-02$ \\
\hline 0.070 & 1.78 & $1.040 \mathrm{E}-01$ \\
\hline 0.090 & 2.29 & $7.500 \mathrm{E}-02$ \\
\hline 0.110 & 2.79 & $5.400 \mathrm{E}-02$ \\
\hline 0.140 & 3.56 & $6.600 \mathrm{E}-02$ \\
\hline 0.180 & 4.57 & $3.340 \mathrm{E}-02$ \\
\hline 0.225 & 5.71 & $2.010 \mathrm{E}-02$ \\
\hline 0.275 & 6.98 & $8.770 \mathrm{E}-03$ \\
\hline 0.325 & 8.25 & $3.802 \mathrm{E}-03$ \\
\hline 0.375 & 9.52 & $1.655 \mathrm{E}-03$ \\
\hline 0.425 & 10.79 & $7.200 \mathrm{E}-04$ \\
\hline 0.475 & 12.06 & $3.130 \mathrm{E}-04$ \\
\hline 0.531 & 13.49 & $1.550 \mathrm{E}-04$ \\
\hline
\end{tabular}

\subsection{Results of Example Calculations}

Results are presented to show how flaw-sizing errors impact the ability of ISIs to reduce leak probabilities. All calculations assumed an interval of 10 years between periodic inspections. The calculations only addressed leak probabilities (e.g., through-wall crack probabilities) and not catastrophic break probabilities. The parameters addressed by the calculations were as follows: 
Table 7.4 Matrix of Inservice Inspection Scenarios

\begin{tabular}{|c|c|c|}
\hline Input Parameter & Vessel & $\begin{array}{c}\text { 6-in. (152.4 mm) Schedule } \\
120 \text { Pipe }\end{array}$ \\
\hline $\begin{array}{l}\text { Probability of Detection } \\
\mathrm{a}^{*}=\text { Flaw Depth for } 50 \% \text { POD, in. }(\mathrm{mm}) \\
\varepsilon=\text { Probability of Nondetection for Very Deep } \\
\quad \text { Flaw }\end{array}$ & $\begin{array}{l}\mathrm{a}^{*}=0.125(3.2) ; \varepsilon=0.001 \\
\mathrm{a}^{*}=0.250(6.35) ; \varepsilon=0.010 \\
\mathrm{a}^{*}=0.500(12.7) ; \varepsilon=0.100 \\
\mathrm{a}^{*}=1.000(25.4) ; \varepsilon=0.250\end{array}$ & $\begin{array}{l}\mathrm{a}^{*}=0.028(0.71) ; \varepsilon=0.005 \\
\mathrm{a}^{*}=0.084(2.13) ; \varepsilon=0.020 \\
\mathrm{a}^{*}=0.225(5.71) ; \varepsilon=0.100\end{array}$ \\
\hline Flaw Sizing Error, in. $(\mathrm{mm})$ & $\begin{array}{l} \pm 0.000( \pm 0.0) \\
\pm 0.125( \pm 3.2) \\
\pm 0.250( \pm 6.35) \\
\pm 0.500( \pm 12.7) \\
\pm 0.750( \pm 19.0) \\
\pm 1.000( \pm 25.4) \\
\pm 1.500( \pm 38.1)\end{array}$ & $\begin{array}{l} \pm 0.000( \pm 0.00) \\
\pm 0.028( \pm 0.71) \\
\pm 0.056( \pm 1.42) \\
\pm 0.112( \pm 2.84) \\
\pm 0.281( \pm 7.14)\end{array}$ \\
\hline Flaw Acceptance Criteria, in. (mm) & $\begin{array}{l}0.00(0.0) \\
0.25(6.35) \\
0.50(12.7) \\
1.00(25.4) \\
1.50(38.1) \\
\text { "Large"(a) }\end{array}$ & $\begin{array}{l}0.000(0.00) \\
0.056(1.42) \\
0.112(2.84) \\
0.281(7.1) \\
\text { "Large"(a) }\end{array}$ \\
\hline
\end{tabular}

- Component Size (e.g., large reactor vessel versus small diameter pipe)

- Number of Stress Cycles (giving low versus high leak probabilities)

- Flaw Locations (surface-breaking versus buried flaws)

- Probability of Flaw Detection

- Flaw-Sizing Error

- Flaw-Acceptance Standard.

\subsubsection{Vessel Calculations}

One set of calculations addressed a vessel with an inner radius of $100 \mathrm{in.}(2540 \mathrm{~mm})$ and a wall thickness of $10 \mathrm{in} .(254 \mathrm{~mm})$ with the flaws having axial orientations. Figure 7.1 provides baseline results that assumed that the flaw was located at the inner surface of the vessel. All detected flaws were assumed to be repaired, which implied no effect of flaw-sizing errors on the vessel leak probabilities. The results of Figure 7.1 considered two POD curves (with the flaws having a 50 percent probability corresponding to $\mathrm{a}^{*}=0.25$ and 0.50 in. [6.35 and $\left.12.7 \mathrm{~mm}\right]$ ). A reference case assumed no inspection. The number of pressure cycles per year was varied from 10 to 1000 to address a wide range of leak probabilities.

Figure 7.1 indicates that the more sensitive of the two POD curves ("very good" POD) can reduce the calculated leak probabilities by as much as two orders of magnitude compared to the case of no ISI. In contrast, the "Good" POD curve reduces the leak probabilities by only about one order of magnitude. 


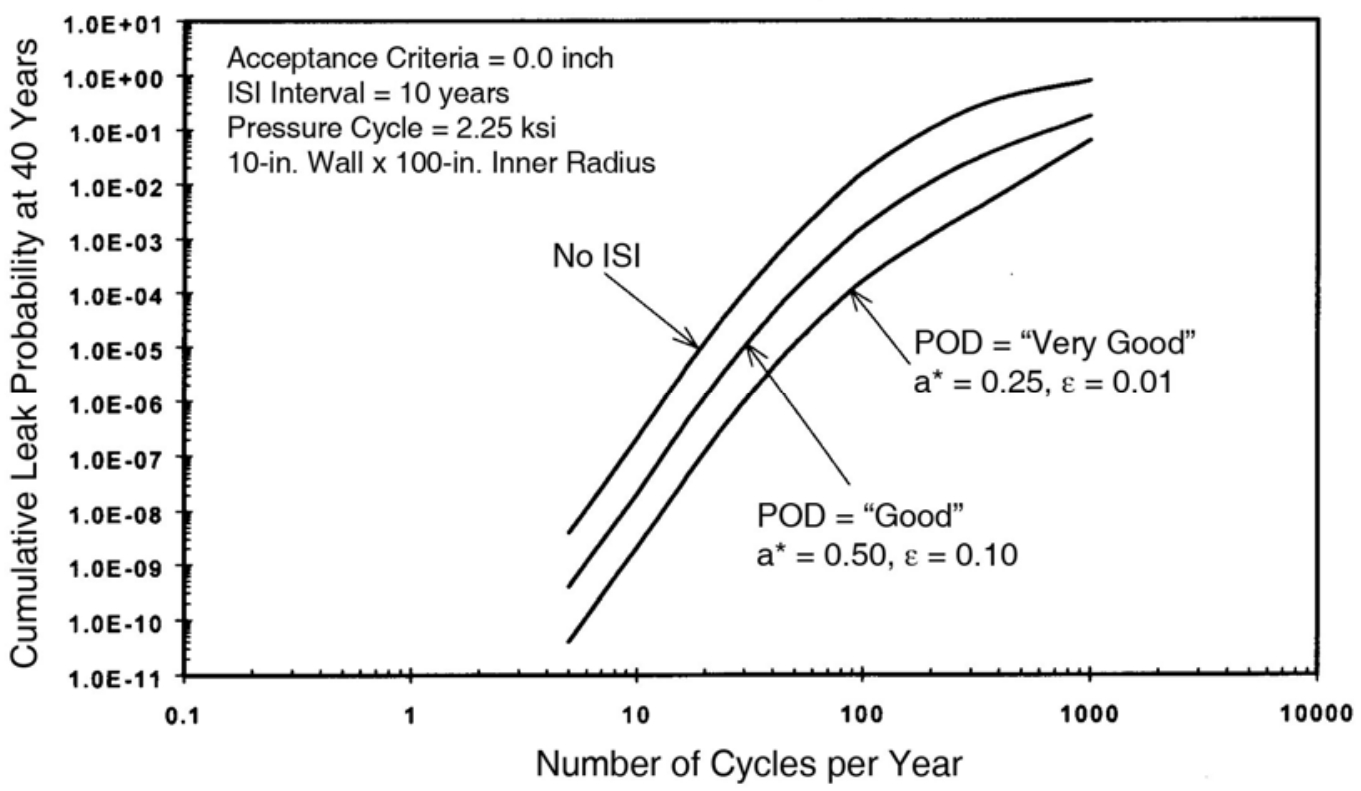

Figure 7.1 Vessel Leak Probability versus Cyclic Stress Frequency Assuming All Detected Flaws are Repaired (Surface Flaws) $(1 \mathrm{inch}=25.4 \mathrm{~mm}, 1 \mathrm{ksi}=6.9 \mathrm{MPa})$

Figures 7.2 to 7.4 address the effects of flaw-sizing errors and also the interactions of flaw-acceptance standards with the errors in flaw sizing. Figures 7.2 and 7.3 both address vessels with surface-breaking flaws, but assumed different levels of flaw-detection capability. It is seen that flaw-sizing errors are a relatively small factor relative to the flaw-detection capability. Flaw-sizing errors of up to \pm 1.5 in. $( \pm 38.1 \mathrm{~mm})$ can be accommodated. Even then, the effects of flaw-sizing errors are insignificant for the more restrictive values of flaw-acceptance standards, which are more typical of those specified by ASME Code Section XI.

Figure 7.4 presents results from other calculations that assumed that the flaws were randomly distributed through the thickness of the vessel wall. Leak probabilities for the buried flaws are much less than the corresponding probabilities for surface-breaking flaws. However, the effects of flaw-sizing errors and of flaw-acceptance standards are much the same as those for the surface-flaw calculations.

Figure 7.5 uses the parameter "Factor of Improvement" to present results from the calculations for inspection effectiveness. This parameter is defined as the ratio of failure probability for no inspection to the corresponding failure probability with inspection. This ratio in Figure 7.5 ranges from 1.0 (limiting case for totally ineffective inspections) to factors as large as 100 when the number of stress cycles per year approaches zero. The failure probabilities are very low for these cases, and the few failures that occur are associated with very deep flaws. It is seen that flaw-sizing errors are relatively unimportant for the two limiting cases of very low cyclic frequencies and very high cyclic frequencies. For high rates of stress cycling, there are many small undetectable flaws that grow to leakage between the 10-year inspections. Between the two extremes of very high and low cyclic frequencies, it is seen that flaw-sizing errors can have a more substantial effect on the calculated factors of improvement. 


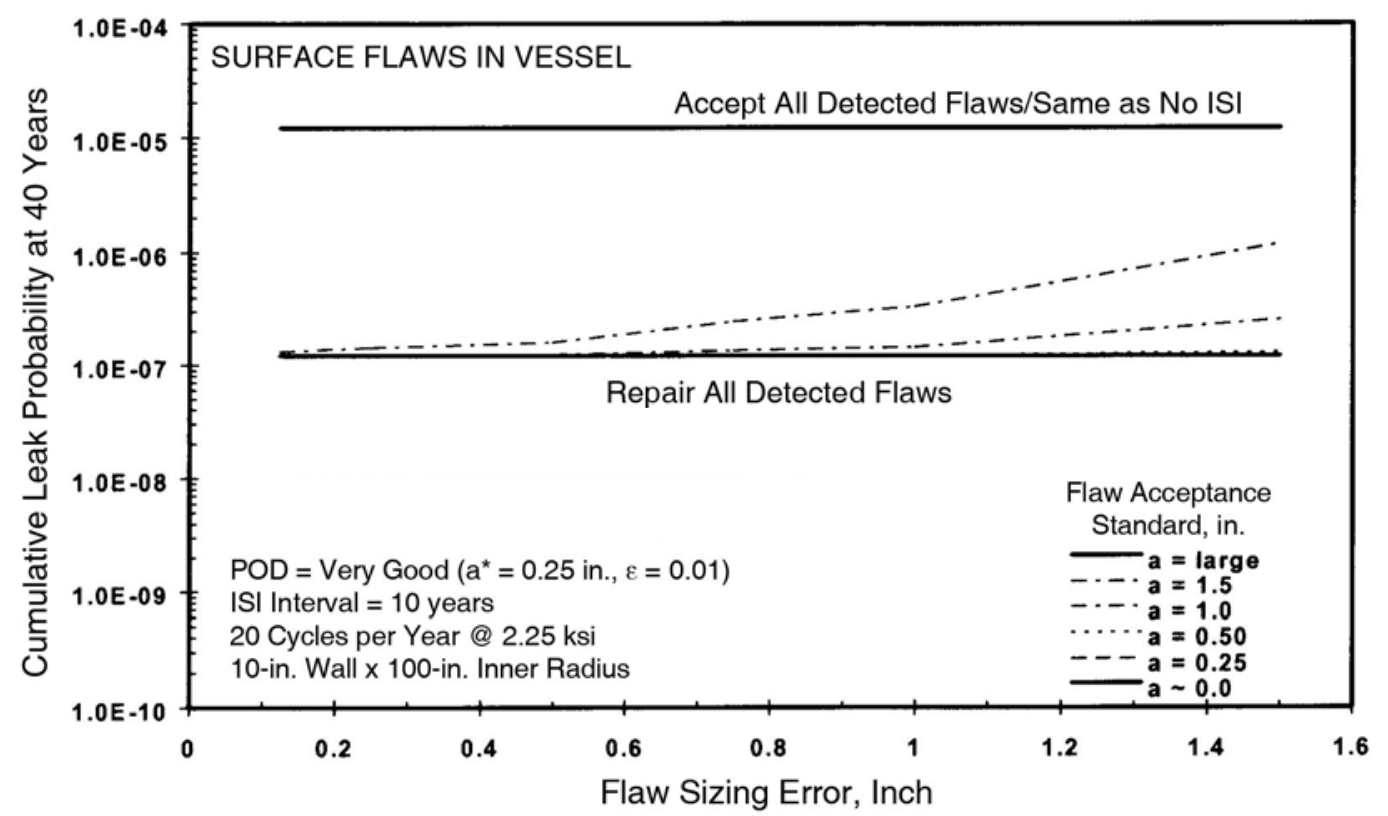

Figure 7.2 Effect of Flaw-Sizing Errors for Vessel Inspected with "Very Good" POD (Surface Flaws $)(1$ inch $=25.4 \mathrm{~mm}, 1 \mathrm{ksi}=6.9 \mathrm{MPa})$

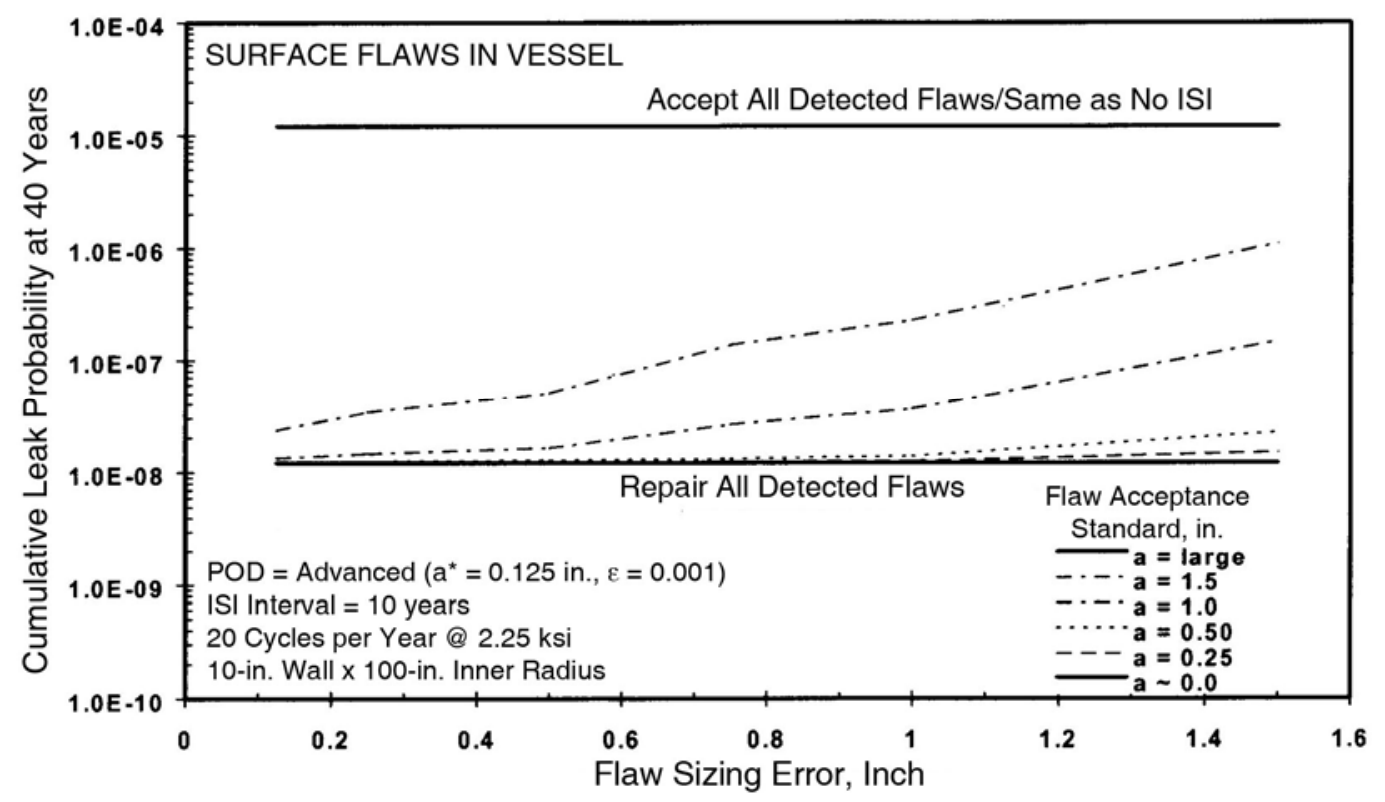

Figure 7.3 Effect of Flaw-Sizing Errors for Vessel Inspected with "Advanced" POD (Surface Flaws $)(1 \mathrm{inch}=25.4 \mathrm{~mm}, 1 \mathrm{ksi}=6.9 \mathrm{MPa})$ 


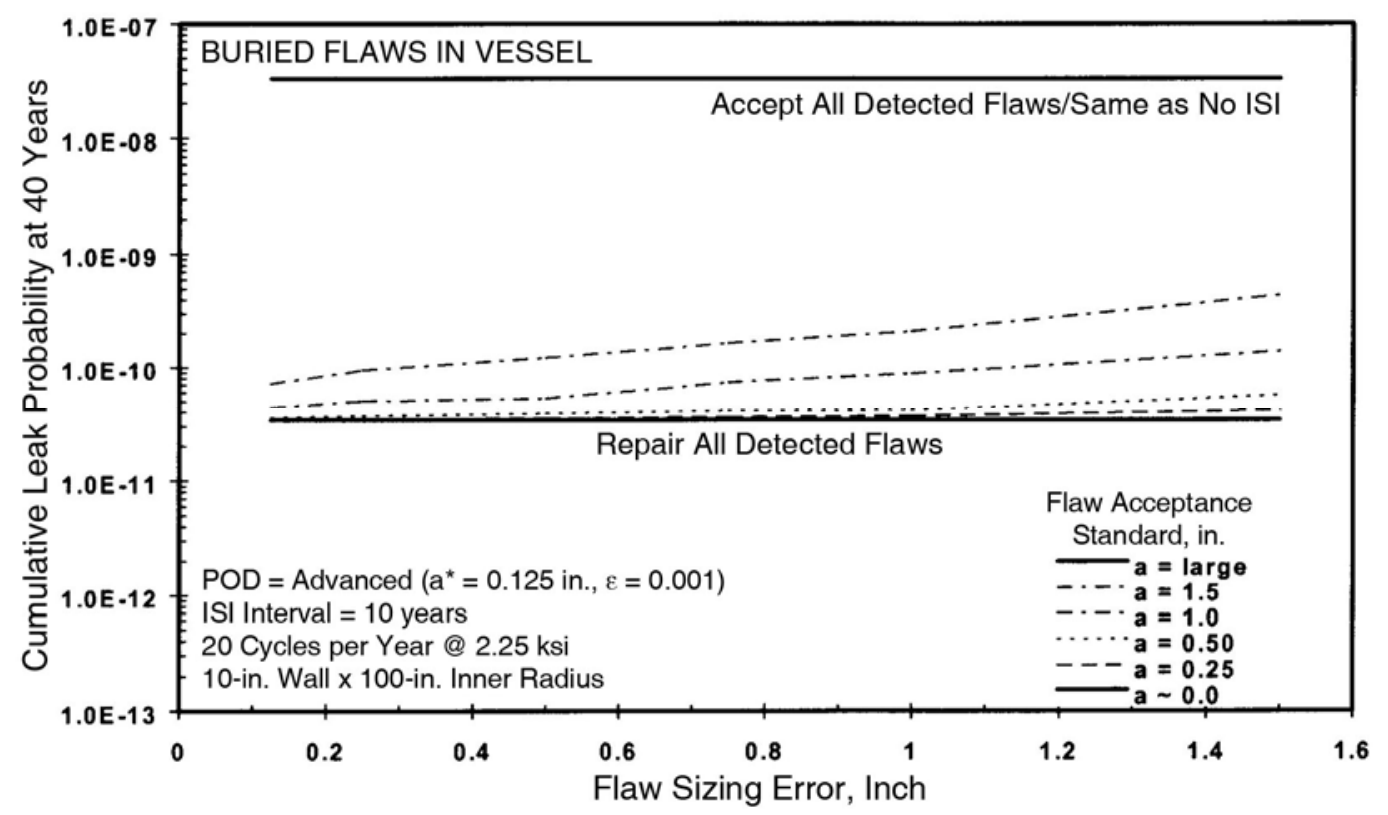

Figure 7.4 Effect of Flaw-Sizing Errors for Vessel Inspected with "Advanced" POD (Buried Flaws $)(1$ inch $=25.4 \mathrm{~mm}, 1 \mathrm{ksi}=6.9 \mathrm{MPa})$

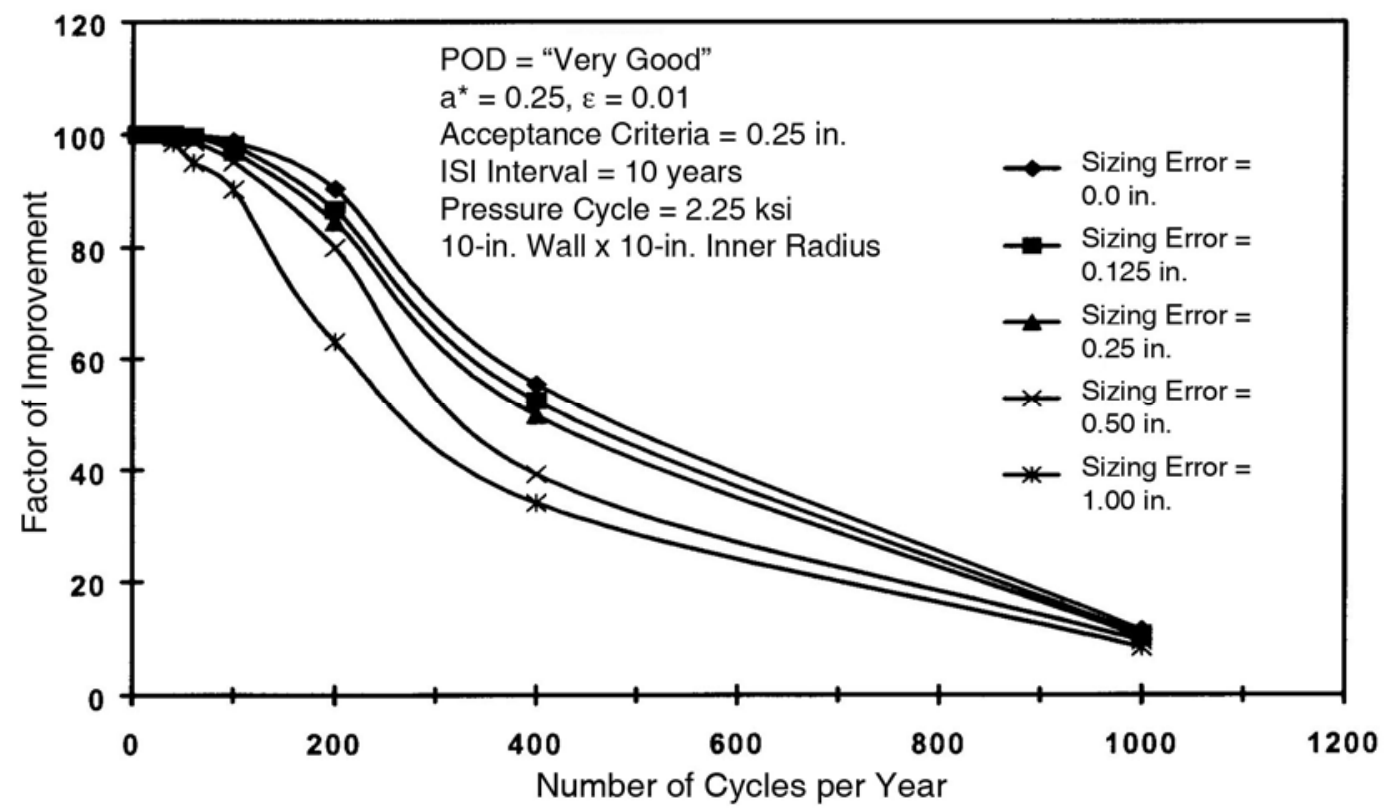

Figure 7.5 Factor of Improvement for Vessel Inspected with "Very Good" POD (Surface Flaws) $(1 \mathrm{inch}=25.4 \mathrm{~mm}, 1 \mathrm{ksi}=6.9 \mathrm{MPa})$ 


\subsubsection{Piping Calculations}

A second set of calculations was for a 6-in. $(152.4 \mathrm{~mm})$ Schedule 120 pipe with an inner radius of $2.75 \mathrm{in}$. (69.8 mm.) and a wall thickness of 0.562 in. $(14.3 \mathrm{~mm})$.

Figures 7.6 through 7.9 address various combinations of inspection scenarios, frequencies of stress cycling, and buried flaws versus surface-breaking flaws. Figures 7.6 through 7.8 address inner surfacebreaking flaws. The calculations indicate that flaw-sizing errors have little effect on the effectiveness of inspections unless the flaw-acceptance standards permit unrealistically large flaws (e.g., flaw depth of 50 percent of the pipe-wall thickness). The effect of sizing errors is somewhat greater for the higher frequency of stress cycling (200 cycles per year) of Figure 7.8, but again, significant effects are associated with unrealistically large values for flaw-sizing errors and acceptance standards.

Figure 7.9 suggests that effects of flaw-sizing errors may be somewhat more significant for buried flaws. However, given that the calculated leak probabilities for the buried flaws are extremely small (less than $1.0 \mathrm{E}-11$ ), any conclusions regarding flaw-sizing errors from these calculations will have little if any practical implications to piping reliability.

\subsection{Summary and Conclusions}

A PFM model was developed to simulate the effects of flaw-sizing errors that may occur during the inspection of vessels and piping. Calculations have been performed for a range of representative values of detection probabilities, flaw-sizing errors, and flaw-acceptance criteria. POD capability appears to be the most limiting factor with regard to the overall capability of ISIs to reduce leak probabilities. The effects of flaw-sizing errors are relatively small when calculations are based on inputs for flaw-sizing capabilities and acceptance standards which are representative of current NDE capabilities and code requirements. However, the calculations show that gross errors in flaw sizing or significant departures from current flaw-acceptance standards could negate the expected benefits of inspection methods that exhibit outstanding performance in the area of flaw detection. 


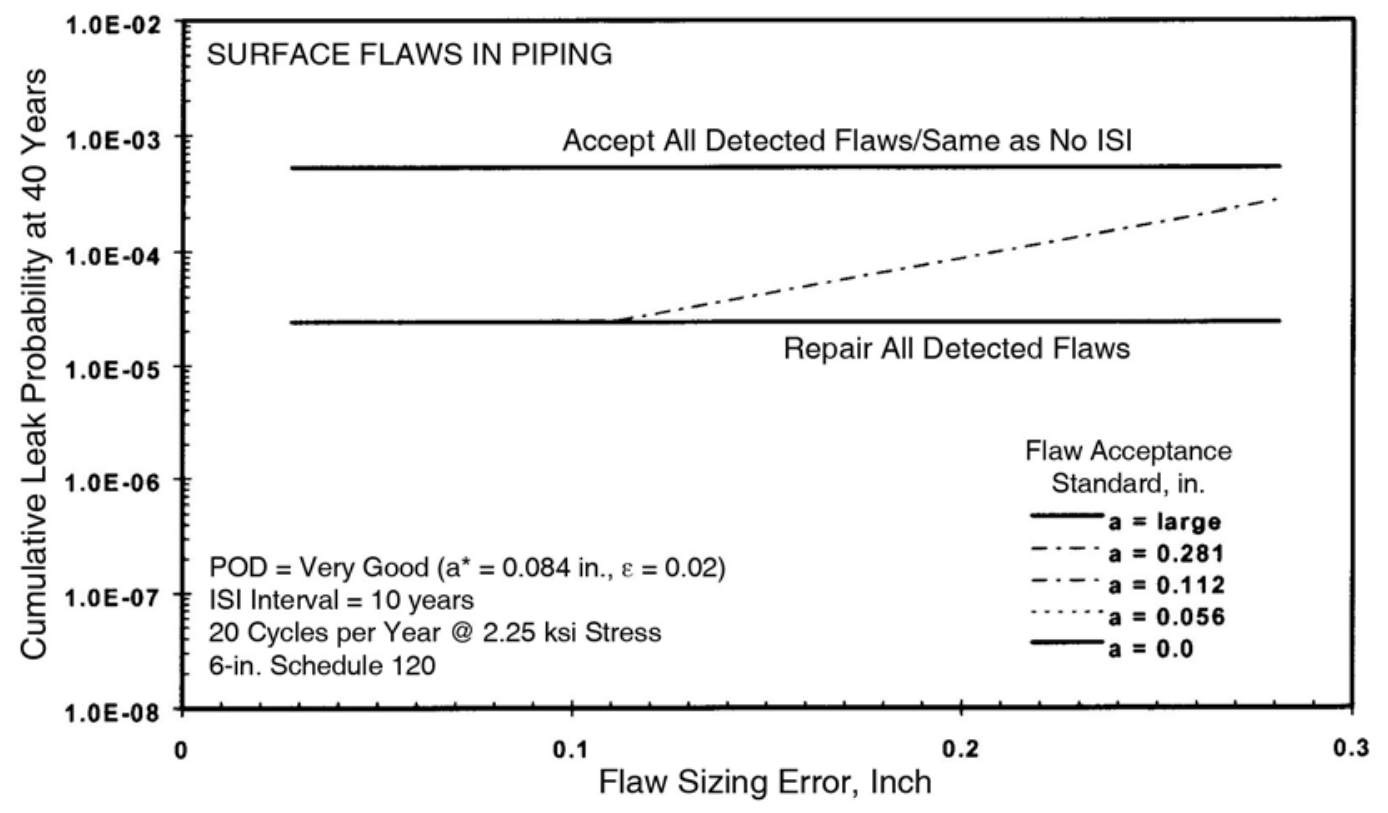

Figure 7.6 Effect of Flaw-Sizing Errors for Low Fatigue of Piping Inspected with "Very Good" POD (Surface Flaws) $(1$ inch $=25.4 \mathrm{~mm}, 1 \mathrm{ksi}=6.9 \mathrm{MPa})$

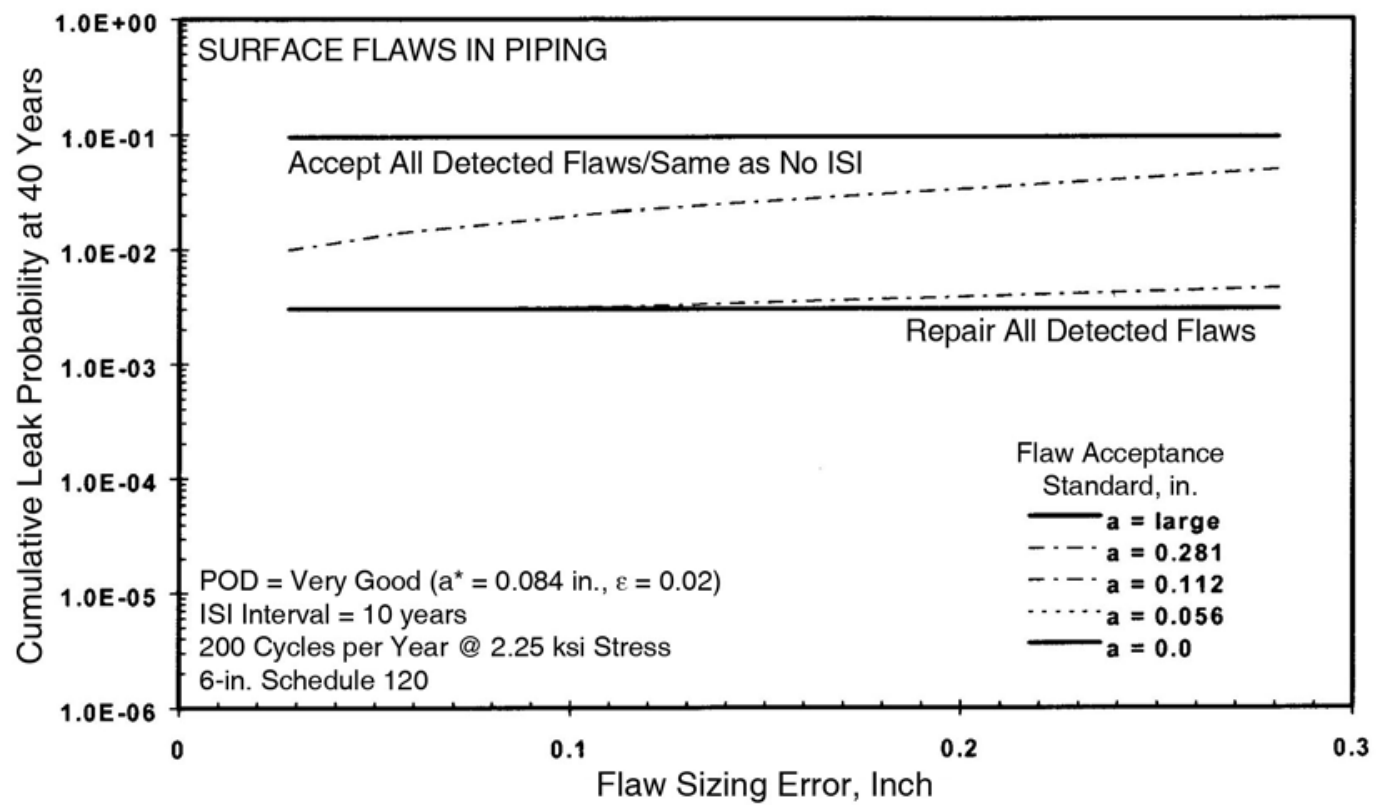

Figure 7.7 Effect of Flaw-Sizing Errors for High Fatigue of Piping Inspected with "Very Good" POD (Surface Flaws) $(1 \mathrm{inch}=25.4 \mathrm{~mm}, 1 \mathrm{ksi}=6.9 \mathrm{MPa})$ 


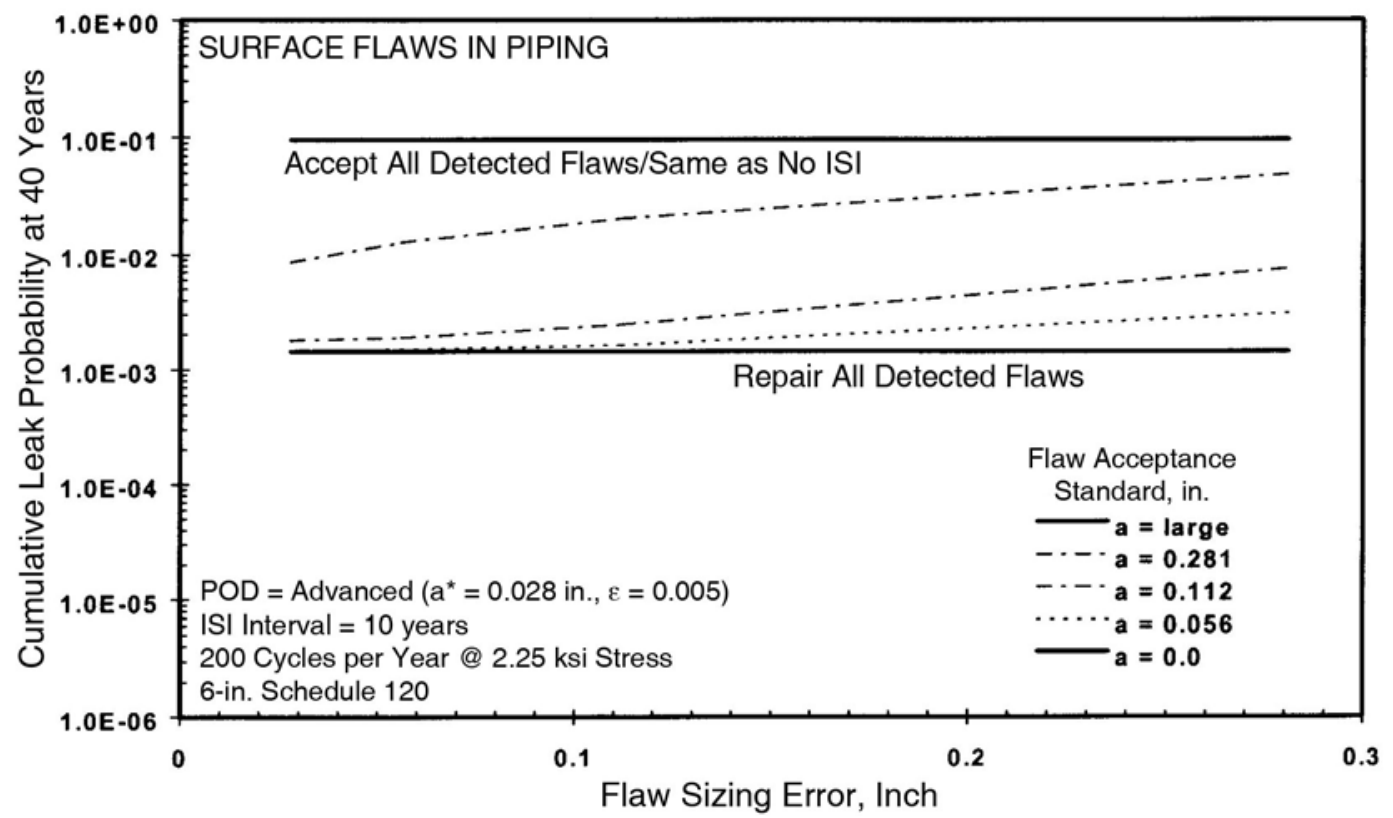

Figure 7.8 Effect of Flaw-Sizing Errors for High Fatigue of Piping Inspected with "Advanced" POD (Surface Flaws) $(1 \mathrm{inch}=25.4 \mathrm{~mm}, 1 \mathrm{ksi}=6.9 \mathrm{MPa})$

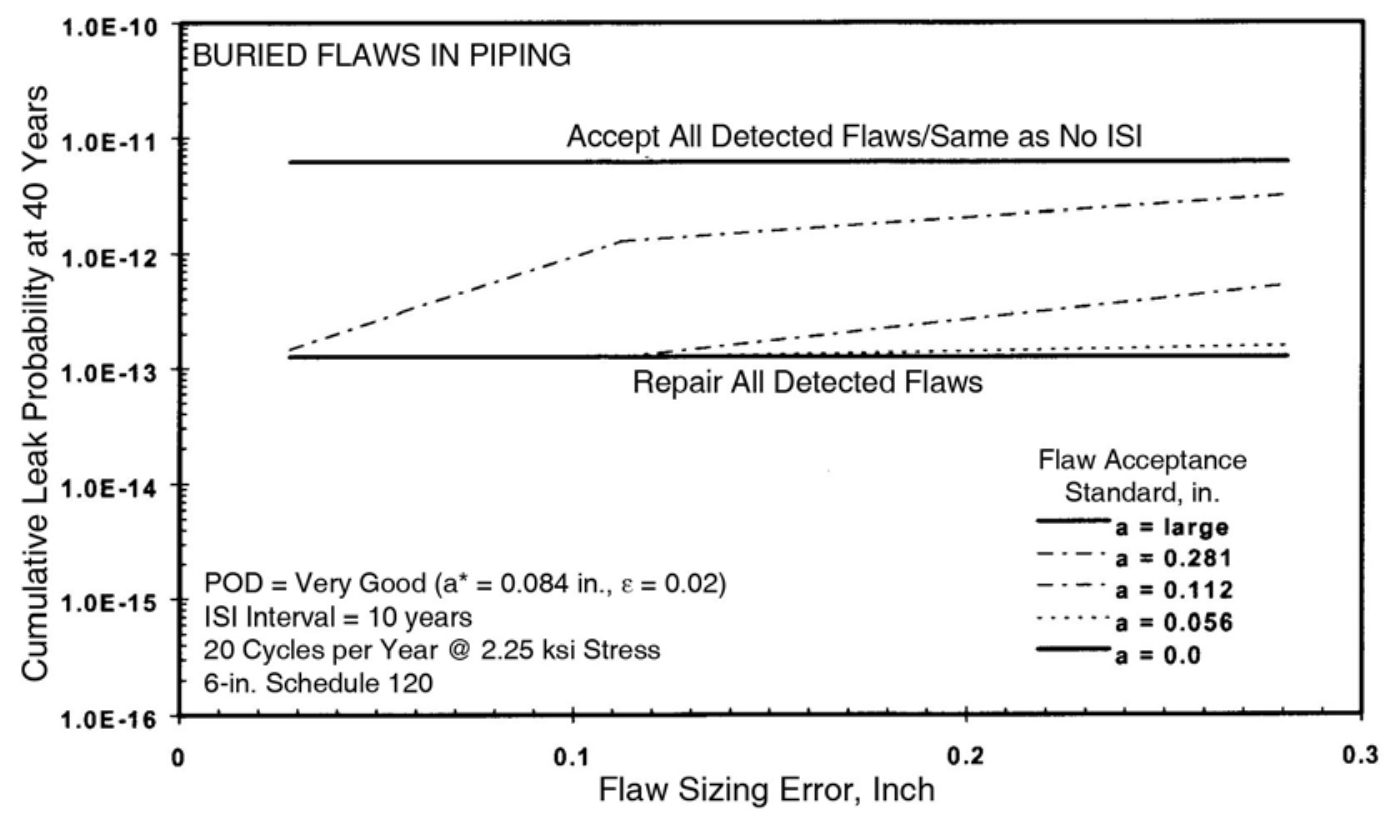

Figure 7.9 Effect of Flaw-Sizing Errors for Low Fatigue of Piping Inspected with "Very Good" POD (Buried Flaws) $(1$ inch $=25.4 \mathrm{~mm}, 1 \mathrm{ksi}=6.9 \mathrm{MPa})$ 


\section{Conclusions}

This report has described a large body of results that demonstrate the capabilities and benefits of probabilistic fracture mechanics calculations. This work has also shown many of the limitations and uncertainties associated with such calculations. Numerical results from the computational effort have supported both the U.S. Nuclear Regulatory Commission and the ASME Section XI Code in the development and implementation of risk-informed inservice inspection for operating nuclear power plants. Another application of the work is to support the development of improved non-destructive examination methods by relating improvements in piping and vessel reliability to improvement in NDE capabilities.

Specific results and conclusions of the present report are:

(1) A simulation model as described in Section 2 has been applied to estimate flaw densities and size distributions for welds in piping systems. Calculations with the model addressed a range of pipe sizes, piping materials, welding methods, and inspection practices. Based on these calculations, recommendations were developed for inputs for the number and sizes of welding fabrication flaws to be used in probabilistic fracture mechanics simulations.

(2) The probabilistic fracture mechanics calculations as described in Section 3 were performed using a structured parametric format in order to study the effects of important design and operating conditions on calculated piping failure probabilities for the damage mechanism of fatigue. Critical inputs included stresses from internal pressure, thermal expansion bending moments, through-wall thermal gradients, and low probability seismic and accident loadings. Other important inputs addressed pipe size, piping material, leak detection sensitivity, and inservice inspection programs as characterized by flaw detection probability and the periodic inspection frequency. The results of Section 3 can be applied to estimate piping failure frequencies without the need to perform detailed probabilistic fracture mechanics calculations.

(3) Additional fatigue calculations as described in Section 4 address stainless steel piping with a focus on inservice inspection programs. The results quantify the benefits of alternative inspection programs that use ultrasonic examinations of welds. The calculations cover a wide range of probability of detection capabilities as well as inservice inspection frequencies. It is shown that high-quality inservice inspections can significantly reduce leak and break probabilities, particularly if the inspections are performed relatively frequently. Results of the probabilistic fracture mechanics calculations are presented as a collection of plots that can be used to select inspection strategies consistent with the goals of risk-informed inservice inspection programs.

(4) Probabilistic fracture mechanics calculations as described in Section 5 also address failure probabilities for stainless steel piping but for the degradation mechanism of intergranular stress corrosion cracking. The fracture mechanics model accounts for material-related factors such as the degree of sensitization for weld locations. Other factors addressed by the model are related to operating conditions including residual stress, temperature, and environmental factors (e.g., oxygen level). Results of calculations are presented with a collection of plots that quantify reductions of failure probabilities as a function of NDE reliability (probability of detection curves) and inspection frequency. It is shown that significant reductions in failure probabilities require both a sensitive ultrasonic examination and a sufficiently small time interval between inspections. 
(5) The calculations of Section 6 quantify the level of uncertainty associated with calculated failure probabilities for piping components. These evaluations supported the development of the methodology for risk-informed inservice inspection programs. The calculations show large uncertainties in calculated failure probabilities that are particularly large when the failure probabilities are very low such as for pipe rupture frequencies. The greatest source of uncertainty was indentified as inputs for initial density and size distributions for fabrication related flaws.

(6) A final set of calculations as presented in Section 7 address the effects of flaw sizing errors on the reliability of vessels and piping systems. A probabilistic fracture mechanics model was developed to simulate the effects of flaw sizing errors along with both flaw detection capabilities and flawacceptance criteria. Inputs to the calculation were selected to represent current NDE capabilities and ASME Section XI Code flaw acceptance standards. The calculations showed that probability of detection is the most limiting factor with regard to the overall capability of an inservice inspection to reduce failure probabilities. The effects of flaw sizing errors are relatively small because of the conservative basis for flaw acceptance standards.

The scope of the present calculations was limited by the available computer codes for probabilistic fracture mechanics simulations. While the results of the calculations as presented in this report have wide-ranging applications, additional calculations can provide further benefits. Future advances in the capabilities of facture mechanics codes should permit more accurate calculations to be performed to address additional degradation mechanisms such as primary water stress corrosion cracking. Improvements are also expected in the capabilities of non-destructive examination methods and additional calculations will be useful to quantify the benefits of improved NDE expressed in terms of potential reductions in failure probabilities for vessel and piping components. 


\section{References}

Andresen PL and FP Ford. 1994. "Fundamental Modeling of Environment Cracking for Improved Design and Lifetime Evaluation in BWRs.” Int J Pres Ves Pip 59(1-3):61-70.

ASME/CRTD. 1992. Risk-Based Inspection - Development of Guidelines: Volume 2 - Part 1 Light Water Reactor (LWR) Nuclear Power Plant Components. CRTD-Vol. 20-2. Center for Research and Technology Development, American Society of Mechanical Engineers.

Balkey KR, NB Closky and RK Perdue. 1997. "Identifying Structural Elements and Examination Requirements in a Risk-Informed Inservice Inspection Program for Piping." In Risk-Informed Decision Making, PVP-Vol. 358, pp. 3-13. American Society of Mechanical Engineers, New York.

Bates DJ, SR Doctor, PG Heasler and E Burck. 1987. Stainless Steel Round Robin Test: Centrifugally Cast Stainless Steel Screening Phase. NUREG/CR-4970, PNL-6266, PISC III Report No. 3. U.S. Nuclear Regulatory Commission, Washington, D.C.

Becher PE and B Hansen. Undated. Statistical Evaluation of Defects in Welds and Design Implications. Danish Welding Institute, Danish Atomic Energy Commission, Research Establishments, RISO.

Berens AP and PW Hovey. 1984. Flaw Detection Reliability Criteria, Vol. 1, Method and Results. AFWALTR844022. Air Force Wright Aeronautical Laboratories, Wright Patterson Air Force Base, Ohio.

Bishop BA. 1997. “An Updated Structural Reliability Model for Piping Risk Informed ISI.” In Fatigue and Fracture - 1997, Volume 2, PVP Vol. 346, pp. 245-252. American Society of Mechanical Engineers, New York.

Bozarth D, CL Mason and JW Minarick. 1985. "Sensitivity and Uncertainty Analyses of Estimated Through Wall Crack Frequencies for H.B. Robinson Unit 2." In Pressurized Thermal Shock Evaluation of the H.B Robinson Unit 2 Nuclear Power Plant, Vol. 1. Ch. 7. U.S. Nuclear Regulatory Commission, Washington, D.C.

Bruckner-Foit A, T Schmidt and J Theodoropoulos. 1989. "A Comparison of the PRAISE Code and the PARIS Code for the Evaluation of Failure Probability of Crack-Containing Components." Nuclear Engineering and Design 110(3):395-411.

Burns ET, DO Harris and RC Erdmann. 1978. Reliability of Nuclear Piping Systems with Consideration of Cases Where Design Points Have Been Exceeded. Report SAI-091-79-PA. Science Applications Inc., Palo Alto, California. Presented at ASME/CSME Pressure Vessels and Piping Conference, Montreal, Canada.

Chang CM, IK Chen, HK Shee, HY Chen and JN Yang. 1994. "Xray Inspection Reliability for Welded Joints." In Structural Safety and Reliability: Proceedings of ICOSSAR '93, 6th International Conference, 9-13 August 1993, pp. 991-996. Innsbruck, Austria. eds: GI Schueller, M Shinozuka and JTP Yao. 
Chapman OJV. 1993. "Simulation of Defects in Weld Construction." In Reliability and Risk in Pressure Vessels and Piping, PVP-Vol. 251, pp. 81-89. American Society of Mechanical Engineers, New York.

Chapman OJV, MA Khaleel and FA Simonen. 1996. “A Simulation Model for Estimating Probabilities of Defects in Weld." In Fatigue and Fracture - 1996 - Volume 1, PVP-Vol. 323, pp. 375-391. American Society of Mechanical Engineers, New York.

Chapman OJV and FA Simonen. 1998. RR-PRODIGAL - A Model for Estimating the Probabilities of Defects in Reactor Pressure Vessel Welds. NUREG/CR-5505, PNNL-11898. U.S. Nuclear Regulatory Commission, Washington, D.C.

Cowfer CD. 1989. "Basis/Background for ASME Code Section XI Proposed Appendix VIII: Ultrasonic Examination Performance Demonstration.” In ASME Conference Publication, NDE-Vol. 5, pp. 1-5. American Society of Mechanical Engineers, New York.

Cramond Jr. R. 1974. A Probabilistic Analysis of Structural Reliability Against Fatigue and Failure. $\mathrm{PhD}$ Thesis, University of Illinois at Urbana, Champaign, Illinois.

Dickson TL. 1994. FAVOR: A Fracture Mechanics Analysis Code for Nuclear Reactor Pressure Vessels, Release 9401. ORNL/NRC/LTR/94/1. Martin Marietta Energy Systems, Inc., Oak Ridge National Laboratory, Oak Ridge, Tennessee.

Dickson TL, JA Keeney and JW Bryson. 1995. "Validation of FAVOR Code Linear Elastic Fracture Solutions for Finite-Length Flaw Geometries." In Fatigue and Fracture Mechanics in Pressure Vessels and Piping, PVP-Vol. 304, pp. 51-60. American Society of Mechanical Engineers, New York.

Doctor SR, FL Becker, PG Heasler and GP Selby. 1983. "Effectiveness of U.S. Inservice Inspection Technologies: A Round Robin Test." In Proceedings of a Specialist Meeting on Defect Detection and Sizing, Vol. 2 (CSNI Report No. 75 and EUR 9066 II EN), pp. 669-678.

EPRI. 1989. Accuracy of Ultrasonic Flaw Sizing Techniques for Reactor Pressure Vessels. NP-6273. Electric Power Research Institute, Palo Alto, California.

Frost NE and K Denton. 1967. "The Fatigue Strength of Butt Welded Joints in Low Alloy Structural Steel." British Welding Journal 14(4):156-161.

General Electric Company. 1982a. The Growth and Stability of Stress Corrosion Cracks in Large Diameter BWR Piping, Volume 1: Summary. EPRI NP-2472, Vol. 1. BWR Owners Groups and Electric Power Research Institute, Palo Alto, California.

General Electric Company. 1982b. The Growth and Stability of Stress Corrosion Cracks in Large Diameter BWR Piping, Volume 2: Appendixes. EPRI NP-2472, Vol. 2. BWR Owners Groups and Electric Power Research Institute, Palo Alto, California. 
Goldberg A, RD Streit and RG Scott. 1980. Evaluation of Cracking in Feedwater Piping Adjacent to the Steam Generators in Nine Pressurized Water Reactor Plant. UCRL-53000, NUREG/CR-1603.

Lawrence Livermore National Laboratory, Livermore, California.

Halmshaw R and CA Hunt. 1975. "Can Cracks Be Found by Radiography?” Brit J Nondestr Test May, pp. 71-75.

Harris DO. 1977. "Relative Rupture Probabilities at Various Locations in Reactor Coolant Piping." T Am Nucl Soc 26:404.

Harris DO. 1978. "Probabilistic Analysis of Rupture in Reactor Coolant Piping." In Proceedings of Topical Meeting, Probabilistic Analysis of Nuclear Reactor Safety, Vol. 2, Paper II.7. American Nuclear Society, Los Angeles, California.

Harris DO. 1981. "Stress Corrosion Crack Growth in the Presence of Residual Stresses." In Proceedings of the 28th Sagamore Army Materials Research Conference, Lake Placid, New York.

Harris DO and DD Dedhia. 1992. A Probabilistic Fracture Mechanics Code for Piping Reliability Analysis (pc-PRAISE Code). NUREG/CR-6237. U.S. Nuclear Regulatory Commission, Washington, D.C.

Harris DO, DD Dedhia and ED Eason. 1986a. "Probabilistic Analysis of Initiation and Growth of Stress Corrosion Cracks in BWR Piping." American Society of Mechanical Engineers, New York. ASME Paper 86-PVP-11.

Harris DO, DD Dedhia, ED Eason and SD Patterson. 1986b. Probability of Failure in BWR Reactor Coolant Piping: Probabilistic Treatment of Stress Corrosion Cracking in 304 and 316NG BWR Piping Weldments. NUREG/CR-4792, Vol. 3. U.S. Nuclear Regulatory Commission, Washington, D.C.

Harris DO, DD Dedhia and SC Lu. 1992. Theoretical and User's Manual for pc-PRAISE, A Probabilistic Fracture Mechanics Computer Code for Piping Reliability Analysis. NUREG/CR-5864. U.S. Nuclear Regulatory Commission, Washington, D.C.

Harris DO and RR Fullwood. 1976. An Analysis of the Relative Probability of Pipe Rupture at Various Locations in the Primary Cooling Loop of a Pressurized Water Reactor Including the Effects of a Periodic Inspection. Report SAI-001-PA. Science Applications, Inc., Palo Alto, California.

Harris DO and EY Lim. 1983. "Application of a Probabilistic Fracture Mechanics Model to the Influence of In-Service Inspection on Structure Reliability." In Probabilistic Fracture Mechanics and Fatigue Methods: Applications for Structural Design and Maintenance. eds: JM Bloom and JC Ekrall. American Society for Testing and Materials, West Conshohocken, Pennsylvania. STP 798. pp. 19-41.

Harris DO, EY Lim and DD Dedhia. 1981. Probability of Pipe Fracture in the Primary Coolant Loop of a PWR Plant Volume 5: Probabilistic Fracture Mechanics Analysis - Load Combination Program Project 1 Final Report. NUREG/CR-2189, Vol. 5. U.S. Nuclear Regulatory Commission, Washington, D.C. 
Hazelton W and WH Koo. 1988. Technical Report on Material Selection and Processing Guidelines for BWR Coolant Pressure Boundary Piping. NUREG-0313, Rev. 2. U.S. Nuclear Regulatory Commission, Washington, D.C.

Heasler PG and SR Doctor. 1996. Piping Inspection Round Robin. NUREG/CR-5068, PNNL-10475. U.S. Nuclear Regulatory Commission, Washington, D.C.

Heasler PG, TT Taylor, JC Spanner, SR Doctor and JD Deffenbaugh. 1990. Ultrasonic Inspection Reliability for Intergranular Stress Corrosion Cracks: A Round Robin Study of the Effects of Personnel, Procedures, Equipment and Crack Characteristics. NUREG/CR-4908, PNL-6179. U.S. Nuclear Regulatory Commission, Washington, D.C.

Khaleel MA, OJV Chapman, DO Harris and FA Simonen. 1999. "Flaw Size Distribution and Flaw Existence Frequencies in Nuclear Piping." In Probabilistic and Environmental Aspects of Fracture and Fatigue, PVP-Vol. 386, pp. 127-144. American Society of Mechanical Engineers, New York.

Khaleel MA and FA Simonen. 1994a. "The Effects of Initial Flaw Sizes and Inservice Inspection on Piping Reliability.” In Service Experience and Reliability Improvement: Nuclear, Fossil, and Petrochemical Plants, PVP-Vol. 288, pp. 95-07. American Society of Mechanical Engineers, New York.

Khaleel MA and FA Simonen. 1994b. "A Parametric Approach to Predicting the Effects of Fatigue on Piping Reliability.” In Service Experience and Reliability Improvement: Nuclear, Fossil, and Petrochemical Plants, PVP-Vol. 288, pp. 117-125. American Society of Mechanical Engineers, New York.

Khaleel MA and FA Simonen. 1995. "A Model for Predicting Vessel Failure Probabilities Due to Fatigue Crack Growth." In Fatigue and Fracture Mechanics in Pressure Vessels and Piping, PVPVol. 304, pp. 401-416. American Society of Mechanical Engineers, New York.

Khaleel MA and FA Simonen. 1997. "Reliability-Based Inspection Planning for Stainless Steel Piping Under Conditions of Intergranular Stress Corrosion Cracking." In Proceedings of ICOSSAR '97 International Conference on Structural Safety and Reliability, November 24-28, 1997, Kyoto, Japan.

Khaleel MA and FA Simonen. 1998. "A Probabilistic Model for Fatigue Crack Initiation and Propagation." In Fatigue, Fracture and Residual Stresses, PVP-373, pp. 27-34. American Society of Mechanical Engineers, New York.

Khaleel MA, FA Simonen, DO Harris and DD Dedhia. 1995. "The Impact of Inspection on Intergranular Stress Corrosion Cracking for Stainless Steel Piping." In Risk and Safety Assessments: Where Is the Balance?, PVP-Vol. 296, pp. 411-422. American Society of Mechanical Engineers, New York.

Marshall Committee. 1976. An Assessment of the Integrity of PWR Pressure Vessels. Report by a Study Group Chaired by Dr. W. Marshall, available from H.M. Stationary Office, London, October 1976. 
Marshall Committee. 1982. An Assessment of the Integrity of PWR Pressure Vessel. Second Report by a Study Group under the Chairmanship of Dr. W. Marshall, published by the UKAEA.

Nichols RW and S Crutzen, Eds. 1988. Ultrasonic Inspection of Heavy Section Steel Components: The PISC-II Final Report. Elsevier Applied Science Publishers, London and New York.

Packman PF, SJ Kilma, RL Davies, J Malpani, J Moyzis, W Walker, BGW Yee and DP Johnson. 1976. "Reliability of Flaw Detection by Nondestructive Inspection." In ASM Metals Handbook, Vol. 11, $8^{\text {th }}$ Ed., Metals Park, Ohio. pp. 214-224.

Parry GW. 1997. "Quality of PRA for Use in Regulatory Decision Making." In Risk Informed Decision Making, PVP-Vol. 358, pp. 81-85. American Society of Mechanical Engineers, New York.

Paul DD, N Ghadiali, J Ahmad and G Wilkowski. 1991. SQUIRT: Seepage Quantification of Upsets in Reactor Tubes. International Piping Integrity Research Group, Battelle, Columbus, Ohio.

Phillips JH, WS Roesener, HL Magleby, WG Reuter and V Geidel. 1991. Development and Demonstration of Methods to Include Aging of Passive Components in RAs. NUREG/CR-5730. U.S. Nuclear Regulatory Commission, Washington, D.C.

Shah NJ, DM Bucheit, A McNeill and EW Throckmorton. 1997. "Implementation of a Risk-Informed ISI Program at Surry Power Station.” In Risk-Formed Decision Making, PVP-Vol. 358, pp. 25-30. American Society of Mechanical Engineers, New York.

Simonen FA. 1990. "An Evaluation of the Impact of Inservice Inspection on Stress Corrosion Cracking of BWR Piping." In Codes and Standards and Applications for Design and Analysis of Pressure Vessel and Piping Components, PVP-Vol. 186, NDE-Vol. 7, pp. 187-193. American Society of Mechanical Engineers, New York.

Simonen FA and OJV Chapman. 1999. "Measured Versus Predicted Distributions of Flaws for Piping Welds." In Probabilistic and Environmental Aspects of Fracture and Fatigue, PVP-Vol. 386, pp. 101-103. August 1-5, 1999, Boston, Massachusetts. American Society of Mechanical Engineers, New York.

Simonen FA, DO Harris and DD Dedhia. 1998. "Effect of Leak Detection on Piping Failure Probabilities.” In Fatigue Fracture and Residual Stress, PVP-Vol. 373, pp. 105-113. American Society of Mechanical Engineers, New York.

Simonen FA, KI Johnson, AM Liebetrau, DW Engel and EP Simonen. 1986. VISA-II - A Computer Code for Predicting the Probability of Reactor Pressure Vessel Failure. NUREG/CR-4486. U.S. Nuclear Regulatory Commission, Washington, D.C.

Simonen FA and MA Khaleel. 1997. "Effects of Flaw Sizing Errors on the Reliability of Vessels and Piping." In Fatigue and Fracture, 1997, PVP-Vol. 346, pp. 233-243. American Society of Mechanical Engineers, New York. 
Simonen FA and MA Khaleel. 1998a. "On the Contribution of Buried Flaws to Piping Failure Probabilities." In Fatigue Fracture and Residual Stress, PVP-Vol. 373, pp. 69-75. American Society of Mechanical Engineers, New York.

Simonen FA and MA Khaleel. 1998b. "Uncertainty Analysis of Probabilistic Fracture Mechanics Calculations of Piping Failure Probabilities." In Proceeding of the 4th International Conference on Probabilistic Safety Assessment and Management, Probabilistic Safety Assessment and Management PSAM 4, Vol. 3, pp. 2040-2045. September 13-18, 1998, New York.

Simonen FA and HH Woo. 1984. Analyses of the Impact of Inservice Inspection Using a Piping Reliability Model. NUREG/CR-3869. U.S. Nuclear Regulatory Commission, Washington, D.C.

Taylor TT, JC Spanner, PG Heasler, SR Doctor and JD Deffenbaugh. 1989. "An Evaluation of Human Reliability in Ultrasonic Inservice Inspection for Intergranular Stress Corrosion Cracking Through Round Robin Testing." Mater Eval 47:338.

Thomas HM. 1979. "In-Service Inspection is Overrated.” J Inst Mech Eng 20(2):43-56.

USNRC. 1997. An Approach for Plant-Specific, Risk-Informed Decisionmaking: Inservice Inspection of Piping. Draft Regulatory Guide DG-1063. U.S. Nuclear Regulatory Commission, Washington, D.C.

Westinghouse Owners Group. 1997. Application of Risk-Informed Methods to Piping Inservice Inspection Topical Report. WCAP-14572, Rev. 1. Westinghouse Electric Co., Pittsburgh, Pennsylvania.

Wilkowski G, G Kramer, P Veith, R Francini and P Scott. 1994. "The Effect of Dynamic and Cyclic Loading During Ductile Tearing on Circumferentially Cracked Pipe: Analytical Results." In Fatigue, Flaw Evaluation and Leak Before Break Assessments, PVP-Vol. 280, pp. 221-239. American Society of Mechanical Engineers, New York.

Wilson AS. 1974. Estimating the Relative Probability of Pipe Severance by Fault Cause. GEAP-20615. General Electric Company, San Jose, California.

Woo HH and CH Chou. 1982. Piping Reliability Model Validation and Potential Use for Licensing Regulation Development. NUREG/CR-2801. U.S. Nuclear Regulatory Commission, Washington, D.C.

Woo HH and FA Simonen. 1984. "The Impact of Inservice Inspection on the Reliability of Nuclear Piping." In Advances in Probabilistic Fracture Mechanics, PVP-Vol. 92. American Society of Mechanical Engineers, New York. 
No. of

Copies

Nuclear Regulatory Commission

TL Chan

NRC/NRR

Mail Stop O-9 H6

SC Dinsmore

NRC/NRR

Mail Stop O-10 C15

BJ Elliot

NRC/NRR

Mail Stop O-9 H3

RO Hardies

NRC/RES

Mail Stop T-10 E10

MT Kirk

NRC/RES

Mail Stop T-10 E10

SNM Malik

NRC/RES

Mail Stop T-10 E10

KA Manoly

NRC/NRR

Mail Stop O-9 D3

MA Mitchell

NRC/NRR

Mail Stop O-9 H6

D Naujock

NRC/NRR

Mail Stop O-9 H6

WE Norris

NRC/RES

Mail Stop T-10 E10

CA Nove

NRC/NRR

Mail Stop O-9 H6

\section{Distribution}

No. of

Copies

IG Prokofiev

NRC/RES

Mail Stop T-10 M5

TJ Sullivan Jr.

NRC/NRR

Mail Stop O-9 H6

D Terao

NRC/NRO

Mail Stop T-7 F1

RL Tregoning

NRC/RES

Mail Stop T-10 M5

JL Uhle

NRC/RES

Mail Stop T-10 M5

\section{Offsite}

BR Bass

Oak Ridge National Laboratory MS 6085

PO Box 2008

Oak Ridge, TN 37831

BA Bishop

Westinghouse Nuclear Services

P.O. Box 158 (Bin 18) or

Exit 54 Interstate 70

Madison, PA 15663

TL Dickson

Oak Ridge National Laboratory

MS 6085

PO Box 2008

Oak Ridge, TN 37831

R. Gamble

Sartrex Corporation

1700 Rockville Pike, Suite 400

Rockville, MD 20852 
No. of

Copies

DO Harris

Structural Integrity Associates, Inc.

3315 Almaden Expressway

Suite 24

San Jose, CA 95118

ST Rosinski

EPRI NDE Center

1300 Harris Blvd.

Charlotte, NC 28262

DL Rudland

Engineering Mechanics Corporation of Columbus

3518 Riverside, Dr., Suite 202

Columbus, $\mathrm{OH} 43220$

PM Scott

Battelle Columbus

505 King Avenue

Columbus, $\mathrm{OH} 43201$

GP Selby

EPRI NDE Center

1300 Harris Blvd.

Charlotte, NC 28262

JC Spanner, Jr.

EPRI NDE Center

1300 Harris Blvd.

Charlotte, NC 28262
No. of

Copies

GM Wilkowski

Engineering Mechanics Corporation of Columbus

3518 Riverside, Dr., Suite 202

Columbus, $\mathrm{OH} 43220$

PT Williams

Oak Ridge National Laboratory

MS 6085

PO Box 2008

Oak Ridge, TN 37831

40 Internal Distribution

Pacific Northwest National Laboratory

P.O. Box 999

Richland, WA 99352

MT Anderson (28)

K5-25

A Ankrum

K6-66

SE Cumblidge

K5-25

AA Diaz

K5-25

SR Doctor

K5-25

PG Heasler

K6-08

M Khaleel

K9-80

AF Pardini

K5-25

FA Simonen (4)

K5-22

TT Taylor

K5-25

Information Release

PDF 